WATER-QUALITY DATA FROM CONTINUOUSLY MONITORED SITES IN THE PAMLICO

AND NEUSE RIVER ESTUARIES, NORTH CAROLINA, 1989-90

By Ronald G. Garrett and Jerad D. Bales

U.S. GEOLOGICAL SURVEY

Open-File Report 91-465
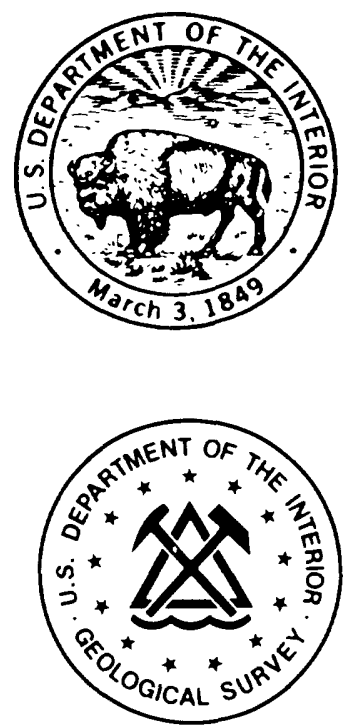

Prepared in cooperation with the

ALBEMARLE-PAMLICO ESTUARINE STUDY, NORTH CAROLINA DEPARTMENT OF ENVIRONMENT, HEALTH, AND NATURAL RESOURCES 


\section{U.S. DEPARTMENT OF THE INTERIOR}

MANUEL LUJAN, JR., Secretary

U.S . GEOLOGICAL SURVEY

Dallas L. Peck, Director

For additional information write to:

District Chief

U.S. Geological Survey

3916 Sunset Ridge Road

Raleigh, North Carolina 27607
Copies of this report can be purchased from:

U.S. Geological Survey Books and Open-File Reports Federal Center, Box 25425

Denver, Colorado 80225 
Abstract . . . . . . . . . . . . . . . . . . . . . . . 1

Introduction . . . . . . . . . . . . . . . . . . . . . . . . . 1

Purpose and scope. . . . . . . . . . . . . . . . . . . . . . . . 3

Acknowledgments . . . . . . . . . . . . . . . . . . . . . . 4

Estuaries and monitored sites. . . . . . . . . . . . . . . . . . . . 4

Pamlico River estuary . . . . . . . . . . . . . . . . . . . . 5

Neuse River estuary . . . . . . . . . . . . . . . . . . . . . . . 6

Data collection and processing . . . . . . . . . . . . . . . . . . . . 9

Instrumentation . . . . . . . . . . . . . . . . . . . . . . . . 9

Field procedures. . . . . . . . . . . . . . . . . . . . 15

Data processing . . . . . . . . . . . . . . . . . . . 16

Water-quality data . . . . . . . . . . . . . . . . . . . . . . 17

Box plots. . . . . . . . . . . . . . . . . . . . . . . . 17

Daily mean values . . . . . . . . . . . . . . . . . . . . . 18

References . . . . . . . . . . . . . . . . . . . . . . . . 149

\section{ILLUSTRATIONS}

Figure 1. Map showing location of Tar-Pamlico and Neuse River drainage basins. . . . . . . . . . . . . . . . . . . 5

2. Map showing location of continuous water-quality monitoring sites in the Pamlico River and Neuse River estuaries . . . 7

3-6. Box plots of salinity concentrations at the Pamlico River estuary monitoring sites for:

3. October 27-31, 1989... . . . . . . . . . . . . . . . 19

4. December $6-10,1989$. . . . . . . . . . . . . . . 20

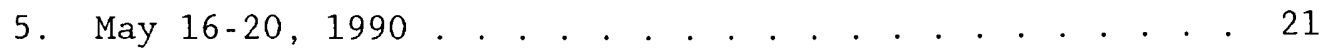

6. Ju1y 26-30, 1990. . . . . . . . . . . . . . . . . 22

7-10. Dissolved-oxygen concentrations at the Pamlico River estuary monitoring sites for:

7. November 21-25, 1989................. 23

8. January $16-20,1990$. . . . . . . . . . . . . . . . . 24

9. May 16-20, 1990 . . . . . . . . . . . . . . . . . . . 25

10. June $21-25,1990$. . . . . . . . . . . . . . . 26 
11-14. Salinity concentrations at the Neuse River estuary monitoring sites for:

11. September 1-5, 1989 . . . . . . . . . . . . . . . . 27

12. December 6-10, 1989 . . . . . . . . . . . . . . . . 28

13. May $26-30,1990$. . . . . . . . . . . . . . . . . 29

14. June $21-25,1990$. . . . . . . . . . . . . . . . . 30

15-18. Dissolved-oxygen concentrations at the Neuse River estuary monitoring sites for:

15. September 1-5, 1989 . . . . . . . . . . . . . . . . . . 31

16. December 6-10, 1989 . . . . . . . . . . . . . . . . 32

17. May 26-30, 1990 . . . . . . . . . . . . . . . . . . 33

18. June $21-25,1990$. . . . . . . . . . . . . . . . . 34

\section{TABLES}

Table 1. Continuous water-quality monitoring sites for salinity, water temperature, and dissolved oxygen . . . . . . . . . . 8

2. Maximum and minimum values recorded for salinity, water temperature, and dissolved oxygen in water at the water-quality monitoring sites, April 1989 to September 1990. . . . . . . . . . . . . . . . 10

3-13. Daily mean values of salinity, water temperature, and dissolved oxygen in water at:

3. Site 1, Pamlico River at Channel Light 16, April 1989 to September 1990 . . . . . . . . . . . . . 36

4. Site 2, Pamlico River at Light 5, April 1989 to September 1990 . . . . . . . . . . . . . 48

5. Site 3, Pamlico River at Light 4, April 1989 to September 1990 . . . . . . . . . . . . . 60

6. Site 4, Pamlico River at Light 3, May 1989 to September 1990 . . . . . . . . . . . . . 72

7. Site 5, Goose Creek at Light 6, May 1989 to September 1990 . . . . . . . . . . . . . . 84 
8. Site 6, Pungo River at Junction Light PR,

May 1989 to September 1990 . . . . . . . . . . . . . . 96

9. Site 7, Neuse River at Channel Light 22,

May to December 1989 . . . . . . . . . . . . . . 107

10. Site 8 , Neuse River at Channel Light 11 ,

May to December 1989 . . . . . . . . . . . . . . . . . 113

11. Site 9, Neuse River at Channel Light 9,

May 1989 to September 1990 . . . . . . . . . . . . . . 119

12. Site 10, Adams Creek at Light 4,

June 1989 to September 1990. . . . . . . . . . . . . . 131

13. Site 11, Neuse River at Junction Light,

May to September 1990. . . . . . . . . . . . . . 143 
CONVERSION FACTORS, VERTICAL DATUM, AND TEMPERATURE
Multiply
By
To obtain
Length
inch (in.)
25.4
foot ( $f t)$
0.3048
mile (mi)
1.609
millimeter meter
Area
square mile $\left(\mathrm{mi}^{2}\right)$
2.590
square kilometer
Mass per unit volume
grain per gallon (gr/gal)
0.05841
milligram per liter
Flow
cubic foot per second $\left(f t^{3} / \mathrm{s}\right)$
0.3048
meter per second
Specific Conductance
micromho per centimeter
1.000
microsiemen per centimeter
at 25 degrees Celsius
at 25 degrees Celsius
( $\mu \mathrm{mho} / \mathrm{cm}$ at $25^{\circ} \mathrm{C}$ )

Sea level: In this report, "sea level" refers to the National Geodetic Vertical Datum of 1929--a geodetic datum derived from a general adjustment of the first-order level nets of the United States and Canada, formerly called Sea Level Datum of 1929.

Temperature: In this report, atmospheric temperature is given in degrees Fahrenheit $\left({ }^{\circ} \mathrm{F}\right)$, which can be converted to degrees Celsius $\left({ }^{\circ} \mathrm{C}\right)$ by the following equation:

$$
{ }^{\circ} \mathrm{C}=5 / 9 \times\left({ }^{\circ} \mathrm{F}-32\right)
$$

Water temperature is given in degrees Celsius $\left({ }^{\circ} \mathrm{C}\right)$, which can be converted to degrees Fahrenheit $\left({ }^{\circ} \mathrm{F}\right)$ by the following equation:

$$
{ }^{\circ} \mathrm{F}=1.8\left({ }^{\circ} \mathrm{C}\right)+32
$$




\title{
WATER-QUALITY DATA FROM CONTINUOUSLY MONITORED SITES IN THE PAMLICO AND NEUSE RIVER ESTUARIES, NORTH CAROLINA, 1989-90
}

\author{
By Ronald G. Garrett and Jerad D. Bales
}

\begin{abstract}
Beginning in April 1989, water-quality measurements were made at six sites in or near the Pamlico River estuary and at five sites in or near the Neuse River estuary. Measurements taken at 15-minute intervals included near-surface and near-bottom specific conductance; near-surface water temperature; and near-surface, mid-depth, and near-bottom dissolved-oxygen concentrations.

Water-quality data obtained from continuously monitored sites in the Pamlico River estuary and the Neuse River estuary are presented for the period April 1989 through September 1990. Instantaneous values for selected periods are summarized in a series of box plots. Instantaneous maximum and minimum values are also tabulated. Daily mean values of salinity, water temperature, and dissolved-oxygen concentrations for the entire period are presented in tables and graphs.
\end{abstract}

\section{INTRODUCTION}

Estuarine environmental monitoring has been defined as "a continuing program of modeling, measurement, analysis, and synthesis that predicts and quantifies environmental conditions or contaminants and incorporates that information effectively into decision making in environmental management" (National Research Council, 1990). Monitoring data can provide information to (1) evaluate pollution abatement activities and control waste discharges, (2) measure trends in water quality, (3) detect new environmental problems in their early stages, potentially leading to lower-cost solutions, 
(4) evaluate human health hazards, and (5) determine the response of organisms to changes in habitat quality (National Academy of Sciences, 1977; Pearce and Despres-Patanjo, 1988; National Research Council, 1990).

The estuarine environment is extremely complex and highly variable, and it is often difficult to detect changes or to separate trends from natural variations. For example, salinity may be affected by tides, variable freshwater inputs, meteorological conditions, bathymetry, sea-level rise, and other factors, all of which are temporally and spatially variable. Moreover, there is a physical coupling between salinity and the flow field. Flows are generated by spatial salinity (density) gradients. The flow field, in turn, advects and mixes salt, and thereby affects the spatial salinity gradients. Salinity is also an important biological factor affecting species productivity and diversity, as well as perhaps the appearance of certain finfish diseases (Noga and Stanley, 1989).

Likewise, dissolved oxygen fluctuates in response to flows, to the salinity-controlled density field, to diurnal and vertical variations in community metabolism, and to variable water-quality conditions. Because vertical density gradients control the transport of oxygen through the water column, salinity distributions and dissolved-oxygen concentrations are closely related. Adequate dissolved-oxygen levels are critical to the biological health of the estuary.

Because of the complex and variable nature of estuaries, continuously recording water-quality instruments can play a key role in monitoring. For example, after evaluating data collected at bimonthly intervals, the Chesapeake Bay Program staff recognized the need for continuously monitored salinity, dissolved oxygen, and temperature data (Martin Marietta Environmental Systems, 1987a, 1987b). The recently initiated Environmental Monitoring and Assessment Program continuously monitored near-bottom dissolved oxygen during the summer of 1990 at 30 estuarine sites from Massachusetts to Virginia (Holland, 1990).

The Albemarle-Pamlico (A-P) Estuarine Study implemented an estuarine monitoring program in 1988 (Holman, 1988). The monitoring program includes an expanded ambient water-quality monitoring network, fish tissue analysis, 
sediment-oxygen demand studies, a citizens monitoring program, and a network of continuous water-quality monitors. The expanded ambient monitoring network and the continuous-monitoring network were designed to provide compatible data, and, in many locations, the ambient-and continuousmonitoring network sites coincide. Information from these and other biological and water-quality monitoring activities in the Sounds provides a comprehensive overview of the health of the estuarine system.

In 1988, the U.S. Geological Survey (USGS), in cooperation with the North Carolina Department of Environment, Health, and Natural Resources, initiated an investigation of water quality in the Pamlico and Neuse River estuaries. The investigation involves the continuous measurement of certain water-quality constituents and analysis of those data to (1) provide information to be used in conjunction with other A-P Study monitoring data to characterize baseline conditions in the two estuaries; (2) evaluate temporal trends after sufficient data are available; (3) characterize the extent, frequency, and duration of density stratification; (4) describe the dissolved-oxygen regime, including the relation of hypoxia to density stratification; and (5) provide data for hydrodynamic and water-quality modeling.

\section{Purpose and Scope}

The purpose of this report is to provide a summary of the continuously monitored water-quality data obtained in the Pamlico River and the Neuse River estuaries. This report covers the period from April 1989, when monitoring first began, through September 1990, which is the end of the first full water year ${ }^{1}$ of data. Water-quality data were measured at 15minute intervals at six sites in the Pamlico River estuary and at five sites in the Neuse River estuary. Measurements include near-surface and nearbottom specific conductance; near-surface water temperature; and nearsurface, mid-depth, and near-bottom dissolved-oxygen concentrations. Specific-conductance data were converted to and reported as salinity values. Basic statistics were used to present selected data as box plots, and instantaneous data are summarized as daily mean values in tables and graphs.

\footnotetext{
${ }^{1}$ Period extending from October 1 through the following September 30 . The water year is determined by the calendar year in which it ends.
} 


\section{Acknowledgments}

This investigation is being conducted in cooperation with the North Carolina Department of Environment, Health, and Natural Resources (EHNR), Albemarle-Pamlico Estuarine Study. Douglas N. Rader (formerly of EHNR) saw the urgent need for continuously monitored water-quality data and was helpful in initiating this study; Robert E. Holman (formerly of EHNR) and Randall G. Waite (EHNR) have helped ensure the continued operation of the monitoring network. Steve Tedder, Jimmie Overton, Dianne Reid, and Jay Sauber of the North Carolina Division of Environmental Management, and Mike Street and Jess Hawkins of the North Carolina Division of Marine Fisheries cooperated in the design of the water-quality monitoring network and have provided continuing support for the study. The U.S. Coast Guard, Fifth District, granted permission for use of existing channel markers to support data-collection instrumentation. The Coast Guard also assisted in instrument installation at some of the sites and participated in the recovery of instrumentation at downed channel markers.

\section{ESTUARIES AND MONITORED SITES}

The Pamlico River estuary and the Neuse River estuary lie within the Coastal Plain physiographic province of North Carolina (fig. 1). Much of the shoreline surrounding these two estuaries is composed of marshes, particularly near the mouths of the estuaries (Bellis and others, 1975; Copeland and others, 1984). Land-surface elevations in the area are generally less than 25 feet ( $f t$ ) above sea level. Streams which drain to the estuaries have small drainage basins with little topographic relief, low sediment loads, and fairly acidic waters (Copeland and others, 1984).

The climate of the region is moderately mild and humid. The annual mean temperature is more than $60^{\circ} \mathrm{F}$, and mean annual precipitation ranges from about 50 inches on the north side of the Pamlico River estuary to about 56 inches on the south side of the Neuse River estuary (Hardy and Hardy, 1971). Interannual variability in precipitation is large, ranging from 35 to 80 inches, but precipitation is relatively uniform throughout the year, with highest rainfall amounts typically occurring in July, August, and September. Evapotranspiration rates average about 34 inches per year and 
exhibit much less variability from year to year than precipitation (Wilder and others, 1978). Average wind speeds are about 10 miles per hour. Winds typically blow from south to southwest between April and August, and from north to northwest between september and February; there is no predominant prevailing wind direction during March.

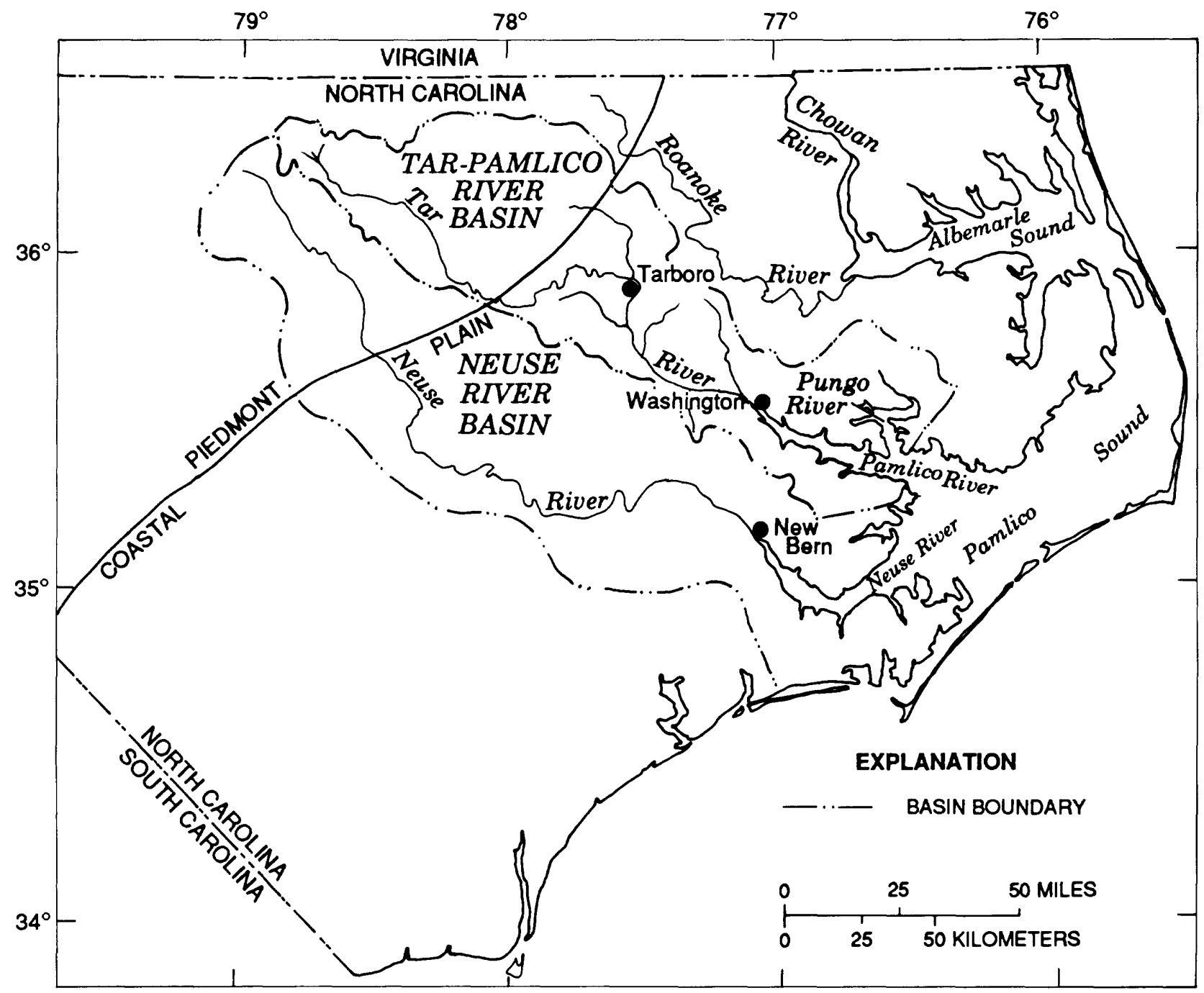

Figure 1.--Location of Tar-Pamlico and Neuse River drainage basins.

\section{Pamlico River Estuary}

Upstream from Washington, the Pamlico River is known as the Tar River and drains a 3,080 square mile $\left(\mathrm{mi}^{2}\right)$ rural area in the Piedmont and Coastal Plain (fig. 1). Downstream from Washington, the Pamlico River estuary 
drains an additional area of $1,220 \mathrm{mi}^{2}$ near the confluence of the Pamlico and Pungo Rivers, giving a total drainage area of 4,300 $\mathrm{mi}^{2}$ for the Tar and Pam1ico basins.

The distance from Washington to the confluence of the Pamlico and Pungo Rivers is about 30 miles ( $\mathrm{mi}$ ). The Pamlico River estuary width increases from about $1,000 \mathrm{ft}$ at Washington to more than $4 \mathrm{mi}$ at the Pamlico-Pungo confluence. Maximum depths range from about $10 \mathrm{ft}$ at Washington to about 18 ft at the mouth. The bottom material near Washington is primarily organicrich mud; in the lower reaches of the estuary, fine-grained materials occur mostly along the channel axis, and sand predominates near the shoreline (We11s, 1989).

Annual average inflow to the Pamlico River estuary at Washington has been estimated to be 3,140 cubic feet per second ( $\mathrm{ft}^{3} / \mathrm{s}$ ) (Harned and Davenport, 1990). Average annual runoff for the $1,220 \mathrm{mi}^{2}$ drainage area downstream from Washington is estimated to be $2,260 \mathrm{ft}^{3} / \mathrm{s}$. Average daily water-level range is $1.0 \mathrm{ft}$ at Washington and $0.6 \mathrm{ft}$ near the mouth of the Pamlico River (Bales, 1990).

Four water-quality monitoring stations were established on channel markers in the Pamlico River estuary; two additional stations were placed at the mouths of Pungo River and Goose Creek, respectively, which flow into the Pam1ico River (fig. 2). Water depth at the monitoring stations ranges from $11 \mathrm{ft}$ at sites 1 and 4 , to $15 \mathrm{ft}$ at site 2 (table 1). Salinities generally ranged from near zero to about 20 parts per thousand (ppt); however, unusually high salinities (up to about 51 ppt, table 2) were observed at site 5 on July 11,1990 . Instrument readings and calibration records were closely checked to verify these high values. Recorded water temperatures in the Pamlico River were between $0^{\circ} \mathrm{C}$ and $33^{\circ} \mathrm{C}$ during the measurement period. Dissolved-oxygen concentrations from less than 1 milligram per liter (mg/L) to $19 \mathrm{mg} / \mathrm{L}$ were recorded (table 2).

\section{Neuse River Estuary}

Like the Tar River, the Neuse River originates in the Piedmont province of North Carolina (fig. 1) and drains upstream from New Bern, a 4,470 $\mathrm{mi}^{2}$ 


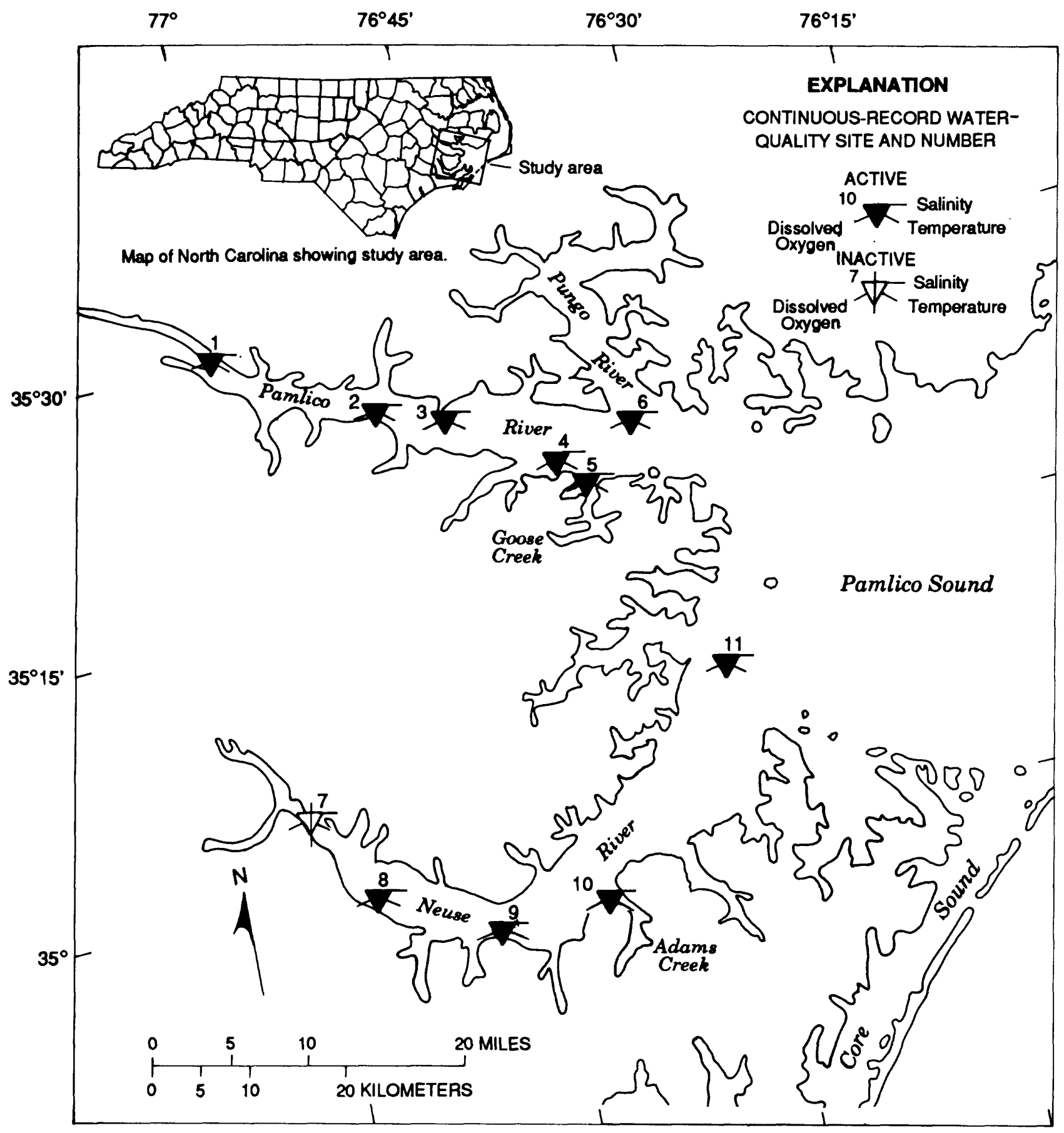

Figure 2.--Location of continuous water-quality monitoring sites in the Pamlico River and Neuse River estuaries. 
area characterized by several large urban areas and widespread agriculture. Downstream from New Bern, an additional 1,130 $\mathrm{mi}^{2}$ drain to the Neuse River estuary giving a total drainage area of $5,600 \mathrm{mi}^{2}$ near site 11 , Neuse River at Junction Light (fig. 2).

Table 1.--Continuous water-quality monitoring sites for salinity, water temperature, and dissolved oxygen

\begin{tabular}{|c|c|c|c|c|c|}
\hline 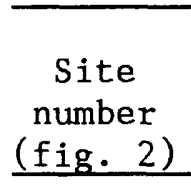 & Location & Latitude & Longitude & $\begin{array}{l}\text { Mean } \\
\text { water } \\
\text { depth } \\
\text { (feet) }\end{array}$ & $\begin{array}{l}\text { Period } \\
\text { of } \\
\text { record }\end{array}$ \\
\hline 1 & $\begin{array}{l}\text { Pamlico River at } \\
\text { Channel Light } 16\end{array}$ & $35^{\circ} 30^{\prime} 30^{\prime \prime}$ & $77^{\circ} 01^{\prime} 12^{\prime \prime}$ & 11 & $04 / 89-09 / 90$ \\
\hline 2 & $\begin{array}{l}\text { Pamlico River at } \\
\text { Light } 5\end{array}$ & $35^{\circ} 25^{\prime} 48^{\prime \prime}$ & $76^{\circ} 50^{\prime} 30^{\prime \prime}$ & 15 & $04 / 89-09 / 90$ \\
\hline 3 & $\begin{array}{l}\text { Pamlico River at } \\
\text { Light } 4\end{array}$ & $35^{\circ} 24^{\prime} 47^{\prime \prime}$ & $76^{\circ} 45^{\prime} 52^{\prime \prime}$ & 14 & $04 / 89-09 / 90$ \\
\hline 4 & $\begin{array}{l}\text { Pamlico River at } \\
\text { Light } 3\end{array}$ & $35^{\circ} 21^{\prime} 24^{\prime \prime}$ & $76^{\circ} 38^{\prime} 48^{\prime \prime}$ & 11 & $05 / 89-09 / 90$ \\
\hline 5 & $\begin{array}{l}\text { Goose Creek at } \\
\text { Light } 6\end{array}$ & $35^{\circ} 19^{\prime} 54^{\prime \prime}$ & $76^{\circ} 37^{\prime} 06^{\prime \prime}$ & 12 & $05 / 89-09 / 90$ \\
\hline 6 & $\begin{array}{l}\text { Pungo River at } \\
\text { Junction Light PR }\end{array}$ & $35^{\circ} 22^{\prime} 42^{\prime \prime}$ & $76^{\circ} 33^{\prime} 24^{\prime \prime}$ & 12 & $05 / 89-09 / 90$ \\
\hline 7 & $\begin{array}{l}\text { Neuse River at } \\
\text { Channe1 Light } 22\end{array}$ & $35^{\circ} 04^{\prime} 48^{\prime \prime}$ & $77^{\circ} 00^{\prime} 24^{\prime \prime}$ & 15 & $05 / 89-12 / 89$ \\
\hline 8 & $\begin{array}{l}\text { Neuse River at } \\
\text { Channel Light } 11\end{array}$ & $34^{\circ} 59^{\prime} 56^{\prime \prime}$ & $76^{\circ} 56^{\prime} 36^{\prime \prime}$ & 13 & $05 / 89-12 / 89$ \\
\hline 9 & $\begin{array}{l}\text { Neuse River at } \\
\text { Channel Light } 9\end{array}$ & $34^{\circ} 56^{\prime} 54^{\prime \prime}$ & $76^{\circ} 48^{\prime} 36^{\prime \prime}$ & 11 & $05 / 89-09 / 90$ \\
\hline 10 & $\begin{array}{l}\text { Adams Creek at } \\
\text { Light } 4\end{array}$ & $34^{\circ} 57^{\prime} 24^{\prime \prime}$ & $76^{\circ} 40^{\prime} 54^{\prime \prime}$ & 8 & $06 / 89-09 / 90$ \\
\hline 11 & $\begin{array}{l}\text { Neuse River at } \\
\text { Junction Light }\end{array}$ & $35^{\circ} 08^{\prime} 42^{\prime \prime}$ & $76^{\circ} 30^{\prime} 06^{\prime \prime}$ & 17 & $05 / 90-09 / 90$ \\
\hline
\end{tabular}

The Neuse River estuary varies in width from $0.9 \mathrm{mi}$ at New Bern to about $6.3 \mathrm{mi}$ at the mouth. Average depths range from $8 \mathrm{ft}$ at New Bern to 17 ft at the mouth (Giese and others, 1985). The bottom material composition of the Neuse River estuary is similar to that of the Pamlico River estuary, where fine-grained materials predominate along the channel axis and sand generally occurs near the shoreline (Wells, 1989). 
Annual average inflow to the Neuse River estuary at New Bern is about 4,910 $\mathrm{ft}^{3} / \mathrm{s}$ (Harned and Davenport, 1990). Giese and others (1985) estimated that the average freshwater outflow from the entire Neuse River basin is about $6,500 \mathrm{ft}^{3} / \mathrm{s}$, meaning that the annual average freshwater inflow from lands adjacent to the estuary is about $1,600 \mathrm{ft}^{3} / \mathrm{s}$. The daily mean waterlevel range is $1.0 \mathrm{ft}$ at New Bern and $0.6 \mathrm{ft}$ near site 10 (fig. 2).

Four water-quality monitoring stations are located along an approximately $35-\mathrm{mi}$ reach in the Neuse River estuary; one additional station was located at the mouth of Adams Creek, a tributary to the Neuse River (fig. 2). Site 7 was in operation from May 1989 until December 1989, when its channel marker platform was destroyed by ice. The multi-pile channel marker which was destroyed was replaced by a single-pile marker, so it was not possible to reinstall the data-collection instrumentation. The channel marker at site 8 was also destroyed by ice in December 1989, but a multipile marker was reconstructed and the data-collection instrumentation was reactivated in November 1990.

Between May 1989 and September 1990, salinities ranged from less than 0.1 ppt to nearly $33 \mathrm{ppt}$. During the same period, recorded water temperatures in the Neuse River estuary were between $0^{\circ} \mathrm{C}$ and $33^{\circ} \mathrm{C}$. Dissolved-oxygen concentrations of less than $1 \mathrm{mg} / \mathrm{L}$ to $20 \mathrm{mg} / \mathrm{L}$ were recorded (table 2).

\section{DATA COLLECTION AND PROCESSING}

Standard U.S. Geological Survey procedures for the collection and analysis of water-quality data were followed. Procedures for servicing data-collection instrumentation and for processing the data were also developed for the specific instrumentation and conditions of this study. The description and function of the instruments used to collect data, as well as field and data-processing procedures, are outlined in this section.

\section{Instrumentation}

The central component of the data-collection system is the U.S. Geological Survey minimonitor (Gordon and Katzenbach, 1983; Ficken and Scott, 1989). The minimonitor consists of (1) a water-tight can (about 
Table 2.--Maximum and minimum values recorded for salinity, water-quality monitoring sites,

[ppt, parts per thousand; ${ }^{\circ} \mathrm{C}$, degrees Celsius;

\begin{tabular}{|c|c|c|c|c|c|}
\hline \multirow{3}{*}{$\begin{array}{l}\text { Site } \\
\text { number } \\
(\text { fig. 2) }\end{array}$} & \multirow{3}{*}{$\begin{array}{c}\text { Station name } \\
\text { and } \\
\text { number } 1 / \\
\text { (Period of record) }\end{array}$} & \multicolumn{4}{|c|}{ Constituent } \\
\hline & & \multicolumn{2}{|c|}{$\begin{array}{l}\text { Salinity, } \\
\text { near } \\
\text { surface } \\
\text { (ppt) } \\
\end{array}$} & \multicolumn{2}{|c|}{$\begin{array}{l}\text { Salinity, } \\
\text { near } \\
\text { bottom } \\
\text { (ppt) } \\
\end{array}$} \\
\hline & & $\begin{array}{l}\text { Maximum } \\
\text { (Date) }\end{array}$ & $\begin{array}{c}\text { Minimum } \\
\text { (Date) }\end{array}$ & $\begin{array}{l}\text { Maximum } \\
\text { (Date) }\end{array}$ & $\begin{array}{c}\text { Minimum } \\
\text { (Date) }\end{array}$ \\
\hline 1 & $\begin{array}{l}\text { Pamlico River at Channel } \\
\text { Light } 16,0208450705 \\
(4 / 89-9 / 90)\end{array}$ & $\begin{array}{c}5.1 \\
(9 / 29 / 90)\end{array}$ & $\begin{array}{l}\quad<0.1 \\
(7 / 20-26 / 89 ; \\
8 / 21,23 / 89 ; \\
12 / 6,15,17- \\
22 / 89 ; \\
4 / 27-30 / 90 ; \\
\text { many days in } \\
\text { May and June } \\
1990 ; \\
7 / 10 / 90 ; \\
8 / 16 / 90)\end{array}$ & $\begin{array}{c}11.0 \\
(8 / 1 / 89)\end{array}$ & $\begin{array}{l}\quad<0.1 \\
\text { (many } \\
\text { days in } \\
\text { June, July, } \\
\text { November, and } \\
\text { December 1989; } \\
\text { January, } \\
\text { April, May, } \\
\text { and June } \\
\text { 1990) }\end{array}$ \\
\hline 2 & $\begin{array}{l}\text { Pamlico River at Light } 5 \text {, } \\
0208453300 \\
(4 / 89-9 / 90)\end{array}$ & $\begin{array}{c}10.5 \\
(8 / 14 / 90)\end{array}$ & $\begin{array}{c}0.10 \\
(3 / 18,21 / 90)\end{array}$ & $\begin{array}{c}16.7 \\
(8 / 24 / 89)\end{array}$ & $\begin{array}{c}<0.1 \\
(4 / 12 / 90)\end{array}$ \\
\hline 3 & $\begin{array}{l}\text { Pam1ico River at Light } 4 \text {, } \\
0208454253 \\
(4 / 89-9 / 90)\end{array}$ & $\begin{array}{c}10.0 \\
(8 / 28 / 90)\end{array}$ & $\begin{array}{c}<0.1 \\
(5 / 6 / 89)\end{array}$ & $\begin{array}{c}14.1 \\
(8 / 30 / 90)\end{array}$ & $\begin{array}{c}0.4 \\
(4 / 21 / 90)\end{array}$ \\
\hline 4 & $\begin{array}{l}\text { Pamlico River at Light } 3 \text {, } \\
0208455155 \\
(5 / 89-9 / 90)\end{array}$ & $\begin{array}{l}11.2 \\
(9 / 4 / 89 \\
9 / 1 / 90)\end{array}$ & $\begin{array}{c}0.9 \\
(5 / 17 / 89)\end{array}$ & $\begin{array}{c}13.1 \\
(8 / 26 / 90)\end{array}$ & $\begin{array}{c}1.7 \\
(5 / 14,16 / 89)\end{array}$ \\
\hline 5 & $\begin{array}{l}\text { Goose Creek at Light } 6 \text {, } \\
0208455615 \\
(5 / 89-9 / 90)\end{array}$ & $\begin{array}{c}14.4 \\
(8 / 6 / 90)\end{array}$ & $\begin{array}{c}2.1 \\
(3 / 13 / 90)\end{array}$ & $\begin{array}{c}50.7 \\
(7 / 11 / 90)\end{array}$ & $\begin{array}{c}2.9 \\
(6 / 14 / 89)\end{array}$ \\
\hline
\end{tabular}

\footnotetext{
1/U.S. Geological Survey downstream order number.
} 


\begin{tabular}{|c|c|c|c|c|c|c|c|}
\hline \multicolumn{8}{|c|}{ Constituent--Continued } \\
\hline \multicolumn{2}{|c|}{$\begin{array}{c}\text { Water } \\
\text { temperature, } \\
\text { near } \\
\text { surface } \\
\left({ }^{\circ} \mathrm{C}\right) \\
\end{array}$} & \multicolumn{2}{|c|}{$\begin{array}{c}\text { Dissolved oxygen, } \\
\text { near } \\
\text { surface } \\
\text { (mg/L) } \\
\end{array}$} & \multicolumn{2}{|c|}{$\begin{array}{l}\text { Dissolved oxygen, } \\
\text { mid-depth } \\
(\mathrm{mg} / \mathrm{L})\end{array}$} & \multicolumn{2}{|c|}{$\begin{array}{c}\text { Dissolved oxygen, } \\
\text { near } \\
\text { bottom } \\
(\mathrm{mg} / \mathrm{L})\end{array}$} \\
\hline $\begin{array}{l}\text { Maximum } \\
\text { (Date) }\end{array}$ & $\begin{array}{r}\text { Minimum } \\
\text { (Date) }\end{array}$ & $\begin{array}{l}\text { Maximum } \\
\text { (Date) }\end{array}$ & $\begin{array}{l}\text { Minimum } \\
\text { (Date) }\end{array}$ & $\begin{array}{l}\text { Maximum } \\
\text { (Date) }\end{array}$ & $\begin{array}{l}\text { Minimum } \\
\text { (Date) }\end{array}$ & $\begin{array}{l}\text { Maximum } \\
\text { (Date) }\end{array}$ & $\begin{array}{l}\text { Minimum } \\
\text { (Date) }\end{array}$ \\
\hline $\begin{array}{c}33.3 \\
(8 / 14 / 90)\end{array}$ & $\begin{array}{l}\quad 1.0 \\
(12 / 4,21 \\
22 / 89 ; \\
1 / 13 / 90)\end{array}$ & $\begin{array}{c}15.0 \\
(12 / 3,4 / 89)\end{array}$ & $\begin{array}{c}1.4 \\
(8 / 7 / 89)\end{array}$ & $\begin{array}{c}15.4 \\
(6 / 17 / 90\end{array}$ & $\begin{array}{l}<1.0 \\
(6 / 28 / 89 ; \\
10 / 28 / 89 ; \\
11 / 2-4 / 89 ; \\
8 / 19-23 / 90 ; \\
9 / 12-17,27 \\
29 / 90)\end{array}$ & $\begin{array}{c}16.4 \\
(7 / 19 / 90)\end{array}$ & $\begin{array}{l}\quad<1.0 \\
(8 / 16,18-31 / 89 ; \\
9 / 1-4,9-11 / 89 ; \\
10 / 28-31 / 89 ; \\
11 / 1-4 / 89 ; \\
7 / 1 / 90 ; 8 / 14 \\
19-25 / 90 ; \\
9 / 11-21,23,26 \\
27,29 / 90)\end{array}$ \\
\hline $\begin{array}{c}33.0 \\
(7 / 23 / 90)\end{array}$ & $\begin{array}{c}0.0 \\
(12 / 3 / 89)\end{array}$ & $\begin{array}{c}16.5 \\
(1 / 4 / 90)\end{array}$ & $\begin{array}{c}2.8 \\
(8 / 30 / 90)\end{array}$ & $\begin{array}{c}18.6 \\
(7 / 19 / 89)\end{array}$ & $\begin{array}{l}<1.0 \\
(11 / 2,8,9 / 89 \\
7 / 7 / 90)\end{array}$ & $\begin{array}{r}18.2 \\
1 / 4 / 90)\end{array}$ & $\begin{array}{l}<1.0 \\
(5 / 12-14,17,20, \\
23,24 / 89 ; 6 / 4, \\
15,20,21, \\
23-30 / 89 ; \\
7 / 1 / 89 ; \\
10 / 17-23,27 \\
28 / 89 ; 11 / 9 / 89 ; \\
4 / 26-28 / 90 ; \\
7 / 27-31 / 90 ; \\
8 / 1-21,27,28 / 90)\end{array}$ \\
\hline $\begin{array}{c}31.8 \\
(7 / 24 / 90)\end{array}$ & $\begin{array}{c}3.5 \\
(1 / 7 / 90)\end{array}$ & $\begin{array}{c}14.7 \\
(1 / 27 / 90)\end{array}$ & $\begin{array}{c}<1.0 \\
(10 / 17 / 89)\end{array}$ & $\begin{array}{c}14.8 \\
(2 / 1 / 90)\end{array}$ & $\begin{array}{l}<1.0 \\
(8 / 24 / 89 ; \\
8 / 28,29 / 90)\end{array}$ & $\begin{array}{c}16.7 \\
(2 / 1 / 90)\end{array}$ & $\begin{array}{l}<1.0 \\
(8 / 24,25 / 89 ; \\
9 / 1 / 89 ; 5 / 29, \\
31 / 89 ; 6 / 1-3 / 89 ; \\
7 / 4,7,8,20-25, \\
27,28 / 89 ; 8 / 1-3, \\
5-31 / 89 ; \\
9 / 1-3 / 90)\end{array}$ \\
\hline $\begin{array}{c}33.3 \\
(8 / 20 / 90)\end{array}$ & $\begin{array}{c}4.1 \\
(12 / 3 / 89)\end{array}$ & $\begin{array}{c}18.7 \\
(1 / 24 / 90)\end{array}$ & $\begin{array}{c}1.5 \\
(10 / 14 / 89)\end{array}$ & $\begin{array}{c}15.8 \\
(1 / 28 / 90)\end{array}$ & $\begin{array}{l}<1.0 \\
(9 / 16 / 89 \\
10 / 16,18 \\
19 / 89)\end{array}$ & $\begin{array}{c}14.1 \\
(5 / 20 / 89)\end{array}$ & $\begin{array}{l}<1.0 \\
(8 / 25,26 / 89 ; \\
9 / 13,15,16 / 89)\end{array}$ \\
\hline $\begin{array}{c}31.7 \\
(7 / 12 / 89)\end{array}$ & $\begin{array}{c}6.4 \\
(2 / 26 / 90)\end{array}$ & $\begin{array}{c}15.9 \\
(1 / 28 / 90)\end{array}$ & $\begin{array}{c}<1.0 \\
(6 / 17-20 / 89)\end{array}$ & $\begin{array}{c}16.0 \\
(1 / 29 / 90)\end{array}$ & $\begin{array}{l}<1.0 \\
(6 / 9,11,12, \\
14,15,18, \\
19 / 89)\end{array}$ & $\begin{array}{c}15.2 \\
(2 / 13 / 90)\end{array}$ & $\begin{array}{l}<1.0 \\
(6 / 10-12 \\
14-26 / 89 \\
7 / 5,14 / 89 \\
9 / 15,16 / 89 ; \\
7 / 21-24 / 90 \\
8 / 4-7 / 90)\end{array}$ \\
\hline
\end{tabular}


Table 2.--Maximum and minimum values recorded for salinity, water-quality monitoring sites, April

[ppt, parts per thousand; ${ }^{\circ} \mathrm{C}$, degrees Celsius;

\begin{tabular}{|c|c|c|c|c|c|}
\hline \multirow{3}{*}{$\begin{array}{c}\text { Site } \\
\text { number } \\
\text { (fig. 2) }\end{array}$} & \multirow{3}{*}{$\begin{array}{c}\text { Station name } \\
\text { and } \\
\text { number } \\
\text { (Period of record) }\end{array}$} & \multicolumn{4}{|c|}{ Constituent } \\
\hline & & \multicolumn{2}{|c|}{$\begin{array}{l}\text { Salinity, } \\
\text { near } \\
\text { surface } \\
\text { (ppt) }\end{array}$} & \multicolumn{2}{|c|}{$\begin{array}{l}\text { Salinity, } \\
\text { near } \\
\text { bottom } \\
\text { (ppt) }\end{array}$} \\
\hline & & $\begin{array}{l}\text { Maximum } \\
\text { (Date) }\end{array}$ & $\begin{array}{c}\text { Minimum } \\
\text { (Date) }\end{array}$ & $\begin{array}{l}\text { Maximum } \\
\text { (Date) }\end{array}$ & $\begin{array}{c}\text { Minimum } \\
(\text { Date })\end{array}$ \\
\hline 6 & $\begin{array}{l}\text { Pungo River at Junction } \\
\text { Light PR, 0208457700 } \\
(5 / 89-9 / 90)\end{array}$ & $\begin{array}{c}13.4 \\
(8 / 23 / 90)\end{array}$ & $\begin{array}{c}1.3 \\
(12 / 24 / 89)\end{array}$ & $\begin{array}{c}12.9 \\
(12 / 23 / 89)\end{array}$ & $\begin{array}{c}4.1 \\
(5 / 6 / 90)\end{array}$ \\
\hline 7 & $\begin{array}{l}\text { Neuse River at Channel } \\
\text { Light } 22,0209258400 \\
(5 / 89-12 / 89)\end{array}$ & $\begin{array}{c}9.2 \\
(8 / 7 / 89)\end{array}$ & $\begin{array}{l}\quad<0.1 \\
(5 / 12-17 / 89 \\
7 / 23,27 \\
28 / 89 ; 12 / 3 \\
6,11,12 / 89)\end{array}$ & $\begin{array}{c}12.0 \\
(8 / 8,9 / 89)\end{array}$ & $\begin{array}{l}\quad<0.1 \\
(5 / 12-17 \\
24 / 89)\end{array}$ \\
\hline 8 & $\begin{array}{l}\text { Neuse River at Channel } \\
\text { Light } 11,0209262905 \\
(5 / 89-12 / 89)\end{array}$ & $\begin{array}{c}8.3 \\
(6 / 28 / 89)\end{array}$ & $\begin{array}{c}<0.1 \\
(5 / 14,15 / 89)\end{array}$ & $\begin{array}{c}13.7 \\
(6 / 28 / 89)\end{array}$ & $\begin{array}{c}0.1 \\
(5 / 15 / 89)\end{array}$ \\
\hline 9 & $\begin{array}{l}\text { Neuse River at Channel } \\
\text { Light } 9,0209265810 \\
(5 / 89-9 / 90)\end{array}$ & $\begin{array}{c}13.0 \\
(8 / 13,14 / 90)\end{array}$ & $\begin{array}{c}0.3 \\
(12 / 30,31 / 89)\end{array}$ & $\begin{array}{c}15.7 \\
(6 / 29 / 89)\end{array}$ & $\begin{array}{c}0.5 \\
(12 / 24 / 89)\end{array}$ \\
\hline 10 & $\begin{array}{l}\text { Adams Creek at Light } 4 \text {, } \\
0209266925 \\
(6 / 89-9 / 90)\end{array}$ & $\begin{array}{c}32.8 \\
(3 / 18 / 90)\end{array}$ & $\begin{array}{c}0.8 \\
(12 / 31 / 89)\end{array}$ & $\begin{array}{c}31.5 \\
(6 / 9 / 89)\end{array}$ & $\begin{array}{c}4.5 \\
(12 / 17 / 89 \\
3 / 10,11 / 90)\end{array}$ \\
\hline 11 & $\begin{array}{l}\text { Neuse River at Junction } \\
\text { Light, } 0209269000 \\
(5 / 90-9 / 90)\end{array}$ & $\begin{array}{c}17.0 \\
(9 / 21 / 90)\end{array}$ & $\begin{array}{c}10.6 \\
(7 / 28 / 90)\end{array}$ & $\begin{array}{c}20.7 \\
(6 / 29 / 90)\end{array}$ & $\begin{array}{c}10.7 \\
(5 / 17 / 90)\end{array}$ \\
\hline
\end{tabular}

$\frac{1}{2} /$ U.S. Geological Survey downstream order number.

$2 /$ Period of record October 1989 to September 1990 . 
water temperature, and dissolved oxygen in water at the 1989 to September 1990--Continued

$\mathrm{mg} / \mathrm{L}$, milligrams per liter; <, less than]

\begin{tabular}{|c|c|c|c|c|c|c|c|}
\hline \multicolumn{8}{|c|}{ Constituent--Continued } \\
\hline \multicolumn{2}{|c|}{$\begin{array}{c}\text { Water } \\
\text { temperature, } \\
\text { near } \\
\text { surface } \\
\left({ }^{\circ} \mathrm{C}\right) \\
\end{array}$} & \multicolumn{2}{|c|}{$\begin{array}{c}\text { Dissolved oxygen, } \\
\text { near } \\
\text { surface } \\
(\mathrm{mg} / \mathrm{L})\end{array}$} & \multicolumn{2}{|c|}{$\begin{array}{c}\text { Dissolved oxygen, } \\
\text { mid-depth } \\
(\mathrm{mg} / \mathrm{L})\end{array}$} & \multicolumn{2}{|c|}{$\begin{array}{c}\text { Dissolved oxygen, } \\
\text { near } \\
\text { bottom } \\
(\mathrm{mg} / \mathrm{L})\end{array}$} \\
\hline $\begin{array}{l}\text { Maximum } \\
\text { (Date) }\end{array}$ & $\begin{array}{r}\text { Minimum } \\
\text { (Date) }\end{array}$ & $\begin{array}{l}\text { Maximum } \\
\text { (Date) }\end{array}$ & $\begin{array}{l}\text { Minimum } \\
\text { (Date) }\end{array}$ & $\begin{array}{l}\text { Maximum } \\
\text { (Date) }\end{array}$ & $\begin{array}{l}\text { Minimum } \\
\text { (Date) }\end{array}$ & $\begin{array}{l}\text { Maximum } \\
\text { (Date) }\end{array}$ & $\begin{array}{l}\text { Minimum } \\
\text { (Date) }\end{array}$ \\
\hline $\begin{array}{c}31.8 \\
(8 / 2 / 90)\end{array}$ & $\begin{array}{c}1.7 \\
(12 / 20 / 89)\end{array}$ & $\begin{array}{c}18.6 \\
(1 / 20 / 90)\end{array}$ & $\begin{array}{c}3.9 \\
(8 / 26 / 89 \\
6 / 19 / 90)\end{array}$ & $\begin{array}{l}\underline{2} / 14.0 \\
(12 / 20 / 89)\end{array}$ & $\begin{array}{c}\underline{2} / 2.4 \\
(6 / 23 / 90)\end{array}$ & $\begin{array}{c}18.4 \\
(1 / 6 / 90)\end{array}$ & $\begin{array}{l}<1.0 \\
(9 / 1 / 89 \\
6 / 5,6 / 90 ; \\
8 / 5,7-16,21 / 90)\end{array}$ \\
\hline $\begin{array}{c}31.3 \\
(7 / 26 / 89)\end{array}$ & $\begin{array}{c}4.4 \\
(12 / 4 / 89)\end{array}$ & $\begin{array}{c}13.3 \\
(9 / 12 / 89)\end{array}$ & $\begin{array}{c}<1.0 \\
(8 / 5,6,9,10 \\
13,14,24 / 89)\end{array}$ & $\begin{array}{c}11.2 \\
(6 / 11 / 89)\end{array}$ & $\begin{array}{l}\quad<1.0 \\
(7 / 21 / 89 ; \\
8 / 28-30 / 89 ; \\
9 / 1,2,4-10 / 89)\end{array}$ & $\begin{array}{c}6.2 \\
(5 / 12 / 89)\end{array}$ & $\begin{array}{l}\quad<1.0 \\
(7 / 21-31 / 89 ; \\
8 / 1-11,13-31 / 89 ; \\
9 / 1-4 / 89 ; \\
10 / 21-25 / 89)\end{array}$ \\
\hline $\begin{array}{c}31.6 \\
(6 / 25 / 89)\end{array}$ & $\begin{array}{c}6.1 \\
(12 / 11 / 89)\end{array}$ & $\begin{array}{c}13.1 \\
(6 / 3 / 89)\end{array}$ & $\begin{array}{c}1.7 \\
(6 / 20 / 89)\end{array}$ & $\begin{array}{c}12.4 \\
(12 / 1 / 89)\end{array}$ & $\begin{array}{l}<1.0 \\
(6 / 18-20 \\
22-24,26 \\
27 / 89)\end{array}$ & $\begin{array}{c}12.5 \\
(11 / 25 / 89)\end{array}$ & $\begin{array}{l}\quad<1.0 \\
(5 / 13 / 89 ; \\
6 / 14,15,18-20, \\
22-29 / 89)\end{array}$ \\
\hline $\begin{array}{c}32.3 \\
(8 / 6 / 89)\end{array}$ & $\begin{array}{c}3.4 \\
(12 / 20 / 89)\end{array}$ & $\begin{array}{c}17.6 \\
(2 / 1 / 90)\end{array}$ & $\begin{array}{c}<1.0 \\
(6 / 15 / 90)\end{array}$ & $\begin{array}{c}17.8 \\
(1 / 31 / 90)\end{array}$ & $\begin{array}{c}<1.0 \\
(6 / 12,13,15 \\
16,20,21 / 89)\end{array}$ & $\begin{array}{c}16.3 \\
(1 / 31 / 90)\end{array}$ & $\begin{array}{l}\quad<1.0 \\
(5 / 31 / 89 ; \\
6 / 1-5,12,13, \\
15-17 / 89 ; \\
8 / 23,29 / 89 ; \\
9 / 2 / 89 ; 3 / 23 / 90 ; \\
4 / 26-30 / 90 ; \\
5 / 1,4,7,16,17, \\
20,21 / 90 ; \\
6 / 15 / 90 ; 7 / 9 / 90)\end{array}$ \\
\hline $\begin{array}{c}32.8 \\
(8 / 19,29 / 90)\end{array}$ & $\begin{array}{c}0.1 \\
(12 / 24 / 89)\end{array}$ & $\begin{array}{c}19.9 \\
(1 / 28 / 90)\end{array}$ & $\begin{array}{c}2.6 \\
(6 / 26 / 89)\end{array}$ & $\begin{array}{c}19.4 \\
(1 / 28 / 90)\end{array}$ & $\begin{array}{l}<1.0 \\
(6 / 25 / 89 \\
7 / 2-5 / 89 \\
5 / 2 / 90)\end{array}$ & $\begin{array}{c}18.6 \\
(1 / 28 / 90)\end{array}$ & $\begin{array}{c}<1.0 \\
(6 / 26 / 89 \\
7 / 3-5 / 89)\end{array}$ \\
\hline $\begin{array}{c}29.5 \\
(6 / 28 / 90)\end{array}$ & $\begin{array}{c}19.8 \\
(5 / 24 / 90)\end{array}$ & $\begin{array}{c}11.2 \\
(7 / 19 / 90)\end{array}$ & $\begin{array}{c}3.0 \\
(7 / 12,13 / 90)\end{array}$ & $\begin{array}{c}11.3 \\
(7 / 23 / 90)\end{array}$ & $\begin{array}{r}4.2 \\
(6 / 5 / 90)\end{array}$ & $\begin{array}{c}14.8 \\
(6 / 16 / 90)\end{array}$ & $\begin{array}{l}\quad<1.0 \\
(5 / 20 / 90 ; \\
7 / 21,27 / 90)\end{array}$ \\
\hline
\end{tabular}


14.5 inches high by 10.5 inches in diameter) containing signal conditioners, (2) cables with waterproof connectors, (3) water-quality sensors, and (4) a 12 -volt (V) battery. Up to eight water-quality sensors may be attached to the minimonitor. Sensors are available to measure specific conductance, temperature, dissolved oxygen, and $\mathrm{pH}$. $\mathrm{pH}$ was not measured in this investigation.

The specific-conductance sensor uses four electrodes to reduce the effects of fouling. Compensation for ambient water temperature is made by a signal conditioner so that all specific-conductance values are referenced to a temperature of $25^{\circ} \mathrm{C}$. Measurement ranges of 0 to 100,0 to $1,000,0$ to 10,000 , or 0 to 100,000 microsiemens per centimeter $(\mu \mathrm{S} / \mathrm{cm})$ may be selected. Following calibration, specific-conductance measurements are accurate to \pm 3 percent of selected full scale in a temperature range of 0 to $40^{\circ} \mathrm{C}$. The standard range for the temperature sensor is from 0 to $50^{\circ} \mathrm{C}$, and for a calibrated system, temperature measurements are accurate to within \pm 1 percent of full scale.

The dissolved-oxygen sensor uses a polarographic membrane and stirrer manufactured by Yellow Springs Instrument Company ${ }^{2}$ and a signal conditioner that was designed by the USGS. The dissolved-oxygen measurement system is potentially subject to more types of errors than the temperature and specific-conductance measurement systems. Potential errors include sensor background error, temperature-compensation uncertainty, and sensorcalibration uncertainty. Measurements are estimated to be accurate within about $0.3 \mathrm{mg} / \mathrm{L}$ for a calibrated system.

The minimonitor is controlled by a CR10 measurement and control module (Campbell Scientific, Inc., 1988). The CR10 is a fully programmable datalogger and controller which will accept voltage inputs from multiple sensors. The CR10 is programmed to turn on the minimonitor at 15-minute intervals, allow the sensors to stabilize for one minute, collect data from each of the sensors, record the time, and turn off the minimonitor. An external $12-\mathrm{V}$ battery provides power to the CR10. The CR10 has sufficient

\footnotetext{
${ }^{2}$ Any use of trade, product, or firm names is for descriptive purposes only and does not imply endorsement by the U.S. Government or State of North Carolina.
} 
internal memory to store about 30 days of minimonitor data collected at 15 minute intervals using six sensors. Data are lost, however, if the CR10 loses power.

To avoid potential problems associated with loss of power to the CR10 and to provide more operational flexibility, an SM192 storage module with nonvolatile memory (Campbell Scientific, Inc., 1987) is connected in series with the CR10. Data are automatically transferred from the CRIO to the SM192, which is not programmable and is only accessible through the CRlo. The SM192 has an internal $3.5-\mathrm{V}$ battery to protect the memory when the module is disconnected from the external power supply. Memory capacity is 192,976 bytes, which is sufficient to store about 3 months of minimonitor data.

All instruments are housed in water-tight shelters attached to U.S. Coast Guard channel markers. The datalogger and storage module are further protected in a moisture-proof container within the shelter. Weights were added to the ends of the sensor cables to minimize current-induced vertical motion of the sensors. A reference mark with a known elevation above the seabed was established at each station to ensure that sensors are returned to the same point in the water column after servicing.

\section{Field Procedures}

Instruments are serviced at approximately 3 -week intervals. During the colder months (October through April), servicing at 4- to 5-week intervals is sufficient, although the 3 -week schedule is generally maintained. More frequent servicing may be necessary during the warmer months because of biofouling problems.

Field meters are calibrated in the laboratory prior to visiting the field installations. Field meters used are the Yellow springs Instrument Company Model 33 S-C-T meter for specific conductance and the Yellow Springs Instrument Company Model 54 dissolved-oxygen meter. Both meters are also capable of measuring temperature. Temperature thermistors are calibrated against an ASTM thermometer at two temperatures. All values are within $0.5^{\circ} \mathrm{C}$ after calibration. Specific-conductance standards are used to develop 
a calibration curve for each conductance meter. Field meter specificconductance values are within 5 percent of the standards after calibration. The dissolved-oxygen meter is calibrated in water-saturated air adjusted for barometric pressure. After calibration, meter values are within $0.1 \mathrm{mg} / \mathrm{L}$ of the saturation value at the measured temperature and barometric pressure. The dissolved-oxygen calibration is for freshwater. Complete calibration records are maintained.

Upon reaching a site, simultaneous field-meter and minimonitor readings are made at coincident locations. If the difference between top and bottom readings for any of the constituents exceeds 5 percent, then field-meter readings are obtained throughout the full depth of the water column. The sensors are then removed from the water and cleaned. Dissolved-oxygen sensors are typically replaced during each visit.

After cleaning, sensors are returned to their original location in the water column, and a new set of field-meter and minimonitor readings is made. If necessary, the minimonitor span control for each measurement channel is adjusted until field and monitor readings agree. The SM192 storage modules containing recorded data are removed and replaced with modules prepared to begin recording a new set of data.

All minimonitors are calibrated with a full set of sensors in the laboratory prior to field installation. Subsequently, if a sensor or sensor cable is replaced, a field calibration must be performed for that particular measurement channel. Standard solutions are used for specific conductance field calibrations. Dissolved-oxygen field calibrations are performed using a sodium sulfite solution to achieve an oxygen-depleted sample.

\section{Data Processing}

The SM192 storage modules are returned to the office and data are transferred to the data base. Corrections for the difference between minimonitor and field-meter readings are applied to the data. Salinity is computed from specific-conductance records using the formulation given by Miller and others (1988). Finally, plots of the data are made. 
Data are reviewed to ensure validity. Outliers are examined and deleted if values are physically unrealistic. Data are examined to ensure that water density at the surface is less than that at the bottom at a given time. Data are rejected if the field-meter and minimonitor readings at the time of servicing are not in reasonable agreement with each constituent. Simple daily statistics are then computed and time-series plots are prepared.

During the early phases of the investigation, various problems resulted in the loss of record. Problems included corrosion of connectors, loss of battery power, improperly calibrated instruments, human errors, and poor agreement between field-meter and minimonitor readings. As the study has progressed, the amount of lost record has substantially decreased. Corrosion problems have been essentially eliminated. Solar panels have been installed to maintain battery voltage at an acceptable level, and SM192 storage modules have been placed at all sites so that if batteries fail, the entire record between site visits will not be lost. Intensive training has resulted in significantly fewer human errors, and more frequent servicing has resulted in less biofouling and measurement drift.

\section{WATER-QUALITY DATA}

Water-quality data collected at continuously monitored sites in the Pamlico and Neuse River estuaries from April 1989 through September 1990 are presented in this section. Instantaneous values of salinity and dissolvedoxygen concentrations are summarized as box plots, whereas daily mean values of salinity and dissolved-oxygen concentrations and water temperature for the entire period are given in tables and graphs.

\section{Box Plots}

Box plots for salinity and dissolved-oxygen concentrations are presented in figures 3 through 18 to show short-term variability at a site, to illustrate longitudinal variations along the estuary, and to give an indication of seasonal variations. Water temperature values were not summarized in this form. The box plots were developed from simple statistics using the 15-minute interval data; each box plot for a selected 
5 -day period represents a maximum of 480 observations. Periods were chosen to illustrate conditions occurring in a week during different seasons of the year.

Each figure includes near-surface and near-bottom box plots for salinity or dissolved oxygen in either the Pamlico or Neuse River estuary. At some sites, no data are available for the selected period.

\section{Daily Mean Values}

Daily mean values of salinity, water temperature, and dissolved-oxygen concentrations are presented for each of the 11 sites (tables 3-13). Any significant changes that occur in these values at time scales of minutes and hours are not represented by the daily mean values. Mean values do, however, give a good indication of general conditions at a site so that a reasonable summary of the large amount of data collected can be presented. The instantaneous maximum and minimum values recorded for the period are listed in each table, and days during which instantaneous dissolved-oxygen concentrations were less than $5.0 \mathrm{mg} / \mathrm{L}$ and $2.0 \mathrm{mg} / \mathrm{L}$ are also noted.

Each numbered table contains all of the data for one site. Within each table, the data, if available, are presented in the form of one constituent per water year per page in the following order:

- near-surface salinity for water year 1989

- near-surface salinity for water year 1990

- near-bottom salinity for water year 1989

- near-bottom salinity for water year 1990

- near-surface water temperature for water year 1989

- near-surface water temperature for water year 1990

- near-surface dissolved-oxygen concentration for water year 1989

- near-surface dissolved-oxygen concentration for water year 1990

- mid-depth dissolved-oxygen concentration for water year 1989

- mid-depth dissolved-oxygen concentration for water year 1990

- near-bottom dissolved-oxygen concentration for water year 1989

- near-bottom dissolved-oxygen concentration for water year 1990

At the bottom of each page, a graph is presented to illustrate the tabulation of data on that page. 

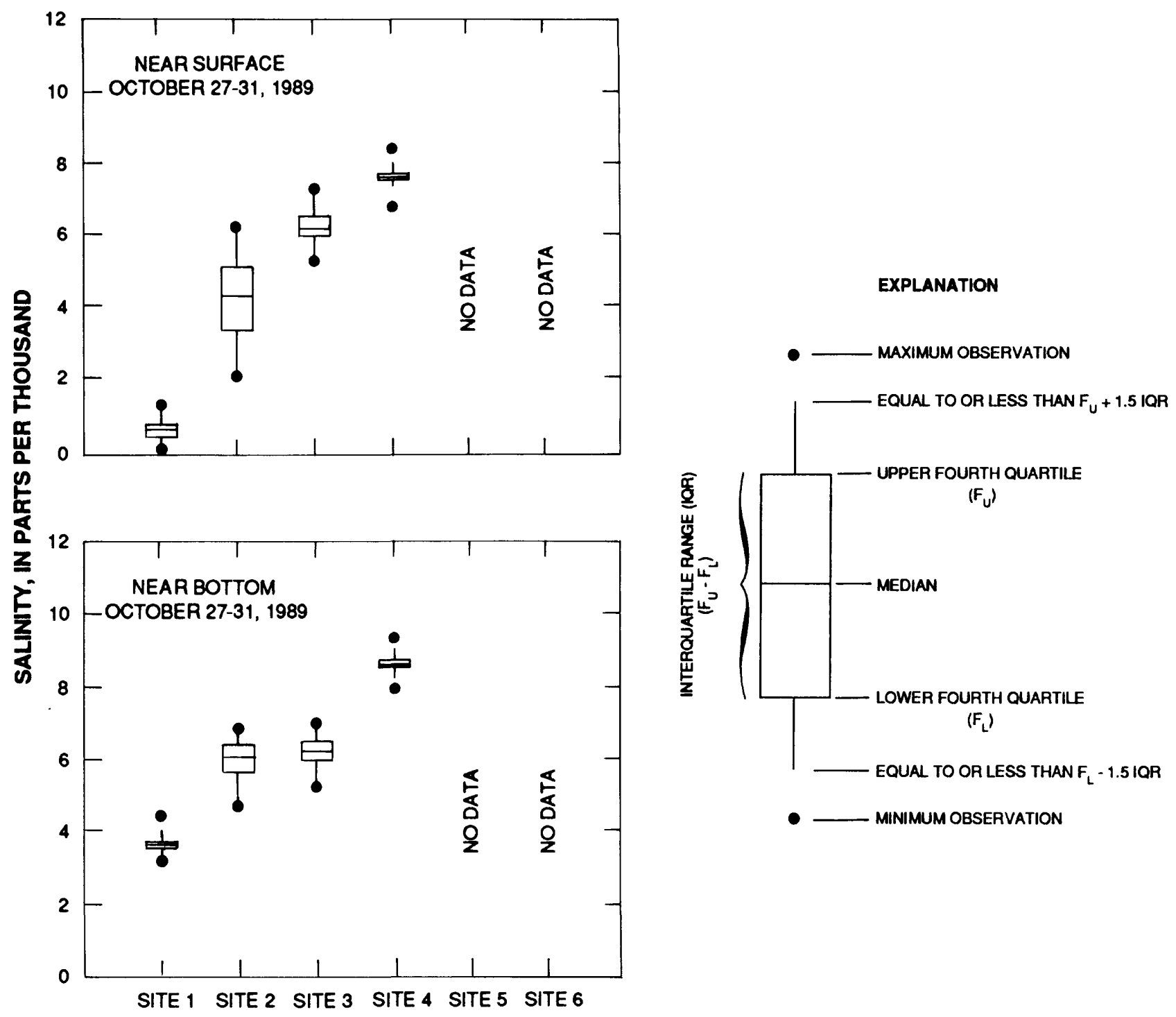

Figure 3.--Salinity concentrations at the Pamlico River estuary monitoring sites for October 27-31, 1989. 


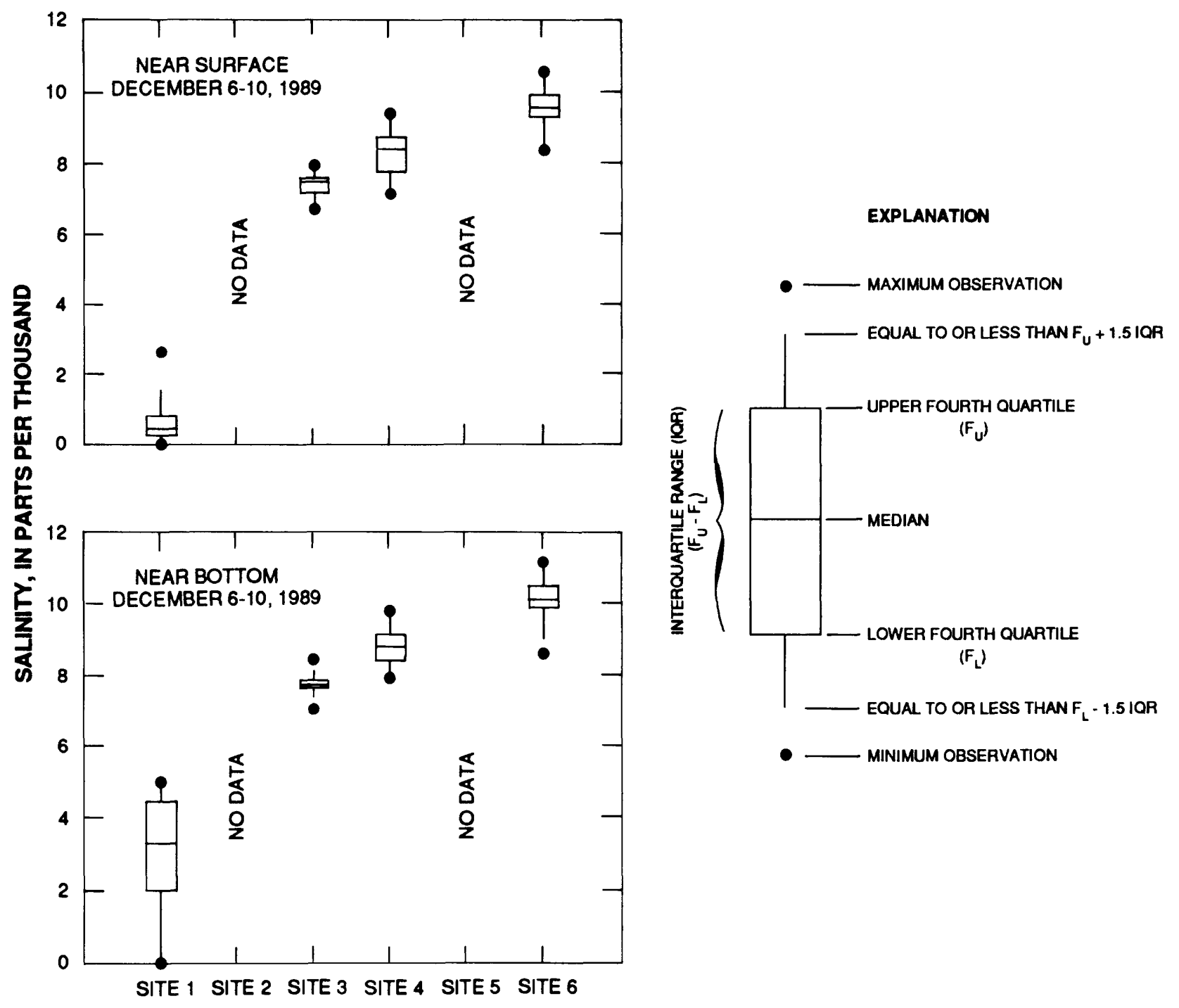

Figure 4.--Salinity concentrations at the Pamlico River estuary monitoring sites for December 6-10, 1989. 

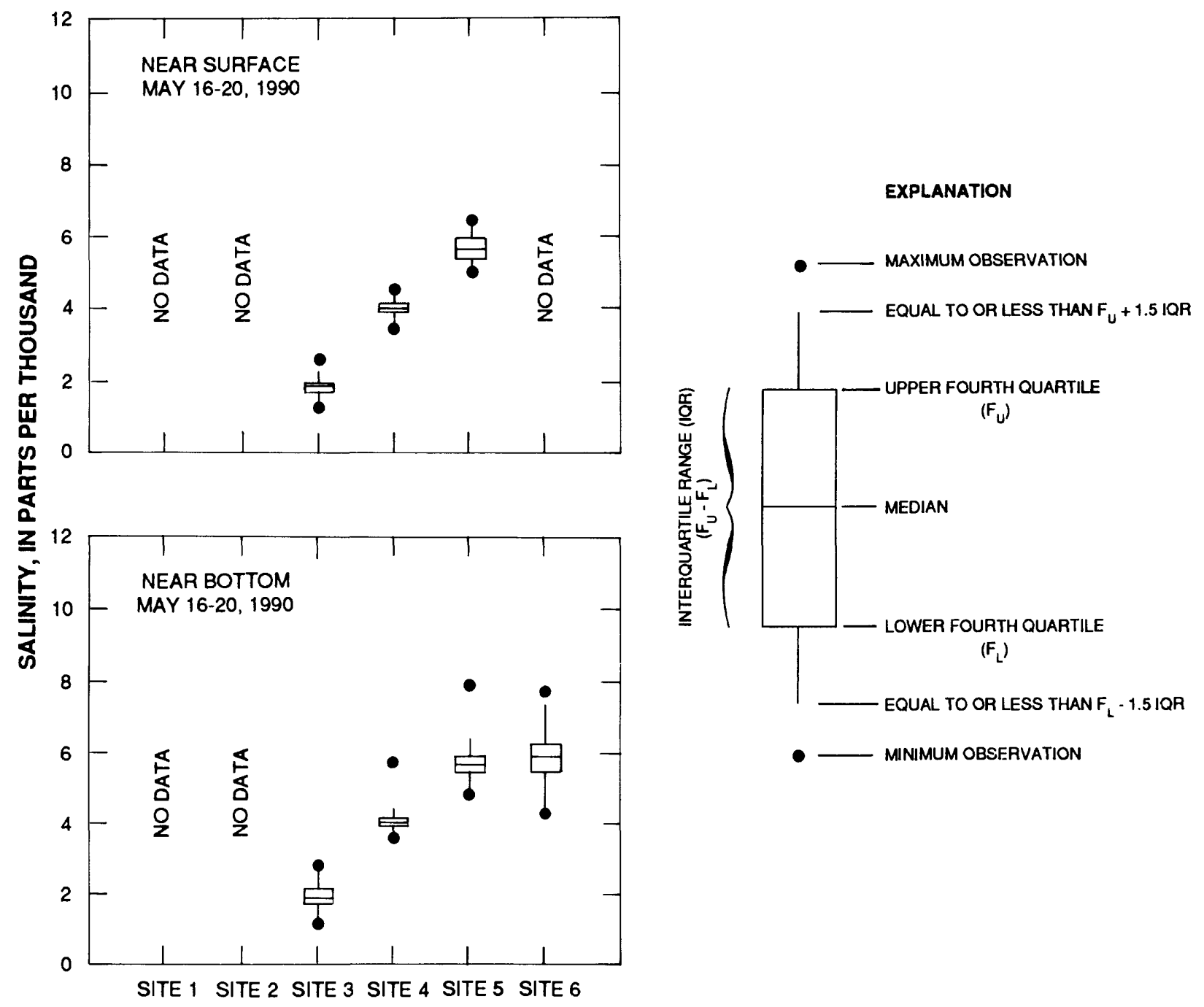

Figure 5.--Salinity concentrations at the Pamlico River estuary monitoring sites for May 16-20, 1990. 


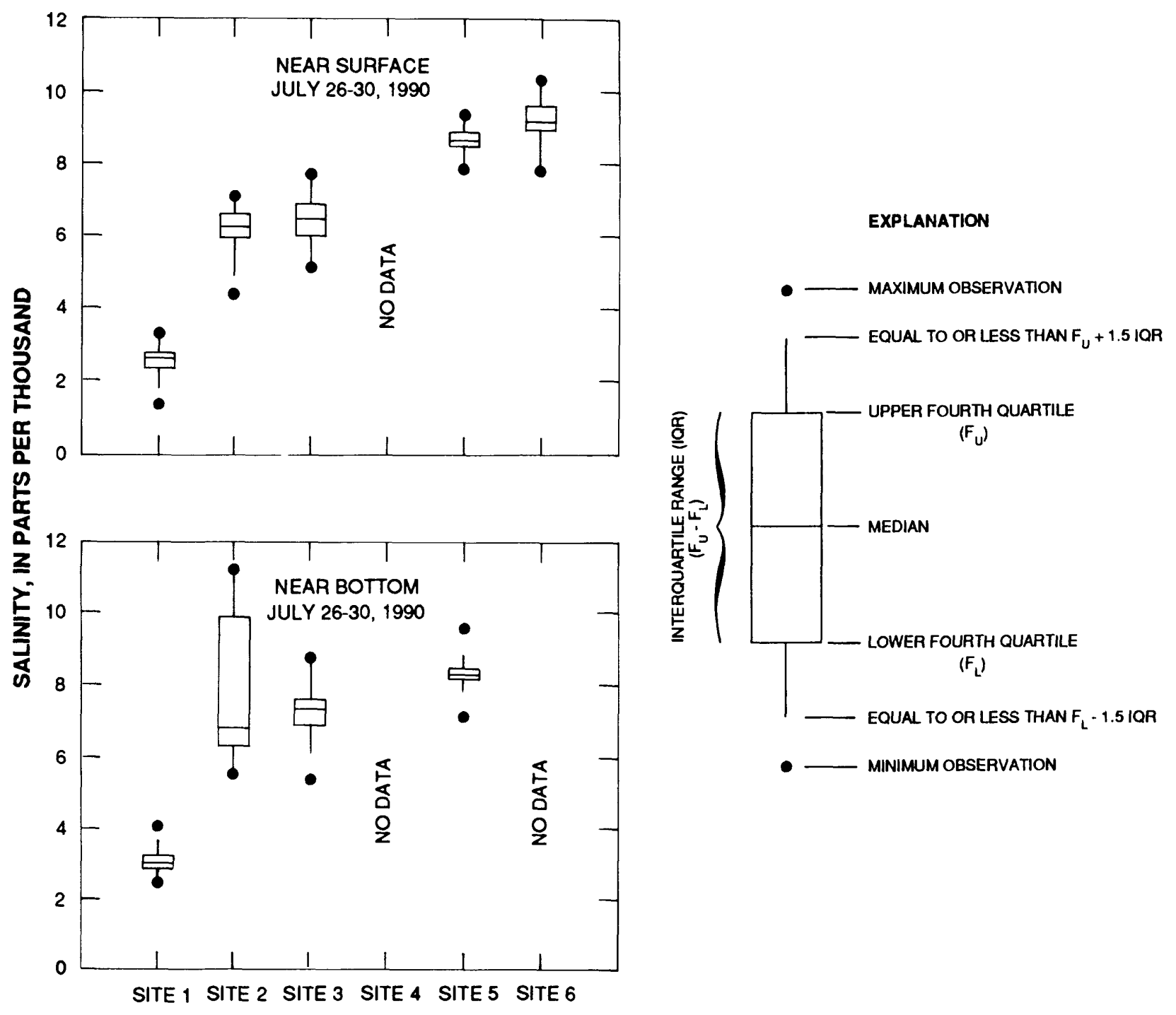

Figure 6.--Salinity concentrations at the Pamlico River estuary monitoring sites for July 26-30, 1990. 

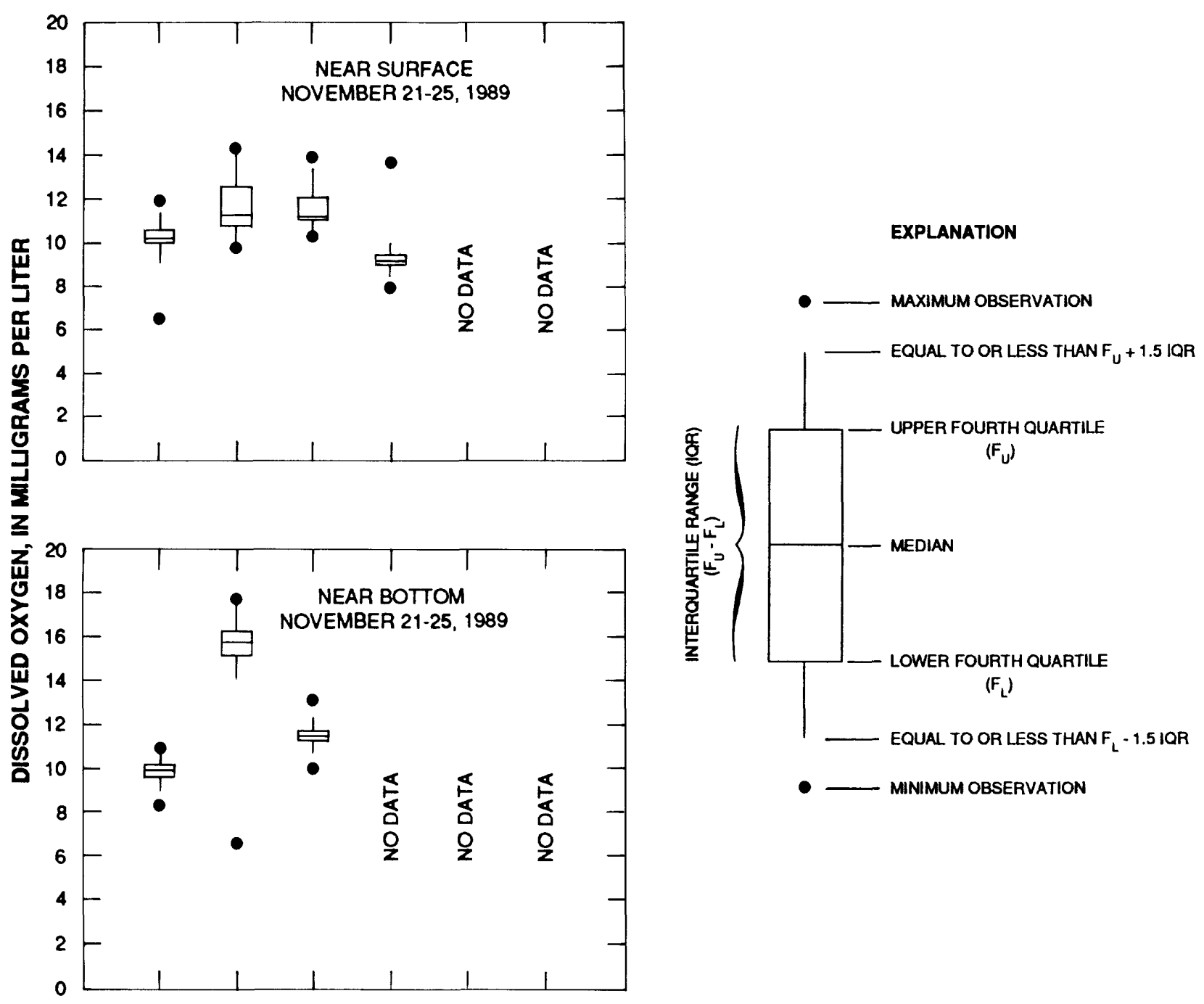

SITE 1 SITE 2 SITE 3 SITE 4 SITE 5 SITE 6

Figure 7.--Dissolved-oxygen concentrations at the Pamlico River estuary monitoring sites for November 21-25, 1989. 


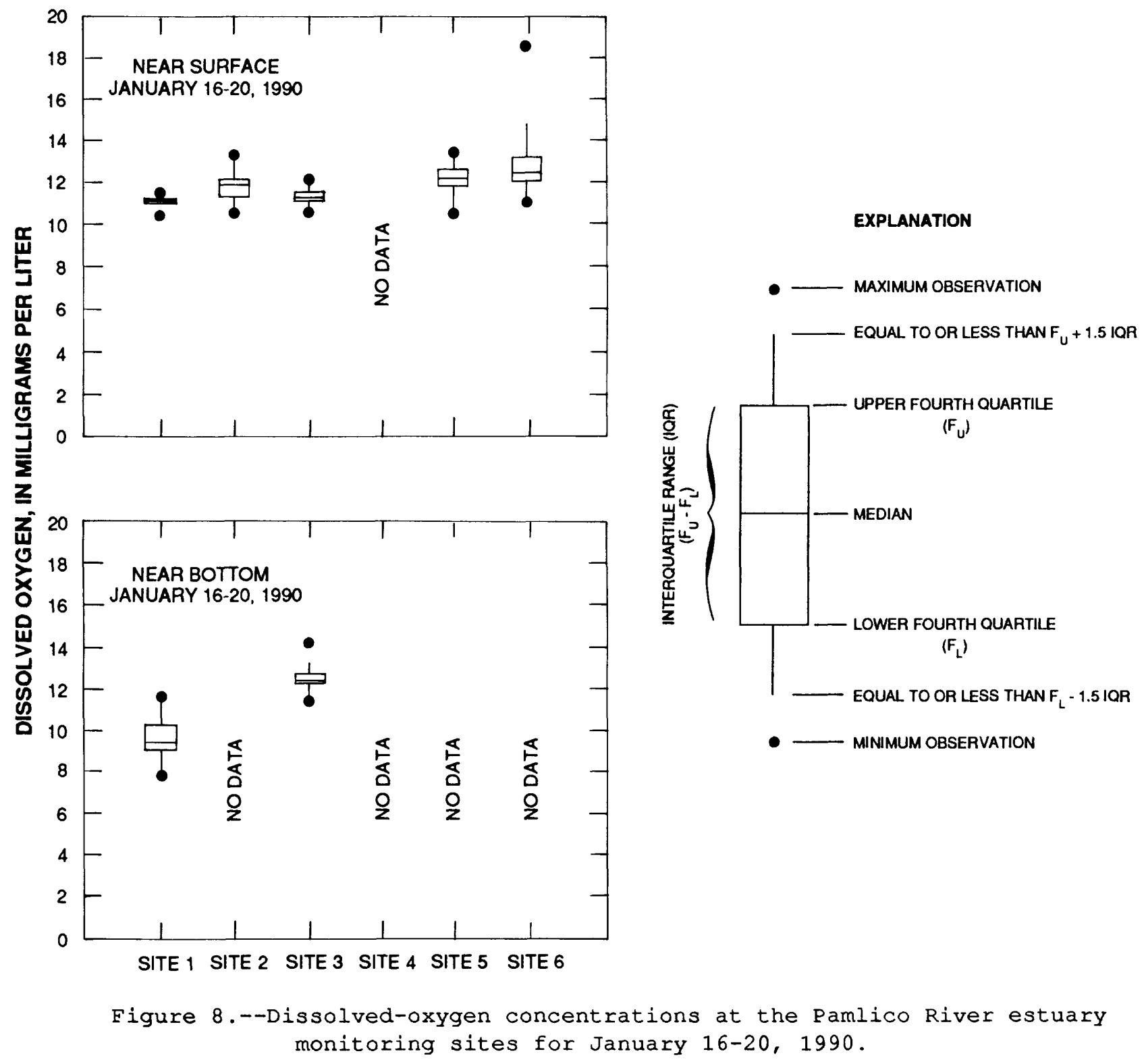



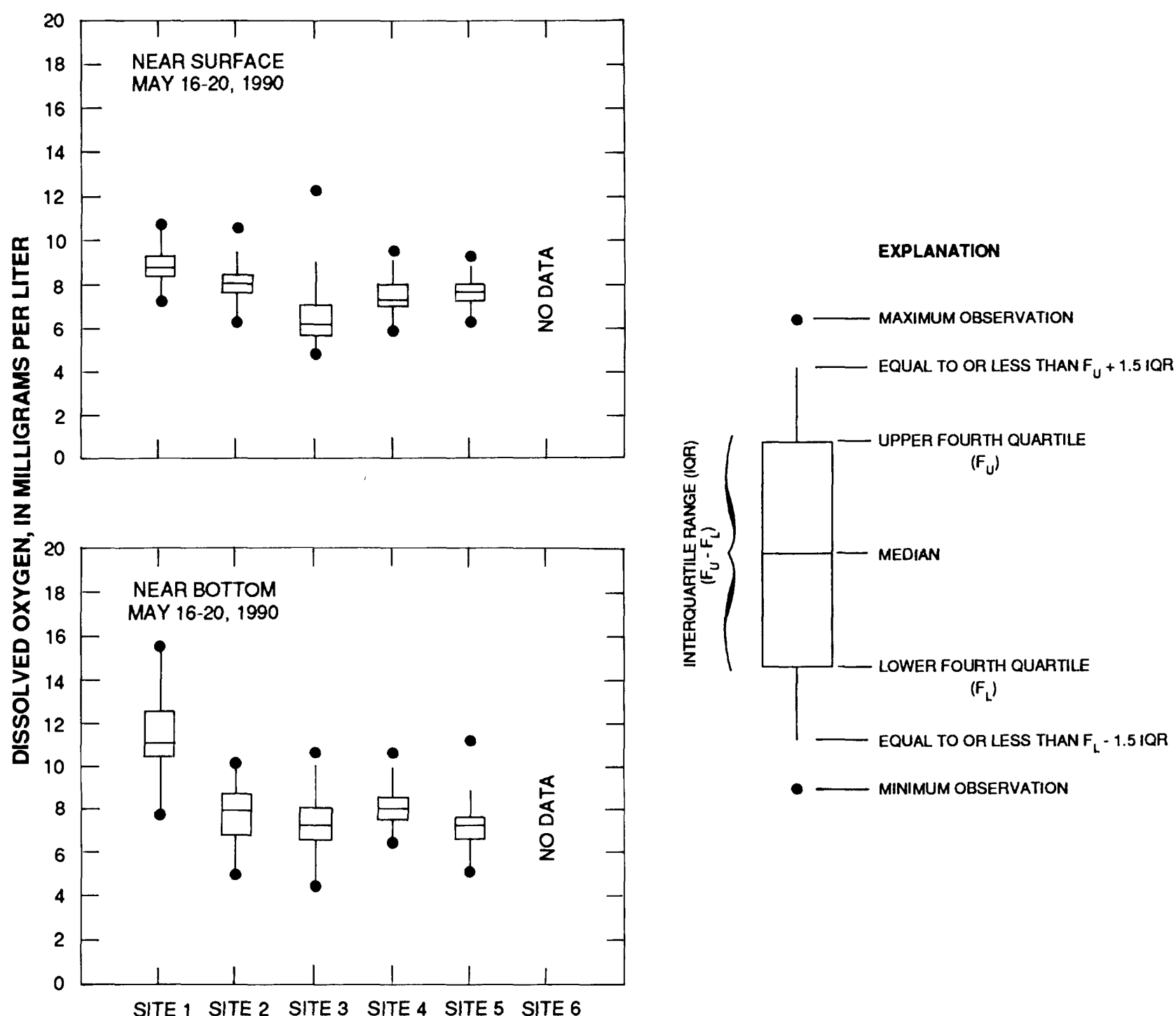

Figure 9.--Dissolved-oxygen concentrations at the Pamlico River estuary monitoring sites for May 16-20, 1990. 


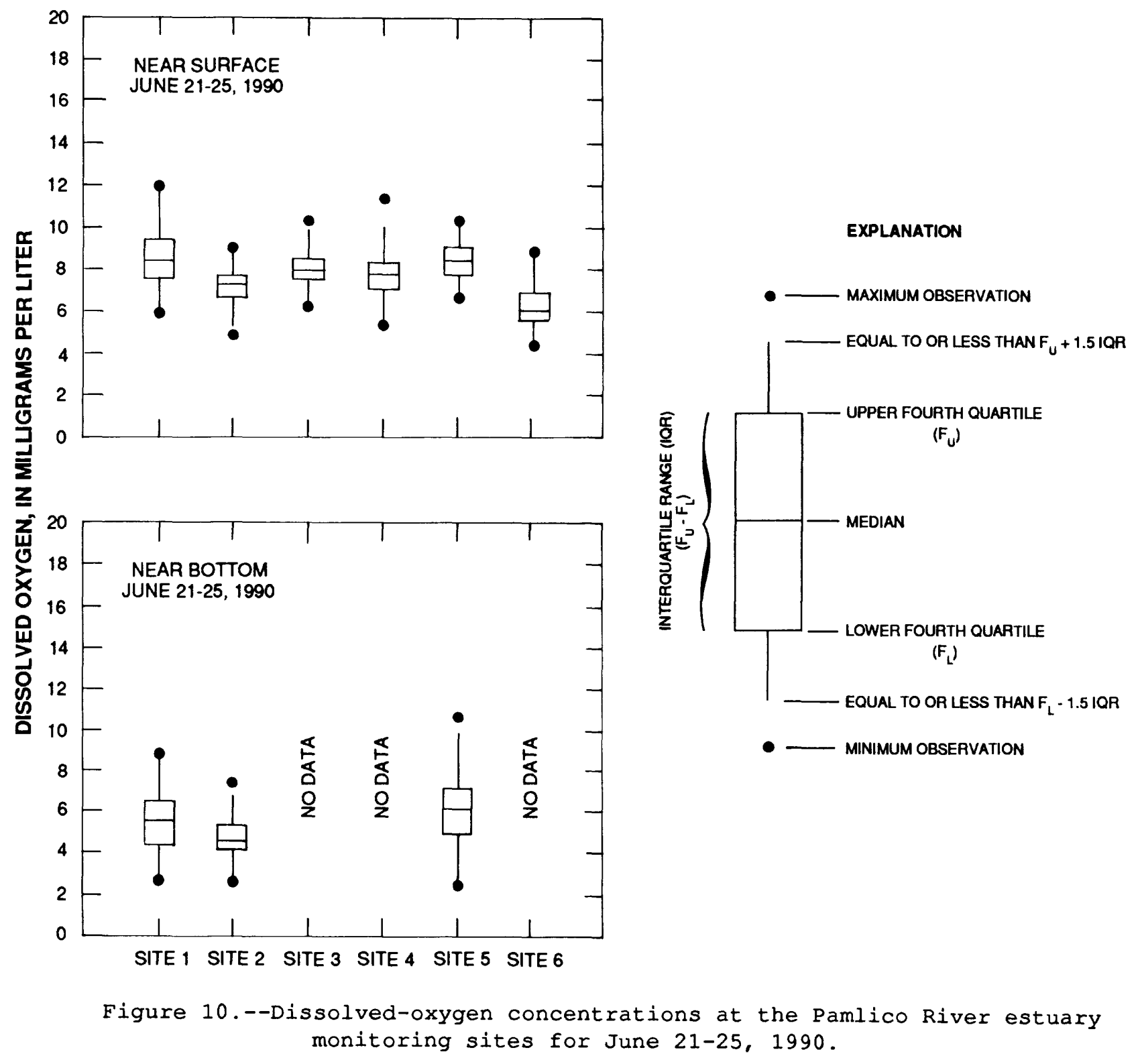




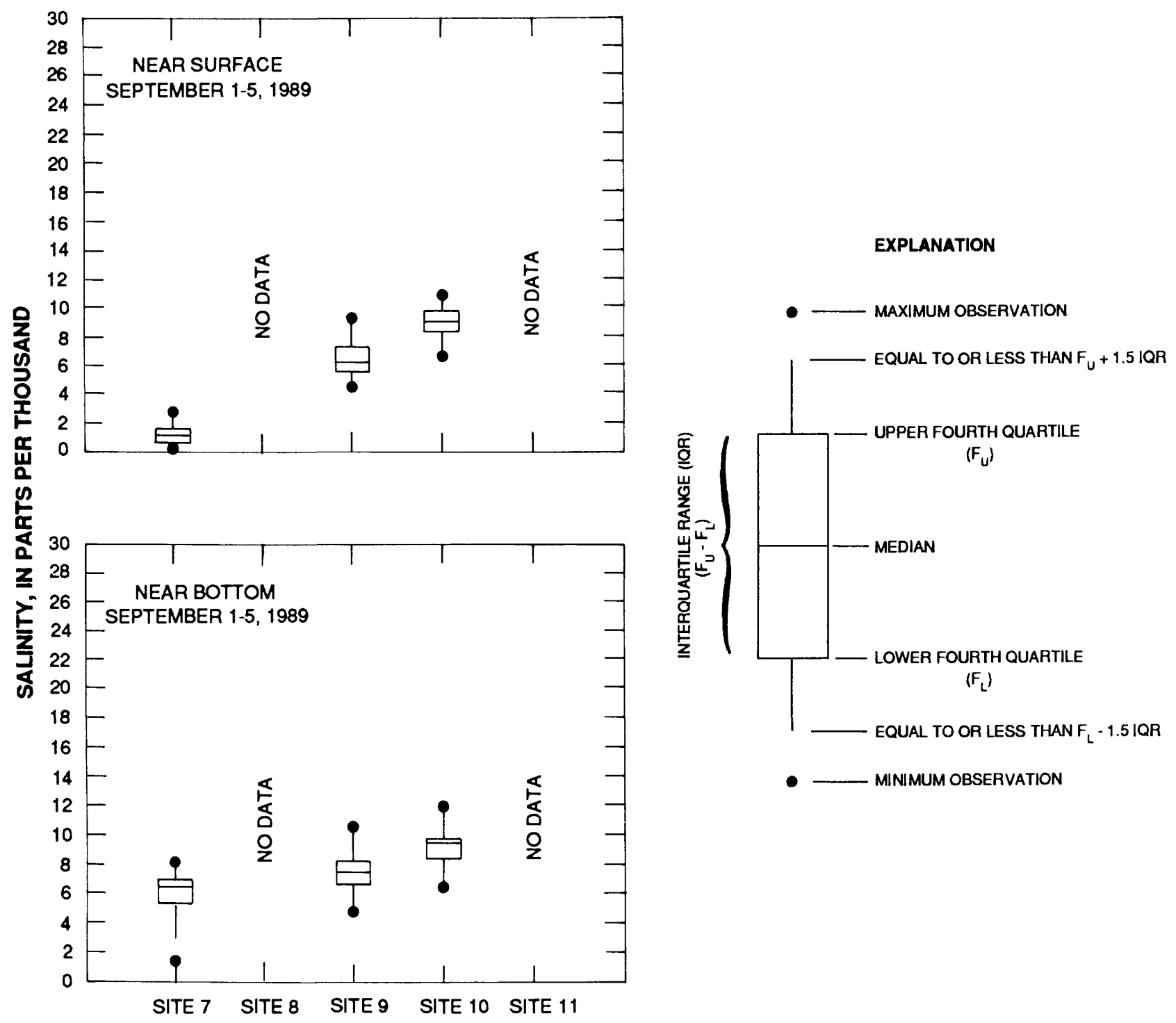

Figure 11.--Salinity concentrations at the Neuse River estuary monitoring sites for September 1-5, 1989. 


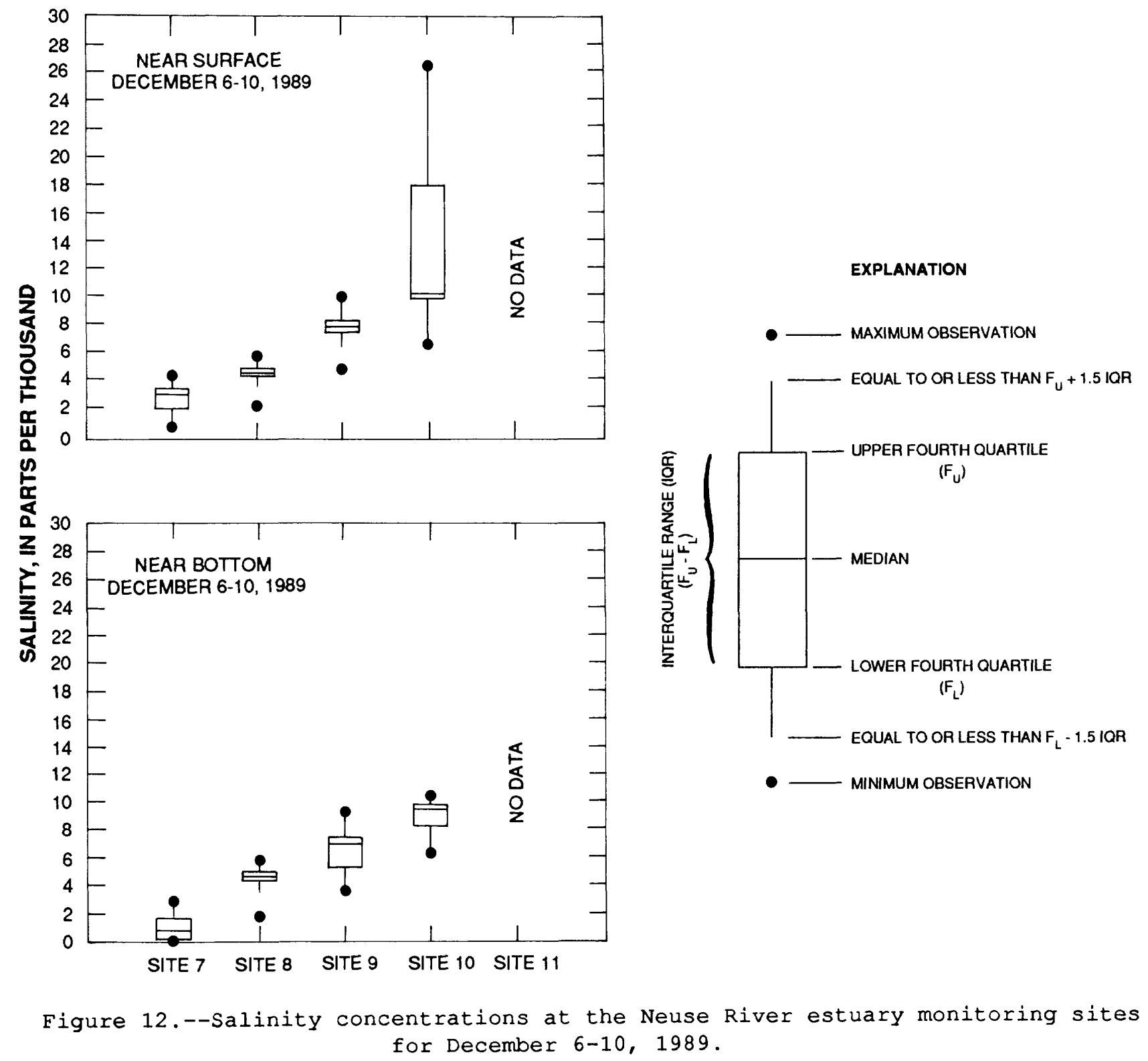




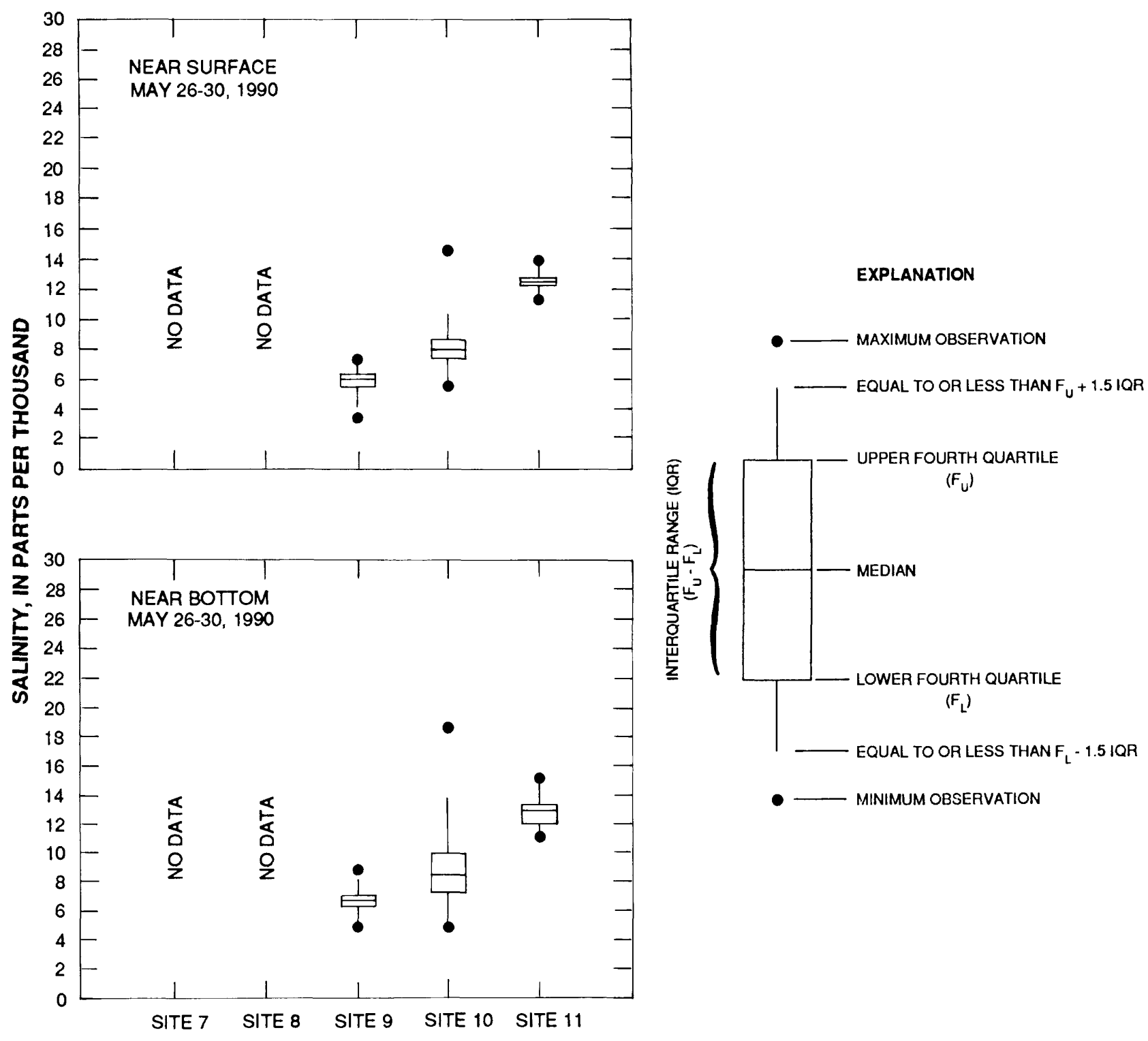

Figure 13.--Salinity concentrations at the Neuse River estuary monitoring sites for May 26-30, 1990. 


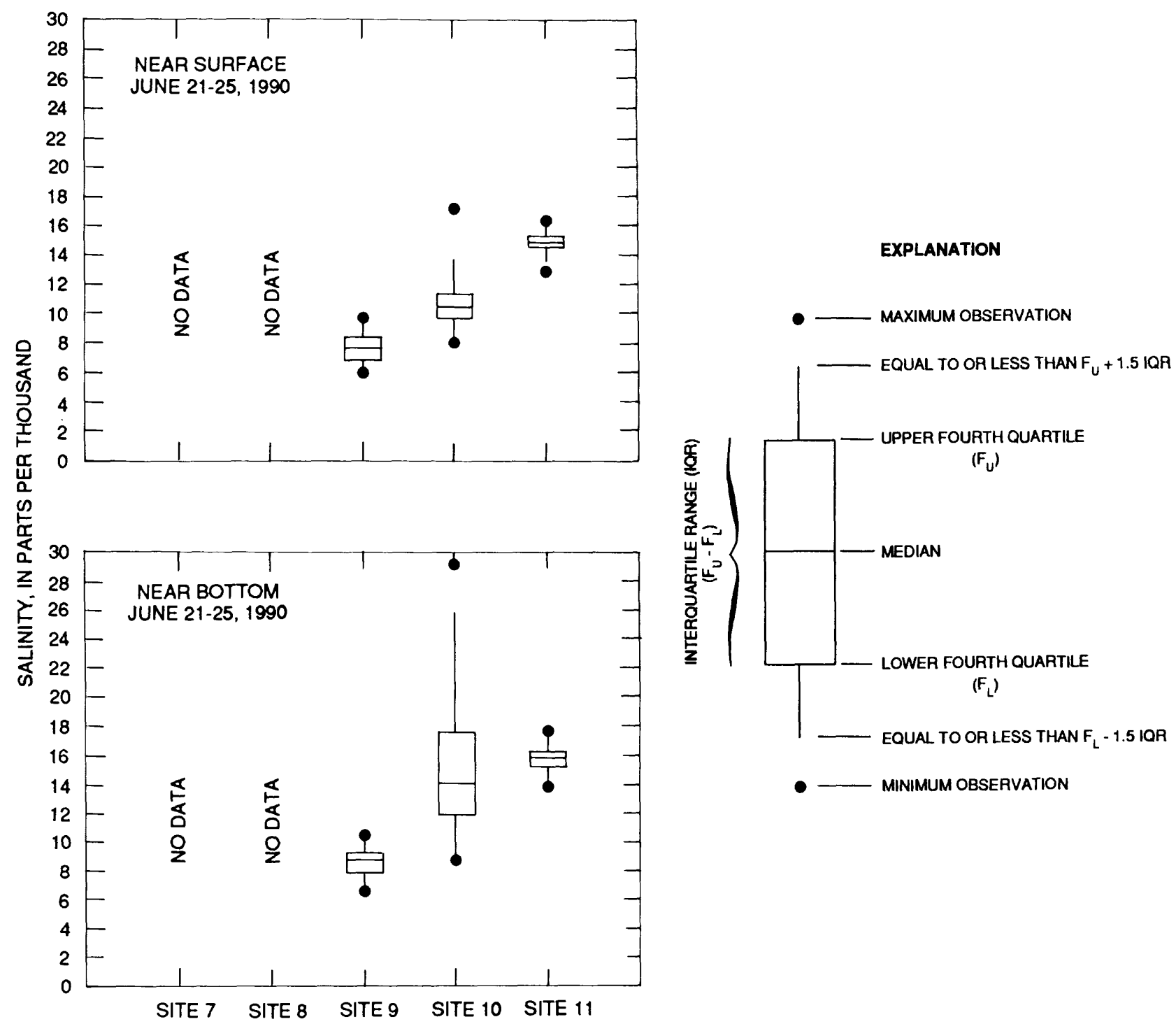

Figure 14.--Salinity concentrations at the Neuse River estuary monitoring sites for June 21-25, 1990. 

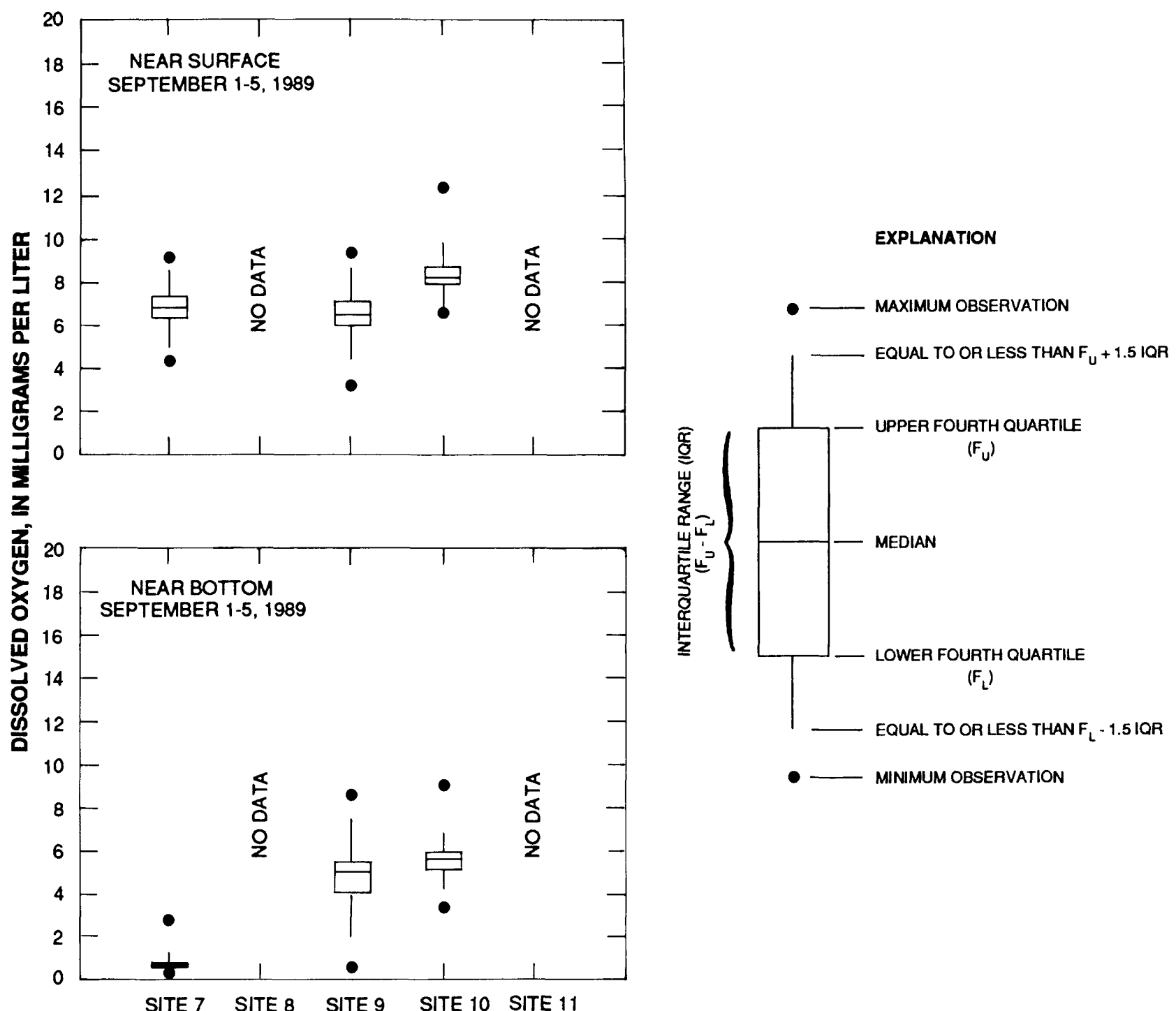

Figure 15.--Dissolved-oxygen concentrations at the Neuse River estuary monitoring sites for september $1-5,1989$. 


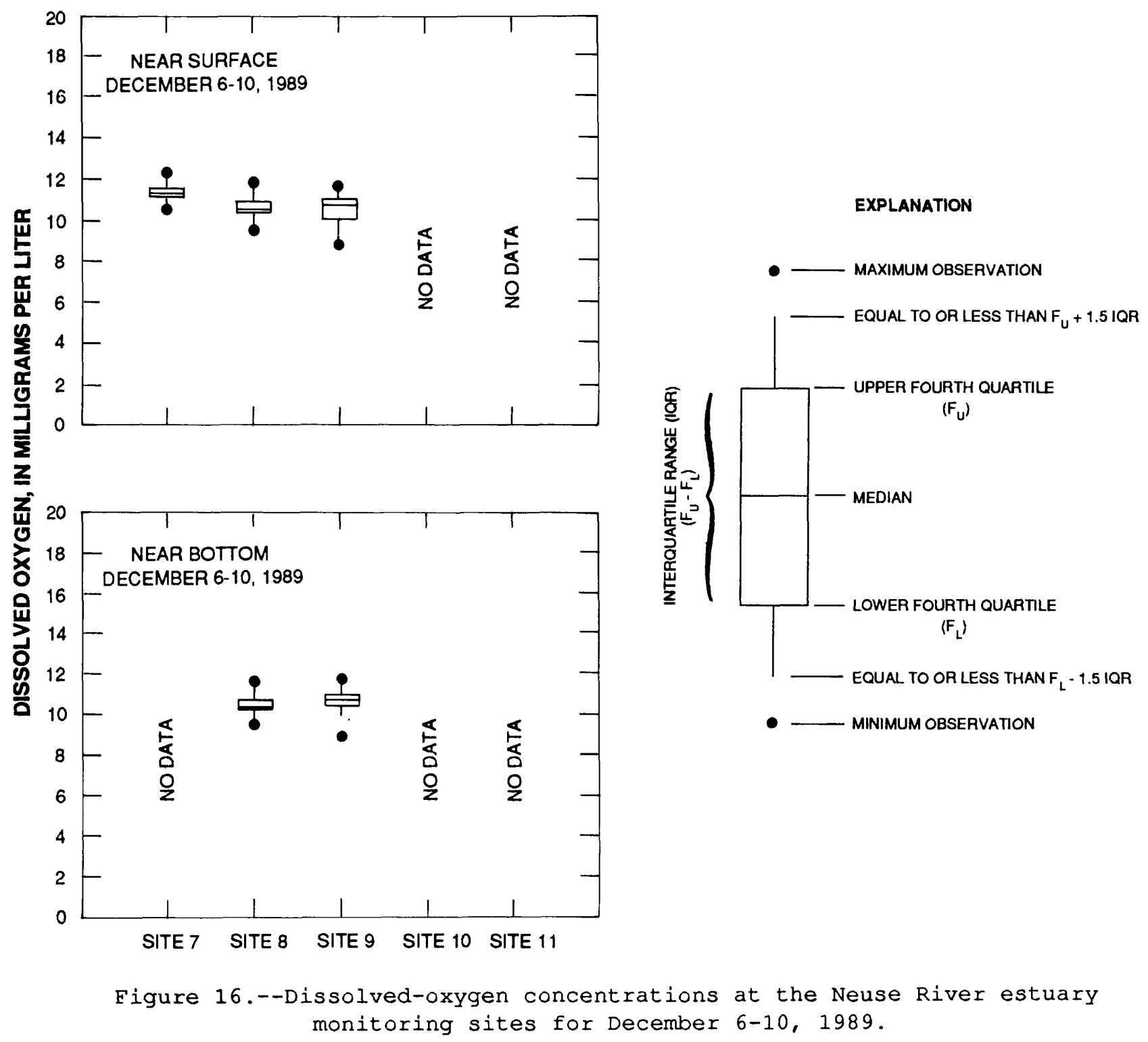



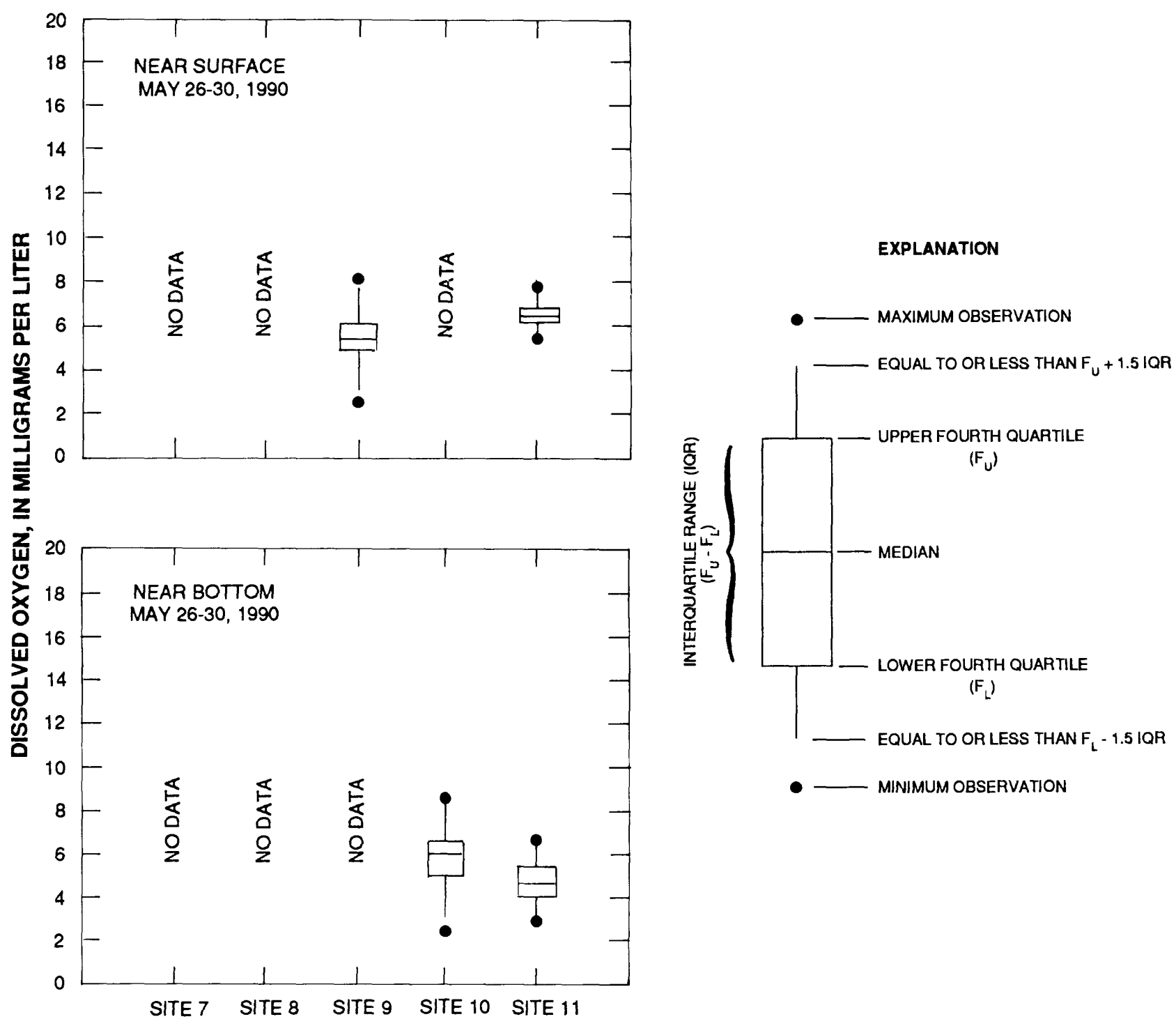

Figure 17.--Dissolved-oxygen concentrations at the Neuse River estuary monitoring sites for May 26-30, 1990. 


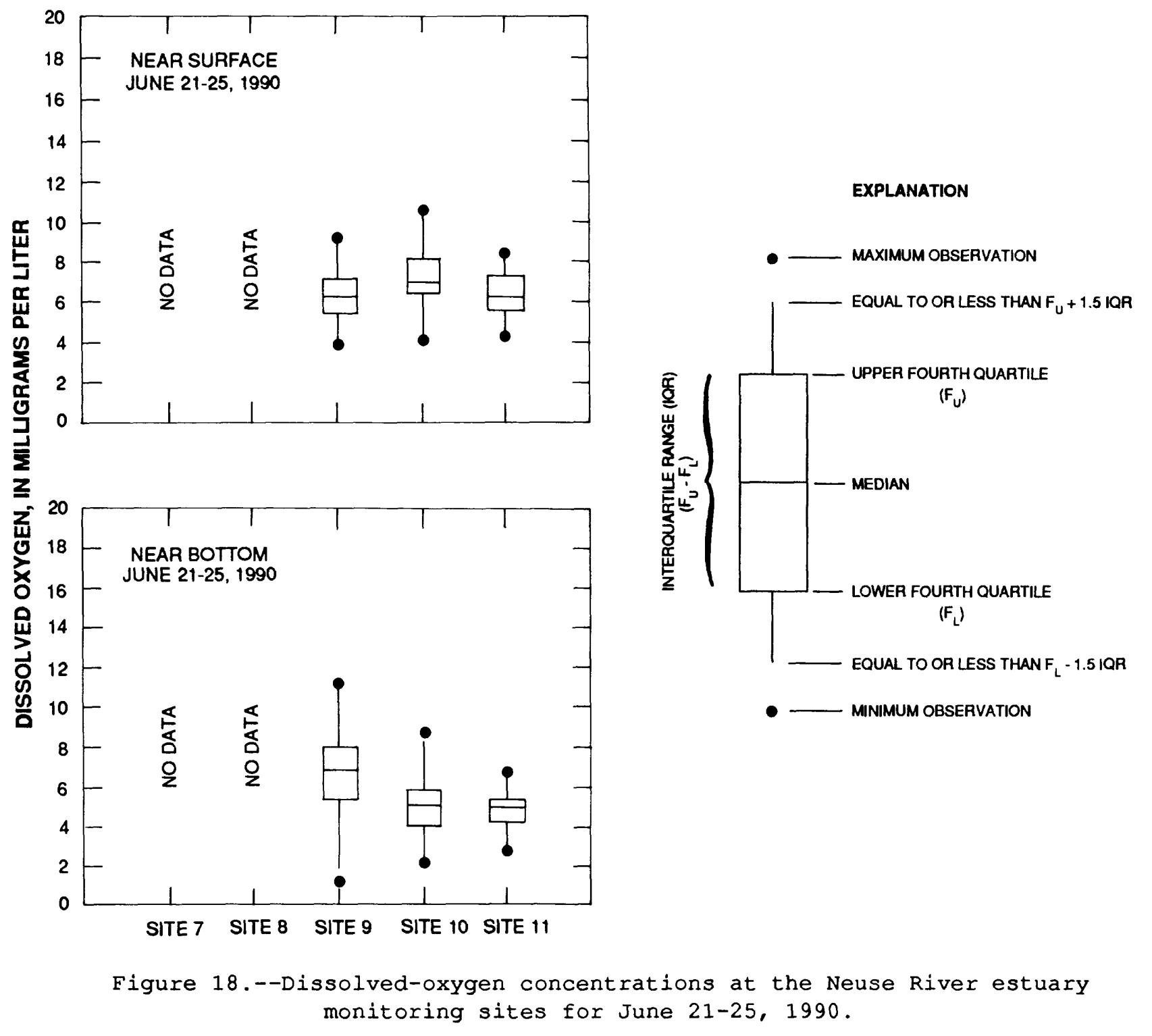


Some exceptions to this format occur in tables $8,9,10$, and 13 . In table 8 , mid-depth dissolved-oxygen concentrations were not recorded at site 6 during water year 1989, thus, there is no page for this water year for that constituent location. At sites 7 and 8 , data collection was interrupted during December 1989, and tables 9 and 10 were arranged to present combined data for the relatively short periods of records spanning water years 1989 and 1990. At site 11, data collection did not begin until late in the 1990 water year, therefore, table 13 presents data for only that water year.

Each page within a given table contains the following information, some of which is repeated from page to page:

- site location given as a latitude and longitude

- explanation of abbreviations used in the table

- constituent for which data are presented in the table

- distance above the streambed at which the sensor is located

- observed extreme recorded values for the given period

- tabulated daily mean values for the given period

- a graph of the daily mean values for the given period

Salinity concentrations are given as parts per thousand, water temperature is expressed as degrees Celsius, and dissolved-oxygen concentrations are presented as milligrams per liter. No correction for the effects of salinity on dissolved oxygen has been made in the data presented in this report. 
Table 3.--Daily mean values of salinity, water temperature, and dissolved oxygen in water at site 1 , Pamlico River at Channel Light 16, April 1989 to September 1990

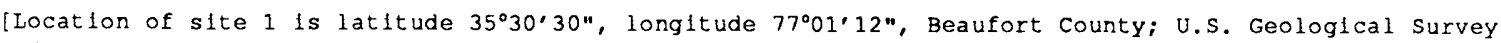
downstream order number 0208450705; ppt, parts per thousand; <, less than; ---, no data; ${ }^{\circ} \mathrm{C}$, degrees Celsfus; mg/L, milligrams per liter; a, dally minlmum value is less than or equal to $2.0 \mathrm{mg} / \mathrm{L}$; b, daily minimum value is less than or equal to $5.0 \mathrm{mg} / \mathrm{L}$ but greater than $2.0 \mathrm{mg} / \mathrm{L}$ ]

SALINITY, NEAR SURFACE

SENSOR POSITION. --6 feet above streambed.

EXTREMES FOR APRIL TO SEPTEMBER 1989.--Maximum value recorded, 3.9 ppt August 10, 13, 1989; m1nimum value recorded, <0.1 ppt July 20-26, August 21, 23, 1989.

DAILY MEAN VALUES OF SALINITY, NEAR SURFACE (IN PARTS PER THOUSAND), APRIL TO SEPTEMBER 1989

\begin{tabular}{|c|c|c|c|c|c|c|}
\hline DAY & APR & MAY & JUNE & JULY & AUG & SEPT \\
\hline 1 & --- & --- & --- & 0.1 & 0.4 & 0.9 \\
\hline 2 & -- & -- & --- & .1 & .3 & .9 \\
\hline 3 & -- & -- & --- & .1 & .2 & 1.5 \\
\hline 4 & -- & --- & -- & .1 & .2 & 2.4 \\
\hline 5 & --- & --- & --- & .1 & .3 & 2.8 \\
\hline 6 & --- & --- & --- & .1 & .3 & 2.3 \\
\hline 7 & --- & --- & 0.1 & .1 & 1.0 & 2.1 \\
\hline 8 & --- & --- & .1 & .1 & 2.4 & 2.0 \\
\hline 9 & --- & --- & .1 & .1 & 3.4 & 2.4 \\
\hline 10 & --- & --- & .1 & .1 & 3.1 & 2.4 \\
\hline 11 & --- & --- & .1 & .1 & 2.4 & 2.2 \\
\hline 12 & -- & --- & .1 & .1 & 2.4 & 2.1 \\
\hline 13 & --- & --- & .1 & .1 & 3.0 & 2.1 \\
\hline 14 & --- & --- & .1 & .3 & 2.9 & -- \\
\hline 15 & --- & --- & .1 & .8 & 1.8 & -- \\
\hline 16 & --- & --- & .1 & 1.3 & 1.6 & --- \\
\hline 17 & --- & -- & .1 & .3 & .7 & --- \\
\hline 18 & -- & -- & .1 & .1 & .5 & -- \\
\hline 19 & -- & --- & .1 & .1 & .5 & -- \\
\hline 20 & --- & --- & .1 & .1 & .4 & --- \\
\hline 21 & -- & --- & .1 & .1 & .2 & --- \\
\hline 22 & --- & --- & .1 & $<.1$ & .1 & --- \\
\hline 23 & --- & --- & .1 & .1 & .2 & --- \\
\hline 24 & --- & --- & .1 & .1 & .8 & -- \\
\hline 25 & --- & --- & .1 & .1 & 1.0 & --- \\
\hline 26 & --- & --- & --- & .1 & .7 & --- \\
\hline 27 & --- & -- & .1 & .1 & .8 & --- \\
\hline 28 & --- & --- & .1 & .1 & .4 & --- \\
\hline 29 & -- & --- & .1 & .3 & .2 & -- \\
\hline 30 & -- & --- & .1 & .5 & .2 & --- \\
\hline 31 & --- & -- & --- & .3 & .5 & -- \\
\hline
\end{tabular}

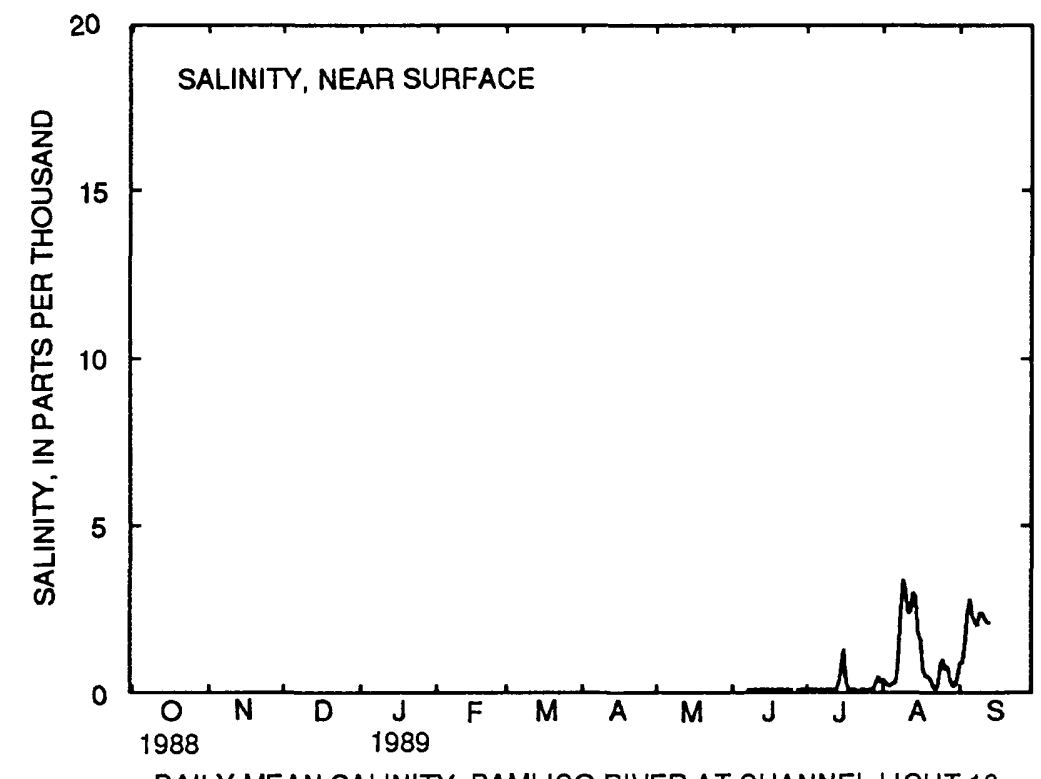


Table 3.--Daily mean values of salinity, water temperature, and dissolved oxygen in water at site 1 , Pamlico River at Channel Light 16, April 1989 to September $1990--$ Continued

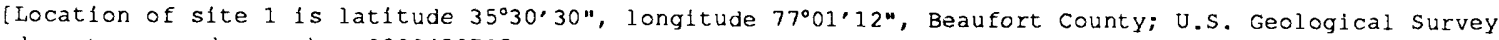
downstream order number 0208450705; ppt, parts per thousand; <, less than; ---, no data; ${ }^{\circ} \mathrm{C}$, degrees Celsius; mg/L, milligrams per liter; a, daily minimum value is less than or equal to $2.0 \mathrm{mg} / \mathrm{L}$; b, daily minimum value is less than or equal to $5.0 \mathrm{mg} / \mathrm{L}$ but greater than $2.0 \mathrm{mg} / \mathrm{L}$ ]

SALINITY, NEAR SURFACE

SENSOR POSITION. --6 feet above streambed.

EXTREMES FOR OCTOBER 1989 TO SEPTEMBER 1990.--Maximum value recorded, 5.1 ppt september 29, 1990 ; minimum value recorded, <0.1 ppt December 6, 15, 17-22, 1989, Apri1 27-30, 1990, on many days in May and June, July 10, August 16, 1990.

DAILY MEAN VALUES OF SALINITY, NEAR SURFACE (IN PARTS PER THOUSAND), WATER YEAR OCTOBER 1989 TO SEPTEMBER 1990

\begin{tabular}{|c|c|c|c|c|c|c|c|c|c|c|c|c|}
\hline DAY & $O C T$ & NOV & DEC & JAN & FEB & MAR & APR & MAY & JUNE & JULY & AUG & SEPT \\
\hline 1 & --- & 0.8 & --- & --- & --- & --- & --- & $<0.1$ & 0.1 & 0.5 & 2.2 & 1.7 \\
\hline 2 & --- & .8 & --- & --- & --- & -- & --- & $<.1$ & $<.1$ & 1.2 & 2.2 & 1.6 \\
\hline 3 & --- & 1.0 & --- & --- & -- & -- & --- & .1 & $<.1$ & 1.4 & 2.6 & 1.3 \\
\hline 4 & --- & 1.2 & --- & --- & -- & --- & --- & .1 & $<.1$ & 1.2 & 2.6 & 2.1 \\
\hline 5 & -- & --- & --- & --- & --- & $-\cdots$ & --- & $<.1$ & --- & .9 & 2.3 & --- \\
\hline 6 & --- & --- & 0.3 & --- & -- & -- & --- & $<.1$ & $<.1$ & .9 & 2.2 & -- \\
\hline 7 & --- & --- & .3 & --- & -- & -- & --- & .1 & --- & 1.3 & 1.8 & 2.0 \\
\hline 8 & -- & --- & .5 & --- & -- & --- & --- & $<.1$ & $<.1$ & 1.1 & 1.6 & 2.1 \\
\hline 9 & --- & --- & 1.5 & --- & -- & --- & --- & .1 & --- & .7 & --- & 2.9 \\
\hline 10 & --- & --- & .6 & --- & --- & --- & --- & $<.1$ & --- & .7 & --- & --- \\
\hline 11 & -- & --- & .2 & --- & $-\cdots$ & -- & --- & $<.1$ & --- & .8 & --- & 2.9 \\
\hline 12 & --- & --- & .1 & --- & --- & --- & --- & .1 & 1.1 & .5 & --- & 2.2 \\
\hline 13 & --- & --- & .1 & --- & --- & -- & --- & .1 & 1.2 & .8 & -- & 2.3 \\
\hline 14 & -- & --- & .1 & --- & --- & $-\infty$ & --- & .1 & 1.3 & .8 & .5 & 2.9 \\
\hline 15 & --- & --- & .1 & --- & -- & --- & --- & $<.1$ & 1.6 & .5 & .6 & 3.5 \\
\hline 16 & --- & --- & $-\cdots$ & --- & $-\cdots$ & --- & --- & --- & 1.2 & .5 & .2 & 3.2 \\
\hline 17 & --- & --- & .1 & --- & --- & $-\infty$ & --- & $<.1$ & .7 & .6 & --- & 3.4 \\
\hline 18 & --- & --- & .1 & --- & -- & $-\sim-$ & --- & $<.1$ & --- & .6 & -- & 4.0 \\
\hline 19 & --- & -- & .1 & --- & --- & -- & --- & $<.1$ & .4 & .3 & 1.2 & 4.1 \\
\hline 20 & --- & --- & .1 & $\cdots$ & --- & $-\infty$ & --- & $<.1$ & .5 & .2 & 1.1 & 3.7 \\
\hline 21 & --- & -- & $<.1$ & --- & --- & --- & --- & --- & .6 & .4 & 1.5 & 4.0 \\
\hline 22 & --- & --- & .1 & --- & -- & --- & --- & .1 & .6 & .5 & 1.4 & 3.6 \\
\hline 23 & --- & --- & -- & --- & -- & --- & --- & $<.1$ & .5 & .4 & 2.1 & 3.2 \\
\hline 24 & --- & --- & $-\cdots$ & --- & --- & -- & --- & $<.1$ & .4 & .8 & 2.2 & 3.2 \\
\hline 25 & --- & --- & --- & --- & --- & -- & --- & $<.1$ & .5 & 1.6 & 1.6 & 3.3 \\
\hline 26 & --- & --- & --- & -- & $\cdots$ & -- & --- & $<.1$ & .5 & 2.3 & --- & 3.1 \\
\hline 27 & --- & --- & -- & --- & -- & -- & $<0.1$ & --- & .2 & 2.6 & -- & 3.2 \\
\hline 28 & 0.3 & --- & --- & --- & $-\cdots$ & --- & $<.1$ & $\cdots$ & .2 & 2.7 & .4 & --- \\
\hline 29 & .7 & --- & --- & --- & --- & $-\infty-$ & .1 & $<.1$ & .2 & 2.7 & .6 & 4.2 \\
\hline 30 & .6 & --- & --- & --- & --- & --- & .1 & $<.1$ & .1 & 2.3 & .8 & --- \\
\hline 31 & .7 & --- & --- & $-\cdots$ & --- & -- & --- & $<.1$ & --- & 2.4 & 1.0 & -- \\
\hline
\end{tabular}

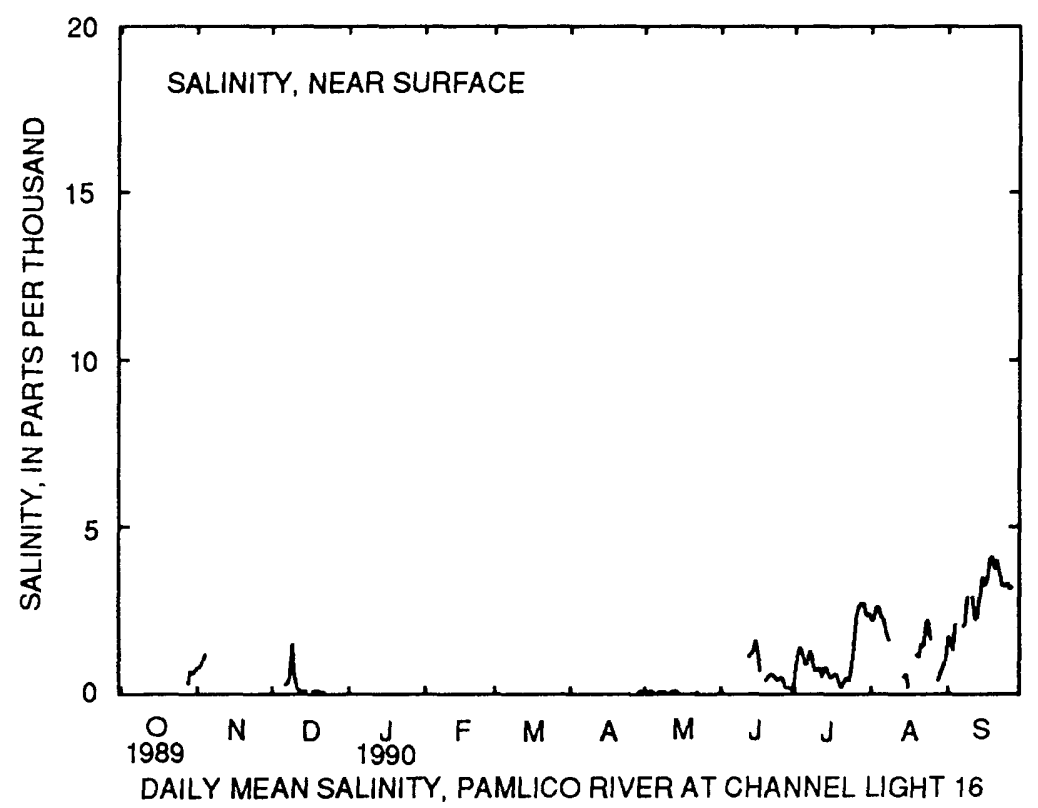


Table 3.--Dally mean values of salinity, water temperature, and dissolved oxygen in water at site 1, Pamlico River at Channel Light 16, April 1989 to September 1990--Continued

[Location of site 1 is latitude $35^{\circ} 30^{\prime} 30^{\prime \prime}$, longitude $77^{\circ} 01^{\prime} 12^{\prime \prime}$, Beaufort County; U.S. Geological Survey downstream order number 0208450705 ; ppt, parts per thousand; <, less than; --., no data; ${ }^{\circ} \mathrm{C}$, degrees Celsius; mg/L, milligrams per liter; a, daily minimum value is less than or equal to $2.0 \mathrm{mg} / \mathrm{L} ; \mathrm{b}$, daily minimum value is less than or equal to $5.0 \mathrm{mg} / \mathrm{L}$ but greater than $2.0 \mathrm{mg} / \mathrm{L}$ ]

SALINITY, NEAR BOTTOM

SENSOR POSITION.--1 foot above streambed.

EXTREMES FOR APRIL TO SEPTEMBER 1989.--Maximum value recorded, 11.0 ppt August 1, 1989; minimum value recorded, $<0.1$ ppt on many days in June and July 1989.

DAILY MEAN VALUES OF SALINITY, NEAR BOTTOM (IN PARTS PER THOUSAND), APRIL TO SEPTEMBER I989

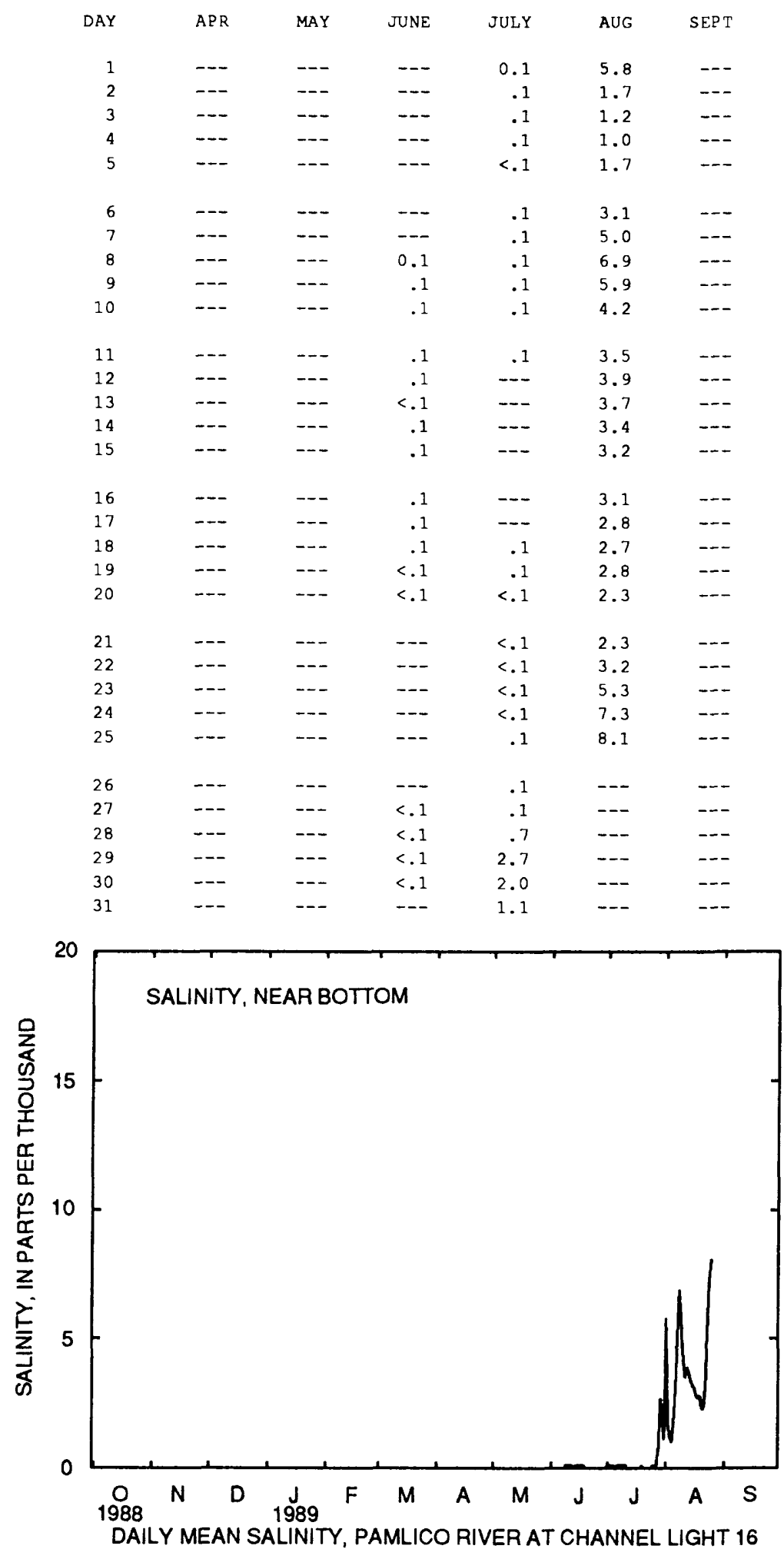


Table 3.--Daily mean values of salinity, water temperature, and dissolved oxygen in water at site 1 , Pamlico River at Channel Light 16, Apr1l 1989 to September 1990--Continued

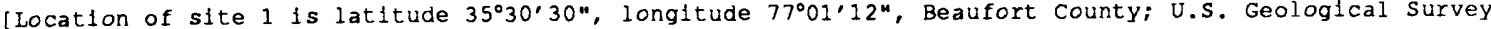
downstream order number 0208450705 ; ppt, parts per thousand; <, less than; ---, no data; ${ }^{\circ} \mathrm{C}$, degrees Celsius; mg/L, milligrams per liter; a, dally minlmum value is less than or equal to $2.0 \mathrm{mg} / \mathrm{L}$; b, daily minlmum value $1 \mathrm{~s}$ less than or equal to $5.0 \mathrm{mg} / \mathrm{L}$ but greater than $2.0 \mathrm{mg} / \mathrm{L}$ ]

SALINITY, NEAR BOTTOM

SENSOR POSITION.--1 foot above streambed.

EXTREMES FOR OCTOBER 1989 TO SEPTEMBER 1990.--Max1mum value recorded, 10.6 ppt August 31, $1990 ;$ minimum value recorded, <0.1 ppt on many days during November, December 1989; January, Apr11, May, and June 1990.

DAILY MEAN VALUES OF SALINITY, NEAR BOTTOM (IN PARTS PER THOUSAND), OCTOBER 1989 TO SEPTEMBER 1990

\begin{tabular}{|c|c|c|c|c|c|c|c|c|c|c|c|c|}
\hline DAY & OCT & NOV & $\mathrm{DEC}$ & JAN & FEB & MAR & APR & MAY & JUNE & JULY & AUG & SEPT \\
\hline 1 & --- & 3.8 & 2.5 & -- & -- & -- & $\cdots$ & $<0.1$ & 0.1 & 2.3 & 3.1 & 10.0 \\
\hline 2 & -- & 3.8 & 4.1 & --- & -- & --- & -- & $<.1$ & .1 & 2.0 & 3.7 & 7.8 \\
\hline 3 & --- & --- & 1.1 & -- & $\cdots$ & -- & --- & .1 & .1 & 2.6 & 2.9 & 6.0 \\
\hline 4 & --- & -- & 1.7 & 6.5 & -- & -- & $-\cdots$ & $<.1$ & .1 & 1.9 & 2.6 & 6.4 \\
\hline 5 & -- & --- & 2.6 & 5.1 & $-\cdots$ & --- & --- & $<.1$ & --- & 1.6 & 2.4 & --- \\
\hline 6 & --- & --- & 3.4 & 9.0 & -- & --- & --- & $<.1$ & .1 & 2.2 & 2.3 & $-\cdots$ \\
\hline 7 & -- & --- & 4.2 & 9.5 & -- & -- & -- & $<.1$ & .1 & 2.5 & 2.2 & 4.0 \\
\hline 8 & $-\infty$ & --- & 3.7 & 7.3 & $-\cdots$ & --- & --- & $<.1$ & .1 & 1.6 & 2.5 & 5.0 \\
\hline 9 & --- & --- & 1.6 & 1.5 & --- & --- & -- & $<.1$ & .1 & 1.5 & --- & 4.4 \\
\hline 10 & --- & --- & 2.5 & .1 & -- & --- & --- & $<.1$ & .3 & 1.4 & --- & -- \\
\hline 11 & --- & -- & 3.4 & .1 & -- & --- & -- & $<.1$ & --- & 1.7 & --- & 5.0 \\
\hline 12 & --- & --- & 2.8 & .1 & --- & --- & --- & .1 & 1.0 & 1.3 & -- & 5.7 \\
\hline 13 & -- & --- & 1.4 & .1 & -- & --- & --- & .1 & 1.2 & 1.3 & -- & 5.8 \\
\hline 14 & --- & -- & 3.1 & .1 & -- & --- & --- & .1 & 1.2 & 1.4 & 3.4 & 5.6 \\
\hline 15 & --- & --- & .1 & .1 & --- & --- & --- & .1 & 1.0 & .9 & 2.8 & 5.3 \\
\hline 16 & -- & $<.1$ & $<.1$ & .1 & -- & --- & -- & --- & .9 & 1.3 & 3.1 & 6.5 \\
\hline 17 & --- & .1 & $<.1$ & .6 & --- & --- & --- & .1 & 1.0 & 1.7 & $-\cdots$ & 7.1 \\
\hline 18 & --- & .1 & .1 & 2.1 & --- & -- & --- & .1 & --- & 1.9 & -- & 6.5 \\
\hline 19 & --- & .1 & .1 & 8.1 & -- & --- & -- & .1 & .8 & 1.6 & 4.8 & 6.0 \\
\hline 20 & --- & .4 & .1 & 7.9 & $\cdots$ & --- & $-\infty$ & .1 & 1.1 & 1.7 & 4.6 & 5.2 \\
\hline 21 & --- & .2 & $<.1$ & 4.2 & --- & -- & --- & --- & 1.4 & 1.9 & 5.3 & 4.9 \\
\hline 22 & --- & .2 & 1.6 & 6.2 & --- & --- & $-\cdots$ & .1 & 1.2 & 1.5 & 5.4 & 4.5 \\
\hline 23 & --- & .2 & --- & --- & --- & --- & --- & .1 & .6 & 2.3 & 5.0 & 5.1 \\
\hline 24 & --- & .6 & --- & --- & -- & --- & -- & .1 & .6 & 3.4 & 3.8 & 4.7 \\
\hline 25 & -- & .7 & -- & $-\cdots$ & --- & --- & --- & .1 & .7 & 3.4 & 3.8 & 5.7 \\
\hline 26 & -- & .2 & -- & --- & -- & --- & -- & $<.1$ & .6 & 3.3 & -- & 5.3 \\
\hline 27 & -- & $<.1$ & --- & --- & $\cdots$ & --- & $<0.1$ & --- & .5 & 3.4 & $-\infty$ & 6.5 \\
\hline 28 & 3.7 & --- & --- & --- & $\cdots$ & --- & $<.1$ & .1 & .6 & 3.0 & 6.6 & --- \\
\hline 29 & 3.8 & .4 & --- & --- & -- & --- & $<.1$ & $<.1$ & .3 & 3.0 & 7.3 & 7.3 \\
\hline 30 & 3.6 & 1.5 & --- & -- & -- & --- & $<.1$ & $<.1$ & .7 & 2.8 & 9.4 & --- \\
\hline 31 & 3.6 & --- & --- & --- & -- & --- & --- & $<.1$ & --- & 2.6 & 10.3 & $-\sim$ \\
\hline
\end{tabular}

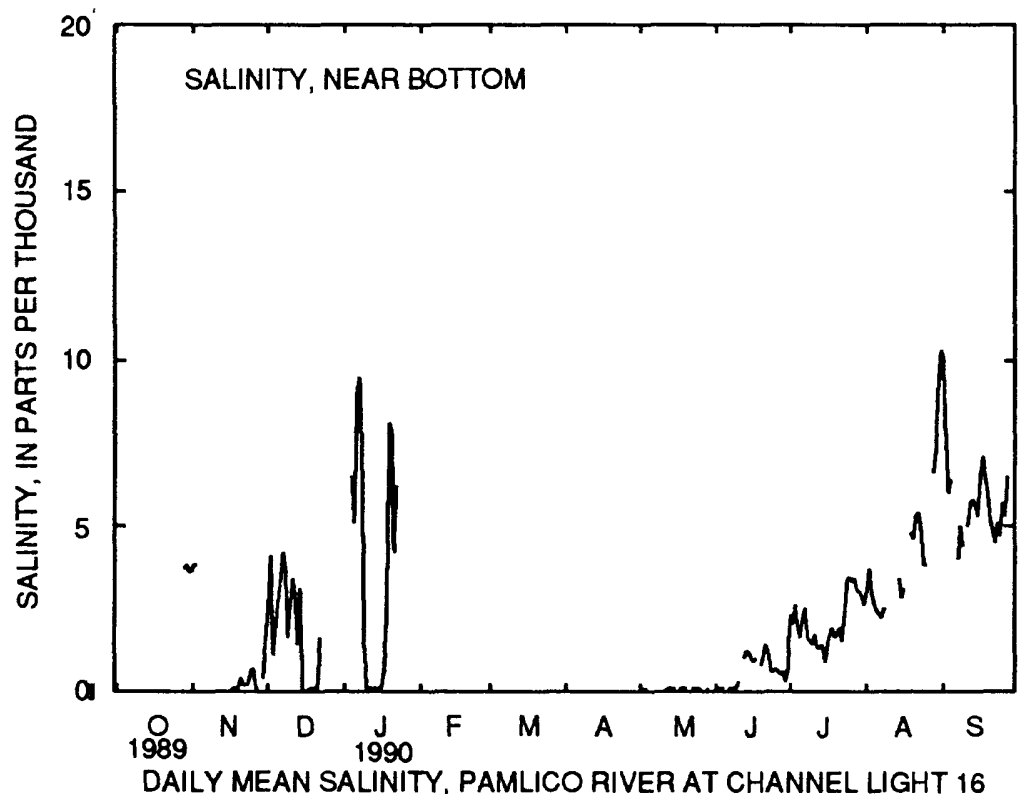


Table 3.--Daily mean values of salinity, water temperature, and dissolved oxygen in water at site 1, Pamlico River at Channel Light 16, April 1989 to September 1990--Continued

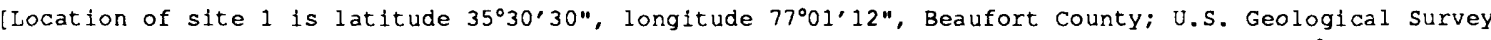
downstream order number 0208450705 ; ppt, parts per thousand; <, less than; ---, no data; ${ }^{\circ} \mathrm{C}$, degrees Celsius; mg/L, milligrams per liter; a, daily minimum value is less than or equal to $2.0 \mathrm{mg} / \mathrm{L} ; \mathrm{b}$, daily minimum value is less than or equal to $5.0 \mathrm{mg} / \mathrm{L}$ but greater than $2.0 \mathrm{mg} / \mathrm{L}$ ]

WATER TEMPERATURE, NEAR SURFACE

SENSOR POSITION.--6 feet above streambed.

EXTREMES FOR APRIL TO SEPTEMBER 1989.--Maximum value recorded, $31.0{ }^{\circ} \mathrm{C}$ July 12 , 1989 ; minimum value recorded, $20.7^{\circ} \mathrm{C}$ April 28, 1989.

DAILY MEAN VALUES OF WATER TEMPERATURE, NEAR SURFACE (IN DEGREES CELSIUS), APRIL TO SEPTEMBER 1989

\begin{tabular}{|c|c|c|c|c|c|c|}
\hline DAY & $A P R$ & MAY & JUNE & JULY & AUG & SEPT \\
\hline 1 & --- & 22.2 & $\cdots$ & 24.9 & 26.0 & 28.1 \\
\hline 2 & --- & 22.9 & --- & 25.3 & 26.7 & 28.4 \\
\hline 3 & -- & 22.5 & --- & 25.7 & 27.9 & 27.9 \\
\hline 4 & --- & --- & --- & 25.4 & 28.0 & 26.3 \\
\hline 5 & --- & --- & --- & 25.3 & 28.1 & 25.6 \\
\hline 6 & --- & --- & --- & 25.2 & 28.6 & 25.6 \\
\hline 7 & --- & --- & --- & 25.6 & 28.9 & 25.5 \\
\hline 8 & --- & --- & 25.8 & 26.4 & 27.0 & 26.0 \\
\hline 9 & --- & --- & 25.4 & 26.7 & 25.4 & 26.0 \\
\hline 10 & --- & -+ & 25.4 & 27.0 & 23.8 & 26.7 \\
\hline 11 & --- & --- & 25.6 & 28.0 & 23.5 & 27.4 \\
\hline 12 & --- & --- & 25.2 & 29.1 & 23.7 & 28.1 \\
\hline 13 & --- & --- & 25.3 & 27.9 & 24.1 & 28.2 \\
\hline 14 & --- & -- & 26.2 & 26.8 & 25.2 & $-\cdots$ \\
\hline 15 & --- & --- & 26.7 & 26.6 & 25.2 & -- \\
\hline 16 & --- & --- & 26.6 & 25.9 & 25.4 & $-\cdots$ \\
\hline 17 & --- & --- & 26.0 & 25.1 & 26.2 & --- \\
\hline 18 & --- & $-\cdots$ & 26.4 & 24.9 & 26.6 & $-\cdots$ \\
\hline 19 & --- & $-\infty$ & 26.9 & 25.1 & 26.3 & -- \\
\hline 20 & --- & --- & 26.5 & 25.5 & 26.6 & --- \\
\hline 21 & --- & $-\cdots$ & 25.8 & 25.5 & 26.8 & -- \\
\hline 22 & --- & --- & 25.7 & 25.3 & 26.7 & --- \\
\hline 23 & --- & --- & 25.6 & 26.7 & 27.5 & --- \\
\hline 24 & --- & --- & 24.9 & 27.2 & 27.2 & --- \\
\hline 25 & --- & --- & 25.3 & 26.7 & 27.3 & --- \\
\hline 26 & --- & --- & --- & 27.2 & 26.8 & -- \\
\hline 27 & --- & --- & --- & 26.9 & 27.0 & --- \\
\hline 28 & 21.7 & --- & --- & 26.8 & 26.7 & $-\cdots$ \\
\hline 29 & 21.8 & --- & --- & 26.6 & 27.3 & $-\cdots$ \\
\hline 30 & 22.1 & --- & --- & 25.7 & 28.0 & -- \\
\hline 31 & --- & --- & --- & 26.0 & --- & -- \\
\hline
\end{tabular}

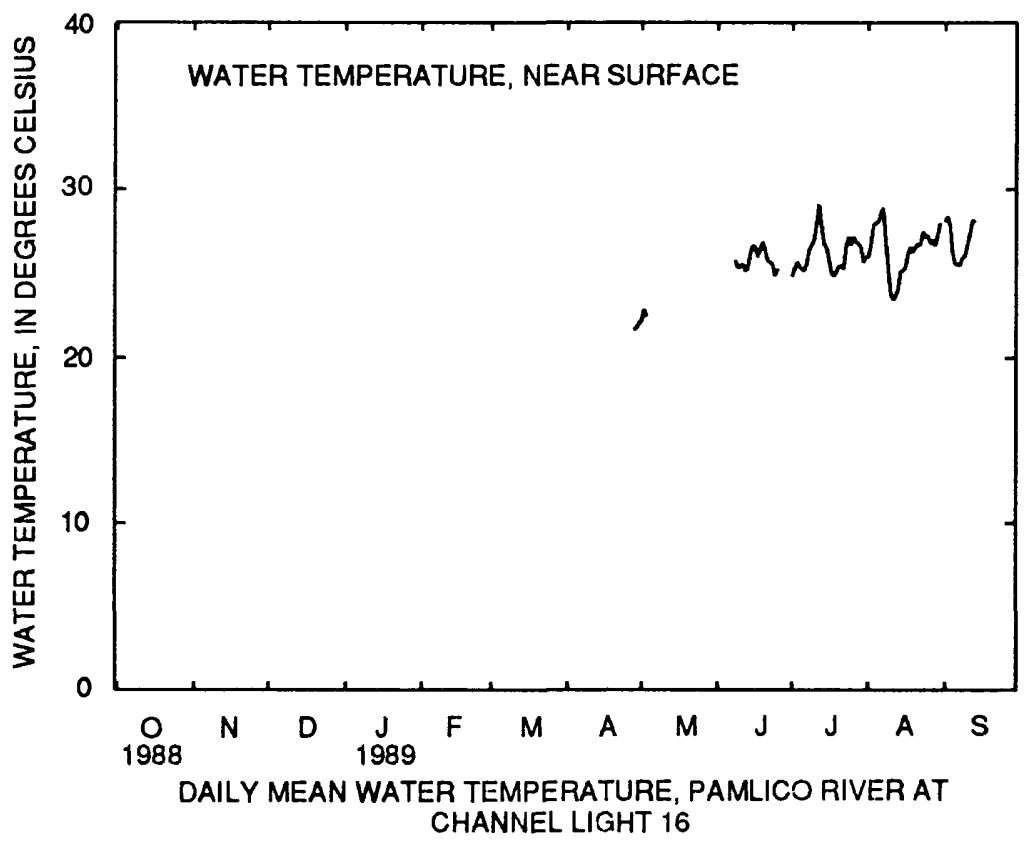


Table 3.--Daily mean values of salinity, water temperature, and dissolved oxygen in water at site 1 , Pamlico River at Channel Light 16, April 1989 to September 1990--Continued

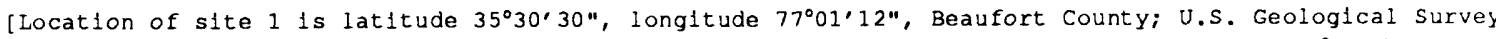
downstream order number 0208450705; ppt, parts per thousand; <, less than; ---, no data; ${ }^{\circ} \mathrm{C}$, degrees Celsius; mg/L, milligrams per liter; a, daily minimum value is less than or equal to $2.0 \mathrm{mg} / \mathrm{L}$; b, daily minimum value is less than or equal to $5.0 \mathrm{mg} / \mathrm{L}$ but greater than $2.0 \mathrm{mg} / \mathrm{L}$ ]

WATER TEMPERATURE, NEAR SURFACE

SENSOR POSITION. --6 feet above streambed.

EXTREMES FOR OCTOBER 1989 TO SEPTEMBER 1990.--Max1mum value recorded, $33.3^{\circ} \mathrm{C}$ August 14 , 1990 ; m1n1mum value recorded, $1.0{ }^{\circ} \mathrm{C}$ December 4, 21, 22, 1989, January 13, 1990.

DAILY MEAN VALUES OF WATER TEMPERATURE, NEAR SURFACE (IN DEGREES CELSIUS), OCTOBER 1989 TO SEPTEMBER 1990

\begin{tabular}{|c|c|c|c|c|c|c|c|c|c|c|c|c|}
\hline DAY & OCT & NOV & $\mathrm{DEC}$ & JAN & FEB & MAR & APR & MAY & JUNE & JULY & AUG & SEPT \\
\hline 1 & --- & 18.4 & 9.2 & --- & 10.5 & 9.0 & -- & 23.8 & 23.7 & 29.1 & 29.9 & 27.1 \\
\hline 2 & --- & 17.8 & 9.1 & --- & 11.5 & 9.4 & --- & 23.9 & 23.6 & 28.3 & 29.5 & 27.4 \\
\hline 3 & $-\cdots$ & 17.2 & 4.7 & --- & 12.3 & 10.3 & -- & 23.5 & 23.9 & 28.4 & 29.2 & 27.8 \\
\hline 4 & --- & 16.4 & 4.2 & 5.9 & 12.8 & 10.8 & -- & 22.5 & 24.8 & 28.4 & 29.2 & 27.3 \\
\hline 5 & --- & --- & 5.2 & 6.4 & 12.1 & 11.3 & --- & 22.4 & --- & 28.6 & 28.4 & $\cdots$ \\
\hline 6 & --- & --- & 6.5 & 6.3 & 11.4 & 12.0 & --- & --- & 24.9 & 29.3 & 28.2 & -- \\
\hline 7 & --- & --- & 7.8 & 6.2 & 11.8 & 11.2 & --- & 21.0 & 25.5 & 29.0 & 27.9 & 28.0 \\
\hline 8 & --- & --- & 7.5 & 6.3 & 12.1 & 10.8 & -- & 21.4 & 27.3 & 28.9 & 27.4 & 28.1 \\
\hline 9 & --- & --- & 6.3 & 6.8 & 12.0 & 11.1 & --- & 21.3 & 27.4 & 29.7 & --- & 27.2 \\
\hline 10 & --- & --- & 6.0 & 7.3 & 13.1 & 12.5 & --- & 21.0 & 28.0 & 29.9 & $\cdots$ & -- \\
\hline 11 & -- & --- & 5.4 & 7.1 & 13.2 & 13.7 & --- & 19.0 & --- & 30.2 & --- & 28.0 \\
\hline 12 & --- & --- & 4.9 & 6.8 & 11.8 & 15.1 & --- & 20.9 & 25.5 & 30.2 & --- & 27.7 \\
\hline 13 & -- & -- & 4.3 & 4.4 & 11.9 & --- & -- & 21.4 & 25.9 & 29.5 & --- & 27.4 \\
\hline 14 & --- & --- & 4.2 & 6.0 & 12.5 & --- & --- & 22.6 & 26.2 & 29.0 & 29.4 & 27.3 \\
\hline 15 & -- & --- & 4.4 & 5.8 & 12.7 & --- & --- & 23.4 & --- & 28.2 & 29.0 & 26.9 \\
\hline 16 & --- & 16.3 & 3.8 & 5.9 & 13.7 & --- & --- & --- & --- & 27.9 & 29.0 & 27.0 \\
\hline 17 & --- & 15.3 & 3.3 & 7.0 & 14.1 & --- & --- & 24.0 & --- & 28.2 & --- & 25.6 \\
\hline 18 & --- & 13.6 & 2.9 & 7.9 & 13.2 & -- & -- & 24.0 & --- & 28.7 & --- & 23.9 \\
\hline 19 & $\cdots$ & 13.1 & 2.5 & 8.4 & 13.4 & --- & -- & 23.8 & 25.8 & 28.9 & 29.3 & 23.2 \\
\hline 20 & --- & 13.0 & 2.4 & 8.5 & 13.0 & --- & --- & 23.9 & 26.1 & 29.0 & 29.5 & 23.8 \\
\hline 21 & --- & 12.0 & 2.2 & 10.5 & 12.1 & --- & $-\cdots$ & -- & 26.6 & 29.3 & 28.8 & 23.3 \\
\hline 22 & -- & 11.1 & 1.4 & 10.3 & 12.1 & --- & --- & 23.9 & 27.4 & 29.7 & 28.5 & 23.2 \\
\hline 23 & --- & 9.6 & --- & 9.9 & 12.3 & --- & -- & 22.2 & 27.0 & 30.6 & 28.5 & 23.4 \\
\hline 24 & -- & 8.5 & --- & 10.4 & 9.9 & --- & $-\cdots$ & 23.1 & 27.2 & 30.3 & 29.0 & 22.0 \\
\hline 25 & --- & 8.7 & --- & 11.4 & --- & --- & -- & 23.4 & 27.7 & 29.6 & 29.2 & 21.4 \\
\hline 26 & --- & 9.6 & --- & 9.5 & 9.7 & --- & -- & 23.6 & 27.5 & 29.0 & -- & 21.9 \\
\hline 27 & -- & 9.8 & --- & 10.0 & 9.2 & --- & 24.7 & --- & 28.1 & 28.8 & -- & 22.3 \\
\hline 28 & 16.0 & --- & --- & 10.4 & 8.8 & $-\cdots$ & 23.7 & 23.9 & 28.5 & 28.7 & 28.5 & -- \\
\hline 29 & 16.8 & 10.1 & --- & 10.6 & --- & -- & 23.3 & 23.2 & 28.7 & 28.9 & 28.1 & 22.3 \\
\hline 30 & 17.2 & 9.1 & -- & 9.9 & $-\cdots$ & -- & 22.7 & 22.9 & 28.9 & 29.6 & 27.1 & -- \\
\hline $3 I$ & 18.1 & --- & $-\cdots$ & 10.1 & -- & --- & --- & 23.4 & +- & 29.5 & 26.8 & $-\cdots$ \\
\hline
\end{tabular}

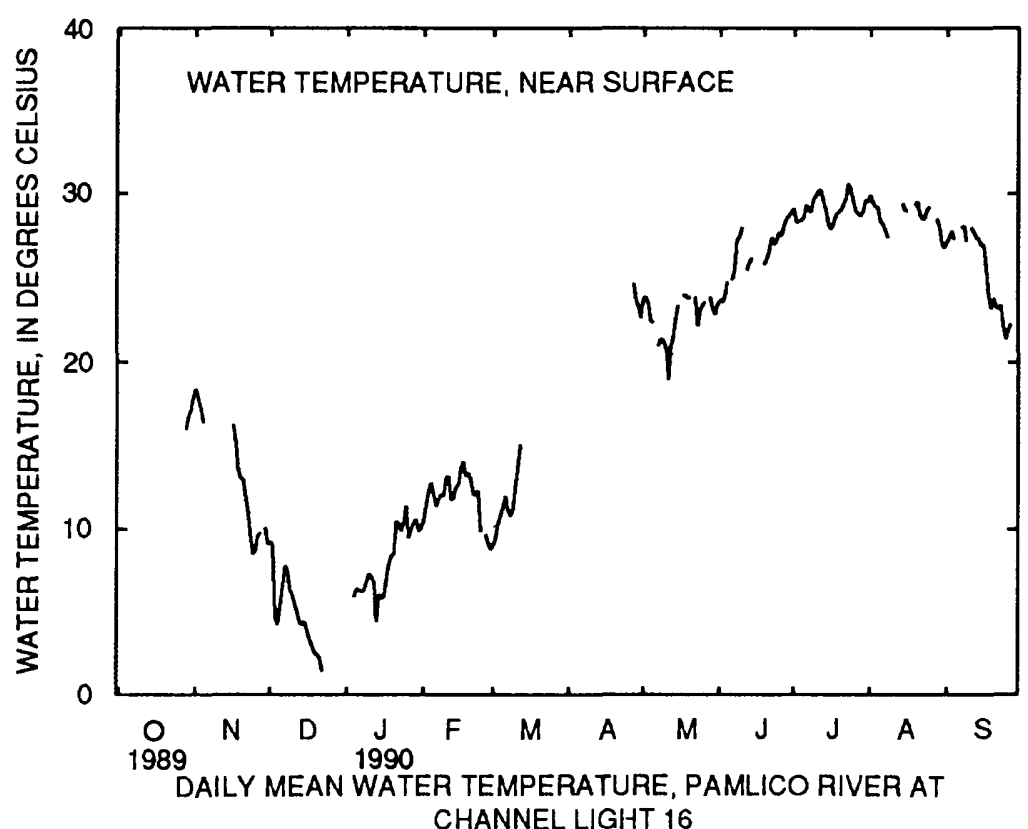


Table 3.--Daily mean values of salinity, water temperature, and dissolved oxygen in water at site 1 , Pamlico River at Channel Light 16, April 1989 to September 1990--Continued

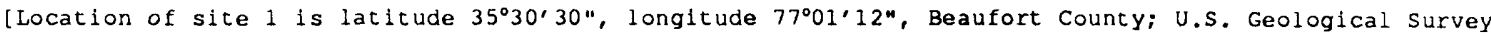
downst ream order number 0208450705 ; ppt, parts per thousand; <, less than; -.-, no data; ${ }^{\circ} \mathrm{C}$, degrees Celsius; mg/L, milligrams per liter; a, daily minimum value is less than or equal to $2.0 \mathrm{mg} / \mathrm{L}$; b, daily $\mathrm{minimum}$ value is less than or equal to $5.0 \mathrm{mg} / \mathrm{L}$ but greater than $2.0 \mathrm{mg} / \mathrm{L}$ ]

DISSOLVED OXYGEN, NEAR SURFACE

SENSOR POSITION. --6 feet above streambed.

EXTREMES FOR APRIL TO SEPTEMBER 1989.--Maximum value recorded, $12.3 \mathrm{mg} / \mathrm{L}$ July 11 , 1989 ; minimum value recorded, $1.4 \mathrm{mg} / \mathrm{L}$ August $7,1989$.

DAILY MEAN VALUES OF DISSOLVED OXYGEN, NEAR SURFACE (IN MILLIGRAMS PER LITER), APRIL TO SEPTEMBER 1989

\begin{tabular}{|c|c|c|c|c|c|c|}
\hline DAY & APR & MAY & JUNE & JULY & AUG & SEPT \\
\hline 1 & --- & 5.5 & $-\infty$ & $5.3 b$ & 8.7 & $6.2 \mathrm{~b}$ \\
\hline 2 & $-\infty$ & $5.4 \mathrm{~b}$ & $-\infty$ & $5.3 b$ & 8.5 & $7.0 \mathrm{~b}$ \\
\hline 3 & $-\infty$ & --- & $-\cdots$ & $5.8 \mathrm{~b}$ & 8.4 & 7.4 \\
\hline 4 & $-\infty$ & -- & -- & -- & 8.2 & 7.2 \\
\hline 5 & $-\infty$ & $-\cdots$ & $-\infty$ & --- & -- & 6.7 \\
\hline 6 & -- & $\ldots$ & $-\cdots$ & 6.7 & $6.4 \mathrm{~b}$ & 7.2 \\
\hline 7 & $-\cdots$ & -- & $5.6 \mathrm{~b}$ & 6.8 & $5.1 a$ & 7.4 \\
\hline 8 & --- & -- & $5.1 \mathrm{~b}$ & 7.4 & $5.5 b$ & $6.8 \mathrm{~b}$ \\
\hline 9 & --- & -- & $4.5 b$ & 8.6 & $5.9 a$ & $7.1 \mathrm{~b}$ \\
\hline 10 & --- & -- & $4.9 b$ & 8.2 & 5.7 & $7.9 b$ \\
\hline 12 & -- & -- & $5.0 \mathrm{~b}$ & 9.2 & 5.8 & $8.1 b$ \\
\hline 12 & -- & --- & $5.1 \mathrm{~b}$ & $8.1 b$ & 6.0 & 8.2 \\
\hline 13 & -- & --- & $5.1 \mathrm{~b}$ & $6.6 b$ & $6.1 \mathrm{~b}$ & 8.2 \\
\hline 14 & --- & --- & $5.5 b$ & 7.0 & 9.3 & -- \\
\hline 15 & --- & $\cdots$ & $5.1 \mathrm{~b}$ & $8.1 \mathrm{~b}$ & 7.5 & --- \\
\hline 16 & $-\cdots$ & -- & $5.1 \mathrm{~b}$ & 7.5 & $6.6 \mathrm{~b}$ & -- \\
\hline 17 & -- & $-\cdots$ & $5.3 b$ & 6.7 & $7.1 \mathrm{~b}$ & $-\cdots$ \\
\hline 18 & -- & -- & $5.2 b$ & $5.6 \mathrm{~b}$ & $6.5 b$ & -- \\
\hline 19 & $\cdots$ & -- & $5.6 \mathrm{~b}$ & $5.2 b$ & $5.3 b$ & -- \\
\hline 20 & --- & --- & 5.8 & $5.3 b$ & $5.1 \mathrm{~b}$ & $\cdots$ \\
\hline 21 & -- & --- & 5.4 & $5.2 \mathrm{~b}$ & $4.9 \mathrm{~b}$ & -- \\
\hline 22 & -- & -- & 5.6 & $5.2 b$ & $5.0 \mathrm{~b}$ & -- \\
\hline 23 & --- & -- & $5.5 b$ & $5.0 \mathrm{~b}$ & $4.7 \mathrm{~b}$ & -- \\
\hline 24 & --- & --- & $5.3 b$ & $5.0 \mathrm{~b}$ & -- & --- \\
\hline 25 & --- & --- & $5.2 b$ & $5.1 \mathrm{~b}$ & $-\infty$ & $-\cdots$ \\
\hline 26 & -- & -- & -- & $6.0 \mathrm{~b}$ & $4.7 \mathrm{~b}$ & -- \\
\hline 27 & --- & -- & $4.8 b$ & 7.5 & $5.8 b$ & -- \\
\hline 28 & 6.8 & --- & $5.0 \mathrm{~b}$ & 8.0 & $5.0 \mathrm{~b}$ & $\ldots$ \\
\hline 29 & 6.4 & --- & $5.0 \mathrm{~b}$ & 8.1 & $5.0 \mathrm{~b}$ & --- \\
\hline 30 & 5.9 & -- & $5.2 \mathrm{~b}$ & 8.5 & $5.9 b$ & $\cdots$ \\
\hline 31 & -- & -- & -- & 8.6 & $5.6 \mathrm{~b}$ & --- \\
\hline
\end{tabular}

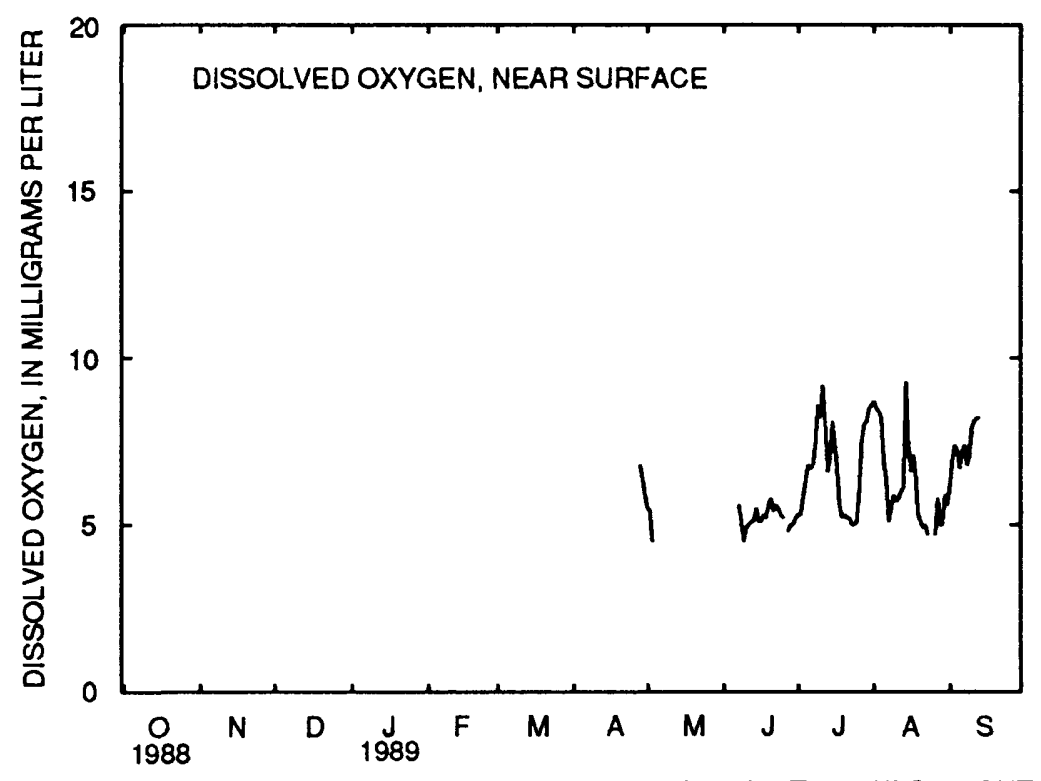


Table 3.--Daily mean values of salinity, water temperature, and dissolved oxygen in water at site 1 , Pamlico River at Channel Light 16, April 1989 to September 1990--Continued

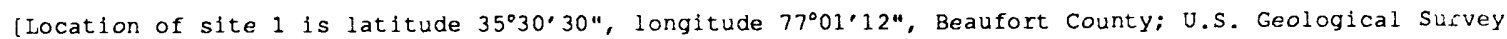
downstream order number 0208450705; ppt, parts per thousand; <, less than; ---, no data; ${ }^{\circ} \mathrm{C}$, degrees Celsius; mg/L, milligrams per liter; a, daily minimum value is less than or equal to $2.0 \mathrm{mg} / \mathrm{L}$; b, daily minimum value is less than or equal to $5.0 \mathrm{mg} / \mathrm{L}$ but greater than $2.0 \mathrm{mg} / \mathrm{L}$ ]

DISSOLVED OXYGEN, NEAR SURFACE

SENSOR POSITION. --6 feet above streambed.

EXTREMES FOR OCTOBER 1989 TO SEPTEMBER 1990.--Maximum value recorded, 15.0 mg/L December 3, 4, 1989 ; minimum value recorded, $2.9 \mathrm{mg} / \mathrm{L}$ September $15,1990$.

DAILY MEAN VALUES OF DISSOLVED OXYGEN, NEAR SURFACE (IN MILLIGRAMS PER LITER), OCTOBER 1989 TO SEPTEMBER 1990

\begin{tabular}{|c|c|c|c|c|c|c|c|c|c|c|c|c|}
\hline DAY & OCT & NOV & DEC & JAN & FEB & MAR & APR & MAY & JUNE & JULY & AUG & SEPT \\
\hline 1 & --- & $7.2 \mathrm{~b}$ & 10.3 & --- & 10.0 & 9.5 & --- & 8.4 & 6.1 & 8.0 & 7.8 & $6.4 \mathrm{~b}$ \\
\hline 2 & -- & 7.8 & 10.2 & -- & -- & 9.5 & -- & 8.3 & 6.4 & 7.4 & 7.9 & 7.5 \\
\hline 3 & --- & 6.9 & 11.4 & --- & --- & 9.3 & --- & 8.2 & 6.9 & 7.8 & 6.8 & 7.3 \\
\hline 4 & --- & 7.0 & 12.2 & 12.7 & $\cdots$ & 9.3 & --- & 7.2 & 7.0 & 7.9 & 7.1 & $7.9 b$ \\
\hline 5 & --- & -- & 11.9 & 12.8 & -- & 9.1 & --- & 6.9 & -- & 8.4 & 6.6 & --- \\
\hline 6 & -- & --- & 11.5 & 13.0 & 9.5 & 8.9 & -- & 7.8 & 7.5 & 8.2 & 6.2 & --- \\
\hline 7 & --- & --- & 11.5 & 12.7 & --- & 9.4 & --- & 7.1 & 6.7 & 7.1 & 6.6 & 9.6 \\
\hline 8 & --- & --- & 11.6 & 12.3 & 9.1 & 9.6 & --- & 6.9 & 7.3 & 7.2 & 6.3 & 9.2 \\
\hline 9 & --- & --- & 11.5 & 11.8 & 9.3 & 9.4 & --- & 6.7 & 7.0 & 7.3 & --- & 7.7 \\
\hline 10 & --- & --- & 11.7 & 11.4 & --- & 9.4 & --- & 7.2 & 7.5 & 7.4 & $\cdots$ & --- \\
\hline 11 & -- & --- & 11.1 & 11.5 & 9.8 & 9.2 & --- & 8.1 & --- & 7.1 & --- & $5.8 b$ \\
\hline 12 & -- & --- & 11.1 & 11.6 & --- & 9.1 & --- & 8.3 & 8.1 & $7.0 \mathrm{~b}$ & --- & $6.5 b$ \\
\hline 13 & -- & --- & 11.9 & 12.1 & 9.1 & --- & --- & 8.0 & 10.1 & 6.7 & $-\cdots$ & $6.1 \mathrm{~b}$ \\
\hline 14 & $\cdots$ & --- & 11.7 & 11.3 & 8.9 & --- & --- & 9.1 & 10.0 & 6.6 & $7.4 \mathrm{~b}$ & $6.1 \mathrm{~b}$ \\
\hline 15 & -- & --- & 11.5 & 11.2 & 8.9 & --- & --- & 10.2 & 9.5 & 6.6 & $7.1 \mathrm{~b}$ & $5.6 b$ \\
\hline 16 & --- & 8.2 & 12.1 & 11.2 & 8.9 & -- & --- & --- & 10.8 & 7.0 & 6.6 & 7.7 \\
\hline 17 & --- & 9.1 & 11.8 & 11.1 & 8.5 & -- & --- & 8.2 & 11.1 & 7.5 & --- & $6.5 b$ \\
\hline 18 & --- & 8.7 & 11.9 & 11.2 & 8.3 & --- & --- & 8.5 & -- & 7.9 & --- & 7.5 \\
\hline 19 & -- & 8.1 & 12.2 & 11.2 & 7.9 & --- & --- & 9.2 & 9.1 & 8.6 & $6.8 \mathrm{~b}$ & 6.9 \\
\hline 20 & --- & 7.9 & 12.4 & 11.0 & 8.0 & -- & -- & 8.9 & 9.0 & 8.9 & $6.9 b$ & 7.1 \\
\hline 21 & --- & 10.0 & 12.4 & 10.5 & 8.6 & --- & -- & --- & 9.1 & 8.9 & E. 4 & $5.9 b$ \\
\hline 22 & -- & 10.0 & 12.8 & 10.1 & 8.7 & $-\cdots$ & --- & 8.5 & 8.9 & 9.1 & $6.6 b$ & $5.6 b$ \\
\hline 23 & -- & 10.5 & -- & 10.0 & 8.5 & --- & --- & 8.5 & 7.7 & 9.2 & 7.0 & 6.2 \\
\hline 24 & -- & 10.7 & -- & 10.0 & 9.2 & -- & -- & 8.9 & 8.3 & 9.4 & $6.9 b$ & 6.4 \\
\hline 25 & --- & 10.2 & --- & 9.9 & 10.6 & --- & --- & 8.7 & 8.8 & 8.8 & 7.2 & 7.6 \\
\hline 26 & --- & 10.1 & --- & --- & 8.5 & $-\cdots$ & -- & 7.7 & 8.0 & 7.7 & --- & 8.0 \\
\hline 27 & --- & 10.6 & -- & 10.2 & 8.6 & --- & 10.6 & -- & 8.8 & 7.1 & --- & 8.2 \\
\hline 28 & 7.1 & --- & -- & 9.3 & 9.0 & --- & 8.7 & 8.4 & 8.4 & 6.7 & $5.1 \mathrm{~b}$ & -- \\
\hline 29 & 7.6 & 10.9 & --- & -- & $\cdots$ & -- & 8.4 & 7.7 & 8.1 & 7.2 & $4.8 \mathrm{~b}$ & 7.1 \\
\hline 30 & 7.2 & 10.7 & --- & --- & --- & --- & 8.2 & 7.6 & 7.8 & 7.8 & $5.1 \mathrm{~b}$ & -- \\
\hline 31 & 7.7 & --- & -- & 10.1 & --- & --- & -- & 6.7 & -- & 7.4 & $5.6 b$ & $-\cdots$ \\
\hline
\end{tabular}

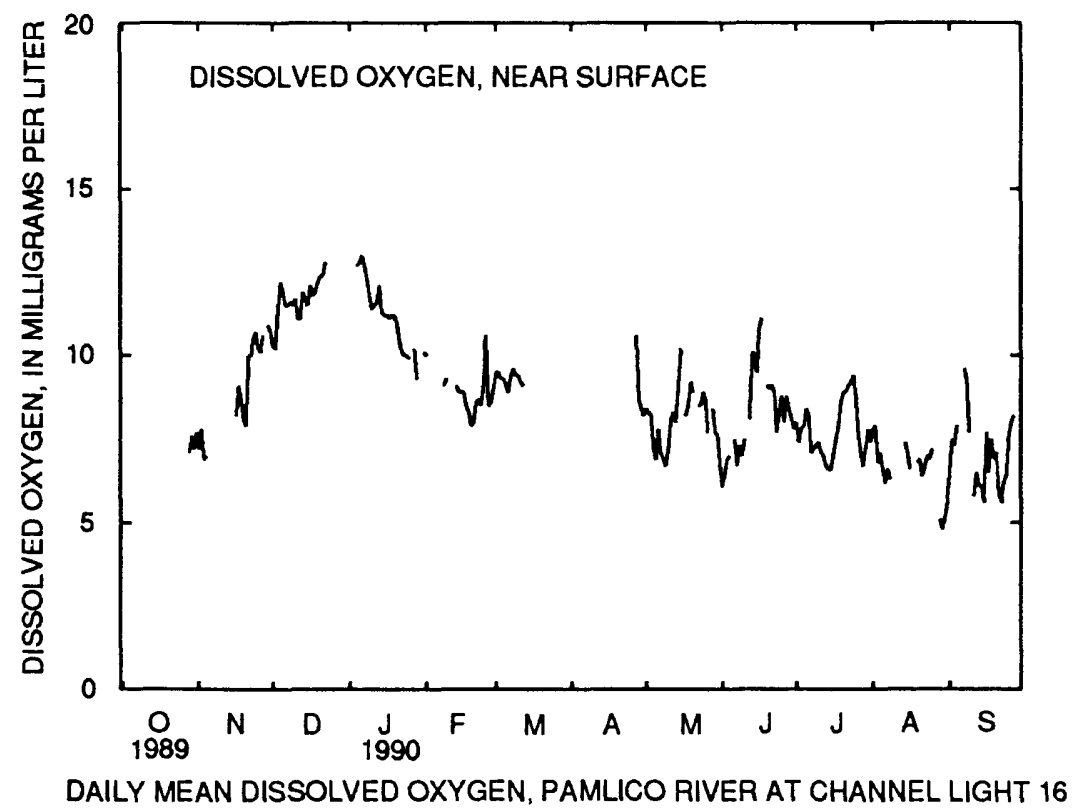


Table 3.--Daily mean values of salinity, water temperature, and dissolved oxygen in water at site 1 , Pamlico River at Channel Light 16, April 1989 to September 1990--Continued

[Location of site 1 is latitude 35\%30'30", longitude $77^{\circ} 01^{\prime} 12^{\prime \prime}$, Beaufort County; U.S. Geological Survey downstream order number 0208450705; ppt, parts per thousand; <, less than; ---, no data; ${ }^{\circ} \mathrm{C}$, degrees Celsius; mg/L, milligrams per liter; a, daily minimum value is less than or equal to $2.0 \mathrm{mg} / \mathrm{L}$; $b$, daily minimum value is less than or equal to $5.0 \mathrm{mg} / \mathrm{L}$ but greater than $2.0 \mathrm{mg} / \mathrm{L}$ ]

DISSOLVED OXYGEN, MID-DEPTH

SENSOR POSITION, --5 feet above streambed.

EXTREMES FOR APRIL TO SEPTEMBER 1989.--Maximum value recorded, 11.9 mg/L August 5, 1989; minimum value recorded, $<1.0 \mathrm{mg} / \mathrm{L}$ June $28,1989$.

DAILY MEAN VALUES OF DISSOLVED OXYGEN, MID-DEPTH (IN MILLIGRAMS PER LITER), APRIL TO SEPTEMBER 1989

\begin{tabular}{|c|c|c|c|c|c|c|}
\hline DAY & APR & MAY & JUNE & JULY & AUG & SEPT \\
\hline 1 & --- & 5.8 & -- & $3.8 \mathrm{a}$ & --- & $4.5 b$ \\
\hline 2 & -- & 5.9 & --- & $4.3 b$ & -- & $6.2 \mathrm{~b}$ \\
\hline 3 & --- & $5.2 \mathrm{~b}$ & -- & $4.5 b$ & -- & $7.2 \mathrm{~b}$ \\
\hline 4 & --- & --- & --- & $4.7 b$ & $2.2 a$ & $7.6 \mathrm{~b}$ \\
\hline 5 & -- & -- & -- & $5.6 b$ & $3.2 \mathrm{~b}$ & $5.4 \mathrm{~b}$ \\
\hline 6 & -- & --- & --- & $5.4 \mathrm{~b}$ & $3.9 b$ & $5.1 \mathrm{~b}$ \\
\hline 7 & --- & --- & $4.0 \mathrm{a}$ & $5.2 b$ & --- & $6.5 b$ \\
\hline 8 & --- & --- & $4.9 b$ & $5.2 b$ & --- & $6.5 b$ \\
\hline 9 & --- & --- & $5.1 b$ & $6.6 b$ & --- & $5.9 \mathrm{~b}$ \\
\hline 10 & --- & -- & $4.6 \mathrm{~b}$ & $4.5 a$ & -- & $6.7 \mathrm{~b}$ \\
\hline 11 & --- & --- & $5.2 b$ & $5.0 a$ & -- & $6.1 \mathrm{~b}$ \\
\hline 12 & -- & --- & $5.3 b$ & $2.9 b$ & -- & 7.2 \\
\hline 13 & --- & --- & $4.6 a$ & $2.7 b$ & --- & 7.6 \\
\hline 14 & --- & --- & $4.9 \mathrm{~b}$ & $4.0 \mathrm{~b}$ & --- & -- \\
\hline 15 & --- & -- & $5.1 b$ & $3.9 \mathrm{~b}$ & -- & --- \\
\hline 16 & --- & --- & $5.4 b$ & $5.2 b$ & -- & --- \\
\hline 17 & --- & -- & $5.9 b$ & -- & -- & --- \\
\hline 18 & --- & --- & $4.6 b$ & --- & -- & --- \\
\hline 19 & --- & --- & $5.0 \mathrm{~b}$ & -- & -- & --- \\
\hline 20 & -- & --- & $5.1 \mathrm{~b}$ & -- & -- & --- \\
\hline 21 & --- & --- & $4.6 \mathrm{~b}$ & --- & --- & -- \\
\hline 22 & -- & --- & $5.2 b$ & -- & -- & --- \\
\hline 23 & --- & --- & $4.9 b$ & --- & -- & -- \\
\hline 24 & --- & --- & $4.5 b$ & --- & $1.3 a$ & -- \\
\hline 25 & $-\cdots$ & --- & $3.4 a$ & --- & $2.2 a$ & -- \\
\hline 26 & --- & -- & --- & -- & $3.6 a$ & --- \\
\hline 27 & --- & -- & $2.4 \mathrm{a}$ & --- & $1.9 a$ & --- \\
\hline 28 & 5.9 & -- & $2.6 a$ & --- & $2.9 a$ & $-\cdots$ \\
\hline 29 & 5.9 & -- & $3.5 b$ & --- & $4.1 \mathrm{a}$ & --- \\
\hline 30 & 5.9 & -- & $3.5 a$ & --- & $4.2 \mathrm{a}$ & --- \\
\hline 31 & --- & -- & -- & -- & $4.2 b$ & --- \\
\hline
\end{tabular}

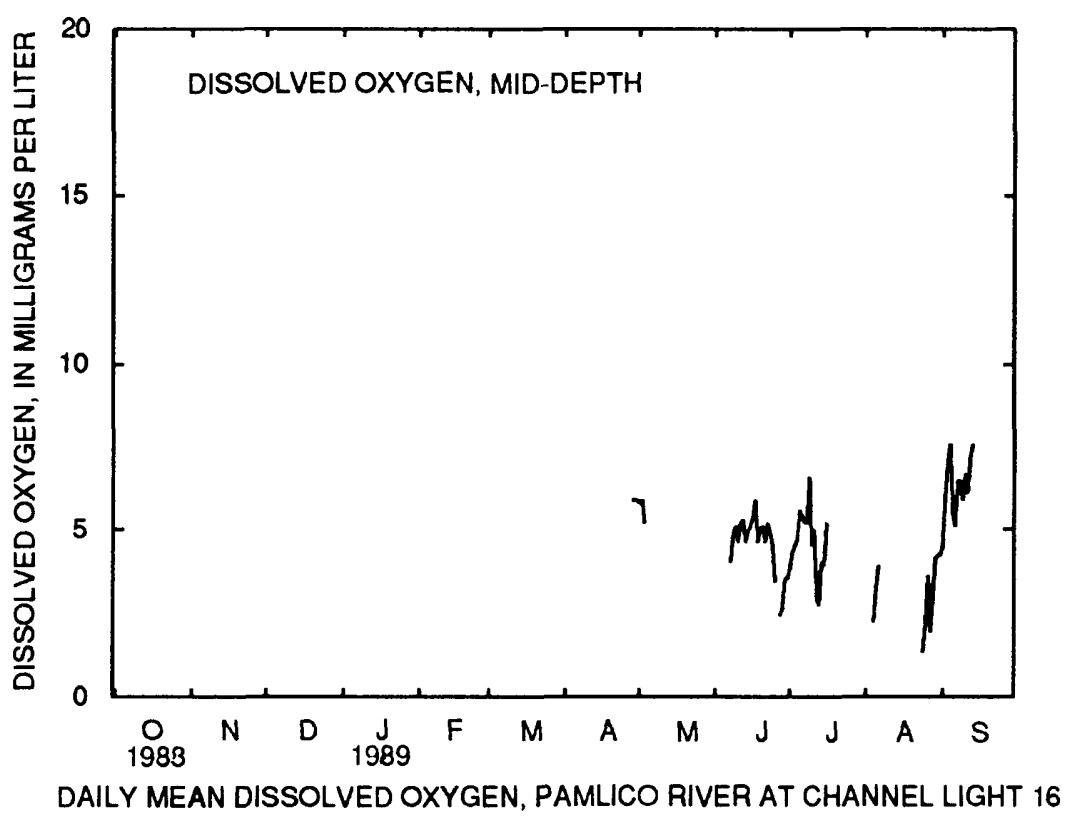


Table 3.--Daily mean values of salinity, water temperature, and dissolved oxygen in water at site 1 , Pamlico River at Channel Light 16, April 1989 to September 1990--Continued

[Location of site 1 is latitude $35^{\circ} 30^{\prime} 30^{\prime \prime}$, longitude $77^{\circ} 01^{\prime} 12^{\prime \prime}$, Beaufort County; U.S. Geological Survey downstream order number 0208450705 ; ppt, parts per thousand; <, less than; ---, no data; ${ }^{\circ} \mathrm{C}$, degrees Celsius; mg/L, milligrams per liter; a, daily minimum value is less than or equal to $2.0 \mathrm{mg} / \mathrm{L}$; b, daily minimum value is less than or equal to $5.0 \mathrm{mg} / \mathrm{L}$ but greater than $2.0 \mathrm{mg} / \mathrm{L}$ )

\section{DISSOLVED OXYGEN, MID-DEPTH}

SENSOR POSITION. --5 feet above streambed.

EXTREMES FOR OCTOBER 1989 TO SEPTEMBER 1990.--Maximum value recorded, 15.4 mg/L June 17, $1990 ;$ minimum value recorded, <1.0 mg/L October 28, November 2-4, 1989, August 19-23, September 12-17, 27, $29,1990$.

DAILY MEAN VALUES OF DISSOLVED OXYGEN, MID-DEPTH (IN MILLIGRAMS PER LITER), OCTOBER 1989 TO SEPTEMBER 1990

\begin{tabular}{|c|c|c|c|c|c|c|c|c|c|c|c|c|}
\hline DAY & OCT & NOV & DEC & JAN & FEB & MAR & APR & MAY & JUNE & JULY & AUG & SEPT \\
\hline 1 & --- & 7.8 & 9.6 & -- & 10.0 & --- & --- & -- & 5.7 & $8.7 b$ & --- & -- \\
\hline 2 & --- & $4.6 a$ & 9.1 & --- & 9.6 & --- & --- & -- & 6.4 & 8.6 & $5.0 b$ & --- \\
\hline 3 & --- & $3.4 a$ & 10.5 & --- & 9.3 & --- & --- & -- & 7.1 & $7.7 \mathrm{~b}$ & $5.8 b$ & --- \\
\hline 4 & --- & $4.6 a$ & 11.6 & 12.0 & 9.5 & --- & --- & --- & 7.4 & 11.3 & $6.1 \mathrm{~b}$ & --- \\
\hline 5 & --- & -- & 11.5 & 12.0 & 10.0 & --- & $-\cdots$ & --- & --- & 11.6 & 6.0 & --- \\
\hline 6 & --- & --- & 11.2 & 12.0 & 9.4 & --- & --- & --- & 7.6 & $9.2 b$ & $5.9 b$ & --- \\
\hline 7 & -- & --- & 11.4 & 11.4 & 8.9 & --- & --- & --- & 7.7 & $9.6 \mathrm{~b}$ & $5.8 b$ & --- \\
\hline 8 & --- & --- & 11.4 & 11.2 & 9.0 & -- & --- & --- & 8.1 & 10.4 & $5.4 \mathrm{~b}$ & --- \\
\hline 9 & --- & -- & 11.2 & 11.2 & 9.2 & --- & --- & --- & 8.6 & 9.5 & --- & --- \\
\hline 10 & --- & --- & 11.5 & 11.0 & 9.7 & --- & --- & --- & 9.1 & 9.0 & --- & --- \\
\hline 11 & --- & --- & 11.0 & 11.2 & 9.8 & --- & --- & --- & --- & $7.2 \mathrm{~b}$ & --- & --- \\
\hline 12 & --- & --- & 10.9 & 11.3 & 9.5 & --- & --- & --- & 10.3 & $7.8 \mathrm{~b}$ & --- & $2.1 a$ \\
\hline 13 & --- & -- & 11.7 & --- & 9.1 & --- & --- & --- & 11.1 & 7.7 & --- & $1.8 a$ \\
\hline 14 & --- & --- & 11.3 & --- & --- & --- & --- & --- & 11.6 & 8.0 & $4.6 a$ & $1.8 \mathrm{a}$ \\
\hline 15 & --- & --- & 11.2 & --- & -- & --- & -- & --- & 12.3 & 8.7 & $4.9 b$ & $5.7 a$ \\
\hline 16 & -- & -- & 11.7 & --- & --- & --- & -- & --- & 13.3 & 8.7 & $3.6 a$ & $4.7 a$ \\
\hline 17 & --- & -- & 11.3 & --- & --- & --- & --- & 8.5 & 12.7 & 8.1 & $-\cdots$ & $6.9 a$ \\
\hline 18 & --- & --- & 11.4 & --- & --- & --- & --- & 9.0 & --- & $7.9 b$ & --- & 8.6 \\
\hline 19 & -- & --- & 11.7 & --- & --- & --- & -- & 9.2 & 9.9 & $7.1 \mathrm{~b}$ & $1.8 \mathrm{a}$ & $6.7 \mathrm{a}$ \\
\hline 20 & --- & --- & 11.9 & --- & -- & -- & -- & 9.2 & 10.2 & $7.3 a$ & $2.2 a$ & $7.6 \mathrm{~b}$ \\
\hline 21 & --- & --- & 11.9 & --- & --- & --- & $\cdots$ & -- & 9.3 & 10.0 & $1.9 a$ & 7.4 \\
\hline 22 & --- & --- & 12.3 & -- & --- & --- & --- & 8.8 & 8.5 & 9.3 & $1.0 \mathrm{a}$ & $6.5 b$ \\
\hline 23 & --- & --- & --- & --- & --- & --- & --- & 8.6 & 9.9 & $6.1 \mathrm{~b}$ & $1.4 a$ & $7.0 \mathrm{~b}$ \\
\hline 24 & --- & --- & -- & 9.6 & --- & -- & --- & 8.5 & 10.6 & $5.5 b$ & 3.5 & 7.3 \\
\hline 25 & --- & --- & -- & 9.8 & --- & --- & --- & 7.9 & 10.5 & $6.8 a$ & --- & $4.7 \mathrm{~b}$ \\
\hline 26 & --- & --- & --- & 10.1 & --- & -- & -- & 7.7 & 10.2 & $9.5 b$ & $-\cdots$ & $5.3 a$ \\
\hline 27 & -- & -- & --- & 10.1 & --- & --- & --- & --- & 10.2 & 9.1 & --- & $4.2 a$ \\
\hline 28 & $2.3 a$ & -- & --- & 9.2 & --- & -- & --- & 8.3 & 9.6 & 8.8 & -- & --- \\
\hline 29 & 8.7 & -- & --- & 9.2 & --- & --- & -- & 7.5 & 9.9 & 9.1 & -- & $1.8 a$ \\
\hline 30 & 9.2 & 10.1 & --- & 10.0 & --- & $-\cdots$ & -- & 7.4 & 9.5 & 9.0 & --- & -- \\
\hline 31 & 8.8 & -- & --- & 10.1 & -- & --- & $-\cdots$ & 6.2 & --- & 8.8 & --- & --- \\
\hline
\end{tabular}

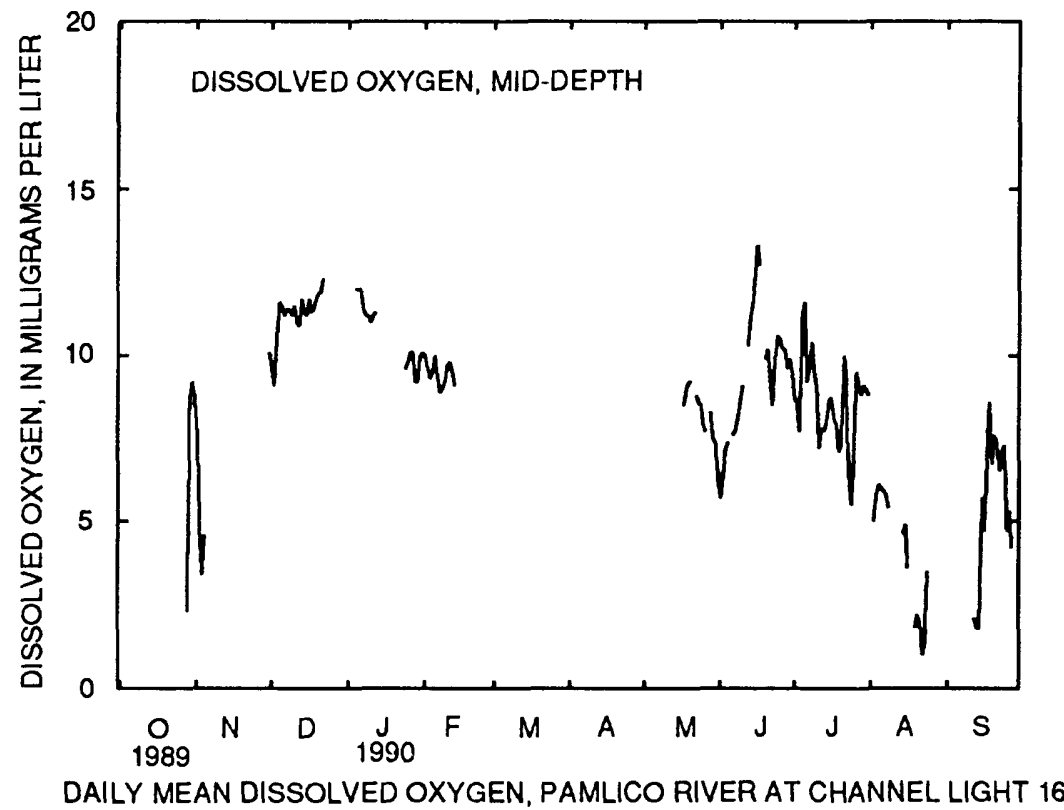


Table 3.--Daily mean values of salinity, water temperature, and dissolved oxygen in water at site 1 , Pamlico River at Channel Light 16, April 1989 to September 1990--Continued

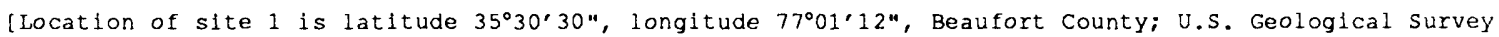
downstream order number 0208450705 ; ppt, parts per thousand; <, less than; ---, no data; ${ }^{\circ} \mathrm{C}$, degrees Celsius; mg/L, milligrams per liter; a, dafly minimum value is less than or equal to $2.0 \mathrm{mg} / \mathrm{L}$; b, daily minimum value is less than or equal to $5.0 \mathrm{mg} / \mathrm{L}$ but greater than $2.0 \mathrm{mg} / \mathrm{L}$ ]

DISSOLVED OXYGEN, NEAR BOTTOM

SENSOR POSITION.--1 foot above streambed.

EXTREMES FOR APRIL TO SEPTEMBER 1989.--Maximum value recorded, $11.8 \mathrm{mg} / \mathrm{L}$ August 2, 1989 ; minimum value recorded, $<1.0 \mathrm{mg} / \mathrm{L}$ August $16,18-31$, September $1-4,9-11,1989$.

DAILY MEAN VALUES OF DISSOLVED OXYGEN, NEAR BOTTOM (IN MILLIGRAMS PER LITER), APRIL TO SEPTEMBER 1989

\begin{tabular}{|c|c|c|c|c|c|c|}
\hline DAY & APR & MAY & JUNE & $J U L Y$ & AUG & SEPT \\
\hline 1 & -- & $5.5 b$ & -- & $4.1 \mathrm{~b}$ & $4.7 b$ & $<1.0 a$ \\
\hline 2 & -- & $5.5 b$ & --- & $3.7 \mathrm{~b}$ & $4.5 b$ & $<1.0 a$ \\
\hline 3 & -- & $4.7 b$ & --- & $4.9 b$ & $3.6 \mathrm{~b}$ & $<1.0 a$ \\
\hline 4 & --- & -- & -- & $6.0 \mathrm{~b}$ & $3.1 a$ & $1.5 a$ \\
\hline 5 & --- & --- & -- & $5.9 b$ & $1.7 a$ & $6.6 \mathrm{~b}$ \\
\hline 6 & -- & --- & --- & 6.3 & $1.5 a$ & $3.5 a$ \\
\hline 7 & --- & -- & $5.0 \mathrm{~b}$ & $5.9 b$ & $1.4 a$ & $5.0 a$ \\
\hline 8 & --- & --- & $4.4 \mathrm{~b}$ & 6.5 & $1.3 a$ & $2.7 a$ \\
\hline 9 & -- & --- & $3.8 \mathrm{~b}$ & 7.4 & $1.2 \mathrm{a}$ & $1.9 a$ \\
\hline 10 & --- & --- & $3.9 b$ & 6.7 & $4.5 a$ & $1.5 a$ \\
\hline 11 & --- & --- & $4.0 \mathrm{~b}$ & $4.7 a$ & $7.3 \mathrm{~b}$ & $3.0 \mathrm{a}$ \\
\hline 12 & --- & --- & $3.8 \mathrm{~b}$ & $2.0 a$ & $4.7 a$ & $4.8 \mathrm{~b}$ \\
\hline 13 & --- & --- & $4.4 \mathrm{~b}$ & $3.7 a$ & $4.3 b$ & $5.9 b$ \\
\hline 14 & --- & --- & $4.2 b$ & $1.6 a$ & $4.3 b$ & $\cdots$ \\
\hline 15 & --- & --- & $4.0 \mathrm{~b}$ & $3.3 a$ & $2.8 \mathrm{a}$ & --- \\
\hline 16 & --- & --- & $4.7 \mathrm{~b}$ & 8.5 & $2.5 a$ & --- \\
\hline 17 & --- & --- & $4.9 \mathrm{~b}$ & 7.8 & $2.3 a$ & --- \\
\hline 18 & --- & --- & $4.2 \mathrm{~b}$ & 6.7 & $1.5 a$ & -- \\
\hline 19 & --- & --- & $4.1 \mathrm{~b}$ & $5.9 b$ & $<1.0 a$ & --- \\
\hline 20 & --- & --- & $4.5 b$ & 6.2 & $<1.0 a$ & -- \\
\hline 21 & --- & --- & $4.7 \mathrm{~b}$ & 6.3 & $<1.0 a$ & -- \\
\hline 22 & -- & $\cdots$ & $4.8 \mathrm{~b}$ & 6.3 & $1.1 a$ & -- \\
\hline 23 & --- & --- & $4.8 \mathrm{~b}$ & 6.3 & $<1.0 \mathrm{a}$ & --- \\
\hline 24 & -- & --- & $4.8 \mathrm{~b}$ & 6.3 & $<1.0 a$ & $-\cdots$ \\
\hline 25 & --- & --- & $4.5 b$ & 6.0 & $<1.0 a$ & -- \\
\hline 26 & --- & --- & --- & $5.1 \mathrm{~b}$ & $<1.0 a$ & --- \\
\hline 27 & --- & --- & $4.1 \mathrm{~b}$ & 6.9 & $<1.0 a$ & --- \\
\hline 28 & 6.4 & --- & $4.3 b$ & $5.6 b$ & $2.7 a$ & --- \\
\hline 29 & 6.1 & --- & $4.5 b$ & $2.6 b$ & $2.9 a$ & --- \\
\hline 30 & 5.9 & --- & $4.8 b$ & $3.8 b$ & $1.5 a$ & --- \\
\hline 31 & --- & --- & --- & $7.5 b$ & $<1.0 \mathrm{a}$ & --- \\
\hline
\end{tabular}

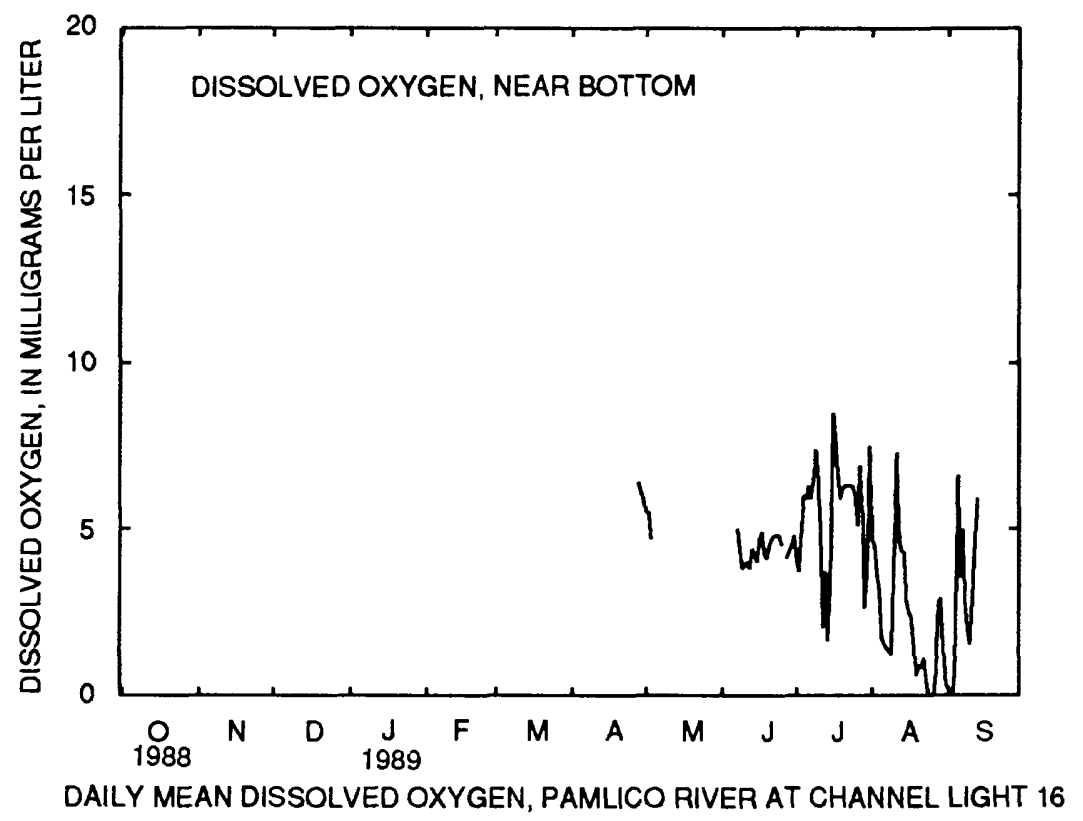


Table 3.--Daily mean values of salinity, water temperature, and dissolved oxygen in water at site 1 , Pamlico River at Channel Light 16, April 1989 to September 1990--Continued

[Location of site 1 is latitude $35^{\circ} 30^{\prime} 30^{\prime \prime}$, longitude $77^{\circ} 01^{\prime} 12^{\prime \prime}$, Beaufort County; U.S. Geological Survey downstream order number 0208450705; ppt, parts per thousand; <, less than; -- -, no data; ${ }^{\circ} \mathrm{C}$, degrees Celsius; mg/L, milligrams per liter; a, daily minimum value 1 s less than or equal to $2.0 \mathrm{mg} / \mathrm{L}$; b, daily minimum value is less than or equal to $5.0 \mathrm{mg} / \mathrm{L}$ but greater than $2.0 \mathrm{mg} / \mathrm{L}$ ]

\section{DISSOLVED OXYGEN, NEAR BOTTOM}

SENSOR POSITION.--1 foot above streambed.

EXTREMES FOR OCTOBER 1989 TO SEPTEMBER 1990.--Maximum value recorded, 16.4 mg/L July 19 , 1990 ; min1mum value recorded, <1.0 mg/L October 28-31, November 1-4, 1989, July 1, August 14, 19-25, September 11-21, 23, 26, 27, $29,1990$.

DAILY MEAN VALUES OF DISSOLVED OXYGEN, NEAR BOTTOM (IN MILLIGRAMS PER LITER), OCTOBER 1989 TO SEPTEMBER 1990

\begin{tabular}{|c|c|c|c|c|c|c|c|c|c|c|c|c|}
\hline DAY & OCT & NOV & $\mathrm{DEC}$ & JAN & FEB & MAR & AP R & MAY & JUNE & JULY & AUG & SEPT \\
\hline 1 & -- & $<1.0 \mathrm{a}$ & $6.2 b$ & --- & 9.5 & 8.7 & --- & 6.4 & 5.5 & $1.5 a$ & --- & --- \\
\hline 2 & --- & $<1.0 \mathrm{a}$ & $5.4 b$ & --- & 9.2 & 8.7 & -- & 7.2 & 5.9 & $2.6 a$ & $2.4 \mathrm{a}$ & --- \\
\hline 3 & --- & $1.6 \mathrm{a}$ & $9.5 b$ & -- & 8.9 & 8.7 & --- & 7.0 & 6.5 & $3.7 \mathrm{~b}$ & $3.0 \mathrm{a}$ & -- \\
\hline 4 & $-\cdots$ & $1.3 a$ & 11.4 & 11.5 & 9.2 & 8.7 & -- & 6.1 & 6.4 & 6.8 & $4.0 \mathrm{~b}$ & --- \\
\hline 5 & --- & --- & 10.6 & 11.0 & 9.7 & 8.6 & -- & 6.0 & --- & 7.7 & $3.2 b$ & --- \\
\hline 6 & -- & --- & 9.9 & 10.8 & 9.2 & 8.4 & -- & 6.8 & 5.8 & $4.9 b$ & $2.7 b$ & --- \\
\hline 7 & --- & --- & 9.7 & 10.7 & 8.8 & 9.0 & --- & 6.2 & 5.7 & $5.2 b$ & $2.3 \mathrm{a}$ & --- \\
\hline 8 & -- & -- & 9.5 & 10.5 & 8.7 & 9.2 & --- & 6.1 & $4.9 b$ & 8.4 & $2.3 a$ & --- \\
\hline 9 & $-\ldots$ & -- & 12.5 & 11.0 & 9.0 & 9.1 & -- & 6.3 & 6.3 & 6.6 & --- & --- \\
\hline 10 & -- & $--\infty$ & 13.1 & 10.9 & 9.5 & 9.2 & --- & 6.8 & $5.3 a$ & $6.2 b$ & --- & --- \\
\hline 11 & --- & --- & 12.6 & 11.0 & 9.6 & 9.0 & --- & 7.5 & --- & 6.7 & -- & $2.5 a$ \\
\hline 12 & --- & -- & 11.6 & 11.3 & 9.4 & 8.9 & --- & 8.0 & $6.5 a$ & 6.7 & --- & $<1.0 \mathrm{a}$ \\
\hline 13 & --- & $\cdots$ & 11.7 & 11.9 & 9.0 & --- & --- & 7.8 & $6.2 \mathrm{a}$ & 7.8 & --- & $<1.0 \mathrm{a}$ \\
\hline 14 & --- & --- & 10.4 & 11.3 & 8.6 & --- & --- & 8.5 & $5.7 b$ & 9.6 & $1.7 a$ & $<1.0 \mathrm{a}$ \\
\hline 15 & --- & --- & 11.8 & 11.3 & 8.4 & --- & -- & 9.3 & 8.9 & 11.2 & $3.5 b$ & $1.0 \mathrm{a}$ \\
\hline 16 & -- & 7.9 & 13.3 & 11.3 & 8.1 & --- & -- & --- & $7.2 \mathrm{~b}$ & 10.5 & $3.1 \mathrm{a}$ & $<1.0 \mathrm{a}$ \\
\hline 17 & --- & 9.0 & 13.0 & 10.1 & 7.4 & --- & --- & 12.0 & $6.4 \mathrm{~b}$ & 8.8 & --- & $<1.0 \mathrm{a}$ \\
\hline 18 & --- & 8.6 & 13.1 & 9.5 & 7.1 & --- & $\cdots$ & 12.7 & --- & 10.8 & -- & $1.1 \mathrm{a}$ \\
\hline 19 & --- & 7.9 & 13.4 & 9.1 & 6.7 & --- & -- & 11.7 & $6.1 \mathrm{~b}$ & 10.6 & $<1.0 \mathrm{a}$ & $<1.0 \mathrm{a}$ \\
\hline 20 & --- & $6.9 b$ & 13.7 & 8.4 & 6.8 & --- & --- & 10.5 & $6.1 \mathrm{~b}$ & $6.9 b$ & $1.0 \mathrm{a}$ & $2.5 a$ \\
\hline 21 & --- & 9.5 & 13.7 & 8.2 & 7.3 & --- & --- & --- & $4.5 b$ & -- & $<1.0 \mathrm{a}$ & $3.4 \mathrm{a}$ \\
\hline 22 & --- & 9.9 & 11.4 & 9.4 & 7.5 & -- & --- & 8.6 & $4.3 b$ & -- & $<1.0 \mathrm{a}$ & $4.0 \mathrm{~b}$ \\
\hline 23 & --- & 10.2 & --- & 8.6 & 7.4 & -- & --- & 8.0 & $6.7 \mathrm{~b}$ & --- & $<1.0 \mathrm{a}$ & $3.2 \mathrm{a}$ \\
\hline 24 & $-\cdots$ & 10.2 & --- & 6.7 & 7.6 & --- & -- & 7.9 & 6.5 & --- & $2.2 \mathrm{a}$ & 6.4 \\
\hline 25 & -- & 9.5 & -- & 8.6 & 8.1 & $\cdots$ & --- & 7.1 & $5.4 \mathrm{~b}$ & -- & $1.3 a$ & $3.8 \mathrm{~b}$ \\
\hline 26 & $\cdots$ & 9.5 & --- & 9.6 & 7.6 & $-\cdots$ & --- & 7.2 & $7.2 \mathrm{~b}$ & -- & --- & $2.1 \mathrm{a}$ \\
\hline 27 & -- & 10.2 & --- & 9.5 & 7.8 & --- & 7.5 & -- & $5.7 b$ & --- & --- & $1.1 \mathrm{a}$ \\
\hline 28 & $<1.0 \mathrm{a}$ & --- & -- & 8.7 & 8.2 & --- & 7.3 & 7.9 & $5.5 b$ & --- & -- & --- \\
\hline 29 & $<1.0 a$ & 9.3 & -- & 8.6 & -- & --- & 7.0 & 7.2 & $6.5 b$ & -- & --- & $<1.0 \mathrm{a}$ \\
\hline 30 & $<1.0 \mathrm{a}$ & 6.2 & -- & 9.5 & -- & --- & 6.6 & 6.9 & $4.7 \mathrm{~b}$ & -- & --- & --- \\
\hline 31 & $<1.0 a$ & --- & --- & 9.6 & -- & --- & -- & 5.9 & --- & -- & --- & --- \\
\hline
\end{tabular}

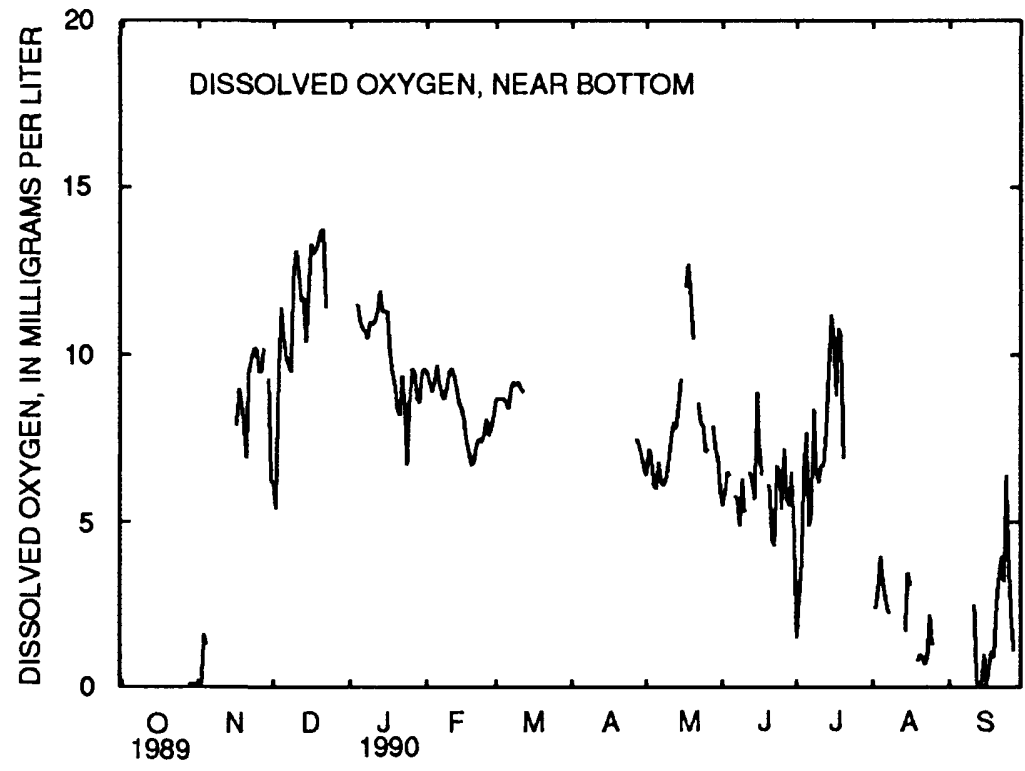


Table 4.--Dally mean values of salinity, water temperature, and dissolved oxygen in water at site 2, Pamlico River at Light 5, April 1989 to september 1990

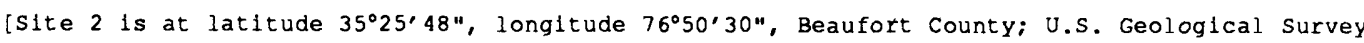
downstream order number 0208453300; ppt, parts per thousand; ---, no data; <, less than; ${ }^{\circ} \mathrm{C}$, degrees Celsius; mg/L, milligrams per liter; a, dally minimum value is less than or equal

to $2.0 \mathrm{mg} / \mathrm{L} ; \mathrm{b}$, dally minimum value is less than or equal to $5.0 \mathrm{mg} / \mathrm{L}$ but greater than $2.0 \mathrm{mg} / \mathrm{I}$ ]

SALINITY, NEAR SURFACE

SENSOR POSITION.--12 feet above streambed.

EXTREMES FOR APRIL TO SEPTEMBER 1989.--Maximum value recorded, 7.5 ppt september 5, 1989; minimum value recorded, 0.3 ppt May 4, 7-14, 1989.

DAILY MEAN VALUES OF SALINITY, NEAR SURFACE (IN PARTS PER THOUSAND), APRIL TO SEPTEMBER 1989

\begin{tabular}{|c|c|c|c|c|c|c|}
\hline DAY & APR & MAY & JUNE & JULY & AUG & SEPT \\
\hline 1 & --- & 1.3 & 2.7 & 2.2 & 2.1 & 6.0 \\
\hline 2 & --- & 1.2 & 2.6 & 2.1 & 2.7 & 5.4 \\
\hline 3 & -- & .6 & 2.8 & 2.1 & --- & 6.4 \\
\hline 4 & --- & .7 & 3.1 & 2.0 & 2.7 & 7.2 \\
\hline 5 & --- & .9 & 3.5 & 1.7 & 2.7 & 6.9 \\
\hline 6 & --- & .8 & 3.2 & 1.5 & 2.7 & 7.0 \\
\hline 7 & --- & .4 & 2.9 & 1.3 & 2.7 & 7.0 \\
\hline 8 & --- & .3 & 2.8 & 1.2 & 4.5 & 7.0 \\
\hline 9 & --- & .3 & 2.7 & 1.3 & 5.2 & 6.4 \\
\hline 10 & --- & .3 & 2.5 & 1.3 & 5.4 & 5.8 \\
\hline 11 & --- & .3 & 2.4 & 1.1 & 5.1 & 5.6 \\
\hline 12 & --- & .4 & --- & .8 & 4.6 & 5.8 \\
\hline 13 & --- & .6 & 2.3 & 1.4 & 4.7 & -- \\
\hline 14 & --- & .5 & 2.1 & 2.2 & 4.8 & --- \\
\hline 15 & --- & .4 & 1.9 & 2.7 & 5.3 & -- \\
\hline 16 & --- & .7 & 2.1 & 3.5 & 5.1 & -- \\
\hline 17 & -- & 1.9 & 2.2 & 4.0 & 4.7 & --- \\
\hline 18 & --- & 2.1 & 1.9 & 3.5 & 4.9 & --- \\
\hline 19 & --- & 1.9 & 1.6 & 3.4 & 4.7 & --- \\
\hline 20 & --- & 1.9 & 1.6 & 2.6 & 4.8 & -- \\
\hline 21 & --- & 1.9 & 1.4 & 1.8 & 4.4 & -- \\
\hline 22 & 1.5 & 2.0 & 1.3 & 1.2 & 4.3 & --- \\
\hline 23 & 1.2 & 2.3 & 1.1 & .7 & -- & --- \\
\hline 24 & 1.2 & 2.5 & .9 & 1.1 & 4.4 & --- \\
\hline 25 & 1.0 & 2.2 & .8 & 1.6 & 5.9 & -- \\
\hline 26 & 1.1 & 2.2 & .8 & 1.5 & 6.4 & -- \\
\hline 27 & 2.0 & 2.2 & .8 & 1.1 & 6.6 & -- \\
\hline 28 & 2.2 & 2.5 & 1.4 & 1.4 & 6.7 & --- \\
\hline 29 & 2.3 & 3.2 & 1.7 & 1.6 & 6.0 & --- \\
\hline 30 & 1.6 & 2.9 & 2.4 & 2.9 & 5.3 & -- \\
\hline 31 & --- & 2.8 & --- & 1.9 & 5.8 & --- \\
\hline
\end{tabular}

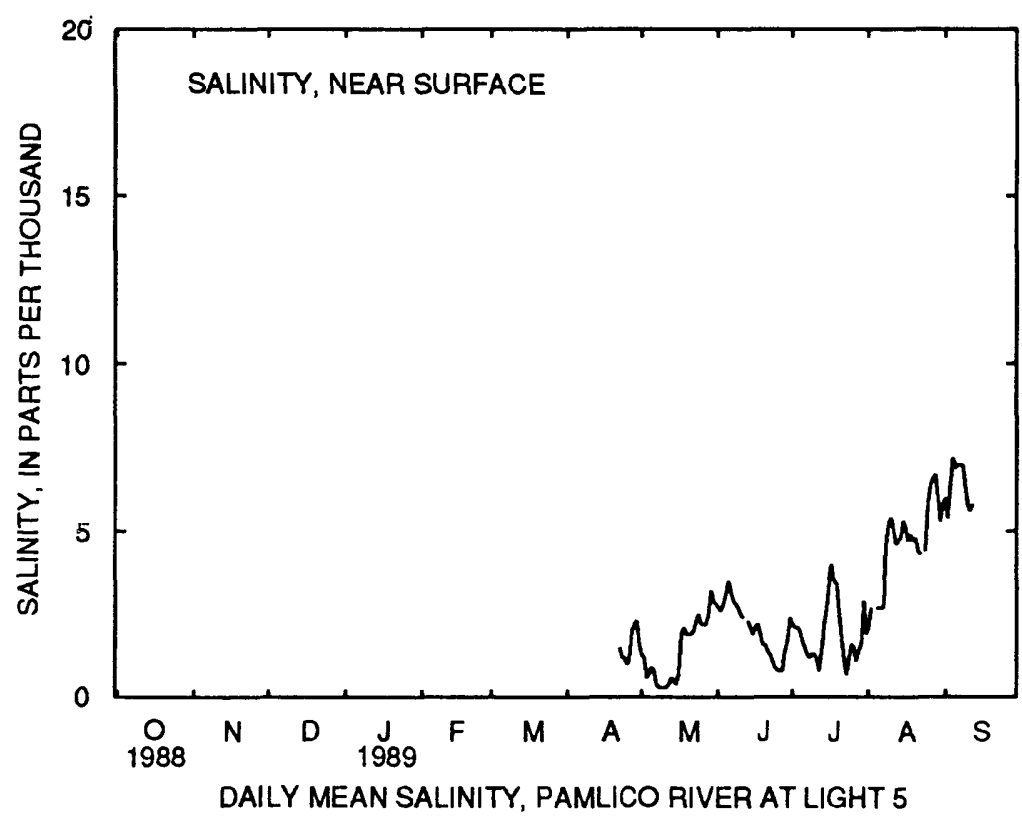


Table 4.--Daily mean values of salinity, water temperature, and dissolved oxygen in water at site 2 , Pamlico River at Light 5, April 1989 to September 1990--Continued

[Site 2 is at latitude $35^{\circ} 25^{\prime} 48^{\prime \prime}$, longltude $76^{\circ} 50^{\prime} 30^{\prime \prime}$, Beaufort County; U.S. Geological Survey downstream order number 0208453300; ppt, parts per thousand; ---, no data; <, less than; ${ }^{\circ} \mathrm{C}$, degrees Celsius; $\mathrm{mg} / \mathrm{L}$, milligrams per liter; a, dally minimum value is less than or equal to $2.0 \mathrm{mg} / \mathrm{L} ; \mathrm{b}$, dally minimum value $1 \mathrm{~s}$ less than or equal to $5.0 \mathrm{mg} / \mathrm{L}$ but greater than $2.0 \mathrm{mg} / \mathrm{L}$ )

SALINITY, NEAR SURFACE

SENSOR POSITION.--12 feet above streambed.

EXTREMES FOR OCTOBER 1989 TO SEPTEMBER 1990.--Maximum value recorded, 10.5 ppt August 14, $1990 ;$ minimum value recorded, 0.1 ppt March 18, 21, 1990.

DAILY MEAN VALUES OF SAIINITY, NEAR SURFACE (IN PARTS PER THOUSAND), OCTOBER 1989 TO SEPTEMBER 1990

\begin{tabular}{|c|c|c|c|c|c|c|c|c|c|c|c|c|}
\hline DAY & OCT & NOV & $\mathrm{DEC}$ & JAN & $F E B$ & MAR & APR & MAY & JUNE & JULY & AUG & SEPT \\
\hline 1 & -- & 3.5 & 3.8 & $--\infty$ & 2.5 & --- & --- & --- & $-\cdots-$ & 4.6 & 4.9 & 7.9 \\
\hline 2 & $-\cdots$ & 4.3 & 3.4 & --- & 1.6 & --- & --- & --- & 0.6 & 4.7 & 5.6 & 7.5 \\
\hline 3 & --- & 4.5 & --- & --- & 1.6 & --- & --- & --- & .5 & --- & 6.2 & 7.5 \\
\hline 4 & --- & 4.6 & --- & 3.1 & 2.4 & --- & --- & --- & .6 & 4.8 & 6.2 & 8.2 \\
\hline 5 & --- & 4.7 & --- & --- & 2.7 & --- & --- & --- & .8 & 5.0 & 6.0 & 7.5 \\
\hline 6 & $-\cdots$ & 4.0 & --- & -- & 2.3 & --- & --- & --- & 1.1 & 5.2 & 6.3 & 6.9 \\
\hline 7 & $-\cdots$ & 3.2 & --- & --- & 2.4 & --- & --- & --- & 1.0 & 5.6 & 6.1 & 5.9 \\
\hline 8 & --- & 3.2 & --- & --- & 2.9 & --- & --- & --- & 1.1 & 5.8 & 5.8 & 5.5 \\
\hline 9 & --- & 3.8 & --- & --- & 2.9 & --- & --- & --- & 1.6 & 5.5 & 5.7 & 6.5 \\
\hline 10 & --- & 4.1 & --- & --- & 2.9 & --- & --- & --- & 2.0 & 5.7 & 5.8 & -- \\
\hline 11 & --- & 4.5 & --- & --- & 3.3 & --- & --- & --- & 2.3 & 5.5 & 5.5 & -- \\
\hline 12 & --- & 4.4 & --- & --- & 2.7 & --- & --- & --- & 2.9 & 5.5 & 5.4 & --- \\
\hline 13 & $\cdots$ & 4.5 & -- & --- & --- & --- & --- & --- & 3.1 & 5.5 & 5.2 & -- \\
\hline 14 & -- & 4.3 & --- & --- & --- & 0.3 & --- & --- & 3.4 & 5.5 & 5.7 & --- \\
\hline 15 & $-\cdots$ & 4.0 & --- & 1.6 & --- & 1.1 & --- & --- & 4.0 & 5.4 & 6.4 & --- \\
\hline 16 & --- & --- & --- & 1.1 & --- & 1.8 & --- & --- & 4.1 & 5.4 & 6.7 & -- \\
\hline 17 & 4.0 & 3.3 & --- & 1.2 & --- & 2.0 & --- & -- & 4.2 & 5.2 & 6.2 & --- \\
\hline 18 & 4.0 & 3.4 & --- & 2.6 & --- & 1.9 & --- & -- & 4.1 & 5.4 & 5.2 & -- \\
\hline 19 & 4.4 & 3.0 & --- & 3.0 & --- & 2.6 & --- & --- & --- & 5.2 & 4.7 & --- \\
\hline 20 & 4.9 & --- & --- & 4.1 & --- & 1.8 & --- & --- & 4.1 & 5.0 & 4.7 & $-\infty$ \\
\hline 21 & 6.0 & --- & --- & --- & --- & 1.5 & --- & --- & 4.0 & 4.8 & 5.5 & --- \\
\hline 22 & 5.5 & 3.7 & --- & 4.2 & --- & 2.4 & --- & --- & 4.0 & 4.0 & 5.9 & --- \\
\hline 23 & 5.3 & 3.2 & -- & --- & --- & 2.2 & -- & --- & 4.3 & 3.9 & 6.5 & -- \\
\hline 24 & 5.4 & 3.0 & --- & 3.6 & --- & 2.5 & --- & -- & 4.3 & 3.9 & 6.9 & --- \\
\hline 25 & 5.6 & 2.3 & --- & 3.9 & --- & 3.3 & --- & --- & 4.4 & 4.6 & 6.1 & --- \\
\hline 26 & 4.1 & 3.2 & --- & --- & --- & 2.9 & --- & --- & 4.6 & 5.6 & 6.4 & --- \\
\hline 27 & 3.7 & 3.8 & -- & 4.1 & -- & -- & --- & --- & 4.4 & 6.1 & 6.1 & $-\infty$ \\
\hline 28 & 3.6 & 3.6 & --- & 3.3 & --- & --- & --- & -- & 4.3 & 6.7 & 5.4 & -- \\
\hline 29 & 5.6 & 2.9 & --- & 3.1 & --- & 2.8 & --- & -- & 4.3 & 6.6 & 4.7 & $\cdots$ \\
\hline 30 & -- & 2.6 & --- & --- & --- & 2.7 & --- & --- & 4.4 & 6.1 & 6.4 & -- \\
\hline 31 & 3.6 & --- & --- & 3.2 & --- & 2.5 & --- & -- & --- & 5.5 & 7.8 & --- \\
\hline
\end{tabular}

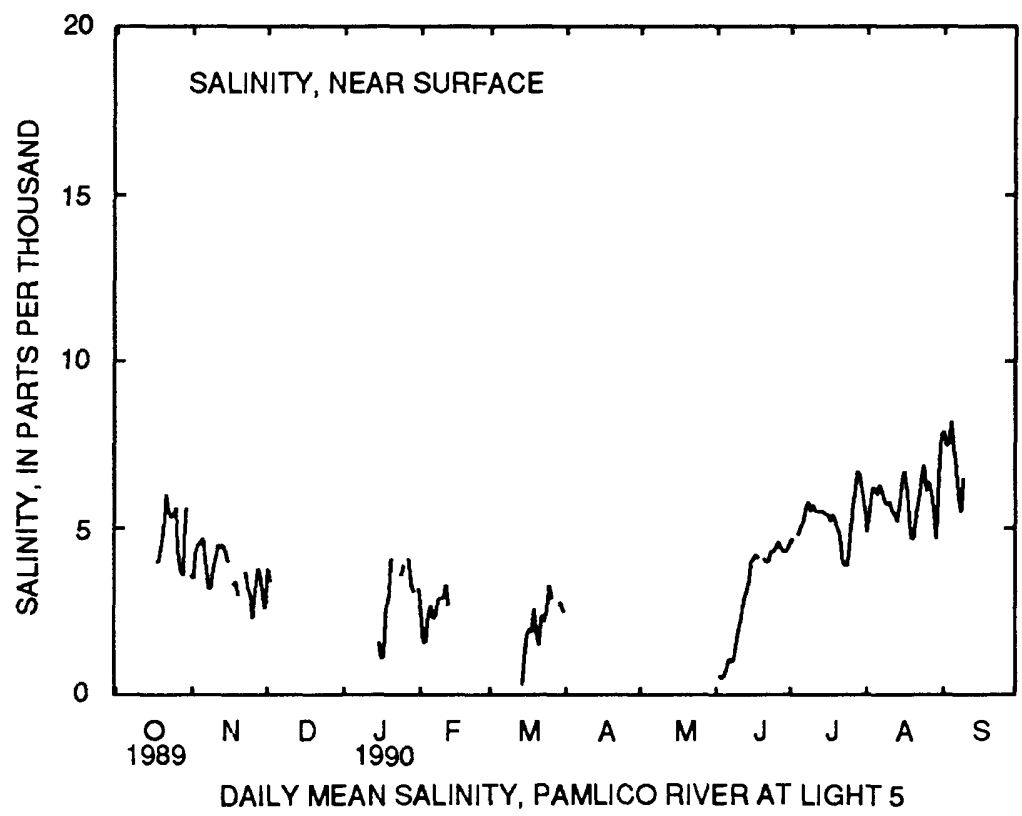


Table 4.--Dally mean values of salinity, water temperature, and dissolved oxygen tn water at site 2, Pamlico River at Light 5, April 1989 to september 1990--Continued

[Site 2 is at latitude $35^{\circ} 25^{\prime} 48^{\prime \prime}$, longitude $76^{\circ} 50^{\prime} 30^{\prime \prime}$, Beaufort County; U.S. Geologlcal Survey downstream order number 0208453300 ; ppt, parts per thousand; ---, no data; <, less than; ${ }^{\circ} \mathrm{C}$, degrees Celsius; $\mathrm{mg} / \mathrm{L}$, mililgrams per liter; a, dally minimum value is less than or equal

to $2.0 \mathrm{mg} / \mathrm{L}$; b, dally minimum value is less than or equal to $5.0 \mathrm{mg} / \mathrm{L}$ but greater than $2.0 \mathrm{mg} / \mathrm{L}$ ]

SALINITY, NEAR BOTTOM

SENSOR POSITION. -4 feet above streambed.

EXTREMES FOR APRIL TO SEPTEMBER 1989.--Maximum value recorded, 16.7 ppt August 24, 1989; minimum value recorded, 0.2 ppt May $7-11,1989$.

DAILY MEAN VALUES OF SALINITY, NEAR BOTTOM (IN PARTS PER THOUSAND), APRIL TO SEPTEMBER 1989

\begin{tabular}{|c|c|c|c|c|c|c|}
\hline DAY & APR & MAY & JUNE & JULY & AUG & SEPT \\
\hline 1 & -- & 4.7 & 2.9 & --- & -- & 7.6 \\
\hline 2 & --- & 4.6 & 3.1 & -- & -- & 7.2 \\
\hline 3 & $\ldots$ & 5.6 & 3.4 & $-\cdots$ & -- & 7.2 \\
\hline 4 & -- & 4.6 & 3.3 & --- & -- & 3.2 \\
\hline 5 & -- & 1.1 & 3.3 & -- & 5.4 & 6.9 \\
\hline 6 & --- & .7 & 3.2 & $-\cdots$ & 7.8 & 6.9 \\
\hline 7 & -- & .3 & 3.1 & -- & 8.9 & 7.0 \\
\hline 8 & --- & .2 & 3.0 & -- & 9.4 & 7.0 \\
\hline 9 & -- & .3 & 2.8 & -- & 6.9 & 7.1 \\
\hline 10 & --- & .3 & 2.6 & --- & 5.7 & 7.3 \\
\hline 11 & --- & .3 & 2.7 & -- & 5.7 & 7.4 \\
\hline 12 & --- & .4 & --- & --- & 5.8 & 7.4 \\
\hline 13 & --- & .8 & 2.5 & --- & 6.4 & -- \\
\hline 14 & --- & .7 & 2.4 & -- & 6.5 & --- \\
\hline 15 & --- & .5 & 2.3 & -- & 6.3 & --- \\
\hline 16 & --- & .9 & 2.3 & -- & 6.3 & --- \\
\hline 17 & --- & 2.1 & 2.3 & -- & 6.4 & -- \\
\hline 18 & --- & 2.4 & 2.1 & -- & 6.5 & -- \\
\hline 19 & --- & 2.7 & 2.2 & -- & 3.4 & -- \\
\hline 20 & --- & 3.1 & 2.1 & -- & 7.8 & -- \\
\hline 21 & --- & 3.4 & 1.7 & $-\cdots$ & 8.9 & -- \\
\hline 22 & 8.8 & 3.5 & 1.6 & -- & 9.9 & -- \\
\hline 23 & 8.6 & 2.6 & 1.5 & -- & --- & -- \\
\hline 24 & 7.6 & 2.4 & 1.4 & -- & 14.8 & -- \\
\hline 25 & 6.1 & 3.0 & 1.7 & --- & 14.4 & -- \\
\hline 26 & 5.9 & 2.8 & --- & --- & 10.7 & -- \\
\hline 27 & 6.7 & 2.7 & --- & --- & 10.3 & -- \\
\hline 28 & 6.0 & 3.5 & --- & --- & 10.7 & $-\cdots$ \\
\hline 29 & 4.7 & 3.2 & --- & -- & 10.7 & --- \\
\hline 30 & 4.8 & 3.0 & --- & --- & 9.7 & -- \\
\hline 31 & --- & 2.9 & -- & --- & 9.3 & -- \\
\hline
\end{tabular}

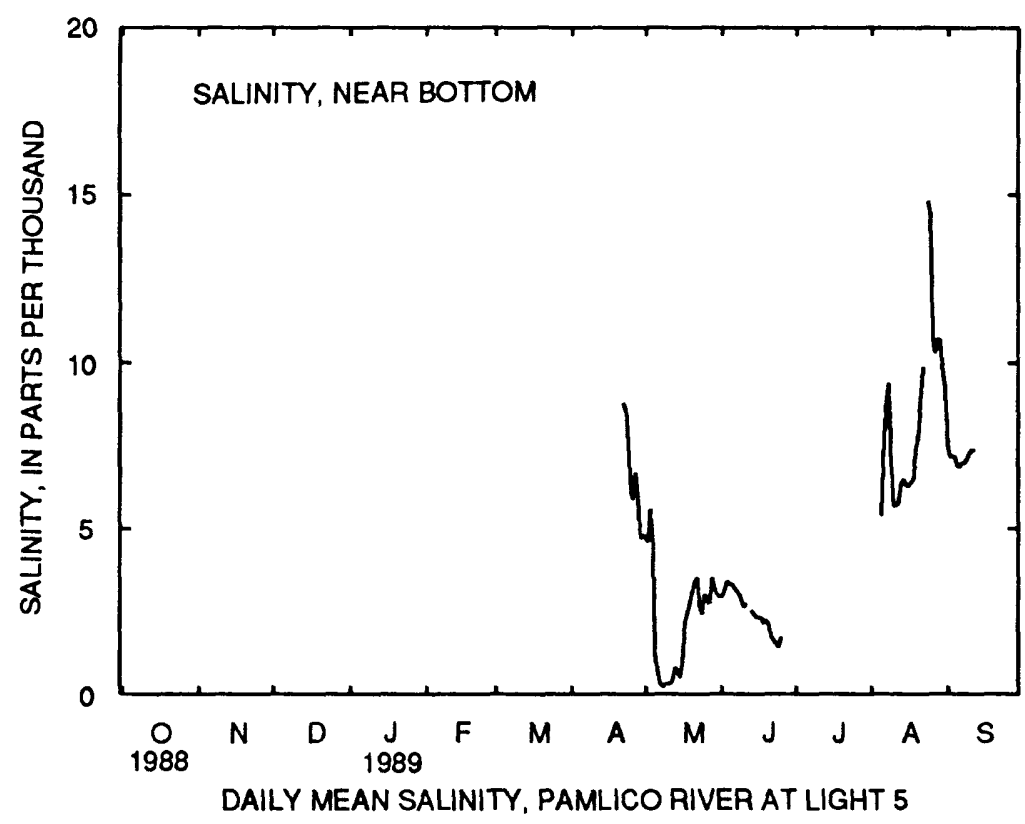


Table 4.--Daily mean values of salinity, water temperature, and dissolved oxygen in water at site 2 , Pamlico River at Light 5, April 1989 to September 1990--Continued

[Site 2 is at latitude $35^{\circ} 25^{\circ} 48^{\prime \prime}$, longitude $76^{\circ} 50^{\prime} 30^{\prime \prime}$, Beaufort County; U.S. Geological Survey downstream order number 0208453300; ppt, parts per thousand; ---, no data; <, less than; ${ }^{\circ} \mathrm{C}$ degrees Celsius; $\mathrm{mg} / \mathrm{L}$, milligrams per liter; a, daily minimum value is less than or equal

to $2.0 \mathrm{mg} / \mathrm{L}$; b, dally minimum value is less than or equal to $5.0 \mathrm{mg} / \mathrm{L}$ but greater than $2.0 \mathrm{mg} / \mathrm{L}$ ]

SALINITY, NEAR BOTTOM

SENSOR POSITION. -4 feet above streambed.

EXTREMES FOR OCTOBER 1989 TO SEPTEMBER 1990.--Maximum value recorded, 14.6 ppt september 3 , 1990 ; minimum value recorded, <0.1 ppt April 12, 1990.

DAILY MEAN VALUES OF SALINITY, NEAR BOTTOM (IN PARTS PER THOUSAND), OCTOBER 1989 TO SEPTEMBER 1990

\begin{tabular}{|c|c|c|c|c|c|c|c|c|c|c|c|c|}
\hline DAY & $\mathrm{OCT}$ & NOV & DEC & JAN & FEB & MAR & APR & MAY & JUNE & JULY & $A U G$ & SEPT \\
\hline 1 & --- & 6.4 & 11.0 & --- & 4.4 & --- & 3.9 & -- & --- & 7.5 & 7.9 & 11.7 \\
\hline 2 & --- & 6.6 & 11.3 & -- & 5.0 & -- & 3.9 & --- & 3.4 & 7.5 & 8.1 & 10.8 \\
\hline 3 & --- & 5.4 & 5.4 & --- & 5.3 & --- & 2.6 & -- & 3.3 & --- & 7.7 & 10.9 \\
\hline 4 & --- & 5.9 & 6.1 & 9.1 & 3.9 & --- & 1.4 & --- & 4.1 & 7.2 & 7.2 & 10.0 \\
\hline 5 & -- & 5.7 & 6.8 & 9.2 & 3.5 & -- & 2.2 & -- & 5.4 & 7.2 & 7.2 & 8.6 \\
\hline 6 & -- & 5.6 & --- & 9.0 & 4.6 & --- & 2.7 & -- & 4.5 & 7.5 & 8.6 & 8.1 \\
\hline 7 & -- & 6.9 & --- & 9.1 & 4.0 & --- & 1.3 & -- & 4.4 & 7.5 & 9.8 & 8.6 \\
\hline 8 & --- & 7.2 & --- & 7.7 & 4.8 & --- & 2.0 & --- & 5.4 & 6.1 & 12.1 & 9.2 \\
\hline 9 & -- & 6.8 & --- & 6.1 & 4.5 & --- & 2.0 & -- & 5.5 & 6.1 & 10.4 & 8.1 \\
\hline 10 & --- & 6.4 & -- & 5.3 & 3.9 & --- & 1.8 & --- & 5.5 & 5.9 & 11.3 & --- \\
\hline 11 & --- & 6.9 & --- & 5.1 & 3.4 & --- & .5 & --- & 5.7 & 5.8 & 12.9 & -- \\
\hline 12 & --- & 6.6 & --- & 4.5 & 3.1 & $-\infty$ & .3 & --- & 6.1 & 5.5 & 13.3 & --- \\
\hline 13 & --- & 7.7 & --- & 4.1 & --- & --- & .8 & --- & 6.7 & 5.5 & 13.1 & -- \\
\hline 14 & --- & --- & --- & 5.9 & --- & 7.1 & .3 & $-\infty$ & 6.4 & 5.3 & 12.1 & --- \\
\hline 15 & --- & --- & --- & 6.1 & 3.3 & 7.1 & .3 & --- & 4.9 & 5.3 & 11.6 & --- \\
\hline 16 & --- & 5.6 & --- & 6.5 & 2.9 & 6.0 & .4 & --- & 4.4 & 5.3 & 11.6 & --- \\
\hline 17 & 6.6 & 4.6 & --- & 6.9 & 2.1 & 4.7 & .3 & --- & 4.5 & 5.4 & 11.3 & --- \\
\hline 18 & 6.8 & 5.4 & --- & 7.0 & 2.0 & 3.6 & .4 & --- & 4.4 & 5.1 & 11.6 & --- \\
\hline 19 & 6.4 & 5.3 & --- & 7.2 & 1.3 & 5.4 & .6 & --- & 4.2 & 5.0 & 11.1 & --- \\
\hline 20 & 5.9 & 5.5 & --- & 6.9 & 1.3 & 2.5 & .5 & -- & 4.2 & 4.7 & 10.6 & $-\cdots$ \\
\hline 21 & 6.2 & 3.8 & --- & 6.5 & 1.4 & 3.5 & .4 & --- & 4.5 & 4.8 & 11.2 & --- \\
\hline 22 & 5.7 & 4.3 & --- & 7.5 & --- & 4.7 & .3 & --- & 4.7 & 5.3 & 10.7 & --- \\
\hline 23 & 5.9 & 3.8 & --- & --- & --- & 4.8 & .6 & --- & 4.9 & 7.7 & 10.7 & -- \\
\hline 24 & 5.5 & 4.9 & --- & 7.2 & -- & 4.4 & .6 & --- & 6.4 & 9.6 & 9.5 & --- \\
\hline 25 & 5.7 & 5.8 & --- & 7.1 & --- & 3.6 & 1.2 & $-\infty$ & 8.1 & 9.7 & 10.2 & --- \\
\hline 26 & 5.7 & 6.7 & --- & --- & --- & 3.8 & 2.6 & --- & 7.1 & 9.5 & 9.8 & --- \\
\hline 27 & 5.9 & 8.2 & --- & 5.0 & --- & --- & 3.0 & --- & 6.0 & 8.7 & 11.0 & -- \\
\hline 28 & 6.6 & 7.9 & --- & 5.6 & --- & --- & --- & --- & 6.5 & 6.9 & 12.2 & --- \\
\hline 29 & 6.2 & 9.1 & --- & 6.1 & --- & 3.1 & --- & --- & 6.6 & 6.8 & 12.7 & --- \\
\hline 30 & --- & 9.3 & --- & 4.4 & --- & 2.5 & --- & --- & 7.5 & 7.5 & 12.5 & --- \\
\hline 31 & 6.0 & --- & --- & 4.1 & --- & 3.2 & --- & --- & --- & 7.8 & 12.0 & --- \\
\hline
\end{tabular}

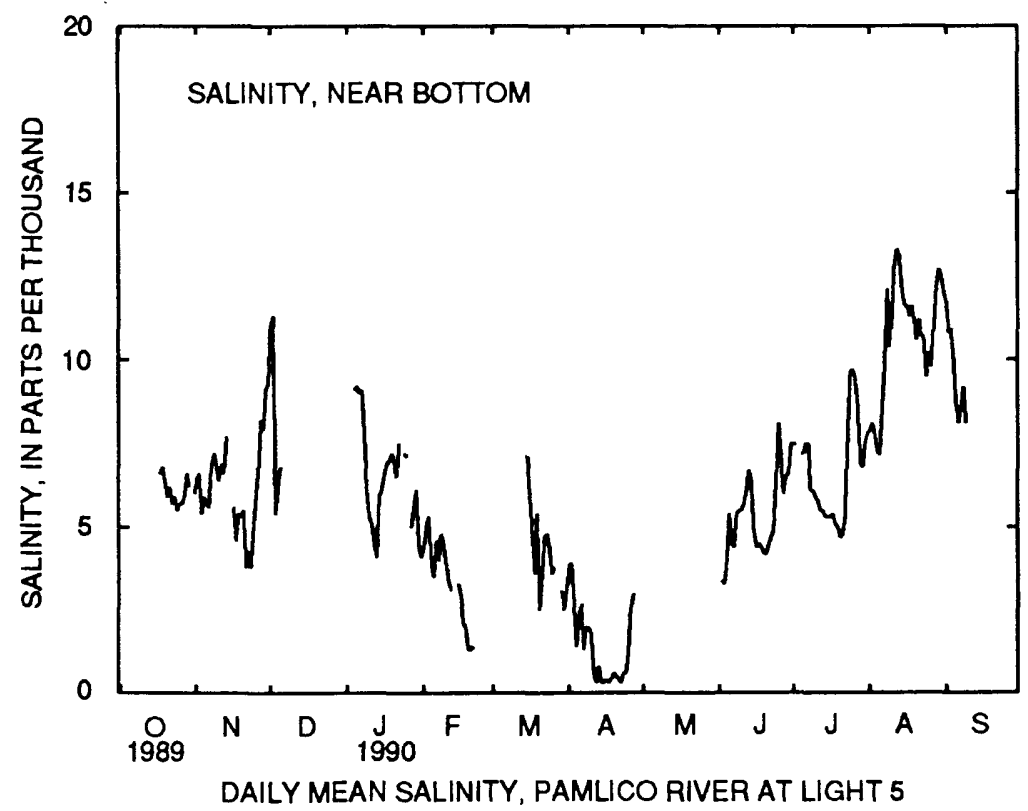


Table 4.--Daily mean values of salinity, water temperature, and dissolved oxygen in water at site 2 , Pamlico River at Light 5, April 1989 to September 1990--Continued

[Site 2 is at latitude $35^{\circ} 25^{\prime} 48^{\prime \prime}$, longitude $76^{\circ} 50^{\prime} 30^{\prime \prime}$, Beaufort County; U.S. Geological Survey downstream order number 0208453300; ppt, parts per thousand; ---, no data; <, less than; ${ }^{\circ} \mathrm{C}$, degrees Celsius; mg/L, milligrams per liter; a, dally minimum value is less than or equal

to $2.0 \mathrm{mg} / \mathrm{L} ; \mathrm{b}$, daily minimum value is less than or equal to $5.0 \mathrm{mg} / \mathrm{L}$ but greater than $2.0 \mathrm{mg} / \mathrm{L}$ l

WATER TEMPERATURE, NEAR SURFACE

SENSOR POSITION.--12 feet above streambed.

EXTREMES FOR APRIL TO SEPTEMBER 1989.--Maximum value recorded, $32.7{ }^{\circ} \mathrm{C}$ July 11, 12 , 1989 ; minimum value recorded, $15.1{ }^{\circ} \mathrm{C}$ April 22, 1989.

DAILY MEAN VALUES OF WATER TEMPERATURE, NEAR SURFACE (IN DEGREES CELSIUS), APRII TO SEPTEMBER 1989

\begin{tabular}{|c|c|c|c|c|c|c|}
\hline DAY & APR & MAY & JUNE & JULY & AUG & SEPT \\
\hline 1 & -- & 22.6 & 26.6 & 26.8 & 29.7 & $-\infty$ \\
\hline 2 & -- & 22.6 & 27.8 & 27.2 & 30.8 & 28.2 \\
\hline 3 & $\cdots$ & 22.4 & 27.7 & 27.1 & $\cdots$ & 27.3 \\
\hline 4 & -- & 21.9 & 26.9 & 27.0 & 29.6 & 26.2 \\
\hline 5 & --- & 21.2 & 26.7 & 27.4 & 29.3 & 25.5 \\
\hline 6 & -- & 21.2 & 26.5 & 27.5 & 30.5 & 25.3 \\
\hline 7 & $\cdots$ & 20.0 & 26.1 & 27.8 & 31.6 & 25.0 \\
\hline 8 & --- & 18.8 & 26.0 & 28.5 & 29.3 & 25.1 \\
\hline 9 & $\cdots$ & 18.3 & 25.8 & 29.2 & 27.4 & 25.2 \\
\hline 10 & --- & 18.1 & 26.5 & 29.8 & 26.0 & 25.5 \\
\hline 11 & $-\cdots$ & 17.9 & 26.7 & 30.8 & 25.4 & 26.3 \\
\hline 12 & $\cdots$ & 18.0 & -- & 31.4 & 25.3 & 26.5 \\
\hline 13 & --- & 18.3 & 26.4 & 30.3 & 25.9 & $\cdots$ \\
\hline 14 & $\cdots$ & 18.3 & 27.5 & 29.4 & 26.8 & --- \\
\hline 15 & --- & 18.7 & 27.8 & 29.4 & 27.0 & --- \\
\hline 16 & --- & 19.3 & 27.7 & 29.0 & 27.6 & $\cdots$ \\
\hline 17 & -- & 19.1 & 27.1 & 28.3 & 28.0 & --- \\
\hline 18 & $-\infty$ & 19.3 & 27.4 & 27.9 & 28.8 & -- \\
\hline 19 & $-\infty$ & 19.9 & 28.0 & 28.3 & 28.9 & -- \\
\hline 20 & --- & 20.9 & 27.5 & 28.8 & 29.0 & $\cdots$ \\
\hline 21 & $m$ & 22.3 & 27.3 & 28.7 & 29.4 & -- \\
\hline 22 & 16.2 & 23.6 & 27.2 & 28.8 & 29.3 & -- \\
\hline 23 & 17.2 & 23.5 & 27.3 & 29.9 & -- & -- \\
\hline 24 & 17.9 & 23.1 & 27.2 & 30.0 & 28.0 & -- \\
\hline 25 & 19.2 & 23.5 & 27.1 & 25.9 & $\cdots$ & $\cdots$ \\
\hline 26 & 21.2 & 23.9 & 27.6 & 21.5 & -- & -- \\
\hline 27 & 20.5 & 25.2 & 28.2 & 21.7 & -- & -- \\
\hline 28 & 20.8 & 24.9 & 28.2 & 25.0 & -- & --- \\
\hline 29 & 20.9 & 23.9 & 27.7 & 29.8 & --- & --- \\
\hline 30 & 22.1 & 24.3 & 27.0 & 27.5 & -- & $\cdots$ \\
\hline 31 & -- & 25.2 & -- & 29.2 & -- & -- \\
\hline
\end{tabular}

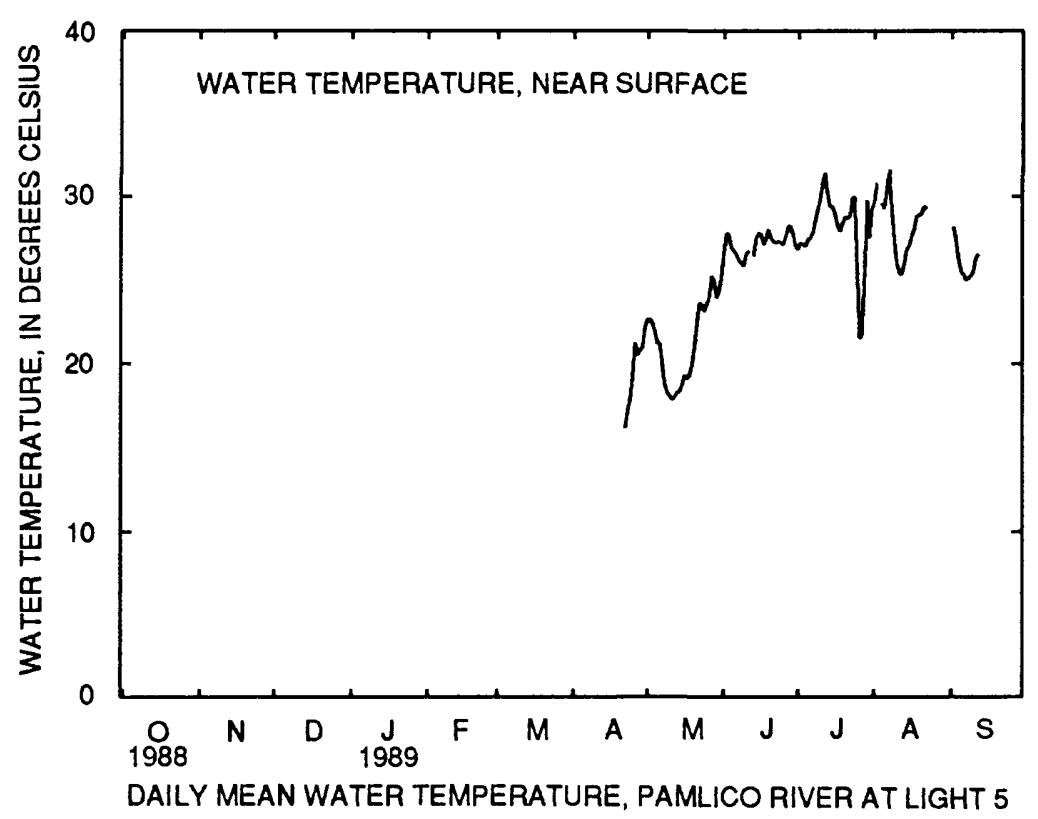


Table 4.--Daily mean values of salinity, water temperature, and dissolved oxygen in water at site 2 , Pamlico River at Light 5, April 1989 to September 1990--Cont inued

[Site 2 is at latitude $35^{\circ} 25^{\prime} 48^{\prime \prime}$, longitude $76^{\circ} 50^{\prime} 30^{\prime \prime}$, Beaufort County; U.S. Geological Survey downstream order number 0208453300; ppt, parts per thousand; ---, no data; <, less than; ${ }^{\circ} \mathrm{C}$, degrees Celsius; $\mathrm{mg} / \mathrm{L}$, milligrams per Iiter; a, daily minimum value is less than or equal

to $2.0 \mathrm{mg} / \mathrm{L} ; \mathrm{b}$, dally minimum value is less than or equal to $5.0 \mathrm{mg} / \mathrm{L}$ but greater than $2.0 \mathrm{mg} / \mathrm{L}$ ]

WATER TEMPERATURE, NEAR SURFACE

SENSOR POSITION. --12 feet above st reambed.

EXTREMES FOR OCTOBER 1989 TO SEPTEMBER 1990.--Maximum value recorded, $33.0{ }^{\circ} \mathrm{C} \mathrm{July} 23$, 1990 ; minimum value recorded, $0.0^{\circ} \mathrm{C}$ December 3, 1989.

DAILY MEAN VALUES OF WATER TEMPERATURE, NEAR SURFACE (IN DEGREES CELSIUS), OCTOBER 1989 TO SEPTEMBER 1990

\begin{tabular}{|c|c|c|c|c|c|c|c|c|c|c|c|c|}
\hline DAY & OCT & NOV & DEC & JAN & FEB & MAR & APR & MAY & JUNE & JULY & AUG & SEPT \\
\hline 1 & -- & 18.7 & 10.0 & --- & 10.5 & --- & 13.7 & -- & 24.0 & 29.8 & 28.9 & 27.0 \\
\hline 2 & -- & 18.4 & 9.4 & --- & 10.7 & --- & 14.4 & -- & 24.7 & 29.4 & 28.3 & 27.1 \\
\hline 3 & --- & 18.0 & 4.6 & --- & 12.5 & --- & 14.3 & -- & 24.9 & --- & 27.8 & 27.9 \\
\hline 4 & --- & 17.0 & 4.8 & 6.6 & 12.2 & -- & 12.2 & $\cdots$ & 25.7 & 29.1 & 27.7 & 27.8 \\
\hline 5 & --- & --- & 6.7 & 9.4 & 11.8 & -- & 13.1 & --- & 25.1 & 29.2 & 27.6 & 26.5 \\
\hline 6 & --- & 17.0 & --- & 9.4 & 10.9 & --- & 13.8 & -- & 24.8 & 30.0 & 27.5 & 26.5 \\
\hline 7 & --- & 18.0 & --- & 9.3 & 12.2 & --- & 13.0 & --- & 25.7 & 30.1 & 27.1 & 26.9 \\
\hline 8 & -- & 18.3 & --- & 8.4 & 10.7 & --- & 12.6 & --- & 27.4 & 29.7 & 26.5 & 27.3 \\
\hline 9 & --- & 18.6 & --- & 7.3 & 11.7 & -- & 12.5 & --- & 27.3 & 29.9 & 26.2 & 26.8 \\
\hline 10 & --- & 17.7 & --- & 7.5 & 13.2 & --- & 12.8 & --- & 27.5 & 30.0 & 26.3 & --- \\
\hline 11 & --- & --- & $-\infty$ & 7.9 & 12.8 & --- & 13.1 & -- & 26.7 & 30.3 & 27.1 & --- \\
\hline 12 & --- & 16.9 & -- & 8.4 & 12.1 & --- & 12.2 & --- & 25.2 & 30.0 & 27.7 & --- \\
\hline 13 & --- & 17.3 & --- & 7.0 & --- & --- & 11.6 & -- & 24.8 & 30.0 & 28.1 & -- \\
\hline 14 & --- & 17.5 & -- & 7.3 & --- & 10.7 & 9.6 & --- & 25.4 & 29.8 & 27.9 & -- \\
\hline 15 & --- & 18.0 & --- & 7.8 & 11.3 & 9.4 & 11.2 & -- & 25.3 & 29.4 & 27.2 & --- \\
\hline 16 & --- & 17.0 & --- & 9.3 & 15.0 & 10.7 & 12.9 & --- & 25.7 & 28.7 & 27.2 & --- \\
\hline 17 & 21.9 & 15.5 & $-\sim$ & 10.1 & 15.3 & 12.3 & 15.2 & 23.7 & 26.1 & 28.7 & 27.1 & --- \\
\hline 18 & 22.7 & 14.3 & -- & 10.4 & 14.5 & 15.9 & 16.6 & 23.5 & 26.8 & 29.0 & 27.3 & --- \\
\hline 19 & 22.8 & 13.1 & --- & 11.0 & 13.0 & 16.4 & 15.4 & 23.7 & --- & 29.1 & 27.7 & -- \\
\hline 20 & 21.4 & 12.1 & --- & 10.8 & 12.7 & 16.5 & 15.5 & 23.5 & 26.7 & 29.0 & 28.3 & --- \\
\hline 21 & 18.7 & 11.2 & -- & 11.9 & 11.3 & 14.5 & 17.5 & --- & 27.6 & 29.1 & 27.7 & --- \\
\hline 22 & 17.7 & 11.7 & --- & 10.5 & -- & 11.7 & 18.7 & --- & 28.5 & 29.6 & 27.2 & --- \\
\hline 23 & 17.3 & 10.6 & --- & --- & --- & 14.0 & 18.5 & --- & 27.3 & 30.4 & 27.2 & --- \\
\hline 24 & 17.2 & 9.5 & --- & 9.0 & -- & 14.2 & 19.6 & -- & 27.5 & 30.6 & 27.3 & --- \\
\hline 25 & 17.4 & 8.6 & --- & 10.5 & --- & 11.1 & 20.4 & -- & 27.8 & 29.8 & 27.5 & --- \\
\hline 26 & 17.0 & 10.2 & --- & --- & -- & 9.2 & 22.2 & -- & 27.6 & 28.8 & 27.6 & --- \\
\hline 27 & 17.0 & 11.2 & --- & 9.7 & --- & --- & 22.8 & --- & 28.1 & 28.3 & 28.1 & --- \\
\hline 28 & 17.2 & 11.8 & --- & 10.0 & -- & -- & --- & --- & 28.6 & 28.1 & 28.3 & --- \\
\hline 29 & 17.9 & 12.2 & --- & 10.4 & --- & 11.5 & --- & --- & 29.0 & 28.0 & 28.5 & --- \\
\hline 30 & --- & 10.9 & $-\cdots$ & 10.1 & --- & 12.9 & --- & --- & 29.3 & 28.2 & 27.8 & --- \\
\hline 31 & 18.6 & --- & --- & 10.4 & --- & 13.2 & --- & --- & --- & 28.7 & 27.1 & --- \\
\hline
\end{tabular}

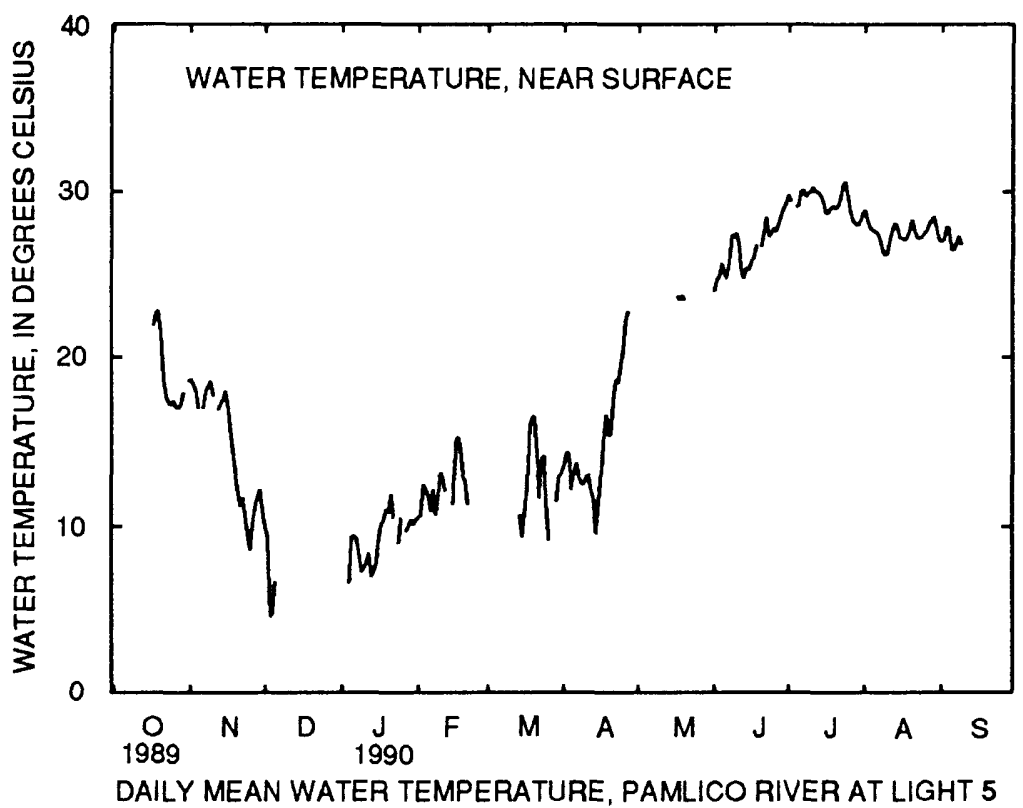


Table 4.--Daily mean values of salinity, water temperature, and dissolved oxygen in water at site 2 , Pamlico River at Light 5, April 1989 to September 1990--Continued

[Site 2 is at latitude $35^{\circ} 25^{\prime} 48^{\prime \prime}$, longitude $76^{\circ} 50^{\prime} 30^{\prime \prime}$, Beaufort County; U.S. Geological Survey downstream order number 0208453300 ; ppt, parts per thousand; -- , no data; <, less than; ${ }^{\circ} \mathrm{C}$, degrees Celsius; mg/L, milligrams per liter; a, dally minimum value is less than or equal

to $2.0 \mathrm{mg} / \mathrm{L} ; \mathrm{b}$, dally minimum value is less than or equal to $5.0 \mathrm{mg} / \mathrm{L}$ but greater than $2.0 \mathrm{mg} / \mathrm{L}$ ]

DISSOLVED OXYGEN, NEAR SURFACE

SENSOR POSITION.--12 feet above streambed.

EXTREMES FOR APRIL TO SEPTEMBER 1989.--Maximum value recorded, $15.6 \mathrm{mg} / \mathrm{L}$ July 19, 1989; minimum value recorded, $2.9 \mathrm{mg} / \mathrm{L}$ June 3, 1989.

DAILY MEAN VALUES OF DISSOLVED OXYGEN, NEAR SURFACE (IN MILLIGRAMS PER LITER), APRIL TO SEPTEMBER 1989

\begin{tabular}{|c|c|c|c|c|c|c|}
\hline DAY & APR & MAY & JUNE & JULY & AUG & SEPT \\
\hline 1 & -- & 7.8 & 9.6 & 8.3 & $6.1 b$ & 7.9 \\
\hline 2 & $-\cdots$ & 6.8 & 9.3 & $\cdots$ & 7.7 & 6.9 \\
\hline 3 & --- & 6.9 & $7.4 \mathrm{~b}$ & -- & --- & $5.3 b$ \\
\hline 4 & -- & 6.9 & $6.5 b$ & 8.5 & $7.8 b$ & $4.9 \mathrm{~b}$ \\
\hline 5 & -- & 6.4 & $5.6 \mathrm{~b}$ & 7.2 & 7.0 & $5.2 b$ \\
\hline 6 & --- & 6.4 & $5.9 b$ & 7.6 & 7.1 & $6.2 \mathrm{~b}$ \\
\hline 7 & -- & 7.0 & $5.9 b$ & 7.7 & 7.5 & 7.0 \\
\hline 8 & -- & 7.1 & $6.6 \mathrm{~b}$ & 10.7 & $5.8 \mathrm{~b}$ & 8.3 \\
\hline 9 & -- & 7.0 & 6.5 & 7.5 & $5.0 \mathrm{~b}$ & 9.5 \\
\hline 10 & -- & 6.8 & 8.1 & $7.2 \mathrm{~b}$ & $5.2 b$ & 10.6 \\
\hline 11 & --- & 6.9 & 9.0 & 6.9 & 6.6 & 9.9 \\
\hline 12 & --- & 7.0 & --- & 6.8 & 7.8 & 9.3 \\
\hline 13 & -- & 7.0 & 7.6 & $5.1 \mathrm{~b}$ & 8.0 & --- \\
\hline 14 & --- & 6.7 & 8.5 & $5.1 b$ & 8.8 & --- \\
\hline 15 & -- & 6.9 & 8.8 & --- & 7.7 & --- \\
\hline 16 & -- & 6.8 & 7.8 & $\cdots$ & 9.0 & --- \\
\hline 17 & -- & 6.8 & 6.9 & $-\cdots$ & 9.1 & --- \\
\hline 18 & $\cdots$ & 7.2 & 7.7 & 10.2 & 8.4 & --- \\
\hline 19 & -. & 7.8 & $8.0 \mathrm{~b}$ & 12.0 & 7.5 & -- \\
\hline 20 & --- & 8.5 & 7.1 & 11.5 & 7.3 & --- \\
\hline 21 & -- & 9.5 & $6.8 \mathrm{~b}$ & 11.8 & 7.6 & --- \\
\hline 22 & 8.7 & 9.2 & 7.5 & 11.4 & 7.6 & --- \\
\hline 23 & 8.6 & 8.1 & 8.1 & -- & -- & -- \\
\hline 24 & 8.4 & 7.5 & 8.5 & -- & $6.2 \mathrm{~b}$ & --- \\
\hline 25 & 8.2 & 8.5 & 8.7 & $10.6 \mathrm{~b}$ & $6.2 b$ & --- \\
\hline 26 & 8.6 & 8.8 & 9.7 & 9.9 & 6.2 & -- \\
\hline 27 & 7.9 & 9.4 & 9.8 & 8.9 & 6.0 & -- \\
\hline 28 & 8.1 & 9.1 & 8.6 & -- & 5.8 & --- \\
\hline 29 & 8.4 & 8.3 & 7.8 & 7.4 & $6.2 b$ & --- \\
\hline 30 & 8.2 & 9.3 & 7.7 & $6.1 \mathrm{~b}$ & 6.5 & --- \\
\hline 31 & --- & 9.1 & --- & $5.2 b$ & 6.7 & --- \\
\hline
\end{tabular}

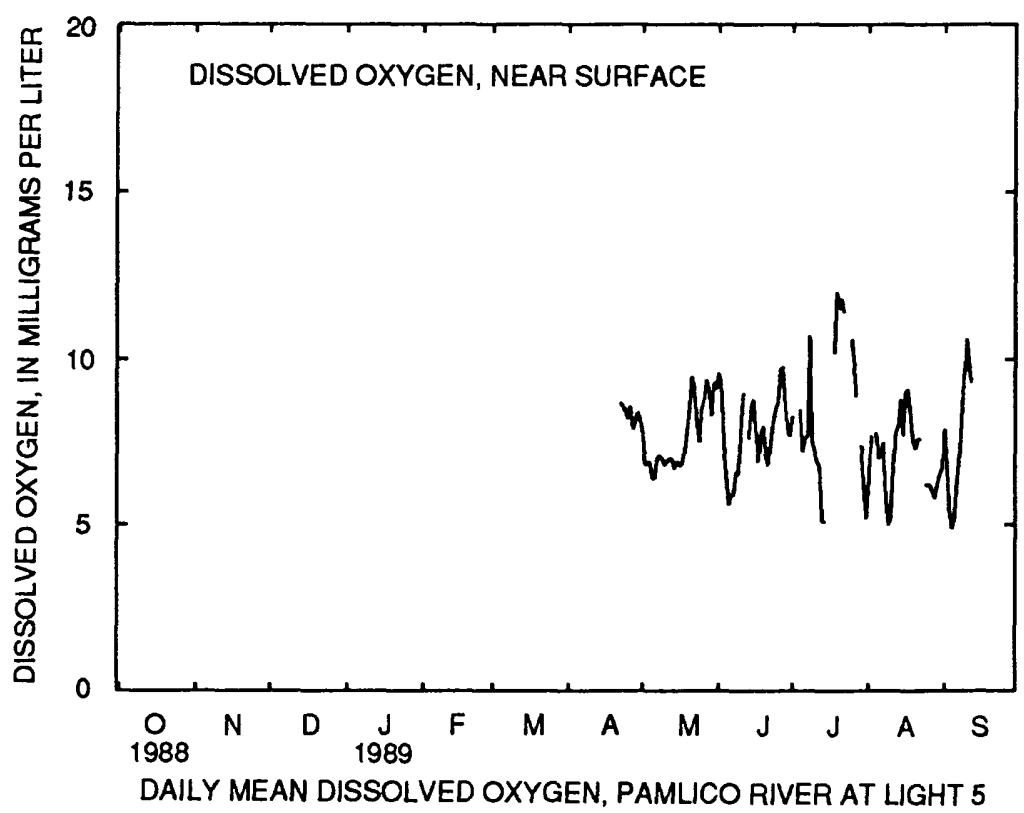


Table 4.--Daily mean values of salinity, water temperature, and dissolved oxygen in water at site 2,

Pamlico River at Light 5, April 1989 to September 1990--Continued

[Site 2 is at latitude $35^{\circ} 25^{\prime} 48^{\prime \prime}$, longitude $76^{\circ} 50^{\prime} 30^{\prime \prime}$, Beaufort County; U.S. Geological Survey downstream order number 0208453300 ; ppt, parts per thousand; ---, no data; <, less than; ${ }^{\circ} \mathrm{C}$, degrees Celsius; mg/L, mllligrams per liter; a, dally minimum value is less than or equal to $2.0 \mathrm{mg} / \mathrm{L} ; \mathrm{b}$, daily minimum value is less than or equal to $5.0 \mathrm{mg} / \mathrm{L}$ but greater than $2.0 \mathrm{mg} / \mathrm{L}$ l

DISSOLVED OXYGEN, NEAR SURFACE

SENSOR POSITION. -12 feet above streambed.

EXTREMES FOR OCTOBER 1989 TO SEPTEMBER 1990.--Maximum value recorded, $16.5 \mathrm{mg} / \mathrm{L} \mathrm{January} 4$, $1990 ; \mathrm{minimum}$ value recorded, $2.8 \mathrm{mg} / \mathrm{L}$ August $30,1990$.

DAILY MEAN VALUES OF DISSOLVED OXYGEN, NEAR SURFACE (IN MILLIGRAMS PER LITER), OCTOBER 1989 TO SEPTEMBER 1990

\begin{tabular}{|c|c|c|c|c|c|c|c|c|c|c|c|c|}
\hline DAY & OCT & NOV & DEC & JAN & FEB & MAR & APR & MAY & JUNE & JULY & $A \cup G$ & SEPT \\
\hline 1 & --- & 10.0 & 10.8 & --- & 11.2 & --- & 10.4 & --- & --- & 7.9 & 7.2 & 8.0 \\
\hline 2 & --- & 9.3 & 11.0 & --- & 10.8 & --- & 10.2 & -- & 8.7 & 7.8 & $6.9 \mathrm{~b}$ & 8.1 \\
\hline 3 & --- & 8.8 & --- & --- & 10.2 & -- & 9.3 & --- & 9.3 & -- & $6.3 \mathrm{~b}$ & 8.7 \\
\hline 4 & --- & 9.2 & --- & 16.0 & 9.6 & --- & 9.1 & --- & 9.4 & 7.2 & $6.2 \mathrm{~b}$ & 7.8 \\
\hline 5 & --- & 9.4 & 11.1 & 15.4 & 9.7 & --- & 9.5 & --- & 9.4 & 6.7 & 6.3 & $7.0 \mathrm{~b}$ \\
\hline 6 & --- & 10.0 & --- & 15.0 & 10.5 & --- & 9.5 & --- & 9.7 & 7.5 & 6.1 & 7.7 \\
\hline 7 & --- & 11.0 & --- & 14.1 & 10.6 & -- & 9.2 & --- & 10.1 & 6.5 & 5.9 & $8.9 b$ \\
\hline 8 & --- & 10.6 & -- & 14.1 & 10.6 & --- & 9.5 & -- & 10.4 & 6.7 & 5.9 & 8.6 \\
\hline 9 & --- & 9.3 & -- & 13.7 & 10.5 & --- & 9.6 & --- & 8.5 & 7.3 & 5.7 & 7.7 \\
\hline 10 & --- & $8.7^{\circ}$ & --- & 13.4 & 10.2 & $\cdots$ & 9.2 & -- & 8.0 & 6.8 & 6.3 & --- \\
\hline 11 & --- & 9.1 & --- & 13.3 & 10.2 & --- & 9.0 & --- & 7.7 & 7.0 & 6.9 & --- \\
\hline 12 & --- & 9.4 & -- & 13.0 & 10.7 & --- & 9.3 & --- & 7.7 & --- & 7.0 & --- \\
\hline 13 & --- & 9.9 & --- & 13.1 & -- & --- & 9.4 & --- & 8.5 & --- & 6.9 & -- \\
\hline 14 & -- & 10.2 & --- & 13.0 & --- & 11.0 & 9.4 & --- & $7.9 b$ & --- & 6.9 & --- \\
\hline 15 & --- & 10.4 & --- & 12.9 & 10.8 & 10.1 & 9.8 & --- & 6.4 & $m$ & 6.5 & --- \\
\hline 16 & --- & 9.0 & --- & 12.2 & 10.4 & 9.4 & 10.3 & --- & 7.2 & -- & 7.1 & --- \\
\hline 17 & --- & 9.4 & --- & 12.1 & 10.0 & 9.0 & 9.8 & 7.8 & 7.8 & --- & 7.1 & $\cdots$ \\
\hline 18 & -- & 10.7 & -- & 11.8 & 10.3 & 9.0 & 9.6 & 7.6 & 7.6 & -- & 7.3 & --- \\
\hline 19 & --- & 11.5 & --- & 11.2 & 9.6 & 9.8 & 10.2 & 8.3 & --- & -- & 7.3 & --- \\
\hline 20 & --- & 11.5 & --- & 11.6 & 9.5 & 9.2 & 10.6 & 7.9 & 7.2 & --- & 7.5 & --- \\
\hline 21 & -- & 10.8 & --- & 10.5 & 9.9 & 9.5 & 10.8 & --- & 7.3 & --- & 7.3 & --- \\
\hline 22 & --- & 11.0 & --- & 10.6 & $-\cdots$ & 10.6 & 10.6 & --- & 7.2 & --- & 7.9 & --- \\
\hline 23 & --- & 11.1 & --- & --- & --- & 10.9 & 11.1 & --- & $6.4 \mathrm{~b}$ & --- & 7.9 & --- \\
\hline 24 & --- & 12.2 & --- & 11.1 & $-\cdots$ & 10.9 & 10.8 & --- & 7.1 & --- & 7.8 & --- \\
\hline 25 & -- & 13.2 & --- & 10.9 & --- & 10.2 & 10.7 & --- & 7.7 & --- & 8.0 & --- \\
\hline 26 & -- & 12.1 & --- & -- & --- & 10.7 & 11.1 & --- & 7.4 & 6.9 & 8.1 & --- \\
\hline 27 & --- & 12.0 & -- & 10.3 & --- & --- & 10.8 & -- & 7.7 & 6.2 & 8.7 & -- \\
\hline 28 & --- & 11.9 & --- & 11.1 & --- & --- & --- & --- & 8.1 & 6.0 & 8.6 & --- \\
\hline 29 & --- & 11.7 & -- & 10.9 & --- & 10.2 & --- & --- & 8.0 & 6.3 & 8.8 & --- \\
\hline 30 & --- & 10.8 & -- & 10.2 & --- & 10.8 & --- & -- & 7.8 & 6.9 & $7.4 \mathrm{~b}$ & --- \\
\hline 31 & --- & --- & --- & 10.7 & --- & 11.2 & --- & --- & --- & 7.2 & $7.5 b$ & --- \\
\hline
\end{tabular}

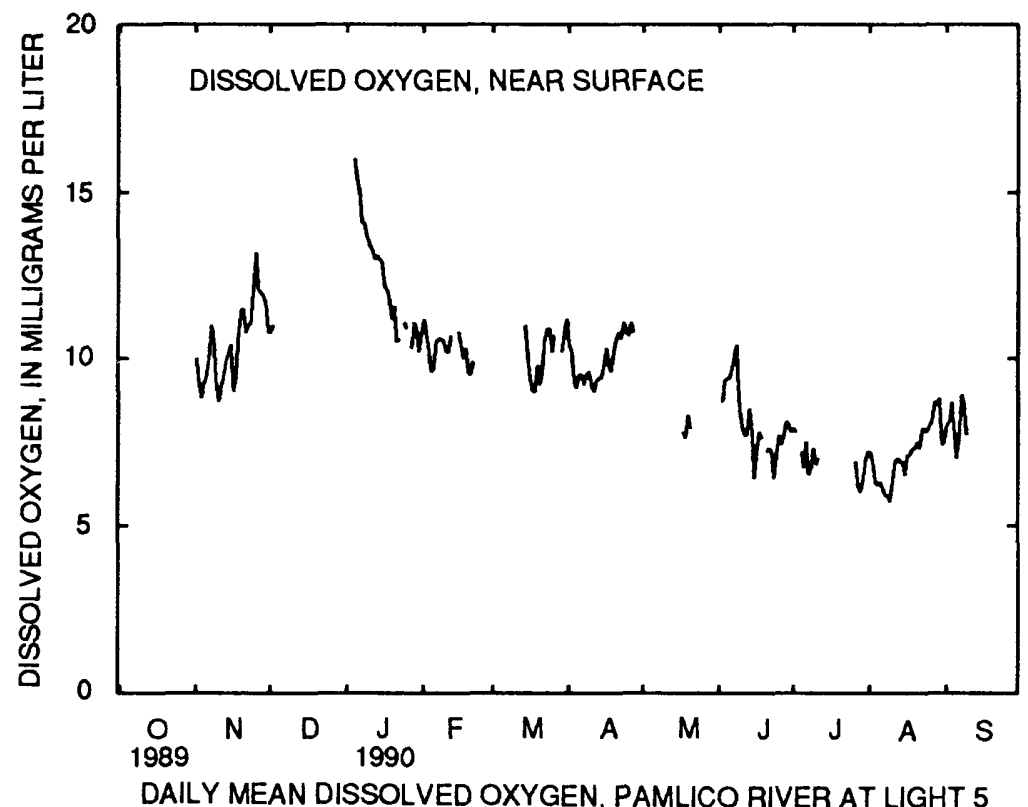


Table 4.--Daily mean values of salinity, water temperature, and dissolved oxygen in water at site 2, Pamlico River at Light 5, April 1989 to September 1990--Continued

[Site 2 is at latitude $35^{\circ} 25^{\prime} 48^{\prime \prime}$, longitude 76 $50^{\prime} 30^{\prime \prime}$, Beaufort County; U.S. Geological Survey downstream order number 0208453300; ppt, parts per thousand; ---, no data; <, less than; ${ }^{\circ} \mathrm{C}$, degrees Celsius; mg/L, milligrams per liter; a, dally minimum value is less than or equal

to $2.0 \mathrm{mg} / \mathrm{L} ; \mathrm{b}$, daily minimum value is less than or equal to $5.0 \mathrm{mg} / \mathrm{L}$ but greater than $2.0 \mathrm{mg} / \mathrm{L}$ ]

DISSOLVED OXYGEN, MID-DEPTH

SENSOR POSITION. --8 feet above streambed.

EXTREMES FOR APRIL TO SEPTEMBER 1989.--Maximum value recorded, $18.6 \mathrm{mg} / \mathrm{L}$ July 19, $1989 ; \mathrm{minlmum}$ value recorded, $2.2 \mathrm{mg} / \mathrm{L}$ August $24,1989$.

DAILY MEAN VALUES OF DISSOLVED OXYGEN, MID-DEPTH (IN MILLIGRAMS PER LITER), APRIL TO SEPTEMBER 1989

\begin{tabular}{|c|c|c|c|c|c|c|}
\hline DAY & APR & MAY & JUNE & JULY & AUG & SEPT \\
\hline 1 & --- & 7.6 & 8.8 & $6.0 \mathrm{~b}$ & $6.5 b$ & $\cdots$ \\
\hline 2 & -- & 6.6 & 8.6 & $6.9 b$ & 7.6 & $\cdots$ \\
\hline 3 & -- & 6.6 & $7.1 \mathrm{~b}$ & $6.2 \mathrm{~b}$ & --- & -- \\
\hline 4 & --- & 6.3 & $5.9 b$ & $5.9 b$ & $7.7 \mathrm{~b}$ & $\cdots$ \\
\hline 5 & --- & 6.1 & $5.1 b$ & $5.0 \mathrm{~b}$ & 6.7 & -- \\
\hline 6 & -- & 6.1 & $5.5 b$ & $4.0 \mathrm{~b}$ & $6.7 \mathrm{~b}$ & $\ldots$ \\
\hline 7 & --- & 6.7 & $5.7 \mathrm{~b}$ & -- & $7.1 \mathrm{~b}$ & $-\cdots$ \\
\hline 8 & --- & 6.9 & $6.1 b$ & --- & $5.0 \mathrm{~b}$ & $-\cdots$ \\
\hline 9 & --- & 6.8 & $6.1 \mathrm{~b}$ & -- & $3.7 b$ & $\cdots$ \\
\hline 10 & -- & 6.6 & 7.7 & -- & -- & $\cdots$ \\
\hline 11 & --- & 6.8 & $8.0 \mathrm{~b}$ & -- & -- & $\ldots$ \\
\hline 12 & -- & 6.8 & -- & --- & -- & -- \\
\hline 13 & --- & 6.8 & 7.3 & $-\infty$ & --- & -- \\
\hline 14 & -- & 6.6 & $7.7 \mathrm{~b}$ & $-\infty$ & 8.1 & $\cdots$ \\
\hline 15 & --- & 6.7 & 8.3 & --- & -- & -- \\
\hline 16 & --- & 6.7 & 7.4 & $6.1 \mathrm{~b}$ & --- & -- \\
\hline 17 & --- & 6.7 & 6.4 & 8.8 & 8.1 & $\cdots$ \\
\hline 18 & --- & 7.0 & $7.0 \mathrm{~b}$ & 11.9 & 6.9 & $\ldots$ \\
\hline 19 & --- & 7.6 & $7.1 \mathrm{~b}$ & 14.8 & $5.4 \mathrm{~b}$ & -- \\
\hline 20 & $-\cdots$ & 8.3 & $6.3 b$ & 13.1 & $5.1 \mathrm{~b}$ & $-\cdots$ \\
\hline 21 & -- & 9.3 & $6.0 \mathrm{~b}$ & 11.1 & $5.3 b$ & $\cdots$ \\
\hline 22 & 8.4 & 9.0 & $6.2 b$ & 10.4 & $5.1 \mathrm{~b}$ & $\cdots$ \\
\hline 23 & 8.3 & 7.8 & $6.1 \mathrm{~b}$ & 11.2 & -- & -- \\
\hline 24 & 8.1 & 7.4 & $6.6 \mathrm{~b}$ & 13.0 & $4.5 b$ & $\cdots$ \\
\hline 25 & 7.9 & 7.9 & 7.5 & $13.2 b$ & $4.6 \mathrm{~b}$ & -- \\
\hline 26 & 8.1 & 8.5 & 7.2 & 12.7 & $4.9 \mathrm{~b}$ & -- \\
\hline 27 & 7.7 & 9.2 & 7.5 & 11.2 & $4.7 \mathrm{~b}$ & --- \\
\hline 28 & 7.8 & 8.9 & $6.7 \mathrm{~b}$ & -- & $5.1 \mathrm{~b}$ & --- \\
\hline 29 & 8.2 & 8.5 & $6.1 \mathrm{~b}$ & $9.2 b$ & $5.9 b$ & -- \\
\hline 30 & 7.8 & 9.2 & $6.3 b$ & 8.8 & 6.2 & --- \\
\hline 31 & $\cdots$ & 9.0 & $\cdots$ & $6.2 b$ & -- & -- \\
\hline
\end{tabular}

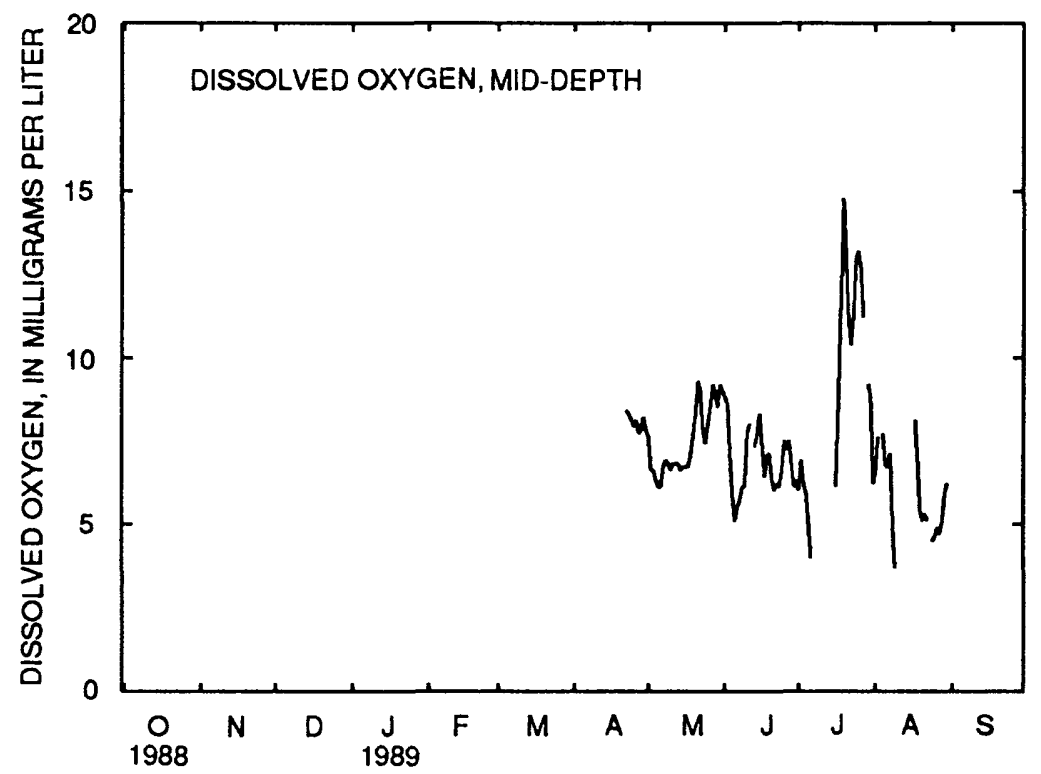

DAILY MEAN DISSOLVED OXYGEN, PAMLICO RIVER AT LIGHT 5 
Table 4.--Daily mean values of salinity, water temperature, and dissolved oxygen in water at site 2 ,

Pamlico River at Light 5, April 1989 to September 1990--Cont inued

[S1te 2 is at latitude $35^{\circ} 25^{\prime} 48^{\prime \prime}$, longitude $76^{\circ} 50^{\prime} 30^{\prime \prime}$, Beaufort County; U.S. Geological Survey downstream order number 0208453300; ppt, parts per thousand; ---, no data; <, less than; ${ }^{\circ} \mathrm{C}$, degrees Celsius; mg/L, milligrams per liter; a, dally minimum value is less than or equal

to $2.0 \mathrm{mg} / \mathrm{L} ; \mathrm{b}$, daliy minimum value is less than or equal to $5.0 \mathrm{mg} / \mathrm{L}$ but greater than $2.0 \mathrm{mg} / \mathrm{L}$ ]

DISSOLVED OXYGEN, MID-DEPTH

SENSOR POSITION. --8 feet above streambed.

EXTREMES FOR OCTOBER 1989 TO SEPTEMBER 1990.--Maximum value recorded, $16.7 \mathrm{mg} / \mathrm{L}$ January 4 , $1990 ; \mathrm{minimum}$ value recorded, <1.0 mg/L November 2, 8, 9, 1989, July 7, 1990.

DAILY MEAN VALUES OF DISSOLVED OXYGEN, MID-DEPTH (IN MILLIGRAMS PER LITER), OCTOBER 1989 TO SEPTEMBER 1990

\begin{tabular}{|c|c|c|c|c|c|c|c|c|c|c|c|c|}
\hline DAY & OCT & NOV & DEC & JAN & FEB & MAR & APR & MAY & JUNE & JULY & AUG & SEPT \\
\hline 1 & --- & 8.8 & 10.4 & -- & 10.9 & --- & 10.8 & --- & --- & 10.1 & 7.8 & 7.5 \\
\hline 2 & --- & $7.4 a$ & 11.0 & -- & 11.0 & --- & 9.1 & --- & 7.5 & $9.4 \mathrm{~b}$ & $4.2 \mathrm{a}$ & $6.6 b$ \\
\hline 3 & --- & 8.4 & 10.9 & -- & 9.9 & --- & 10.3 & -- & $7.8 \mathrm{~b}$ & -- & $5.8 b$ & 7.7 \\
\hline 4 & --- & 8.1 & 11.6 & 16.1 & 9.6 & --- & 9.9 & --- & 8.9 & $7.4 b$ & $6.7 a$ & --- \\
\hline 5 & --- & -- & 12.1 & 15.6 & 9.8 & -- & 10.3 & -- & $7.4 \mathrm{a}$ & 7.6 & $6.8 b$ & $-\cdots$ \\
\hline 6 & -- & 8.9 & -- & 15.4 & 10.5 & --- & 10.3 & - & $6.2 \mathrm{~b}$ & $7.3 b$ & $7.5 b$ & - \\
\hline 7 & -- & $7.7 a$ & -- & 13.8 & 10.2 & -- & 9.9 & $\ldots$ & 9.7 & 5.6 & 7.5 & -- \\
\hline 8 & -- & $5.0 a$ & --- & --- & 10.1 & --- & 10.1 & -- & $7.6 \mathrm{~b}$ & 7.3 & $6.9 b$ & --- \\
\hline 9 & --- & $4.9 a$ & -- & -- & 10.2 & --- & 10.0 & --- & $7.5 b$ & 7.7 & $6.7 b$ & --- \\
\hline 10 & -- & $6.7 \mathrm{~b}$ & $\ldots$ & $-\cdots$ & 10.2 & --- & 9.7 & --- & $7.9 b$ & 7.3 & $7.9 \mathrm{~b}$ & --- \\
\hline 11 & --- & 7.2 & -- & --- & 10.1 & --- & 9.5 & --- & 8.0 & 7.4 & $7.7 \mathrm{~b}$ & --- \\
\hline 12 & -- & 7.6 & --- & -- & 10.7 & --- & 9.7 & --- & 8.0 & 7.3 & $6.2 b$ & -- \\
\hline 13 & --- & 7.7 & -- & --- & --- & --- & 9.8 & --- & $7.9 \mathrm{~b}$ & 7.2 & $4.7 b$ & --- \\
\hline 14 & --- & 7.8 & --- & --- & -- & 7.5 & 9.8 & --- & $6.6 b$ & 7.0 & $6.3 b$ & $-\cdots$ \\
\hline 15 & -- & $6.4 \mathrm{~b}$ & -- & --- & 10.4 & 8.7 & 9.9 & --- & $6.4 \mathrm{~b}$ & 7.0 & $5.0 b$ & --- \\
\hline 16 & -- & $4.5 b$ & --- & --- & 10.2 & 9.0 & $9.7 \mathrm{~b}$ & $-\ldots$ & 7.1 & 6.9 & 7.5 & --- \\
\hline 17 & -- & $5.3 b$ & --- & --- & 9.9 & 9.6 & 9.8 & 7.4 & 7.4 & $7.0 \mathrm{~b}$ & $7.2 b$ & --- \\
\hline 18 & --- & 6.3 & --- & -- & 10.2 & 9.4 & 9.8 & 7.2 & 7.3 & 8.5 & $7.2 \mathrm{~b}$ & $\ldots$ \\
\hline 19 & --- & 7.0 & --- & --- & 9.6 & 9.8 & 10.5 & 7.3 & 7.5 & 8.0 & $5.2 b$ & $\cdots$ \\
\hline 20 & -- & 7.4 & --- & --- & 9.5 & 9.6 & 10.9 & 7.5 & 6.9 & $8.8 b$ & $5.6 b$ & --- \\
\hline 21 & --- & 7.3 & --- & -- & 10.0 & 9.7 & 11.1 & --- & 7.0 & $8.3 b$ & $6.3 b$ & -- \\
\hline 22 & -- & 8.0 & --- & -- & -- & 10.1 & 10.8 & -- & $5.8 b$ & $7.1 \mathrm{~b}$ & $5.0 \mathrm{~b}$ & -- \\
\hline 23 & -- & 8.3 & --- & -- & --- & 11.0 & 11.0 & $\cdots$ & $7.0 \mathrm{~b}$ & $8.1 b$ & $4.4 b$ & --- \\
\hline 24 & --- & 9.1 & --- & 11.0 & --- & 10.0 & 10.4 & --- & $7.8 \mathrm{~b}$ & $7.4 \mathrm{~b}$ & $6.7 \mathrm{~b}$ & --- \\
\hline 25 & --- & 9.5 & --- & 11.0 & --- & 10.1 & 10.6 & --- & 8.2 & $7.6 \mathrm{~b}$ & $6.4 \mathrm{~b}$ & -- \\
\hline 26 & -- & 9.6 & --- & -- & --- & 10.6 & 9.8 & -- & 8.1 & $7.2 \mathrm{~b}$ & $6.4 \mathrm{~b}$ & -- \\
\hline 27 & --- & 9.6 & --- & 9.8 & -- & -- & 10.6 & -- & 8.0 & $6.9 \mathrm{~b}$ & --- & -- \\
\hline 28 & $-\cdots$ & 10.1 & -- & 11.1 & --- & --- & --- & $-\cdots$ & 8.8 & 7.2 & $\cdots$ & $\cdots$ \\
\hline 29 & --- & 10.3 & -- & 10.1 & --- & 11.8 & -- & --- & $9.4 \mathrm{~b}$ & 7.8 & --- & --- \\
\hline 30 & --- & 10.1 & --- & 10.1 & --- & 11.9 & --- & --- & 9.9 & 8.0 & --- & --- \\
\hline 31 & 8.3 & --- & --- & 10.6 & --- & 11.3 & --- & --- & --- & 8.2 & --- & --- \\
\hline
\end{tabular}

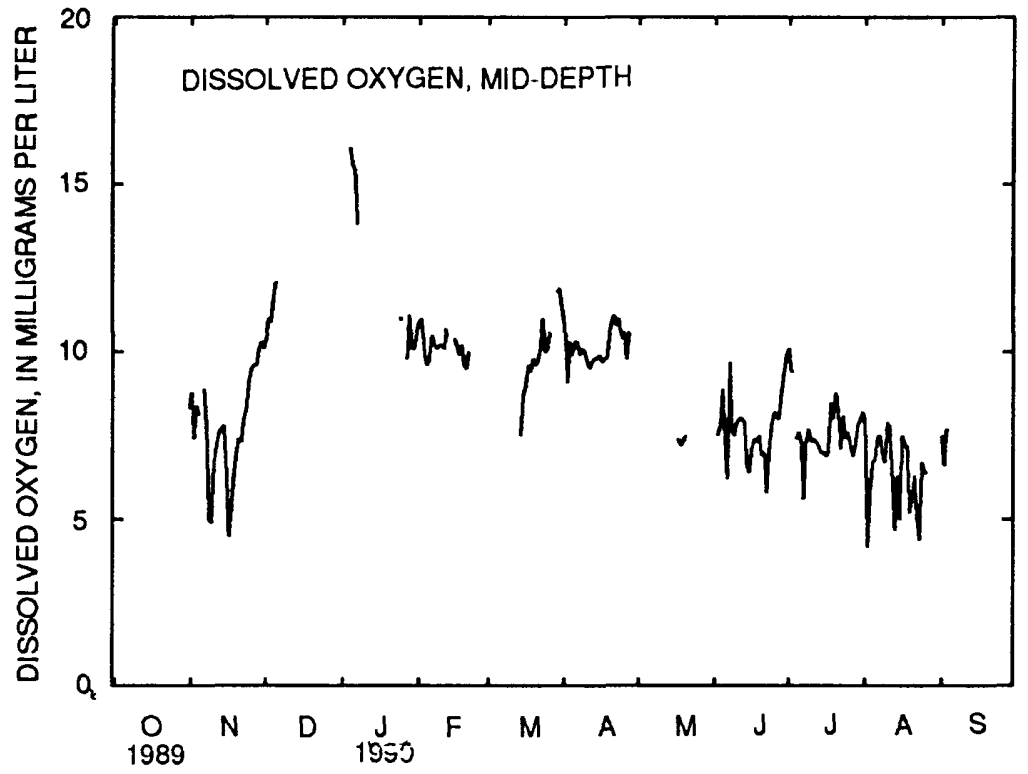

DAILY MEAN DISSOLVED OXYGEN, PAMLICO RIVER AT LIGHT 5 
Table 4.--Daily mean values of salinity, water temperature, and dissolved oxygen in water at site 2, Pamlico River at Light 5, April 1989 to September 1990--Continued

[Site 2 is at latitude $35^{\circ} 25^{\prime} 48^{\prime \prime}$, longitude $76^{\circ} 50^{\prime} 30^{\prime \prime}$, Beaufort County: U.S. Geological Survey downstream order number 0208453300; ppt, parts per thousand; ---, no data; <, less than; ${ }^{\circ} \mathrm{C}$, degrees Celsius; mg/L, milligrams per liter; a, dally minimum value is less than or equal to $2.0 \mathrm{mg} / \mathrm{L} ; \mathrm{b}$, dally minimum value is less than or equal to $5.0 \mathrm{mg} / \mathrm{L}$ but greater than $2.0 \mathrm{mg} / \mathrm{L}$ ]

DISSOLVED OXYGEN, NEAR BOTTOM

SENSOR POSITION.--4 feet above streambed.

EXTREMES FOR APRIL TO SEPTEMBER 1989.--Maximum value recorded, $10.0 \mathrm{mg} / \mathrm{L} \mathrm{July} \mathrm{2,} 1989 ; \mathrm{min} 1 \mathrm{mum}$ value recorded, $<1.0 \mathrm{mg} / \mathrm{L}$ May 12-14, 17, 20, 23, 24, June 4, 15, 20, 21, 23-30, July 1, 1989.

DAILY MEAN VALUES OF DISSOLVED OXYGEN, NEAR BOTTOM (IN MILLIGRAMS PER LITER), APRIL TO SEPTEMBER 1989

\begin{tabular}{|c|c|c|c|c|c|c|}
\hline DAY & AP R & MAY & JUNE & JULY & AUG & SEPT \\
\hline 1 & --- & --- & --- & $5.9 a$ & --- & --- \\
\hline 2 & --- & --- & --- & 7.8 & --- & --- \\
\hline 3 & --- & --- & --- & $6.0 \mathrm{~b}$ & -- & $-\cdots$ \\
\hline 4 & --- & -- & $2.7 a$ & $5.3 a$ & --- & -- \\
\hline 5 & --- & $5.1 \mathrm{~b}$ & $3.3 a$ & $4.7 \mathrm{~b}$ & --- & -- \\
\hline 6 & --- & $5.9 b$ & $4.5 b$ & -- & $-\infty$ & -- \\
\hline 7 & -- & 6.5 & $5.4 b$ & --- & --- & --- \\
\hline 8 & -- & 6.6 & $5.1 b$ & --- & -- & -- \\
\hline 9 & -- & 6.3 & $5.4 b$ & -- & -- & $\cdots$ \\
\hline 10 & -- & 6.1 & $7.0 \mathrm{~b}$ & --- & --- & -- \\
\hline 11 & --- & 6.1 & $5.8 \mathrm{a}$ & --- & --- & --- \\
\hline 12 & -- & $4.8 \mathrm{a}$ & -- & --- & $-\cdots$ & -- \\
\hline 13 & --- & $5.1 \mathrm{a}$ & $5.9 b$ & --- & --- & $\cdots$ \\
\hline 14 & --- & $4.8 \mathrm{a}$ & $6.2 b$ & -- & --- & -- \\
\hline 15 & -- & $4.8 \mathrm{~b}$ & $4.5 a$ & -- & --- & $-\cdots$ \\
\hline 16 & --- & --- & $5.2 a$ & --- & --- & $\cdots$ \\
\hline 17 & --- & $5.0 \mathrm{a}$ & 5.8 & $-\cdots$ & --- & --- \\
\hline 18 & --- & $5.4 \mathrm{~b}$ & $4.9 b$ & --- & --- & $-\cdots$ \\
\hline 19 & --- & $6.4 \mathrm{~b}$ & $3.7 a$ & --- & -- & --- \\
\hline 20 & --- & $4.9 a$ & $3.1 \mathrm{a}$ & --- & --- & --- \\
\hline 21 & --- & --- & $3.3 a$ & --- & --- & --- \\
\hline 22 & -- & $\cdots$ & $3.7 a$ & --- & --- & --- \\
\hline 23 & --- & $4.5 a$ & $3.4 a$ & --- & $\cdots$ & --- \\
\hline 24 & $\cdots$ & $4.9 a$ & $3.6 a$ & --- & $-\cdots$ & --- \\
\hline 25 & -- & --- & $2.5 a$ & --- & $-\cdots$ & -- \\
\hline 26 & --- & --- & $1.9 a$ & --- & --- & --- \\
\hline 27 & --- & -- & $<1.0 \mathrm{a}$ & --- & --- & -- \\
\hline 28 & --- & --- & $<1.0 \mathrm{a}$ & --- & --- & $\cdots$ \\
\hline 29 & --- & $5.2 b$ & $<1.0 a$ & --- & --- & --- \\
\hline 30 & $\cdots$ & $5.3 b$ & $2.7 a$ & --- & --- & --- \\
\hline 31 & -- & --- & --- & --- & -- & --- \\
\hline
\end{tabular}

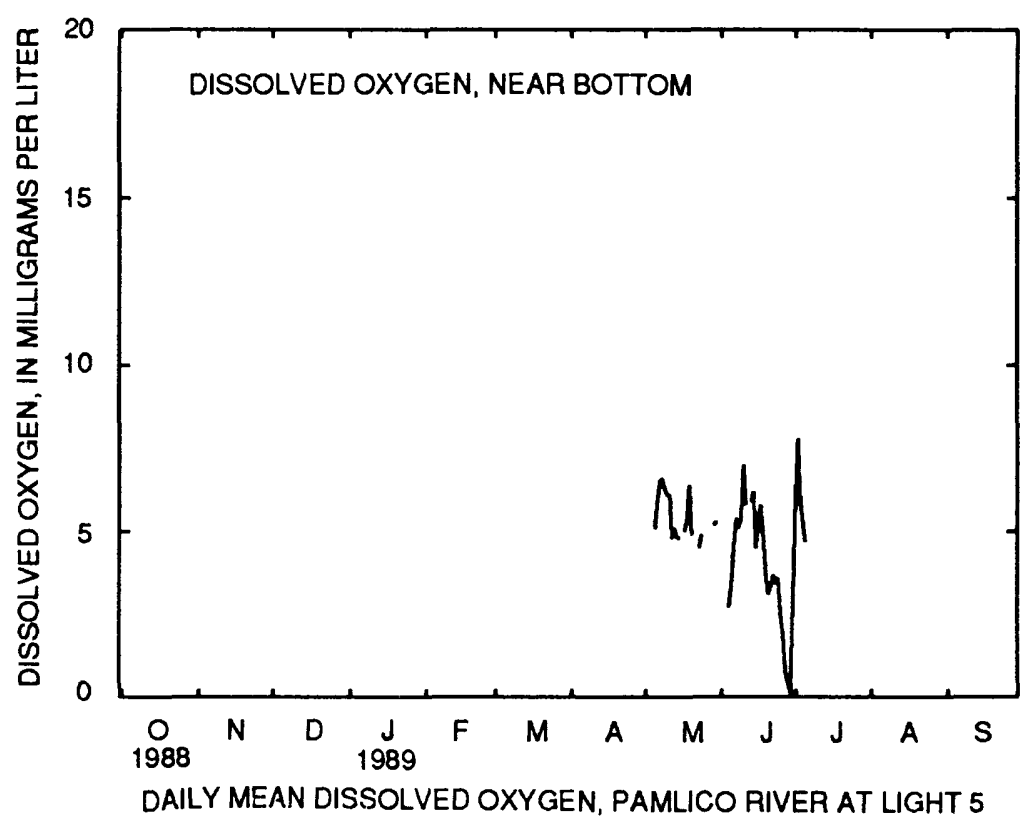


Table 4.--Daily mean values of salinity, water temperature, and dissolved oxygen in water at site 2 ,

Pamlico River at Light 5, April 1989 to September 1990--Continued

[S1te 2 is at latitude $35^{\circ} 25^{\prime} 48^{\prime \prime}$, longltude $76^{\circ} 50^{\prime} 30^{\prime \prime}$, Beaufort County; U.S. Geological Survey downstream order number 0208453300; ppt, parts per thousand; ---, no data; <, less than; ${ }^{\circ} \mathrm{C}$, degrees Celsius; $\mathrm{mg} / \mathrm{L}$, milligrams per liter; a, daily minimum value is less than or equal

to $2.0 \mathrm{mg} / \mathrm{L} ; \mathrm{b}$, daily minimum value is less than or equal to $5.0 \mathrm{mg} / \mathrm{L}$ but greater than $2.0 \mathrm{mg} / \mathrm{L}$ ]

DISSOLVED OXYGEN, NEAR BOTTOM

SENSOR POSITION. -4 feet above streambed.

EXTREMES FOR OCTOBER 1989 TO SEPTEMBER 1990.--Maximum value recorded, 18.2 mg/L January 4, 1990; minimum value recorded, <1.0 mg/L October 17-23, 27, 28, November 9, 1989, Apri1 26-28, July 27-31, August 1-21, 27, 28, 1990.

DAILY MEAN VALUES OF DISSOLVED OXYGEN, NEAR BOTTOM (IN MILLIGRAMS PER LITER), OCTOBER 1989 TO SEPTEMBER I990

\begin{tabular}{|c|c|c|c|c|c|c|c|c|c|c|c|c|}
\hline DAY & OCT & NOV & DEC & JAN & FEB & MAR & APR & MAY & JUNE & JULY & AUG & SEPT \\
\hline 1 & --- & $3.1 \mathrm{a}$ & $-\infty$ & --- & 7.5 & -- & 7.4 & 7.0 & --- & -- & $1.0 \mathrm{a}$ & -- \\
\hline 2 & --- & --- & --- & --- & 7.4 & -- & 6.6 & 7.1 & --- & --- & $<1.0 \mathrm{a}$ & --- \\
\hline 3 & -- & -- & $-\infty$ & --- & 7.1 & -- & $7.1 \mathrm{~b}$ & 7.2 & --- & --- & $<1.0 a$ & -- \\
\hline 4 & -- & -- & -- & 17.1 & 7.5 & --- & 10.2 & 7.2 & --- & --- & $1.6 a$ & -- \\
\hline 5 & $-\cdots$ & $4.4 a$ & -- & 15.5 & 8.5 & -- & 9.8 & 7.3 & --- & --- & $<1.0 a$ & -- \\
\hline 6 & -- & --- & --- & 15.6 & 8.3 & --- & 7.4 & -- & --- & --- & $<1.0 a$ & --- \\
\hline 7 & -- & --- & --- & 15.3 & 8.1 & -- & $8.3 b$ & --- & --- & --- & $<1.0 \mathrm{a}$ & --- \\
\hline 8 & $\cdots$ & $3.2 a$ & --- & --- & 7.1 & --- & $6.8 b$ & --- & -- & --- & $<1.0 \mathrm{a}$ & --- \\
\hline 9 & --- & $3.2 a$ & $-\cdots$ & --- & 6.9 & -- & 7.9 & -- & --- & --- & $<1.0 a$ & --- \\
\hline 10 & -- & $5.2 b$ & -- & --- & $8.2 b$ & --- & 7.3 & --- & --- & --- & $<1.0 \mathrm{a}$ & --- \\
\hline 11 & $-\cdots$ & $4.7 b$ & --- & --- & 9.2 & -- & $9.7 \mathrm{~b}$ & --- & -- & -- & $<1.0 \mathrm{a}$ & --- \\
\hline 12 & --- & $4.2 \mathrm{a}$ & --- & -- & 9.7 & -- & 9.3 & --- & -- & -- & $<1.0 a$ & --- \\
\hline 13 & -- & $2.7 a$ & --- & --- & --- & -- & $7.2 \mathrm{~b}$ & --- & -- & --- & $<1.0 \mathrm{a}$ & --- \\
\hline 14 & --- & -- & --- & --- & $-\cdots$ & 6.4 & 10.1 & --- & -- & --- & $<1.0 \mathrm{a}$ & -- \\
\hline 15 & --- & $-\cdots$ & -- & --- & 7.4 & $6.0 \mathrm{~b}$ & 10.6 & --- & $\cdots$ & -- & $<1.0 \mathrm{a}$ & -- \\
\hline 16 & --- & --- & --- & --- & 7.5 & $5.6 \mathrm{~b}$ & 8.7 & --- & --- & --- & $<1.0 a$ & --- \\
\hline 17 & $<1.0 \mathrm{a}$ & 12.0 & --- & --- & 7.8 & $6.5 b$ & 9.6 & 7.1 & --- & --- & $<1.0 \mathrm{a}$ & --- \\
\hline 18 & $<1.0 \mathrm{a}$ & 10.4 & --- & --- & 8.3 & $7.8 \mathrm{~b}$ & 11.2 & 9.0 & -- & --- & $<1.0 \mathrm{a}$ & $-\infty-$ \\
\hline 19 & $1.4 \mathrm{a}$ & 9.9 & --- & $\cdots$ & $7.3 b$ & $6.9 b$ & 11.9 & 8.5 & $5.6 b$ & --- & $<1.0 a$ & --- \\
\hline 20 & $1.9 \mathrm{a}$ & 8.7 & -- & -- & 7.4 & 9.2 & 12.5 & 6.9 & 7.0 & --- & $1.0 \mathrm{a}$ & $-\cdots$ \\
\hline 21 & $6.7 \mathrm{a}$ & 14.5 & --- & -- & 8.5 & 8.4 & 11.8 & --- & $5.4 \mathrm{~b}$ & --- & $1.0 \mathrm{a}$ & $-\cdots$ \\
\hline 22 & $7.2 \mathrm{a}$ & 15.6 & --- & -- & $6.4 a$ & 8.0 & 11.8 & --- & $4.3 b$ & -- & $1.1 \mathrm{a}$ & $\cdots$ \\
\hline 23 & 4.02 & 16.5 & --- & --- & --- & $7.1 \mathrm{~b}$ & $9.3 b$ & -- & --- & --- & $1.3 a$ & $\cdots$ \\
\hline 24 & $7.3 a$ & 16.1 & --- & 7.8 & -- & $7.9 \mathrm{~b}$ & $9.1 \mathrm{~b}$ & --- & $-\cdots$ & -- & $1.7 \mathrm{a}$ & $-m$ \\
\hline 25 & 8.6 & 15.0 & --- & 6.8 & -- & $9.2 b$ & $6.0 \mathrm{~b}$ & -- & $\cdots$ & --- & $1.6 a$ & $\cdots$ \\
\hline 26 & $7.0 \mathrm{~b}$ & --- & --- & -- & $\cdots$ & $8.3 b$ & $1.9 a$ & --- & -- & --- & $1.5 \mathrm{a}$ & -- \\
\hline 27 & $5.3 a$ & -- & --- & 7.8 & --- & --- & $<1.0 a$ & --- & -- & $1.7 a$ & $1.2 \mathrm{a}$ & --- \\
\hline 28 & $1.6 a$ & -- & --- & 7.4 & $-\cdots$ & -- & $1.3 a$ & --- & -- & $3.8 \mathrm{a}$ & $<1.0 \mathrm{a}$ & $-\cdots$ \\
\hline 29 & $5.3 a$ & --- & --- & 7.2 & --- & 12.4 & 6.8 & -- & $-\cdots$ & $5.5 a$ & -- & $-\cdots$ \\
\hline 30 & -- & --- & --- & 8.4 & --- & 11.4 & 7.0 & -- & --- & $3.3 \mathrm{a}$ & --- & -- \\
\hline 31 & -- & --- & -- & 8.4 & $-\cdots$ & 10.1 & --- & --- & --- & $1.7 a$ & -- & $\cdots-$ \\
\hline
\end{tabular}

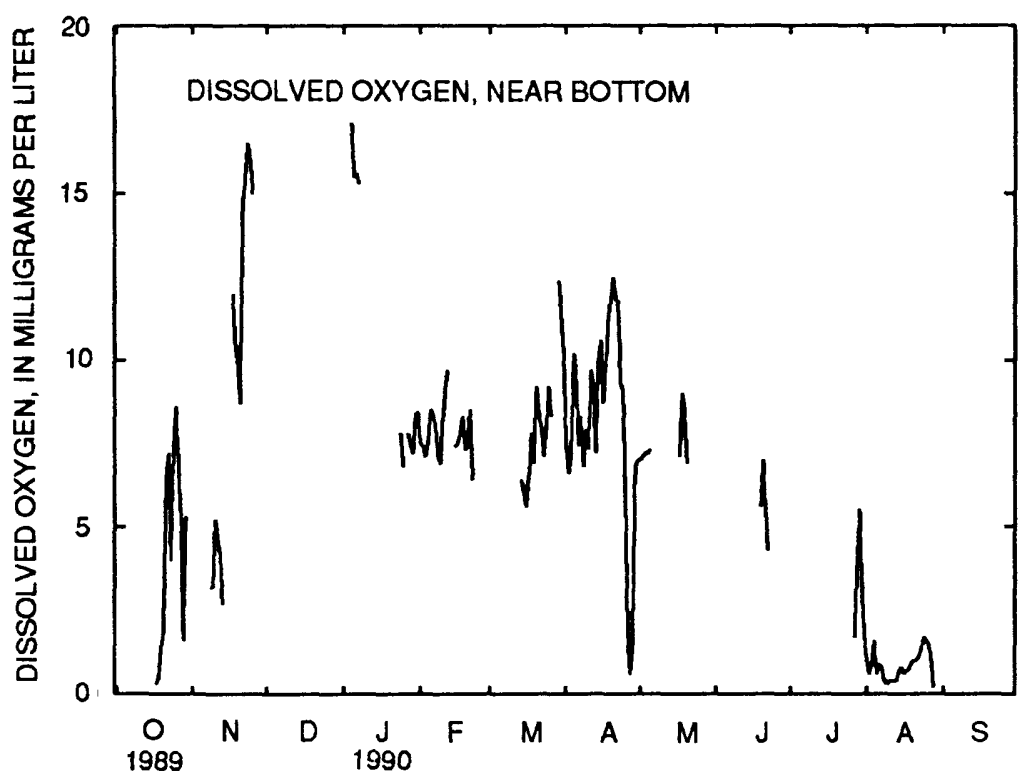


Table 5.--Daily mean values of salinity, water temperature, and dissolved oxygen in water at site 3 , Pamlico River at Light 4, April 1989 to september 1990

[S1te 3 is at latitude $35^{\circ} 24^{\prime} 47^{\prime \prime}$, longitude 76 $45^{\prime} 52^{\prime \prime}$, Beaufort County; U.S. Geological Survey downstream order number 0208454253; ppt, parts per thousand; <, less than; ---, no data;

${ }^{\circ} \mathrm{C}$, degrees Celsius; mg/L, milligrams per liter; a, daily minimum value is less than or equal

to $2.0 \mathrm{mg} / \mathrm{L} ; \mathrm{b}$, daily minimum value is less than or equal to $5.0 \mathrm{mg} / \mathrm{L}$ but greater than $2.0 \mathrm{mg} / \mathrm{L}$ l

\section{SALINITY, NEAR SURFACE}

SENSOR POSITION.--8 feet above streambed.

EXTREMES FOR APRIL TO SEPTEMBER 1989.--Maximum value recorded, 8.7 ppt September 9, 1989; minimum value recorded, $<0.1$ ppt May 6, 1989.

DAILY MEAN VALUES OF SALINITY, NEAR SURFACE (IN PARTS PER THOUSAND), APRIL TO SEPTEMBER 1989

\begin{tabular}{|c|c|c|c|c|c|c|}
\hline DAY & APR & MAY & JUNE & JULY & AUG & SEPT \\
\hline 1 & -- & 3.0 & --- & --- & --- & 7.4 \\
\hline 2 & --- & --- & --- & -- & --- & 7.2 \\
\hline 3 & --- & 2.8 & --- & -- & --- & 7.7 \\
\hline 4 & -- & 2.1 & --- & $\cdots$ & -- & 8.0 \\
\hline 5 & --- & 2.1 & --- & --- & --- & 7.9 \\
\hline 6 & -- & 1.4 & -- & --- & -- & 8.2 \\
\hline 7 & --- & 2.1 & -- & --- & --- & 8.2 \\
\hline 8 & --- & 1.8 & -- & --- & --- & 8.4 \\
\hline 9 & --- & 1.0 & --- & --- & --- & 8.4 \\
\hline 10 & --- & .8 & $\cdots$ & --- & --- & 8.0 \\
\hline 11 & --- & 1.1 & --- & --- & --- & 7.9 \\
\hline 12 & --- & 1.0 & --- & --- & $\cdots$ & 7.9 \\
\hline 13 & -- & .9 & --- & -- & --- & $-\cdots$ \\
\hline 14 & --- & .8 & --- & --- & $\cdots$ & --- \\
\hline 15 & -- & .7 & --- & --- & -- & -- \\
\hline 16 & --- & 1.4 & $-\cdots$ & --- & --- & -- \\
\hline 17 & --- & 2.8 & -- & --- & $\cdots$ & -- \\
\hline 18 & --- & --- & --- & --- & --- & $-\cdots$ \\
\hline 19 & $\cdots$ & --- & -- & --- & --- & --- \\
\hline 20 & $-\infty$ & -- & --- & --- & -- & --- \\
\hline 21 & --- & -- & --- & --- & --- & --- \\
\hline 22 & --- & $\cdots$ & -- & -- & --- & --- \\
\hline 23 & $-\infty$ & --- & --- & --- & --- & -- \\
\hline 24 & --- & -- & --- & --- & 7.1 & --- \\
\hline 25 & $\cdots$ & -- & -- & $\cdots$ & 7.4 & -- \\
\hline 26 & --- & $\cdots$ & --- & --- & 7.2 & -- \\
\hline 27 & --- & --- & --- & -- & 7.6 & --- \\
\hline 28 & --- & -- & --- & --- & 7.8 & --- \\
\hline 29 & 3.8 & --- & --- & --- & 7.4 & --- \\
\hline 30 & 3.4 & -- & --- & --- & 7.4 & -- \\
\hline 31 & -- & --- & --- & --- & 7.6 & --- \\
\hline
\end{tabular}

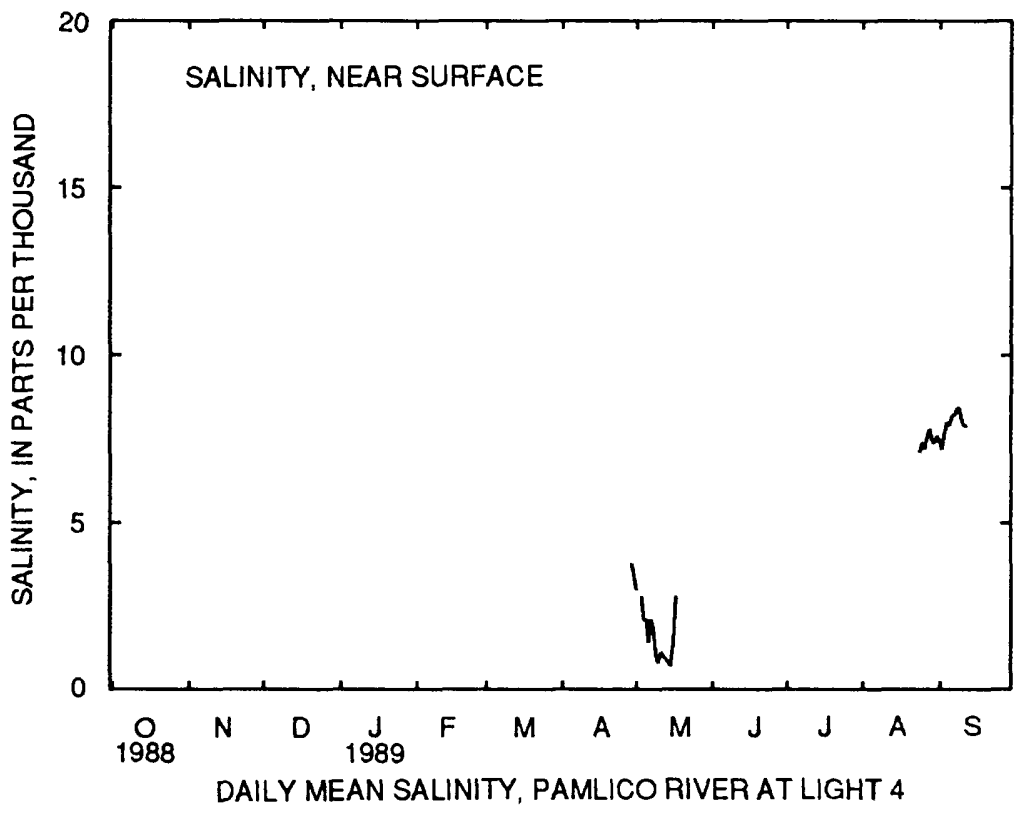


Table 5.--Daily mean values of salinity, water temperature, and dissolved oxygen in water at site 3 , Pamlico River at Light 4, April 1989 to september 1990--Continued

[Site 3 is at latitude $35^{\circ} 24^{\prime} 47^{\prime \prime}$, longitude $76^{\circ} 45^{\prime} 52^{\prime \prime}$, Beaufort County; U.S. Geological Survey downstream order number 0208454253; ppt, parts per thousand; <, less than; ---, no data;

${ }^{\circ} \mathrm{C}$, degrees Celsius; mg/L, milligrams per liter; a, daily minimum value is less than or equal to $2.0 \mathrm{mg} / \mathrm{L}$; b, daily minimum value is less than or equal to $5.0 \mathrm{mg} / \mathrm{L}$ but greater than $2.0 \mathrm{mg} / \mathrm{L}$ )

\section{SALINITY, NEAR SURFACE}

SENSOR POSITION. --8 feet above streambed.

EXTREMES FOR OCTOBER 1989 TO SEPTEMBER 1990.--Maximum value recorded, 10.0 ppt August 28, $1990 ;$ minimum value recorded, 0.4 ppt April 21-22, 1990.

DAILY MEAN VALUES OF SALINITY, NEAR SURFACE (IN PARTS PER THOUSAND), OCTOBER 1989 TO SEPTEMBER 1990

\begin{tabular}{|c|c|c|c|c|c|c|c|c|c|c|c|c|}
\hline DAY & $\mathrm{OCT}$ & NOV & DEC & JAN & $\mathrm{FEB}$ & MAR & $A P R$ & MAY & JUNE & JULY & AUG & SEPT \\
\hline 1 & --- & -- & 5.0 & --- & 5.0 & --- & 4.4 & 2.2 & 2.2 & 6.4 & 6.9 & 8.2 \\
\hline 2 & -- & -- & 4.6 & -- & 3.9 & -- & 4.9 & 2.7 & 1.6 & 6.7 & 6.7 & 7.7 \\
\hline 3 & -- & -- & 6.3 & -- & -- & --- & 4.2 & 2.7 & 1.4 & 7.2 & 6.6 & 7.7 \\
\hline 4 & --- & -- & 7.4 & --- & 4.1 & --- & 3.2 & 2.5 & 2.5 & 7.3 & 7.2 & 7.9 \\
\hline 5 & --- & -- & 7.2 & --- & 4.9 & --- & 3.1 & 2.6 & 4.8 & -- & 7.0 & 7.6 \\
\hline 6 & -- & -- & 7.0 & -- & 3.9 & -- & 2.9 & 2.6 & 3.2 & --- & 6.5 & 7.4 \\
\hline 7 & --- & --- & 7.3 & 5.5 & 3.8 & --- & 3.1 & 2.6 & 3.3 & 7.9 & 6.6 & 7.0 \\
\hline 8 & -- & --- & 7.6 & 5.3 & 4.3 & -- & 2.6 & 2.2 & 3.1 & 8.0 & 6.4 & 6.9 \\
\hline 9 & $-\cdots$ & --- & 7.5 & 6.8 & --- & --- & 2.1 & 2.1 & 3.2 & 7.9 & 6.4 & 6.9 \\
\hline 10 & --- & --- & 7.4 & 6.9 & 4.5 & --- & 1.3 & 2.1 & 3.2 & 7.7 & 6.3 & --- \\
\hline 11 & -- & --- & 7.0 & 6.6 & 4.9 & --- & 1.4 & 2.1 & 4.5 & 7.7 & 6.1 & --- \\
\hline 12 & --- & $-\cdots$ & --- & 6.3 & 4.5 & -- & 1.5 & 2.3 & 5.2 & 7.5 & 5.9 & --- \\
\hline 13 & -- & --- & --- & 6.4 & 4.3 & -- & 1.4 & 2.0 & 5.3 & 7.3 & 5.9 & --- \\
\hline 14 & --- & --- & --- & 5.1 & 3.9 & 1.7 & 1.0 & 2.0 & 4.3 & 7.0 & 6.2 & --- \\
\hline 15 & --- & --- & --- & 3.5 & --- & 1.9 & .8 & 2.1 & 5.5 & 6.6 & 6.7 & -- \\
\hline 16 & --- & --- & --- & 4.6 & 4.1 & --- & 1.1 & 1.7 & --- & 6.4 & 7.1 & --- \\
\hline 17 & 4.8 & --- & -- & 3.9 & 3.8 & --- & .9 & 1.8 & --- & 6.2 & 7.0 & --- \\
\hline 18 & 5.8 & --- & --- & 2.6 & 4.0 & --- & 1.7 & 1.9 & 5.3 & 6.2 & 6.6 & --- \\
\hline 19 & 5.6 & -- & --- & 6.0 & 3.4 & --- & 1.3 & 2.0 & -- & 6.3 & 6.5 & --- \\
\hline 20 & 5.9 & --- & --- & 5.4 & 3.6 & --- & 1.0 & 1.8 & --- & 6.0 & 6.1 & --- \\
\hline 21 & 5.7 & 5.2 & -- & 5.2 & --- & --- & .5 & 1.7 & 5.3 & 5.6 & 6.7 & $\ldots$ \\
\hline 22 & 6.1 & 5.7 & -- & 5.7 & -- & --- & 1.0 & 2.5 & 5.4 & 6.0 & 6.7 & --- \\
\hline 23 & 6.5 & 5.8 & --- & 5.5 & $\cdots$ & --- & 1.0 & 2.5 & 5.3 & 5.6 & 6.7 & --- \\
\hline 24 & 6.7 & 5.7 & --- & 5.1 & --- & --- & 1.1 & 2.4 & 5.5 & 6.0 & 6.8 & --- \\
\hline 25 & 6.8 & 4.3 & --- & --- & --- & --- & 1.0 & 2.0 & 5.8 & 5.8 & 7.1 & $-\cdots$ \\
\hline 26 & 7.0 & 5.2 & --- & 5.4 & --- & --- & --- & 1.9 & 6.0 & 5.8 & 7.5 & --- \\
\hline 27 & 6.6 & 6.3 & --- & 5.9 & --- & --- & 1.5 & 2.7 & 6.3 & 6.3 & 7.6 & --- \\
\hline 28 & 6.1 & 5.1 & -- & -- & --- & 3.9 & 1.4 & 2.9 & 6.3 & 6.4 & 8.0 & --- \\
\hline 29 & 6.0 & 5.8 & --- & 5.2 & --- & 4.0 & 1.6 & 2.6 & 6.6 & 6.8 & 7.0 & --- \\
\hline 30 & $\cdots$ & 4.9 & --- & 5.3 & --- & 4.5 & 1.8 & 2.9 & 6.3 & 7.1 & 8.2 & -- \\
\hline 31 & --- & --- & --- & 5.4 & --- & 4.3 & --- & 2.4 & -- & 6.5 & 8.2 & -- \\
\hline
\end{tabular}

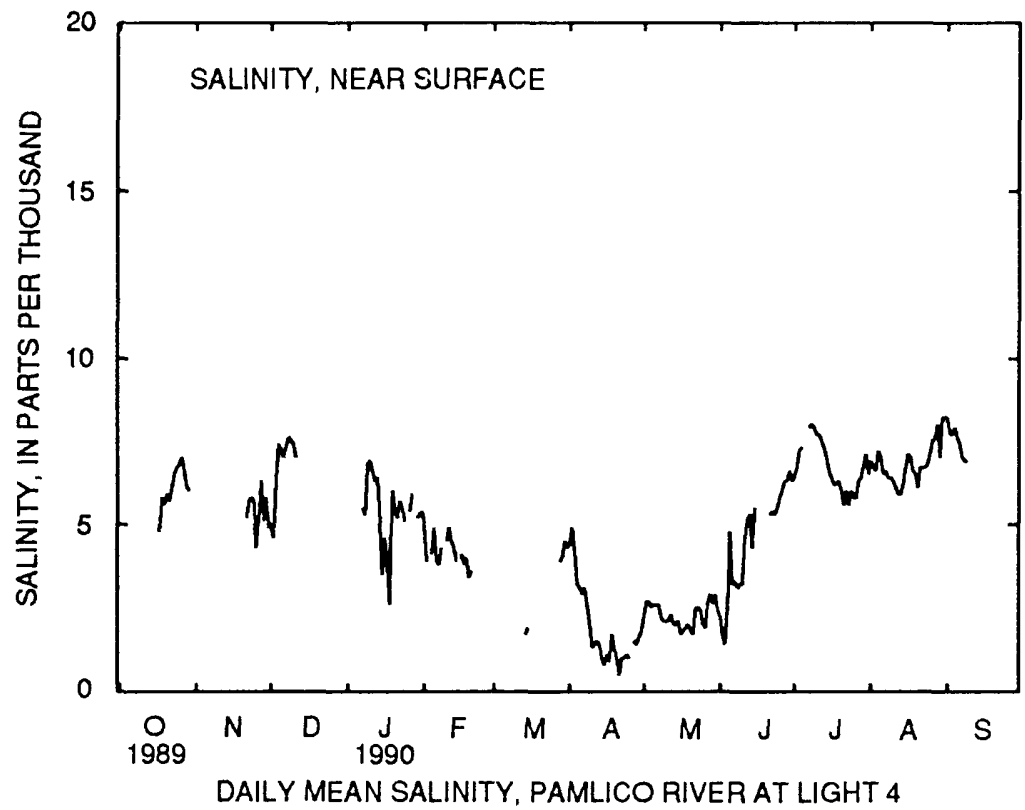


Table 5.--Daily mean values of salinity, water temperature, and dissolved oxygen 1n water at site 3 , Pamlico River at Light 4, April 1989 to september 1990--Continued

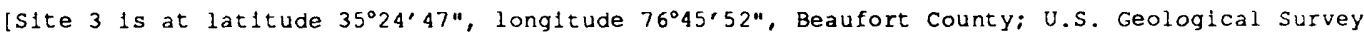
downstream order number 0208454253; ppt, parts per thousand; <, less than; ---, no data;

${ }^{\circ} \mathrm{C}$, degrees Celsius; $\mathrm{mg} / \mathrm{L}$, milligrams per liter; a, dally minimum value is less than or equal

to $2.0 \mathrm{mg} / \mathrm{L} ; \mathrm{b}$, dally minlmum value is less than or equal to $5.0 \mathrm{mg} / \mathrm{L}$ but greater than $2.0 \mathrm{mg} / \mathrm{i}$ :

SALINITY, NEAR BOTTOM

SENSOR POSITION. - - 2 feet above streambed.

EXTREMES FOR APRIL TO SEPTEMBER 1989.--Maximum value recorded, 12.4 ppt August 24, $1989 ;$ minlmum value reccrceg, 0.5 ppt May 10, 1989.

DAILY MEAN VALUES OF SALINITY, NEAR BOTTOM (IN PARTS PER THOUSAND), APRIL TO SEPTEMBER 1989

\begin{tabular}{|c|c|c|c|c|c|c|}
\hline DAY & APR & MAY & JUNE & JULY & AUG & SEPT \\
\hline 1 & --- & 3.3 & -- & -- & $-\cdots$ & 8.0 \\
\hline 2 & $-\infty$ & 3.4 & -- & -- & $\cdots$ & 8.0 \\
\hline 3 & -- & 3.2 & --- & -- & -- & 7.3 \\
\hline 4 & -- & 2.5 & $\cdots$ & -- & -- & 7.1 \\
\hline 5 & --- & 2.1 & -- & -- & -- & 7.1 \\
\hline 6 & --- & 1.7 & $\ldots$ & -- & -- & 6.9 \\
\hline 7 & -- & 2.1 & -- & -- & -- & 6.1 \\
\hline 8 & -- & 1.8 & -- & --- & --- & 6.1 \\
\hline 9 & --- & 1.0 & -- & --- & --- & 5.9 \\
\hline 10 & --- & .7 & -- & --- & -- & 5.4 \\
\hline 11 & --- & .8 & -- & -- & -- & 4.6 \\
\hline 12 & --- & .9 & --- & -- & -- & 4.6 \\
\hline 13 & --- & 1.0 & -- & $-\cdots$ & --- & --- \\
\hline 14 & --- & .8 & --- & --- & --- & -- \\
\hline 15 & -- & .9 & --- & --- & -- & --- \\
\hline 16 & -- & 1.6 & -- & -- & -- & -- \\
\hline 17 & -- & 2.8 & -- & -- & $-\cdots$ & -- \\
\hline 18 & --- & --- & $\cdots$ & -- & $\cdots$ & --- \\
\hline 19 & --- & -- & --- & -- & --- & -- \\
\hline 20 & --- & --- & --- & -- & --- & --- \\
\hline 21 & $-\infty$ & -- & --- & -- & -- & --- \\
\hline 22 & -- & $-\cdots$ & -- & -- & -- & -- \\
\hline 23 & --- & -- & -- & -- & -- & -- \\
\hline 24 & --- & $-\cdots$ & -- & -- & 10.1 & $-\cdots$ \\
\hline 25 & -- & --- & --- & --- & 8.1 & $-\infty$ \\
\hline 26 & --- & --- & --- & -- & 8.0 & --- \\
\hline 27 & -- & -- & -- & -- & 7.7 & --- \\
\hline 28 & 4.0 & --- & --- & $-\cdots$ & 7.8 & --- \\
\hline 29 & 3.8 & --- & --- & --- & 7.9 & -- \\
\hline 30 & 3.7 & $--\infty$ & --- & $-\cdots$ & 7.5 & --- \\
\hline 31 & --- & -- & --- & --- & 8.1 & -- \\
\hline
\end{tabular}

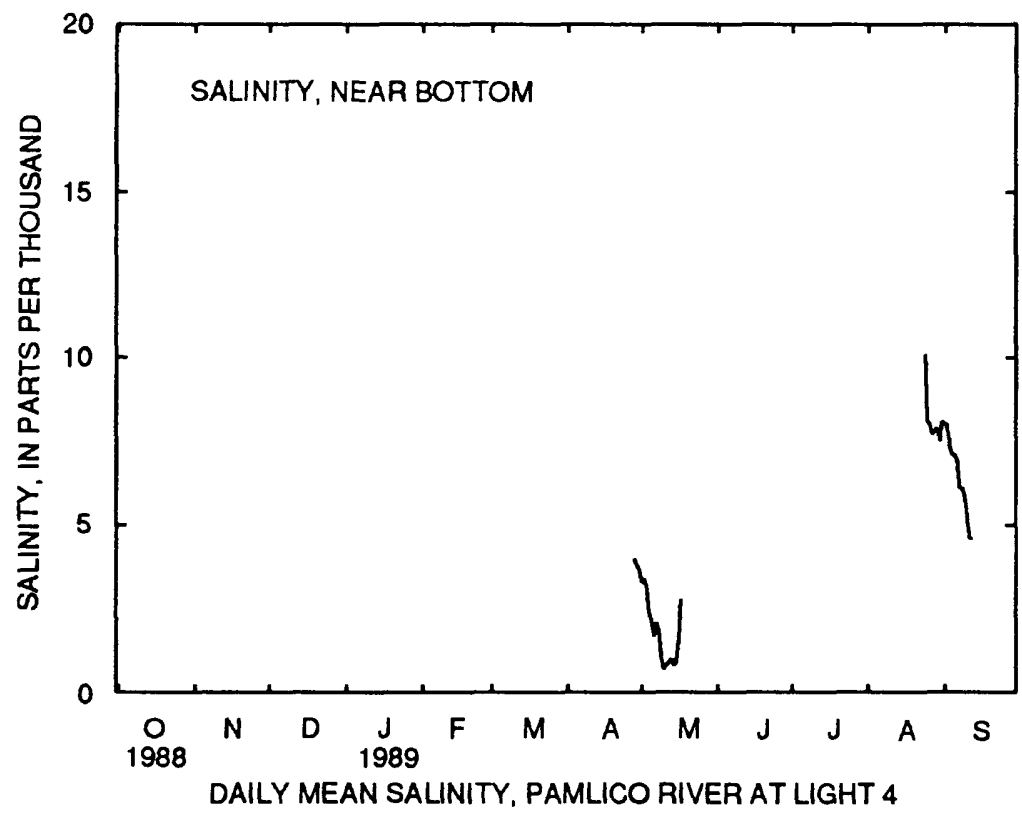


Table 5.--Dally mean values of salinity, water temperature, and dissolved oxygen in water at site 3 , Pam11co River at Light 4, Apri1 1989 to September 1990--Continued

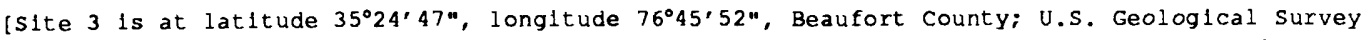
downstream order number 0208454253; ppt, parts per thousand; <, less than; ---, no data;

${ }^{\circ} \mathrm{C}$, degrees Celsius; mg/L, milligrams per liter; a, dally minimum value is less than or equal

to $2.0 \mathrm{mg} / \mathrm{L} ; \mathrm{b}$, dally minimum value is less than or equal to $5.0 \mathrm{mg} / \mathrm{L}$ but greater than $2.0 \mathrm{mg} / \mathrm{L}$ ]

SALINITY, NEAR BOTTOM

SENSOR POSITION.--2 feet above streambed.

EXTREMES FOR OCTOBER 1989 TO SEPTEMBER 1990.--Maximum value recorded, 14.1 ppt August 30, 1990 ; minimum value recorded, 0.4 ppt April 21, 1990.

DAILY MEAN VALUES OF SALINITY, NEAR BOTTOM (IN PARTS PER THOUSAND), OCTOBER 1989 TO SEPTEMBER 1990

\begin{tabular}{|c|c|c|c|c|c|c|c|c|c|c|c|c|}
\hline DAY & OCT & NOV & DEC & JAN & FEB & MAR & APR & MAY & JUNE & JULY & AUG & SEPT \\
\hline 1 & --- & --- & 8.8 & --- & 5.8 & --- & 4.3 & 4.8 & 3.5 & 7.7 & 8.3 & 9.2 \\
\hline 2 & --- & -- & 9.3 & $-\cdots-$ & 5.9 & --- & 4.9 & 3.7 & 4.2 & 8.4 & 9.1 & 8.6 \\
\hline 3 & --- & --- & 8.3 & -- & -- & --- & 4.1 & 3.3 & 3.2 & --- & 7.9 & 8.9 \\
\hline 4 & -- & --- & 7.7 & --- & 4.8 & --- & 3.2 & 2.8 & 5.7 & -- & 7.8 & 8.1 \\
\hline 5 & --- & --- & 7.9 & $-\cdots$ & 5.6 & --- & 3.1 & 2.8 & 5.4 & $-\dot{-}$ & 8.0 & 7.8 \\
\hline 6 & --- & --- & 7.7 & --- & 5.3 & --- & 2.9 & 3.0 & 5.3 & -- & 8.5 & 7.6 \\
\hline 7 & --- & -- & 7.8 & 9.1 & 5.8 & -- & 3.0 & 3.0 & 5.1 & --- & 9.3 & 7.6 \\
\hline 8 & -- & -- & 7.8 & 8.2 & 5.1 & -- & 2.6 & 2.6 & 5.1 & -- & 8.9 & 7.7 \\
\hline 9 & -- & -- & 7.7 & 7.0 & -- & --- & 2.0 & 2.5 & 6.4 & $\cdots$ & 8.7 & 6.9 \\
\hline 10 & -- & -- & 7.8 & 6.7 & 4.8 & -- & 1.3 & 2.4 & 6.6 & -- & 9.7 & $-\cdots$ \\
\hline 11 & --- & --- & 8.1 & 6.6 & 5.4 & --- & 1.5 & 2.5 & 6.5 & --- & 10.5 & --- \\
\hline 12 & --- & -- & --- & 6.3 & 4.9 & --- & 1.6 & 2.9 & 6.5 & $\cdots$ & 11.0 & -- \\
\hline 13 & --- & --- & --- & 6.5 & 4.5 & --- & 1.4 & 2.2 & 5.9 & -- & 10.4 & --- \\
\hline 14 & --- & -- & --- & 6.6 & 4.2 & 7.0 & 1.0 & 2.3 & 6.1 & --- & 11.0 & --- \\
\hline 15 & -- & -- & -- & 7.3 & --- & 7.2 & 1.1 & 2.2 & 5.2 & --- & 9.3 & -- \\
\hline 16 & -- & -- & --- & 7.7 & 4.1 & --- & 1.3 & 2.0 & --- & -- & 9.2 & --- \\
\hline 17 & 6.6 & --- & --- & 8.0 & 4.2 & -- & 1.3 & 1.7 & --- & -- & 8.7 & --- \\
\hline 18 & 6.8 & --- & --- & 8.1 & 4.0 & --- & 1.6 & 1.9 & 5.1 & --- & 8.7 & -- \\
\hline 19 & 6.6 & -- & --- & 7.6 & 3.6 & --- & 1.2 & 2.3 & 5.0 & $-\cdots$ & 8.7 & --- \\
\hline 20 & 6.1 & --- & --- & 7.6 & 3.8 & -- & 1.0 & 1.7 & 5.3 & --- & 9.4 & $-\cdots$ \\
\hline 21 & 6.0 & 5.3 & --- & 6.0 & --- & --- & .6 & 1.8 & 5.4 & --- & 8.9 & --- \\
\hline 22 & 6.5 & 6.0 & --- & 6.7 & --- & --- & 1.2 & 2.3 & 5.7 & $-\cdots$ & 9.5 & --- \\
\hline 23 & 6.7 & 5.8 & -- & 6.5 & --- & --- & 1.1 & 2.5 & 6.5 & 8.5 & 9.8 & --- \\
\hline 24 & 6.7 & 6.6 & --- & 7.3 & -- & --- & 1.1 & 2.4 & 6.0 & 8.7 & 9.8 & --- \\
\hline 25 & 6.7 & 8.0 & --- & $-\cdots$ & --- & --- & 1.2 & 3.1 & 7.2 & 8.6 & 10.2 & --- \\
\hline 26 & 6.8 & 8.6 & -- & 6.4 & --- & -- & -- & 2.7 & 7.7 & 7.8 & 10.1 & -- \\
\hline 27 & 6.4 & 7.9 & -- & 7.2 & $-\cdots$ & --- & 3.3 & 3.0 & 6.7 & 7.3 & 11.7 & $-\cdots$ \\
\hline 28 & 6.1 & 8.9 & --- & $\cdots$ & $\cdots$ & 3.9 & 4.4 & 2.8 & 7.2 & 6.9 & 12.8 & -- \\
\hline 29 & 6.3 & 8.9 & --- & 6.3 & --- & 4.0 & 5.3 & 2.7 & 7.5 & 7.4 & 12.8 & --- \\
\hline 30 & -- & 9.4 & --- & 5.8 & -- & 4.5 & 5.1 & 3.0 & 7.7 & 7.4 & 11.6 & -- \\
\hline 31 & --- & --- & $-\cdots$ & 6.2 & --- & 4.2 & --- & 3.3 & -- & 7.5 & 10.7 & -- \\
\hline
\end{tabular}

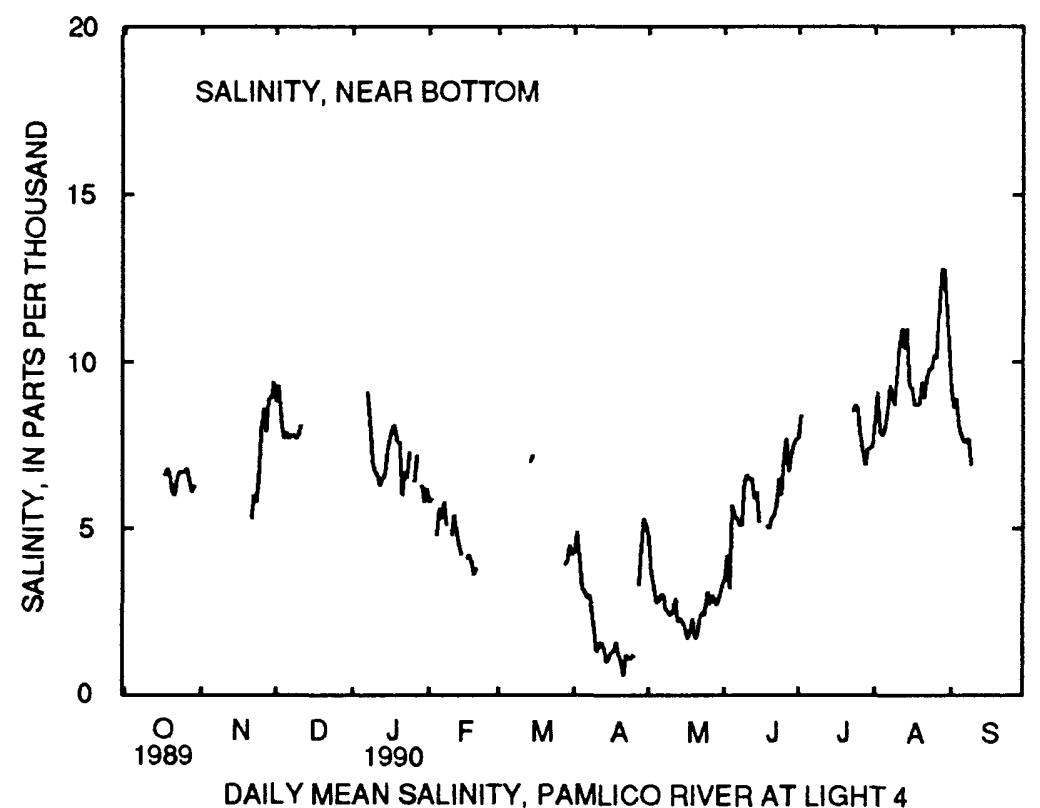


Table 5.--Dally mean values of salinity, water temperature, and dissolved oxygen in water at site 3 , Pamlico River at Light 4, April 1989 to september 1990--Continued

ISite 3 is at latitude $35^{\circ} 24^{\prime} 47^{\prime \prime}$, longitude $76^{\circ} 45^{\prime} 52^{\prime \prime}$, Beaufort County; U.S. Geological Survey downstream order number 0208454253; ppt, parts per thousand; <, less than; ---, no data;

${ }^{\circ} \mathrm{C}$, degrees Celsius; mg/L, milligrams per liter; a, dally minimum value is less than or equal to $2.0 \mathrm{mg} / \mathrm{L} ; \mathrm{b}$, daliy minimum value is less than or equal to $5.0 \mathrm{mg} / \mathrm{L}$ but greater than $2.0 \mathrm{mg} / \mathrm{L}$ ]

WATER TEMPERATURE, NEAR SURFACE

SENSOR POSITION. -8 feet above streambed.

EXTREMES FOR APRIL TO SEPTEMBER 1989.--Maximum value recorded, $30.1{ }^{\circ} \mathrm{C}$ August 24, $1989 ;$ minimum value recorded, $14.1{ }^{\circ} \mathrm{C}$ MaY 17, 1989.

DAILY MEAN VALUES OF WATER TEMPERATURE, NEAR SURFACE (IN DEGREES CELSIUS), APRIL TO SEPTEMBER 1989

\begin{tabular}{|c|c|c|c|c|c|c|}
\hline DAY & APR & MAY & JUNE & JULY & AUG & SEPT \\
\hline 1 & -- & 22.5 & --- & -- & -- & 29.2 \\
\hline 2 & --- & 22.0 & --- & --- & --- & 29.2 \\
\hline 3 & -- & 22.3 & -- & -- & -- & -- \\
\hline 4 & -- & 22.3 & --- & --- & --- & $\cdots$ \\
\hline 5 & --- & 21.6 & --- & --- & --- & --- \\
\hline 6 & --- & 21.5 & --- & --- & --- & -- \\
\hline 7 & --- & 20.7 & --- & --- & --- & -- \\
\hline 8 & -- & 19.3 & -- & --- & --- & $-\cdots$ \\
\hline 9 & --- & 19.0 & --- & --- & --- & $-\cdots$ \\
\hline 10 & --- & 18.8 & --- & --- & --- & -- \\
\hline 11 & -- & 18.1 & -- & -- & -- & $\ldots$ \\
\hline 12 & -- & 17.6 & --- & --- & --- & --- \\
\hline 13 & --- & 17.9 & --- & --- & $-\sim$ & --- \\
\hline 14 & -- & 17.7 & --- & --- & -- & -- \\
\hline 15 & --- & 18.3 & --- & --- & --- & $\cdots$ \\
\hline 16 & -- & 18.5 & --- & --- & --- & --- \\
\hline 17 & -- & 17.6 & --- & --- & --- & -- \\
\hline 18 & -- & --- & --- & --- & --- & -- \\
\hline 19 & -- & $-\cdots$ & --- & -- & -- & -- \\
\hline 20 & --- & --- & --- & -- & -- & --- \\
\hline 21 & $-\cdots$ & $-\cdots$ & --- & -- & -- & -- \\
\hline 22 & --- & --- & --- & -- & --- & --- \\
\hline 23 & --- & --- & --- & -- & --- & -- \\
\hline 24 & --- & --- & --- & --- & 28.9 & $\cdots$ \\
\hline 25 & --- & --- & --- & -- & 28.8 & --- \\
\hline 26 & --- & --- & --- & --- & 28.4 & -- \\
\hline 27 & --- & --- & --- & -- & 28.1 & $\cdots$ \\
\hline 28 & 21.1 & --- & --- & --- & 28.1 & --- \\
\hline 29 & 21.4 & --- & --- & -- & 28.4 & $-\cdots$ \\
\hline 30 & 22.2 & --- & --- & --- & 28.4 & $-\cdots$ \\
\hline 31 & --- & --- & --- & --- & 28.6 & --- \\
\hline
\end{tabular}

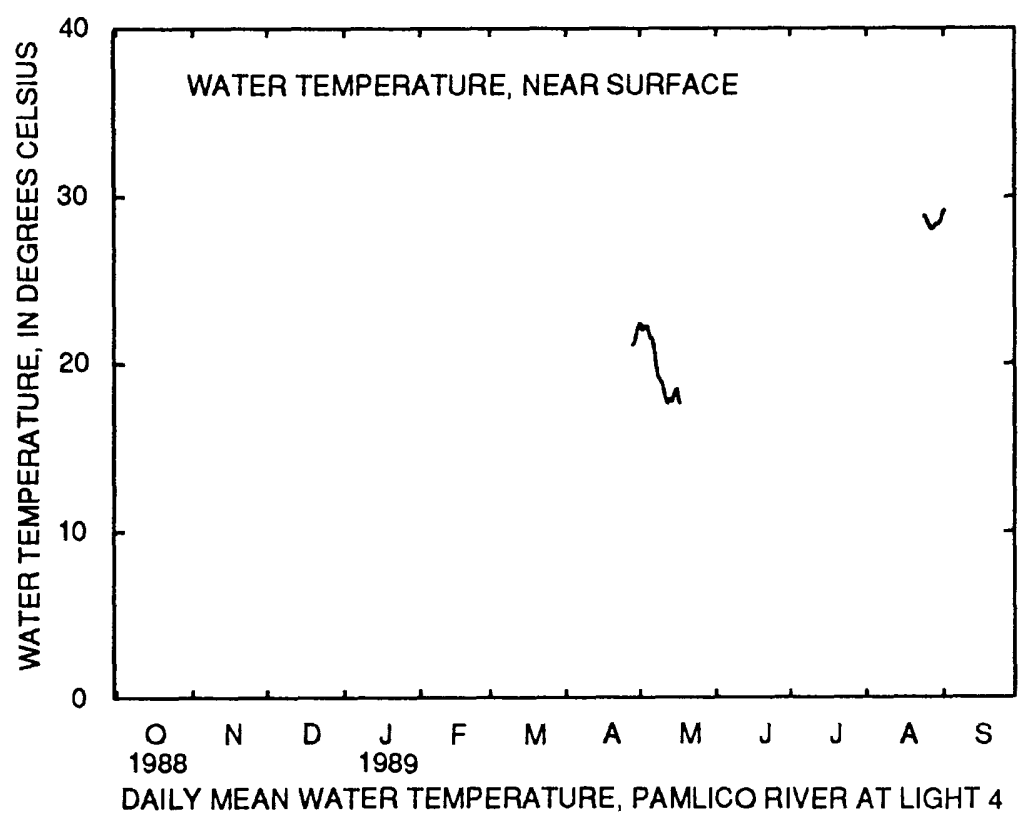


Table 5.--Daily mean values of salinity, water temperature, and dissolved oxygen in water at site 3 , Pamlico River at Light 4, April 1989 to september 1990--Continued

[Site 3 is at latitude $35^{\circ} 24^{\prime} 47^{\prime \prime}$, longitude 76 $45^{\prime} 52^{\prime \prime}$, Beaufort County; U.S. Geological Survey downstream order number 0208454253; ppt, parts per thousand; <, less than; ---, no data;

${ }^{\circ} \mathrm{C}$, degrees celsius; mg/L, milligrams per liter; a, dally minimum value is less than or equal

to $2.0 \mathrm{mg} / \mathrm{L} ; \mathrm{b}$, daily minimum value is less than or equal to $5.0 \mathrm{mg} / \mathrm{L}$ but greater than $2.0 \mathrm{mg} / \mathrm{L}$ ]

WATER TEMPERATURE, NEAR SUREACE

SENSOR POSITION. --8 feet above streambed.

EXTREMES FOR OCTOBER 1989 TO SEPTEMBER 1990.--Maximum value recorded, $31.8{ }^{\circ} \mathrm{C} \mathrm{JUly} 24$, $1990 ; \mathrm{mln} 1 \mathrm{mum}$ value recorded, $3.5^{\circ} \mathrm{C}$ January $7,1990$.

DAILY MEAN VALUES OF WATER TEMPERATURE, NEAR SURFACE (IN DEGREES CELSIUS), OCTOBER 1989 TO SEPTEMBER 1990

\begin{tabular}{|c|c|c|c|c|c|c|c|c|c|c|c|c|}
\hline DAY & OCT & NOV & DEC & JAN & FEB & MAR & APR & MAY & JUNE & JULY & AUG & SEPT \\
\hline 1 & -- & -- & $\ldots$ & -- & 11.9 & -- & 14.3 & 23.4 & 23.3 & 27.5 & 29.3 & 27.3 \\
\hline 2 & -- & -- & -- & -- & 13.0 & --- & 14.8 & 24.0 & 23.8 & 27.6 & 29.2 & 27.1 \\
\hline 3 & -- & -- & -- & $-\cdots$ & --- & -- & 14.9 & 22.9 & 24.4 & 27.6 & 29.6 & 27.2 \\
\hline 4 & -- & $\cdots$ & --- & -- & 14.8 & -- & 13.9 & 22.9 & 24.8 & 27.6 & 29.6 & 27.0 \\
\hline 5 & --- & -- & --- & -- & 13.7 & --- & 14.2 & 23.5 & 24.3 & -- & 29.1 & 26.7 \\
\hline 6 & $\ldots$ & -- & -- & -- & 12.8 & -- & 14.9 & 22.7 & 24.9 & $\ldots$ & 28.4 & 26.8 \\
\hline 7 & --- & -- & $-\cdots$ & 5.2 & 13.1 & -- & 15.1 & 22.2 & 25.1 & 28.2 & 27.8 & 27.1 \\
\hline 8 & $-\ldots$ & -- & -- & 6.1 & 13.3 & $\ldots$ & 14.9 & 22.7 & 26.4 & 28.2 & 27.3 & 27.8 \\
\hline 9 & $\ldots$ & $\ldots$ & -- & 5.7 & -- & -- & 15.0 & 22.6 & 27.0 & 28.6 & 27.0 & 27.5 \\
\hline 10 & --- & $-\cdots$ & --- & 5.9 & 14.5 & $\cdots$ & 15.5 & 22.6 & 27.1 & 29.0 & 26.7 & -- \\
\hline 11 & -- & -- & -- & 6.1 & 14.4 & $-\cdots$ & 16.0 & 21.9 & 26.4 & 29.3 & 27.1 & $-\cdots$ \\
\hline 12 & --- & -- & --- & 6.6 & 13.7 & -- & 15.9 & 22.0 & 25.3 & 29.0 & 27.6 & -- \\
\hline 13 & -- & -- & --- & 6.0 & 13.2 & -- & 16.1 & 22.4 & 25.2 & 29.0 & 28.6 & -- \\
\hline 14 & $\ldots$ & $\ldots$ & -- & 5.8 & 13.6 & 15.3 & 16.2 & 22.9 & 25.2 & 29.1 & 28.4 & -- \\
\hline 15 & -- & -- & --- & 6.5 & --- & 17.8 & 16.5 & 23.4 & 25.3 & 28.6 & 27.7 & -- \\
\hline 16 & $-\ldots$ & -- & $\ldots$ & 6.7 & 15.3 & 18.3 & 17.5 & 24.2 & -- & 28.4 & 27.4 & -- \\
\hline 17 & 22.2 & -- & -- & 7.4 & 16.1 & 18.0 & 18.0 & 24.2 & -- & 28.6 & 27.8 & -- \\
\hline 18 & 21.2 & --- & -- & 8.6 & 15.2 & 17.5 & 17.2 & 24.0 & 26.8 & 29.1 & 27.8 & --- \\
\hline 19 & 22.1 & -- & $\ldots$ & 8.4 & 14.9 & $-\cdots$ & 17.0 & 23.5 & 27.0 & 29.5 & 28.6 & -- \\
\hline 20 & 21.1 & -- & --- & 8.9 & 14.2 & -- & 17.5 & 23.7 & $-\cdots$ & 29.4 & 28.6 & --- \\
\hline 21 & 18.6 & -- & -- & 10.0 & -- & -- & 18.0 & 24.0 & 26.8 & 29.6 & 280 & --- \\
\hline 22 & 17.5 & -- & -- & 10.3 & --- & -- & 18.6 & 23.2 & 26.8 & 29.3 & 27.6 & --- \\
\hline 23 & 17.1 & -- & -- & 10.2 & -- & -- & 18.8 & 21.7 & 27.5 & 30.3 & 27.5 & -- \\
\hline 24 & 17.2 & -- & -- & 10.5 & --- & $-\cdots$ & 19.1 & 21.7 & 27.6 & 30.8 & 27.5 & --- \\
\hline 25 & 17.1 & -- & -- & $-\cdots$ & --- & --- & 19.6 & 21.8 & 27.3 & 30.8 & 27.6 & -- \\
\hline 26 & 17.0 & --- & $-\cdots$ & 11.7 & $\cdots$ & -- & -- & 22.3 & 27.2 & 30.2 & 28.1 & -- \\
\hline 27 & 17.1 & -- & --- & 10.7 & $\cdots$ & -- & 22.6 & 22.8 & 27.4 & 29.5 & 28.2 & --- \\
\hline 28 & 17.1 & -- & -- & -- & --- & 14.1 & 23.2 & 22.1 & 27.1 & 29.4 & 28.5 & --- \\
\hline 29 & 17.4 & --- & -- & 11.0 & --- & 13.8 & 23.5 & 22.2 & 27.4 & 29.0 & 28.8 & -- \\
\hline 30 & -- & -- & -- & 11.2 & -- & 13.8 & 22.8 & 22.0 & 27.8 & 29.1 & 28.1 & --- \\
\hline 31 & -- & -- & -- & 11.5 & --- & 14.1 & -- & 22.6 & -- & 29.5 & 27.6 & --- \\
\hline
\end{tabular}

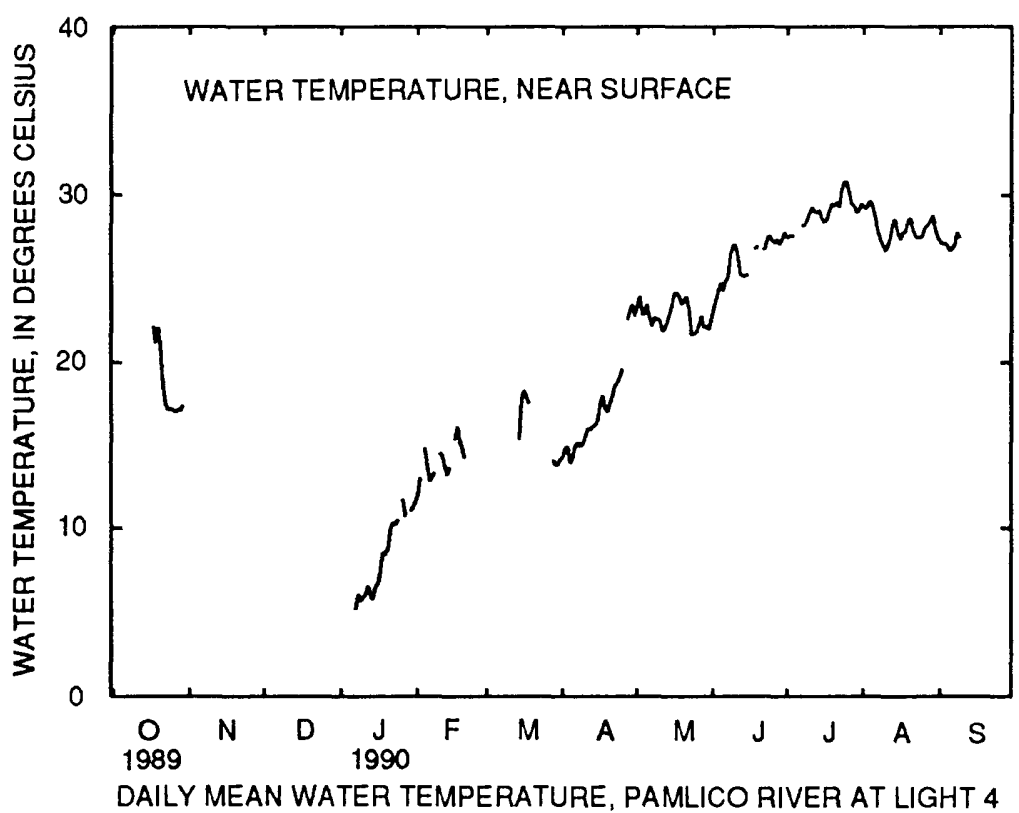


Table 5.--Daliy mean values of salinity, water temperature, and dissolved oxygen in water at site 3 , Pamlico River at Light 4, April 1989 to September 1990--Cont inued

[Site 3 is at latitude $35^{\circ} 24^{\prime} 47^{\prime \prime}$, longitude $76^{\circ} 45^{\prime} 52^{\prime \prime}$, Beaufort County; U.S. Geological Survey downstream order number 0208454253; ppt, parts per thousand; <, less than; ---, no data;

${ }^{\circ} \mathrm{C}$, degrees Celslus; mg/L, milligrams per liter; a, daily minlmum value is less than or equal

to $2.0 \mathrm{mg} / \mathrm{L} ; \mathrm{b}$, dally minimum value $1 \mathrm{~s}$ less than or equal to $5.0 \mathrm{mg} / \mathrm{L}$ but greater than $2.0 \mathrm{mg} / \mathrm{L}$

DISSOLVED OXYGEN, NEAR SURFACE

SENSOR POSITION. --8 feet above streambed.

EXTREMES FOR APRIL TO SEPTEMBER 1989.--Maximum value recorded, 13.6 mg/L September 1, 1989; minimum value recorded, $2.6 \mathrm{mg} / \mathrm{L}$ september 3,1989 .

DAILY MEAN VALUES OF DISSOLVED OXYGEN, NEAR SURFACE (IN MILLIGRAMS PER LITER), APRIL TO SEPTEMBER 1989

\begin{tabular}{|c|c|c|c|c|c|c|}
\hline DAY & APR & MAY & JUNE & JULY & AUG & SEPT \\
\hline 1 & --- & 8.3 & -- & -- & -- & 9.4 \\
\hline 2 & $-\infty$ & 7.1 & -- & -- & -- & $6.3 \mathrm{~b}$ \\
\hline 3 & -- & 7.9 & -- & -- & --- & $4.4 \mathrm{~b}$ \\
\hline 4 & -- & 8.5 & -- & -- & -- & $5.4 b$ \\
\hline 5 & -- & 7.9 & -- & -- & -- & $4.8 b$ \\
\hline 6 & -- & 7.5 & -- & -- & -- & $4.6 b$ \\
\hline 7 & --- & 7.3 & -- & -- & -- & $5.5 b$ \\
\hline 8 & -- & 7.1 & --- & $-\infty$ & -- & 6.5 \\
\hline 9 & --- & 7.2 & -- & -- & $\cdots$ & 6.9 \\
\hline 10 & -- & 7.3 & -- & --- & -- & 9.5 \\
\hline 11 & -- & 7.0 & -- & -- & -- & 9.1 \\
\hline 12 & --- & 6.9 & -- & -- & -- & 8.6 \\
\hline 13 & -- & 7.7 & -- & -- & -- & -- \\
\hline 14 & --- & 7.6 & $-\cdots$ & -- & -- & --- \\
\hline 15 & --- & 8.0 & --- & -- & $-\cdots$ & --- \\
\hline 16 & --- & 7.5 & --- & --- & --- & -- \\
\hline 17 & -- & 7.6 & --- & -- & --- & --- \\
\hline 18 & --- & -- & -- & -- & --- & --- \\
\hline 19 & --- & --- & --- & -- & --- & -- \\
\hline 20 & -- & $-\cdots$ & --- & -- & --- & --- \\
\hline 21 & --- & --- & --- & --- & --- & --- \\
\hline 22 & -- & $\sim-$ & -- & -- & -- & -- \\
\hline 23 & -- & -- & -- & -- & --- & --- \\
\hline 24 & --- & $\sim$ & $-\sim-$ & -- & 7.3 & --- \\
\hline 25 & --- & -- & --- & -- & 7.0 & --- \\
\hline 26 & -- & -- & $-\sim$ & $-\infty$ & $7.6 \mathrm{~b}$ & -- \\
\hline 27 & -- & -- & --- & -- & 6.6 & --- \\
\hline 28 & 8.9 & --- & $-\sim$ & -- & $6.2 \mathrm{~b}$ & --- \\
\hline 29 & 9.1 & $\cdots$ & -- & -- & $6.3 b$ & --- \\
\hline 30 & 8.7 & $-\cdots$ & $-\sim$ & --- & $5.7 b$ & --- \\
\hline 31 & $-\sim$ & -- & --- & $-\cdots$ & $6.0 \mathrm{~b}$ & --- \\
\hline
\end{tabular}

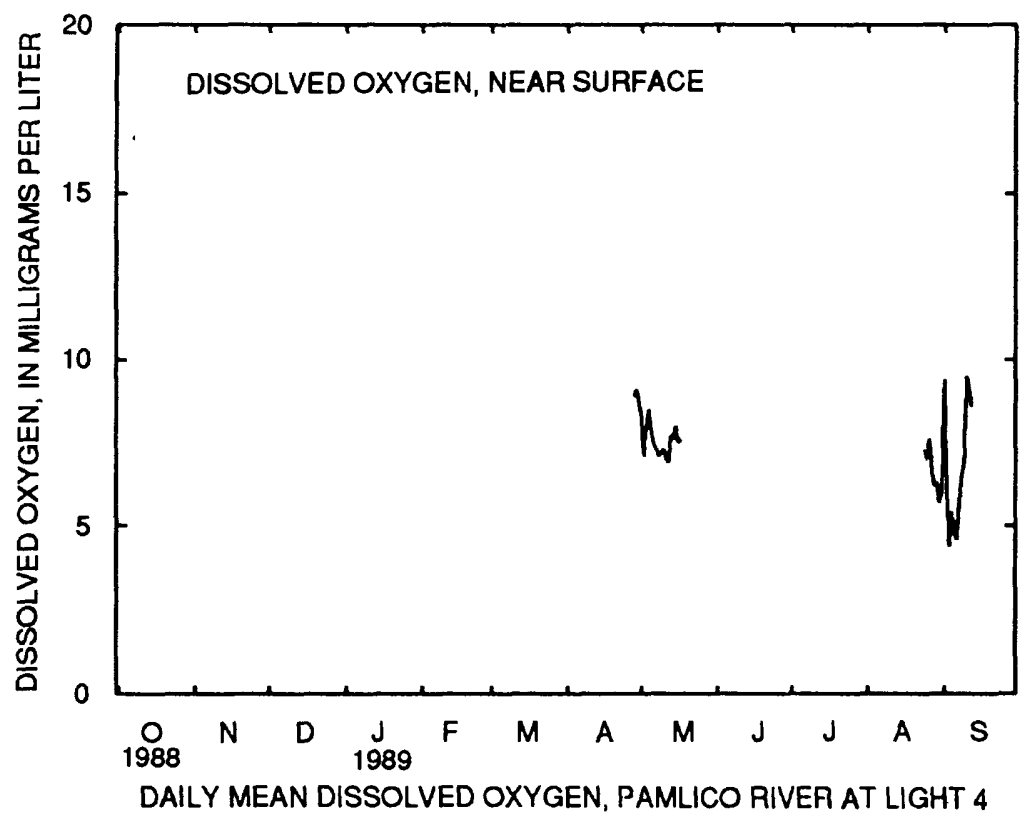


Table 5.--Daily mean values of salinity, water temperature, and dissolved oxygen in water at site 3 , Pamlico River at Light 4, April 1989 to september 1990--Continued

[Site 3 is at latitude $35^{\circ} 24^{\prime} 47^{\prime \prime}$, longitude 76 45'52", Beaufort County; U.S. Geological Survey downstream order number 0208454253 ; ppt, parts per thousand; <, less than; --., no data;

${ }^{\circ} \mathrm{C}$, degrees Celsius; mg/L, milligrams per liter; a, daily minimum value is less than or equal

to $2.0 \mathrm{mg} / \mathrm{L} ; \mathrm{b}$, daily minimum value is less than or equal to $5.0 \mathrm{mg} / \mathrm{L}$ but greater than $2.0 \mathrm{mg} / \mathrm{L}$ ]

DISSOLVED OXYGEN, NEAR SURFACE

SENSOR POSITION. --8 feet above streambed.

EXTREMES FOR OCTOBER 1989 TO SEPTEMBER 1990.--Maximum value recorded, 14.7 mg/L January 27, 1990 ; minimum value recorded, <1.0 mg/L October $17,1989$.

DAILY MEAN VALUES OF DISSOLVED OXYGEN, NEAR SUREACE (IN MILLIGRAMS PER LITER), OCTOBER 1989 TO SEPTEMBER 1990

\begin{tabular}{|c|c|c|c|c|c|c|c|c|c|c|c|c|}
\hline DAY & OCT & NOV & DEC & JAN & $\mathrm{FEB}$ & MAR & APR & MAY & JUNE & JULY & AUG & SEPT \\
\hline 1 & --- & $\ldots$ & 11.3 & --- & 12.5 & -- & 7.8 & 9.6 & 8.5 & 7.9 & 7.5 & 6.8 \\
\hline 2 & -- & -- & 12.2 & -- & 12.1 & -- & 8.1 & 8.2 & 8.4 & 8.1 & 8.2 & $7.0 \mathrm{~b}$ \\
\hline 3 & -- & --- & 10.5 & -- & --- & -- & $6.6 \mathrm{~b}$ & 7.2 & 8.3 & --- & 8.6 & 7.3 \\
\hline 4 & --- & --- & 10.8 & -- & 11.7 & --- & $6.0 \mathrm{~b}$ & 7.7 & 7.8 & 7.5 & 8.2 & 7.0 \\
\hline 5 & -- & -- & 10.9 & --- & 10.5 & --- & 7.1 & 7.7 & $7.2 \mathrm{~b}$ & --- & 7.8 & 7.5 \\
\hline 6 & --- & --- & 11.2 & --- & 11.4 & --- & 7.3 & 7.7 & 8.2 & --- & 7.1 & 7.9 \\
\hline 7 & -- & -- & 10.9 & 12.1 & 10.9 & --- & 7.1 & 8.3 & 7.7 & 7.5 & 6.5 & 8.4 \\
\hline 8 & -- & -- & 10.3 & 11.3 & 11.5 & --- & 7.7 & 8.8 & 8.2 & 7.3 & 6.3 & 8.3 \\
\hline 9 & -- & -- & 10.4 & 10.7 & -- & --- & 8.3 & 8.7 & 7.7 & 8.3 & 6.1 & 7.7 \\
\hline 10 & -- & -- & 10.7 & 10.8 & 9.9 & -- & 7.9 & 8.4 & 7.5 & 8.3 & 6.2 & --- \\
\hline 11 & --- & --- & 10.8 & 10.8 & 9.9 & $-\cdots$ & 7.2 & 8.4 & 7.4 & 8.3 & 6.4 & --- \\
\hline 12 & $-\infty$ & --- & -- & 10.8 & 10.3 & --- & 7.5 & 8.5 & 7.6 & 7.8 & 6.7 & --- \\
\hline 13 & -- & --- & -- & 11.0 & 11.3 & -- & 8.3 & 8.4 & 8.1 & 7.8 & 6.9 & --- \\
\hline 14 & --- & --- & -- & 11.1 & 11.4 & 11.6 & 8.0 & 8.5 & 8.4 & 7.7 & 6.9 & --- \\
\hline 15 & --- & --- & --- & 11.2 & , & 11.3 & 7.7 & 8.7 & 7.8 & 7.6 & 6.6 & $-\cdots$ \\
\hline 16 & --- & --- & -- & 11.3 & 10.6 & 10.7 & 8.3 & 9.7 & 8.0 & 7.9 & $5.6 \mathrm{~b}$ & --- \\
\hline 17 & $5.5 a$ & -- & --- & 11.3 & 10.6 & 10.0 & 8.7 & 6.3 & -- & 8.7 & 6.0 & --- \\
\hline 18 & $5.6 \mathrm{~b}$ & -- & --- & 11.2 & 10.5 & 10.1 & 7.8 & $5.5 b$ & 8.1 & 9.3 & 6.7 & --- \\
\hline 19 & $6.6 a$ & --- & --- & 11.5 & 10.5 & --- & 8.4 & 5.9 & 7.9 & 9.5 & 7.5 & --- \\
\hline 20 & 7.2 & $-\cdots$ & --- & 11.5 & 10.1 & --- & 9.2 & 6.1 & 7.5 & 9.0 & 7.5 & --- \\
\hline 21 & 6.4 & 11.2 & $-\cdots$ & 11.2 & $-\cdots$ & $--\infty$ & 9.4 & 5.9 & 7.9 & 8.6 & 6.6 & --- \\
\hline 22 & 6.8 & 11.3 & -- & 11.6 & --- & -- & 9.5 & $5.4 \mathrm{~b}$ & 8.2 & 8.0 & 7.2 & --- \\
\hline 23 & $6.5 b$ & 11.1 & -- & 12.1 & $-\cdots$ & -- & 10.2 & 6.0 & 7.7 & 8.4 & 7.7 & -- \\
\hline 24 & $6.9 b$ & 11.8 & -- & 12.1 & --- & --- & 9.8 & 6.9 & 7.9 & 7.6 & 7.9 & --- \\
\hline 25 & 7.2 & 12.7 & -- & --- & --- & $-\infty$ & 9.6 & 7.3 & 8.6 & 7.3 & 7.8 & $\cdots$ \\
\hline 26 & 7.2 & 12.3 & --- & 10.4 & -- & -- & -- & 7.1 & 8.2 & 7.3 & 7.6 & --- \\
\hline 27 & 7.5 & 12.0 & -- & 11.2 & --- & -- & 9.6 & 6.7 & 7.9 & 6.9 & 7.7 & --- \\
\hline 28 & 7.8 & 12.9 & -- & --- & -- & 7.6 & 9.5 & 6.5 & 8.2 & 7.1 & $7.3 \mathrm{~b}$ & --- \\
\hline 29 & 8.1 & 12.1 & --- & 12.5 & --- & 6.6 & 9.1 & 6.9 & 8.5 & 6.7 & 7.6 & --- \\
\hline 30 & -- & 12.0 & -- & 11.1 & $-\cdots$ & 6.9 & 8.8 & 7.1 & 7.8 & 7.3 & $6.4 \mathrm{~b}$ & --- \\
\hline 31 & --- & -- & -- & 11.7 & -- & 7.5 & -- & 7.9 & --- & 7.8 & 7.1 & -- \\
\hline
\end{tabular}

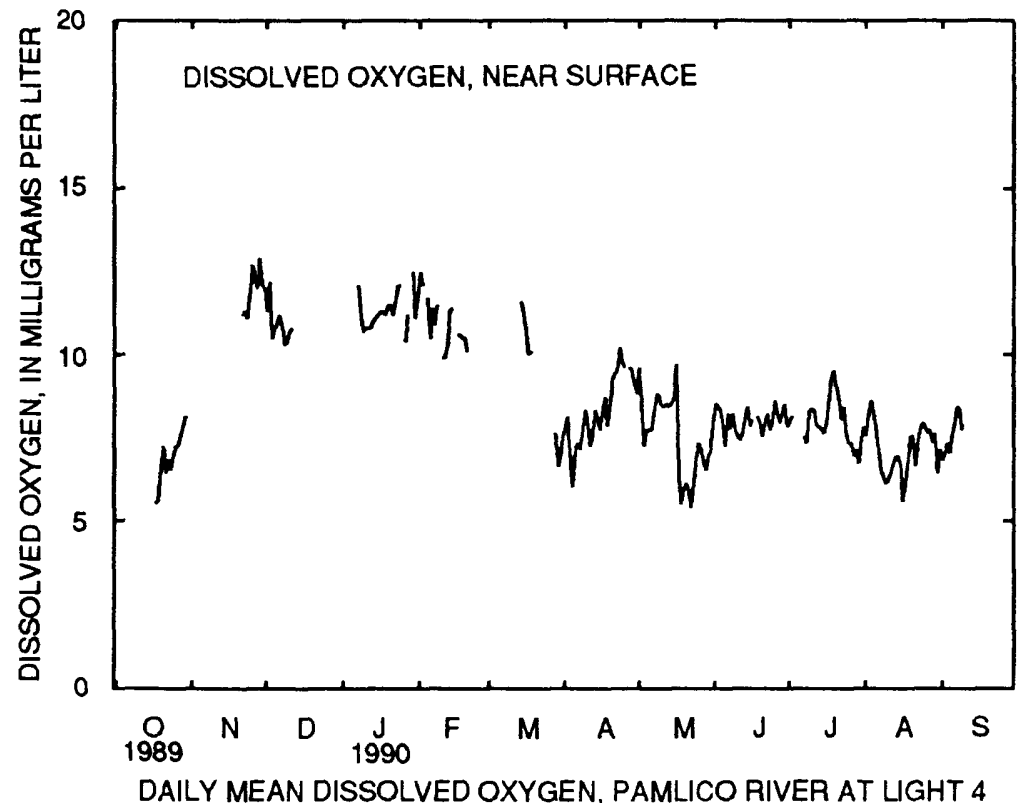


Table 5.- Daily mean values of salinity, water temperature, and dissolved oxygen in water at site 3 , Pamlico River at Light 4, April 1989 to September 1990--Continued

[Site 3 is at latitude $35^{\circ} 24^{\prime} 47^{\prime \prime}$, longitude 76 $45^{\prime} 52^{\prime \prime}$, Beaufort County; U.S. Geological Survey downstream order number 0208454253; ppt, parts per thousand; <, less than; ---, no data;

${ }^{\circ} \mathrm{C}$, degrees Celsius; mg/L, milligrams per liter; a, daily minimum value is less than or equal

to $2.0 \mathrm{mg} / \mathrm{L} ; \mathrm{b}$, daily minimum value is less than or equal to $5.0 \mathrm{mg} / \mathrm{L}$ but greater than $2.0 \mathrm{mg} / \mathrm{L}$ ]

DISSOLVED OXYGEN, MID-DEPTH

SENSOR POSITION. --6 feet above streambed.

EXTREMES FOR APRIL TO SEPTEMBER 1989.--Maximum value recorded, $11.9 \mathrm{mg} / \mathrm{L}$ August 28 , 1989 ; minimum value recorded, $<1.0 \mathrm{mg} / \mathrm{L}$ August $24,1989$.

DAILY MEAN VALUES OF DISSOLVED OXYGEN, MID-DEPTH (IN MILLIGRAMS PER LITER), APRIL TO SEPTEMBER 1989

\begin{tabular}{|c|c|c|c|c|c|c|}
\hline DAY & APR & MAY & JUNE & JULY & AUG & SEPT \\
\hline 1 & $\cdots$ & 8.2 & -- & $\cdots$ & -- & $5.9 \mathrm{~b}$ \\
\hline 2 & $-m$ & 7.5 & --- & $\cdots$ & -- & $4.6 \mathrm{~b}$ \\
\hline 3 & $m$ & 8.2 & -- & --- & $\cdots$ & $5.3 b$ \\
\hline 4 & -- & 8.4 & -- & $\cdots$ & $-\cdots$ & $5.1 \mathrm{~b}$ \\
\hline 5 & $\cdots$ & 7.5 & -- & -- & -- & $4.4 b$ \\
\hline 6 & --- & 6.5 & --- & $\cdots$ & -- & $5.1 \mathrm{~b}$ \\
\hline 7 & $m$ & 6.4 & --- & $\cdots$ & --- & $5.8 b$ \\
\hline 8 & --- & 6.2 & --- & --- & -- & 6.8 \\
\hline 9 & --- & 6.2 & $\cdots$ & --- & --- & 6.9 \\
\hline 10 & -- & 6.2 & $-\infty$ & --- & --- & 7.9 \\
\hline 11 & --- & 5.6 & --- & --- & $-\cdots$ & 8.4 \\
\hline 12 & --- & 5.4 & --- & -- & $-\cdots$ & 7.8 \\
\hline 13 & -- & $5.7 \mathrm{~b}$ & -- & --- & --- & --- \\
\hline 14 & $-\cdots$ & 5.9 & --- & -- & -- & --- \\
\hline 15 & -- & 6.0 & --- & $-\cdots$ & --- & --- \\
\hline 16 & -- & $5.4 b$ & --- & --- & --- & --- \\
\hline 17 & --- & $5.6 \mathrm{~b}$ & --- & $\cdots$ & -- & --- \\
\hline 18 & --- & --- & --- & $\cdots$ & -- & -- \\
\hline 19 & --- & --- & --- & -- & -- & --- \\
\hline 20 & -- & --- & --- & --- & --- & --- \\
\hline 21 & --- & --- & --- & -- & --- & -- \\
\hline 22 & -- & --- & -- & --- & --- & --- \\
\hline 23 & --- & --- & --- & --- & $-\cdots$ & --- \\
\hline 24 & $\cdots$ & --- & --- & --- & $3.3 a$ & --- \\
\hline 25 & $\cdots$ & --- & $\cdots$ & $\cdots$ & $6.7 a$ & -- \\
\hline 26 & $\cdots$ & --- & -- & --- & $7.1 \mathrm{a}$ & -- \\
\hline 27 & --- & --- & --- & -- & 8.7 & --- \\
\hline 28 & 7.8 & --- & --- & $-\cdots$ & $8.1 \mathrm{~b}$ & --- \\
\hline 29 & 8.5 & -- & --- & -- & 7.8 & --- \\
\hline 30 & 7.8 & --- & -- & --- & $6.8 \mathrm{~b}$ & --- \\
\hline 31 & --- & --- & --- & --- & $7.1 \mathrm{~b}$ & -- \\
\hline
\end{tabular}

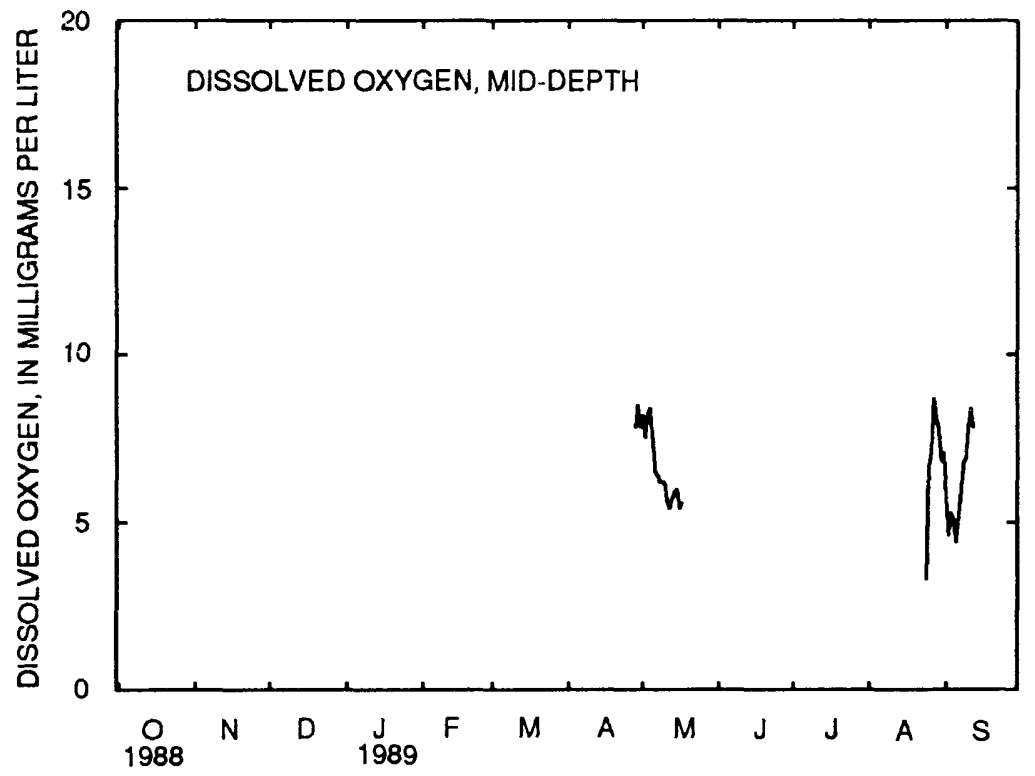


Table 5.--Daily mean values of salinity, water temperature, and dissolved oxygen in water at site 3 , Pamlico River at Light 4, April 1989 to September 1990--Continued

[Site 3 is at latitude $35^{\circ} 24^{\prime} 47^{\prime \prime}$, longitude 76 $45^{\prime} 52^{\prime \prime}$, Beaufort County; U.S. Geological Survey downstream order number 0208454253; ppt, parts per thousand; <, less than; ---, no data;

${ }^{\circ} \mathrm{C}$, degrees Celsius; mg/L, milligrams per liter; a, dally minimum value is less than or equal

to $2.0 \mathrm{mg} / \mathrm{L} ; \mathrm{b}$, daily minimum value is less than or equal to $5.0 \mathrm{mg} / \mathrm{L}$ but greater than $2.0 \mathrm{mg} / \mathrm{L}$ ]

DISSOLVED OXYGEN, MID-DEPTH

SENSOR POSITION. --6 feet above streambed.

EXTREMES FOR OCTOBER 1989 TO SEPTEMBER 1990.--Maximum value recorded, 14.8 mg/L February 1 , 1990 ; minlmum value recorded, $<1.0 \mathrm{mg} / \mathrm{L}$ August $28,29,1990$.

DAILY MEAN VALUES OF DISSOLVED OXYGEN, MID-DEPTH (IN MILLIGRAMS PER LITER), OCTOBER 1989 TO SEPTEMBER ?990

\begin{tabular}{|c|c|c|c|c|c|c|c|c|c|c|c|c|}
\hline DAY & OCT & NOV & DEC & JAN & FEB & MAR & $A P R$ & MAY & JUNE & JULY & AUG & SEPT \\
\hline 1 & --- & --- & 11.7 & --- & 12.6 & --- & 10.6 & 8.1 & 9.1 & 7.7 & $6.1 \mathrm{~b}$ & $5.6 b$ \\
\hline 2 & --- & $\cdots$ & 12.5 & --- & 12.0 & -- & 9.9 & $7.0 \mathrm{~b}$ & 9.1 & 7.9 & $6.7 b$ & $5.4 a$ \\
\hline 3 & $\ldots$ & -- & 10.9 & --- & -- & --- & $8.9 b$ & 6.5 & 8.7 & --- & 7.7 & $5.7 b$ \\
\hline 4 & --- & $\ldots$ & 11.3 & --- & 11.9 & --- & 8.9 & 7.1 & 7.0 & 7.2 & 7.7 & $5.6 b$ \\
\hline 5 & --- & --- & 11.4 & -- & 10.7 & -- & 9.6 & 7.1 & 7.6 & $-\cdots$ & 7.5 & $5.7 b$ \\
\hline 6 & --- & --- & 11.9 & $-\cdots$ & 11.2 & --- & 9.9 & 7.0 & 8.7 & --- & 7.1 & 6.0 \\
\hline 7 & --- & -- & 11.5 & 12.4 & 11.1 & --- & 9.5 & 7.6 & 8.1 & 7.2 & $6.1 \mathrm{~b}$ & $6.1 b$ \\
\hline 8 & --- & --- & 10.9 & 11.9 & 11.9 & --- & 9.9 & 8.2 & 8.7 & 6.7 & $6.2 \mathrm{~b}$ & 5.70 \\
\hline 9 & --- & --- & 11.0 & 11.4 & -- & --- & 10.5 & 8.2 & 8.3 & 7.3 & 6.3 & 5.3 \\
\hline 10 & --- & --- & 11.4 & 11.6 & 10.2 & --- & 9.9 & 8.1 & 8.0 & 7.5 & $5.9 b$ & $-\cdots$ \\
\hline 11 & --- & --- & 11.5 & 11.7 & 10.1 & --- & 9.1 & 8.2 & 7.8 & 7.4 & 5.90 & $-\cdots$ \\
\hline 12 & --- & --- & --- & 11.6 & 10.5 & --- & 9.2 & 8.4 & 8.1 & 7.3 & 5.80 & --- \\
\hline 13 & --- & --- & --- & 11.8 & 11.6 & --- & 9.8 & 8.5 & 8.5 & 7.4 & $5.7 b$ & $\cdots$ \\
\hline 14 & --- & --- & -- & 11.9 & 11.6 & 9.9 & 9.4 & 8.6 & 8.7 & 7.6 & $4.6 a$ & --- \\
\hline 15 & --- & --- & --- & 11.8 & -- & 9.4 & 8.9 & 8.7 & 8.5 & 7.4 & $4.7 \mathrm{~b}$ & --- \\
\hline 16 & --- & --- & --- & 11.9 & 10.8 & 10.0 & 9.3 & 9.1 & 8.3 & 7.5 & $3.7 b$ & $\ldots$ \\
\hline 17 & $4.3 a$ & $-\cdots$ & --- & 11.8 & 10.6 & 10.0 & 9.5 & 8.1 & --- & 8.2 & $4.5 \mathrm{~b}$ & -- \\
\hline 18 & $3.6 \mathrm{a}$ & --- & --- & 11.8 & 10.5 & 10.4 & 8.8 & 8.0 & 8.1 & 8.7 & $5.3 b$ & $\cdots$ \\
\hline 19 & $4.0 \mathrm{a}$ & --- & --- & 12.0 & 10.5 & -- & 9.2 & 8.2 & 8.2 & 8.4 & $6.0 \mathrm{~b}$ & --- \\
\hline 20 & $6.1 \mathrm{a}$ & --- & -- & 11.9 & 10.3 & --- & 9.9 & 8.7 & 8.3 & 8.2 & $5.5 b$ & -- \\
\hline 21 & $6.4 \mathrm{~b}$ & 11.6 & --- & 11.5 & -- & -- & 10.1 & 8.1 & 8.5 & 8.1 & $4.9 b$ & --- \\
\hline 22 & 7.5 & 11.8 & --- & 11.8 & --- & --- & 10.1 & 7.2 & 8.7 & 7.8 & $5.1 b$ & --- \\
\hline 23 & 7.8 & 11.6 & --- & 12.3 & --- & --- & 10.2 & 7.9 & 8.4 & 8.4 & 5.80 & $\cdots$ \\
\hline 24 & 8.6 & 12.0 & --- & 12.3 & --- & --- & 9.9 & 8.9 & 8.3 & 7.9 & $5.6 b$ & --- \\
\hline 25 & 8.7 & 12.4 & --- & --- & --- & --- & 9.5 & 9.0 & 8.8 & 7.4 & 4.90 & --- \\
\hline 26 & 8.3 & 12.5 & --- & 10.6 & --- & --- & --- & 9.2 & 8.5 & 7.2 & 5.30 & $\ldots$ \\
\hline 27 & 8.3 & 12.3 & --- & 11.2 & --- & --- & 8.2 & 8.2 & 8.0 & $6.5 b$ & $5.4 \mathrm{~b}$ & $\ldots$ \\
\hline 28 & 8.3 & 13.2 & --- & -- & --- & 10.6 & $7.5 b$ & 7.8 & 8.1 & $6.6 b$ & $6.0 a$ & --- \\
\hline 29 & 8.4 & 12.5 & --- & 12.5 & --- & 10.1 & $7.4 \mathrm{~b}$ & 8.1 & 8.5 & $6.1 b$ & $6.1 a$ & -- \\
\hline 30 & --- & 12.4 & $\cdots$ & 11.4 & --- & 10.2 & $7.1 \mathrm{~b}$ & 8.3 & 7.6 & 6.6 & $5.5 b$ & --- \\
\hline 31 & --- & -- & --- & 11.8 & --- & 10.5 & $\cdots$ & 9.2 & --- & 6.9 & $6.3 b$ & -- \\
\hline
\end{tabular}

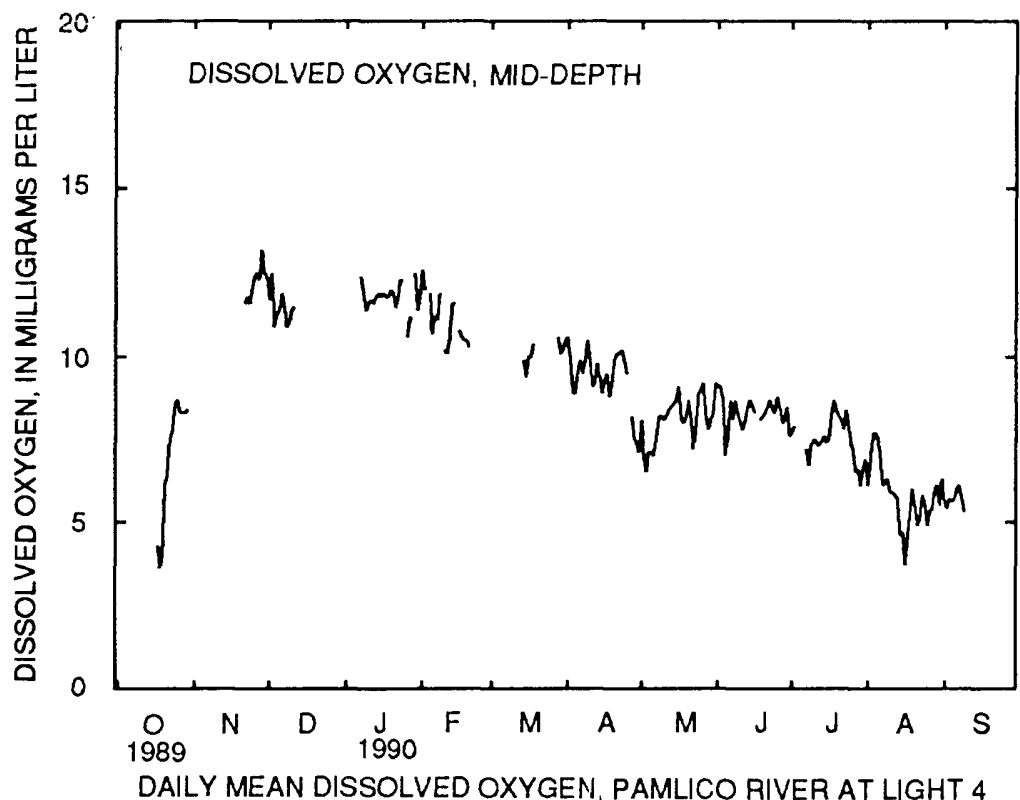


Table 5.--Daily mean values of salinity, water temperature, and dissolved oxygen in water at site 3 , Pamlico River at Light 4, April 1989 to September 1990--Continued

[Site 3 is at latitude $35^{\circ} 24^{\prime} 47^{\prime \prime}$, longitude $76^{\circ} 45^{\prime} 52^{\prime \prime}$, Beaufort County; U.S. Geological Survey downstream order number 0208454253; ppt, parts per thousand; <, less than; ---, no data;

${ }^{\circ} \mathrm{C}$, degrees Celsius; mg/L, milligrams per I1ter; a, daily minimum value is less than or equal

to $2.0 \mathrm{mg} / \mathrm{L}$; b, dally minimum value is less than or equal to $5.0 \mathrm{mg} / \mathrm{L}$ but greater than $2.0 \mathrm{mg} / \mathrm{L}$ ]

DISSOLVED OXYGEN, NEAR BOTTOM

SENSOR POSITION. --2 feet above streambed.

EXTREMES FOR APRIL TO SEPTEMBER 1989.--Maximum value recorded, $11.5 \mathrm{mg} / \mathrm{L}$ May 1, 1989; minlmum value recorded, $<1.0 \mathrm{mg} / \mathrm{L}$ August 24, 25, september 1, 1989.

DAILY MEAN VALUES OF DISSOLVED OXYGEN, NEAR BOTTOM (IN MILLIGRAMS PER LITER), APRIL TO SEPTEMBER 1989

\begin{tabular}{|c|c|c|c|c|c|c|}
\hline DAY & APR & MAY & JUNE & JULY & AUG & SEPT \\
\hline 1 & -- & 9.5 & $\ldots$ & $-\ldots$ & $-\infty$ & $4.2 a$ \\
\hline 2 & -- & 9.2 & -- & $\cdots$ & -- & $5.0 a$ \\
\hline 3 & -- & 9.6 & -- & $\ldots$ & $-\cdots$ & $4.9 a$ \\
\hline 4 & --- & 9.6 & $\ldots$ & -- & $-\cdots$ & $4.9 b$ \\
\hline 5 & $\cdots$ & 8.8 & $\ldots$ & -- & -- & $3.7 a$ \\
\hline 6 & -- & 7.6 & -- & $\cdots$ & -- & $4.4 \mathrm{~b}$ \\
\hline 7 & --- & 7.6 & $\ldots$ & $-\infty$ & -- & $5.5 b$ \\
\hline 8 & -- & 7.5 & -- & -- & --- & 7.0 \\
\hline 9 & --- & 7.6 & $-\ldots$ & $-\cdots$ & -- & 6.7 \\
\hline 10 & --- & 7.4 & -- & $\cdots$ & --- & 8.0 \\
\hline 11 & -- & $6.4 \mathrm{~b}$ & $\ldots$ & -- & --- & $8.3 b$ \\
\hline 12 & -- & $6.0 \mathrm{~b}$ & -- & -- & -- & $7.6 \mathrm{~b}$ \\
\hline 13 & -- & $6.8 b$ & $\ldots$ & -- & --- & --- \\
\hline 14 & --- & $5.0 \mathrm{~b}$ & -- & $\cdots$ & -- & -- \\
\hline 15 & --- & $3.7 \mathrm{~b}$ & -- & -- & --- & $-\cdots$ \\
\hline 16 & -- & $4.7 b$ & -- & $\ldots$ & -- & $-\cdots$ \\
\hline 17 & --- & 6.7 & -- & $\cdots$ & $\cdots$ & -- \\
\hline 18 & --- & --- & --- & $\cdots$ & -- & $-\cdots$ \\
\hline 19 & --- & --- & --- & -- & --- & --- \\
\hline 20 & --- & --- & --- & -- & --- & --- \\
\hline 21 & --- & -- & -- & $\ldots$ & -- & --- \\
\hline 22 & --- & --- & $\cdots$ & $\ldots$ & --- & --- \\
\hline 23 & $-\infty$ & --- & --- & - & $-\cdots$ & --- \\
\hline 24 & -- & $-\infty$ & $\cdots$ & -- & $2.4 \mathrm{a}$ & --- \\
\hline 25 & $\cdots$ & -- & --- & $\cdots$ & $5.2 a$ & --- \\
\hline 26 & $-\cdots$ & -- & --- & $\cdots$ & $5.7 \mathrm{a}$ & --- \\
\hline 27 & --- & -- & --- & -- & $6.8 \mathrm{~b}$ & --- \\
\hline 28 & $-\cdots$ & -- & --- & $\cdots$ & $6.6 a$ & --- \\
\hline 29 & 9.7 & --- & --- & -- & $6.4 \mathrm{~b}$ & --- \\
\hline 30 & 8.8 & --- & --- & -- & $5.7 b$ & --- \\
\hline 31 & -- & -- & -- & -- & $4.9 b$ & --- \\
\hline
\end{tabular}

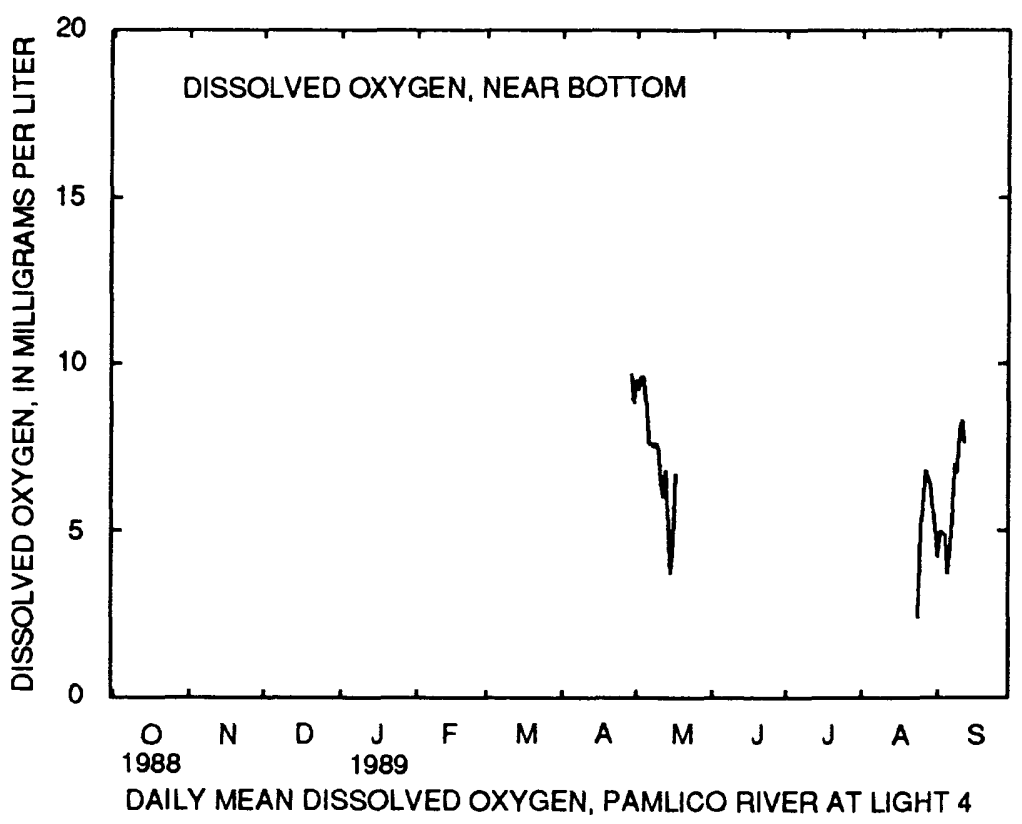


Table 5.--Dally mean values of salinity, water temperature, and dissolved oxygen in water at site 3 ,

Pamlico River at Light 4, April 1989 to September 1990--Continued

[S1te 3 is at latitude $35^{\circ} 24^{\prime} 47^{\prime \prime}$, longltude 76 $45^{\prime} 52^{\prime \prime}$, Beaufort County; U.S. Geologlcal Survey

downstream order number 0208454253 ; ppt, parts per thousand; <, less than; ---, no data;

${ }^{\circ} \mathrm{C}$, degrees Celsius; mg/L, mllligrams per liter; a, dally minimum value is less than or equal

to $2.0 \mathrm{mg} / \mathrm{L} ; \mathrm{b}$, dally minimum value is less than or equal to $5.0 \mathrm{mg} / \mathrm{L}$ but greater than $2.0 \mathrm{mg} / \mathrm{L}]$

DISSOLVED OXYGEN, NEAR BOTTOM

SENSOR POSITION. --2 feet above streambed.

EXTREMES FOR OCTOBER 1989 TO SEPTEMBER 1990.--Maximum value recorded, 16.7 mg/L February 1 , $1990 ;$ minimum value recorded, <1.0 mg/L May 29, 31, June 1-3, July 4, 7, 8, 20-25, 27, 28, August 1-3, 5-31, September 1-3, 1990.

DAILY MEAN VALUES OF DISSOLVED OXYGEN, NEAR BOTTOM (IN MILLIGRAMS PER LITER), OCTOBER 1989 TO SEPTEMBER 1990

\begin{tabular}{|c|c|c|c|c|c|c|c|c|c|c|c|c|}
\hline DAY & $\mathrm{OCT}$ & NOV & DEC & JAN & FEB & MAR & APR & MAY & JUNE & JULY & AUG & SEPT \\
\hline 1 & --- & --- & 9.1 & --- & 13.4 & --- & $7.3 b$ & --- & $3.8 \mathrm{a}$ & --- & $3.3 a$ & $3.2 a$ \\
\hline 2 & --- & --- & $7.9 b$ & --- & 13.5 & --- & $5.7 b$ & --- & $2.4 a$ & --- & $2.5 a$ & $4.3 a$ \\
\hline 3 & --- & --- & $8.6 \mathrm{~b}$ & --- & --- & --- & $7.4 b$ & --- & $2.3 a$ & --- & $4.4 a$ & $4.7 \mathrm{a}$ \\
\hline 4 & --- & --- & 10.6 & --- & 12.7 & --- & 9.2 & --- & --- & $4.6 a$ & $6.4 a$ & $6.0 \mathrm{~b}$ \\
\hline 5 & --- & --- & 10.5 & --- & 12.0 & --- & 8.8 & --- & --- & $\therefore--$ & $4.8 a$ & $6.0 \mathrm{~b}$ \\
\hline 6 & --- & --- & 10.8 & --- & 12.4 & --- & 8.8 & 9.2 & --- & --- & $1.8 \mathrm{a}$ & 6.5 \\
\hline 7 & --- & --- & 10.4 & 12.0 & 10.9 & --- & 9.5 & 9.6 & --- & $6.9 a$ & $<1.0 a$ & $6.5 b$ \\
\hline 8 & --- & --- & 10.0 & 11.6 & 12.8 & --- & 10.5 & 10.2 & --- & $6.7 a$ & $1.1 \mathrm{a}$ & $6.0 \mathrm{~b}$ \\
\hline 9 & --- & --- & 10.2 & 11.8 & --- & --- & 11.1 & 10.0 & --- & $7.4 \mathrm{~b}$ & $1.9 a$ & $6.0 \mathrm{~b}$ \\
\hline 10 & --- & --- & 10.4 & 12.2 & 11.7 & --- & 11.0 & 10.2 & --- & 8.1 & $<1.0 \mathrm{a}$ & --- \\
\hline 11 & --- & -- & 10.1 & 12.4 & 11.2 & --- & 9.9 & 9.7 & --- & 8.0 & $<1.0 a$ & --- \\
\hline 12 & --- & --- & --- & 12.4 & 12.0 & --- & 9.9 & 9.4 & --- & 8.1 & $<1.0 a$ & --- \\
\hline 13 & --- & --- & --- & 12.7 & 13.3 & --- & 11.0 & 10.0 & --- & 8.0 & $<1.0 \mathrm{a}$ & --- \\
\hline 14 & --- & --- & --- & 12.8 & 13.0 & 11.0 & 11.0 & 9.6 & --- & 7.9 & $1.0 \mathrm{a}$ & --- \\
\hline 15 & --- & --- & --- & 12.6 & --- & 11.3 & 10.0 & 9.5 & --- & 7.3 & $2.9 a$ & --- \\
\hline 16 & --- & --- & --- & 12.5 & 12.5 & 11.0 & 10.0 & 8.4 & --- & $6.7 \mathrm{~b}$ & $1.7 \mathrm{a}$ & --- \\
\hline 17 & --- & --- & --- & 12.3 & 11.9 & 13.1 & 9.3 & 7.9 & --- & $4.3 b$ & $2.9 a$ & --- \\
\hline 18 & --- & --- & --- & 12.4 & 12.3 & 12.0 & 10.4 & 6.9 & --- & $5.6 b$ & $1.9 a$ & --- \\
\hline 19 & --- & --- & --- & 13.3 & 11.5 & --- & 11.2 & 6.6 & --- & $5.8 a$ & $2.1 \mathrm{a}$ & --- \\
\hline 20 & --- & --- & --- & 12.9 & 11.8 & --- & 11.7 & $7.0 \mathrm{~b}$ & --- & $3.6 a$ & $<1.0 a$ & --- \\
\hline 21 & $5.8 b$ & 11.8 & --- & 13.0 & --- & --- & 11.1 & $6.4 \mathrm{~b}$ & --- & $2.7 a$ & $<1.0 \mathrm{a}$ & --- \\
\hline 22 & $6.0 \mathrm{~b}$ & 11.4 & --- & 12.8 & --- & --- & 11.4 & 6.2 & --- & $<1.0 a$ & $<1.0 \mathrm{a}$ & --- \\
\hline 23 & $6.1 b$ & 11.6 & --- & --- & --- & --- & 11.4 & 6.6 & --- & $<1.0 \mathrm{a}$ & $<1.0 \mathrm{a}$ & --- \\
\hline 24 & $6.2 b$ & 11.5 & --- & --- & --- & --- & 10.5 & $7.0 \mathrm{~b}$ & --- & $<1.0 a$ & $<1.0 a$ & -- \\
\hline 25 & 6.6 & 11.1 & --- & --- & --- & --- & 10.0 & $4.7 b$ & --- & $<1.0 a$ & $<1.0 \mathrm{a}$ & --- \\
\hline 26 & $6.3 b$ & 10.8 & --- & --- & --- & --- & --- & $4.3 a$ & --- & --- & $<1.0 \mathrm{a}$ & --- \\
\hline 27 & 6.4 & 11.2 & --- & 12.7 & --- & --- & --- & $5.5 a$ & --- & $5.2 a$ & $<1.0 a$ & $-\cdots-$ \\
\hline 28 & $6.3 \mathrm{~b}$ & 9.3 & --- & --- & --- & 9.6 & --- & $5.8 b$ & --- & $4.7 a$ & $<1.0 \mathrm{a}$ & --- \\
\hline 29 & $7.1 \mathrm{~b}$ & 9.4 & --- & 13.3 & --- & 10.0 & --- & $4.9 a$ & -- & $6.6 \mathrm{~b}$ & $<1.0 a$ & --- \\
\hline 30 & --- & 8.9 & --- & 13.1 & --- & 9.5 & --- & $6.0 \mathrm{~b}$ & --- & 7.1 & $1.9 a$ & --- \\
\hline 31 & --- & --- & -- & 12.8 & --- & 9.2 & --- & $4.9 a$ & -- & $6.3 b$ & $2.2 a$ & -- \\
\hline
\end{tabular}

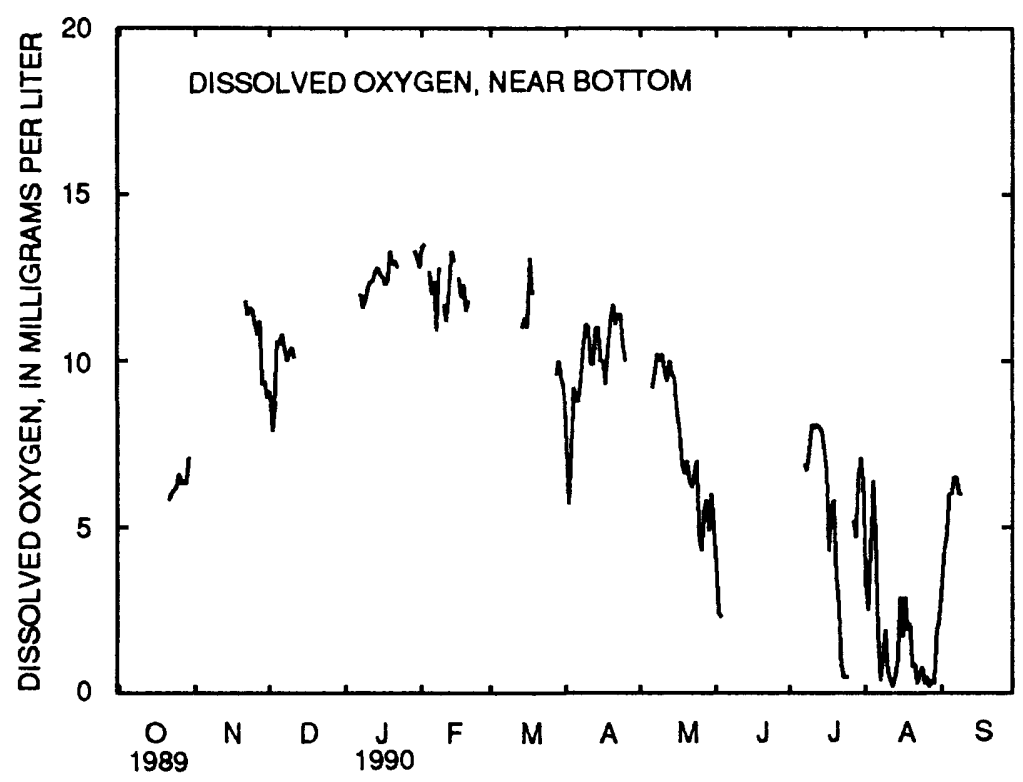

DAILY MEAN DISSOLVED OXYGEN, PAMLICO RIVER AT LIGHT 4 
Table 6.--Daily mean values of salinity, water temperature, and dissolved oxygen in water at site 4, Pamlico River at Light 3, May 1989 to september 1990

[Site 4 is at latitude $35^{\circ} 21^{\prime} 24^{\prime \prime}$, longitude 76 38'48", Beaufort County; U.S. Geological Survey downstream order number 0208455155 ; ppt, parts per thousand; ---, no data; ${ }^{\circ} \mathrm{C}$, degrees

Celsius; mg/L, milligrams per liter; a, dally minimum value is less than or equal to $2.0 \mathrm{mg} / \mathrm{L}$; b, dally minimum value is less than or equal to $5.0 \mathrm{mg} / \mathrm{L}$ but greater than $2.0 \mathrm{mg} / \mathrm{L}$; <, less thanl

SALINITY, NEAR SURFACE

SENSOR POSITION.--8 feet above streambed.

EXTREMES FOR MAY TO SEPTEMBER 1989.--Maximum value recorded, 11.2 ppt September 4, 1989; minimum value recorded, 0.9 ppt MaY 17, 1989.

DAILY MEAN VALUES OF SALINITY, NEAR SURFACE (IN PARTS PER THOUSAND), MAY TO SEPTEMBER 1989

\begin{tabular}{|c|c|c|c|c|c|}
\hline DAY & MAY & JUNE & JULY & AUG & SEPT \\
\hline 1 & -- & 6.1 & --- & --- & 10.1 \\
\hline 2 & --- & 5.9 & --- & --- & 10.0 \\
\hline 3 & $-\cdots$ & 5.7 & -- & -- & 10.1 \\
\hline 4 & 4.6 & 5.8 & -- & -- & 10.8 \\
\hline 5 & 5.6 & 6.1 & -- & -- & 10.4 \\
\hline 6 & 4.3 & 6.4 & --- & --- & 9.9 \\
\hline 7 & 3.8 & 6.2 & --- & -- & 10.1 \\
\hline 8 & 3.3 & --- & --- & --- & 10.1 \\
\hline 9 & 2.8 & --- & --- & --- & 9.9 \\
\hline 10 & 2.5 & 5.7 & -- & --- & 9.9 \\
\hline 11 & 2.3 & 5.8 & --- & --- & 9.6 \\
\hline 12 & 2.4 & --- & --- & --- & 9.6 \\
\hline 13 & 2.3 & -- & --- & --- & 9.6 \\
\hline 14 & 2.0 & 5.5 & --- & --- & 9.7 \\
\hline 15 & 1.9 & 5.6 & --- & --- & 9.8 \\
\hline 16 & 2.0 & --- & --- & --- & 9.7 \\
\hline 17 & 3.2 & --- & --- & -- & 9.5 \\
\hline 18 & 4.4 & 5.5 & --- & --- & 9.8 \\
\hline 19 & 4.7 & 5.5 & --- & --- & 10.2 \\
\hline 20 & 3.8 & 5.3 & -- & --- & 10.0 \\
\hline 21 & 3.8 & 4.8 & --- & $-\cdots$ & 10.2 \\
\hline 22 & 4.5 & 4.6 & --- & --- & 10.3 \\
\hline 23 & 5.2 & 4.5 & --- & -- & 9.1 \\
\hline 24 & 5.6 & 4.2 & --- & --- & 9.9 \\
\hline 25 & 5.6 & $\cdots$ & --- & --- & 9.8 \\
\hline 26 & 5.7 & --- & --- & 9.2 & 8.6 \\
\hline 27 & 5.7 & --- & --- & 9.3 & 8.8 \\
\hline 28 & 6.3 & -- & --- & --- & 8.8 \\
\hline 29 & 7.0 & --- & --- & 9.3 & 8.8 \\
\hline 30 & 6.7 & --- & --- & 9.3 & 8.9 \\
\hline 31 & 6.2 & --- & --- & --- & $-\cdots$ \\
\hline
\end{tabular}

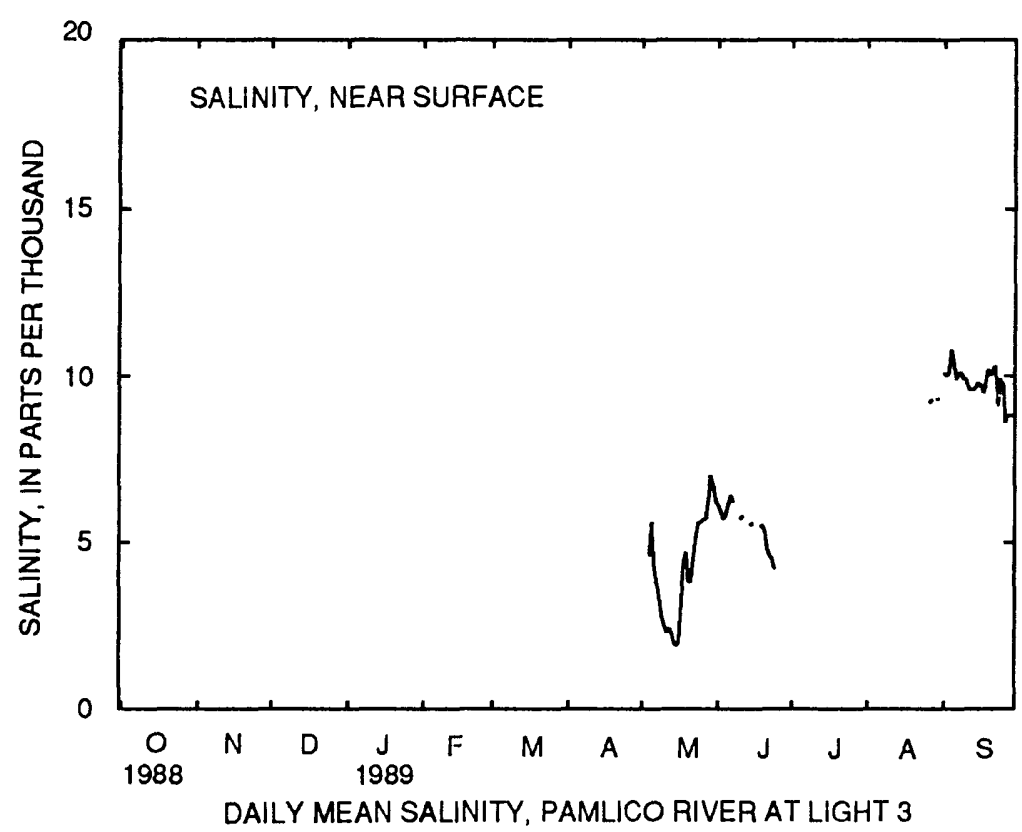


Table 6.--Daily mean values of salinity, water temperature, and dissolved oxygen in water at site 4, Pamlico River at Llght 3, May 1989 to September 1990--Continued

[Site 4 is at latitude $35^{\circ} 21^{\prime} 24^{\prime \prime}$, longitude $76^{\circ} 38^{\prime} 48^{\prime \prime}$, Beaufort County; U.S. Geological Survey downst ream order number 0208455155 ; ppt, parts per thousand; ---, no data; ${ }^{\circ} \mathrm{C}$, degrees

Celsius; mg/L, milligrams per liter; a, dally minimum value is less than or equal to $2.0 \mathrm{mg} / \mathrm{L}$; b, daily minimum value is less than or equal to $5.0 \mathrm{mg} / \mathrm{L}$ but greater than $2.0 \mathrm{mg} / \mathrm{L}$; <, less than]

SALINITY, NEAR SURFACE

SENSOR POSITION. --8 feet above streambed.

EXTREMES FOR OCTOBER 1989 TO SEPTEMBER 1990.--Maximum value recorded, 11.2 ppt September 1, 1990 ; minimum value recorded, 1.1 ppt May 6, 1990.

DAILY MEAN VALUES OF SALINITY, NEAR SURFACE (IN PARTS PER THOUSAND), OCTOBER 1989 TO SEPTEMBER 1990

\begin{tabular}{|c|c|c|c|c|c|c|c|c|c|c|c|c|}
\hline DAY & $\mathrm{OCT}$ & Nov & DEC & JAN & FEB & MAR & $A P R$ & MAY & JUNE & JULY & AUG & SEPT \\
\hline 1 & 8.9 & 6.6 & 6.3 & -- & 7.4 & 4.1 & -- & 3.2 & 4.1 & --- & 7.0 & 10.8 \\
\hline 2 & 8.6 & 7.4 & 5.6 & -- & 6.4 & --- & -- & 4.0 & 4.1 & --- & 7.3 & 9.8 \\
\hline 3 & 8.2 & 7.5 & $-\cdots$ & -- & -- & --- & --- & 4.0 & 4.5 & --- & 7.6 & 9.8 \\
\hline 4 & 7.9 & 7.4 & -- & 5.6 & 6.7 & --- & -- & 4.1 & 4.2 & -- & 8.0 & 10.0 \\
\hline 5 & 6.3 & 7.6 & 7.1 & 5.5 & 7.1 & --- & -- & 4.0 & 4.7 & --- & 7.7 & 9.8 \\
\hline 6 & 6.2 & 7.2 & 7.5 & 5.4 & 6.4 & --- & -- & 3.9 & 4.7 & $--\infty$ & 7.7 & 9.6 \\
\hline 7 & 7.1 & 6.4 & 7.9 & 5.2 & 6.0 & --- & --- & 4.2 & 4.8 & --- & 7.7 & 9.0 \\
\hline 8 & 7.5 & 5.5 & 8.6 & 6.1 & 6.2 & $\cdots$ & --- & 4.0 & 4.2 & --- & 7.9 & 8.9 \\
\hline 9 & 6.8 & 6.3 & 8.7 & 7.3 & 6.3 & --- & -- & 4.0 & 4.5 & -- & 8.0 & 9.2 \\
\hline 10 & 7.2 & 6.5 & 8.7 & 7.6 & -- & --- & --- & --- & 4.7 & -- & 7.8 & --- \\
\hline 11 & 7.2 & 7.1 & 8.5 & 7.7 & --- & --- & --- & --- & 5.0 & --- & 8.1 & -- \\
\hline 12 & 6.1 & 7.0 & -- & 7.6 & 6.9 & --- & -- & 4.0 & 5.2 & -- & 7.9 & --- \\
\hline 13 & 5.7 & 7.2 & -- & 7.5 & 6.6 & -- & -- & 4.0 & 4.7 & --- & 7.9 & --- \\
\hline 14 & 5.5 & 6.8 & --- & 6.5 & 6.3 & --- & --- & 4.2 & 4.5 & --- & 8.1 & -- \\
\hline 15 & 4.9 & 7.0 & -- & 5.3 & 6.3 & -- & -- & 4.1 & 5.2 & --- & 8.1 & -- \\
\hline 16 & 5.2 & 7.3 & --- & 5.0 & 6.1 & --- & $-\cdots$ & --- & 5.0 & --- & 8.8 & $-\cdots$ \\
\hline 17 & --- & 7.1 & -- & 4.6 & -- & $-\cdots$ & -- & 4.0 & 5.0 & --- & 9.2 & $\cdots$ \\
\hline 18 & 6.4 & 6.5 & -- & 4.7 & 6.4 & --- & -- & 4.0 & 5.2 & --- & 9.1 & -- \\
\hline 19 & 6.9 & 6.4 & -- & 5.6 & 5.5 & --- & -- & 4.0 & 5.3 & --- & 8.9 & -- \\
\hline 20 & 7.2 & 6.2 & -- & 5.9 & 5.7 & --- & 3.2 & 3.8 & 5.4 & --- & 8.7 & -- \\
\hline 21 & 7.8 & 6.7 & --- & 6.8 & 5.7 & --- & 3.4 & 4.1 & 5.4 & --- & 9.1 & -- \\
\hline 22 & 7.9 & 6.5 & --- & 7.1 & 5.4 & --- & 3.4 & 5.5 & 5.6 & --- & 8.5 & -- \\
\hline 23 & 7.7 & 6.7 & -- & 6.9 & 5.9 & --- & 2.9 & 5.4 & 6.0 & --- & 8.2 & $\cdots$ \\
\hline 24 & 7.8 & 6.2 & -- & 6.6 & 4.8 & -- & 2.9 & 4.5 & 6.5 & -- & 8.6 & $-\cdots$ \\
\hline 25 & 7.5 & 4.2 & --- & 6.9 & 4.3 & --- & 3.1 & 4.0 & 6.9 & --- & 8.5 & --- \\
\hline 26 & 7.3 & -- & --- & 7.3 & 4.1 & --- & 2.8 & 4.3 & 7.2 & -- & 8.3 & -- \\
\hline 27 & 7.7 & 6.1 & --- & 7.3 & 4.0 & --- & 2.3 & 5.3 & 7.1 & -- & 8.7 & -- \\
\hline 28 & 7.5 & 5.9 & --- & 7.4 & 3.3 & --- & 2.2 & 5.9 & --- & --- & --- & -- \\
\hline 29 & 7.8 & 6.1 & $-\cdots$ & 7.4 & -- & --- & 2.9 & 5.1 & -- & -- & 7.7 & -- \\
\hline 30 & 7.7 & 6.3 & --- & --- & -- & -- & 3.1 & 5.4 & $-\cdots$ & -- & 8.3 & -- \\
\hline 31 & 7.5 & -- & --- & $-\cdots$ & $\ldots$ & --- & -- & 4.4 & -- & -- & 10.1 & --- \\
\hline
\end{tabular}

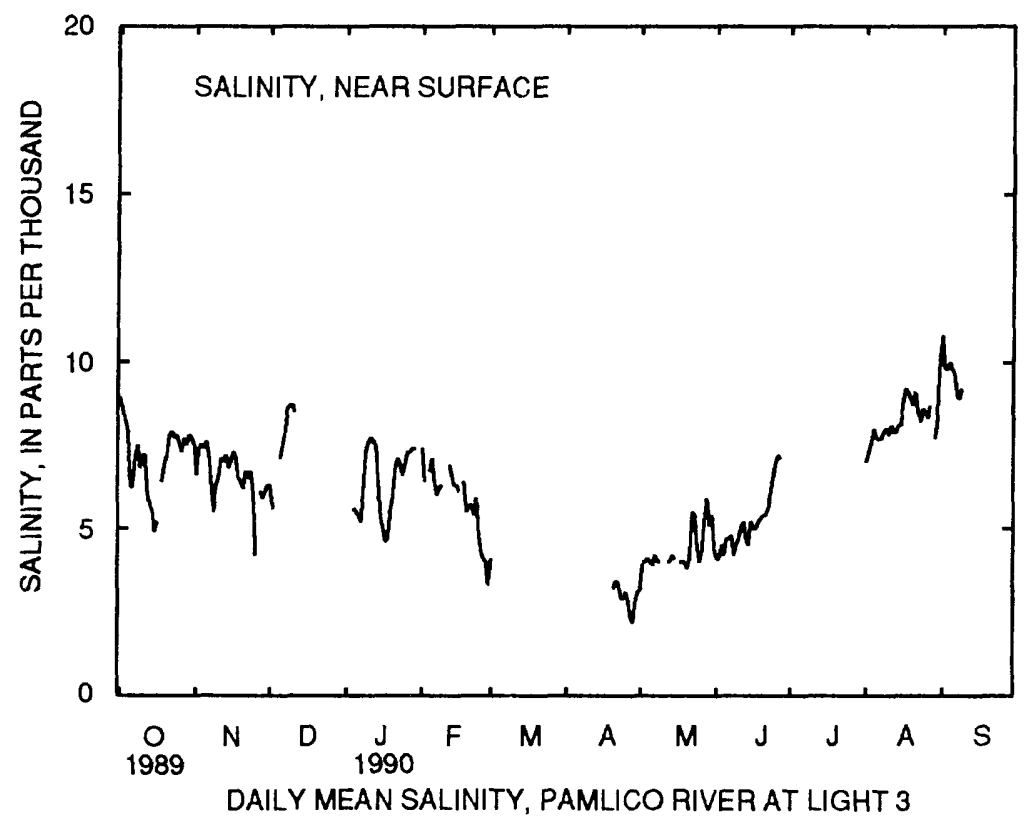


Table 6.--Daily mean values of salinity, water temperature, and dissolved oxygen in water at site 4, Pamlico River at Light 3, May 1989 to september 1990--Continued

[Site 4 is at latitude $35^{\circ} 21^{\prime} 24^{\prime \prime}$, longitude $76^{\circ} 38^{\prime} 48^{\prime \prime}$, Beaufort County; U.S. Geological Survey downstream order number 0208455155 ; ppt, parts per thousand; ---, no data; ${ }^{\circ} \mathrm{C}$, degrees

Celsius; mg/L, milligrams per Ilter; a, dally minimum value is less than or equal to $2.0 \mathrm{mg} / \mathrm{L}$; $b$, daily minimum value is less than or equal to $5.0 \mathrm{mg} / \mathrm{L}$ but greater than $2.0 \mathrm{mg} / \mathrm{L}$; <, less than]

\section{SALINITY, NEAR BOTTOM}

SENSOR POSITION. --2 feet above streambed.

EXTREMES FOR MAY TO SEPTEMBER 1989.--Maximum value recorded, 11.9 ppt September 13, 1989; minimum value recorded, 1.7 ppt May 14, 16, 1989.

DAILY MEAN VALUES OF SALINITY, NEAR BOTTOM (IN PARTS PER THOUSAND), MAY TO SEPTEMBER 1989

\begin{tabular}{|c|c|c|c|c|c|}
\hline DAY & MAY & JUNE & JULY & AUG & SEPT \\
\hline 1 & --- & 5.7 & --- & --- & 9.8 \\
\hline 2 & --- & 5.6 & --- & -- & 9.5 \\
\hline 3 & --- & 5.4 & --- & -- & 9.3 \\
\hline 4 & 5.2 & 5.5 & --- & -- & 9.9 \\
\hline 5 & 6.5 & 5.9 & --- & -- & 9.7 \\
\hline 6 & --- & 6.2 & --- & -- & 9.2 \\
\hline 7 & --- & 6.1 & --- & -- & 9.3 \\
\hline 8 & --- & 5.8 & --- & --- & 9.5 \\
\hline 9 & --- & 5.7 & --- & --- & 9.6 \\
\hline 10 & 2.9 & 5.8 & --- & -- & 9.7 \\
\hline 11 & 2.7 & 5.7 & -- & -- & 9.6 \\
\hline 12 & 2.8 & 5.4 & --- & -- & 9.7 \\
\hline 13 & 2.6 & 5.6 & --- & --- & 10.2 \\
\hline 14 & 2.2 & 5.6 & -- & -- & 9.5 \\
\hline 15 & 3.0 & 5.8 & --- & --- & 9.6 \\
\hline 16 & 2.3 & 5.9 & -- & --- & 9.9 \\
\hline 17 & 3.3 & 5.8 & --- & -- & 9.1 \\
\hline 18 & 4.5 & 5.8 & --- & -- & 9.1 \\
\hline 19 & 5.3 & 5.7 & --- & -- & 9.5 \\
\hline 20 & 6.0 & 5.6 & -- & --- & 9.6 \\
\hline 21 & 6.1 & 5.3 & --- & -- & 9.7 \\
\hline 22 & 6.1 & 4.8 & --- & -- & 10.1 \\
\hline 23 & 5.4 & 4.7 & --- & -- & 9.8 \\
\hline 24 & 5.6 & 4.6 & -- & -- & 10.1 \\
\hline 25 & 5.5 & --- & --- & -- & 10.3 \\
\hline 26 & 5.5 & -- & -- & 9.4 & 9.4 \\
\hline 27 & 5.6 & --- & -- & 9.4 & 9.0 \\
\hline 28 & 6.2 & -- & $-\cdots$ & -- & 9.1 \\
\hline 29 & 6.7 & --- & --- & 9.0 & 9.2 \\
\hline 30 & 6.3 & --- & --- & 8.7 & 9.0 \\
\hline 31 & 5.9 & --- & -- & -- & -- \\
\hline
\end{tabular}

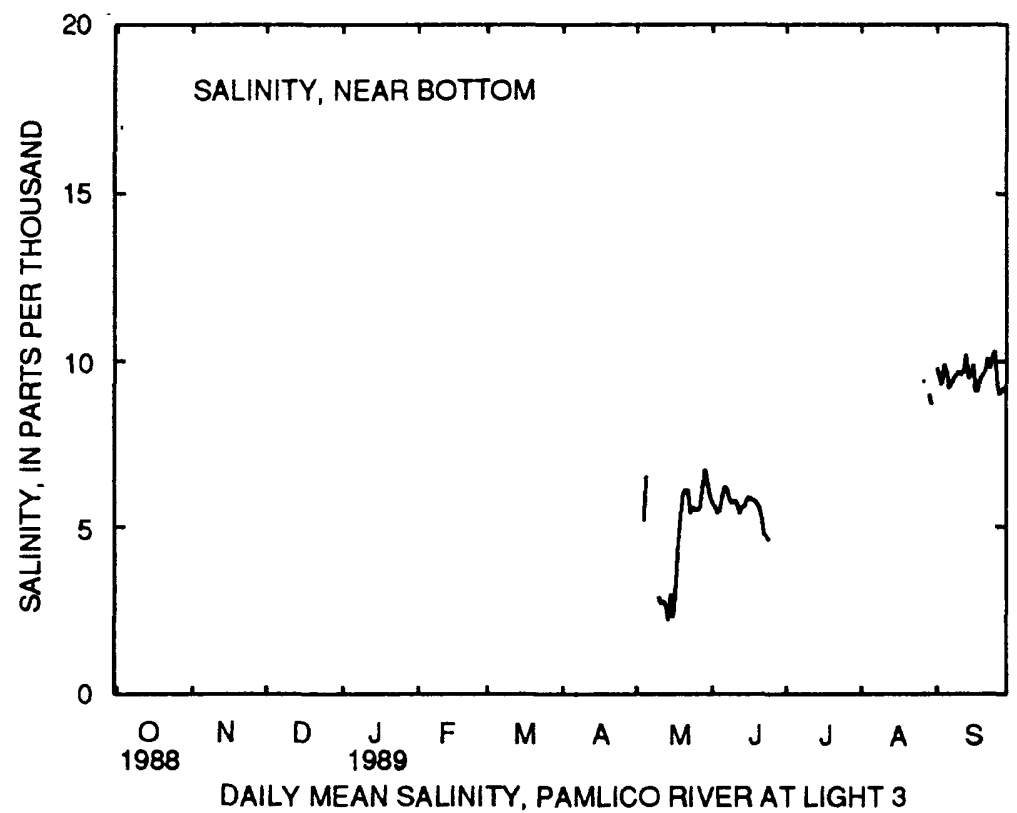


Table 6.--Da1ly mean values of salinity, water temperature, and dissolved oxygen in water at site 4, Pamlico River at Light 3, May 1989 to september 1990--Continued

[Site 4 is at latitude $35^{\circ} 21^{\prime} 24^{\prime \prime}$, longltude $76^{\circ} 38^{\prime} 48^{\prime \prime}$, Beaufort County; U.S. Geological Survey downstream order number 0208455155 ; ppt, parts per thousand; ---, no data; ${ }^{\circ} \mathrm{C}$, degrees

Celslus; mg/L, milligrams per liter; a, dally minimum value is less than or equal to $2.0 \mathrm{mg} / \mathrm{L}$; b, daily minimum value $1 \mathrm{~s}$ less than or equal to $5.0 \mathrm{mg} / \mathrm{L}$ but greater than $2.0 \mathrm{mg} / \mathrm{L}$; <, less thanl

\section{SALINITY, NEAR BOTTOM}

SENSOR POSITION.--2 feet above streambed.

EXTREMES FOR OCTOBER 1989 TO SEPTEMBER 1990.--Maximum value recorded, 13.1 ppt August 26 , 1990 ; minimum value recorded, 2.3 ppt Apr11 24, 26-28, 1990.

DAILY MEAN VALUES OF SALINITY, NEAR BOTTOM (IN PARTS PER THOUSAND), OCTOBER 1989 TO SEPTEMBER 1990

\begin{tabular}{|c|c|c|c|c|c|c|c|c|c|c|c|c|}
\hline DAY & $\mathrm{OCT}$ & NOV & DEC & JAN & FEB & MAR & APR & MAY & JUNE & JULY & AUG & SEPT \\
\hline 1 & 9.4 & 8.2 & 7.0 & --- & 7.1 & 4.5 & -- & 3.5 & 4.5 & --- & 8.0 & $\cdots$ \\
\hline 2 & 9.6 & 8.7 & 6.9 & -- & 6.7 & --- & --- & 4.0 & 4.3 & -- & 8.7 & $-\cdots$ \\
\hline 3 & 9.0 & 8.7 & 7.0 & --- & 6.9 & -- & --- & 4.0 & 4.1 & --- & 9.4 & $\cdots$ \\
\hline 4 & 8.8 & 8.6 & 7.5 & 7.0 & 6.5 & -- & --- & 4.1 & 4.2 & -- & 8.8 & -- \\
\hline 5 & 8.4 & 9.0 & 8.1 & 6.6 & 6.8 & --- & --- & 4.1 & 4.7 & --- & 8.5 & -- \\
\hline 6 & 7.4 & 8.7 & 8.3 & 6.4 & 6.8 & -- & --- & 4.1 & 5.6 & --- & 8.8 & $\cdots$ \\
\hline 7 & 8.1 & 8.2 & 8.5 & 6.0 & 6.4 & -- & --- & 4.2 & 4.7 & -- & 8.3 & $-\cdots$ \\
\hline 8 & 8.5 & 8.8 & 9.1 & 7.2 & 6.5 & --- & -- & 3.9 & 5.0 & $\cdots$ & 9.0 & -- \\
\hline 9 & 7.1 & 9.3 & 9.2 & 7.7 & 6.4 & --- & -- & 4.0 & 4.4 & --- & 9.4 & -- \\
\hline 20 & 7.8 & 8.3 & 9.1 & 7.6 & --- & --- & --- & 4.1 & 4.7 & -- & 8.7 & $\cdots$ \\
\hline 11 & 8.0 & 8.2 & 8.9 & 8.2 & --- & --- & --- & 3.6 & 4.7 & -- & 8.9 & 10.1 \\
\hline 12 & 8.3 & 7.8 & --- & 8.1 & 6.7 & -- & --- & 4.1 & 5.0 & --- & 9.3 & 10.1 \\
\hline 13 & 8.3 & 8.0 & --- & 8.0 & 6.6 & -- & -- & 4.0 & 4.7 & -- & 10.0 & 10.3 \\
\hline 14 & 9.0 & 8.1 & -- & 7.9 & 6.3 & -- & --- & 4.2 & 5.1 & -- & 8.6 & 10.3 \\
\hline 15 & 10.4 & 8.2 & --- & 7.3 & 6.3 & --- & --- & 4.3 & 5.7 & --- & 9.7 & 9.9 \\
\hline 16 & 10.0 & --- & -- & 6.1 & 6.0 & --- & -- & 4.1 & 5.4 & --- & 9.1 & 10.6 \\
\hline 17 & --- & -- & -- & 7.9 & -- & --- & --- & 4.0 & 5.5 & --- & 9.2 & 10.3 \\
\hline 18 & 9.2 & -- & -- & 7.6 & 6.4 & -- & -- & 4.0 & 5.6 & --- & 8.9 & 10.5 \\
\hline 19 & 10.5 & -- & -- & 7.2 & 5.7 & --- & --- & 4.1 & 5.7 & --- & 8.8 & 10.6 \\
\hline 20 & 8.2 & --- & --- & 7.1 & 5.7 & --- & 3.0 & 4.0 & 5.8 & --- & 8.5 & 10.0 \\
\hline 21 & 8.7 & --- & -- & 7.0 & 6.1 & --- & 3.2 & 4.1 & 6.1 & --- & 8.7 & 11.4 \\
\hline 22 & 8.9 & 7.6 & -- & 7.2 & 5.7 & --- & 3.2 & 5.4 & 6.4 & -- & 8.8 & 11.3 \\
\hline 23 & 9.0 & 7.6 & -- & 7.1 & 5.7 & --- & 3.0 & 5.4 & 6.3 & --- & 9.3 & 10.6 \\
\hline 24 & 9.0 & 8.1 & -- & 7.0 & 4.5 & -- & 2.6 & 5.3 & 6.7 & -- & 10.0 & 11.3 \\
\hline 25 & 8.7 & 8.7 & --- & 7.4 & 3.8 & --- & 2.8 & 4.9 & 7.4 & --- & 10.2 & 10.8 \\
\hline 26 & 8.3 & --- & --- & 7.6 & 4.2 & -- & 2.8 & 5.0 & 7.7 & -- & 10.1 & 10.1 \\
\hline 27 & 8.7 & 7.5 & $\cdots$ & 7.9 & 4.6 & -- & 2.9 & 5.1 & 7.7 & --- & 8.7 & 10.7 \\
\hline 28 & 8.6 & 7.5 & --- & 7.7 & 5.5 & --- & 3.3 & 5.9 & --- & --- & --- & 11.2 \\
\hline 29 & 8.8 & 6.6 & -- & 7.6 & -- & -- & 3.0 & 5.2 & -- & --- & --- & 11.2 \\
\hline 30 & 8.7 & 6.7 & -- & -- & --- & -- & 3.2 & 5.1 & --- & -- & -- & 9.9 \\
\hline 31 & 8.6 & $-\cdots$ & --- & --- & --- & --- & --- & 5.3 & -- & -- & --- & -- \\
\hline
\end{tabular}

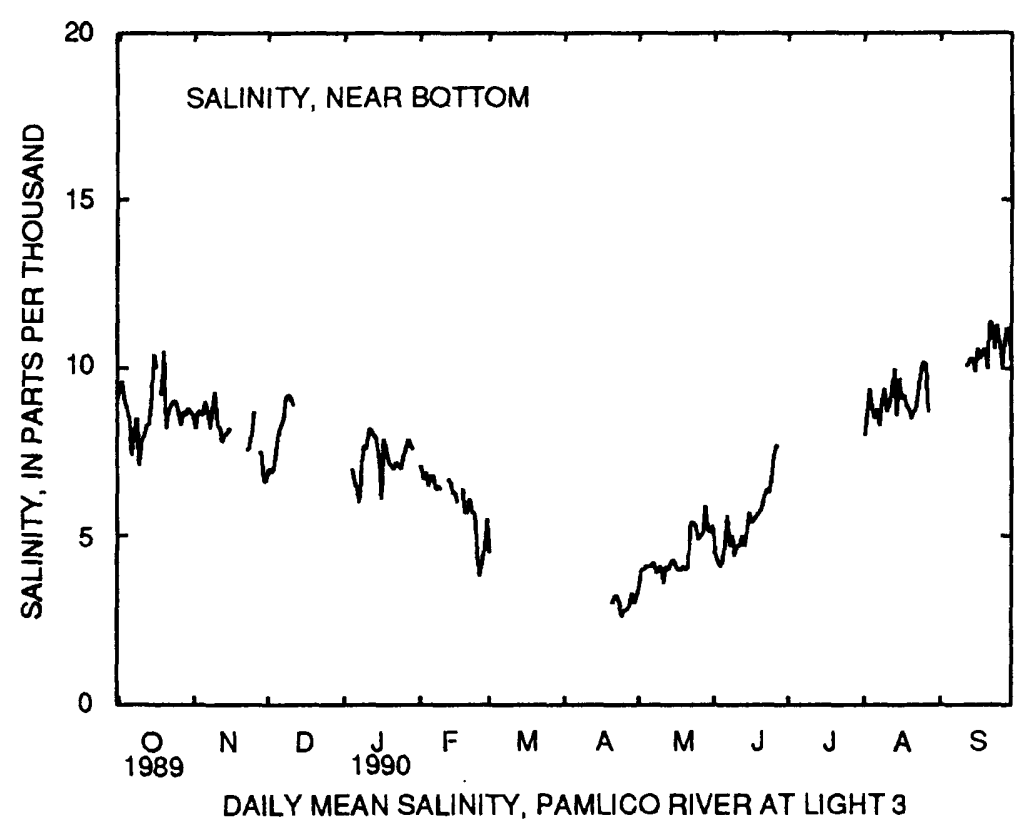


Table 6.--Daily mean values of salinity, water temperature, and dissolved oxygen in water at site 4, Pamlico River at Light 3, May 1989 to september 1990--Continued

[Site 4 is at latitude $35^{\circ} 21^{\prime} 24^{\prime \prime}$, longitude $76^{\circ} 38^{\prime} 48^{\prime \prime}$, Beaufort County; U.S. Geological Survey downstream order number 0208455155 ; ppt, parts per thousand; ---, no data; ${ }^{\circ} \mathrm{C}$, degrees

Celsius; mg/L, milligrams per liter; a, dally minimum value is less than or equal to $2.0 \mathrm{mg} / \mathrm{L}$; b, daily minimum value is less than or equal to $5.0 \mathrm{mg} / \mathrm{L}$ but greater than $2.0 \mathrm{mg} / \mathrm{L} ;<$, less thanl

WATER TEMPERATURE, NEAR SURFACE

SENSOR POSITION. --8 feet above streambed.

EXTREMES FOR MAY TO SEPTEMBER 1989.--Maximum value recorded, $30.6{ }^{\circ} \mathrm{C}$ september 1 , 1989 ; minimum value recorded, $17.1{ }^{\circ} \mathrm{C}$ May $12,13,1989$.

DAILY MEAN VALUES OF WATER TEMPERATURE, NEAR SURFACE (IN DEGREES CELSIUS), MAY TO SEPTEMBER 1989

\begin{tabular}{|c|c|c|c|c|c|}
\hline DAY & MAY & JUNE & JULY & AUG & SEPT \\
\hline 1 & --- & 24.6 & 27.0 & --- & 29.0 \\
\hline 2 & --- & 25.8 & 26.7 & --- & 28.8 \\
\hline 3 & --- & 25.8 & 26.6 & --- & 28.2 \\
\hline 4 & 20.6 & 25.8 & -- & --- & 27.1 \\
\hline 5 & 20.1 & 25.6 & --- & --- & 26.2 \\
\hline 6 & 20.4 & 25.3 & --- & --- & 26.0 \\
\hline 7 & 19.6 & 25.1 & -- & --- & 25.5 \\
\hline 8 & 18.5 & 25.1 & --- & --- & 25.6 \\
\hline 9 & 18.4 & 25.1 & --- & --- & 25.8 \\
\hline 10 & 18.2 & 25.5 & --- & --- & 26.3 \\
\hline 11 & 18.0 & 25.9 & -- & --- & 26.6 \\
\hline 12 & 17.7 & 25.5 & -- & --- & 26.6 \\
\hline 13 & 17.8 & 25.5 & --- & --- & 26.6 \\
\hline 14 & 17.9 & 26.5 & --- & --- & 26.4 \\
\hline 15 & 18.2 & 26.7 & --- & --- & 26.4 \\
\hline 16 & 18.8 & 26.6 & --- & --- & 26.5 \\
\hline 17 & 18.3 & 26.4 & --- & --- & 26.4 \\
\hline 18 & 18.5 & 26.7 & --- & --- & 26.0 \\
\hline 19 & 18.9 & 27.0 & --- & --- & 25.5 \\
\hline 20 & 19.6 & 26.9 & --- & --- & 25.3 \\
\hline 21 & 20.6 & 26.7 & --- & --- & 25.8 \\
\hline 22 & 21.3 & 26.8 & --- & --- & 26.0 \\
\hline 23 & 21.8 & 26.7 & --- & --- & 25.9 \\
\hline 24 & 21.1 & 26.9 & --- & --- & 24.2 \\
\hline 25 & 22.0 & 27.1 & --- & --- & 22.6 \\
\hline 26 & 22.5 & 27.7 & --- & 28.1 & 22.9 \\
\hline 27 & 23.9 & 28.2 & --- & 27.7 & 22.2 \\
\hline 28 & 23.5 & 28.2 & --- & --- & 21.3 \\
\hline 29 & 22.7 & 27.7 & --- & 28.1 & 21.6 \\
\hline 30 & 23.0 & 26.8 & --- & 28.1 & 21.8 \\
\hline 31 & 23.7 & -- & --- & -- & -- \\
\hline
\end{tabular}

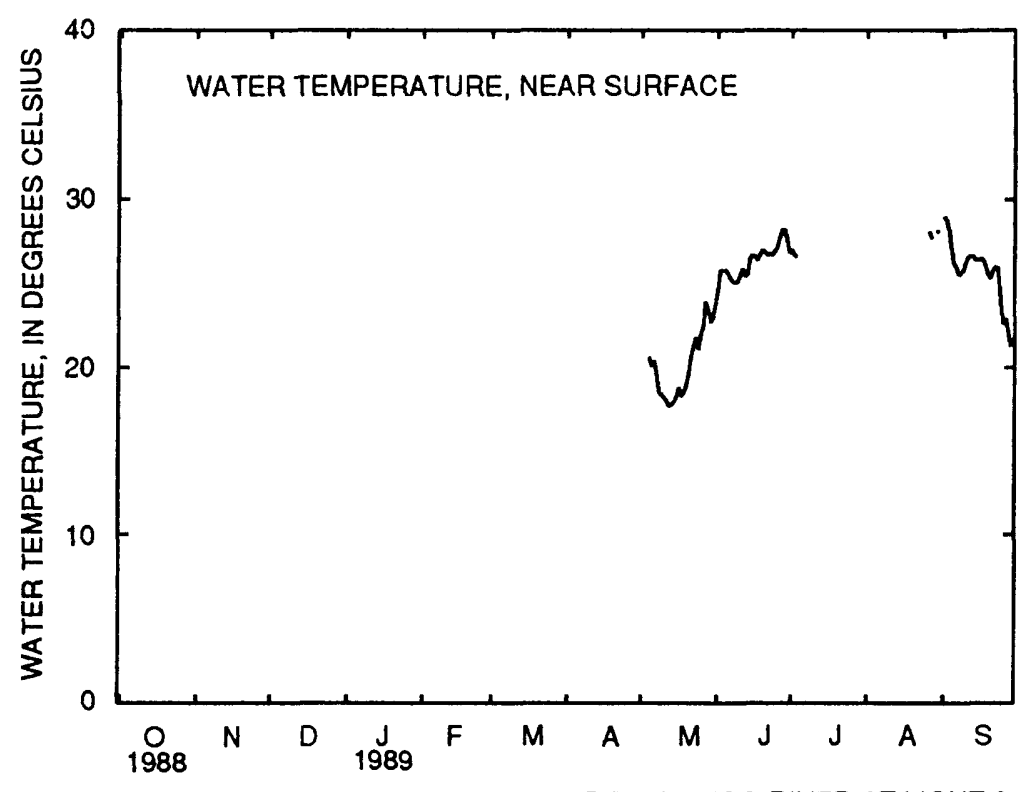


Table 6.--Daily mean values of salinity, water temperature, and dissolved oxygen in water at site 4,

Pamlico River at Light 3, May 1989 to september 1990--Continued

[Site 4 is at latitude $35^{\circ} 21^{\prime} 24^{\prime \prime}$, longitude $76^{\circ} 38^{\prime} 48^{\prime \prime}$, Beaufort County; U.S. Geologlcal Survey downst ream order number 0208455155 ; ppt, parts per thousand; ---, no data; ${ }^{\circ} \mathrm{C}$, degrees

Celsius; mg/L, mllilgrams per liter; a, daily minimum value is less than or equal to $2.0 \mathrm{mg} / \mathrm{L} ; \mathrm{b}$, daily minimum value $1 \mathrm{~s}$ less than or equal to $5.0 \mathrm{mg} / \mathrm{L}$ but greater than $2.0 \mathrm{mg} / \mathrm{L}$; <, less than]

WATER TEMPERATURE, NEAR SURFACE

SENSOR POSITION.--8 feet above streambed.

EXTREMES FOR OCTOBER 1989 TO SEPTEMBER 1990.--Maximum value recorded, $33.3^{\circ} \mathrm{C}$ August 20 , 1990 ; minimum value recorded, $4.1{ }^{\circ} \mathrm{C}$ December 3, 1989.

DAILY MEAN VALUES OF WATER TEMPERATURE, NEAR SUREACE (IN DEGREES CELSIUS), OCTOBER 1989 TO SEPTEMBER 1990

\begin{tabular}{|c|c|c|c|c|c|c|c|c|c|c|c|c|}
\hline DAY & OCT & NoV & DEC & JAN & FEB & MAR & $A P R$ & MAY & JUNE & JULY & AUG & SEPT \\
\hline 1 & 21.3 & 18.3 & 9.2 & --- & 11.5 & 10.3 & -- & 22.5 & 23.5 & --- & 29.6 & 27.0 \\
\hline 2 & 21.6 & 17.9 & 9.5 & --- & 12.5 & 11.0 & $-\infty$ & 22.3 & 24.2 & --- & 29.8 & 25.9 \\
\hline 3 & 22.4 & 17.8 & 8.2 & --- & 13.1 & 12.7 & --- & 22.5 & 24.7 & --- & 29.2 & 25.8 \\
\hline 4 & 22.5 & 17.2 & 6.9 & -- & 13.5 & 12.8 & --- & 22.3 & 25.4 & -- & 29.3 & 26.7 \\
\hline 5 & 21.7 & 16.8 & 7.0 & 6.1 & 13.3 & 11.3 & -- & 22.8 & 24.9 & -- & 28.8 & 26.3 \\
\hline 6 & 21.1 & 17.3 & 7.3 & 6.4 & 12.4 & 12.2 & -- & 22.0 & 24.5 & $-\cdots$ & 28.5 & 26.2 \\
\hline 7 & 21.4 & 18.2 & 7.9 & 6.7 & 12.7 & 11.8 & --- & 21.8 & 24.9 & --- & 28.0 & 26.8 \\
\hline 8 & 20.4 & 18.2 & 7.9 & 6.2 & 12.8 & 11.3 & -- & 22.0 & 26.6 & $-\cdots$ & 28.0 & 26.7 \\
\hline 9 & 19.2 & 18.4 & 7.4 & 5.8 & 13.1 & 11.5 & --- & 21.9 & 26.5 & -- & 27.6 & 26.3 \\
\hline 10 & 18.4 & 17.7 & 6.9 & 5.8 & --- & 12.7 & $-\cdots$ & 21.9 & 26.8 & --- & 27.9 & 27.0 \\
\hline 11 & 18.6 & 17.3 & 6.3 & 6.0 & -- & 14.5 & --- & 21.0 & 26.3 & -- & 28.4 & 26.8 \\
\hline 12 & 18.6 & 17.2 & --- & 6.6 & 13.2 & -- & $-\cdots$ & 21.3 & 25.1 & --- & 29.4 & 26.6 \\
\hline 13 & 19.7 & 17.4 & --- & 5.9 & 12.8 & 16.2 & --- & 21.9 & 24.5 & $\cdots$ & 29.4 & 26.8 \\
\hline 14 & 20.1 & 17.8 & --- & 5.5 & 13.1 & -- & --- & 22.6 & 24.6 & --- & 29.7 & 26.8 \\
\hline 15 & 20.3 & 18.3 & --- & 6.0 & 13.5 & --- & --- & 23.1 & 24.7 & --- & 29.4 & 26.5 \\
\hline 16 & 20.9 & 16.9 & --- & 7.2 & 15.0 & --- & $\ldots$ & 23.5 & 24.8 & $-\cdots$ & 29.6 & 26.5 \\
\hline 17 & --- & 15.5 & --- & 8.0 & --- & $\cdots$ & --- & 23.1 & 25.3 & 28.1 & 29.4 & 26.2 \\
\hline 18 & 21.9 & 14.0 & -- & 9.0 & 14.4 & --- & --- & 23.2 & 25.7 & 28.2 & 29.0 & 24.6 \\
\hline 19 & 22.1 & 12.0 & --- & 9.4 & 13.9 & -+ & 16.5 & 23.4 & 26.3 & 28.3 & 29.3 & 23.6 \\
\hline 20 & 20.7 & 10.7 & $-\cdots$ & 9.4 & 13.1 & -- & 17.0 & 23.2 & 26.7 & 28.3 & 30.8 & 23.7 \\
\hline 21 & 17.9 & 9.5 & -- & 9.8 & 12.7 & -- & 17.5 & 23.6 & 27.3 & 28.6 & 30.5 & 23.4 \\
\hline 22 & 17.0 & 11.5 & --- & 10.0 & 12.8 & $-\cdots$ & 18.0 & 22.9 & 28.2 & 27.5 & 30.6 & 23.5 \\
\hline 23 & 16.9 & 9.9 & $-\cdots$ & 10.0 & 13.8 & --- & 18.9 & 21.7 & 27.6 & --- & 30.4 & 23.5 \\
\hline 24 & 17.0 & 9.9 & -- & 10.6 & 13.2 & --- & 19.3 & 22.0 & 27.4 & -- & 30.1 & 22.6 \\
\hline 25 & 17.0 & 8.5 & --- & 11.1 & 11.3 & -- & 20.0 & 22.1 & 27.4 & --- & 29.5 & 22.0 \\
\hline 26 & 16.8 & -- & -- & 11.2 & 10.3 & --- & 21.4 & 22.6 & 27.1 & --- & 29.2 & 21.8 \\
\hline 27 & 16.9 & 10.9 & $-\cdots$ & 10.3 & 9.7 & --- & 22.9 & 23.0 & 27.2 & --- & 29.0 & 22.1 \\
\hline 28 & 16.9 & 12.3 & --- & 10.4 & 9.9 & -- & 22.5 & 22.5 & --- & --- & --- & 22.8 \\
\hline 29 & 17.2 & 12.0 & $\cdots$ & 10.5 & -- & --- & 22.5 & 22.7 & --- & $-\cdots$ & 29.2 & 22.8 \\
\hline 30 & 17.4 & 10.4 & $\cdots$ & $--\sim$ & --- & --- & 21.9 & 22.7 & --- & --- & 28.7 & 23.0 \\
\hline 31 & 18.0 & --- & --- & --- & --- & $-\cdots$ & --- & 22.9 & --- & -- & 28.0 & -- \\
\hline
\end{tabular}

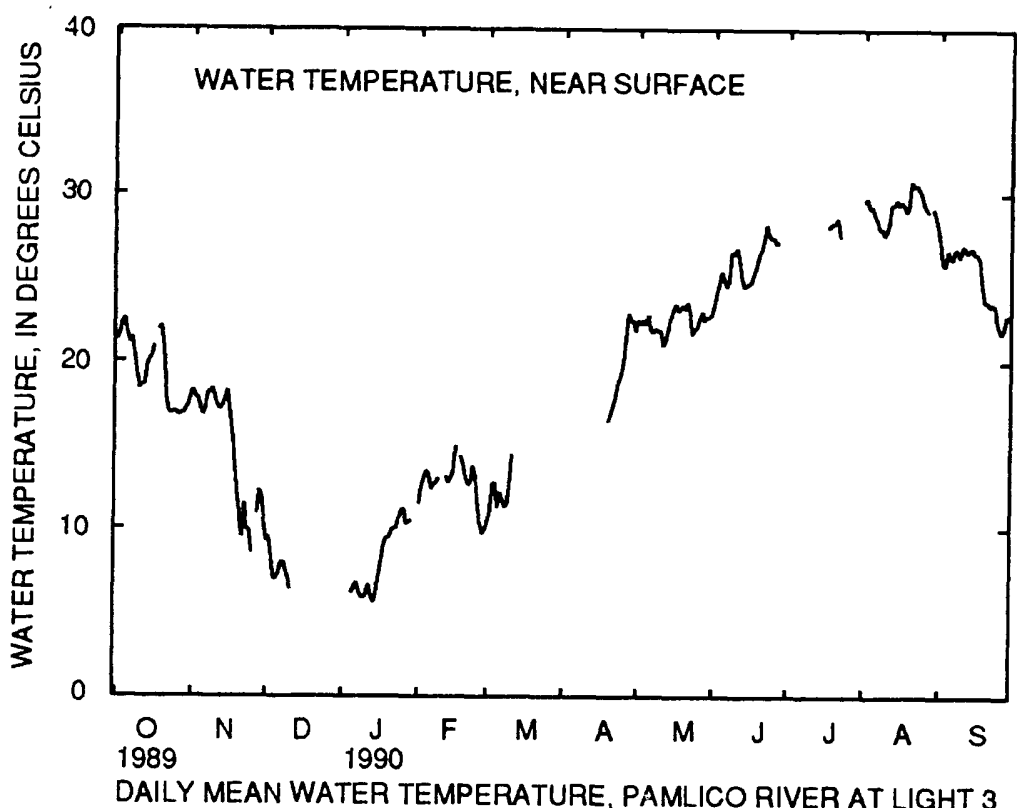


Table 6.--Dally mean values of salinity, water temperature, and dissolved oxygen in water at site 4, Pamlico River at Light 3, May 1989 to september 1990--Continued

[Site 4 is at latitude $35^{\circ} 21^{\prime} 24^{\prime \prime}$, longltude $76^{\circ} 38^{\prime} 48^{\prime \prime}$, Beaufort County; U.S. Geological Survey downst ream order number 0208455155 ; ppt, parts per thousand; - - , no data; ${ }^{\circ} \mathrm{C}$, degrees

Celsius; mg/L, milligrams per liter; a, dally minimum value is less than or equal to $2.0 \mathrm{mg} / \mathrm{L}$; b, daily minimum value is less than or equal to $5.0 \mathrm{mg} / \mathrm{L}$ but greater than $2.0 \mathrm{mg} / \mathrm{L} ;<$, less than

DISSOLVED OXYGEN, NEAR SURFACE

SENSOR POSITION. --8 feet above streambed.

EXTREMES FOR MAY TO SEPTEMBER 1989.--Maximum value recorded, $15.8 \mathrm{mg} / \mathrm{L}$ May 20, 1989 ; minimum value recorded, 4.7 $\mathrm{mg} / \mathrm{L}$ September $18,1989$.

DAILY MEAN VALUES OF DISSOLVED OXYGEN, NEAR SURFACE (IN MILLIGRAMS PER LITER), MAY TO SEPTEMBER 1989

\begin{tabular}{|c|c|c|c|c|c|}
\hline DAY & MAY & JUNE & JULY & AUG & SEPT \\
\hline 1 & -- & 9.7 & 7.4 & --- & -- \\
\hline 2 & $-\cdots$ & 10.2 & 8.0 & $\ldots$ & $-\ldots$ \\
\hline 3 & --- & 10.0 & 7.6 & --- & -- \\
\hline 4 & -- & -- & --- & --- & -- \\
\hline 5 & -- & --- & --- & --- & --- \\
\hline 6 & 8.2 & --- & $\ldots$ & --- & --- \\
\hline 7 & 8.0 & --- & -- & -- & --- \\
\hline 8 & 8.8 & -- & --- & -- & --- \\
\hline 9 & 9.2 & --- & --- & --- & -- \\
\hline 10 & 8.8 & --- & --- & $-\cdots$ & $\cdots$ \\
\hline 11 & 8.7 & --- & --- & -- & -- \\
\hline 12 & 8.7 & --- & --- & $-\sim$ & --- \\
\hline 13 & 9.0 & --- & --- & --- & -- \\
\hline 14 & 9.3 & --- & --- & --- & 9.1 \\
\hline 15 & 9.6 & -- & $\cdots$ & -- & 7.8 \\
\hline 16 & 8.8 & --- & --- & --- & 7.1 \\
\hline 17 & 8.3 & -- & $-\cdots$ & --- & 6.5 \\
\hline 18 & 8.5 & -- & --- & $-\cdots$ & $5.5 b$ \\
\hline 19 & 11.0 & --- & --- & --- & $5.5 b$ \\
\hline 20 & 12.9 & --- & --- & --- & $6.0 \mathrm{~b}$ \\
\hline 21 & 13.1 & --- & --- & --- & $5.6 \mathrm{~b}$ \\
\hline 22 & 12.0 & --- & -- & $\ldots$ & 5.9 \\
\hline 23 & 10.5 & --- & --- & $-\cdots$ & 6.4 \\
\hline 24 & 8.7 & --- & --- & $-\sim$ & 6.8 \\
\hline 25 & 10.1 & -- & $\cdots$ & $\ldots$ & 6.9 \\
\hline 26 & 9.9 & --- & -- & $\ldots$ & 7.1 \\
\hline 27 & 11.4 & 8.4 & --- & --- & 7.0 \\
\hline 28 & 10.5 & 7.6 & --- & -- & 6.8 \\
\hline 29 & 10.2 & 7.3 & --- & --- & 6.9 \\
\hline 30 & 10.3 & 7.1 & -- & -- & 7.3 \\
\hline 31 & 10.5 & -- & -- & -- & --- \\
\hline
\end{tabular}

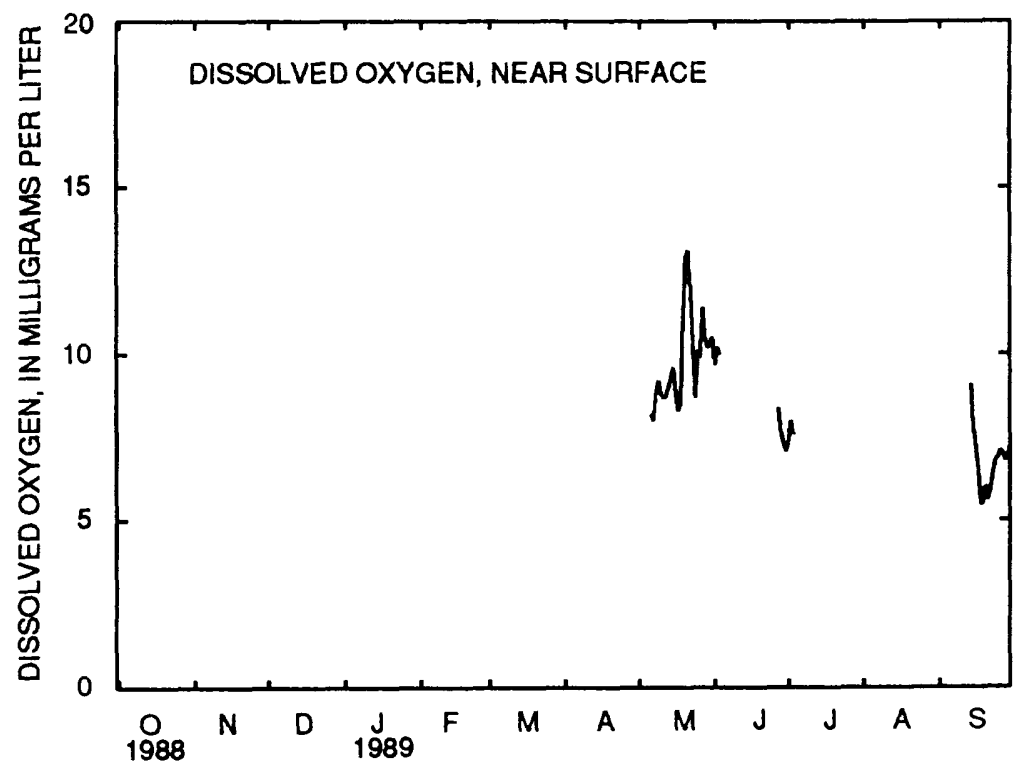

DAILY MEAN DISSOLVED OXYGEN, PAMLICO RIVER AT LIGHT 3 
Table 6.--Daily mean values of salinity, water temperature, and dissolved oxygen in water at site 4, Pamilico River at Light 3, May 1989 to September 1990--Continued

[Site 4 is at latitude $35^{\circ} 21^{\prime} 24^{\prime \prime}$, longitude $76^{\circ} 38^{\prime} 48^{\prime \prime}$, Beaufort County; U.S. Geological Survey downst ream order number 0208455155 ; ppt, parts per thousand; ---, no data; ${ }^{\circ} \mathrm{C}$, degrees

Celsius; $\mathrm{mg} / \mathrm{L}$, milligrams per liter; a, dally minimum value is less than or equal to $2.0 \mathrm{mg} / \mathrm{L}$; b, daily minimum value is less than or equal to $5.0 \mathrm{mg} / \mathrm{L}$ but greater than $2.0 \mathrm{mg} / \mathrm{L}$; $<$, less than]

DISSOLVED OXYGEN, NEAR SURFACE

SENSOR POSITION. --8 feet above streambed.

EXTREMES FOR OCTOBER 1989 TO SEPTEMBER 1990.--Maximum value recorded, $18.7 \mathrm{mg} / \mathrm{L}$ January 24 , $1990 ; \mathrm{minimum}$ value recorded, $1.5 \mathrm{mg} / \mathrm{L}$ October $14,1989$.

DAILY MEAN VALUES OF DISSOLVED OXYGEN, NEAR SURFACE (IN MILLIGRAMS PER LITER), OCTOBER 1989 TO SEPTEMBER 1990

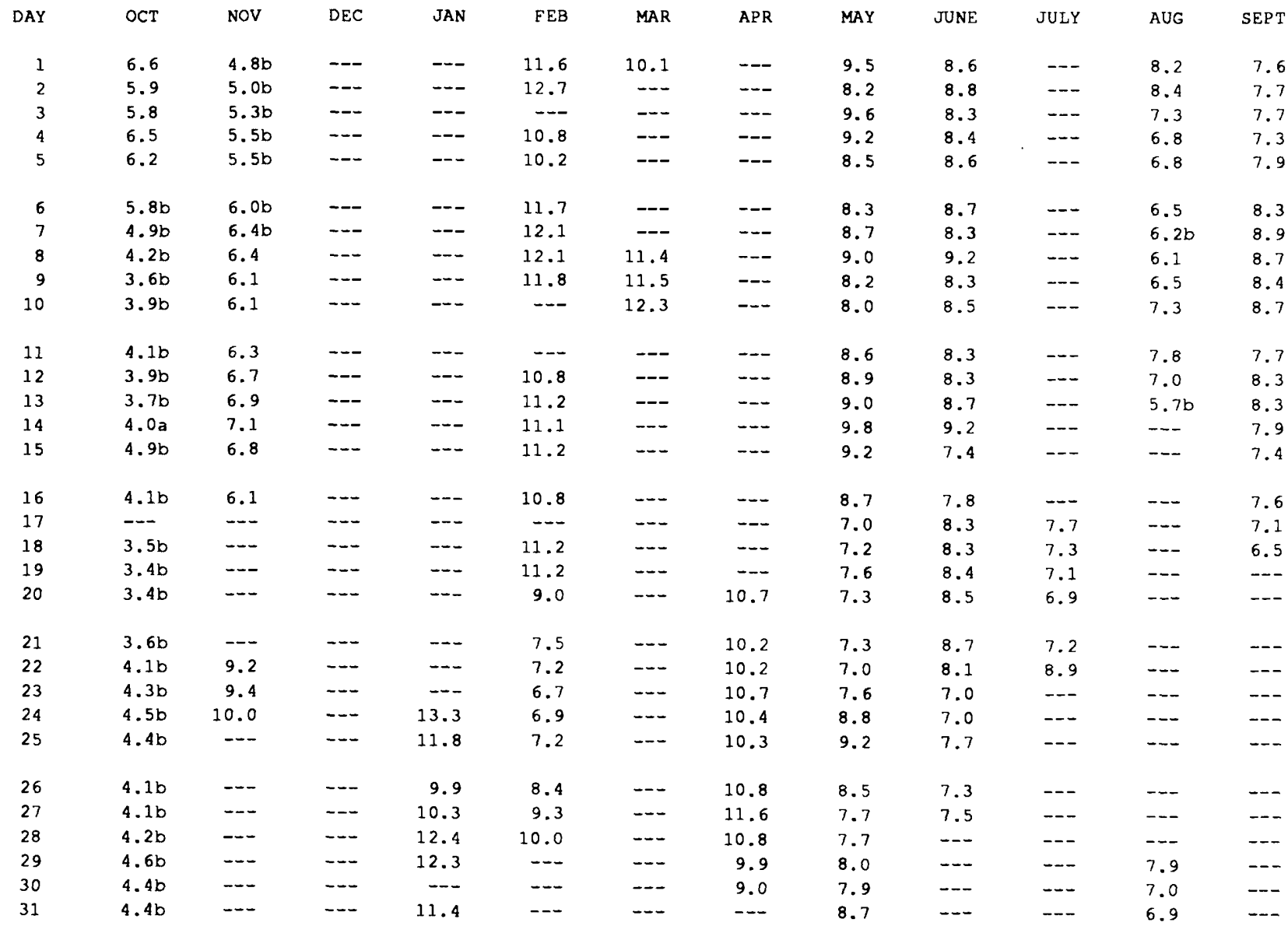

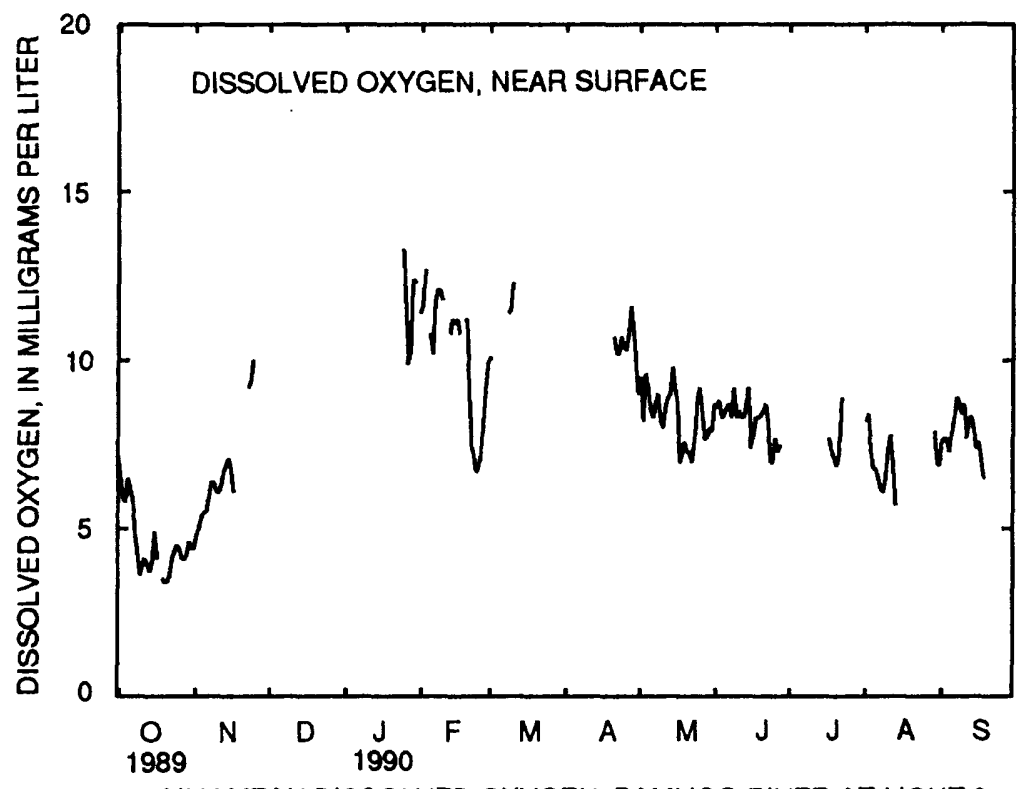

DAILY MEAN DISSOLVED OXYGEN, PAMLICO RIVER AT LIGHT 3 
Table 6.--Daily mean values of salinity, water temperature, and dissolved oxygen in water at site 4, Pamlico River at Light 3, May 1989 to September 1990--Continued

[Site 4 is at latitude $35^{\circ} 21^{\prime} 24^{\prime \prime}$, long1tude $76^{\circ} 38^{\prime} 48^{\prime \prime}$, Beaufort County; U.S. Geological Survey downst ream order number 0208455155; ppt, parts per thousand; ---, no data; ${ }^{\circ} \mathrm{C}$, degrees

Celsius; mg/L, mllligrams per liter; a, dally minimum value is less than or equal to $2.0 \mathrm{mg} / \mathrm{L}$; b, da1ly minimum value is less than or equal to $5.0 \mathrm{mg} / \mathrm{L}$ but greater than $2.0 \mathrm{mg} / \mathrm{L}$; <, less than]

DISSOLVED OXYGEN, MID-DEPTH

SENSOR POSITION, --6 feet above streambed.

EXTREMES FOR MAY TO SEPTEMBER 1989.--MaXimum value recorded, $15.6 \mathrm{mg} / \mathrm{L}$ May 20,$1989 ; \mathrm{minimum}$ value recorded, <1.0 mg/L September $16,1989$.

DAILY MEAN VALUES OF DISSOLVED OXYGEN, MID-DEPTH (IN MILLIGRAMS PER LITER), MAY TO SEPTEMBER 1989

\begin{tabular}{|c|c|c|c|c|c|}
\hline DAY & MAY & JUNE & JULY & AUG & SEPT \\
\hline 1 & --- & $5.5 a$ & -- & --- & 7.5 \\
\hline 2 & --- & $5.8 \mathrm{a}$ & -- & --- & 7.3 \\
\hline 3 & --- & $6.2 \mathrm{a}$ & --- & --- & 6.8 \\
\hline 4 & -- & 7.1 & --- & --- & 6.8 \\
\hline 5 & --- & --- & --- & --- & 7.0 \\
\hline 6 & 7.0 & -- & --- & --- & 7.2 \\
\hline 7 & 7.7 & --- & -- & --- & 7.7 \\
\hline 8 & 8.3 & -- & -- & --- & 7.9 \\
\hline 9 & 8.4 & --- & --- & --- & 7.9 \\
\hline 10 & 8.0 & -- & -- & --- & 7.3 \\
\hline 11 & 8.3 & --- & -- & -- & 7.0 \\
\hline 12 & 7.7 & -- & --- & --- & $6.5 b$ \\
\hline 13 & $7.5 b$ & --- & -- & -- & $6.8 b$ \\
\hline 14 & $7.3 \mathrm{~b}$ & --- & --- & -- & 6.6 \\
\hline 15 & $6.1 \mathrm{~b}$ & --- & --- & -- & 6.6 \\
\hline 16 & $7.6 a$ & --- & -- & -- & $6.8 \mathrm{a}$ \\
\hline 17 & 8.2 & -- & --- & --- & 7.3 \\
\hline 18 & 8.4 & --- & --- & -- & 6.8 \\
\hline 19 & 11.1 & --- & --- & --- & 6.7 \\
\hline 20 & 11.7 & --- & --- & --- & 7.1 \\
\hline 21 & 9.9 & --- & --- & --- & 7.0 \\
\hline 22 & 8.7 & --- & --- & --- & 7.1 \\
\hline 23 & 9.8 & --- & --- & --- & 7.1 \\
\hline 24 & 8.5 & --- & --- & --- & 7.4 \\
\hline 25 & 8.6 & $\cdots$ & --- & 7.1 & 7.2 \\
\hline 26 & 9.3 & --- & -- & 7.1 & 7.4 \\
\hline 27 & 10.0 & --- & -- & 7.1 & 7.4 \\
\hline 28 & 9.1 & --- & --- & 7.3 & 7.3 \\
\hline 29 & 9.0 & --- & --- & 7.7 & 6.8 \\
\hline 30 & 8.2 & --- & -- & 8.1 & 7.8 \\
\hline 31 & 7.4 & --- & --- & 7.5 & --- \\
\hline
\end{tabular}

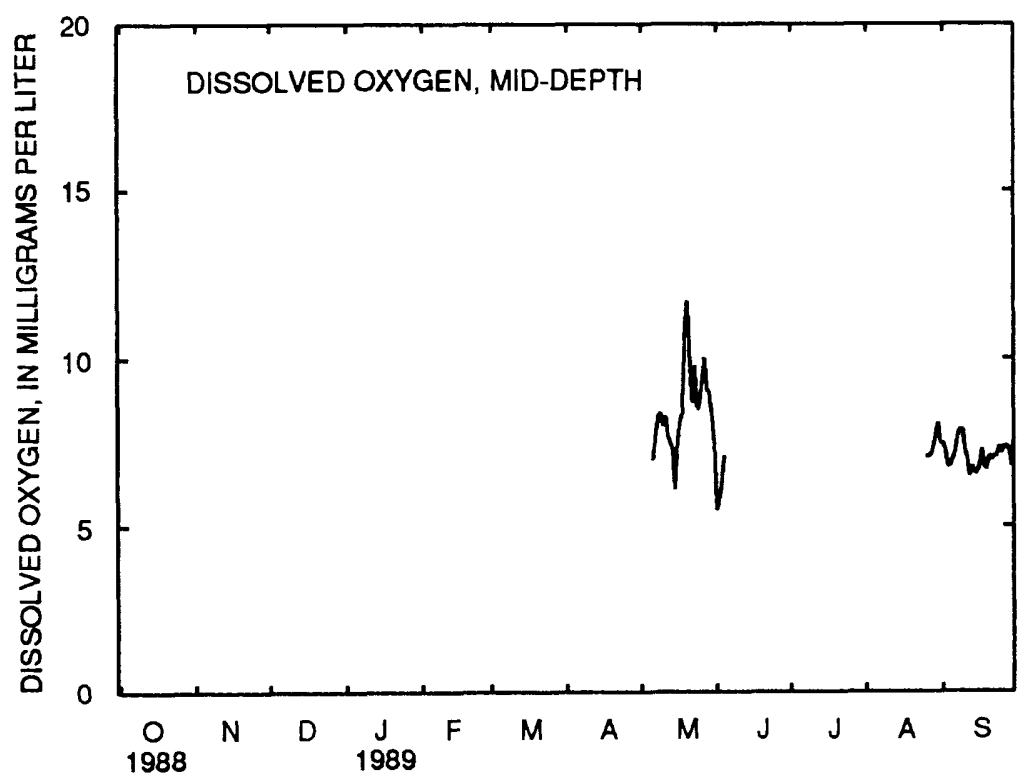

DAILY MEAN DISSOLVED OXYGEN, PAMLICO RIVER AT LIGHT 3 
Table 6.--Daily mean values of salinity, water temperature, and dissolved oxygen in water at site 4, Pamlico River at Light 3, May 1989 to september 1990--Continued

[Site 4 is at latitude $35^{\circ} 21^{\prime} 24^{\prime \prime}$, Iongitude $76^{\circ} 38^{\prime} 48^{\prime \prime}$, Beaufort County; U.S. Geological Survey downstream order number 0208455155 ; ppt, parts per thousand; --., no data; ${ }^{\circ} \mathrm{C}$, degrees

Celsius; mg/L, milligrams per liter; a, daily minimum value is less than or equal to $2.0 \mathrm{mg} / \mathrm{L}$; b, daily minimum value is less than or equal to $5.0 \mathrm{mg} / \mathrm{L}$ but greater $=11 \mathrm{n}^{2.0} \mathrm{mg} / \mathrm{L}$; <, less than)

DISSOLVED OXYGEN, MID-DEPTH

SENSOR POSITION.--6 feet above streambed.

EXTREMES FOR OCTOBER 1989 TO SEPTEMBER 1990.--Maximum value recorded, 15.8 mg/L January 28, $1990 ; \mathrm{minimum} \mathrm{value}$ recorded, <1.0 October 16, 18, 19, 1989.

DAILY MEAN VALUES OF DISSOLVED OXYGEN, MID-DEPTH (IN MILLIGRAMS PER LITER), OCTOBER 1989 TO SEPTEMBER 1990

\begin{tabular}{|c|c|c|c|c|c|c|c|c|c|c|c|c|}
\hline DAY & OCT & NOV & DEC & JAN & FEB & MAR & APR & MAY & JUNE & JULY & AUG & SEPT \\
\hline 1 & 6.8 & 6.5 & 9.1 & --- & 11.6 & 11.4 & --- & 9.0 & 8.6 & --- & 7.7 & 7.9 \\
\hline 2 & $6.1 b$ & 7.1 & 8.1 & --- & 12.8 & --- & --- & 8.1 & 9.1 & -- & 8.1 & 7.8 \\
\hline 3 & $6.7 b$ & 7.4 & 11.6 & $-\cdots$ & --- & --- & -- & 8.7 & 8.6 & --- & 6.9 & 7.9 \\
\hline 4 & 7.6 & 7.2 & 11.5 & $-\cdots$ & 10.8 & -- & --- & 9.2 & 8.8 & --- & 6.3 & 7.6 \\
\hline 5 & 7.4 & 7.1 & 11.1 & --- & 10.0 & --- & --- & 8.4 & 8.5 & --- & 6.5 & 8.3 \\
\hline 6 & 7.1 & 7.6 & 10.2 & --- & 11.3 & --- & --- & 8.2 & 7.9 & -- & 6.3 & 8.5 \\
\hline 7 & $6.3 b$ & 7.1 & 11.2 & --- & 11.9 & 11.0 & -- & 8.5 & 7.8 & -- & 5.9 & 8.6 \\
\hline 8 & $5.5 b$ & 6.4 & 11.9 & -- & 11.5 & 11.2 & --- & 9.0 & 8.2 & -- & $5.9 b$ & 9.0 \\
\hline 9 & $5.7 b$ & 6.6 & 11.8 & --- & 11.3 & 11.0 & -- & 8.2 & 7.6 & --- & $6.0 \mathrm{~b}$ & 8.8 \\
\hline 10 & $5.3 b$ & 7.2 & 11.7 & --- & --- & 11.5 & --- & 7.8 & 7.7 & -- & 6.8 & 8.4 \\
\hline 11 & $6.1 \mathrm{~b}$ & 7.3 & 10.8 & --- & -- & -- & -- & 8.2 & 7.6 & -- & 7.8 & 7.2 \\
\hline 12 & $5.7 \mathrm{~b}$ & 8.1 & -- & --- & 10.1 & --- & --- & 8.5 & 7.5 & --- & 6.9 & 8.0 \\
\hline 13 & $5.2 b$ & 8.5 & $-\cdots$ & --- & 10.3 & -- & --- & 8.3 & 7.8 & --- & $5.8 \mathrm{~b}$ & 8.1 \\
\hline 14 & $3.6 a$ & 8.2 & -- & -- & 10.1 & -- & --- & 8.9 & 8.1 & --- & -- & 7.9 \\
\hline 15 & $2.3 a$ & $7.3 b$ & --- & --- & 10.2 & -- & --- & 8.8 & 6.8 & --- & -- & 7.4 \\
\hline 16 & $2.2 a$ & $7.0 \mathrm{~b}$ & -- & --- & 9.8 & -- & -- & 9.1 & 7.2 & -- & --- & 7.7 \\
\hline 17 & -- & 7.8 & --- & --- & -- & -- & -- & 8.6 & 7.3 & 8.1 & --- & 7.8 \\
\hline 18 & $1.8 \mathrm{a}$ & 8.5 & --- & --- & 9.9 & -- & --- & 8.2 & 7.6 & 8.0 & --- & 7.7 \\
\hline 19 & $2.3 a$ & 8.9 & --- & -- & 9.9 & -- & -- & 7.7 & 7.8 & 7.3 & -- & 8.4 \\
\hline 20 & $4.8 a$ & 9.7 & --- & --- & 9.5 & --- & -- & 7.6 & 8.0 & 7.2 & $-\infty$ & 7.9 \\
\hline 21 & $5.6 b$ & 9.0 & --- & -- & 9.8 & --- & 12.2 & 7.5 & 8.2 & 7.6 & -- & 7.3 \\
\hline 22 & 6.4 & 9.1 & --- & --- & 9.8 & --- & 13.3 & 7.2 & 8.1 & 8.6 & --- & 6.6 \\
\hline 23 & 6.5 & 9.4 & -- & --- & 9.2 & --- & 13.1 & 7.7 & 7.6 & --- & -- & 6.7 \\
\hline 24 & 6.6 & 9.5 & -- & 13.2 & 9.2 & --- & -- & 8.6 & 8.1 & -- & --- & 6.6 \\
\hline 25 & 6.7 & 8.7 & --- & 12.4 & 9.4 & --- & --- & 9.2 & 8.7 & --- & --- & $6.8 b$ \\
\hline 26 & 6.5 & -- & -- & 10.4 & 10.1 & --- & 11.4 & 8.7 & 8.0 & $-\cdots$ & -- & 6.5 \\
\hline 27 & 6.5 & 9.6 & --- & 10.4 & 10.7 & --- & 11.5 & 7.8 & 8.0 & -- & --- & 6.3 \\
\hline 28 & 6.7 & 10.2 & --- & 12.3 & 11.6 & --- & 10.8 & 7.9 & --- & -- & --- & 6.6 \\
\hline 29 & 7.3 & 10.5 & --- & 12.5 & --- & --- & 10.0 & 8.4 & $-\cdots$ & -- & 8.0 & 6.0 \\
\hline 30 & 7.0 & 10.6 & --- & --- & --- & --- & 8.9 & 8.2 & --- & --- & 7.1 & $5.6 b$ \\
\hline 31 & 6.9 & $-\cdots$ & -- & 11.7 & --- & --- & --- & 8.4 & -- & -- & 7.3 & --- \\
\hline
\end{tabular}

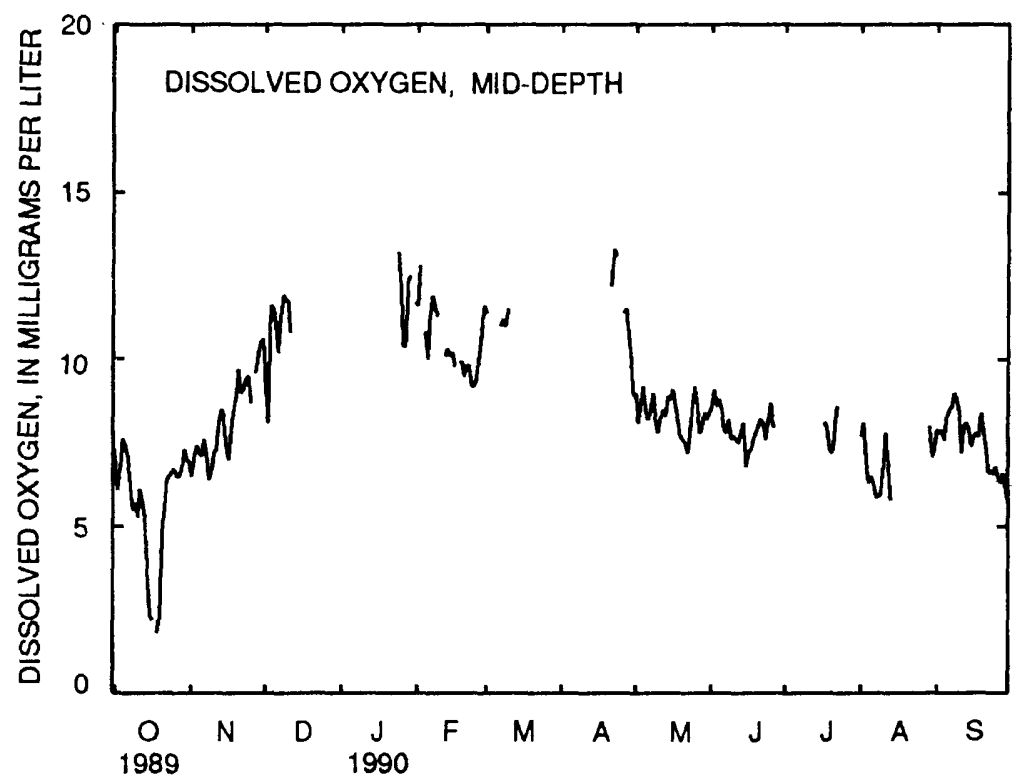

DAILY MEAN DISSOLVED OXYGEN, PAMLICO RIVER AT LIGHT 3 
Table 6.--Daily mean values of salinity, water temperature, and dissolved oxygen in water at site 4, Pamlico River at Light 3, May 1989 to september 1990--Continued

[Site 4 is at latitude $35^{\circ} 21^{\prime} 24^{\prime \prime}$, longltude $76^{\circ} 38^{\prime} 48^{\prime \prime}$, Beaufort County; U.S. Geological Survey downstream order number 0208455155 ; ppt, parts per thousand; ---, no data; ${ }^{\circ} \mathrm{C}$, degrees

Celsius; mg/L, milligrams per liter; a, dally minimum value is less than or equal to $2.0 \mathrm{mg} / \mathrm{L} ; \mathrm{b}$, daily minimum value is less than or equal to $5.0 \mathrm{mg} / \mathrm{L}$ but greater than $2.0 \mathrm{mg} / \mathrm{L}$; <, less than)

DISSOLVED OXYGEN, NEAR BOTTOM

SENSOR POSITION.--2 feet above streambed.

EXTREMES FOR MAY TO SEPTEMBER 1989.--Maximum value recorded, 14.1 mg/L May 20, 1989; minimum value recorded, <1.0 mg/L August 25, 26, September 13, 15, 16, 1989.

DAILY MEAN VALUES OF DISSOLVED OXYGEN, NEAR BOTTOM (IN MILLIGRAMS PER LITER), MAY TO SEPTEMBER 1989

\begin{tabular}{|c|c|c|c|c|c|}
\hline DAY & MAY & JUNE & JULY & AUG & SEPT \\
\hline 1 & --- & $7.4 b$ & --- & --- & $6.1 \mathrm{~b}$ \\
\hline 2 & --- & $7.6 b$ & --- & --- & $5.7 \mathrm{~b}$ \\
\hline 3 & --- & $8.2 b$ & --- & -- & $6.1 b$ \\
\hline 4 & --- & $7.6 \mathrm{~b}$ & --- & --- & 6.4 \\
\hline 5 & --- & $6.9 \mathrm{~b}$ & -- & -- & 6.7 \\
\hline 6 & 6.8 & 8.9 & --- & --- & 6.8 \\
\hline 7 & 7.3 & -- & --- & --- & 7.5 \\
\hline 8 & 7.9 & --- & --- & --- & 7.4 \\
\hline 9 & 8.1 & --- & -- & --- & 7.6 \\
\hline 10 & 8.0 & --- & --- & --- & $7.0 \mathrm{~b}$ \\
\hline 11 & 7.9 & --- & --- & --- & $6.7 \mathrm{~b}$ \\
\hline 12 & 7.8 & --- & --- & --- & $5.9 b$ \\
\hline 13 & 7.7 & --- & --- & --- & $3.8 \mathrm{a}$ \\
\hline 14 & 7.8 & --- & -- & --- & $6.0 \mathrm{a}$ \\
\hline 15 & $6.5 b$ & -- & -- & --- & $6.1 \mathrm{a}$ \\
\hline 16 & $7.7 \mathrm{~b}$ & --- & --- & --- & $4.8 \mathrm{a}$ \\
\hline 17 & 7.7 & --- & --- & --- & $5.3 a$ \\
\hline 18 & 8.5 & --- & --- & --- & $6.3 b$ \\
\hline 19 & 11.1 & --- & --- & --- & 6.9 \\
\hline 20 & 11.4 & --- & -- & -- & 7.1 \\
\hline 21 & 10.2 & --- & -- & -- & 7.3 \\
\hline 22 & 9.0 & -- & --- & --- & 7.9 \\
\hline 23 & 10.4 & --- & --- & -- & 7.4 \\
\hline 24 & 9.1 & --- & -- & --- & 7.9 \\
\hline 25 & 9.9 & --- & --- & $2.9 a$ & 7.8 \\
\hline 26 & 10.4 & -- & --- & $3.9 \mathrm{a}$ & 7.5 \\
\hline 27 & 11.5 & --- & -- & $4.8 a$ & 8.1 \\
\hline 28 & 10.8 & --- & --- & $4.8 b$ & 7.6 \\
\hline 29 & 10.8 & --- & -- & $5.3 b$ & $6.6 \mathrm{~b}$ \\
\hline 30 & 10.1 & -- & --- & $5.9 b$ & 7.9 \\
\hline 31 & 9.6 & --- & --- & $5.7 b$ & --- \\
\hline
\end{tabular}

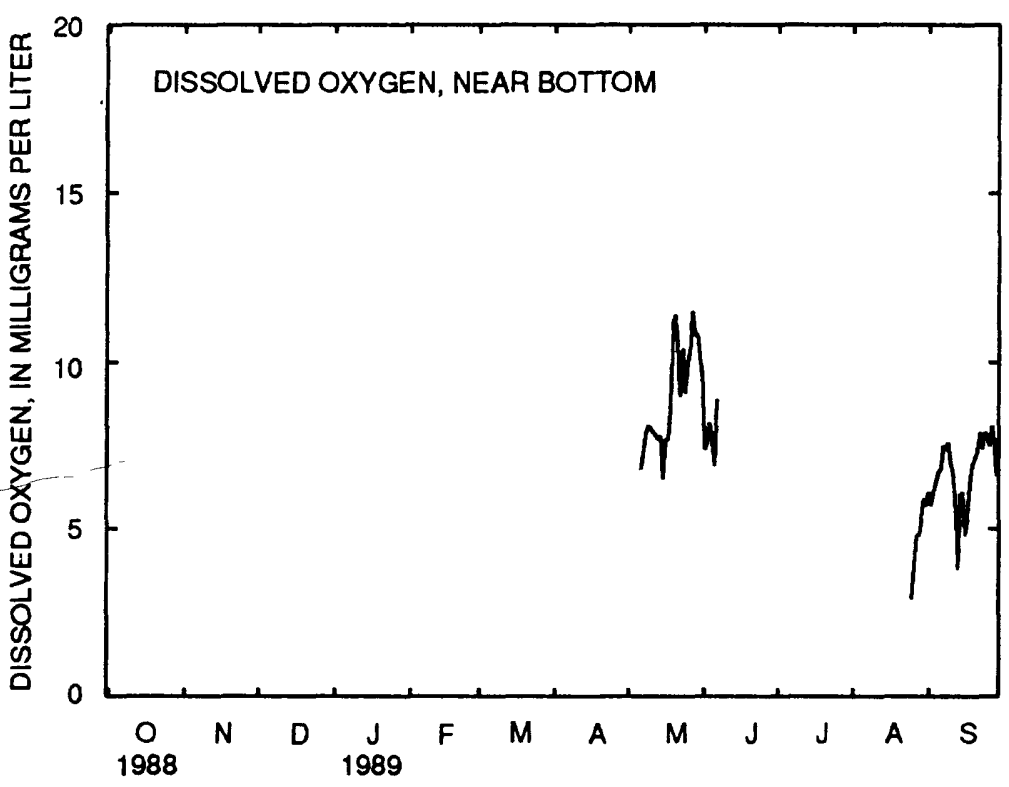


Table 6.--Dafly mean values of salinity, water temperature, and dissolved oxygen in water at site 4, Pamlico River at Light 3, May 1989 to September 1990--Continued

[Site 4 is at latitude $35^{\circ} 21^{\prime} 24^{\prime \prime}$, longitude $76^{\circ} 38^{\prime} 48^{\prime \prime}$, Beaufort County; U.S. Geological Survey downstream order number 0208455155; ppt, parts per thousand; ---, no data; ${ }^{\circ} \mathrm{C}$, degrees

Celsius; mg/L, milligrams per liter; a, daily minimum value is less than or equal to $2.0 \mathrm{mg} / \mathrm{L}$; b, daily minimum value is less than or equal to $5.0 \mathrm{mg} / \mathrm{L}$ but greater than $2.0 \mathrm{mg} / \mathrm{L}$; <, less thanl

DISSOLVED OXYGEN, NEAR BOTTOM

SENSOR POSITION. --2 feet above streambed.

EXTREMES FOR OCTOBER 1989 TO SEPTEMBER 1990.--Maximum value recorded, $13.3 \mathrm{mg} / \mathrm{L}$ February 27 , 1990 ; minimum value recorded, $1.0 \mathrm{mg} / \mathrm{L}$ July $21,1990$.

DAILY MEAN VALUES OF DISSOLVED OXYGEN, NEAR BOTTOM (IN MILLIGRAMS PER LITER), OCTOBER 1989 TO SEPTEMBER 1990

\begin{tabular}{|c|c|c|c|c|c|c|c|c|c|c|c|c|}
\hline DAY & $\mathrm{OCT}$ & NOV & DEC & JAN & FEB & MAR & APR & MAY & JUNE & JULY & AUG & SEPT \\
\hline 1 & --- & -- & --- & --- & --- & --- & --- & 8.4 & --- & -- & 6.8 & 8.0 \\
\hline 2 & -- & --- & 10.6 & --- & 11.5 & --- & -- & 7.9 & --- & --- & $6.8 b$ & 7.6 \\
\hline 3 & --- & --- & 10.1 & --- & 10.5 & --- & --- & 8.2 & --- & --- & $5.2 b$ & 7.7 \\
\hline 4 & --- & --- & 10.4 & --- & 10.6 & --- & --- & 8.9 & --- & --- & $5.0 \mathrm{~b}$ & 7.5 \\
\hline 5 & -- & --- & 10.2 & --- & 10.3 & --- & --- & 8.2 & 8.3 & --- & $5.3 b$ & 7.9 \\
\hline 6 & --- & --- & 10.3 & --- & 9.8 & --- & --- & 8.0 & 7.4 & --- & $5.0 \mathrm{~b}$ & 8.2 \\
\hline 7 & --- & --- & 10.6 & --- & 10.2 & --- & --- & 8.3 & 7.8 & --- & $4.6 b$ & 8.1 \\
\hline 8 & --- & -- & 10.7 & --- & 9.9 & --- & --- & 8.7 & 8.3 & --- & $4.3 a$ & 8.5 \\
\hline 9 & --- & --- & 10.6 & --- & $9.8 \mathrm{~b}$ & --- & --- & 7.9 & 7.9 & --- & $3.9 b$ & 8.0 \\
\hline 10 & --- & -- & 10.9 & --- & --- & --- & --- & 7.5 & 8.0 & $\cdots$ & $4.8 b$ & $7.1 \mathrm{~b}$ \\
\hline 11 & --- & --- & 11.2 & --- & -- & --- & --- & 7.9 & 8.3 & $-\cdots$ & $5.4 \mathrm{~b}$ & $4.0 \mathrm{~b}$ \\
\hline 12 & --- & --- & --- & --- & 9.8 & --- & --- & 8.2 & 8.2 & --- & --- & $3.1 \mathrm{~b}$ \\
\hline 13 & --- & --- & --- & --- & 9.4 & --- & --- & 8.2 & 8.3 & --- & -- & --- \\
\hline 14 & --- & --- & --- & --- & 9.3 & --- & --- & 8.8 & $8.1 b$ & $-\sim$ & --- & --- \\
\hline 15 & --- & --- & --- & --- & 9.1 & --- & --- & 8.8 & $6.6 b$ & $-\cdots$ & --- & $\cdots$ \\
\hline 16 & --- & --- & --- & --- & 8.8 & --- & --- & 9.1 & 7.0 & -- & --- & --- \\
\hline 17 & --- & --- & --- & --- & --- & --- & --- & 8.1 & 7.2 & $3.1 \mathrm{a}$ & --- & $-\infty$ \\
\hline 18 & --- & --- & --- & --- & 9.1 & --- & --- & 7.9 & 7.5 & $3.9 b$ & --- & --- \\
\hline 19 & --- & --- & --- & --- & 9.1 & --- & --- & 7.7 & 8.1 & $3.3 a$ & --- & $\cdots$ \\
\hline 20 & --- & --- & --- & --- & 9.8 & --- & --- & 7.8 & $7.8 \mathrm{~b}$ & $3.7 \mathrm{~b}$ & $-\cdots$ & -- \\
\hline 21 & --- & --- & --- & --- & 10.3 & --- & --- & 7.8 & --- & $3.2 \mathrm{a}$ & --- & -- \\
\hline 22 & --- & --- & --- & --- & 10.9 & --- & --- & 7.7 & --- & $3.0 \mathrm{a}$ & --- & --- \\
\hline 23 & --- & --- & --- & -- & 10.1 & --- & --- & 8.3 & -- & --- & --- & -- \\
\hline 24 & --- & --- & --- & -- & 11.1 & --- & --- & 9.1 & -- & $-\cdots$ & --- & --- \\
\hline 25 & --- & --- & --- & --- & 11.7 & --- & -- & 9.7 & --- & --- & --- & --- \\
\hline 26 & --- & --- & --- & --- & 11.8 & --- & --- & --- & -- & --- & $-\cdots$ & -- \\
\hline 27 & --- & --- & --- & --- & 11.3 & --- & --- & -- & --- & -- & --- & --- \\
\hline 28 & --- & --- & --- & --- & 11.3 & --- & 10.4 & --- & --- & --- & --- & -- \\
\hline 29 & --- & --- & --- & --- & --- & --- & 10.0 & --- & --- & --- & --- & -- \\
\hline 30 & --- & --- & --- & --- & --- & --- & 8.7 & --- & -- & $\cdots$ & 7.2 & --- \\
\hline 31 & --- & --- & --- & --- & --- & --- & --- & -- & --- & --- & 7.2 & --- \\
\hline
\end{tabular}

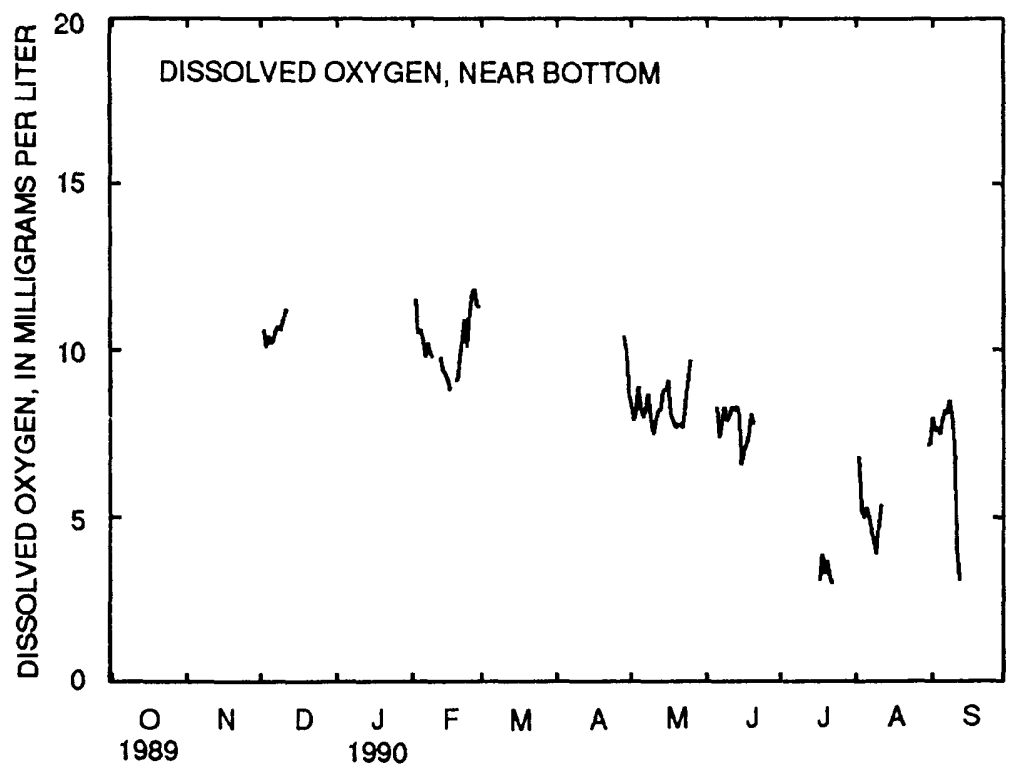

DAILY MEAN DISSOLVED OXYGEN, PAMLICO RIVER AT LIGHT 3 
Table 7.--Daily mean values of salinity, water temperature, and dissolved oxygen in water at site 5, Goose Creek at Light 6, May 1989 to september 1990

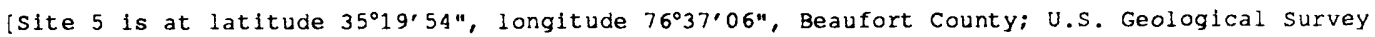
downstream order number 0208455615 ; ppt, parts per thousand; ---, no data; ${ }^{\circ} \mathrm{C}$, degrees Celsius; mg/L, milligrams per liter; a, daily minimum value is less than or equal to $2.0 \mathrm{mg} / \mathrm{L}$; b. daily minimum value is less than or equal to $5.0 \mathrm{mg} / \mathrm{L}$ but greater than $2.0 \mathrm{mg} / \mathrm{L}$; <, less than]

SALINITY, NEAR SURFACE

SENSOR POSITION. -9 feet above streambed.

EXTREMES FOR MAY TO SEPTEMBER 1989.--Maximum value recorded, 11.3 ppt September 17, 1989; minimum value recorded, 4.4 ppt June $27,1989$.

DAILy MEAN VALUES OF SALINITY, NEAR SURFACE (IN PARTS PER THOUSAND), MAY TO SEPTEMBER 1989

\begin{tabular}{|c|c|c|c|c|c|}
\hline DAY & MAY & JUNE & JULY & AUG & SEPT \\
\hline 1 & -- & -- & 6.0 & 7.5 & 9.8 \\
\hline 2 & -- & --- & 6.6 & --- & 9.9 \\
\hline 3 & -- & --- & 6.7 & 7.5 & 9.8 \\
\hline 4 & -- & --- & 6.9 & 7.6 & 9.8 \\
\hline 5 & -- & --- & 7.0 & 7.3 & 10.2 \\
\hline 6 & -- & --- & 7.6 & 6.7 & 10.5 \\
\hline 7 & -- & --- & 7.6 & 6.5 & 10.7 \\
\hline 8 & -- & --- & 7.3 & 6.6 & 10.8 \\
\hline 9 & -- & 7.5 & 6.9 & 6.6 & 10.7 \\
\hline 10 & 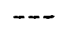 & 7.2 & 7.3 & 7.2 & 10.8 \\
\hline 11 & $-\cdots$ & 7.0 & 7.1 & 7.0 & 10.8 \\
\hline 12 & --- & 7.0 & 6.5 & 6.5 & 10.9 \\
\hline 13 & --- & 7.3 & 6.6 & 6.5 & 10.9 \\
\hline 14 & --- & 7.3 & 6.8 & 6.6 & 11.0 \\
\hline 15 & --- & 7.4 & 6.7 & 6.9 & 11.0 \\
\hline 16 & -- & 8.1 & 7.4 & 6.9 & 11.1 \\
\hline 17 & --- & 8.1 & 7.8 & 7.0 & 11.1 \\
\hline 18 & --- & 7.8 & 7.8 & 7.2 & 10.8 \\
\hline 19 & -- & 7.6 & 7.6 & 7.4 & -- \\
\hline 20 & --- & 7.5 & 7.9 & 7.6 & -- \\
\hline 21 & --- & 7.6 & 8.0 & 8.0 & -- \\
\hline 22 & --- & 7.4 & 8.1 & 8.6 & --- \\
\hline 23 & -- & 6.8 & 8.1 & 8.7 & --- \\
\hline 24 & --- & 6.0 & 7.4 & --- & -- \\
\hline 25 & --- & 5.9 & 7.3 & 8.9 & --- \\
\hline 26 & -- & 6.1 & 7.4 & 9.2 & --- \\
\hline 27 & --- & 5.5 & 7.5 & 9.3 & -- \\
\hline 28 & --- & 5.5 & 7.2 & 9.6 & --- \\
\hline 29 & --- & 5.4 & 7.1 & 9.7 & -- \\
\hline 30 & --- & 5.8 & 7.1 & 9.8 & -- \\
\hline 31 & -- & $\cdots$ & 7.4 & 9.9 & -- \\
\hline
\end{tabular}

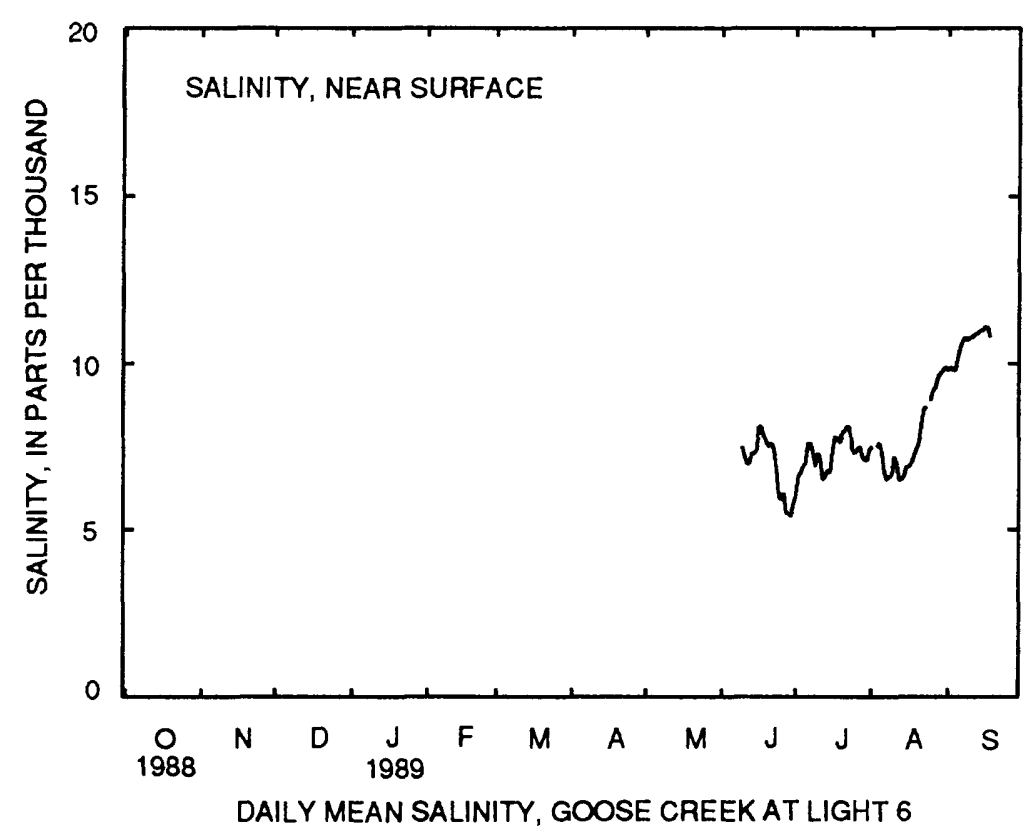


Table 7.--Daily mean values of salinity, water temperature, and dissolved oxygen in water at site 5, Goose Creek at Light 6, May 1989 to September 1990--Continued

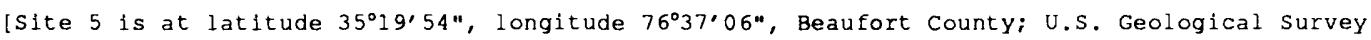
downstream order number 0208455615; ppt, parts per thousand; ---, no data; ${ }^{\circ} \mathrm{C}$, degrees Celsius; mg/L, milligrams per liter; a, daily minlmum value is less than or equal to $2.0 \mathrm{mg} / \mathrm{L}$; b, daily minimum value is less than or equal to $5.0 \mathrm{mg} / \mathrm{L}$ but greater than $2.0 \mathrm{mg} / \mathrm{L}$; <, less thanl

SALINITY, NEAR SURFACE

SENSOR POSITION. --9 feet above streambed.

EXTREMES FOR OCTOBER 1989 TO SEPTEMBER 1990.--Maximum value recorded, 14.4 ppt August 6, $1990 ;$ minimum value recorded, 2.1 ppt March 13, 1990.

DAILY MEAN VALUES OF SALINITY, NEAR SURFACE (IN PARTS PER THOUSAND), OCTOBER 1989 TO SEPTEMBER 1990

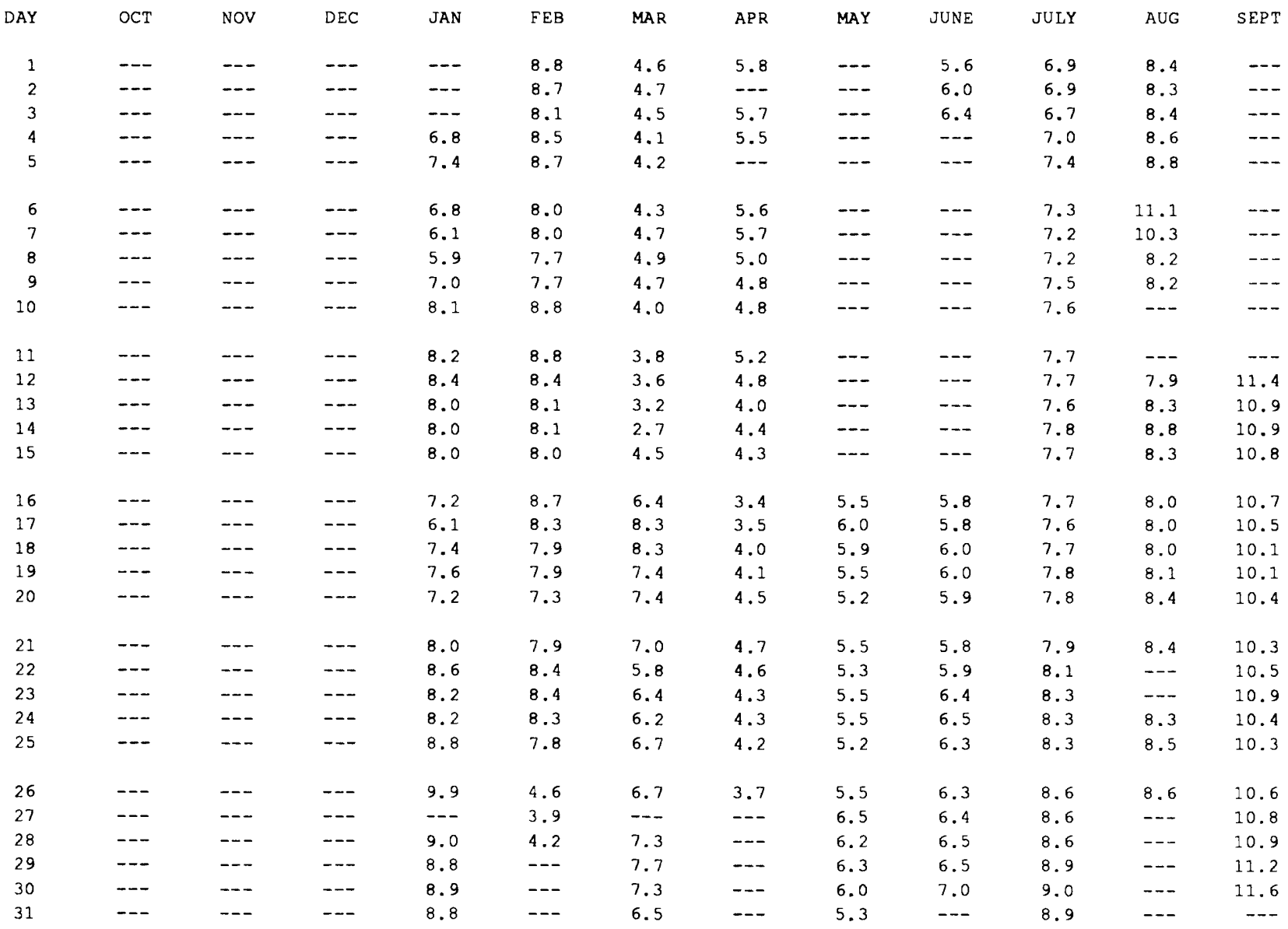

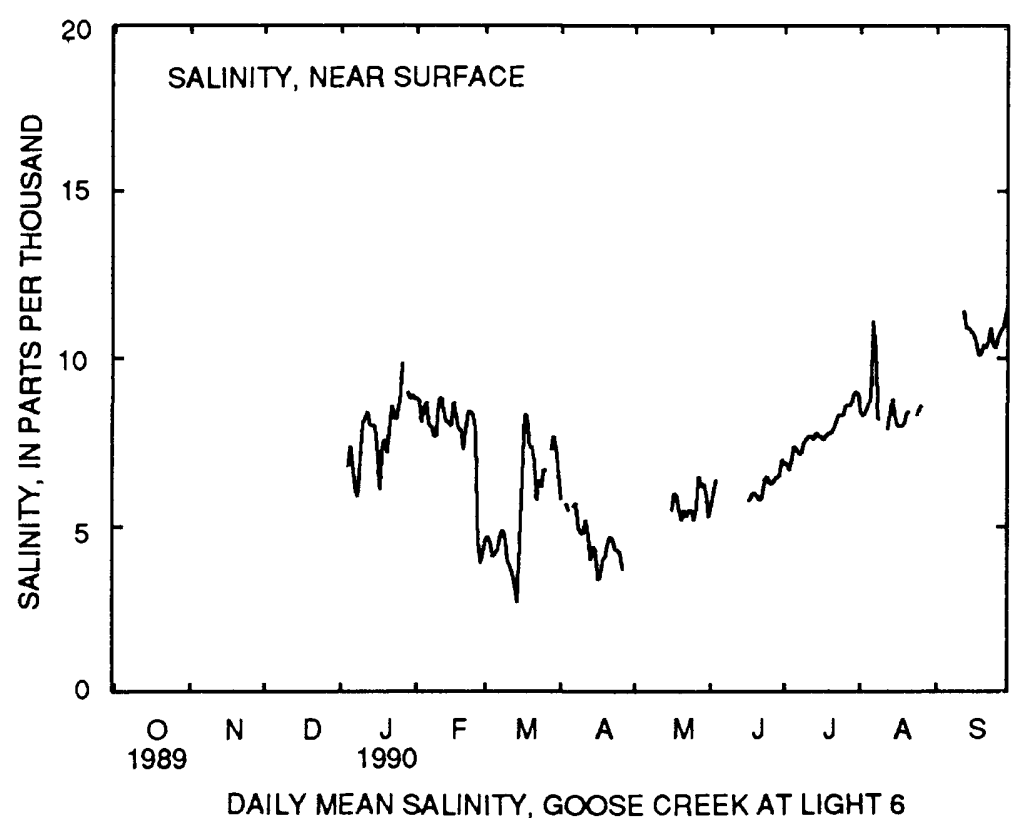


Table 7.--Daily mean values of salinity, water temperature, and dissolved oxygen in water at site 5, Goose Creek at Light 6, May 1989 to September 1990--Cont inued

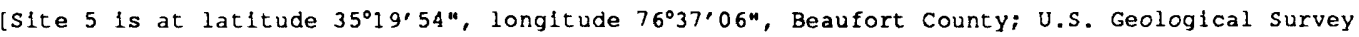
downstream order number 0208455615 ; ppt, parts per thousand; - --, no data; ${ }^{\circ} \mathrm{C}$, degrees Celsius; mg/L, milligrams per liter; a, dally minimum value ls less than or equal to $2.0 \mathrm{mg} / \mathrm{L}$; b, dally minimum value $1 \mathrm{~s}$ less than or equal to $5.0 \mathrm{mg} / \mathrm{L}$ but greater than $2.0 \mathrm{mg} / \mathrm{L}$; <, less thanl

SALINITY, NEAR BOTTOM

SENSOR POSITION. -2 feet above streambed.

EXTREMES FOR MAY TO SEPTEMBER 1989.--Max1mum value recorded, 13.8 ppt August 22, 1989; m1nimum value recorded, 2.9 ppt June 14, 1989.

DAILY MEAN VALUES OF SALINITY, NEAR BOTTOM (IN PARTS PER THOUSAND), MAY TO SEPTEMBER 1989

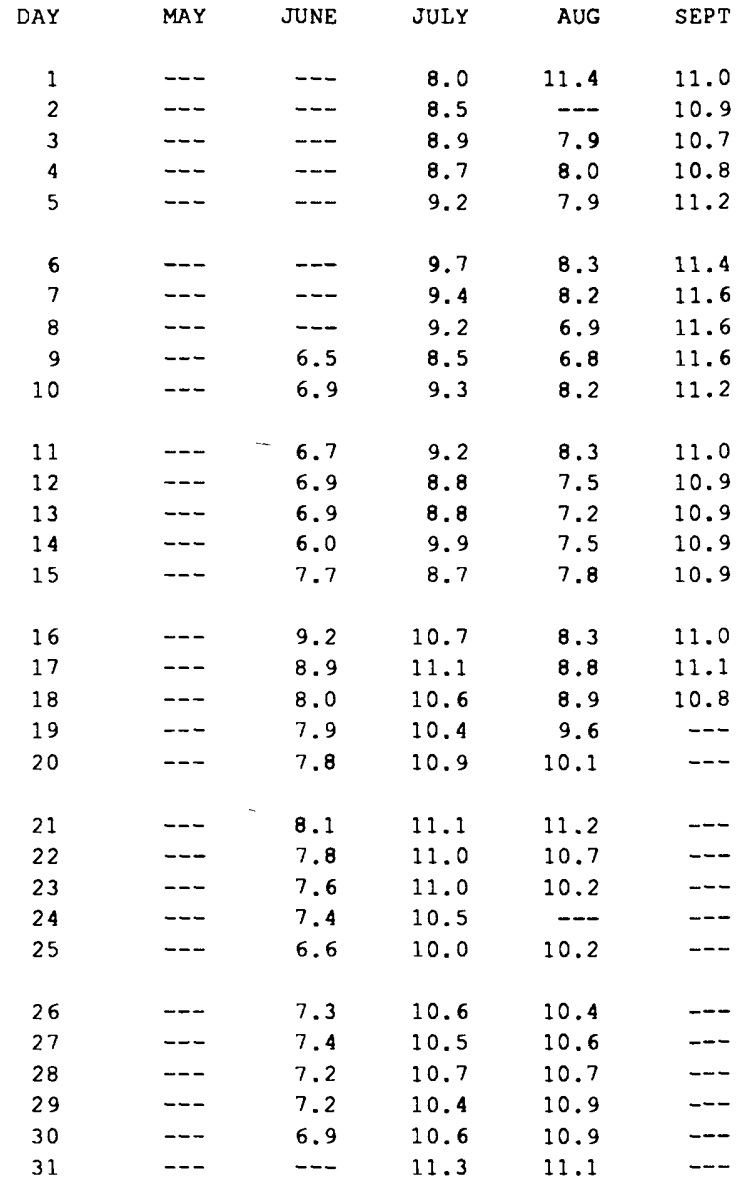

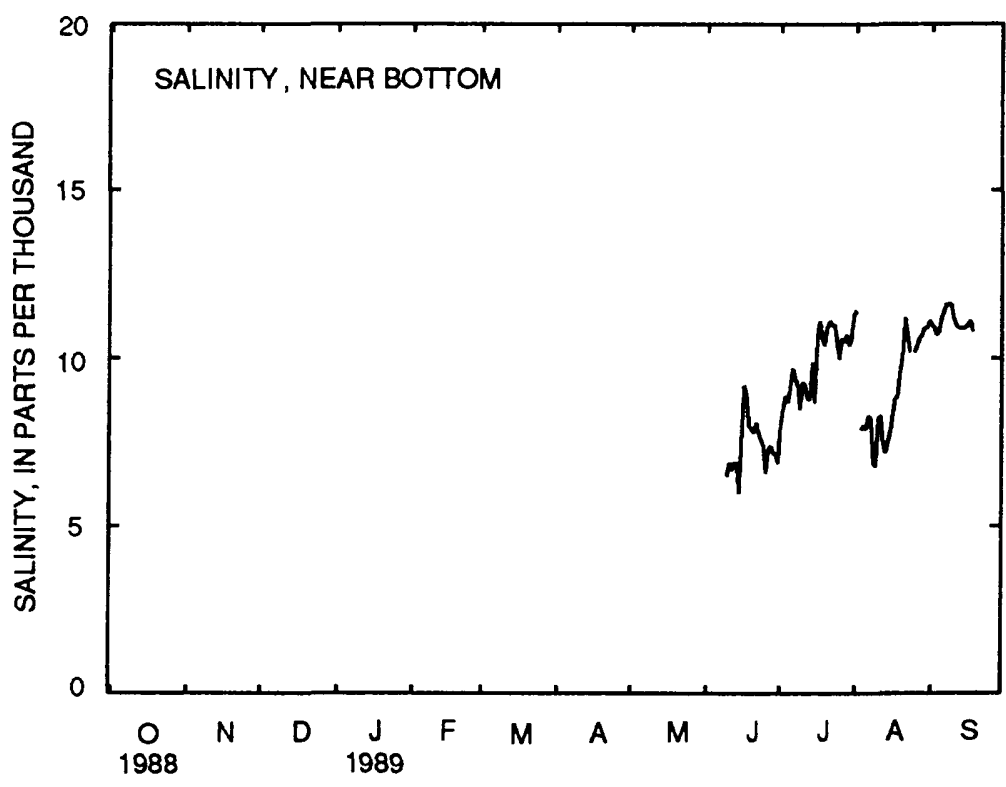


Table 7.--Daily mean values of salinity, water temperature, and dissolved oxygen in water at site 5, Goose Creek at Light 6, May 1989 to September 1990--Cont Inued

[Site 5 is at latitude $35^{\circ} 19^{\prime} 54^{\prime \prime}$, longitude $76^{\circ} 37^{\prime} 06^{\prime \prime}$, Beaufort County; U.S. Geological Survey downst ream order number 0208455615 ; ppt, parts per thousand; ---, no data; ${ }^{\circ} \mathrm{C}$, degrees

Celsius; mg/L, milligrams per liter; a, dally minimum value is less than or equal to $2.0 \mathrm{mg} / \mathrm{L}$; b, daily minimum value $1 \mathrm{~s}$ less than or equal to $5.0 \mathrm{mg} / \mathrm{L}$ but greater than $2.0 \mathrm{mg} / \mathrm{L}$; $<$, less thanl

SALINITY, NEAR BOTTOM

SENSOR POSITION. --2 feet above streambed.

EXTREMES FOR OCTOBER 1989 TO SEPTEMBER 1990.--Maximum value recorded, 50.7 ppt July 11, $1990 ;$ minimum value recorded, 3.7 ppt Apr11 19, 1990.

DAILY MEAN VALUES OF SALINITY, NEAR BOTTOM (IN PARTS PER THOUSAND), OCTOBER 1989 TO SEPTEMBER 1990

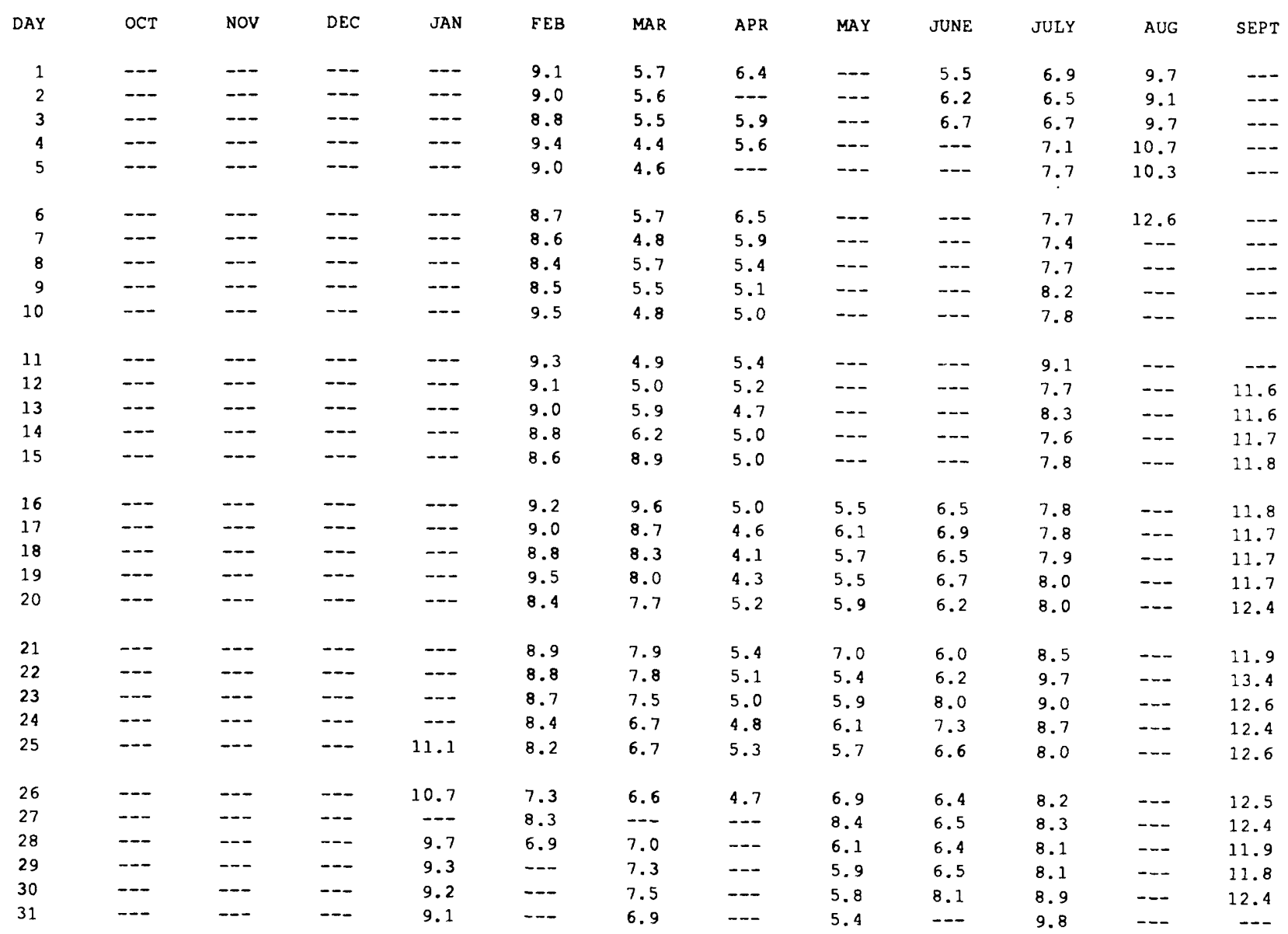

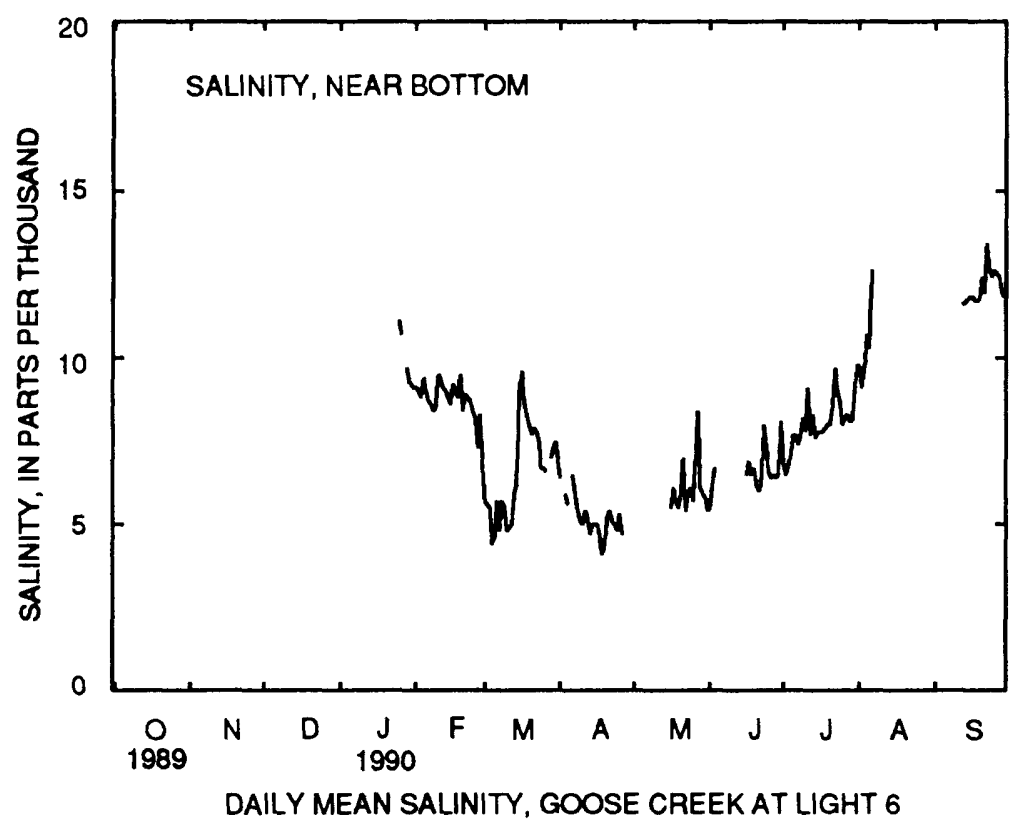


Table 7.--Daily mean values of salinity, water temperature, and dissolved oxygen in water at site 5, Goose Creek at Light 6, May 1989 to September 1990--Cont inued

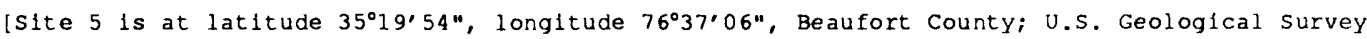
downstream order number 0208455615; ppt, parts per thousand; ---, no data; ${ }^{\circ} \mathrm{C}$, degrees

Celsius; mg/L, milligrams per liter; a, dally minimum value is less than or equal to $2.0 \mathrm{mg} / \mathrm{L} ; \mathrm{b}$, daily minimum value is less than or equal to $5.0 \mathrm{mg} / \mathrm{L}$ but greater than $2.0 \mathrm{mg} / \mathrm{L} ;<$, less thanl

WATER TEMPERATURE, NEAR SURFACE

SENSOR POSITION. --9 feet above streambed.

EXTREMES FOR MAY TO SEPTEMBER 1989.--Maximum recorded, $31.7{ }^{\circ} \mathrm{C}$ July 12,$1989 ; \mathrm{minimum} \mathrm{recorded,} 19.5{ }^{\circ} \mathrm{C}$ MaY 13 , 1989.

DAILY MEAN VALUES OF WATER TEMPERATURE, NEAR SURFACE (IN DEGREES CELSIUS), MAY TO SEPTEMBER 1989

\begin{tabular}{|c|c|c|c|c|c|}
\hline DAY & MAY & JUNE & JULY & AUG & SEPT \\
\hline 1 & -- & 25.9 & 25.2 & 27.5 & 28.3 \\
\hline 2 & - & 27.2 & 25.6 & -- & -- \\
\hline 3 & $\cdots$ & 27.3 & 26.0 & 27.0 & -- \\
\hline 4 & $\ldots$ & 26.4 & 25.7 & 27.1 & 26.1 \\
\hline 5 & -- & 25.8 & 25.7 & 27.2 & 25.6 \\
\hline 6 & $\ldots$ & 25.4 & 25.6 & 27.9 & 25.5 \\
\hline 7 & $-\cdots$ & 25.0 & 26.0 & 28.6 & 25.5 \\
\hline 8 & $-\infty$ & 25.0 & 26.9 & 27.0 & 25.7 \\
\hline 9 & 21.4 & 24.7 & 27.3 & 24.0 & 26.7 \\
\hline 10 & 21.4 & 24.8 & 27.6 & 22.8 & 27.4 \\
\hline 11 & 21.1 & 25.9 & 28.6 & 22.6 & 27.9 \\
\hline 12 & 20.6 & 25.7 & 29.8 & 22.7 & 27.6 \\
\hline 13 & 20.6 & 25.3 & 28.6 & 23.2 & 27.1 \\
\hline 14 & 20.3 & 26.2 & 27.5 & 23.8 & 27.1 \\
\hline 15 & 20.5 & 26.7 & 27.4 & 24.2 & 26.6 \\
\hline 16 & 20.9 & 26.2 & 26.8 & 25.2 & 26.9 \\
\hline 17 & 20.1 & 25.3 & 26.0 & 26.1 & 26.6 \\
\hline 18 & -- & 26.1 & 25.8 & 26.1 & 26.1 \\
\hline 19 & -- & 26.4 & 26.1 & 25.7 & $-\cdots$ \\
\hline 20 & --- & 26.5 & 26.5 & 25.9 & $\cdots$ \\
\hline 21 & 23.9 & 26.2 & 26.5 & 26.3 & - \\
\hline 22 & 24.4 & 25.9 & 26.4 & 26.6 & $-\cdots$ \\
\hline 23 & 24.2 & 26.0 & 27.9 & 26.9 & -- \\
\hline 24 & 22.9 & 26.1 & 28.4 & --- & - \\
\hline 25 & 23.8 & 26.6 & 28.0 & 28.1 & -- \\
\hline 26 & 23.9 & 27.1 & 28.5 & 27.1 & $-\cdots$ \\
\hline 27 & 24.8 & 27.3 & 28.2 & 27.2 & -- \\
\hline 28 & 24.8 & 27.2 & 28.1 & 27.6 & --- \\
\hline 29 & 23.8 & 26.8 & 28.0 & 27.9 & $\ldots$ \\
\hline 30 & 23.9 & 25.6 & 27.2 & 27.7 & $-\cdots$ \\
\hline 31 & 24.3 & -- & 27.5 & 27.8 & --- \\
\hline
\end{tabular}

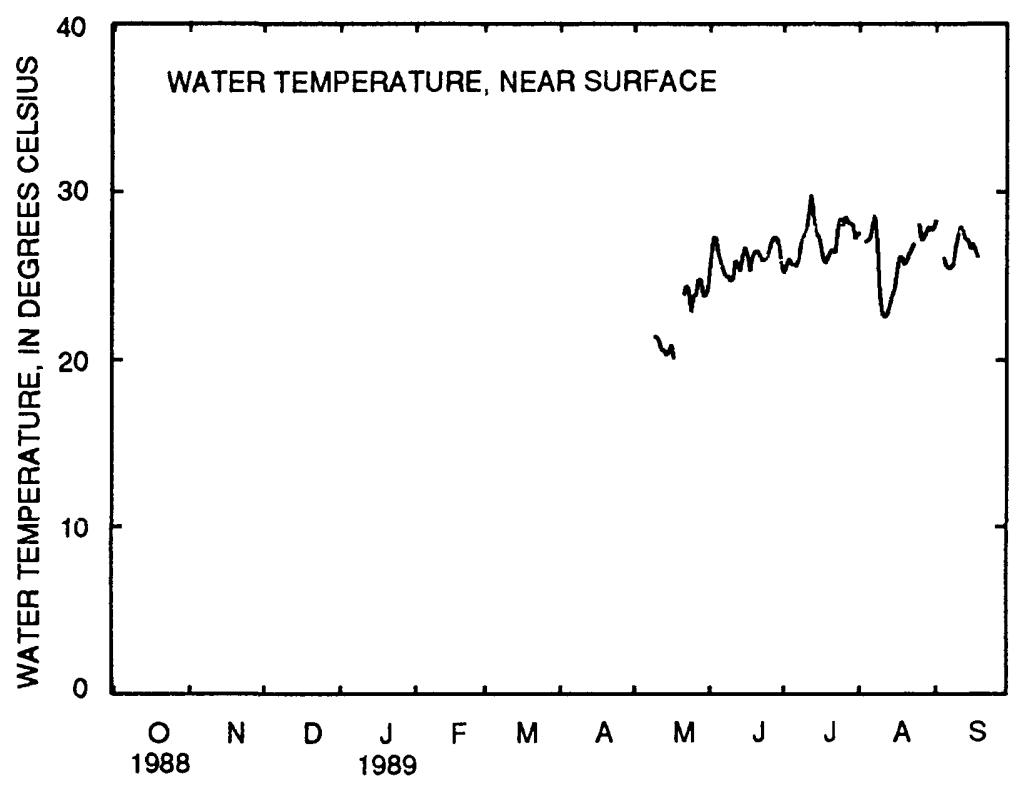


Table 7.--Dally mean values of salinity, water temperature, and dissolved oxygen in water at site 5, Goose Creek at Light 6, May 1989 to September 1990--Continued

[S1te 5 is at latitude $35^{\circ} 19^{\prime} 54^{\prime \prime}$, long1tude $76^{\circ} 37^{\prime} 06^{\prime \prime}$, Beaufort County; U.S. Geological Survey downst ream order number 0208455615 ; ppt, parts per thousand; ---, no data; ${ }^{\circ} \mathrm{C}$, degrees

Celsius; mg/L, milligrams per liter; a, dally minimum value is less than or equal to $2.0 \mathrm{mg} / \mathrm{L}$; b, daily minimum value is less than or equal to $5.0 \mathrm{mg} / \mathrm{L}$ but greater than $2.0 \mathrm{mg} / \mathrm{L} ;<$, less than]

WATER TEMPERATURE, NEAR SURFACE

SENSOR POSITION.--9 feet above streambed.

EXTREMES FOR OCTOBER 1989 TO SEPTEMBER 1990.--Maximum value recorded, $31.6{ }^{\circ} \mathrm{C} \mathrm{Ju}$ Y 24 , 1990 ; minimum value recorded, $6.4{ }^{\circ} \mathrm{C}$ February 26, 1990.

DAILY MEAN VALUES OF WATER TEMPERATURE, NEAR SURFACE (IN DEGREES CELSIUS), OCTOBER 1989 TO SEPTEMBER 1990

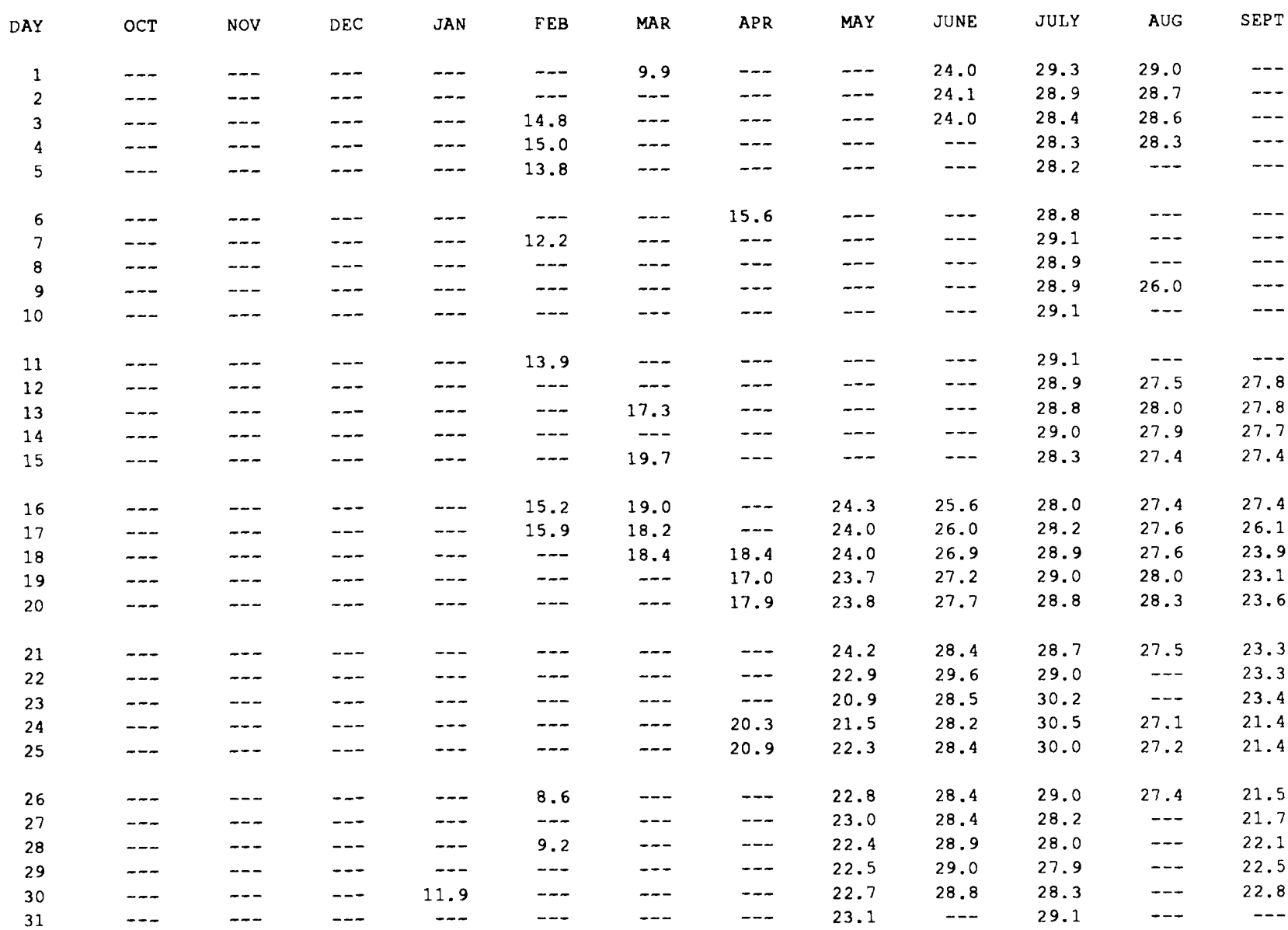

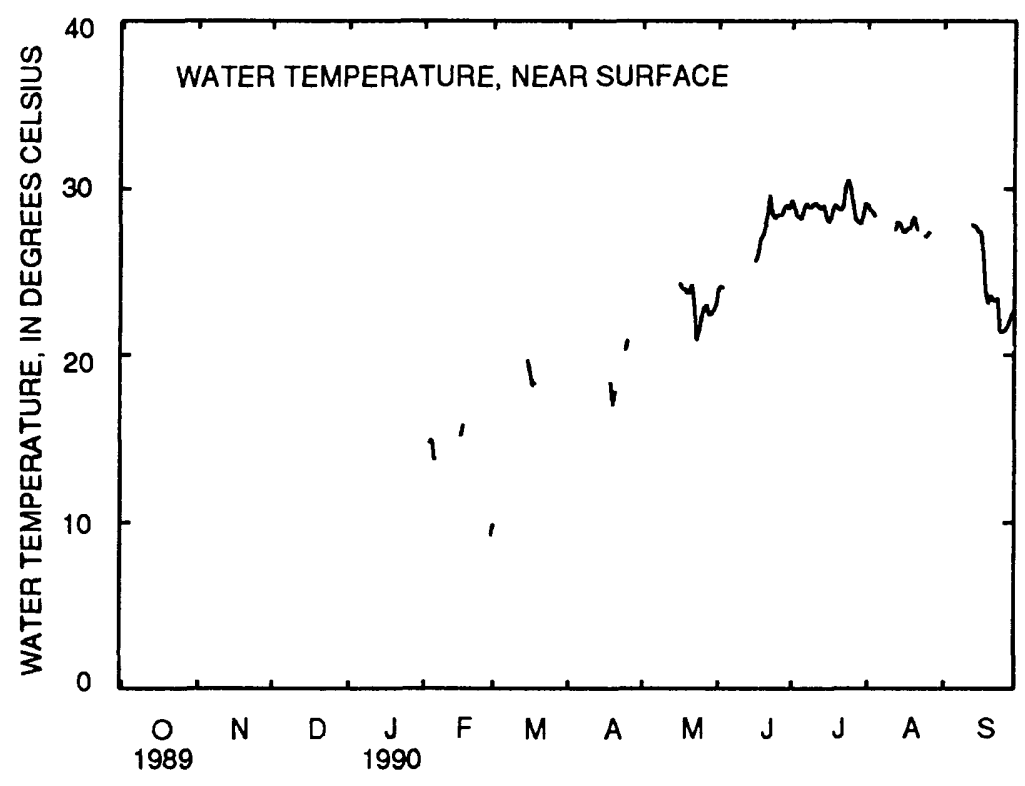


Table 7.--Daily mean values of salinity, water temperature, and dissolved oxygen in water at site 5, Goose Creek at Light 6, May 1989 to September 1990--Continued

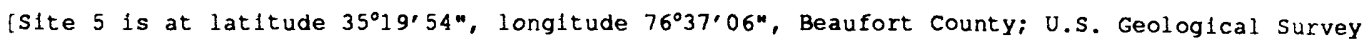
downst ream order number 0208455615 ; ppt, parts per thousand; ---, no data; ${ }^{\circ} \mathrm{C}$, degrees Celsius; mg/L, milligrams per liter; a, dally minimum value is less than or equal to $2.0 \mathrm{mg} / \mathrm{L}$; b, daily minimum value is less than or equal to $5.0 \mathrm{mg} / \mathrm{L}$ but greater than $2.0 \mathrm{mg} / \mathrm{L}$; <, less than)

DISSOLVED OXYGEN, NEAR SURFACE

SENSOR POSITION.--9 feet above streambed.

EXTREMES FOR MAY TO SEPTEMBER 1989.--Max1mum value recorded, 11.4 mg/L June 23, 27, July 23, 1989; m1n1mum value recorded, <1.0 mg/L June $17-20,1989$.

DAILY MEAN VALUES OF DISSOLVED OXYGEN, NEAR SURFACE (IN MILLIGRAMS PER LITER), MAY TO SEPTEMBER 1989

\begin{tabular}{|c|c|c|c|c|c|}
\hline DAY & MAY & JUNE & JULY & AUG & SEPT \\
\hline 1 & --- & --- & 8.4 & 7.7 & $6.6 \mathrm{~b}$ \\
\hline 2 & --- & -- & 8.3 & --- & $6.5 b$ \\
\hline 3 & --- & --- & 7.4 & 8.4 & $5.6 b$ \\
\hline 4 & --- & --- & 6.0 & 8.3 & 6.5 \\
\hline 5 & --- & -- & $4.8 b$ & 8.3 & 6.3 \\
\hline 6 & --- & --- & $4.4 b$ & 9.1 & 6.2 \\
\hline 7 & --- & --- & $5.1 \mathrm{~b}$ & 7.9 & 6.6 \\
\hline 8 & -- & --- & $6.0 \mathrm{~b}$ & 6.3 & 7.4 \\
\hline 9 & --- & -+- & 7.0 & 6.3 & 8.2 \\
\hline 10 & --- & --- & 6.7 & 6.8 & 8.6 \\
\hline 11 & -- & $-\cdots$ & 7.3 & 7.0 & 8.5 \\
\hline 12 & --- & -- & 8.1 & 7.5 & $7.1 b$ \\
\hline 13 & --- & --- & 6.8 & 7.9 & $5.2 b$ \\
\hline 14 & --- & --- & 7.3 & 8.6 & $4.2 b$ \\
\hline 15 & --- & --- & 7.4 & 8.1 & $4.2 b$ \\
\hline 16 & --- & $6.0 a$ & 6.1 & 8.3 & $4.5 b$ \\
\hline 17 & -- & $5.0 a$ & $6.0 \mathrm{~b}$ & 9.2 & $4.3 b$ \\
\hline 18 & -- & $5.1 a$ & 6.6 & 9.0 & $4.5 b$ \\
\hline 19 & -- & $4.8 a$ & 7.6 & 8.9 & --- \\
\hline 20 & --- & $6.0 a$ & 7.0 & 9.0 & --- \\
\hline 21 & -- & $6.0 \mathrm{~b}$ & 6.2 & 9.0 & -- \\
\hline 22 & -- & $6.4 \mathrm{~b}$ & $6.3 b$ & 8.9 & --- \\
\hline 23 & --- & $7.4 \mathrm{~b}$ & $7.7 \mathrm{~b}$ & 8.8 & --- \\
\hline 24 & -- & 8.6 & 8.0 & --- & -- \\
\hline 25 & --- & 8.5 & 6.9 & 6.9 & --- \\
\hline 26 & -- & 8.6 & $6.9 \mathrm{~b}$ & 7.6 & -- \\
\hline 27 & -- & 9.1 & $6.6 \mathrm{~b}$ & 7.6 & -- \\
\hline 28 & $-\cdots$ & 8.6 & 6.7 & 7.6 & --- \\
\hline 29 & --- & 8.2 & 7.5 & 7.0 & --- \\
\hline 30 & $-\cdots$ & 7.6 & 6.8 & 6.0 & --- \\
\hline 31 & -- & -- & 7.1 & $6.2 b$ & --- \\
\hline
\end{tabular}

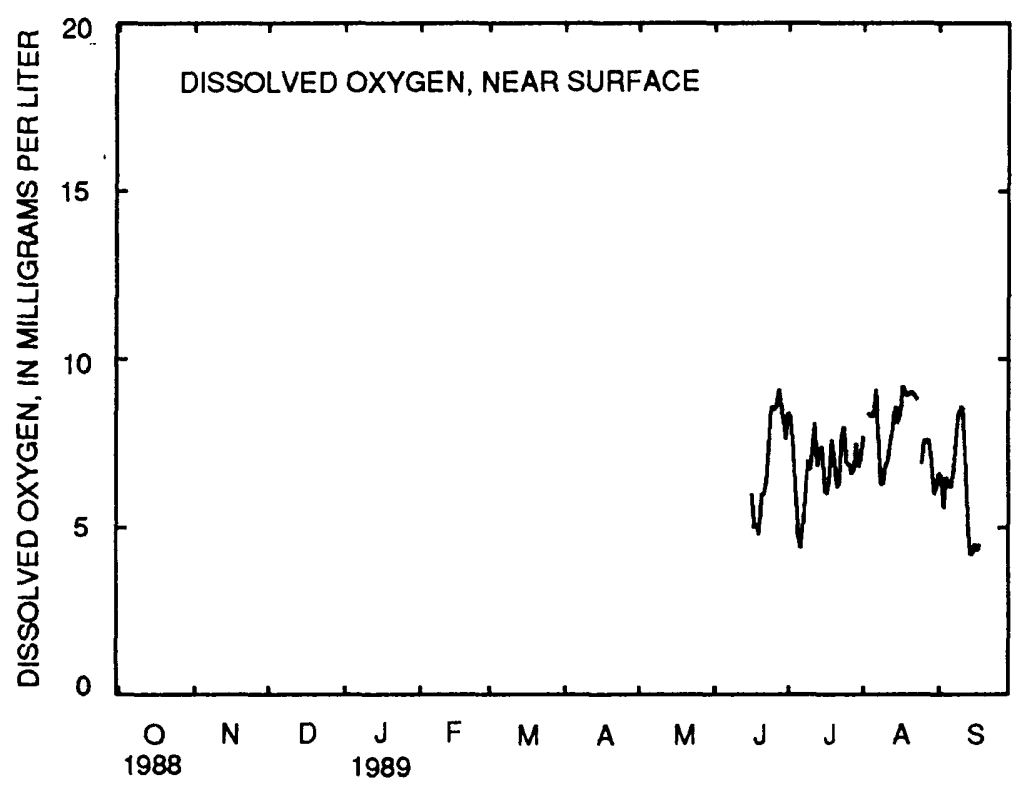


Table 7.--Daily mean values of salinity, water temperature, and dissolved oxygen in water at site 5, Goose Creek at Light 6, May 1989 to september 1990--Cont inued

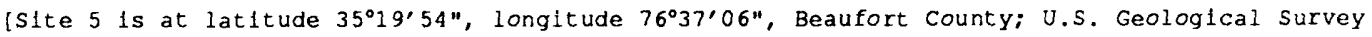
downstream order number 0208455615 ; ppt, parts per thousand; ---, no data; ${ }^{\circ} \mathrm{C}$, degrees

Celsius; mg/L, milligrams per liter; a, dally minimum value is less than or equal to $2.0 \mathrm{mg} / \mathrm{L}$; $\mathrm{b}$, daily minimum value is less than or equal to $5.0 \mathrm{mg} / \mathrm{L}$ but greater than $2.0 \mathrm{mg} / \mathrm{L}$; <, less than]

DISSOLVED OXYGEN, NEAR SURFACE

SENSOR POSITION. --9 feet above streambed.

EXTREMES FOR OCTOBER 1989 TO SEPTEMBER 1990.--Maximum value recorded, $15.9 \mathrm{mg} / \mathrm{L} \mathrm{January} 28$, $1990 ; \mathrm{minimum} \mathrm{value}$ recorded, $2.3 \mathrm{mg} / \mathrm{L}$ August 9, 1990.

DAILY MEAN VALUES OF DISSOLVED OXYGEN, NEAR SURFACE (IN MILLIGRAMS PER LITER), OCTOBER 1989 TO SEPTEMBER 1990

\begin{tabular}{|c|c|c|c|c|c|c|c|c|c|c|c|c|}
\hline DAY & OCT & NOV & $\mathrm{DEC}$ & JAN & $F E B$ & MAR & APR & MAY & JUNE & JULY & AUG & SEPT \\
\hline 1 & -- & -- & --- & --- & 12.5 & 11.2 & 10.2 & -- & 8.6 & 8.0 & $6.2 b$ & --- \\
\hline 2 & -- & --- & -- & -- & 12.2 & 11.7 & --- & -- & 8.2 & 6.9 & $5.6 \mathrm{~b}$ & --- \\
\hline 3 & -- & --- & --- & -- & 12.5 & 11.7 & 9.5 & -- & 8.2 & 7.3 & $5.4 \mathrm{~b}$ & --- \\
\hline 4 & -- & --- & --- & 14.7 & 11.1 & 10.8 & 9.7 & -- & -- & 7.0 & $4.8 \mathrm{~b}$ & --- \\
\hline 5 & -- & --- & --- & 14.2 & 9.6 & 10.5 & --- & --- & $m$ & 6.6 & $4.6 \mathrm{~b}$ & --- \\
\hline 6 & -- & --- & --- & 14.2 & 10.4 & 11.1 & 9.7 & --- & -- & 6.8 & $4.7 \mathrm{~b}$ & --- \\
\hline 7 & -- & -- & --- & 13.8 & 10.4 & 9.9 & 9.4 & --- & -- & 6.4 & $4.3 b$ & --- \\
\hline 8 & --- & -- & $\cdots$ & 12.7 & 11.0 & 9.9 & 10.2 & -- & -- & 6.0 & $4.1 \mathrm{~b}$ & $-\cdots-$ \\
\hline 9 & $\cdots$ & -- & --- & 12.0 & 10.8 & 9.9 & 10.9 & -- & -- & 6.4 & $3.7 \mathrm{~b}$ & --- \\
\hline 10 & $\cdots$ & --- & -- & 12.2 & 9.2 & 10.1 & 10.1 & -- & -- & 6.5 & --- & --- \\
\hline 11 & -- & -- & -- & 12.1 & 9.4 & 10.6 & 9.2 & -- & -- & 6.5 & -- & -- \\
\hline 12 & -- & --- & --- & 12.0 & 9.9 & 10.5 & 9.7 & -- & -- & 6.3 & $4.1 b$ & $6.3 b$ \\
\hline 13 & -- & --- & -- & 11.9 & 10.4 & 11.0 & 10.7 & -- & -- & 6.3 & --- & $6.0 \mathrm{~b}$ \\
\hline 14 & -- & --- & -- & 12.0 & 10.2 & 11.4 & 10.1 & -- & -- & 6.5 & -- & 6.4 \\
\hline 15 & --- & -- & --- & 12.1 & 10.3 & 9.3 & 10.4 & --- & --- & 6.3 & --- & 6.9 \\
\hline 16 & --- & -- & --- & 12.5 & 9.3 & 8.1 & 11.6 & 8.1 & 8.7 & 6.3 & -- & 7.0 \\
\hline 17 & --- & --- & --- & 12.8 & 9.5 & 7.8 & 10.7 & 7.4 & 9.1 & 6.7 & --- & 6.6 \\
\hline 18 & --- & --- & --- & 12.3 & 9.8 & 7.8 & 9.4 & 7.4 & 8.9 & 7.0 & --- & 7.1 \\
\hline 19 & --- & --- & --- & 11.8 & 10.0 & 8.6 & 9.7 & 7.8 & 8.1 & 6.7 & -- & 7.6 \\
\hline 20 & --- & --- & --- & 11.6 & 9.8 & 8.1 & 10.1 & 7.5 & 8.3 & 6.3 & -- & 7.7 \\
\hline 21 & --- & -- & --- & 10.9 & 10.0 & 9.1 & 9.6 & 7.4 & 8.4 & 6.7 & -- & 7.0 \\
\hline 22 & -- & --- & -- & 11.1 & 9.8 & 10.1 & 10.7 & 7.3 & 8.9 & 7.4 & --- & 7.0 \\
\hline 23 & --- & --- & --- & 12.1 & 9.2 & 9.5 & 11.7 & 8.2 & 8.0 & 7.8 & --- & 7.1 \\
\hline 24 & -- & -- & --- & 12.8 & 9.1 & 9.3 & 11.1 & 9.0 & 8.2 & 7.6 & -- & --- \\
\hline 25 & -- & -- & --- & 11.5 & 9.9 & 9.0 & 10.3 & 9.5 & 8.6 & 6.7 & --- & --- \\
\hline 26 & --- & -- & --- & 9.4 & 11.1 & 9.0 & 10.4 & 8.6 & 7.7 & 6.1 & --- & -- \\
\hline 27 & --- & --- & --- & --- & 11.4 & --- & --- & 7.8 & 7.6 & 5.9 & --- & --- \\
\hline 28 & -- & -- & --- & 13.5 & 11.8 & 9.6 & -- & 7.7 & 8.0 & 6.0 & -- & -- \\
\hline 29 & -- & -- & $-\cdots$ & 14.1 & --- & 9.8 & --- & 7.7 & 7.6 & 6.4 & -- & -- \\
\hline 30 & -- & -- & --- & 11.7 & --- & 9.9 & --- & 8.1 & 7.5 & 6.6 & -- & 7.0 \\
\hline 31 & --- & $-\cdots$ & --- & 12.7 & --- & 10.2 & $--\infty$ & 8.8 & -- & 6.9 & -- & --- \\
\hline
\end{tabular}

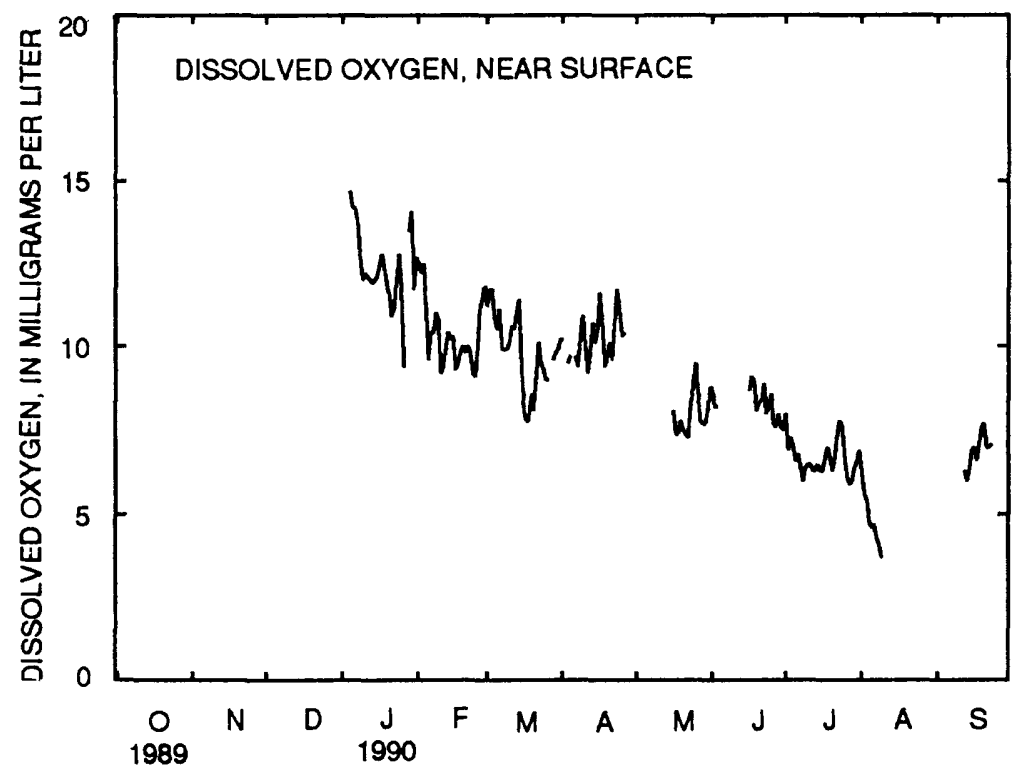

DAILY MEAN DISSOLVED OXYGEN, GOOSE CREEK AT LIGHT 6 
Table 7.--Daily mean values of salinity, water temperature, and dissolved oxygen in water at site 5, Goose Creek at Light 6, May 1989 to September 1990--Cont inued

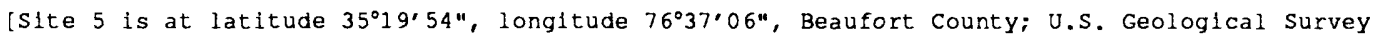
downst ream order number 0208455615; ppt, parts per thousand; ---, no data; ${ }^{\circ} \mathrm{C}$, degrees

Celslus; mg/L, mllligrams per liter; a, dally minimum value is less than or equal to $2.0 \mathrm{mg} / \mathrm{L}$; b, dally minimum value is less than or equal to $5.0 \mathrm{mg} / \mathrm{L}$ but greater than $2.0 \mathrm{mg} / \mathrm{L}$; <, less than]

DISSOLVED OXYGEN, MID-DEPTH

SENSOR POSITION. -6 feet above streambed.

EXTREMES FOR MAY TO SEPTEMBER 1989.--Maximum value recorded, $11.6 \mathrm{mg} / \mathrm{l}$ May 21, $1989 ; \mathrm{m} 1 \mathrm{n} 1 \mathrm{mum}$ value recorded, <1.0 mg/L June 9, 11, 12, 14, 15, 18, 19, 1989.

DAILY MEAN VALUES OF DISSOLVED OXYGEN, MID-DEPTH (IN MILIIGRAMS PER LITER), MAY TO SEPTEMBER 1989

\begin{tabular}{|c|c|c|c|c|c|}
\hline DAY & MAY & JUNE & JULY & AUG & SEPT \\
\hline 1 & --- & $6.9 b$ & $6.1 \mathrm{~b}$ & 7.0 & $5.3 b$ \\
\hline 2 & -- & 7.2 & $6.4 \mathrm{~b}$ & -- & $5.4 b$ \\
\hline 3 & --- & $7.0 \mathrm{~b}$ & $6.0 \mathrm{~b}$ & $6.2 b$ & $5.4 b$ \\
\hline 4 & -- & 6.9 & $5.8 b$ & $6.6 b$ & 6.2 \\
\hline 5 & -- & $6.7 \mathrm{~b}$ & $5.2 b$ & $6.0 \mathrm{~b}$ & 6.2 \\
\hline 6 & -- & 7.9 & $5.4 b$ & $6.0 \mathrm{~b}$ & 6.2 \\
\hline 7 & --- & $6.1 b$ & $5.6 b$ & $5.3 b$ & 6.6 \\
\hline 8 & --- & $3.3 a$ & $5.5 b$ & 6.9 & 7.0 \\
\hline 9 & 7.8 & $4.3 a$ & $6.1 \mathrm{~b}$ & 6.8 & 7.0 \\
\hline 10 & $6.7 b$ & $3.6 a$ & $5.8 b$ & 7.3 & 7.1 \\
\hline 11 & $6.7 b$ & $2.5 a$ & $6.0 \mathrm{~b}$ & 6.8 & 7.0 \\
\hline 12 & $7.3 b$ & $2.1 a$ & $6.4 b$ & 7.7 & $6.8 b$ \\
\hline 13 & 8.3 & $3.5 a$ & $5.7 b$ & 7.8 & $6.7 b$ \\
\hline 14 & 8.5 & $1.8 a$ & $5.8 b$ & 7.8 & 6.2 \\
\hline 15 & 7.9 & $3.1 \mathrm{a}$ & $6.4 \mathrm{~b}$ & 8.3 & $6.4 \mathrm{~b}$ \\
\hline 16 & $6.8 b$ & $3.9 a$ & $5.1 b$ & 7.7 & $6.8 b$ \\
\hline 17 & 8.3 & $3.8 \mathrm{a}$ & $5.5 b$ & 7.4 & $6.2 \mathrm{~b}$ \\
\hline 18 & --- & $3.2 a$ & 6.2 & $7.3 b$ & 6.4 \\
\hline 19 & -- & $4.1 \mathrm{a}$ & 6.5 & 7.8 & -- \\
\hline 20 & --- & $4.9 a$ & 6.4 & 8.2 & -- \\
\hline 21 & $6.9 b$ & $4.9 b$ & $5.9 b$ & 8.1 & --- \\
\hline 22 & $7.0 \mathrm{~b}$ & $5.2 b$ & $5.8 b$ & 8.2 & $\cdots$ \\
\hline 23 & 7.0 & $6.0 \mathrm{~b}$ & $6.0 \mathrm{~b}$ & 8.4 & --- \\
\hline 24 & 7.5 & $6.5 b$ & 7.0 & -- & --- \\
\hline 25 & 7.9 & $6.6 \mathrm{~b}$ & 6.7 & $5.3 b$ & --- \\
\hline 26 & 7.7 & $5.6 b$ & $5.7 b$ & $5.7 \mathrm{~b}$ & --- \\
\hline 27 & 8.0 & $5.5 b$ & 6.4 & $5.5 b$ & --- \\
\hline 28 & 8.7 & $5.7 b$ & $6.5 b$ & $5.7 \mathrm{~b}$ & -- \\
\hline 29 & 8.5 & $5.6 b$ & 7.2 & $5.6 b$ & --- \\
\hline 30 & 8.3 & $5.7 b$ & 6.8 & $4.9 b$ & --- \\
\hline 31 & 8.0 & --- & 6.8 & $5.2 b$ & -- \\
\hline
\end{tabular}

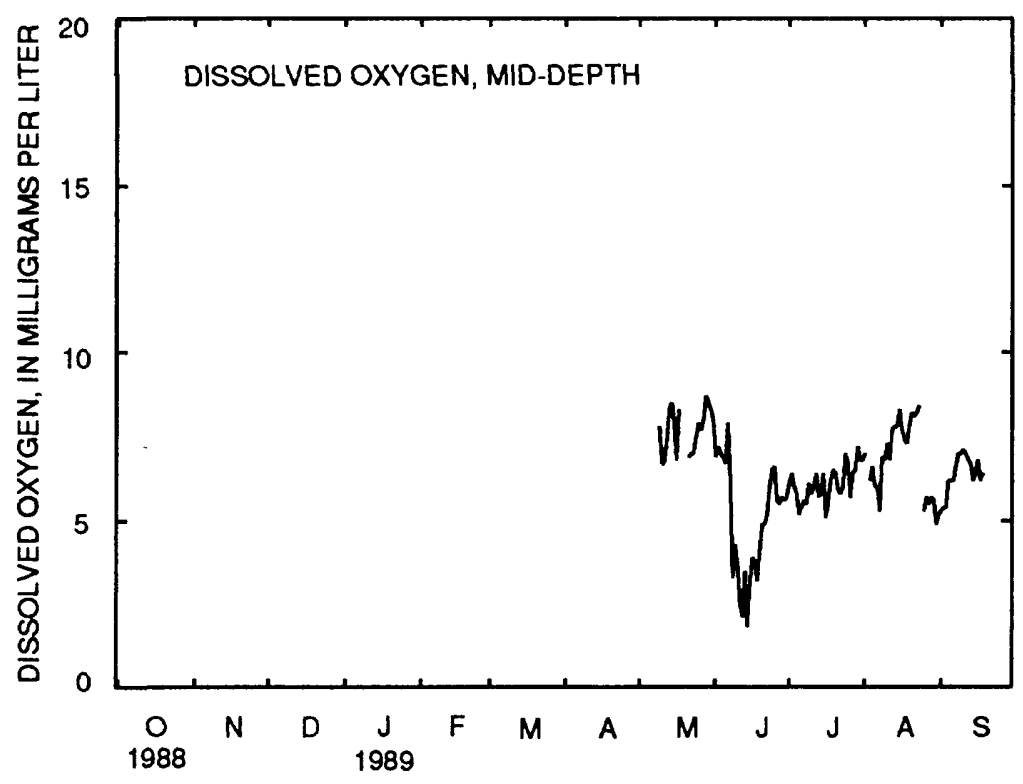


Table 7.--Daily mean values of salinity, water temperature, and dissolved oxygen in water at site 5 , Goose Creek at Light 6, May 1989 to september 1990--Continued

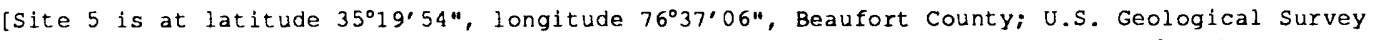
downstream order number 0208455615; ppt, parts per thousand; ---, no data; ${ }^{\circ} \mathrm{C}$, degrees

Celsius; mg/L, milligrams per llter; a, dally minimum value is less than or equal to $2.0 \mathrm{mg} / \mathrm{L}$; b, dally minimum value is less than or equal to $5.0 \mathrm{mg} / \mathrm{L}$ but greater than $2.0 \mathrm{mg} / \mathrm{L}$; <, less than]

DISSOLVED OXYGEN, MID-DEPTH

SENSOR POSITION.--6 feet above streambed.

EXTREMES FOR OCTOBER 1989 TO SEPTEMBER 1990.--Maximum value recorded, 16.0 mg/L January 29, 1990; minimum value recorded, $1.0 \mathrm{mg} / \mathrm{L}$ August 4, 1990.

DAILY MEAN VALUES OF DISSOLVED OXYGEN, MID-DEPTH (IN MILLIGRAMS PER LITER), OCTOBER 1989 TO SEPTEMBER 1990

\begin{tabular}{|c|c|c|c|c|c|c|c|c|c|c|c|c|}
\hline DAY & OCT & NOV & DEC & JAN & FEB & MAR & APR & MAY & JUNE & JULY & AUG & SEPT \\
\hline 1 & --- & --- & --- & --- & 12.6 & 11.2 & 10.7 & --- & --- & $6.4 \mathrm{~b}$ & $4.4 \mathrm{~b}$ & --- \\
\hline 2 & --- & --- & --- & --- & 12.2 & --- & -- & --- & --- & 6.1 & $4.0 \mathrm{~b}$ & $-\cdots$ \\
\hline 3 & -- & --- & --- & --- & $: 2.6$ & -- & 9.8 & --- & --- & $6.3 \mathrm{~b}$ & $3.4 \mathrm{a}$ & --- \\
\hline 4 & --- & --- & --- & 14.5 & 11.2 & --- & 9.9 & --- & --- & $6.4 \mathrm{~b}$ & $2.5 a$ & --- \\
\hline 5 & --- & --- & --- & 14.1 & 9.8 & --- & -- & --- & --- & 6.3 & $2.5 a$ & --- \\
\hline 6 & --- & -- & --- & 13.4 & 10.3 & --- & 11.1 & --- & --- & $6.1 \mathrm{~b}$ & $2.5 a$ & $\cdots$ \\
\hline 7 & --- & --- & --- & 12.8 & 10.3 & --- & 10.3 & --- & -- & 6.2 & --- & -- \\
\hline 8 & --- & --- & --- & 12.2 & 10.8 & --- & 10.6 & -- & --- & $5.8 b$ & --- & --- \\
\hline 9 & --- & -- & --- & 12.3 & 10.7 & --- & 10.7 & --- & -- & 6.2 & -- & -- \\
\hline 10 & --- & --- & --- & 12.4 & 9.2 & --- & 9.9 & --- & --- & 6.0 & --- & -- \\
\hline 11 & --- & --- & --- & 12.4 & 9.5 & --- & 8.7 & --- & --- & $5.8 \mathrm{~b}$ & -- & --- \\
\hline 12 & --- & --- & --- & 12.3 & 9.9 & -- & 9.1 & --- & --- & 5.9 & -- & 6.5 \\
\hline 13 & -- & --- & --- & 12.2 & 10.3 & -- & 9.9 & --- & --- & 5.9 & --- & $6.1 \mathrm{~b}$ \\
\hline 14 & --- & --- & --- & 12.3 & 10.1 & --- & 9.7 & -- &.- & 6.1 & -- & $6.2 \mathrm{~b}$ \\
\hline 15 & -- & --- & --- & 12.5 & 10.2 & --- & 9.4 & --- & --- & 6.0 & -- & 6.9 \\
\hline 16 & --- & --- & --- & 12.6 & 9.2 & 8.7 & 9.9 & 8.0 & 7.6 & 6.0 & -- & 7.0 \\
\hline 17 & --- & -- & --- & 12.6 & 9.3 & 8.6 & 9.3 & --- & 7.7 & 6.6 & -- & 6.9 \\
\hline 18 & --- & --- & -- & 12.6 & 9.7 & 8.8 & 8.7 & --- & $7.7 \mathrm{~b}$ & 6.8 & -- & 7.6 \\
\hline 19 & --- & --- & $-\cdots$ & 12.4 & 9.5 & 9.4 & 8.9 & --- & $7.4 \mathrm{~b}$ & 6.5 & -- & 7.8 \\
\hline 20 & --- & -- & --- & 11.8 & 9.8 & 9.1 & 9.1 & --- & 7.6 & 6.7 & --- & 7.7 \\
\hline 21 & --- & --- & --- & 11.7 & 10.0 & 9.8 & 8.9 & --- & 7.3 & 6.8 & -- & 7.6 \\
\hline 22 & --- & --- & --- & 11.7 & 9.9 & 10.4 & 8.6 & --- & 7.2 & 6.9 & $-\cdots$ & 7.5 \\
\hline 23 & --- & --- & --- & 12.8 & 9.4 & 10.3 & 9.0 & --- & 6.9 & $6.9 \mathrm{~b}$ & --- & 7.8 \\
\hline 24 & --- & -- & --- & 13.3 & 9.2 & 10.4 & 9.1 & --- & $7.2 \mathrm{~b}$ & $6.0 \mathrm{~b}$ & --- & 7.9 \\
\hline 25 & --- & --- & --- & 12.0 & 10.0 & 10.3 & 9.6 & --- & 7.7 & 6.2 & -- & 8.4 \\
\hline 26 & --- & --- & --- & 9.8 & 10.6 & 10.3 & 10.6 & -- & 6.6 & 5.9 & -- & 8.1 \\
\hline 27 & --- & --- & --- & --- & 11.3 & --- & --- & --- & $6.1 \mathrm{~b}$ & 5.9 & --- & 8.3 \\
\hline 28 & --- & --- & --- & 13.0 & 11.3 & 10.9 & --- & --- & $6.5 b$ & 5.8 & -- & 7.8 \\
\hline 29 & --- & --- & --- & 14.2 & --- & 11.1 & --- & --- & $6.6 \mathrm{~b}$ & 6.1 & --- & 7.7 \\
\hline 30 & -- & --- & --- & 11.9 & --- & 11.0 & $-\infty$ & $-\infty$ & $6.1 \mathrm{~b}$ & $6.3 b$ & $-\cdots$ & 7.1 \\
\hline 31 & --- & --- & --- & 13.0 & -- & 11.0 & --- & -- & -- & $6.1 b$ & -- & -- \\
\hline
\end{tabular}

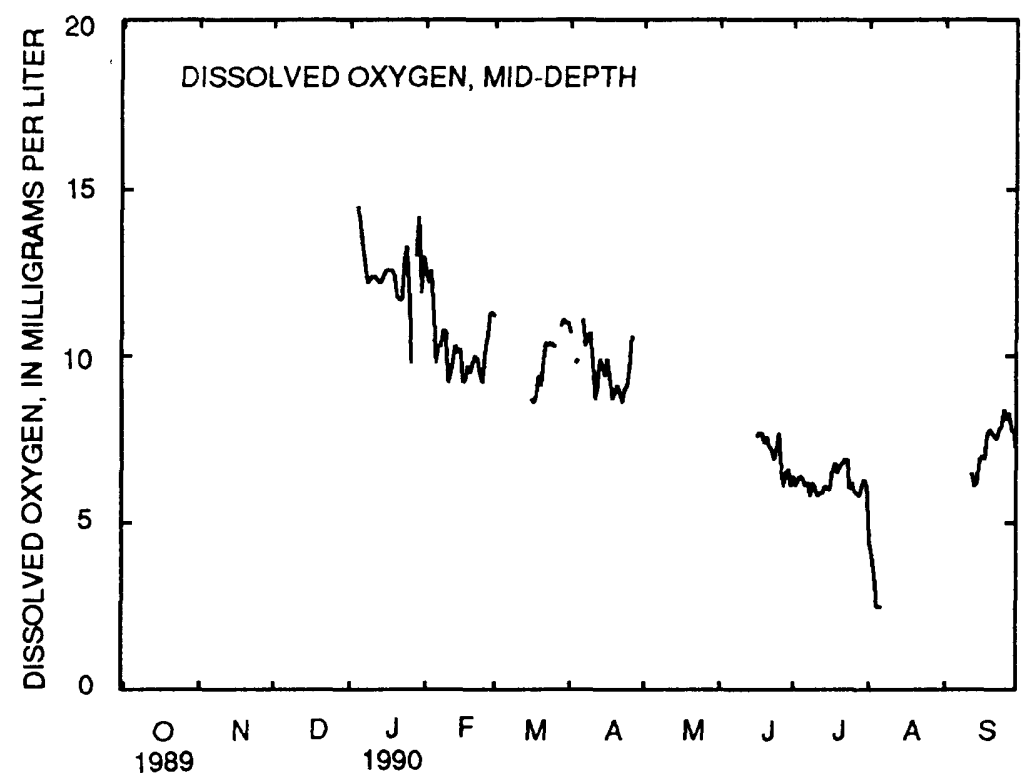

DAILY MEAN DISSOLVED OXYGEN, GOOSE CREEK AT LIGHT 6 
Table 7.--Daily mean values of salinity, water temperature, and dissolved oxygen in water at site 5, Goose Creek at Light 6, May 1989 to September 1990--Cont inued

[Site 5 is at latitude $35^{\circ} 19^{\prime} 54^{\prime \prime}$, Longitude $76^{\circ} 37^{\prime} 06^{\prime \prime}$, Beaufort County; U.S. Geological Survey downstream order number 0208455615 ; ppt, parts per thousand; ---, no data; ${ }^{\circ} \mathrm{C}$, degrees

Celsius; mg/L, milligrams per liter; a, dally minimum value is less than or equal to $2.0 \mathrm{mg} / \mathrm{L}$; b, daily minimum value is less than or equal to $5.0 \mathrm{mg} / \mathrm{L}$ but greater than $2.0 \mathrm{mg} / \mathrm{L}$; <, less than]

DISSOLVED OXYGEN, NEAR BOTTOM

SENSOR POSITION. -2 feet above streambed.

EXTREMES FOR MAY TO SEPTEMBER 1989.--Maximum value recorded, 9.4 mg/L May 15, 1989; minimum value recorded, <1.0 June 10-12, 14-26, July 5, 14, September 15, 16, 1989.

DAILY MEAN VALUES OF DISSOLVED OXYGEN, NEAR BOTTOM (IN MILLIGRAMS PER LITER), MAY TO SEPTEMBER 1989

\begin{tabular}{|c|c|c|c|c|c|}
\hline DAY & MAY & JUNE & JULY & AUG & SEPT \\
\hline 1 & -- & $5.0 \mathrm{~b}$ & $5.4 \mathrm{~b}$ & $5.7 b$ & -- \\
\hline 2 & --- & $4.9 b$ & $5.2 b$ & $5.5 b$ & --- \\
\hline 3 & --- & $5.1 \mathrm{~b}$ & $5.0 \mathrm{~b}$ & $4.9 b$ & --- \\
\hline 4 & -- & $5.1 \mathrm{~b}$ & $5.3 b$ & $5.0 b$ & -- \\
\hline 5 & --- & $5.3 b$ & $4.3 a$ & $4.4 b$ & -- \\
\hline 6 & -- & $6.1 \mathrm{~b}$ & $5.1 \mathrm{~b}$ & $3.9 b$ & -- \\
\hline 7 & --- & $5.7 \mathrm{~b}$ & $5.4 b$ & $2.9 a$ & --- \\
\hline 8 & --- & $3.9 \mathrm{~b}$ & $5.5 b$ & 6.1 & --- \\
\hline 9 & $5.6 b$ & $4.8 \mathrm{~b}$ & $5.5 b$ & 6.5 & -- \\
\hline 10 & $4.4 \mathrm{~b}$ & $3.6 a$ & $4.4 \mathrm{~b}$ & $6.1 \mathrm{~b}$ & -- \\
\hline 11 & $5.0 \mathrm{~b}$ & $3.8 \mathrm{a}$ & $4.9 b$ & $5.1 \mathrm{~b}$ & $\cdots-$ \\
\hline 12 & $6.1 \mathrm{~b}$ & $2.7 a$ & $4.4 \mathrm{~b}$ & $6.1 \mathrm{~b}$ & --- \\
\hline 13 & $6.1 \mathrm{~b}$ & $4.1 \mathrm{a}$ & $3.0 \mathrm{a}$ & $5.6 b$ & $3.7 \mathrm{a}$ \\
\hline 14 & $5.1 a$ & $2.2 a$ & $2.9 a$ & $5.2 b$ & $2.9 \mathrm{~b}$ \\
\hline 15 & $5.0 \mathrm{~b}$ & $1.8 \mathrm{a}$ & $4.4 a$ & $6.1 \mathrm{~b}$ & $2.2 a$ \\
\hline 16 & $3.9 a$ & $1.9 a$ & $2.7 a$ & $5.5 b$ & $2.5 a$ \\
\hline 17 & $7.3 b$ & $2.4 a$ & $3.5 a$ & $4.6 \mathrm{~b}$ & --- \\
\hline 18 & -- & $1.4 \mathrm{a}$ & $4.9 b$ & $4.2 b$ & $2.9 a$ \\
\hline 19 & --- & $1.7 a$ & $5.1 b$ & $4.0 \mathrm{~b}$ & -- \\
\hline 20 & --- & $2.3 a$ & $4.9 b$ & $3.4 \mathrm{~b}$ & --- \\
\hline 21 & $5.1 b$ & $1.4 \mathrm{a}$ & $4.1 \mathrm{~b}$ & $3.0 \mathrm{~b}$ & -- \\
\hline 22 & $4.1 \mathrm{~b}$ & $2.1 \mathrm{a}$ & $4.2 b$ & $3.6 b$ & --- \\
\hline 23 & $4.9 b$ & $2.2 \mathrm{a}$ & $4.5 b$ & $4.6 b$ & --- \\
\hline 24 & $4.8 b$ & $1.8 \mathrm{a}$ & $4.2 b$ & --- & --- \\
\hline 25 & $5.7 b$ & $2.5 a$ & $4.0 \mathrm{~b}$ & $-\infty$ & --- \\
\hline 26 & $5.0 \mathrm{~b}$ & $3.0 \mathrm{a}$ & $3.1 \mathrm{~b}$ & -- & --- \\
\hline 27 & $4.4 \mathrm{~b}$ & $5.0 \mathrm{a}$ & $3.9 \mathrm{~b}$ & $-\infty$ & --- \\
\hline 28 & 6.8 & $4.2 a$ & $4.2 \mathrm{~b}$ & --- & -- \\
\hline 29 & 6.4 & $5.0 \mathrm{a}$ & $5.0 b$ & --- & --- \\
\hline 30 & $6.3 b$ & $5.9 b$ & $5.4 b$ & --- & --- \\
\hline 31 & $6.0 \mathrm{~b}$ & --- & $4.3 b$ & --- & --- \\
\hline
\end{tabular}

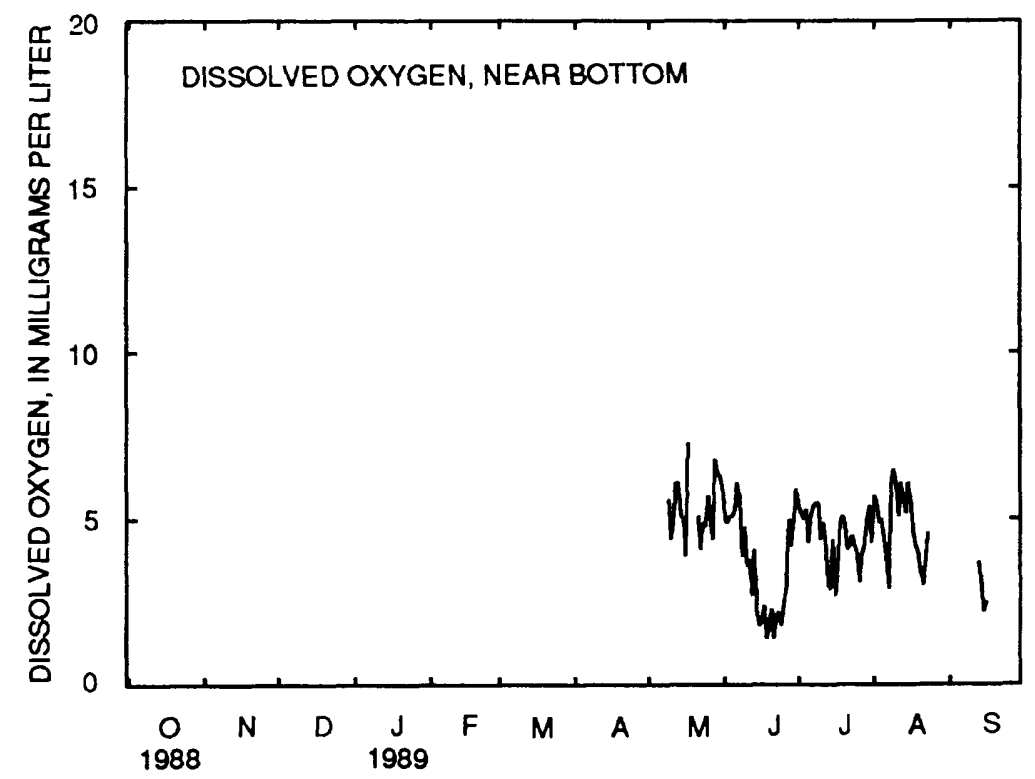


Table 7.--Dally mean values of salinity, water temperature, and dissolved oxygen in water at site 5, Goose Creek at Light 6, May 1989 to September 1990--Cont inued

[Site 5 is at latitude $35^{\circ} 19^{\prime} 54^{\prime \prime}$, longltude 76.37'06", Beaufort County; U.S. Geological Survey downstream order number 0208455615 ; ppt, parts per thousand; ---, no data; ${ }^{\circ} \mathrm{C}$, degrees

Celsius; mg/L, milligrams per liter; a, dally minimum value is less than or equal to $2.0 \mathrm{mg} / \mathrm{L}$; b, daily minimum value is less than or equal to $5.0 \mathrm{mg} / \mathrm{L}$ but greater than $2.0 \mathrm{mg} / \mathrm{L}$; <, less than]

DISSOLVED OXYGEN, NEAR BOTTOM

SENSOR POSITION. --2 feet above streambed.

EXTREMES FOR OCTOBER 1989 TO SEPTEMBER 1990.--Maximum value recorded, $15.2 \mathrm{mg} / \mathrm{L}$ February 13, 1990 ; minimum value recorded, <1.0 mg/L July 21-24, August 4-7, 1990.

DAILY MEAN VALUES OF DISSOLVED OXYGEN, NEAR BOTTOM (IN MILLIGRAMS PER LITER), OCTOBER 1989 TO SEPTEMBER 1990

\begin{tabular}{|c|c|c|c|c|c|c|c|c|c|c|c|c|}
\hline DAY & OCT & NoV & DEC & JAN & FEB & MAR & APR & MAY & JUNE & JULY & AUG & SEPT \\
\hline 1 & -- & -- & -- & $-\infty$ & --- & 10.6 & -- & -- & -- & $5.5 b$ & $6.4 \mathrm{~b}$ & -- \\
\hline 2 & -- & -- & --- & -- & -- & 10.3 & -- & -- & -- & 7.1 & $6.2 \mathrm{~b}$ & --- \\
\hline 3 & -- & --- & -- & $\cdots$ & --- & 10.2 & -- & --- & --- & 6.7 & $4.8 a$ & --- \\
\hline 4 & $-\infty$ & -- & $-\cdots$ & --- & --- & 11.1 & $-\infty$ & --- & --- & $6.0 \mathrm{~b}$ & $2.7 a$ & --- \\
\hline 5 & --- & -- & -- & $\cdots$ & $-\cdots$ & 10.7 & -- & $-\cdots$ & --- & $5.7 \mathrm{~b}$ & $3.0 \mathrm{a}$ & -- \\
\hline 6 & --- & --- & -- & -- & $-m$ & 10.3 & -- & --- & -- & $5.3 b$ & $4.1 \mathrm{a}$ & --- \\
\hline 7 & -- & --- & $-\cdots$ & -- & -- & 10.7 & 8.8 & --- & -- & $6.7 b$ & $3.7 a$ & -- \\
\hline 8 & $\ldots$ & --- & --- & $\cdots$ & $-\cdots$ & 10.7 & 8.8 & -- & -- & $6.0 \mathrm{~b}$ & $5.0 \mathrm{~b}$ & -- \\
\hline 9 & $-\cdots$ & -- & $-\cdots$ & -- & -- & 10.5 & 8.9 & -- & --- & $6.2 b$ & $3.8 \mathrm{~b}$ & --- \\
\hline 10 & -- & --- & -- & --- & $-\infty$ & 10.5 & 8.3 & --- & -- & $5.9 b$ & --- & --- \\
\hline 11 & -- & -- & --- & -- & -- & 9.8 & 7.7 & --- & -- & $5.7 \mathrm{~b}$ & --- & -- \\
\hline 12 & -- & --- & $-\cdots$ & -- & --- & 9.5 & 7.7 & -- & -- & 6.5 & $5.4 b$ & $4.7 \mathrm{~b}$ \\
\hline 13 & $\cdots$ & --- & -- & --- & 12.1 & 9.2 & 8.2 & --- & -- & 6.5 & $4.7 a$ & $4.7 b$ \\
\hline 14 & -- & -- & --- & --- & 10.4 & -- & 7.8 & -- & -- & 6.8 & $4.9 a$ & $4.6 b$ \\
\hline 15 & -- & -- & -- & --- & 9.4 & --- & 7.2 & --- & -- & 6.6 & 6.5 & $5.4 b$ \\
\hline 16 & --- & -- & --- & --- & 7.9 & --- & 6.3 & 7.2 & $6.3 \mathrm{~b}$ & 6.3 & $5.7 \mathrm{~b}$ & $5.1 \mathrm{~b}$ \\
\hline 17 & -- & -- & --- & -- & 7.2 & -- & $6.1 \mathrm{~b}$ & 6.9 & $6.8 \mathrm{~b}$ & $6.4 \mathrm{~b}$ & 7.0 & $5.5 b$ \\
\hline 18 & -- & --- & -- & -- & 7.6 & --- & 7.6 & 7.2 & $6.8 \mathrm{~b}$ & $5.8 \mathrm{~b}$ & $6.6 b$ & 5.9 \\
\hline 19 & -- & --- & --- & $=--$ & $7.1 \mathrm{~b}$ & -- & 9.9 & 7.7 & $6.7 \mathrm{~b}$ & $4.7 a$ & $6.5 b$ & $5.9 \mathrm{~b}$ \\
\hline 20 & -- & -- & --- & --- & 9.1 & -- & 9.1 & $6.9 \mathrm{~b}$ & 7.8 & $5.7 b$ & $4.7 b$ & $5.7 \mathrm{~b}$ \\
\hline 21 & -- & -- & $-\cdots$ & --- & 9.2 & --- & 9.9 & $5.9 b$ & $6.8 \mathrm{~b}$ & $4.7 a$ & 6.4 & 6.4 \\
\hline 22 & -- & -- & $-\infty$ & -- & 9.3 & -- & 8.8 & 7.7 & $6.1 \mathrm{~b}$ & $4.6 a$ & -- & $5.8 \mathrm{~b}$ \\
\hline 23 & --- & -- & -- & -- & 9.1 & -- & 6.7 & 9.1 & $4.7 b$ & $2.6 a$ & --- & $6.1 \mathrm{~b}$ \\
\hline 24 & -- & -- & --- & -- & 9.1 & $\cdots$ & $6.5 b$ & --- & $5.6 b$ & $3.1 \mathrm{a}$ & $4.5 b$ & $6.3 \mathrm{~b}$ \\
\hline 25 & -- & --- & --- & --- & 9.8 & -- & $7.1 \mathrm{~b}$ & --- & $7.2 \mathrm{~b}$ & $5.8 b$ & $4.4 \mathrm{~b}$ & $5.5 b$ \\
\hline 26 & -- & --- & --- & -- & 10.3 & --- & 8.1 & $-\infty$ & $7.6 \mathrm{~b}$ & $5.7 \mathrm{~b}$ & $4.2 b$ & $5.6 \mathrm{~b}$ \\
\hline 27 & -- & $-\infty$ & --- & -- & 10.0 & -- & --- & --- & $6.9 b$ & $5.7 b$ & --- & $6.0 \mathrm{~b}$ \\
\hline 28 & --- & -- & --- & -- & 9.0 & -- & -- & --- & $6.6 \mathrm{~b}$ & 6.3 & $-\cdots$ & $6.4 \mathrm{~b}$ \\
\hline 29 & -- & --- & --- & --- & --- & -- & -- & -- & $6.1 \mathrm{~b}$ & $6.0 \mathrm{~b}$ & -- & 6.7 \\
\hline 30 & -- & -- & $-\infty$ & -- & --- & $-\infty$ & -- & --- & $4.8 \mathrm{a}$ & $5.4 b$ & -- & 6.5 \\
\hline 31 & --- & --- & --- & --- & --- & --- & --- & --- & --- & $4.7 a$ & -- & -- \\
\hline
\end{tabular}

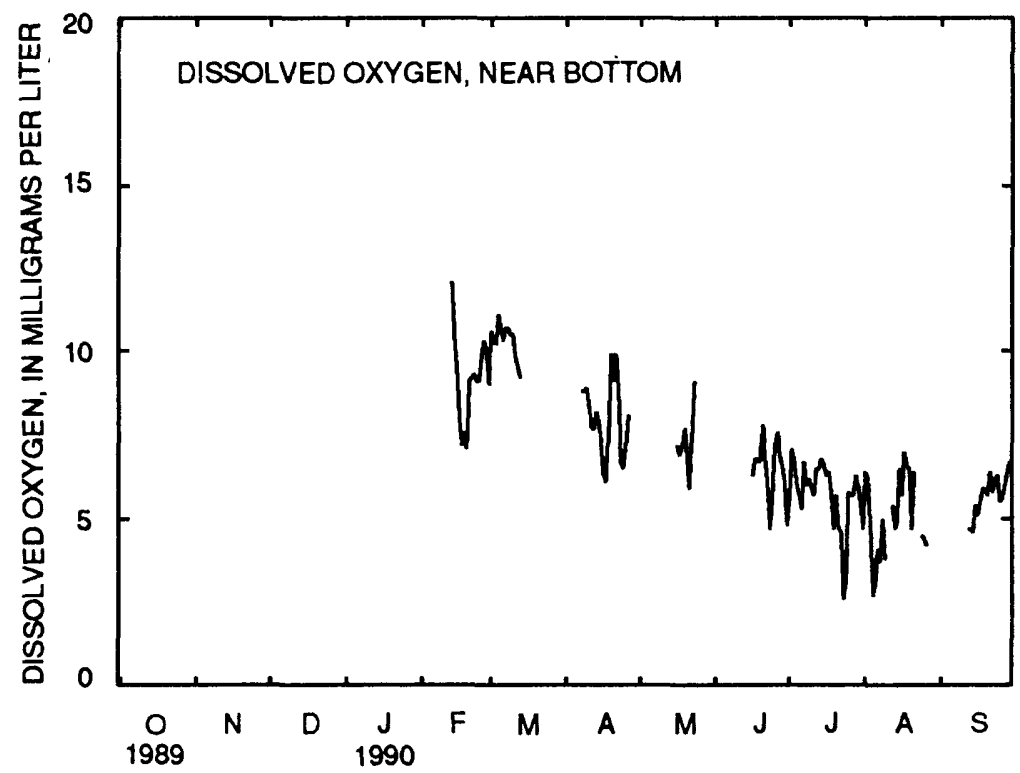

DAILY MEAN DISSOLVED OXYGEN, GOOSE CREEK AT LIGHT 6 
Table 8.--Daily mean values of salinity, water temperature, and dissolved oxygen in water at site 6, Pungo River at Junction Light PR, May 1989 to September 1990

[Site 6 is at latitude $35^{\circ} 22^{\prime} 42^{\prime \prime}$, longitude $76^{\circ} 33^{\prime} 24^{\prime \prime}$, Beaufort County; U.S. Geological Survey downstream order number 0208457700; ppt, parts per thousand; ---, no data; ${ }^{\circ} \mathrm{C}$, degrees

Celsius; mg/L, mllilgrams per liter; a, dally minimum value $1 \mathrm{~s}$ less than or equal to $2.0 \mathrm{mg} / \mathrm{L} ; \mathrm{b}$, daily minimum value is less than or equal to $5.0 \mathrm{mg} / \mathrm{L}$ but greater than $2.0 \mathrm{mg} / \mathrm{L}$; <, less than)

\section{SALINITY, NEAR SURFACE}

SENSOR POSITION. --8 feet above streambed.

EXTREMES FOR MAY TO SEPTEMBER 1989.--Maximum value recorded, 11.1 ppt September 2, 3, 1989; minimum value recorded, 2.4 ppt May 14, 15, 1989.

DAILY MEAN VALUES OF SALINITY, NEAR SURFACE (IN PARTS PER THOUSAND), MAY TO SEPTEMBER 1989

\begin{tabular}{|c|c|c|c|c|c|}
\hline DAY & MAY & JUNE & JULY & AUG & SEPT \\
\hline 1 & -- & 4.9 & -- & $--\infty$ & 10.6 \\
\hline 2 & -- & 5.0 & -- & --- & 10.5 \\
\hline 3 & --- & 5.2 & -- & --- & 10.9 \\
\hline 4 & 7.5 & 5.1 & $-\infty$ & --- & 10.8 \\
\hline 5 & 7.7 & 5.1 & --- & --- & 10.3 \\
\hline 6 & 6.0 & 5.2 & --- & --- & 10.4 \\
\hline 7 & 6.7 & 5.3 & -- & $--\infty$ & 10.6 \\
\hline 8 & 6.1 & 5.2 & --- & --- & 10.7 \\
\hline 9 & 4.6 & 5.3 & --- & $-\cdots$ & 10.5 \\
\hline 10 & 4.6 & 5.3 & --- & $-\infty$ & 10.6 \\
\hline 11 & 5.6 & 5.6 & -- & -- & 10.7 \\
\hline 12 & 5.5 & 5.8 & -- & -- & --- \\
\hline 13 & 4.0 & 5.9 & -- & -- & -- \\
\hline 14 & 3.4 & 6.0 & --- & -- & --- \\
\hline 15 & 3.5 & 6.1 & $--\infty$ & -- & -- \\
\hline 16 & 5.2 & 6.4 & --- & $--\infty$ & --- \\
\hline 17 & 6.8 & 6.4 & --- & --- & -- \\
\hline 18 & 7.2 & 6.2 & -- & -- & --- \\
\hline 19 & 7.7 & --- & --- & -- & --- \\
\hline 20 & 6.9 & --- & -- & $-\infty$ & --- \\
\hline 21 & 6.0 & --- & -- & --- & --- \\
\hline 22 & 6.2 & --- & -- & -- & -- \\
\hline 23 & 5.8 & -- & -- & -- & -- \\
\hline 24 & 6.1 & -- & --- & -- & --- \\
\hline 25 & 6.6 & --- & --- & -- & -- \\
\hline 26 & 6.3 & --- & --- & 10.1 & --- \\
\hline 27 & 5.7 & --- & -- & 10.4 & -- \\
\hline 28 & 6.0 & --- & --- & 10.5 & --- \\
\hline 29 & 5.6 & -- & --- & 10.5 & --- \\
\hline 30 & 5.1 & -- & -- & 10.4 & --- \\
\hline 31 & 4.8 & --- & -- & 10.3 & --- \\
\hline
\end{tabular}

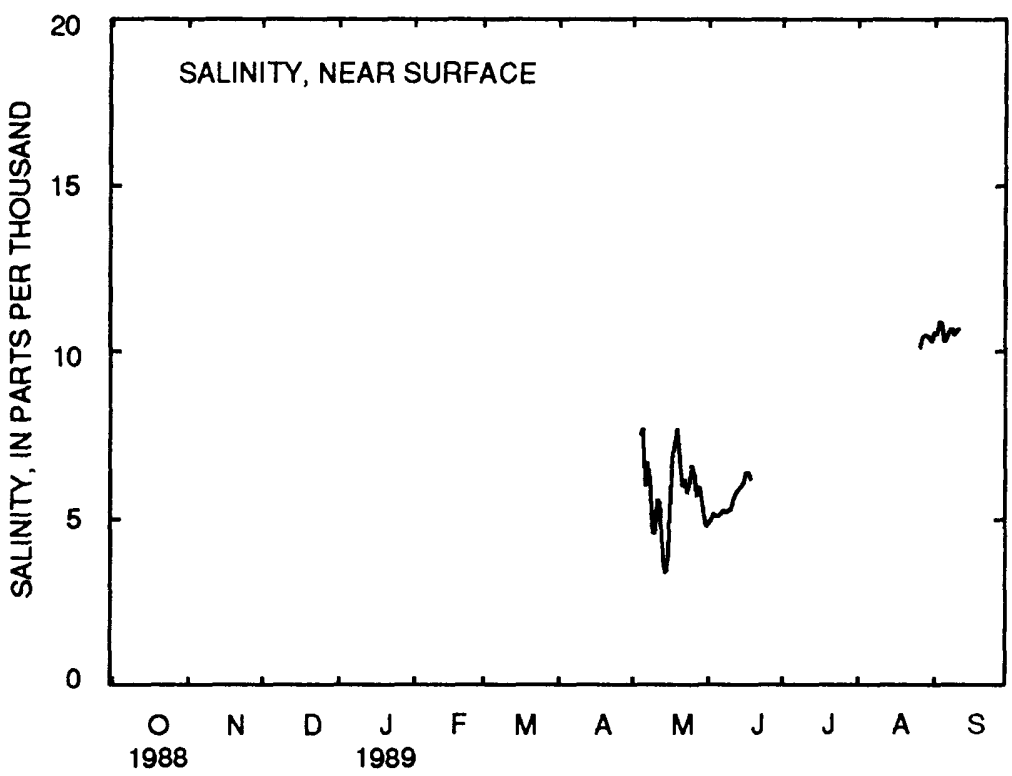


Table 8.--Dally mean values of salinity, water temperature, and dissolved oxygen in water at site 6, Pungo River at Junction Light $P R$, May 1989 to September 1990--Continued

[Site 6 is at latitude $35^{\circ} 22^{\prime} 42^{\prime \prime}$, longltude $76^{\circ} 33^{\prime} 24^{\prime \prime}$, Beaufort County; U.S. Geological Survey downstream order number 0208457700; ppt, parts per thousand; ---, no data; ${ }^{\circ} \mathrm{C}$, degrees

Celsius; mg/L, milligrams per liter; a, daily minimum value is less than or equal to $2.0 \mathrm{mg} / \mathrm{L}$; b, daily minimum value is less than or equal to $5.0 \mathrm{mg} / \mathrm{L}$ but greater than $2.0 \mathrm{mg} / \mathrm{L}$; <, less thanl

\section{SALINITY, NEAR SURFACE}

SENSOR POSITION. --8 feet above streambed.

EXTREMES FOR OCTOBER 1989 TO SEPTEMBER 1990.--Maximum value recorded, 13.4 ppt August 23, 1990; minimum value recorded, 1.3 ppt December $24,1989$.

DAILY MEAN VALUES OF SALINITY, NEAR SURFACE (IN PARTS PER THOUSAND), OCTOBER 1989 TO SEPTEMBER 1990

\begin{tabular}{|c|c|c|c|c|c|c|c|c|c|c|c|c|}
\hline DAY & OCT & NOV & DEC & JAN & FEB & MAR & APR & MAY & JUNE & JULY & AUG & SEPT \\
\hline 1 & --- & --- & 8.8 & -- & --- & 5.9 & -- & 5.5 & --- & $-\cdots$ & 9.9 & -- \\
\hline 2 & --- & $-\cdots$ & 8.1 & -- & -- & 5.5 & -- & 5.8 & --- & --- & 9.6 & -- \\
\hline 3 & -- & --- & 8.7 & --- & 8.4 & 5.8 & -- & 5.7 & --- & $-\infty$ & 9.4 & -- \\
\hline 4 & -- & --- & 9.4 & -- & 8.2 & 6.7 & --- & -- & --- & -- & 9.8 & -- \\
\hline 5 & --- & -- & 8.8 & 7.7 & 8.6 & 5.9 & -- & -- & 6.4 & $-\cdots$ & 9.3 & --- \\
\hline 6 & -- & 7.2 & 9.2 & 8.4 & 8.5 & 5.6 & 5.3 & -- & 6.3 & --- & 9.2 & $-\cdots$ \\
\hline 7 & --- & 7.1 & 9.3 & --- & -- & -- & 5.4 & --- & 6.1 & -- & 8.7 & -- \\
\hline 8 & --- & 6.7 & 10.0 & --- & -- & 6.7 & 5.8 & $-\cdots$ & 6.1 & -- & 9.1 & --- \\
\hline 9 & -- & 6.0 & 9.9 & 9.3 & 7.9 & 5.7 & 6.0 & -- & 5.7 & -- & 8.5 & $-\cdots$ \\
\hline 10 & -- & 6.8 & 9.5 & 9.0 & 8.1 & 6.3 & 5.0 & --- & 5.7 & -- & 8.6 & -- \\
\hline 11 & -- & 7.4 & 9.5 & 9.2 & 8.3 & 5.8 & 4.9 & --- & 6.3 & --- & 8.3 & -- \\
\hline 12 & -- & 6.9 & 9.2 & 9.1 & 7.8 & --- & 4.9 & -- & 6.3 & -- & 8.2 & --- \\
\hline 13 & --- & 7.1 & 9.6 & -- & -- & 5.5 & 5.3 & -- & 6.6 & --- & 8.0 & -- \\
\hline 14 & -- & 7.1 & --- & 9.1 & 8.1 & -- & --- & -- & 6.6 & -- & 8.0 & -- \\
\hline 15 & -- & 7.3 & 8.8 & 8.9 & 8.0 & --- & --- & --- & 6.9 & --- & 8.3 & -- \\
\hline 16 & -- & 6.9 & 9.5 & 8.6 & 7.8 & -- & 5.0 & --- & 6.8 & -- & 8.2 & $\cdots$ \\
\hline 17 & -- & 7.1 & -- & 7.7 & 7.7 & -- & 4.2 & -- & 6.1 & 8.7 & 8.4 & --- \\
\hline 18 & --- & 7.1 & 10.0 & 6.8 & 7.8 & -- & 6.1 & --- & 5.7 & 8.8 & 8.2 & -- \\
\hline 19 & --- & 7.1 & 9.1 & 8.2 & 7.9 & -- & 5.6 & -- & 5.7 & 8.4 & 7.7 & --- \\
\hline 20 & --- & 6.9 & 8.3 & 8.7 & 8.2 & -- & 4.9 & --- & 6.4 & 8.5 & 7.5 & --- \\
\hline 21 & --- & 6.5 & 10.8 & 8.0 & 7.2 & -- & 4.0 & --- & 6.2 & 8.8 & 8.0 & --- \\
\hline 22 & -- & 7.3 & 11.6 & 8.7 & 6.6 & --- & 4.7 & -- & 6.2 & 8.9 & 8.2 & --- \\
\hline 23 & --- & 7.1 & 7.3 & 8.6 & 6.3 & -- & 4.9 & -- & 6.1 & 8.7 & 11.4 & -- \\
\hline 24 & -- & 7.3 & 2.6 & 8.3 & 6.2 & -- & 4.9 & --- & 6.3 & 9.0 & 13.1 & --- \\
\hline 25 & --- & 6.4 & --- & 8.1 & 6.8 & -- & 4.7 & --- & 6.2 & 8.6 & 9.3 & --- \\
\hline 26 & --- & 5.9 & $-\cdots$ & -- & 7.3 & -- & 5.1 & $\cdots$ & 6.2 & 8.9 & 9.3 & -- \\
\hline 27 & -- & 7.3 & -- & --- & 7.2 & --- & 4.9 & -- & 6.4 & 9.6 & 9.5 & -- \\
\hline 28 & -- & 8.2 & --- & --- & 6.0 & -- & 4.7 & -- & -- & 9.4 & -- & --- \\
\hline 29 & --- & 9.3 & -- & -- & $-\cdots$ & -- & 5.0 & -- & --- & --- & --- & -- \\
\hline 30 & -- & 9.0 & -- & --- & $-\cdots$ & -- & 5.1 & -- & -- & -- & -- & -- \\
\hline 31 & -- & --- & -- & 8.8 & --- & -- & -- & -- & --- & -- & --- & -- \\
\hline
\end{tabular}

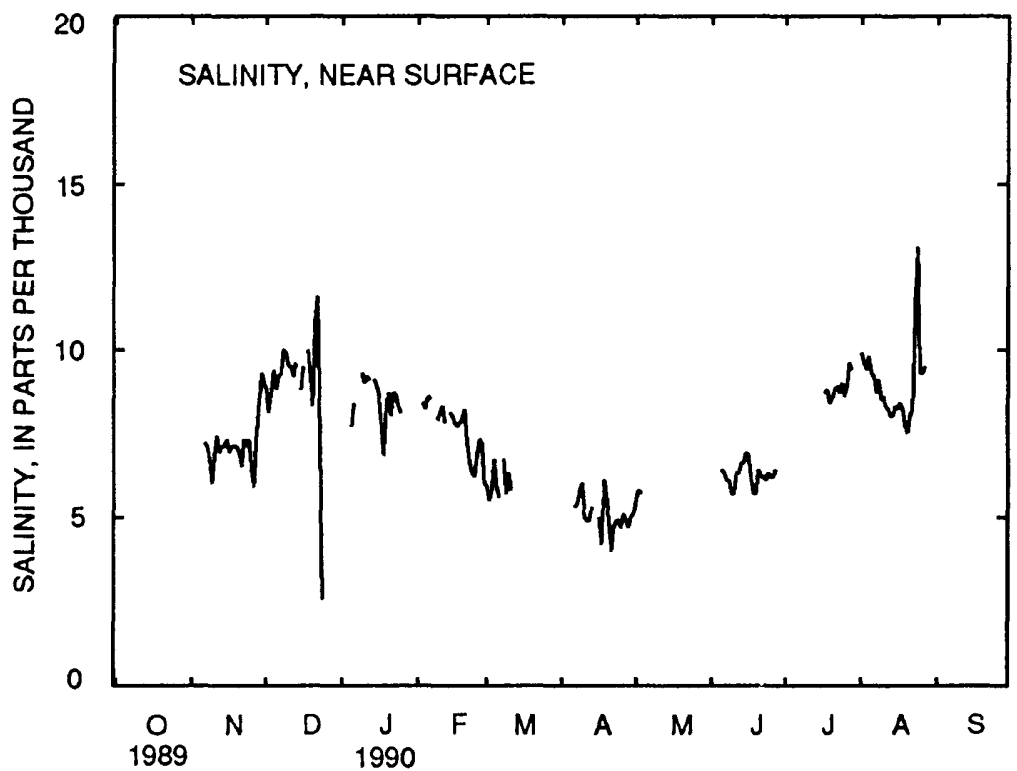

DAILY MEAN SALINITY, PUNGO RIVER AT JUNCTION LIGHT PR 
Table 8.--Dally mean values of salintty, water temperature, and dissolved oxygen in water at site 6 , Pungo River at Junction Light PR, May 1989 to September 1990--Continued

[Site 6 is at latitude $35^{\circ} 22^{\prime} 42^{\prime \prime}$, longitude $76^{\circ} 33^{\prime} 24^{\prime \prime}$, Beaufort County; U.S. Geological Survey downstream order number 0208457700; ppt, parts per thousand; ---, no data; ${ }^{\circ} \mathrm{C}$, degrees

Celsius; mg/L, milligrams per liter; a, daily minimum value is less than or equal to $2.0 \mathrm{mg} / \mathrm{L}$; b, daily minimum value is less than or equal to $5.0 \mathrm{mg} / \mathrm{L}$ but greater than $2.0 \mathrm{mg} / \mathrm{L}$; <, less than]

SALINITY, NEAR BOTTOM

SENSOR POSITION. --2 feet above streambed.

EXTREMES FOR MAY TO SEPTEMBER 1989.--Maximum value recorded, 11.3 ppt August 29, 1989 ; minimum value recorded, 5.5 ppt May 8, 1989.

DAILY MEAN VALUES OF SALINITY, NEAR BOTTOM (IN PARTS PER THOUSAND), MAY TO SEPTEMBER 1989

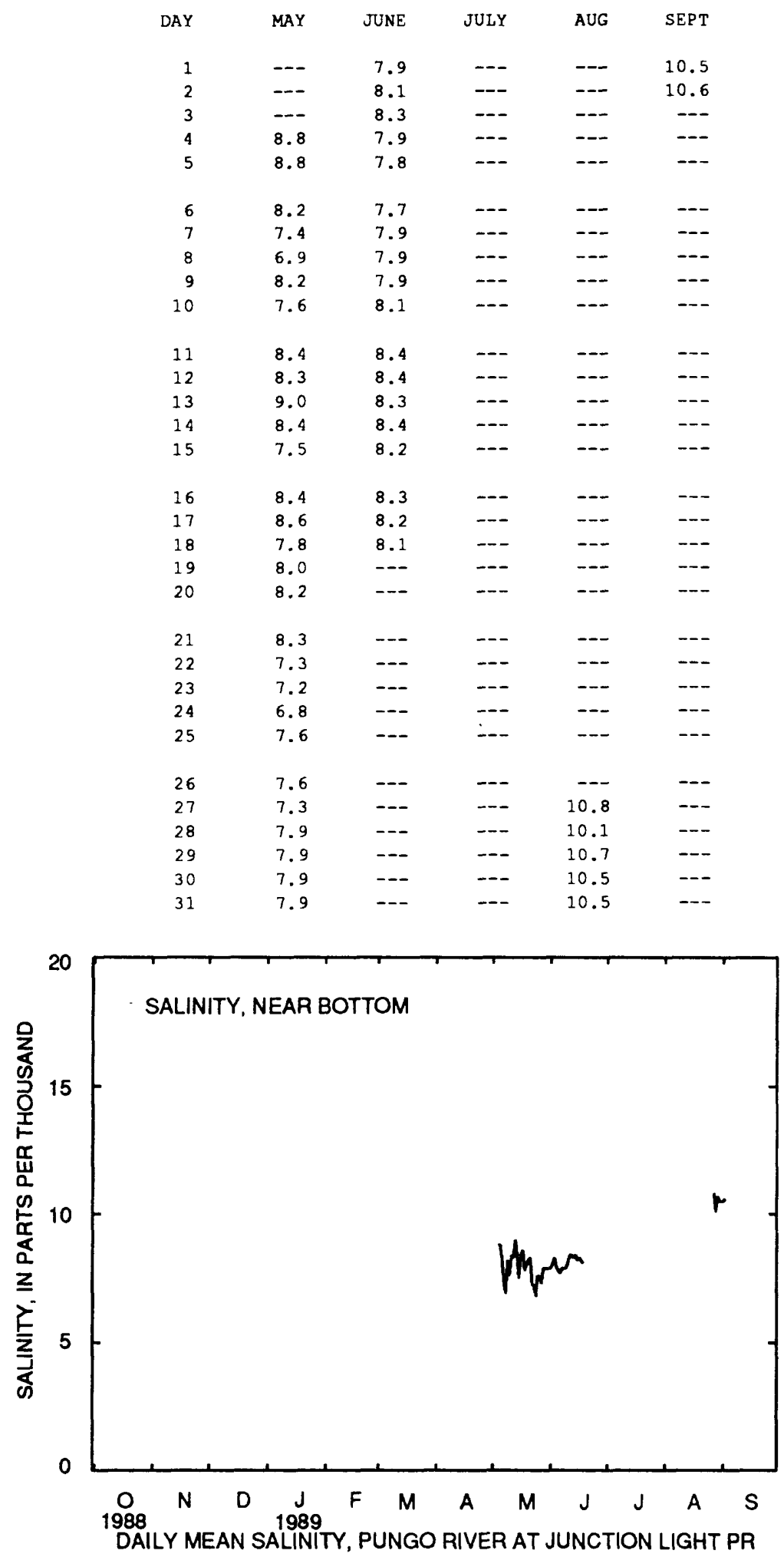


Table 8.--Dally mean values of salinlty, water temperature, and dissolved oxygen in water at site 6 , Pungo River at Junction Light PR, May 1989 to September 1990--Continued

[Site 6 is at latitude $35^{\circ} 22^{\prime} 42^{\prime \prime}$, longitude $76^{\circ} 33^{\prime} 24^{\prime \prime}$, Beaufort County; U.S. Geological Survey downst ream order number 0208457700 ; ppt, parts per thousand; ---, no data; ${ }^{\circ} \mathrm{C}$, degrees

Celsius; mg/L, milligrams per liter; a, dally minimum value is less than or equal to $2.0 \mathrm{mg} / \mathrm{L}$; b, daily minimum value is less than or equal to $5.0 \mathrm{mg} / \mathrm{L}$ but greater than $2.0 \mathrm{mg} / \mathrm{L}$; <, less than]

SALINITY, NEAR BOTTOM

SENSOR POSITION. --2 feet above streambed.

EXTREMES FOR OCTOBER 1989 TO SEPTEMBER 1990.--Maximum value recorded, 12.9 ppt December 23, 1989; minimum value recorded, 4.1 ppt May 6, 1990.

DAILY MEAN VALUES OF SALINITY, NEAR BOTTOM (IN PARTS PER THOUSAND), OCTOBER 1989 TO SEPTEMBER 1990

\begin{tabular}{|c|c|c|c|c|c|c|c|c|c|c|c|c|}
\hline DAY & OCT & NOV & DEC & JAN & FEB & MAR & $A P R$ & MAY & JUNE & JULY & AUG & SERT \\
\hline 1 & -- & -- & 10.3 & -- & $\cdots$ & 7.0 & -- & -- & 7.9 & -- & --- & --- \\
\hline 2 & -- & -- & 9.7 & --- & -- & 7.5 & --- & -- & 7.5 & --- & --- & --- \\
\hline 3 & -- & --- & 9.7 & --- & 9.1 & -- & --- & 5.8 & $-\cdots$ & -- & --- & -- \\
\hline 4 & --- & --- & 10.4 & $\cdots$ & 8.8 & 6.8 & --- & 5.7 & 6.2 & $-\cdots$ & --- & $\cdots$ \\
\hline 5 & --- & --- & 9.7 & 8.6 & 8.7 & 6.8 & --- & 5.0 & 7.0 & --- & -- & -- \\
\hline 6 & --- & 11.5 & 10.1 & 8.9 & 8.8 & 7.0 & 6.2 & 5.2 & 7.3 & $\cdots$ & --- & --- \\
\hline 7 & -- & 11.5 & 9.9 & --- & -- & 6.7 & 6.1 & 5.9 & 7.0 & -- & -- & -- \\
\hline 8 & --- & 11.1 & 10.6 & -- & -- & 7.3 & 6.4 & 5.7 & 7.6 & $\cdots$ & --- & -- \\
\hline 9 & --- & 9.6 & 10.4 & 10.0 & 8.8 & 7.0 & 6.4 & 5.3 & 7.1 & --- & --- & -- \\
\hline 10 & --- & 10.4 & 9.9 & 9.6 & 8.1 & 7.3 & 6.1 & 5.4 & 6.3 & --- & --- & -- \\
\hline 11 & -- & 11.0 & 9.8 & 9.9 & 8.3 & 7.8 & 5.7 & 5.2 & 6.6 & --- & --- & --- \\
\hline 12 & -- & 10.5 & 9.4 & 9.9 & 8.0 & 8.4 & 5.6 & 6.1 & 6.5 & --- & -- & -- \\
\hline 13 & --- & 10.8 & 9.7 & -- & -- & 8.9 & 5.8 & 6.1 & 7.4 & --- & -- & -- \\
\hline 14 & -- & 10.8 & -- & 10.2 & 8.1 & -- & --- & 6.0 & 9.5 & --- & --- & -- \\
\hline 15 & -- & 10.9 & 9.2 & 10.3 & 8.1 & --- & --- & 6.1 & 8.0 & --- & --- & --- \\
\hline 16 & -- & 10.4 & 9.7 & 10.3 & 7.8 & -- & 6.4 & 6.1 & 7.9 & --- & --- & -- \\
\hline 17 & --- & 10.5 & -- & 9.9 & 7.9 & --- & 6.9 & 5.4 & 7.9 & -- & --- & -- \\
\hline 18 & --- & 10.4 & 10.1 & 9.8 & 7.7 & --- & 6.8 & 5.5 & 8.1 & --- & --- & -- \\
\hline 19 & --- & 10.5 & 9.4 & 10.2 & 7.8 & --- & 6.3 & 6.5 & 7.3 & --- & --- & -- \\
\hline 20 & --- & 10.2 & 9.6 & 10.4 & 8.0 & --- & 5.9 & 6.1 & 8.5 & --- & --- & -- \\
\hline 21 & -- & 10.1 & 10.9 & 9.8 & 7.5 & --- & 5.9 & 5.9 & $\cdots$ &.-- & $-\cdots$ & --- \\
\hline 22 & --- & 10.8 & 11.7 & 10.5 & 7.0 & -- & 5.4 & 7.0 & --- & --- & $\cdots$ & $\cdots$ \\
\hline 23 & --- & 10.4 & 7.9 & 10.5 & 6.6 & --- & 6.3 & 7.0 & --- & --- & --- & --- \\
\hline 24 & -- & 10.7 & -- & 10.0 & 6.3 & --- & 5.7 & 8.2 & --- & --- & --- & --- \\
\hline 25 & --- & 10.3 & --- & 9.2 & 7.0 & --- & 5.8 & 8.2 & --- & --- & --- & --- \\
\hline 26 & --- & 9.2 & -- & -- & 7.9 & --- & 6.2 & 7.4 & --- & --- & --- & $-\cdots$ \\
\hline 27 & -- & 9.6 & -- & -- & 7.8 & -- & 6.8 & 6.9 & --- & --- & -- & -- \\
\hline 28 & -- & 9.9 & -- & -- & 8.4 & --- & 7.0 & --- & --- & --- & -- & -- \\
\hline 29 & -- & 10.7 & -- & --- & -- & --- & 6.3 & 7.6 & --- & --- & $-m$ & -- \\
\hline 30 & --- & 10.3 & --- & --- & --- & --- & 6.2 & -- & --- & -- & --- & -- \\
\hline 31 & --- & -- & -- & 9.1 & --- & --- & $\cdots$ & 7.7 & --- & --- & --- & -- \\
\hline
\end{tabular}

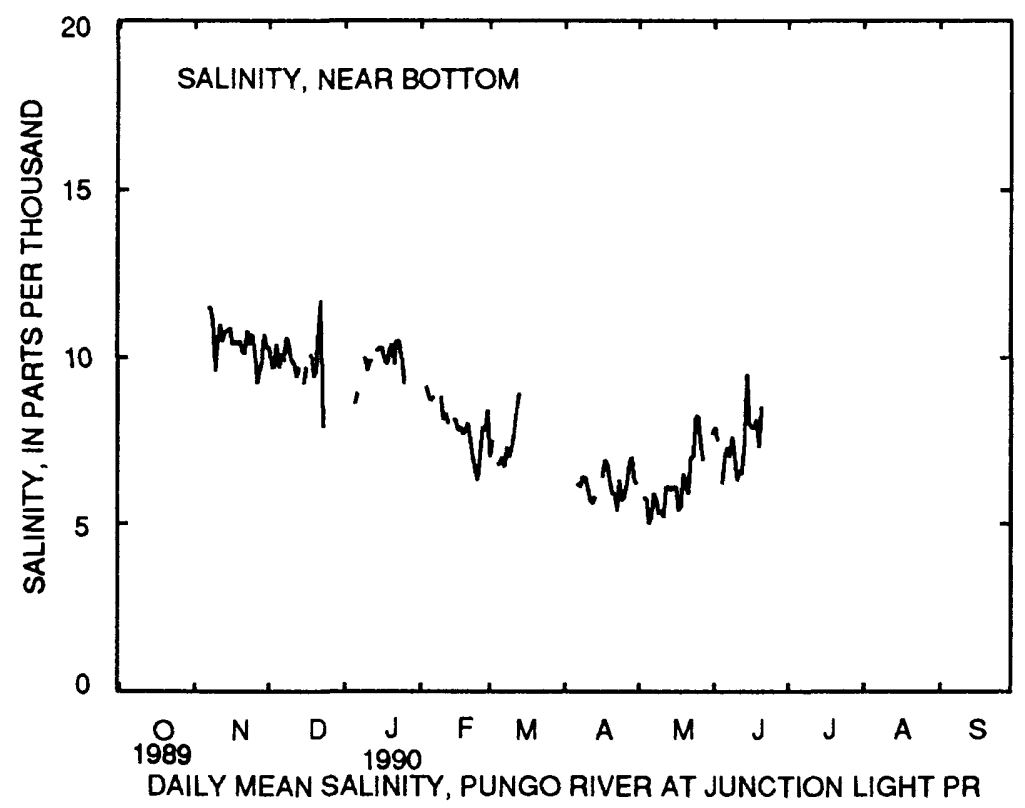


Table 8.--Daily mean values of salinity, water temperature, and dissolved oxygen in water at site 6, Pungo River at Junction Light PR, May 1989 to september 1990 -Continued

[Site 6 is at latitude $35^{\circ} 22^{\prime} 42^{\prime \prime}$, longitude $76^{\circ} 33^{\prime} 24^{\prime \prime}$, Beaufort County; U.S. Geological Survey downstream order number 0208457700; ppt, parts per thousand; ---, no data; ${ }^{\circ} \mathrm{C}$, degrees

Celslus; mg/L, milligrams per liter; a, dally minimum value is less than or equal to $2.0 \mathrm{mg} / \mathrm{L}$; b, daily minimum value is less than or equal to $5.0 \mathrm{mg} / \mathrm{L}$ but greater than $2.0 \mathrm{mg} / \mathrm{L} ;<$, less than]

WATER TEMPERATURE, NEAR SUREACE

SENSOR POSITION. --8 feet above streambed.

EXTREMES FOR MAY TO SEPTEMBER 1989.--Maximum value recorded, $29.6{ }^{\circ} \mathrm{C}$ September 2, 1989 ; minimum value recorded, $17.3{ }^{\circ} \mathrm{C}$ May $12,1989$.

DAILY MEAN VALUES OF WATER TEMPERATURE, NEAR SURFACE (IN DEGREES CELSIUS), MAY TO SEPTEMBER 1989

\begin{tabular}{|c|c|c|c|c|c|}
\hline DAY & MAY & JUNE & JULY & AUG & SEPT \\
\hline 1 & -- & 24.2 & --- & -- & 28.1 \\
\hline 2 & $\ldots$ & 24.6 & - & $-\infty$ & 28.5 \\
\hline 3 & -- & 24.9 & -- & -- & 27.8 \\
\hline 4 & 20.2 & 25.3 & -- & -- & 27.1 \\
\hline 5 & 19.8 & 25.3 & $-\infty$ & -- & 26.3 \\
\hline 6 & 20.2 & 25.3 & -- & -- & 26.2 \\
\hline 7 & 19.5 & 25.0 & $-\infty$ & -- & 25.9 \\
\hline 8 & 18.5 & 24.8 & -- & -- & 26.0 \\
\hline 9 & 18.4 & 24.8 & -- & $-\infty$ & 26.2 \\
\hline 10 & 18.3 & 25.2 & -- & -- & 26.3 \\
\hline 11 & 18.1 & 25.3 & -- & -- & 27.1 \\
\hline 12 & 17.9 & 25.5 & -- & -- & -- \\
\hline 13 & 17.9 & 25.6 & -- & -- & -- \\
\hline 14 & 18.0 & 26.2 & --- & $-\infty$ & $-\infty$ \\
\hline 15 & 18.5 & 26.4 & -- & -- & -- \\
\hline 16 & 19.1 & 26.4 & -- & -- & -- \\
\hline 17 & 18.6 & 26.3 & --- & --- & -- \\
\hline 18 & 18.6 & 26.3 & -- & -- & --- \\
\hline 19 & 19.0 & --- & -- & --- & --- \\
\hline 20 & 19.9 & --- & -- & --- & --- \\
\hline 21 & 20.8 & -- & $-\ldots$ & -- & -- \\
\hline 22 & 21.8 & --- & --- & -- & --- \\
\hline 23 & 22.3 & -- & --- & -- & -- \\
\hline 24 & 21.9 & -- & -- & --- & -- \\
\hline 25 & 22.2 & --- & -- & $-\infty$ & --- \\
\hline 26 & 22.6 & -- & -- & 26.9 & --- \\
\hline 27 & 23.3 & --- & --- & 26.7 & --- \\
\hline 28 & 23.1 & --- & -- & 27.2 & --- \\
\hline 29 & 22.4 & -- & -- & 27.9 & -- \\
\hline 30 & 22.6 & --- & --- & 28.0 & -- \\
\hline 31 & 23.5 & -- & --- & 28.2 & -- \\
\hline
\end{tabular}

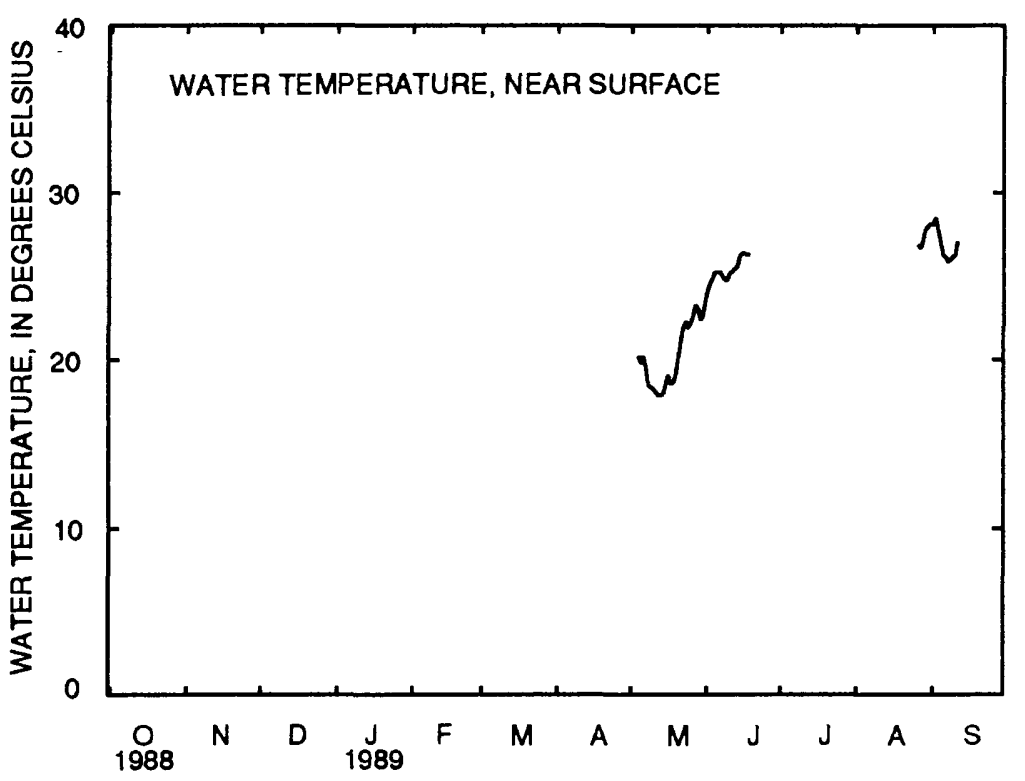


Table 8.--Daily mean values of salinity, water temperature, and dissolved oxygen in water at site 6, Pungo River at Junction Light PR, May 1989 to September 1990--Continued

[Site 6 is at latitude $35^{\circ} 22^{\prime} 42^{\prime \prime}$, Iongitude $76^{\circ} 33^{\prime} 24^{\prime \prime}$, Beaufort County; U.S. Geological Survey downst ream order number 0208457700 ; ppt, parts per thousand; ---, no data; ${ }^{\circ} \mathrm{C}$, degrees

Celsius; mg/L, milligrams per liter; a, daily minimum value is less than or equal to $2.0 \mathrm{mg} / \mathrm{L}$; b, daily minimum value is less than or equal to $5.0 \mathrm{mg} / \mathrm{L}$ but greater than $2.0 \mathrm{mg} / \mathrm{L}$; <, less than]

WATER TEMPERATURE, NEAR SURFACE

SENSOR POSITION. --8 feet above streambed.

EXTREMES FOR OCTOBER 1989 TO SEPTEMBER 1990.--Maximum value recorded, $31.8{ }^{\circ} \mathrm{C}$ August 2 , 1990 ; minimum value recorded, $1.7^{\circ} \mathrm{C}$ December $20,1989$.

DAILY MEAN VALUES OF WATER TEMPERATURE, NEAR SURFACE (IN DEGREES CELSIUS), OCTOBER 1989 TO SEPTEMBER I990

\begin{tabular}{|c|c|c|c|c|c|c|c|c|c|c|c|c|}
\hline DAY & OCT & NOV & DEC & JAN & FEB & MAR & $A P R$ & MAY & JUNE & JULY & AUG & SEPT \\
\hline 1 & --- & --- & 10.0 & -- & --- & 9.5 & --- & --- & 23.3 & --- & 29.7 & $\cdots$ \\
\hline 2 & --- & -- & 9.6 & --- & -- & 9.7 & --- & --- & 24.1 & --- & 29.6 & $-\cdots$ \\
\hline 3 & --- & -- & 9.0 & --- & 13.0 & 10.1 & --- & --- & --- & --- & 29.1 & --- \\
\hline 4 & --- & $-\cdots$ & 7.7 & --- & 13.7 & 10.4 & --- & 23.0 & 24.5 & --- & 29.1 & --- \\
\hline 5 & --- & --- & 7.2 & 4.0 & 13.1 & 10.0 & --- & 23.2 & 23.7 & --- & 29.1 & -- \\
\hline 6 & --- & 17.3 & 7.2 & 4.3 & 12.6 & 10.7 & 16.6 & 22.5 & 24.0 & --- & 28.8 & -- \\
\hline 7 & --- & 17.6 & 7.7 & --- & --- & 10.2 & 16.9 & 22.0 & 24.9 & -- & 28.4 & -- \\
\hline 8 & -- & 17.8 & 7.5 & --- & -- & 9.9 & 16.4 & 22.4 & 25.5 & --- & 28.0 & -- \\
\hline 9 & --- & 18.1 & 6.9 & 4.6 & 13.2 & 10.1 & 16.6 & 22.4 & 26.2 & --- & 27.6 & -- \\
\hline 10 & --- & 17.5 & 6.3 & 4.9 & 14.1 & 10.8 & 17.0 & 23.1 & 26.3 & -- & 27.7 & --- \\
\hline 11 & --- & 17.2 & 6.0 & 5.1 & 13.9 & 12.6 & 17.3 & 22.5 & 25.9 & -- & 28.0 & -- \\
\hline 12 & --- & 17.0 & 5.8 & 5.5 & 13.1 & 13.7 & 16.9 & 22.9 & 24.8 & -- & 28.6 & -- \\
\hline 13 & --- & 17.0 & 5.6 & --- & -- & 14.7 & 17.0 & 23.1 & 24.1 & --- & 29.0 & --- \\
\hline 14 & --- & 17.4 & -- & 4.9 & 13.4 & --- & --- & 23.8 & 24.4 & --- & 29.0 & --- \\
\hline 15 & --- & 17.9 & 5.2 & 5.4 & 13.8 & --- & --- & 24.1 & 24.1 & --- & 28.8 & -- \\
\hline 16 & --- & 17.5 & 5.0 & 6.1 & 14.9 & --- & 18.4 & 24.5 & 24.4 & --- & 29.2 & $-\cdots$ \\
\hline 17 & --- & 16.2 & -- & 6.9 & 15.5 & --- & 19.3 & 24.6 & 25.0 & 27.3 & 28.9 & --- \\
\hline 18 & --- & 15.2 & 3.2 & 8.2 & 14.3 & --- & 18.2 & 24.6 & 25.4 & 27.6 & 29.1 & -- \\
\hline 19 & --- & 14.1 & 3.1 & 8.3 & 14.0 & -- & 18.0 & 24.2 & 25.2 & 27.6 & 29.4 & -- \\
\hline 20 & --- & 12.9 & 2.8 & 8.6 & 13.5 & --- & 18.5 & 24.2 & 25.7 & 27.7 & 29.8 & --- \\
\hline 21 & --- & 11.6 & --- & 8.9 & 12.6 & --- & 19.6 & 24.5 & 26.4 & 27.8 & 29.3 & --- \\
\hline 22 & --- & 12.2 & --- & 9.2 & 12.8 & -- & 20.1 & 23.6 & 27.7 & 27.8 & 29.5 & --- \\
\hline 23 & --- & 11.1 & --- & 9.3 & 13.5 & --- & 20.8 & 22.3 & 27.0 & 28.2 & -- & -- \\
\hline 24 & --- & 10.0 & --- & 10.0 & 13.4 & --- & 20.9 & 22.4 & 26.8 & 29.1 & -- & --- \\
\hline 25 & --- & 9.4 & --- & 10.7 & 11.7 & --- & 21.4 & 22.8 & 26.8 & 28.8 & -- & --- \\
\hline 26 & --- & 10.2 & --- & --- & 9.8 & --- & 22.6 & 22.9 & 26.7 & 27.9 & --- & -- \\
\hline 27 & --- & 10.8 & --- & --- & 9.3 & --- & --- & 23.3 & 26.7 & 27.7 & --- & -- \\
\hline 28 & --- & 11.5 & --- & --- & 9.3 & --- & --- & --- & -- & 27.4 & --- & -- \\
\hline 29 & --- & 11.4 & --- & --- & -- & --- & --- & 22.8 & -- & -- & --- & - \\
\hline 30 & --- & 10.7 & --- & --- & --- & --- & --- & -- & --- & --- & --- & -- \\
\hline 31 & --- & --- & --- & 10.9 & $--\infty$ & -- & --- & 22.7 & --- & --- & --- & --- \\
\hline
\end{tabular}

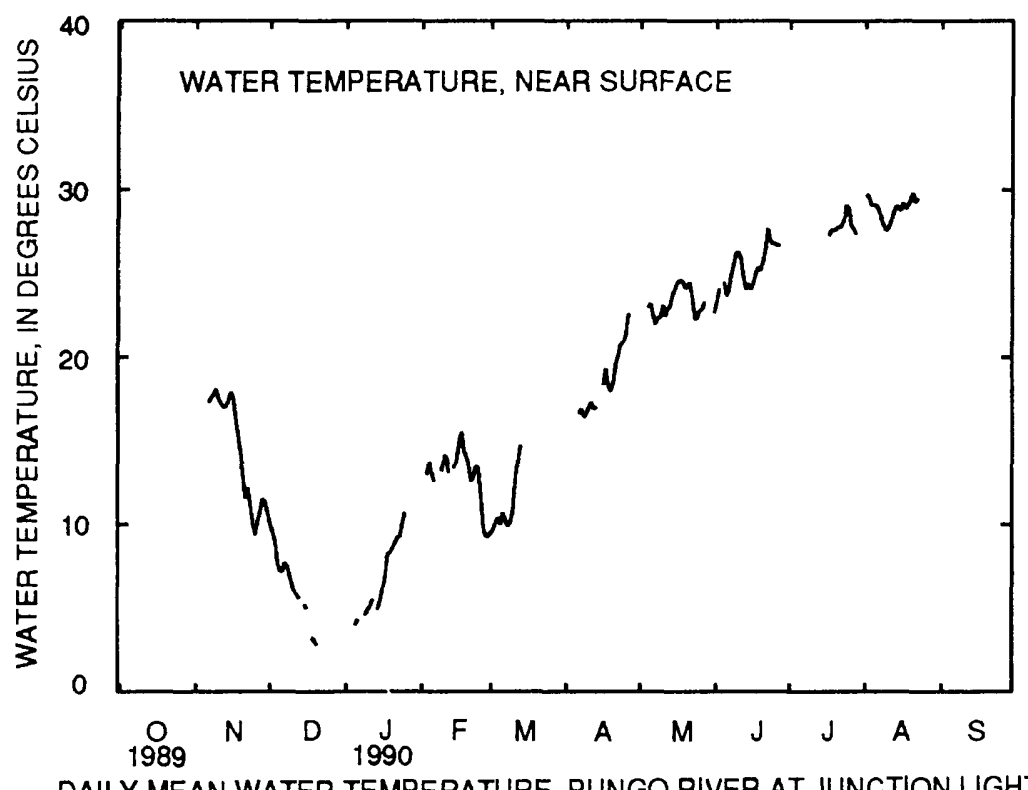


Table 8.--Daily mean values of salinity, water temperature, and dissolved oxygen in water at site 6, Pungo River at Junction Light PR, May 1989 to September 1990--Continued

[S1te 6 is at lat1tude $35^{\circ} 22^{\prime} 42^{\prime \prime}$, long1tude $76^{\circ} 33^{\prime} 24^{\prime \prime}$, Beaufort County; U.S. Geological Survey downstream order number 0208457700 ; ppt, parts per thousand; ---, no data; ${ }^{\circ} \mathrm{C}$, degrees Celsius; mg/L, m1lligrams per 11ter; a, da1ly minimum value is less than or equal to $2.0 \mathrm{mg} / \mathrm{L}$; b, daily minimum value is less than or equal to $5.0 \mathrm{mg} / \mathrm{L}$ but greater than $2.0 \mathrm{mg} / \mathrm{L}$; <, less than]

\section{DISSOLVED OXYGEN, NEAR SURFACE}

SENSOR POSITION.--8 feet above streambed.

EXTREMES FOR MAY TO SEPTEMBER 1989.--Max1mum value recorded, 14.6 mg/L May 20, 1989; m1n1mum value recorded, 3.9 mg/L August 26, 1989.

DAILY MEAN VALUES OF DISSOLVED OXYGEN, NEAR SURFACE (IN MILLIGRAMS PER LITER), MAY TO SEPTEMBER 1989

\begin{tabular}{|c|c|c|c|c|c|}
\hline DAY & MAY & JUNE & JULY & AUG & SEPT \\
\hline 1 & --- & -- & --- & --- & $5.7 \mathrm{~b}$ \\
\hline 2 & --- & --- & --- & -- & 5.8 \\
\hline 3 & --- & -- & --- & --- & $5.5 b$ \\
\hline 4 & 7.1 & --- & --- & -- & 6.5 \\
\hline 5 & 6.9 & --- & --- & -- & 6.8 \\
\hline 6 & 7.2 & $-\infty$ & --- & --- & 7.6 \\
\hline 7 & 7.3 & -- & --- & --- & 7.8 \\
\hline 8 & 7.7 & --- & -- & -- & 7.9 \\
\hline 9 & 8.9 & -- & $\cdots$ & -- & 7.7 \\
\hline 10 & 8.3 & -- & --- & --- & 7.7 \\
\hline 11 & 7.9 & $-\infty$ & --- & -- & 8.3 \\
\hline 12 & 8.4 & --- & --- & --- & -- \\
\hline 13 & 8.4 & -- & -- & --- & --- \\
\hline 14 & 9.0 & -- & --- & --- & -- \\
\hline 15 & 9.7 & -- & --- & --- & -- \\
\hline 16 & 10.2 & -- & -- & -- & -- \\
\hline 17 & 9.2 & --- & --- & -- & -- \\
\hline 18 & 9.9 & --- & --- & -- & -- \\
\hline 19 & 10.9 & --- & -- & -- & --- \\
\hline 20 & 10.9 & -- & --- & --- & --- \\
\hline 21 & 11.7 & $\ldots$ & -- & -- & -- \\
\hline 22 & 11.6 & --- & --- & --- & $-\infty$ \\
\hline 23 & 11.1 & -- & --- & -- & --- \\
\hline 24 & 8.5 & -- & --- & --- & --- \\
\hline 25 & 7.7 & --- & --- & --- & -- \\
\hline 26 & 7.5 & --- & --- & $5.3 b$ & -- \\
\hline 27 & 7.4 & -- & --- & $5.5 b$ & --- \\
\hline 28 & 6.9 & -- & --- & $5.8 b$ & - \\
\hline 29 & 6.6 & --- & --- & $5.9 b$ & --- \\
\hline 30 & 6.6 & -- & --- & 5.9 & -- \\
\hline 31 & 6.3 & --- & -- & $6.1 \mathrm{~b}$ & -- \\
\hline
\end{tabular}

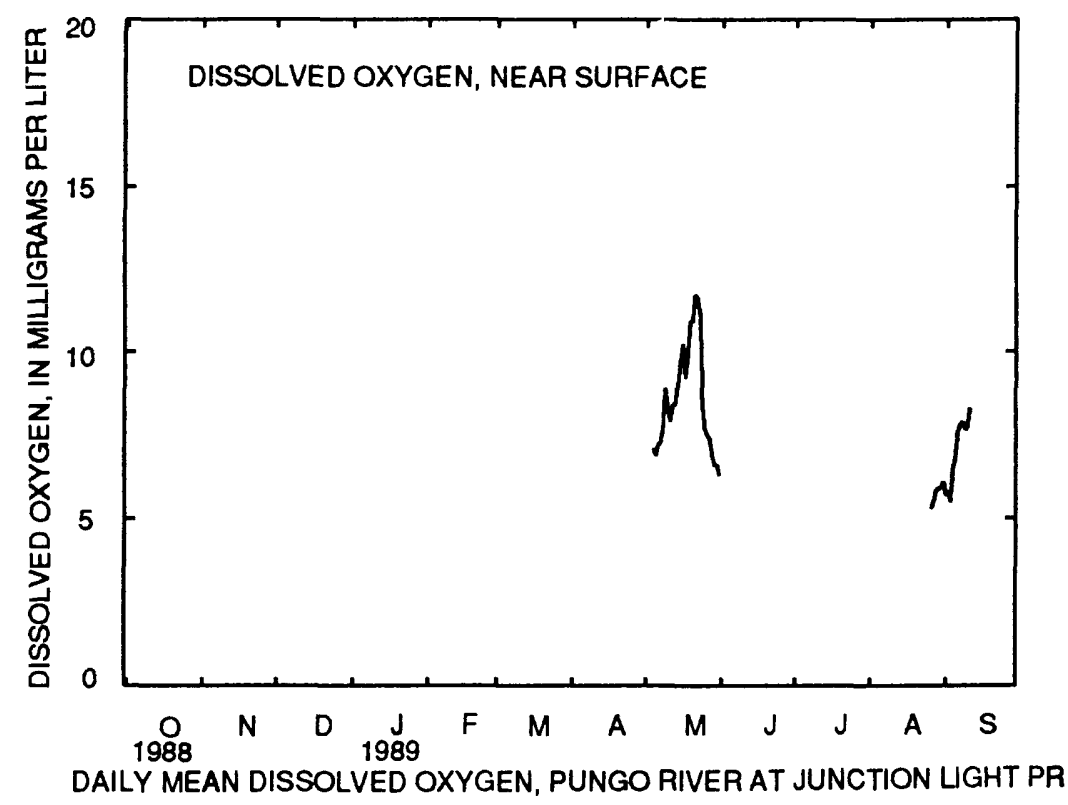


Table 8.--Dally mean values of salinity, water temperature, and dissolved oxygen in water at site 6, Pungo River at Junction Light PR, May 1989 to September 1990--Continued

[Site 6 is at latitude $35^{\circ} 22^{\prime} 42^{\prime \prime}$, longitude $76^{\circ} 33^{\prime} 24^{\prime \prime}$, Beaufort County; U.S. Geological Survey downstream order number 0208457700 ; ppt, parts per thousand; ---, no data; ${ }^{\circ} \mathrm{C}$, degrees

Celsius; $\mathrm{mg} / \mathrm{L}$, milligrams per liter; a, dally minimum value is less than or equal to $2.0 \mathrm{mg} / \mathrm{L} ; \mathrm{b}$, daily minimum value $1 \mathrm{~s}$ less than or equal to $5.0 \mathrm{mg} / \mathrm{L}$ but greater than $2.0 \mathrm{mg} / \mathrm{L} ;<$, less than]

DISSOLVED OXYGEN, NEAR SURFACE

SENSOR POSITION. --8 feet above streambed.

EXTREMES FOR OCTOBER 1989 TO SEPTEMBER 1990.--Maximum value.recorded, $18.6 \mathrm{mg} / \mathrm{L}$ January 20 , 1990 ; min1mum value recorded, $3.9 \mathrm{mg} / \mathrm{L}$ June $19,1990$.

DAILY MEAN VALUES OF DISSOLVED OXYGEN, NEAR SURFACE (IN MILLIGRAMS PER LITER), OCTOBER 1989 TO SEPTEMBER 1990

\begin{tabular}{|c|c|c|c|c|c|c|c|c|c|c|c|c|}
\hline DAY & OCT & NOV & DEC & JAN & FEB & MAR & APR & MAY & JUNE & JULY & AUG & SEPT \\
\hline 1 & -- & --- & 11.7 & -- & -- & 10.7 & --- & 8.6 & --- & --- & 8.7 & --- \\
\hline 2 & --- & --- & 11.9 & --- & --- & 10.7 & -- & 7.9 & --- & -- & 8.9 & --- \\
\hline 3 & -- & --- & 11.3 & -- & 12.6 & 10.6 & --- & 8.1 & --- & -- & 8.8 & -- \\
\hline 4 & --- & --- & 11.4 & -- & 11.4 & 9.9 & --- & --- & -- & -- & 9.1 & -- \\
\hline 5 & --- & --- & 11.4 & 15.7 & 9.9 & 10.0 & --- & --- & 6.8 & -- & 8.9 & --- \\
\hline 6 & --- & --- & 11.5 & 15.2 & 10.6 & 10.2 & 9.1 & -- & 6.5 & --- & 7.9 & --- \\
\hline 7 & --- & --- & 11.5 & --- & --- & 9.5 & 8.5 & -- & 6.4 & -- & 8.1 & -- \\
\hline 8 & --- & --- & 10.9 & -- & -- & 9.9 & 8.7 & --- & 7.0 & -- & 8.1 & -- \\
\hline 9 & --- & -- & 10.9 & 12.9 & 11.0 & 9.6 & 9.0 & -- & 7.5 & -- & 8.5 & -- \\
\hline 10 & --- & --- & 11.1 & 12.5 & 10.0 & 9.7 & 9.5 & -- & 7.7 & -- & 9.4 & -- \\
\hline 11 & -- & --- & 11.0 & 12.3 & 9.6 & 10.6 & 9.0 & --- & $5.8 b$ & -- & 9.4 & --- \\
\hline 12 & -- & -- & 11.0 & 12.0 & 9.5 & 10.9 & 8.6 & -- & 5.6 & -- & 9.2 & $\cdots$ \\
\hline 13 & -- & --- & 10.8 & -- & --- & 11.1 & 8.8 & -- & $6.1 b$ & -- & 8.8 & --- \\
\hline 14 & -- & -- & -- & 12.1 & 10.0 & -- & --- & -- & 6.5 & -- & 8.1 & -- \\
\hline 15 & -- & --- & 11.8 & 12.4 & 10.2 & -- & -- & --- & 6.5 & $\cdots$ & 7.8 & --- \\
\hline 16 & -- & --- & 11.1 & 12.6 & 9.9 & -- & 9.4 & --- & 6.5 & --- & 7.8 & -- \\
\hline 17 & --- & -- & --- & 12.9 & 9.6 & --- & 9.8 & -- & $6.4 \mathrm{~b}$ & -- & 6.7 & --- \\
\hline 18 & -- & --- & 11.7 & 12.1 & 9.6 & -- & 8.1 & --- & 6.1 & -- & 6.6 & -- \\
\hline 19 & $\cdots$ & --- & 11.9 & 12.7 & 9.6 & --- & 8.2 & --- & $5.1 \mathrm{~b}$ & --- & 6.8 & $\cdots$ \\
\hline 20 & -- & --- & 12.6 & 13.9 & 9.6 & -- & 8.7 & -- & $5.2 b$ & -- & 6.5 & --- \\
\hline 21 & -- & --- & 12.2 & 11.7 & 10.0 & --- & 9.1 & -- & $6.0 \mathrm{~b}$ & --- & $5.8 \mathrm{~b}$ & -- \\
\hline 22 & -- & --- & 12.4 & 12.6 & 9.5 & -- & 8.9 & -- & 7.1 & --- & -- & --- \\
\hline 23 & --- & --- & -- & 13.3 & 9.0 & --- & 8.9 & -- & $5.8 b$ & --- & $-\cdots$ & $\cdots-$ \\
\hline 24 & --- & --- & -- & 14.1 & 8.6 & --- & 8.5 & -- & $5.7 b$ & --- & -- & -- \\
\hline 25 & -- & -- & -- & 13.2 & 8.7 & -- & 8.4 & -- & 6.5 & $\cdots$ & $\cdots$ & --- \\
\hline 26 & --- & --- & -- & -- & 9.6 & --- & 8.6 & --- & 6.1 & --- & $\cdots$ & -- \\
\hline 27 & -- & --- & $-\infty$ & -- & 10.5 & --- & 9.0 & --- & 6.0 & --- & $\cdots$ & --- \\
\hline 28 & --- & 13.1 & -- & -- & 10.4 & -- & 8.8 & --- & --- & --- & $\cdots$ & $\cdots$ \\
\hline 29 & --- & 12.3 & $-\infty$ & --- & --- & --- & 8.7 & --- & --- & $-\cdots$ & -- & $\cdots$ \\
\hline 30 & --- & 11.9 & --- & --- & -- & --- & 8.3 & -- & --- & --- & --- & $\cdots$ \\
\hline 31 & -- & -- & -- & 11.3 & --- & --- & --- & -- & --- & --- & $\cdots$ & $\cdots$ \\
\hline
\end{tabular}

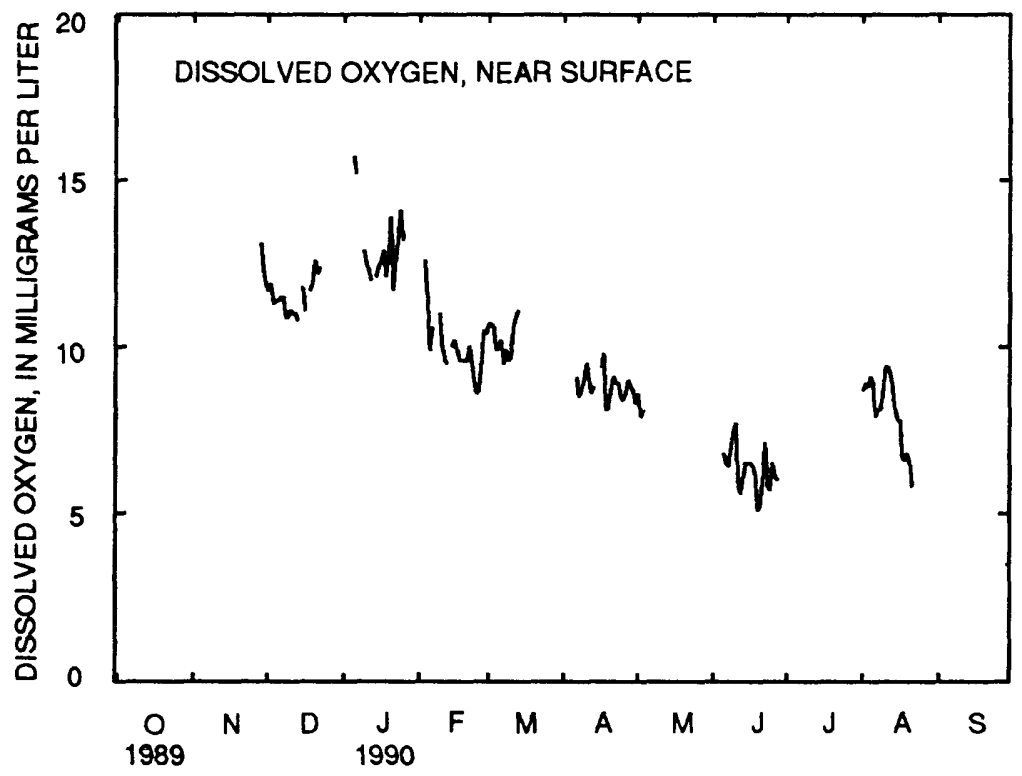


Table 8.--Dally mean values of salinity, water temperature, and dissolved oxygen in water at site 6, Pungo River at Junction Light PR, May 1989 to September 1990--Continued

[S1te 6 is at latitude $35^{\circ} 22^{\prime} 42^{\prime \prime}$, longitude $76^{\circ} 33^{\prime} 24^{\prime \prime}$, Beaufort County; U.S. Geological Survey downstream order number 0208457700; ppt, parts per thousand; ---, no data; ${ }^{\circ} \mathrm{C}$, degrees

Celsius; mg/L, mllilgrams per liter; a, dally minimum value is less than or equal to $2.0 \mathrm{mg} / \mathrm{L}$; b, daily minimum value is less than or equal to $5.0 \mathrm{mg} / \mathrm{L}$ but greater than $2.0 \mathrm{mg} / \mathrm{L}$; <, less than]

DISSOLVED OXYGEN, MID-DEPTH

SENSOR POSITION. --6 feet above streambed.

EXTREMES FOR OCTOBER 1989 TO SEPTEMBER 1990.--Maximum value recorded, 14.0 mg/L December 20, 1989 ; minimum value recorded, $2.4 \mathrm{mg} / \mathrm{L}$ June $23,1990$.

DAILY MEAN VALUES OF DISSOLVED OXYGEN, MID-DEPTH (IN MILLIGRAMS PER LITER), OCTOBER 1989 TO SEPTEMBER 1990

\begin{tabular}{|c|c|c|c|c|c|c|c|c|c|c|c|c|}
\hline DAY & OCT & NOV & DEC & JAN & FEB & MAR & $A P R$ & MAY & JUNE & JULY & AUG & SEPT \\
\hline 1 & -- & -- & 12.0 & -- & --- & 10.9 & -- & $-\cdots$ & 8.8 & -- & 7.8 & --- \\
\hline 2 & --- & -- & 12.1 & --- & --- & 10.3 & $-\cdots$ & $-\infty$ & 8.9 & -- & 7.7 & --- \\
\hline 3 & -- & -- & 11.5 & -- & 12.6 & 10.4 & -- & -- & $-\infty$ & -- & 7.9 & --- \\
\hline 4 & -- & -- & 11.7 & -- & 11.7 & 10.4 & -- & 8.6 & 8.0 & --- & 8.1 & -- \\
\hline 5 & -- & -- & 11.7 & --- & 10.1 & 10.4 & -- & 8.0 & 7.6 & --- & $7.1 \mathrm{~b}$ & $-\cdots$ \\
\hline 6 & -- & -- & 11.9 & -- & 10.3 & 10.7 & 9.8 & 7.8 & 7.8 & -- & $6.9 b$ & --- \\
\hline 7 & -- & -- & 11.8 & -- & -- & 10.4 & 9.6 & 7.7 & 7.9 & -- & 6.7 & -- \\
\hline 8 & --- & -- & 11.1 & -- & --- & 10.7 & 9.5 & 8.3 & 7.8 & --- & $7.2 \mathrm{~b}$ & -- \\
\hline 9 & --- & --- & 11.0 & --- & 11.1 & 10.5 & 10.2 & 8.5 & 7.7 & -- & $6.3 b$ & --- \\
\hline 10 & --- & --- & 11.1 & -- & 10.0 & 10.5 & 10.6 & 8.2 & 7.2 & -- & 8.9 & -- \\
\hline 11 & -- & -- & 11.0 & -- & 9.6 & 11.1 & 9.9 & 8.2 & $6.7 \mathrm{~b}$ & --- & 8.7 & $\cdots$ \\
\hline 12 & -- & --- & 11.1 & -- & 9.5 & 11.2 & 9.3 & 8.1 & 6.9 & --- & $8.1 b$ & -- \\
\hline 13 & -- & -- & 11.0 & -- & --- & 11.1 & 9.7 & 8.3 & 7.5 & --- & $8.1 \mathrm{~b}$ & -- \\
\hline 14 & --- & -- & --- & -- & 9.9 & --- & --- & 8.6 & 7.6 & --- & 7.7 & --- \\
\hline 15 & --- & --- & 11.8 & --- & 10.1 & --- & --- & 9.5 & 7.7 & --- & 7.6 & --- \\
\hline 16 & --- & -- & 11.3 & -- & 9.9 & --- & 10.0 & 8.9 & 7.7 & -- & 7.9 & -- \\
\hline 17 & -- & -- & -- & -- & 9.6 & -- & 10.1 & 8.3 & 7.6 & $6.3 b$ & 6.7 & -- \\
\hline 18 & --- & -- & 12.0 & -- & 9.5 & --- & 9.0 & 8.0 & $6.9 \mathrm{~b}$ & 6.6 & 7.4 & --- \\
\hline 19 & --- & --- & 12.1 & -- & 9.5 & --- & 9.1 & 8.1 & 6.3 & $6.1 \mathrm{~b}$ & 7.2 & -- \\
\hline 20 & --- & --- & 12.6 & --- & 9.5 & --- & 9.7 & 8.6 & 6.2 & $6.3 b$ & 7.2 & -- \\
\hline 21 & --- & -- & 12.4 & -- & 9.3 & -- & 9.9 & 8.2 & $6.2 \mathrm{~b}$ & $6.3 b$ & 6.4 & -- \\
\hline 22 & --- & --- & 12.6 & -- & 9.0 & $m$ & 9.7 & 7.4 & $5.8 b$ & $5.3 b$ & -- & -- \\
\hline 23 & -- & -- & -- & -- & 8.9 & -- & 9.4 & 8.2 & $5.7 \mathrm{~b}$ & $5.8 \mathrm{~b}$ & -- & -- \\
\hline 24 & -- & --- & --- & -- & 8.7 & --- & 9.0 & 8.5 & 5.8 & $6.0 \mathrm{~b}$ & $\cdots$ & -- \\
\hline 25 & --- & -- & --- & --- & 8.9 & --- & --- & 9.3 & $5.8 b$ & $5.6 b$ & -- & --- \\
\hline 26 & --- & -- & --- & -- & 9.7 & --- & --- & 9.7 & 6.2 & $5.0 \mathrm{~b}$ & $-\cdots$ & -- \\
\hline 27 & -- & -- & --- & --- & 10.5 & --- & -- & 8.8 & $-\cdots$ & $4.6 \mathrm{~b}$ & --- & -- \\
\hline 28 & -- & 13.4 & -- & -- & 10.5 & --- & -- & -- & -- & $4.3 b$ & --- & -- \\
\hline 29 & --- & 12.7 & --- & -- & --- & -- & -- & 8.0 & $-\cdots$ & --- & -- & $-\cdots$ \\
\hline 30 & --- & 12.2 & -- & -- & --- & -- & -- & -- & -- & -- & $-\cdots$ & -- \\
\hline 31 & -- & --- & --- & 11.7 & --- & --- & --- & 8.2 & --- & --- & --- & --- \\
\hline
\end{tabular}

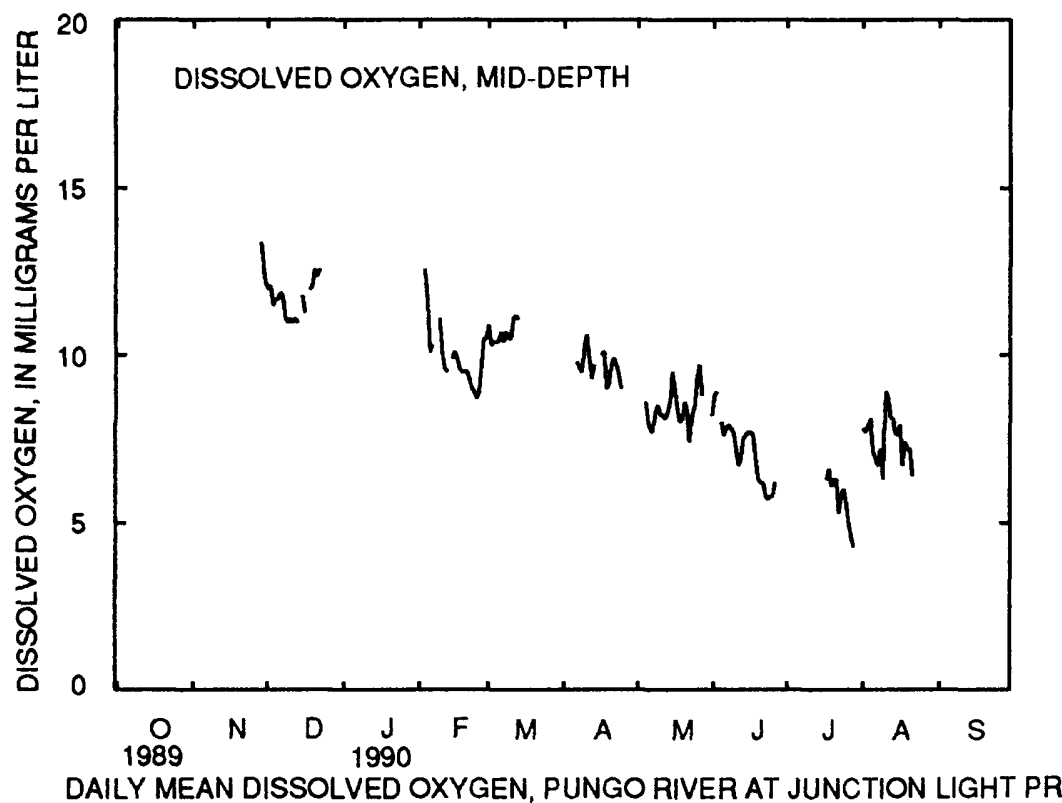


Table 8.--Daily mean values of salinity, water temperature, and dissolved oxygen in water at site 6, Pungo River at Junction Light PR, May 1989 to september 1990--Continued

[Site 6 is at latitude $35^{\circ} 22^{\prime} 42^{\prime \prime}$, longltude $76^{\circ} 33^{\prime} 24^{\prime \prime}$, Beaufort County; U.S. Geological Survey downstream order number 0208457700; ppt, parts per thousand; ---, no data; ${ }^{\circ} \mathrm{C}$, degrees

Celsius; mg/L, milligrams per liter; a, dally minimum value is less than or equal to $2.0 \mathrm{mg} / \mathrm{L}$; b, daily minimum value is less than or equal to $5.0 \mathrm{mg} / \mathrm{L}$ but greater than $2.0 \mathrm{mg} / \mathrm{L} ;<$, less than]

DISSOLVED OXYGEN, NEAR BOTTOM

SENSOR POSITION. --2 feet above streambed.

EXTREMES FOR MAY TO SEPTEMBER 1989.--Maximum value recorded, $12.9 \mathrm{mg} / \mathrm{L}$ May 20, $1989 ; \mathrm{min} 1 \mathrm{mum}$ value recorded, <1.0 $\mathrm{mg} / \mathrm{L}$ September 1, 1989.

DAILY MEAN VALUES OF DISSOLVED OXYGEN, NEAR BOTTOM (IN MILLIGRAMS PER LITER), MAY TO SEPTEMBER 1989

\begin{tabular}{|c|c|c|c|c|c|}
\hline DAY & MAY & JUNE & JULY & AUG & SEPT \\
\hline 1 & --- & $4.9 a$ & --- & --- & $3.0 \mathrm{a}$ \\
\hline 2 & --- & $5.2 a$ & -- & --- & $4.2 b$ \\
\hline 3 & --- & $5.5 b$ & --- & -- & $4.7 b$ \\
\hline 4 & $5.6 b$ & 6.8 & --- & --- & $6.0 \mathrm{~b}$ \\
\hline 5 & --- & $7.0 \mathrm{~b}$ & --- & --- & $6.1 \mathrm{~b}$ \\
\hline 6 & -- & 7.6 & --- & --- & $6.2 b$ \\
\hline 7 & --- & $6.6 b$ & --- & --- & $7.0 \mathrm{~b}$ \\
\hline 8 & --- & $4.7 \mathrm{a}$ & --- & --- & $6.7 \mathrm{~b}$ \\
\hline 9 & $\cdots$ & 8.0 & --- & --- & $4.6 \mathrm{~b}$ \\
\hline 10 & --- & $6.2 \mathrm{a}$ & --- & --- & $4.4 b$ \\
\hline 11 & -- & $6.2 b$ & --- & --- & $4.6 b$ \\
\hline 12 & --- & $7.1 \mathrm{~b}$ & --- & --- & --- \\
\hline 13 & --- & 8.5 & --- & --- & --- \\
\hline 14 & --- & $5.9 b$ & --- & --- & --- \\
\hline 15 & -- & $8.2 b$ & --- & --- & -- \\
\hline 16 & --- & 8.0 & --- & --- & -- \\
\hline 17 & --- & 7.8 & --- & --- & --- \\
\hline 18 & --- & $7.4 b$ & --- & --- & --- \\
\hline 19 & 10.8 & $-\cdots$ & --- & --- & --- \\
\hline 20 & 10.1 & --- & --- & --- & --- \\
\hline 21 & 10.2 & --- & --- & --- & --- \\
\hline 22 & 10.8 & --- & --- & --- & -- \\
\hline 23 & 10.2 & --- & --- & --- & --- \\
\hline 24 & 9.1 & --- & --- & --- & --- \\
\hline 25 & 8.1 & --- & --- & --- & --- \\
\hline 26 & 7.8 & --- & --- & $4.6 \mathrm{~b}$ & --- \\
\hline 27 & 7.8 & --- & --- & $5.3 b$ & --- \\
\hline 28 & 7.8 & --- & --- & $4.6 a$ & --- \\
\hline 29 & 7.7 & $-\cdots$ & --- & $3.5 a$ & --- \\
\hline 30 & 7.6 & --- & --- & $4.6 \mathrm{~b}$ & --- \\
\hline 31 & $6.9 \mathrm{~b}$ & --- & --- & $4.7 \mathrm{~b}$ & --- \\
\hline
\end{tabular}

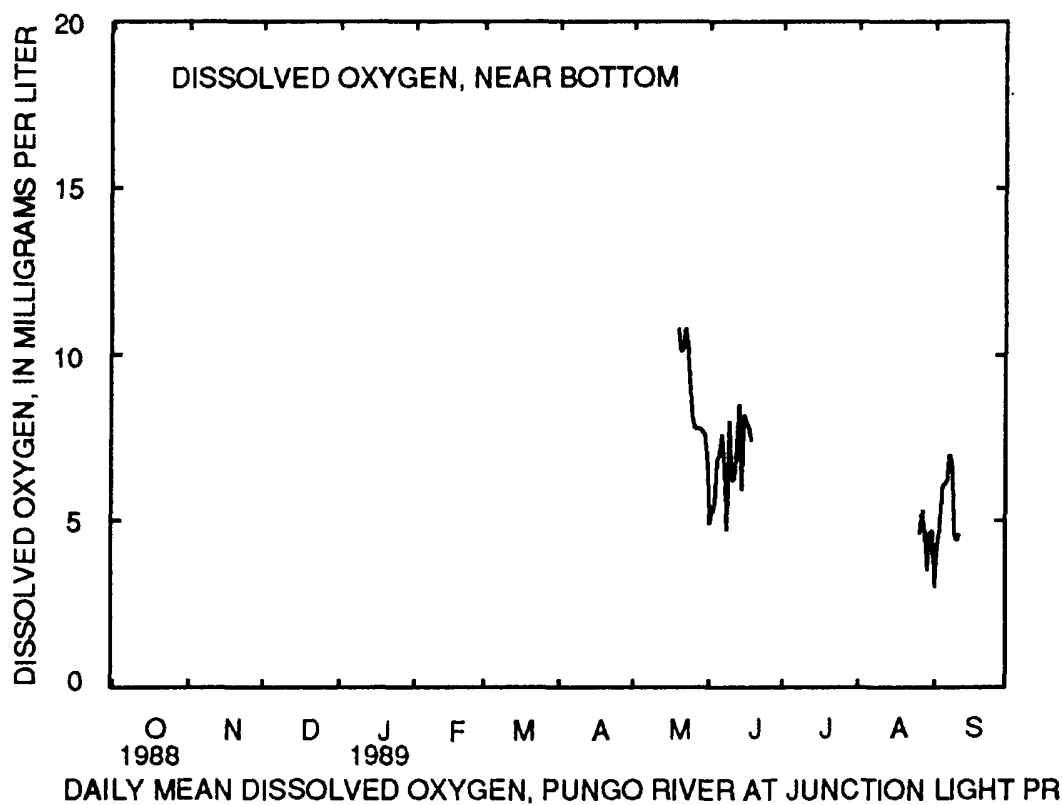


Table 8.--Daily mean values of salinity, water temperature, and dissolved oxygen in water at site 6, Pungo River at Junction Light PR, May 1989 to September 1990--Continued

[Site 6 is at latitude $35^{\circ} 22^{\prime} 42^{\prime \prime}$, longltude $76^{\circ} 33^{\prime} 24^{\prime \prime}$, Beaufort County; U.S. Geological Survey downstream order number 0208457700 ; ppt, parts per thousand; ---, no data; ${ }^{\circ} \mathrm{C}$, degrees

Celsius; mg/L, milligrams per liter; a, dally minimum value is less than or equal to $2.0 \mathrm{mg} / \mathrm{L}$; $\mathrm{b}$, daily minimum value is less than or equal to $5.0 \mathrm{mg} / \mathrm{L}$ but greater than $2.0 \mathrm{mg} / \mathrm{L}$; <, less than]

DISSOLVED OXYGEN, NEAR BOTTOM

SENSOR POSITION.--2 feet above streambed.

EXTREMES FOR OCTOBER 1989 TO SEPTEMBER 1990.--Maximum value recorded, 18.4 mg/L January 6, 1990; minlmum value recorded, <1.0 mg/L June 5, 6, August 5, 7-16, 21, 1990.

DAILY MEAN VALUES OF DISSOLVED OXYGEN, NEAR BOTTOM (IN MILLIGRAMS PER LITER), OCTOBER 1989 TO SEPTEMBER 1990

\begin{tabular}{|c|c|c|c|c|c|c|c|c|c|c|c|c|}
\hline DAY & OCT & Nov & $D E C$ & JAN & FEB & MAR & APR & MAY & JUNE & JULY & AUG & SEPT \\
\hline 1 & --- & --- & 11.4 & -- & -- & 9.6 & $-\infty$ & -- & --- & --- & $4.6 b$ & -- \\
\hline 2 & -- & -- & 11.6 & -- & -- & 9.9 & -- & -- & --- & --- & $4.5 b$ & -- \\
\hline 3 & -- & -- & 11.2 & -- & 11.1 & 9.3 & -- & -- & --- & -- & $4.2 b$ & --- \\
\hline 4 & -- & -- & 11.4 & --- & 9.7 & 10.1 & $-\infty$ & -- & -- & --- & $4.3 b$ & -- \\
\hline 5 & --- & --- & 11.5 & 17.8 & 11.1 & 9.3 & -- & -- & $5.3 a$ & --- & $<1.0 \mathrm{a}$ & -- \\
\hline 6 & --- & --- & 11.7 & 17.7 & 10.2 & 9.6 & 9.4 & -- & $4.4 \mathrm{a}$ & -- & $3.7 b$ & --- \\
\hline 7 & --- & -- & 11.7 & -- & --- & 10.1 & 9.7 & -- & $4.8 a$ & --- & $3.9 a$ & -+ \\
\hline 8 & --- & --- & 11.0 & -- & -- & 10.0 & 9.5 & -- & $4.2 b$ & -- & $5.0 \mathrm{a}$ & --- \\
\hline 9 & --- & --- & 10.9 & 15.8 & 8.8 & 9.2 & 9.3 & -- & $3.4 \mathrm{~b}$ & --- & $2.1 \mathrm{a}$ & -- \\
\hline 10 & --- & --- & 11.0 & 15.4 & 10.5 & 9.6 & 9.8 & --- & $4.9 a$ & --- & $3.9 a$ & --- \\
\hline 11 & --- & -- & 11.0 & 15.5 & 10.1 & 9.9 & 9.8 & -- & $5.4 \mathrm{~b}$ & --- & $1.8 a$ & -- \\
\hline 12 & -- & --- & 10.8 & 15.3 & 9.9 & 8.4 & 8.9 & -- & $6.0 \mathrm{~b}$ & --- & $2.0 a$ & -- \\
\hline 13 & --- & -- & 10.2 & -- & -- & 8.7 & 9.4 & $-\infty$ & $5.3 b$ & --- & $2.5 a$ & --- \\
\hline 14 & -- & --- & 10.2 & 15.8 & 10.0 & -- & -- & -- & $4.3 a$ & --- & $2.8 \mathrm{a}$ & --- \\
\hline 15 & --- & --- & 10.8 & 16.2 & 9.8 & --- & -- & $\cdots$ & $4.6 a$ & --- & $2.6 a$ & $-\cdots$ \\
\hline 16 & -- & --- & 10.5 & -- & 10.3 & --- & 7.8 & -- & $4.3 b$ & --- & $1.1 \mathrm{a}$ & -- \\
\hline 17 & -- & -- & --- & -- & 9.5 & --- & 6.8 & -- & $3.6 a$ & --- & $2.4 \mathrm{a}$ & -- \\
\hline 18 & --- & --- & 11.0 & -- & 9.4 & --- & 8.9 & --- & --- & -- & -- & --- \\
\hline 19 & -- & --- & 11.2 & -- & 9.4 & -- & 9.5 & -- & --- & -- & --- & -- \\
\hline 20 & --- & --- & 11.5 & -- & 9.6 & --- & 9.3 & --- & -- & --- & $\cdots$ & -- \\
\hline 21 & -- & --- & 11.4 & --- & 9.2 & -- & --- & --- & -- & --- & $2.3 a$ & $\ldots$ \\
\hline 22 & -- & --- & 11.5 & --- & 8.8 & --- & --- & --- & -- & --- & --- & --- \\
\hline 23 & --- & --- & --- & --- & 8.9 & -- & -- & --- & -- & --- & --- & --- \\
\hline 24 & -- & --- & --- & --- & 8.7 & -- & -- & --- & --- & -- & -- & -- \\
\hline 25 & --- & --- & --- & 11.8 & 8.9 & -- & --- & --- & -- & -- & -- & $\cdots$ \\
\hline 26 & $-\cdots$ & -- & --- & --- & 9.3 & -- & -- & --- & --- & --- & -- & -- \\
\hline 27 & --- & --- & --- & -- & 9.5 & --- & -- & --- & --- & -- & -- & --- \\
\hline 28 & -- & 13.0 & --- & --- & 9.0 & --- & --- & --- & -- & -- & -- & --- \\
\hline 29 & --- & 12.3 & --- & -- & --- & --- & -- & --- & --- & --- & $\cdots$ & -- \\
\hline 30 & --- & 11.8 & --- & --- & --- & --- & -- & -- & --- & --- & -- & --- \\
\hline 31 & --- & --- & --- & 12.2 & --- & --- & --- & --- & -- & --- & -- & -- \\
\hline
\end{tabular}

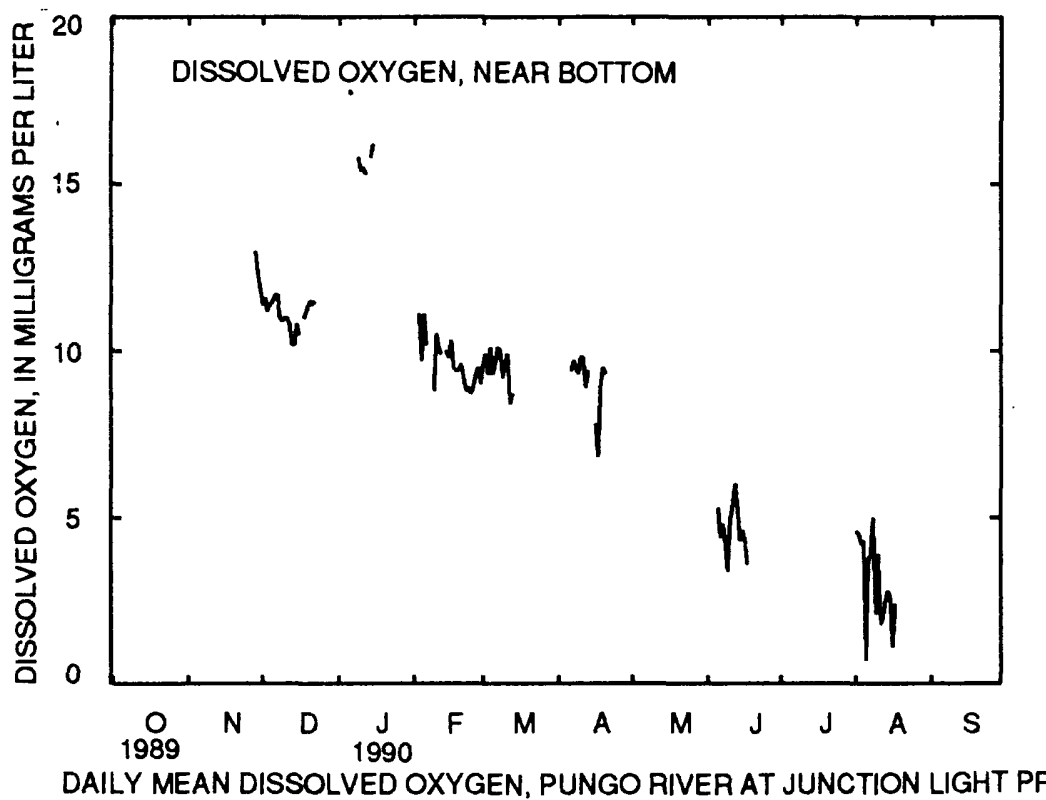


Table 9.--Daily mean values of salinity, water temperature, and dissolved oxygen in water at site 7, Neuse River at Channel Light 22, May to December 1989

[Site 7 is at latitude $35^{\circ} 04^{\prime} 48^{\prime \prime}$, longitude $77^{\circ} 00^{\prime} 24^{\prime \prime}$, Craven County; U. S. Geological Survey
downstream order number 0209258400 ; ppt, parts per thousand; <, less than; - - no data; ${ }^{\circ} \mathrm{C}$,
degrees Celsius; mg/L, milligrams per liter; a, daily minimum value is less than or equal

to $2.0 \mathrm{mg} / \mathrm{L} ; \mathrm{b}$, dally minimum value $1 \mathrm{~s}$ less than or equal to $5.0 \mathrm{mg} / \mathrm{L}$ but greater than $2.0 \mathrm{mg} / \mathrm{L}\}$

SALINITY, NEAR SURFACE

SENSOR POSITION. --12 feet above st reambed.

REMARKS.--Station discontinued December 1989.

EXTREMES FOR MAY TO DECEMBER 1989.--Maximum value recorded, 9.2 ppt August 7,1989 ; minimum value recorded, <0.1 ppt May 12-17, July 23, 27, 28, December 3, 6, 11, 12, 1989.

DAILY MEAN VALUES OF SALINITY, NEAR SURFACE (IN PARTS PER THOUSAND), MAY TO DECEMBER 1989

\begin{tabular}{|c|c|c|c|c|c|c|c|c|}
\hline DAY & MAY & JUNE & JULY & AUG & SEPT & OCT & NOV & DEC \\
\hline 1 & --- & 0.2 & -- & 0.2 & 0.6 & --- & -- & 0.5 \\
\hline 2 & --- & .2 & -- & .2 & .6 & -- & $\ldots$ & .2 \\
\hline 3 & -- & .6 & -- & .2 & 1.2 & --- & -- & .3 \\
\hline 4 & -- & .4 & -- & .2 & 1.9 & -- & - - & .8 \\
\hline 5 & --- & .3 & $\cdots$ & .4 & 1.6 & -- & --- & .5 \\
\hline 6 & $\ldots$ & .2 & -- & .8 & 1.5 & -- & --- & .3 \\
\hline 7 & $\ldots$ & .2 & - - & 2.1 & 1.4 & -- & -- & .6 \\
\hline 8 & -- & .3 & -- & 6.8 & 1.3 & -- & -- & 1.9 \\
\hline 9 & $\ldots$ & -- & -- & 6.1 & 1.1 & --- & - - & 1.8 \\
\hline 10 & --- & -- & -- & 7.1 & 1.1 & -- & --- & .5 \\
\hline 11 & --- & .7 & -- & 3.8 & 1.1 & --- & $\ldots$ & .1 \\
\hline 12 & $<0.1$ & .4 & -- & 2.9 & 1.2 & --- & --- & .1 \\
\hline 13 & $<.1$ & $\ldots$ & $\sim$ & 3.4 & 1.5 & -- & --- & .7 \\
\hline 14 & $<.1$ & --- & -- & 2.7 & 1.2 & -- & -- & -- \\
\hline 15 & $<.1$ & --- & --- & 1.5 & 1.3 & --- & --- & -- \\
\hline 16 & $<.1$ & -- & -- & 1.6 & 1.4 & -- & -- & -- \\
\hline 17 & .1 & - & -- & 1.7 & 1.4 & -- & -- & $\ldots$ \\
\hline 18 & .5 & -- & -- & 1.4 & - & - - & --- & $\ldots$ \\
\hline 19 & .3 & - & -- & 1.2 & -- & - - & --- & -- \\
\hline 20 & .2 & -- & $\ldots$ & .7 & -- & --- & --- & --- \\
\hline 21 & .1 & $\ldots$ & 0.4 & -- & -- & 0.5 & --- & -- \\
\hline 22 & .1 & - & .2 & -- & -- & .8 & - - & $\ldots$ \\
\hline 23 & .1 & -- & .1 & .6 & -- & 1.1 & -- & -- \\
\hline 24 & .1 & -- & .2 & 1.1 & $\cdots$ & 1.5 & --- & -- \\
\hline 25 & .1 & --- & .2 & 1.7 & -- & 2.6 & --- & -- \\
\hline 26 & .1 & -- & .2 & 1.1 & $\ldots$ & -- & $\ldots$ & - - \\
\hline 27 & .1 & -- & .1 & .9 & - & $-\cdots$ & -- & - \\
\hline 28 & .9 & $\ldots$ & .1 & .5 & -- & -- & -- & -- \\
\hline 29 & .3 & --- & .5 & .4 & --- & -- & -- & -- \\
\hline 30 & .2 & -- & .3 & .4 & -- & - - & 0.5 & -- \\
\hline 31 & .2 & -- & .2 & .7 & --- & -- & -- & -- \\
\hline
\end{tabular}

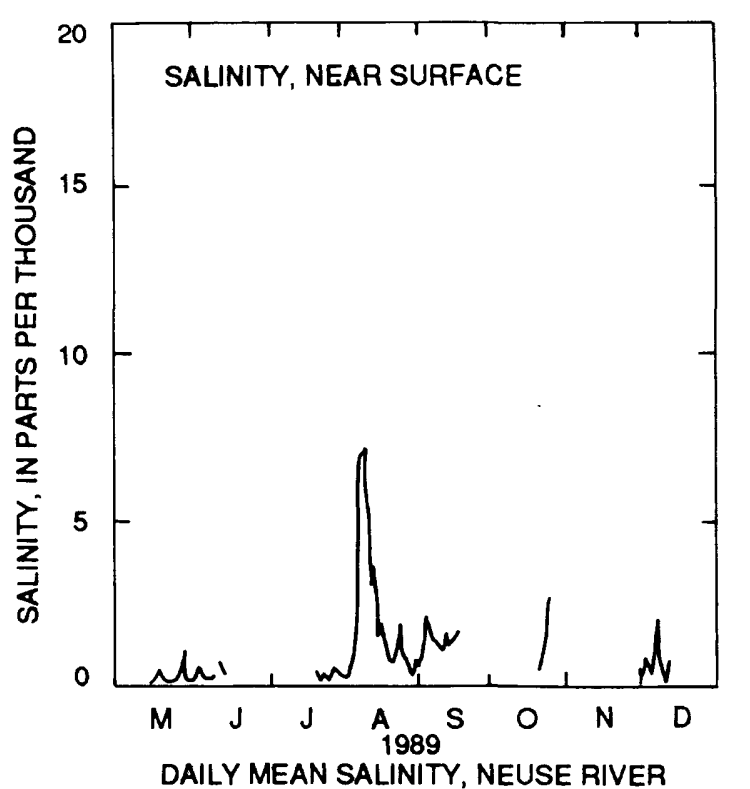


Table 9.--Daily mean values of salinity, water temperature, and dissolved oxygen in water at site 7, Neuse River at Channel Light 22, May to December 1989--Continued

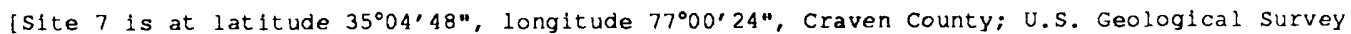
downstream order number 0209258400 ; ppt, parts per thousand; <, less than; ---, no data; ${ }^{\circ} \mathrm{C}$, degrees Celsius; mg/L, milligrams per liter; a, dally minimum value is less than or equal to $2.0 \mathrm{mg} / \mathrm{L} ; \mathrm{b}$, daily minimum value is less than or equal to $5.0 \mathrm{mg} / \mathrm{L}$ but greater than $2.0 \mathrm{mg} / \mathrm{L}$ ]

SALINITY, NEAR BOTTOM

SENSOR POSITION.--2 feet above streambed.

REMARKS.--Station discontinued December 1989.

EXTREMES FOR MAY TO DECEMBER 1989.--Maximum value recorded, 12.0 ppt August 8, 9, 1989; minimum value recorded, $<0.1$ ppt May 12-17, 24, 1989.

DAILY MEAN VALUES OF SALINITY, NEAR BOTTOM (IN PARTS PER THOUSAND), MAY TO DECEMBER 1989

\begin{tabular}{|c|c|c|c|c|c|c|c|c|}
\hline DAY & MAY & JUNE & JULY & AUG & SERT & $O C T$ & NOV & DEC \\
\hline 1 & --- & 0.9 & --- & 6.2 & 7.1 & --- & --- & 2.6 \\
\hline 2 & -- & 2.7 & --- & 6.3 & 6.8 & -- & --- & 4.0 \\
\hline 3 & --- & 1.9 & -- & 6.4 & 6.5 & --- & --- & .9 \\
\hline 4 & --- & .5 & -- & 7.3 & 5.7 & -- & --- & 3.2 \\
\hline 5 & --- & .4 & -- & 9.2 & 2.7 & --- & $-\cdots$ & 3.1 \\
\hline 6 & -- & .2 & --- & 11.0 & 2.8 & --- & -- & 2.7 \\
\hline 7 & -- & .2 & --- & 11.2 & 2.3 & --- & --- & 3.2 \\
\hline 8 & -- & .6 & --- & 11.9 & 2.2 & -- & -- & 3.0 \\
\hline 9 & --- & .4 & -- & 11.8 & 2.2 & --- & --- & 2.7 \\
\hline 10 & --- & 1.0 & --- & 9.3 & 2.2 & --- & --- & 1.4 \\
\hline 11 & --- & 2.4 & --- & 8.4 & 2.6 & -- & --- & 2.3 \\
\hline 12 & $<0.1$ & 1.2 & --- & 9.2 & 3.2 & --- & --- & 5.5 \\
\hline 13 & $<.1$ & $\cdots$ & --- & 9.2 & 3.7 & -- & -- & 4.3 \\
\hline 14 & $<.1$ & --- & --- & 9.6 & 4.0 & -- & -- & -- \\
\hline 15 & $<.1$ & $-\cdots$ & -- & 10.0 & 4.3 & --- & --- & --- \\
\hline 16 & $<.1$ & $\cdots$ & -- & 9.9 & 3.1 & --- & -- & --- \\
\hline 17 & 5.1 & -- & $-\cdots$ & 9.5 & 2.8 & -- & --- & --- \\
\hline 18 & 9.8 & --- & --- & 8.9 & -- & --- & --- & -- \\
\hline 19 & 9.1 & --- & --- & 8.3 & --- & -- & --- & --- \\
\hline 20 & 9.4 & --- & --- & 7.7 & -- & $\cdots$ & --- & --- \\
\hline 21 & 8.3 & --- & 3.3 & 5.3 & --- & 6.1 & --- & --- \\
\hline 22 & 5.4 & -- & 3.4 & 5.0 & -- & 7.4 & --- & -- \\
\hline 23 & .2 & $\cdots$ & 4.5 & 6.6 & -- & 7.8 & --- & --- \\
\hline 24 & 1.2 & --- & 6.6 & 9.1 & --- & 7.7 & -- & -- \\
\hline 25 & 3.1 & $\cdots$ & $\cdot 7.5$ & 10.6 & --- & 7.2 & --- & $-\cdots$ \\
\hline 26 & .1 & --- & 7.4 & 10.4 & -- & --- & -- & --- \\
\hline 27 & 2.3 & --- & 6.8 & 9.7 & --- & --- & -- & -- \\
\hline 28 & 3.3 & --- & 5.2 & 8.2 & --- & --- & --- & --- \\
\hline 29 & 1.4 & -- & 7.8 & 7.5 & --- & -- & --- & -- \\
\hline 30 & .4 & -- & 7.3 & 6.3 & -- & -- & 1.5 & -- \\
\hline 31 & .6 & --- & 2.8 & 7.6 & --- & --- & --- & --- \\
\hline
\end{tabular}

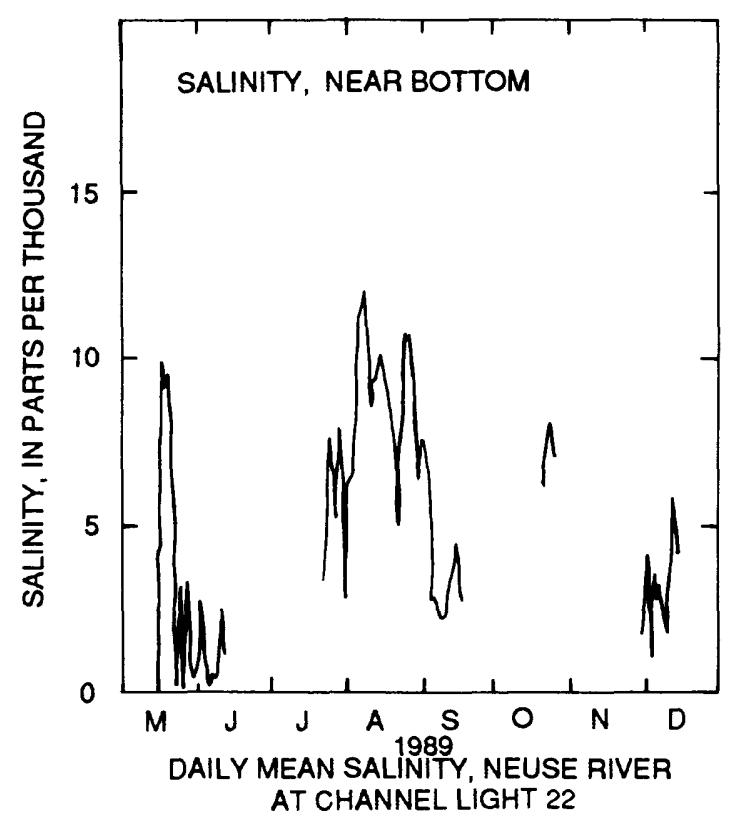


Table 9.--Daily mean values of salinity, water temperature, and dissolved oxygen in water at site 7, Neuse River at Channel Light 22, May to December 1989--Continued

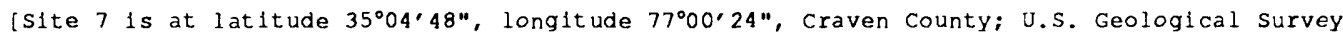
downstream order number 0209258400 ; ppt, parts per thousand; <, less than; ---, no data; ${ }^{\circ} \mathrm{C}$, degrees Celsius; $\mathrm{mg} / \mathrm{L}$, milligrams per liter; a, daily minimum value is less than or equal

to $2.0 \mathrm{mg} / \mathrm{L} ; \mathrm{b}$, daily minimum value is less than or equal to $5.0 \mathrm{mg} / \mathrm{L}$ but greater than $2.0 \mathrm{mg} / \mathrm{L}$ ]

WATER TEMPERATURE, NEAR SURFACE

SENSOR POSITION. --12 feet above streambed.

REMARKS.--Station discontinued December 1989.

EXTREMES FOR MAY TO DECEMBER 1989.--Maximum value recorded, $31.3{ }^{\circ} \mathrm{C}$ July 26,$1989 ;$ minimum value recorded, $4.4{ }^{\circ} \mathrm{C}$ December 4,1989 .

DAILY MEAN VALUES OF WATER TEMPERATURE, NEAR SURFACE (IN DEGREES CELSIUS), MAY TO DECEMBER 1989

\begin{tabular}{|c|c|c|c|c|c|c|c|c|}
\hline DAY & MAY & JUNE & JULY & AUG & SEPT & OCT & NOV & DEC \\
\hline 1 & -- & 25.2 & --- & 26.9 & 26.9 & --- & --- & 10.6 \\
\hline 2 & -- & 25.8 & --- & 26.3 & 27.2 & --- & --- & 10.3 \\
\hline 3 & -- & 25.7 & --- & 26.7 & 26.8 & --- & --- & 8.7 \\
\hline 4 & -- & 25.6 & --- & 27.1 & 26.1 & --- & --- & 5.9 \\
\hline 5 & -- & 25.4 & --- & 27.3 & 25.4 & --- & --- & 6.2 \\
\hline 6 & -- & 24.8 & --- & 28.0 & 25.5 & --- & --- & 7.1 \\
\hline 7 & --- & 24.5 & --- & 28.5 & 25.3 & --- & --- & 8.3 \\
\hline 8 & -- & 24.4 & --- & 26.6 & 25.5 & --- & $\cdots$ & 8.5 \\
\hline 9 & --- & 23.8 & --- & 24.4 & 25.9 & $-\cdots$ & --- & 7.8 \\
\hline 10 & $-\cdots$ & 24.7 & --- & 23.9 & 26.2 & --- & --- & 7.7 \\
\hline 11 & --- & 24.7 & --- & 23.1 & 26.9 & --- & --- & 7.7 \\
\hline 12 & 17.2 & 24.9 & --- & 23.3 & 27.8 & --- & -- & 7.8 \\
\hline 13 & 17.0 & --- & --- & 24.1 & 27.9 & --- & --- & 7.6 \\
\hline 14 & 17.2 & --- & --- & 24.8 & 27.3 & --- & --- & --- \\
\hline 15 & 17.6 & --- & --- & 24.3 & 27.2 & --- & --- & --- \\
\hline 16 & 17.7 & --- & --- & 24.9 & 27.4 & --- & --- & -- \\
\hline 17 & 17.3 & --- & --- & 26.2 & 27.2 & --- & --- & --- \\
\hline 18 & 18.0 & --- & --- & 26.7 & --- & --- & --- & --- \\
\hline 19 & 18.6 & --- & --- & 26.2 & --- & --- & --- & --- \\
\hline 20 & 18.6 & --- & --- & 26.2 & --- & --- & --- & -- \\
\hline 21 & 19.6 & --- & -- & 27.0 & --- & 19.7 & --- & -- \\
\hline 22 & 20.7 & --- & 26.5 & 27.7 & --- & 18.2 & --- & --- \\
\hline 23 & 21.1 & --- & 27.2 & 28.5 & --- & 17.6 & --- & -- \\
\hline 24 & 21.3 & --- & 27.2 & 28.2 & -- & 17.6 & --- & --- \\
\hline 25 & 21.5 & --- & 26.4 & 27.5 & $\cdots$ & 17.8 & --- & -- \\
\hline 26 & 22.7 & --- & 26.4 & 26.8 & --- & -- & --- & --- \\
\hline 27 & 23.8 & --- & 26.5 & 26.4 & --- & --- & --- & -- \\
\hline 28 & 23.2 & --- & 26.3 & 26.3 & -- & --- & --- & --- \\
\hline 29 & 22.9 & --- & 26.8 & 27.0 & --- & --- & --- & --- \\
\hline 30 & 23.2 & --- & 26.2 & 26.7 & --- & --- & 10.9 & --- \\
\hline 31 & 24.0 & --- & 26.6 & 26.8 & --- & --- & --- & --- \\
\hline
\end{tabular}

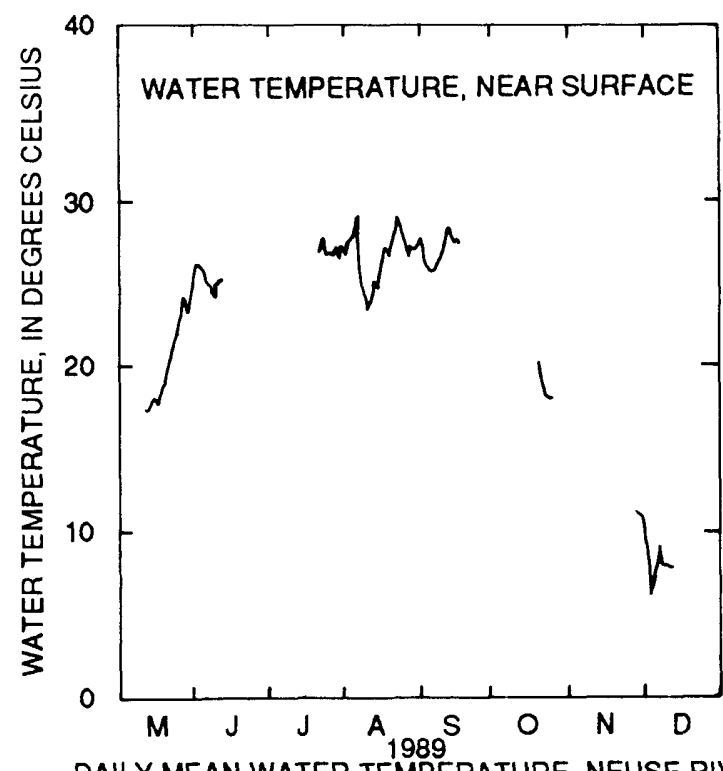


Table 9.--Daily mean values of salinity, water temperature, and dissolved oxygen in water at site 7, Neuse River at Channel Light 22, May to December 1989--Continued

IS1te 7 is at lat 1 tude $35^{\circ} 04^{\prime} 48^{\prime \prime}$, long1tude $77^{\circ} 00^{\prime} 24^{\prime \prime}$, Craven County; U.S. Geological Survey downstream order number 0209258400; ppt, parts per thousand; <, less than; ---, no data;

${ }^{\circ} \mathrm{C}$, degrees Celsius; mg/L, milligrams per liter; a, dally minimum value is less than or equal

to $2.0 \mathrm{mg} / \mathrm{L} ; \mathrm{b}$, dally minimum value $1 \mathrm{~s}$ less than or equal to $5.0 \mathrm{mg} / \mathrm{L}$ but greater than $2.0 \mathrm{mg} / \mathrm{L}$ ]

\section{DISSOLVED OXYGEN, NEAR SURFACE}

SENSOR POSITION.--12 feet above streambed.

REMARKS.--Station discontinued December 1989.

EXTREMES FOR MAY TO DECEMBER 1989.--Maximum value recorded, $13.3 \mathrm{mg} / \mathrm{L}$ September 12 , 1989 ; minimum value recorded, $<1.0 \mathrm{mg} / \mathrm{L}$ August $5,6,9,10,13,14,24,1989$.

DAILY MEAN VALUES OF DISSOLVED OXYGEN, NEAR SURFACE (IN MILLIGRAMS PER LITER), MAY TO DECEMBER 1989

\begin{tabular}{|c|c|c|c|c|c|c|c|c|}
\hline DAY & MAY & JUNE & JULY & AUG & SEPT & OCT & NOV & DEC \\
\hline 1 & --- & 8.5 & -- & $3.9 b$ & $6.3 \mathrm{~b}$ & -- & --- & 9.8 \\
\hline 2 & --- & 8.1 & --- & $3.5 b$ & 6.3 & -- & -- & 9.6 \\
\hline 3 & -- & 7.4 & --- & $3.2 \mathrm{~b}$ & $6.7 \mathrm{~b}$ & -- & --- & 10.3 \\
\hline 4 & -- & 7.3 & -- & $3.5 b$ & 7.6 & --- & $\ldots$ & 11.1 \\
\hline 5 & $-\cdots$ & 7.4 & --- & $3.1 \mathrm{a}$ & 7.3 & -- & -- & 11.3 \\
\hline 6 & --- & 7.3 & --- & $2.8 a$ & 7.1 & --- & -- & 11.0 \\
\hline 7 & --- & 7.2 & --- & --- & 7.4 & -- & --- & 11.1 \\
\hline 8 & --- & 7.4 & --- & --- & 7.6 & --- & --- & 11.2 \\
\hline 9 & --- & --- & --- & $2.7 a$ & 8.1 & -- & --- & 11.5 \\
\hline 10 & -- & 8.2 & -- & $1.9 a$ & 8.7 & --- & --- & 11.9 \\
\hline 11 & --- & 8.4 & -- & $2.4 a$ & 9.4 & -- & --- & 10.8 \\
\hline 12 & 6.1 & 9.0 & $-\cdots$ & $2.6 a$ & 10.8 & -- & -- & 10.3 \\
\hline 13 & 6.5 & -- & $-\cdots$ & $2.0 a$ & 10.7 & -- & --- & 10.9 \\
\hline 14 & 6.4 & -- & --- & $2.6 a$ & 8.8 & --- & --- & -- \\
\hline 15 & 6.7 & --- & -- & $4.1 \mathrm{~b}$ & 9.4 & -- & --- & -- \\
\hline 16 & 6.9 & -- & --- & $4.2 b$ & 9.3 & --- & --- & --- \\
\hline 17 & 7.2 & $\cdots$ & --- & $5.0 \mathrm{~b}$ & 9.0 & -- & $\cdots$ & -- \\
\hline 18 & 6.7 & --- & --- & $7.3 b$ & $\cdots$ & --- & --- & -- \\
\hline 19 & 6.7 & --- & --- & $6.8 b$ & -- & --- & --- & - \\
\hline 20 & 6.2 & --- & --- & $7.4 b$ & --- & --- & --- & --- \\
\hline 21 & 6.2 & --- & $5.0 \mathrm{~b}$ & -- & --- & 7.5 & -- & -- \\
\hline 22 & 6.2 & -- & $4.5 b$ & -- & -- & 8.1 & $\ldots$ & -- \\
\hline 23 & 6.3 & --- & $4.2 \mathrm{~b}$ & 6.5 & -. & 8.5 & -- & --- \\
\hline 24 & 6.8 & $\cdots$ & $3.9 \mathrm{~b}$ & $5.3 a$ & $-\cdots$ & 8.3 & -- & -- \\
\hline 25 & 6.3 & --- & $3.8 b$ & $3.8 \mathrm{a}$ & -- & 8.4 & --- & -- \\
\hline 26 & 6.6 & --- & $3.6 \mathrm{~b}$ & $5.2 b$ & $\cdots$ & --- & -- & -- \\
\hline 27 & 7.0 & -- & $3.8 \mathrm{~b}$ & $5.0 \mathrm{~b}$ & $-\cdots$ & -- & $-\cdots$ & --- \\
\hline 28 & 7.1 & --- & $3.9 b$ & $5.3 b$ & -- & --- & --- & --- \\
\hline 29 & 6.9 & --- & $3.7 b$ & 6.0 & -- & -- & --- & -- \\
\hline 30 & 7.3 & --- & $3.6 \mathrm{~b}$ & $6.1 b$ & -- & --- & 9.7 & -- \\
\hline 31 & 7.8 & --- & $3.9 \mathrm{~b}$ & 6.5 & -- & -- & $\cdots$ & -- \\
\hline
\end{tabular}

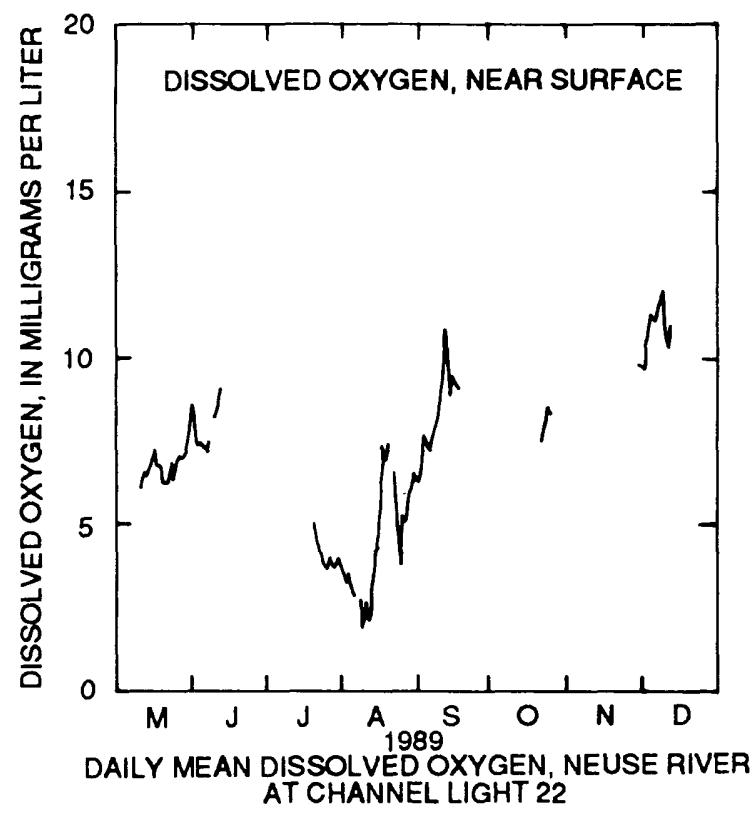


Table 9.--Daily mean values of salinity, water temperature, and dissolved oxygen in water at site 7, Neuse River at Channel Light 22, May to December 1989--Continued

[Site 7 is at lat1tude $35^{\circ} 04^{\prime} 48^{\prime \prime}$, longitude $77^{\circ} 00^{\prime} 24^{\prime \prime}$, Craven County; U.S. Geological Survey downstream order number 0209258400; ppt, parts per thousand; <, less than; ---, no data;

${ }^{\circ} \mathrm{C}$, degrees Celsius; mg/L, milligrams per liter; a, dally minimum value is less than or equal

to $2.0 \mathrm{mg} / \mathrm{L} ; \mathrm{b}$, daily minimum value is less than or equal to $5.0 \mathrm{mg} / \mathrm{L}$ but greater than $2.0 \mathrm{mg} / \mathrm{L}$ ]

DISSOLVED OXYGEN, MID-DEPTH

SENSOR POSITION, --6 feet above streambed.

REMARKS.--Station discontinued December 1989.

EXTREMES FOR MAY TO DECEMBER 1989. - Maximum value recorded, $11.2 \mathrm{mg} / \mathrm{L}$ June 11, 1989; minlmum value recorded, <1.0 July 21, August 28-30, september 1, 2, 4-10, 1989.

DAILY MEAN VALUES OF DISSOLVED OXYGEN, MID-DEPTH (IN MILLIGRAMS PER LITER), MAY TO DECEMBER 1989

\begin{tabular}{|c|c|c|c|c|c|c|c|c|}
\hline DAY & MAY & JUNE & JULY & AUG & SEPT & OCT & NOV & $\mathrm{DEC}$ \\
\hline 1 & -- & 8.1 & --- & $4.8 a$ & $1.3 a$ & --- & --- & $-\cdots$ \\
\hline 2 & --- & 7.2 & --- & $4.7 b$ & $1.3 a$ & --- & --- & $-\cdots$ \\
\hline 3 & -- & $6.5 b$ & --- & $4.1 \mathrm{a}$ & --- & --- & -- & -- \\
\hline 4 & --- & 7.2 & --- & --- & $1.7 a$ & --- & -- & $-\cdots$ \\
\hline 5 & --- & 7.4 & -- & --- & $1.6 a$ & --- & --- & -- \\
\hline 6 & --- & 7.2 & --- & --- & $1.2 \mathrm{a}$ & --- & --- & -- \\
\hline 7 & --- & 7.1 & -- & --- & $1.3 a$ & --- & --- & $-\cdots$ \\
\hline 8 & --- & 7.2 & --- & --- & $1.3 a$ & --- & --- & -- \\
\hline 9 & -- & 7.7 & --- & --- & $1.4 \mathrm{a}$ & --- & --- & -- \\
\hline 10 & --- & 7.7 & --- & --- & $1.9 a$ & --- & --- & -- \\
\hline 11 & -- & $7.3 \mathrm{~b}$ & --- & -- & $2.3 a$ & --- & --- & -- \\
\hline 12 & 6.2 & 8.4 & -- & --- & $3.1 \mathrm{a}$ & --- & $-\cdots-$ & -- \\
\hline 13 & 6.6 & --- & --- & --- & $2.7 a$ & --- & --- & --- \\
\hline 14 & 6.4 & --- & --- & --- & --- & --- & -- & -- \\
\hline 15 & 6.6 & --- & --- & --- & --- & --- & --- & --- \\
\hline 16 & 6.9 & --- & -- & --- & --- & --- & --- & -- \\
\hline 17 & 6.8 & --- & --- & --- & --- & --- & --- & --- \\
\hline 18 & $5.8 b$ & -- & -- & -- & --- & --- & -- & -- \\
\hline 19 & $5.9 b$ & -- & -- & -- & --- & -- & --- & --- \\
\hline 20 & 5.7 & --- & --- & --- & --- & --- & --- & -- \\
\hline 21 & 5.7 & --- & $4.3 a$ & --- & --- & --- & --- & -- \\
\hline 22 & 5.8 & -- & $4.8 b$ & -- & --- & --- & --- & -- \\
\hline 23 & 6.2 & --- & $4.0 \mathrm{~b}$ & -- & --- & -- & --- & -- \\
\hline 24 & 6.6 & -- & --- & --- & --- & -- & -- & -- \\
\hline 25 & 6.2 & --- & --- & --- & -- & --- & --- & --- \\
\hline 26 & 6.6 & --- & $4.6 \mathrm{~b}$ & -- & --- & --- & --- & -- \\
\hline 27 & $6.8 b$ & -- & $5.1 b$ & -- & -- & -- & --- & --- \\
\hline 28 & $6.8 b$ & -- & $5.1 b$ & $1.0 a$ & -- & --- & --- & --- \\
\hline 29 & 6.8 & --- & --- & $1.3 a$ & -- & --- & --- & -- \\
\hline 30 & 7.4 & -- & $4.9 b$ & $1.5 \mathrm{a}$ & --- & --- & --- & --- \\
\hline 31 & 7.9 & --- & $5.6 b$ & --- & --- & -- & --- & --- \\
\hline
\end{tabular}

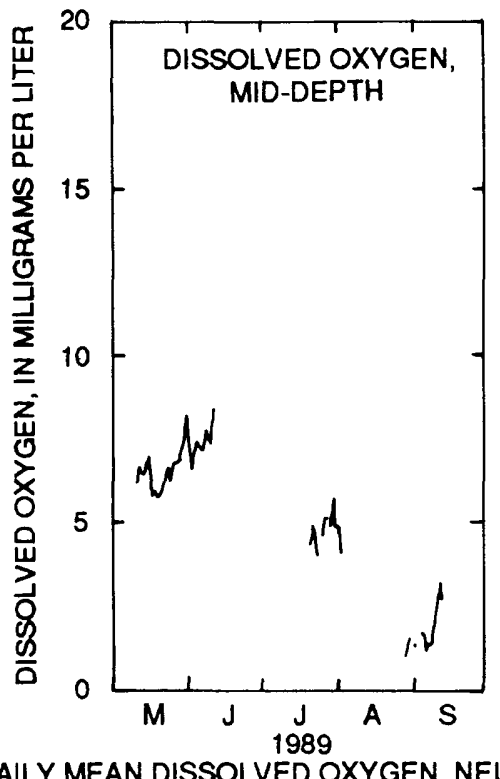


Table 9.--Daily mean values of salinity, water temperature, and dissolved oxygen in water at site 7 , Neuse River at Channel Light 22, May to December 1989--Continued

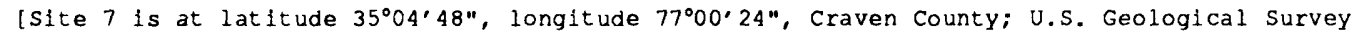
downstream order number 0209258400; ppt, parts per thousand; <, less than; ---, no data;

${ }^{\circ} \mathrm{C}$, degrees Celsius; mg/L, milligrams per liter; a, dally minimum value is less than or equal to $2.0 \mathrm{mg} / \mathrm{L} ; \mathrm{b}$, daily minimum value is less than or equal to $5.0 \mathrm{mg} / \mathrm{L}$ but greater than $2.0 \mathrm{mg} / \mathrm{L}$ ]

\section{DISSOLVED OXYGEN, NEAR BOTTOM}

SENSOR POSITION. --2 feet above streambed.

REMARKS. - Station discontinued December 1989.

EXTREMES EOR MAY TO DECEMBER 1989. - Maximum value recorded, 6.2 mg/L May 12, 1989; minimum value recorded, $<1.0 \mathrm{mg} / \mathrm{L}$ July 21-31, August 1-11, 13-31, September 1-4, october 21-25, 1989.

DAILY MEAN VALUES OF DISSOLVED OXYGEN, NEAR BOTTOM (IN MILLIGRAMS PER LITER), MAY TO DECEMBER 1989

\begin{tabular}{|c|c|c|c|c|c|c|c|c|}
\hline DAY & MAY & JUNE & JULY & AUG & SEPT & OCT & NOV & DEC \\
\hline 1 & --- & -- & -- & $<1.0 \mathrm{a}$ & $<1.0 \mathrm{a}$ & --- & --- & -- \\
\hline 2 & --- & --- & -- & $<1.0 \mathrm{a}$ & $<1.0 \mathrm{a}$ & --- & -- & -- \\
\hline 3 & -- & $\cdots$ & --- & $<1.0 \mathrm{a}$ & $<1.0 \mathrm{a}$ & --- & --- & -- \\
\hline 4 & --- & --- & -- & $<1.0 \mathrm{a}$ & $<1.0 \mathrm{a}$ & --- & --- & --- \\
\hline 5 & -- & $\cdots$ & -- & $<1.0 \mathrm{a}$ & $2.0 \mathrm{a}$ & -- & -- & -- \\
\hline 6 & --- & --- & $-\cdots$ & $<1.0 \mathrm{a}$ & $1.8 a$ & --- & --- & -- \\
\hline 7 & --- & --- & --- & $<1.0 \mathrm{a}$ & $1.9 a$ & --- & -- & -- \\
\hline 8 & -- & -- & --- & $<1.0 \mathrm{a}$ & $2.1 \mathrm{a}$ & -- & -- & -- \\
\hline 9 & -- & -- & --- & $<1.0 \mathrm{a}$ & $2.1 \mathrm{a}$ & --- & --- & -- \\
\hline 10 & --- & --- & -- & $<1.0 \mathrm{a}$ & $1.7 a$ & -- & --- & --- \\
\hline 11 & --- & -- & -- & $1.2 \mathrm{a}$ & $1.6 a$ & -- & -- & -- \\
\hline 12 & 6.0 & $-\cdots$ & -- & $1.3 a$ & $1.3 a$ & -- & --- & -- \\
\hline 13 & -- & $-\cdots$ & -- & $<1.0 \mathrm{a}$ & $1.4 a$ & $-\cdots$ & -- & --- \\
\hline 14 & --- & --- & --- & $<1.0 \mathrm{a}$ & $1.3 a$ & --- & --- & --- \\
\hline 15 & --- & --- & -- & $<1.0 \mathrm{a}$ & $1.4 \mathrm{a}$ & -- & -- & --- \\
\hline 16 & -. & $-\cdots$ & --- & $<1.0 \mathrm{a}$ & $1.8 \mathrm{a}$ & --- & -- & -- \\
\hline 17 & -- & --- & --- & $<1.0 a$ & $2.0 a$ & -- & --- & -- \\
\hline 18 & -- & --- & --- & $<1.0 a$ & -- & --- & -- & --- \\
\hline 19 & --- & -- & -- & $<1.0 \mathrm{a}$ & --- & --- & -- & -- \\
\hline 20 & --- & --- & --- & $<1.0 a$ & --- & --- & --- & --- \\
\hline 21 & $-\cdots$ & --- & $1.0 \mathrm{a}$ & $<1.0 \mathrm{a}$ & --- & $<1.0 a$ & -- & -- \\
\hline 22 & -- & --- & $<1.0 a$ & $<1.0 a$ & --- & $<1.0 a$ & -- & $-\cdots$ \\
\hline 23 & -- & -- & $<1.0 \mathrm{a}$ & $<1.0 \mathrm{a}$ & -- & $<1.0 a$ & $-\cdots$ & -- \\
\hline 24 & --- & -- & $<1.0 \mathrm{a}$ & $<1.0 \mathrm{a}$ & --- & $<1.0 a$ & --- & $-\cdots$ \\
\hline 25 & --- & --- & $<1.0 \mathrm{a}$ & $<1.0 \mathrm{a}$ & --- & $<1.0 a$ & --- & -- \\
\hline 26 & --- & --- & $<1.0 a$ & $<1.0 \mathrm{a}$ & -- & --- & --- & --- \\
\hline 27 & --- & --- & $<1.0 \mathrm{a}$ & $<1.0 \mathrm{a}$ & -- & -- & --- & -- \\
\hline 28 & --- & --- & $<1.0 \mathrm{a}$ & $<1.0 \mathrm{a}$ & --- & -- & -- & -- \\
\hline 29 & $-\infty$ & --- & $<1.0 \mathrm{a}$ & $<1.0 \mathrm{a}$ & $-\cdots$ & --- & -- & $-\cdots$ \\
\hline 30 & --- & --- & $<1.0 \mathrm{a}$ & $<1.0 \mathrm{a}$ & -- & --- & -- & -- \\
\hline 31 & --- & --- & $<1.0 \mathrm{a}$ & $<1.0 \mathrm{a}$ & -- & --- & --- & $-\cdots$ \\
\hline
\end{tabular}

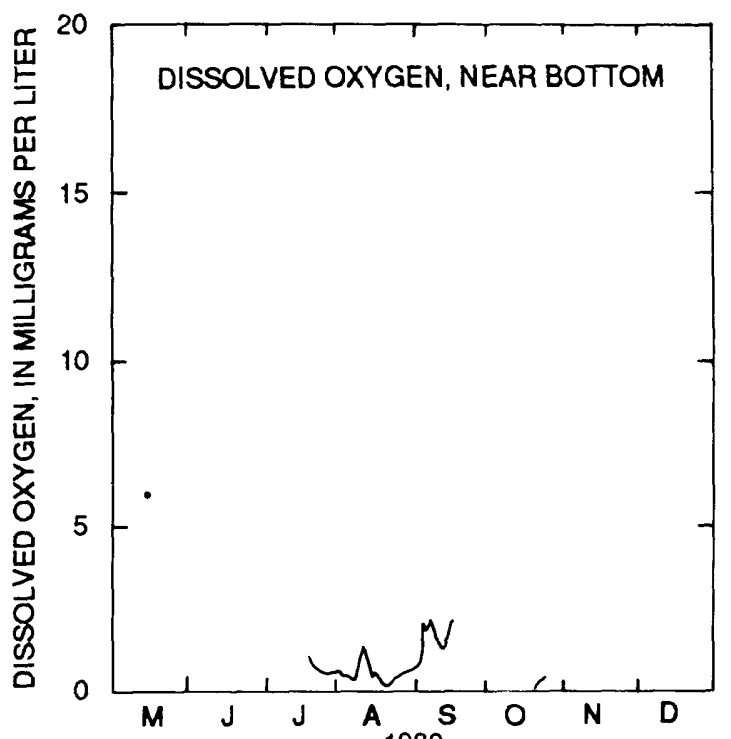


Table 10.--Dally mean values of salinity, water temperature, and dissolved oxygen in water at site 8 ,

Neuse River at Channel Light 11, May to December 1989

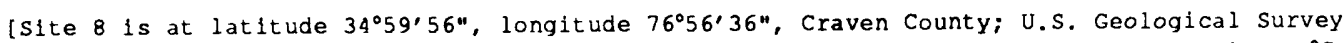
downstream order number 0209262905; ppt, parts per thousand; <, less than; ---, no data; ${ }^{\circ} \mathrm{C}$, degrees Celsius; mg/L, milligrams per liter; a, dally minimum value is less than or equal

to $2.0 \mathrm{mg} / \mathrm{L} ; \mathrm{b}$, dally minimum value $1 \mathrm{~s}$ less than or equal to $5.0 \mathrm{mg} / \mathrm{L}$ but greater $\mathrm{than} 2.0 \mathrm{mg} / \mathrm{L}$ )

SALINITY, NEAR SURFACE

SENSOR POSITION.--10 feet above st reambed.

EXTREMES FOR MAY TO DECEMBER 1989.--Maximum value recorded, 8.3 ppt June 28, 1989 ; minimum value recorded, <0.1 ppt May 14, 15, 1989.

DAILY MEAN VALUES OF SALINITY, NEAR SURFACE (IN PARTS PER THOUSAND), MAY TO DECEMBER 1989

\begin{tabular}{|c|c|c|c|c|c|c|c|c|}
\hline DAY & MAY & JUNE & JULY & AUG & SEPT & OCT & NOV & DEC \\
\hline 1 & --- & 2.6 & 3.9 & --- & --- & --- & 3.3 & 3.3 \\
\hline 2 & --- & 2.8 & 3.6 & --- & --- & --- & 3.5 & 2.3 \\
\hline 3 & --- & 3.4 & 3.9 & --- & --- & --- & 3.7 & --- \\
\hline 4 & --- & 3.2 & 3.4 & --- & -- & --- & 4.0 & -- \\
\hline 5 & --- & 3.3 & 2.6 & 1.5 & --- & --- & 3.9 & 4.5 \\
\hline 6 & --- & 3.4 & 2.8 & 1.8 & --- & --- & 3.4 & 4.5 \\
\hline 7 & --- & 3.4 & 3.5 & 1.7 & --- & --- & 3.4 & 4.4 \\
\hline 8 & -- & 2.8 & --- & 1.4 & --- & --- & 3.7 & 5.0 \\
\hline 9 & -- & --- & --- & 1.2 & --- & --- & 3.9 & 4.9 \\
\hline 10 & -- & --- & --- & 1.3 & --- & --- & 3.9 & 4.1 \\
\hline 11 & --- & 3.7 & --- & 1.2 & -- & --- & 3.8 & 1.5 \\
\hline 12 & 0.5 & 3.3 & $\cdots-$ & --- & -- & -- & 3.5 & 1.5 \\
\hline 13 & .5 & --- & --- & -- & --- & --- & 2.5 & 1.7 \\
\hline 14 & .2 & --- & --- & --- & --- & --- & 2.6 & -- \\
\hline 15 & .1 & 3.5 & --- & --- & --- & --- & 2.4 & -- \\
\hline 16 & .4 & 2.4 & --- & --- & --- & --- & 2.4 & --- \\
\hline 17 & 1.2 & 2.3 & --- & --- & --- & -- & 3.4 & --- \\
\hline 18 & 2.1 & 1.5 & --- & --- & -- & -- & 3.6 & -- \\
\hline 19 & 2.2 & 1.3 & -- & -- & --- & -- & 4.1 & $\cdots$ \\
\hline 20 & 1.3 & 1.4 & --- & --- & --- & --- & 3.6 & --- \\
\hline 21 & 1.2 & 1.8 & --- & -- & --- & -- & 4.6 & --- \\
\hline 22 & .8 & 1.2 & -- & --- & --- & 6.3 & 4.0 & -- \\
\hline 23 & .7 & 1.1 & -- & --- & -- & --- & 3.7 & -- \\
\hline 24 & 1.7 & 1.7 & --- & --- & -- & 3.9 & 3.7 & --- \\
\hline 25 & 2.3 & 2.1 & --- & --- & --- & 5.1 & 3.0 & --- \\
\hline 26 & 1.7 & 2.4 & --- & --- & --- & -- & 3.8 & -- \\
\hline 27 & 1.6 & 4.9 & -- & --- & -- & 4.1 & 3.3 & -- \\
\hline 28 & 2.0 & 5.9 & -- & -- & -- & 4.0 & 3.1 & --- \\
\hline 29 & 2.4 & 4.8 & -- & --- & -- & 4.4 & 3.5 & -- \\
\hline 30 & 1.9 & 5.2 & -- & -- & -- & 4.1 & 3.6 & --- \\
\hline 31 & 2.0 & -- & -- & --- & -- & 3.5 & -- & $\cdots$ \\
\hline
\end{tabular}

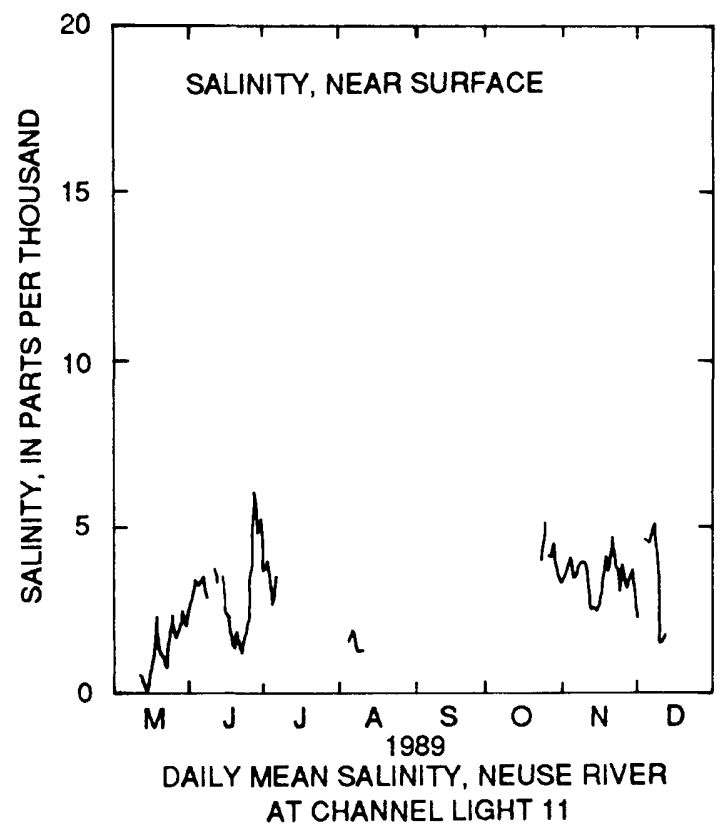


Table 10.--Daily mean values of salinity, water temperature, and dissolved oxygen in water at site 8 , Neuse River at Channel Light 11, May to December 1989--Cont inued

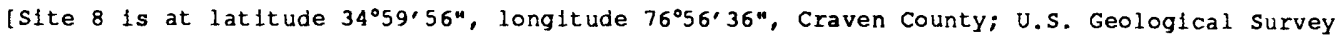
downstream order number 0209262905 ; ppt, parts per thousand; <, less than; ---, no data; ${ }^{\circ} \mathrm{C}$, degrees Celsius; mg/L, mllifgrams per liter; a, dally minimum value is less than or equal

to $2.0 \mathrm{mg} / \mathrm{L}$; b, dally minlmum value is less than or equal to $5.0 \mathrm{mg} / \mathrm{L}$ but greater than $2.0 \mathrm{mg} / \mathrm{L}$ ]

SALINITY, NEAR BOTTOM

SENSOR POSITION. -2 feet above streambed.

EXTREMES FOR MAY TO DECEMBER 1989.--Maximum value recorded, 13.7 ppt June 28, 1989; minimum value recorded, 0.1 ppt MaY 15, 1989.

DAILY MEAN VALUES OF SALINITY, NEAR BOTTOM (IN PARTS PER THOUSAND), MAY TO DECEMBER 1989

\begin{tabular}{|c|c|c|c|c|c|c|c|c|}
\hline DAY & MAY & JUNE & JULY & AUG & SEPT & OCT & NOV & DEC \\
\hline 1 & --- & 4.0 & 4.5 & --- & -- & -- & 4.2 & 3.5 \\
\hline 2 & --- & 4.5 & 4.4 & --- & --- & --- & 4.6 & 2.8 \\
\hline 3 & -- & 4.0 & 4.9 & --- & -- & --- & 4.7 & 4.1 \\
\hline 4 & --- & 3.3 & 4.5 & --- & --- & --- & 5.1 & 4.0 \\
\hline 5 & --- & 4.1 & 3.7 & 10.4 & --- & -- & 5.0 & 4.4 \\
\hline 6 & --- & 3.2 & 3.8 & 10.3 & --- & -- & 4.2 & 4.4 \\
\hline 7 & --- & 3.1 & 4.6 & 9.5 & --- & --- & 4.3 & 4.4 \\
\hline 8 & -- & 4.0 & --- & 9.0 & --- & --- & 4.6 & 4.8 \\
\hline 9 & --- & 2.9 & --- & 8.9 & -- & -- & 4.8 & 4.6 \\
\hline 10 & --- & 3.6 & --- & 9.4 & --- & --- & 4.8 & 4.2 \\
\hline 11 & --- & 4.5 & --- & 10.2 & --- & --- & 4.6 & 2.2 \\
\hline 12 & 0.7 & 4.1 & --- & --- & --- & --- & 4.2 & 1.7 \\
\hline 13 & 1.3 & 3.0 & --- & --- & --- & --- & 2.8 & 1.4 \\
\hline 14 & .8 & 4.2 & --- & --- & --- & --- & 2.8 & $-\cdots$ \\
\hline 15 & .7 & 4.1 & --- & -- & --- & --- & 2.6 & --- \\
\hline 16 & 3.5 & 2.8 & -- & -- & --- & $--\infty$ & 2.5 & -- \\
\hline 17 & 5.2 & 2.7 & -- & --- & --- & $-\cdots$ & 3.8 & --- \\
\hline 18 & 2.2 & 3.0 & --- & $-\cdots$ & -- & --- & 4.1 & -- \\
\hline 19 & 1.8 & 4.5 & --- & --- & --- & --- & 4.7 & $-\cdots$ \\
\hline 20 & 2.0 & 3.6 & --- & --- & --- & --- & 4.0 & -- \\
\hline 21 & 2.5 & 3.0 & --- & --- & -- & -- & 5.3 & $-\cdots$ \\
\hline 22 & 2.3 & 3.5 & --- & --- & --- & 7.1 & 4.5 & -- \\
\hline 23 & 2.3 & 5.3 & -- & --- & --- & -- & 4.0 & -- \\
\hline 24 & 4.6 & 4.4 & --- & --- & --- & 4.7 & 3.9 & $-\cdots$ \\
\hline 25 & 4.1 & 4.3 & --- & --- & --- & 6.3 & 3.0 & -- \\
\hline 26 & 4.9 & 6.2 & --- & --- & --- & -- & 4.0 & $\ldots$ \\
\hline 27 & 6.8 & 8.4 & --- & --- & --- & 5.5 & 3.4 & --- \\
\hline 28 & 3.3 & 8.9 & --- & --- & --- & 5.4 & 3.1 & -- \\
\hline 29 & 2.2 & 6.5 & --- & --- & --- & 5.9 & 3.5 & -- \\
\hline 30 & 2.3 & 5.8 & --- & --- & --- & 5.4 & 3.6 & -- \\
\hline 31 & 3.2 & --- & --- & --- & --- & 4.6 & --- & -- \\
\hline
\end{tabular}

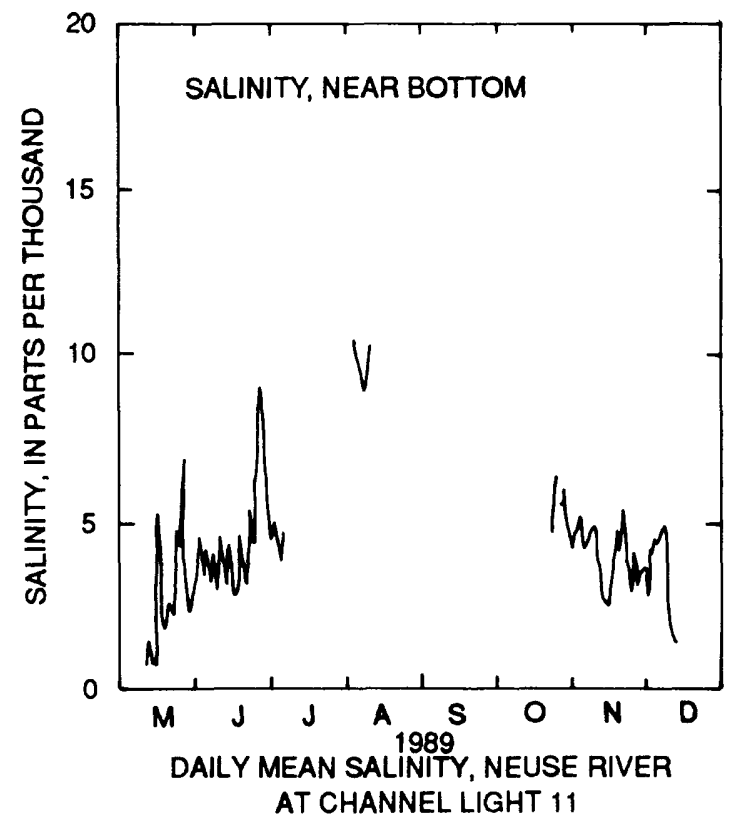


Table 10.--Dally mean values of salinity, water temperature, and dissolved oxygen in water at site 8 , Neuse River at Channel Light 11, May to December 1989--Cont inued

[Site 8 is at latitude $34^{\circ} 59^{\prime} 56^{\prime \prime}$, longitude 7656'36", Craven County; U.S. Geological Survey downstream order number 0209262905; ppt, parts per thousand; <, less than; ---, no data; ${ }^{\circ} \mathrm{C}$, degrees Celsius; mg/L, milligrams per liter; a, dally minimum value is less than or equal

to $2.0 \mathrm{mg} / \mathrm{L} ; \mathrm{b}$, dally minimum value is less than or equal to $5.0 \mathrm{mg} / \mathrm{L}$ but greater than $2.0 \mathrm{mg} / \mathrm{L}$ ]

WATER TEMPERATURE, NEAR SURFACE

SENSOR POSITION. --10 feet above streambed.

EXTREMES FOR MAY TO DECEMBER 1989. --Maximum value recorded, $31.6{ }^{\circ} \mathrm{C}$ june 25,$1989 ;$ minimum value recorded, $6.1{ }^{\circ} \mathrm{C}$ December $11,1989$.

DAILY MEAN VALUES OF WATER TEMPERATURE, NEAR SURFACE (IN DEGREES CELSIUS), MAY TO DECEMBER 1989

\begin{tabular}{|c|c|c|c|c|c|c|c|c|}
\hline DAY & MAY & JUNE & JULY & AUG & SEPT & OCT & NOV & DEC \\
\hline 1 & -- & 25.3 & 26.6 & --- & --- & --- & 18.0 & 11.0 \\
\hline 2 & --- & 26.2 & 26.7 & --- & -- & --- & 18.0 & 10.2 \\
\hline 3 & --- & 26.1 & 26.6 & --- & -- & --- & 17.8 & 10.1 \\
\hline 4 & --- & 25.7 & 26.5 & -- & -- & --- & 17.0 & 8.3 \\
\hline 5 & -- & 25.7 & 27.1 & 27.5 & -- & --- & 16.8 & 8.0 \\
\hline 6 & --- & 25.4 & 27.2 & 28.4 & -- & --- & 16.9 & 8.2 \\
\hline 7 & --- & 24.9 & 27.3 & 28.8 & --- & --- & 17.6 & 9.1 \\
\hline 8 & -- & 25.4 & --- & 29.3 & -- & -- & 17.7 & 9.0 \\
\hline 9 & --- & 24.2 & --- & 29.2 & --- & -- & 17.7 & 8.3 \\
\hline 10 & --- & 25.3 & --- & 29.4 & --- & --- & 17.5 & 7.4 \\
\hline 11 & --- & 26.1 & --- & 29.2 & $\cdots$ & --- & 17.3 & 6.8 \\
\hline 12 & 18.5 & 26.0 & --- & --- & -- & --- & 17.4 & 7.0 \\
\hline 13 & 18.2 & 26.1 & --- & --- & --- & $-\cdots$ & 18.3 & 6.9 \\
\hline 14 & 18.0 & 26.8 & --- & --- & --- & -- & 18.5 & $\cdots$ \\
\hline 15 & 18.4 & 27.1 & $\div--$ & $-\cdots$ & $\cdots$ & -- & 18.7 & -- \\
\hline 16 & 19.0 & 27.5 & $\cdots$ & --- & --- & -- & 18.5 & -- \\
\hline 17 & 18.9 & 26.8 & --- & --- & --- & -- & 16.6 & $\cdots$ \\
\hline 18 & 19.4 & 27.1 & --- & --- & --- & -- & 15.1 & $\ldots$ \\
\hline 19 & 19.8 & 27.7 & --- & $-\cdots$ & --- & $-\cdots$ & 14.0 & $\ldots$ \\
\hline 20 & 20.4 & 27.6 & $\cdots$ & --- & --- & -- & 13.1 & $\cdots$ \\
\hline 21 & 21.9 & 27.6 & --- & --- & -- & -- & 13.5 & --- \\
\hline 22 & 22.7 & 27.0 & --- & -- & --- & 17.7 & 12.2 & $\ldots$ \\
\hline 23 & 22.6 & 27.2 & --- & --- & -- & -- & 11.5 & -- \\
\hline 24 & 22.3 & 27.1 & $\cdots$ & -- & --- & 16.3 & 10.3 & $\cdots$ \\
\hline 25 & 23.5 & 27.8 & $-\cdots$ & --- & --- & 16.4 & 9.8 & $-\cdots$ \\
\hline 26 & 23.9 & 28.0 & -- & --- & --- & --- & 10.8 & $\ldots$ \\
\hline 27 & 25.2 & 27.5 & -- & -- & --- & 16.8 & 11.7 & -- \\
\hline 28 & 25.1 & 27.3 & --- & --- & -- & 17.0 & 12.4 & -- \\
\hline 29 & 24.1 & 27.6 & --- & --- & --- & 17.0 & 12.9 & $\cdots$ \\
\hline 30 & 24.0 & 27.0 & --- & --- & -- & 17.4 & 11.8 & -- \\
\hline 31 & 24.4 & -- & -- & $\ldots$ & --- & 17.8 & --- & -- \\
\hline
\end{tabular}

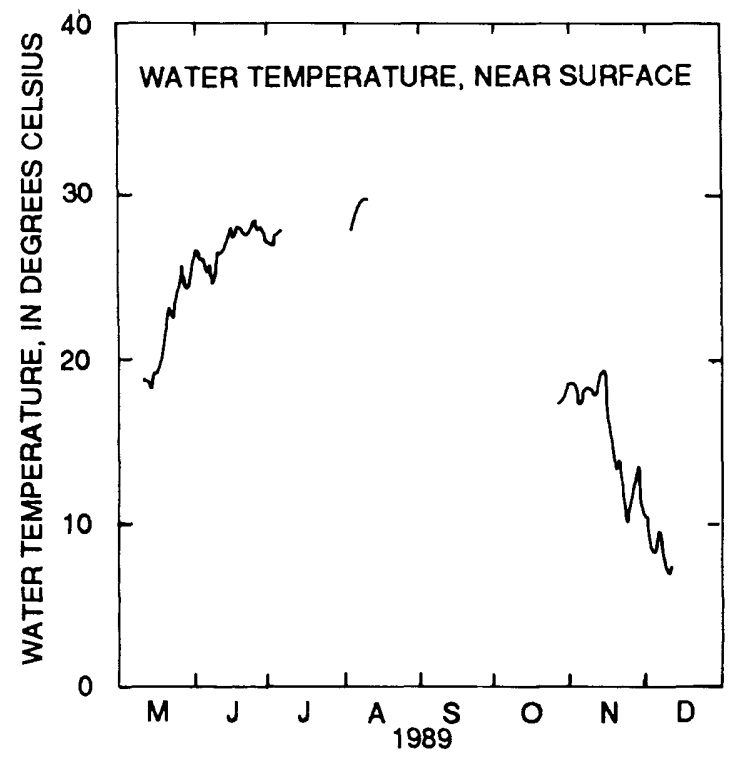

DAILY MEAN WATER TEMPERATURE, NEUSE RIVER

AT CHANNEL LIGHT 11 
Table 10.--Daily mean values of salinity, water temperature, and dissolved oxygen in water at site 8 , Neuse River at Channel Light 11, May to December 1989--Continued

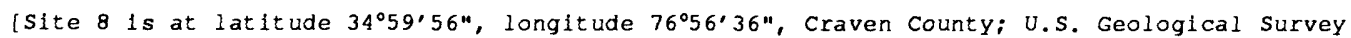
downstream order number 0209262905; ppt, parts per thousand; <, less than; ---, no data;

${ }^{\circ} \mathrm{C}$, degrees Celsius; $\mathrm{mg} / \mathrm{L}$, milligrams per liter; a, dally minimum value is less than or equal

to $2.0 \mathrm{mg} / \mathrm{L} ; \mathrm{b}$, daily minimum value is less than or equal to $5.0 \mathrm{mg} / \mathrm{L}$ but greater than $2.0 \mathrm{mg} / \mathrm{L}$ ]

DISSOLVED OXYGEN, NEAR SURFACE

SENSOR POSITION.--10 feet above streambed.

EXTREMES FOR MAY TO DECEMBER 1989.--Maximum value recorded, $13.1 \mathrm{mg} / \mathrm{L}$ June 3, 1989; minimum value recorded, 1.7 $\mathrm{mg} / \mathrm{L}$ June 20, 1989.

DAILY MEAN VALUES OF DISSOLVED OXYGEN, NEAR SURFACE (IN MILLIGRAMS PER LITER), MAY TO DECEMBER 1989

\begin{tabular}{|c|c|c|c|c|c|c|c|c|}
\hline DAY & MAY & JUNE & JULY & AUG & SEPT & OCT & NOV & DEC \\
\hline 1 & --- & 9.1 & -- & --- & --- & -- & 8.9 & 10.0 \\
\hline 2 & --- & 9.3 & -- & --- & -- & $-\cdots$ & 9.2 & 10.4 \\
\hline 3 & $\cdots$ & 8.4 & -- & $\cdots$ & -- & --- & 8.7 & 8.3 \\
\hline 4 & -- & 8.0 & --- & -- & --- & -- & 8.4 & 9.5 \\
\hline 5 & -- & 7.9 & --- & -- & -- & -- & 8.3 & 10.1 \\
\hline 6 & -- & 8.2 & -- & -- & -.. & $\cdots$ & 8.2 & 10.3 \\
\hline 7 & --- & 8.7 & $\cdots$ & --- & -- & -- & 8.9 & 10.7 \\
\hline 8 & --- & 9.6 & -- & -- & -- & --- & 8.2 & 10.5 \\
\hline 9 & -- & 7.7 & -- & --- & -- & -- & 6.5 & 10.5 \\
\hline 10 & -- & 9.6 & --- & -- & -.- & $\cdots$ & 6.8 & 11.0 \\
\hline 11 & -- & 10.7 & --- & -- & -- & -- & 7.6 & 11.2 \\
\hline 12 & 7.7 & 10.6 & -- & --- & -- & -- & 7.8 & 11.2 \\
\hline 13 & 6.9 & 9.2 & -- & $-\cdots$ & -- & -- & 8.4 & 11.0 \\
\hline 14 & 6.8 & 8.6 & -- & -- & -- & -- & 7.9 & $\cdots$ \\
\hline 15 & 6.6 & 7.7 & -- & --- & -- & -- & 7.9 & -.. \\
\hline 16 & 6.8 & 7.4 & --- & -- & -- & -- & 7.8 & --- \\
\hline 17 & 7.7 & 7.0 & $\ldots$ & -- & --- & --- & 7.6 & -- \\
\hline 18 & 7.9 & 7.5 & --- & -- & --- & --- & 9.3 & --- \\
\hline 19 & 8.1 & $6.7 \mathrm{~b}$ & --- & -- & -.. & -- & 8.9 & -- \\
\hline 20 & 7.5 & $6.2 a$ & --- & --- & -- & --- & 9.5 & --- \\
\hline 21 & 8.4 & $6.0 \mathrm{~b}$ & -- & --- & --- & -- & 8.5 & --- \\
\hline 22 & 7.8 & -- & --- & --- & -- & --- & 8.7 & --- \\
\hline 23 & 7.5 & -- & --- & --- & -- & -- & 9.4 & -- \\
\hline 24 & 7.3 & $\cdots$ & -- & -- & -- & 9.7 & 8.5 & -- \\
\hline 25 & 9.6 & -- & --- & -- & --- & 8.2 & 8.0 & --- \\
\hline 26 & 9.0 & $\cdots$ & -- & -- & -- & --- & 8.6 & --- \\
\hline 27 & 9.3 & -- & -- & $\cdots$ & -- & 8.6 & 9.4 & $-\cdots$ \\
\hline 28 & 9.4 & $\cdots$ & -- & $\cdots$ & --- & 9.1 & 9.3 & --- \\
\hline 29 & 9.7 & -- & --- & --- & -- & 8.1 & 9.6 & -- \\
\hline 30 & 9.7 & $\cdots$ & -- & -- & -- & 8.0 & 9.2 & $\cdots$ \\
\hline 31 & 9.3 & -- & -- & -- & -- & 8.3 & --- & --- \\
\hline
\end{tabular}

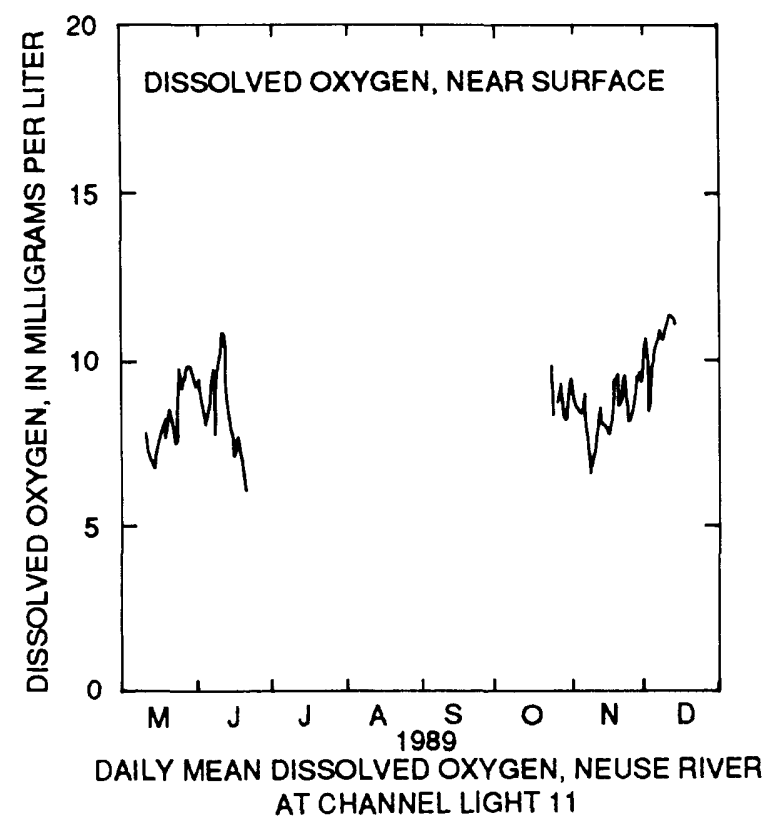


Table 10.--Daily mean values of salinity, water temperature, and dissolved oxygen in water at site 8 , Neuse River at Channel Light 11, May to December 1989--Continued

[S1te 8 is at latitude $34^{\circ} 59^{\prime} 56^{\prime \prime}$, longitude $76^{\circ} 56^{\prime} 36^{\prime \prime}$, Craven County; U. S. Geological Survey downstream order number 0209262905; ppt, parts per thousand; <, less than; ---, no data;

${ }^{\circ} \mathrm{C}$, degrees Celsius; $\mathrm{mg} / \mathrm{L}$, milligrams per liter; a, dally minimum value is less than or equal to $2.0 \mathrm{mg} / \mathrm{L} ; \mathrm{b}$, dally minimum value $1 \mathrm{~s}$ less than or equal to $5.0 \mathrm{mg} / \mathrm{L}$ but greater than $2.0 \mathrm{mg} / \mathrm{L}$ ]

DISSOLVED OXYGEN, MID-DEPTH

SENSOR POSITION. -6 feet above streambed.

EXTREMES FOR MAY TO DECEMBER 1989,--Maximum value recorded, $12.4 \mathrm{mg} / \mathrm{L}$ December 1, 1989; minimum value recorded, $<1.0$ June $18-20,22-24,26-27,1989$.

DAILY MEAN VALUES OF DISSOLVED OXYGEN, MID-DEPTH (IN MILLIGRAMS PER LITER), MAY TO DECEMBER 1989

\begin{tabular}{|c|c|c|c|c|c|c|c|c|}
\hline DAY & MAY & JUNE & JULY & AUG & SEPT & OCT & NOV & DEC \\
\hline 1 & --- & 8.2 & 7.8 & --- & --- & -- & 9.1 & 10.8 \\
\hline 2 & --- & $7.6 \mathrm{~b}$ & 5.7 & -- & --- & -- & 9.1 & 11.0 \\
\hline 3 & --- & $6.8 \mathrm{~b}$ & 5.9 & --- & -- & -- & 8.5 & 8.5 \\
\hline 4 & -- & 7.5 & 7.2 & -- & -- & -- & 8.5 & 9.6 \\
\hline 5 & --- & $7.5 b$ & 6.3 & --- & --- & --- & 8.7 & 10.0 \\
\hline 6 & -- & 8.0 & 4.4 & --- & -- & -- & 9.4 & 10.0 \\
\hline 7 & --- & 8.9 & 4.7 & --- & --- & --- & 10.3 & 10.2 \\
\hline 8 & -- & 7.9 & $\cdots$ & --- & --- & --- & 9.4 & 9.7 \\
\hline 9 & -- & $7.3 \mathrm{~b}$ & -- & --- & --- & -- & 6.9 & 9.6 \\
\hline 10 & $\cdots$ & 8.7 & $-\cdots$ & --- & -- & --- & 7.3 & 9.9 \\
\hline 11 & -- & 10.1 & -- & -- & --- & --- & 9.0 & 9.8 \\
\hline 12 & 8.3 & 9.2 & --- & -- & --- & --- & 8.9 & 9.6 \\
\hline 13 & 7.3 & 8.9 & --- & --- & --- & --- & 9.8 & 9.1 \\
\hline 14 & 7.4 & $7.2 \mathrm{~b}$ & --- & --- & --- & --- & 9.9 & --- \\
\hline 15 & 7.1 & $5.4 a$ & --- & --- & --- & --- & 9.1 & --- \\
\hline 16 & 7.2 & 7.2 & --- & --- & --- & --- & 7.9 & --- \\
\hline 17 & 8.1 & 6.9 & --- & --- & --- & --- & 8.3 & --- \\
\hline 18 & 8.3 & $4.4 a$ & --- & --- & -- & --- & 10.3 & --- \\
\hline 19 & 8.6 & $1.1 \mathrm{a}$ & $\cdots$ & --- & --- & --- & 10.0 & -- \\
\hline 20 & 8.0 & $3.8 a$ & --- & --- & --- & --- & 10.2 & -- \\
\hline 21 & 8.0 & $6.0 \mathrm{~b}$ & --- & --- & --- & --- & 8.8 & $\cdots$ \\
\hline 22 & 8.2 & $1.9 a$ & --- & --- & --- & --- & 9.5 & -- \\
\hline 23 & 7.7 & $1.2 \mathrm{a}$ & --- & --- & --- & --- & 9.7 & $-\cdots$ \\
\hline 24 & 7.2 & $2.2 \mathrm{a}$ & --- & --- & --- & 10.1 & 10.0 & --- \\
\hline 25 & 9.2 & $3.8 a$ & --- & $-\cdots$ & --- & 8.4 & 10.7 & --- \\
\hline 26 & 9.5 & $2.3 \mathrm{a}$ & --- & -- & -- & --- & 10.4 & --- \\
\hline 27 & 8.3 & $1.1 \mathrm{a}$ & --- & --- & -- & 8.7 & 10.6 & --- \\
\hline 28 & 9.6 & $1.5 a$ & --- & --- & --- & 9.1 & 10.4 & --- \\
\hline 29 & 9.6 & $3.8 a$ & -- & -- & --- & 8.1 & 10.6 & --- \\
\hline 30 & 9.8 & $7.4 \mathrm{a}$ & -- & --- & --- & 7.9 & 10.2 & --- \\
\hline 31 & 8.8 & $\cdots$ & --- & $-\cdots$ & --- & 8.2 & --- & --- \\
\hline
\end{tabular}

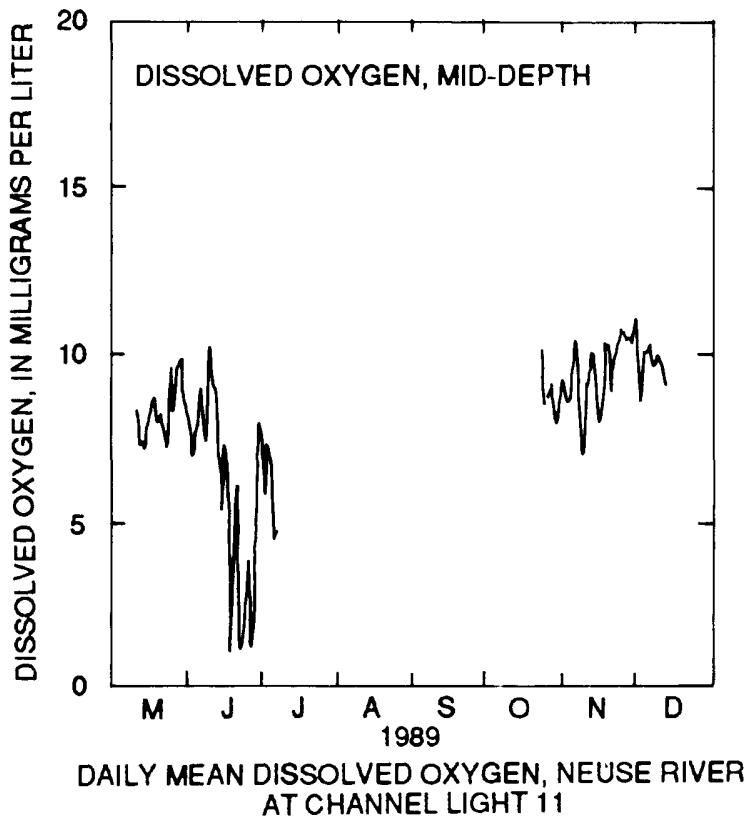


Table 10.--Daily mean values of salinity, water temperature, and dissolved oxygen in water at site 8 , Neuse River at Channel Light 11, May to December 1989--Cont inued

[Site 8 is at latitude $34^{\circ} 59^{\prime} 56^{\prime \prime}$, longitude $76^{\circ} 56^{\prime} 36^{\prime \prime}$, Craven County; U.S. Geological Survey downstream order number 0209262905; ppt, parts per thousand; <, less than; ---, no data;

${ }^{\circ} \mathrm{C}$, degrees Celsius; mg/L, milligrams per liter; a, daily minimum value is less than or equal

to $2.0 \mathrm{mg} / \mathrm{L}$; b, dally minimum value is less than or equal to $5.0 \mathrm{mg} / \mathrm{L}$ but greater than $2.0 \mathrm{mg} / \mathrm{L}$ ]

DISSOLVED OXYGEN, NEAR BOTTOM

SENSOR POSITION. --2 feet above streambed.

EXTREMES FOR MAY TO DECEMBER 1989.--Maximum value recorded, 12.5 mg/L November 25, 1989 ; minimum value recorded, $<1.0$ May 13, June 14, 15, 18-20, 22-29, 1989.

DAILY MEAN VALUES OF DISSOLVED OXYGEN, NEAR BOTTOM (IN MILLIGRAMS PER LITER), MAY TO DECEMBER 1989

\begin{tabular}{|c|c|c|c|c|c|c|c|c|}
\hline DAY & MAY & JUNE & JULY & AUG & SEPT & OCT & Nov & DEC \\
\hline 1 & -- & -- & 8.4 & -- & -- & -- & 9.0 & 9.8 \\
\hline 2 & --- & --- & $7.0 \mathrm{a}$ & --- & --- & --- & 9.1 & 10.4 \\
\hline 3 & -- & --- & $6.1 \mathrm{a}$ & --- & --- & -- & 8.6 & 8.6 \\
\hline 4 & -- & --- & $7.2 \mathrm{~b}$ & --- & -- & -- & 8.5 & 9.6 \\
\hline 5 & -- & --- & $6.5 b$ & --- & -- & -- & 8.6 & 10.1 \\
\hline 6 & --- & --- & $4.0 \mathrm{a}$ & --- & -- & $-\infty$ & 9.4 & 10.2 \\
\hline 7 & --- & -- & $4.5 a$ & -- & --- & --- & 10.2 & 10.5 \\
\hline 8 & --- & -- & --- & --- & -- & -- & 9.3 & 10.4 \\
\hline 9 & --- & -- & --- & --- & --- & -- & 6.7 & 10.4 \\
\hline 10 & -- & -- & --- & --- & --- & -- & 7.1 & 10.8 \\
\hline 11 & --- & --- & $\ldots$ & -- & $m$ & -- & 8.7 & 11.0 \\
\hline 12 & $6.8 a$ & -- & --- & --- & -- & -- & 8.6 & 11.1 \\
\hline 13 & $4.6 a$ & -- & --- & -- & -- & --- & 9.7 & 10.8 \\
\hline 14 & --- & 7.0 & $-\cdots$ & -- & -- & -- & 9.8 & --- \\
\hline 15 & --- & 4.4 & --- & --- & --- & -- & 9.0 & --- \\
\hline 16 & -- & $7.7 \mathrm{~b}$ & --- & --- & --- & --- & 7.7 & --- \\
\hline 17 & --- & $7.1 d$ & --- & -- & --- & -- & 8.2 & -- \\
\hline 18 & --- & 3.6 & -- & -- & -- & -- & 10.5 & -- \\
\hline 19 & 8.5 & $<1.0$ & -- & --- & $m$ & -- & 10.2 & --- \\
\hline 20 & 8.1 & 3.5 & -- & -- & -- & -- & 10.4 & -- \\
\hline 21 & 7.8 & $6.6 a$ & --- & -- & $m$ & --- & 8.9 & -- \\
\hline 22 & $6.0 a$ & $1.0 a$ & --- & -- & -- & --- & 9.7 & -- \\
\hline 23 & $3.8 \mathrm{a}$ & $<1.0 a$ & -- & $-\cdots$ & --- & --- & 9.8 & -- \\
\hline 24 & --- & $1.3 a$ & --- & --- & --- & 9.0 & 10.4 & --- \\
\hline 25 & --- & $2.6 a$ & $\cdots$ & -- & --- & 8.1 & 11.2 & --- \\
\hline 26 & -- & $3.1 \mathrm{a}$ & --- & -- & --- & --- & 10.7 & --- \\
\hline 27 & --- & $1.0 \mathrm{a}$ & --- & -- & -- & 8.8 & 10.8 & -- \\
\hline 28 & --- & $1.1 \mathrm{a}$ & -- & -- & --- & 9.2 & 10.7 & -- \\
\hline 29 & --- & $3.7 a$ & -- & --- & -- & 8.2 & 10.2 & --- \\
\hline 30 & -- & 7.9 & --- & --- & --- & 8.0 & 9.1 & --- \\
\hline 31 & --- & -- & $-\cdots$ & -- & -- & 8.2 & $\cdots$ & -- \\
\hline
\end{tabular}

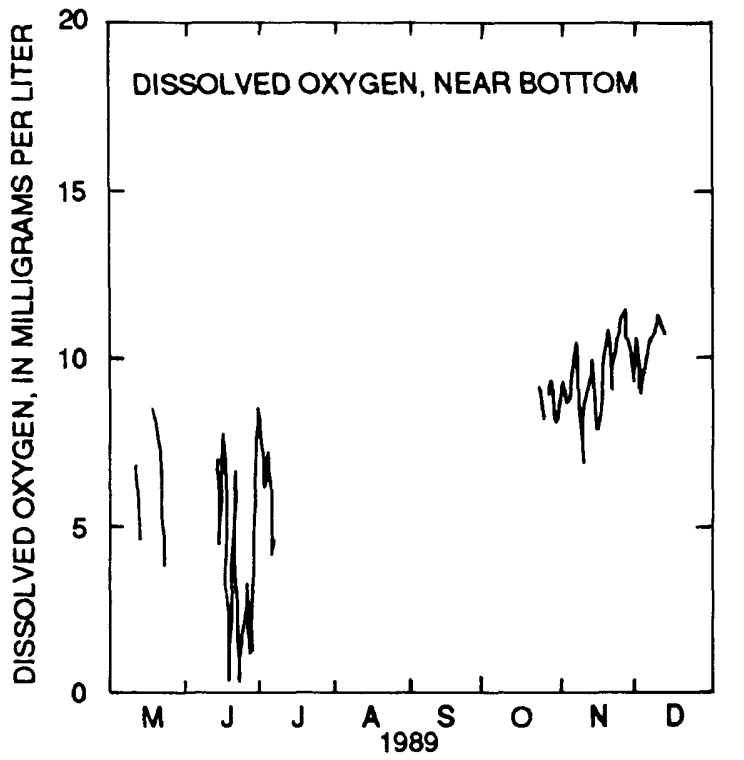

DAILY MEAN DISSOLVED OXYGEN, NEUSE RIVER

AT CHANNEL LIGHT 11 
Table 11.--Daily mean values of salinity, water temperature, and dissolved oxygen in water at site 9, Neuse River at Channel Light 9, May 1989 to September 1990

[Site 9 is at latitude $34^{\circ} 56^{\prime} 54^{\prime \prime}$, longitude $76^{\circ} 48^{\prime} 36^{\prime \prime}$, Craven County; U.S. Geological Survey downst ream order number 0209265810; ppt, parts per thousand; ---, no data; ${ }^{\circ} \mathrm{C}$, degrees

Celsius; $\mathrm{mg} / \mathrm{L}$, milifgrams per liter; a, dally minimum value 1 s less than or equal to $2.0 \mathrm{mg} / \mathrm{L}$; b, daily minimum value is less than or equal to $5.0 \mathrm{mg} / \mathrm{L}$ but greater than $2.0 \mathrm{mg} / \mathrm{L}$; <, less than]

SALINITY, NEAR SURFACE

SENSOR POSITION. --8 feet above streambed.

EXTREMES FOR MAY TO SEPTEMBER 1989.--Maximum value recorded, 10.9 ppt September 25, 1989; minimum value recorded, 2.2 ppt August 4, 1989.

DAILY MEAN VALUES OF SALINITY, NEAR SURFACE (IN PARTS PER THOUSAND), MAY TO SEPTEMBER 1989

\begin{tabular}{|c|c|c|c|c|c|}
\hline DAY & MAY & JUNE & JULY & AUG & SEPT \\
\hline 1 & --- & -- & --- & 4.0 & 6.0 \\
\hline 2 & -- & --- & -- & 3.9 & 5.3 \\
\hline 3 & --- & --- & -- & 3.4 & 5.7 \\
\hline 4 & --- & --- & --- & 2.4 & 7.9 \\
\hline 5 & --- & -- & --- & 2.8 & 7.2 \\
\hline 6 & --- & --- & --- & 3.1 & 7.1 \\
\hline 7 & --- & --- & --- & 3.2 & 6.6 \\
\hline 8 & -- & -- & --- & 4.4 & 6.6 \\
\hline 9 & -- & -- & --- & 5.7 & 5.9 \\
\hline 10 & -- & --- & --- & 5.4 & 5.1 \\
\hline 11 & --- & --- & --- & 5.0 & 5.2 \\
\hline 12 & --- & --- & --- & 5.7 & 5.8 \\
\hline 13 & -- & -- & -- & 5.6 & 6.7 \\
\hline 14 & --- & --- & --- & 5.7 & 6.7 \\
\hline 15 & --- & --- & --- & 6.0 & 6.3 \\
\hline 16 & $-\cdots$ & -- & --- & 6.0 & 7.4 \\
\hline 17 & --- & --- & --- & 5.3 & 7.7 \\
\hline 18 & --- & --- & --- & 6.4 & 7.9 \\
\hline 19 & -- & --- & --- & 6.5 & 6.9 \\
\hline 20 & -- & --- & --- & 6.7 & 7.8 \\
\hline 21 & --- & --- & 8.1 & 6.9 & 9.1 \\
\hline 22 & -- & --- & 7.7 & 7.1 & 9.6 \\
\hline 23 & -- & --- & 6.4 & 7.4 & B. 4 \\
\hline 24 & --- & --- & 4.6 & 7.2 & 8.4 \\
\hline 25 & --- & --- & 5.9 & 7.8 & 9.0 \\
\hline 26 & --- & --- & 5.1 & 8. 6 & 8.0 \\
\hline 27 & --- & -- & 4.0 & 8.5 & 8. 2 \\
\hline 28 & -- & --- & 3.4 & 7.7 & 7.7 \\
\hline 29 & --- & --- & 3.8 & 7.2 & 6.7 \\
\hline 30 & --- & --- & 4.6 & 6.5 & 6.2 \\
\hline 31 & --- & --- & 4.0 & 5.9 & --- \\
\hline
\end{tabular}

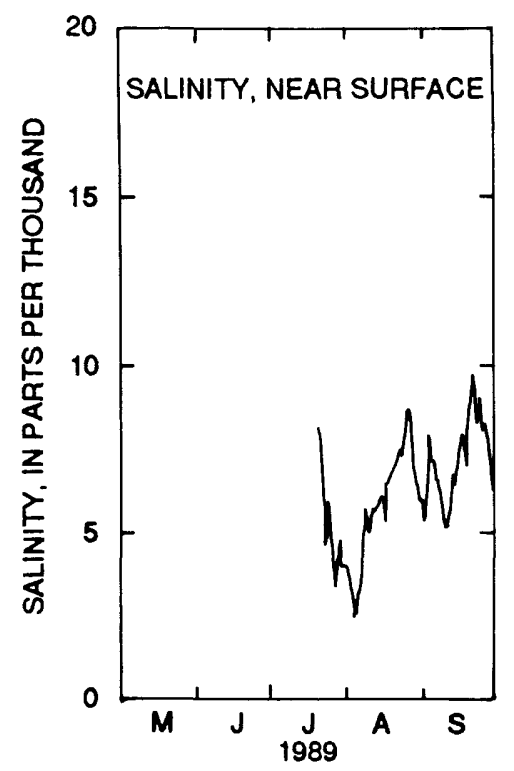

DAILY MEAN SALINITY, NEUSE RIVER 
Table 11.--Daily mean values of salinity, water temperature, and dissolved oxygen tn water at site 9, Neuse River at Channel Light 9, May 1989 to September 1990--Continued

[S1te 9 is at latitude $34^{\circ} 56^{\prime} 54^{\prime \prime}$, longitude $76^{\circ} 48^{\prime} 36^{\prime \prime}$, Craven County; U.s. Geological Survey downst ream order number 0209265810; ppt, parts per thousand; ---, no data; ${ }^{\circ} \mathrm{C}$, degrees Celsius; mg/L, mllilgrams per liter; a, dally minimum value is less than or equal to $2.0 \mathrm{mg} / \mathrm{L}$; b, daily minimum value is less than or equal to $5.0 \mathrm{mg} / \mathrm{L}$ but greater than $2.0 \mathrm{mg} / \mathrm{L} ;<$, less than]

SALINITY, NEAR SURFACE

SENSOR POSITION.--8 feet above streambed.

EXTREMES FOR OCTOBER 1989 TO SEPTEMBER 1990.--Maximum value recorded, 13.0 ppt August 13, 14, 1990; m1nimum value recorded, 0.3 ppt December $30,31,1989$.

DAILY MEAN VALUES OF SALINITY, NEAR SURFACE (IN PARTS PER THOUSAND), OCTOBER 1989 TO SEPTEMBER 1990

\begin{tabular}{|c|c|c|c|c|c|c|c|c|c|c|c|c|}
\hline DAY & OCT & NoV & DEC & JAN & FEB & MAR & APR & MAY & JUNE & JULY & AUG & SEPT \\
\hline 1 & 6.6 & --- & 5.4 & 4.6 & 6.3 & 2.2 & --- & 5.0 & 3.9 & 9.9 & $-\cdots$ & 10.1 \\
\hline 2 & 6.1 & --- & 4.9 & --- & --- & 1.3 & --- & 5.2 & 4.0 & -- & 10.4 & 10.4 \\
\hline 3 & 5.3 & --- & 5.5 & --- & --- & 1.1 & --- & 6.5 & 4.3 & 10.8 & 11.0 & 9.8 \\
\hline 4 & 4.6 & --- & 6.2 & --- & 5.8 & 1.5 & --- & 5.5 & 4.1 & 10.2 & 10.7 & 10.0 \\
\hline 5 & 3.5 & -- & 5.5 & --- & -- & 1.9 & --- & 5.6 & 5.1 & 10.0 & 10.9 & 10.0 \\
\hline 6 & 3.7 & --- & 5.9 & --- & 4.3 & 1.0 & --- & 4.9 & 5.5 & 9.8 & 11.1 & 9.6 \\
\hline 7 & 4.4 & -- & 6.9 & --- & 4.2 & 2.0 & --- & 4.4 & 5.0 & 9.7 & 11.3 & $\cdots$ \\
\hline 8 & 5.5 & -- & 7.4 & $\cdots$ & 4.6 & 1.5 & --- & 4.3 & 4.3 & 8.6 & 11.6 & -- \\
\hline 9 & 4.8 & --- & 7.7 & --- & 4.8 & .8 & -- & 4.7 & 5.4 & 8.6 & 11.5 & -- \\
\hline 10 & --- & --- & 4.9 & --- & 5.9 & 1.1 & $-\cdots$ & 5.4 & 5.6 & 9.1 & 11.5 & $-\cdots$ \\
\hline 11 & 4.6 & -- & 4.5 & --- & 5.6 & .8 & --- & 5.1 & 5.4 & 9.4 & 11.7 & $\cdots$ \\
\hline 12 & 4.4 & --- & 5.2 & -- & 5.5 & 1.0 & --- & 4.7 & 5.1 & 9.7 & 12.0 & -- \\
\hline 13 & 4.1 & --- & 4.5 & --- & 5.3 & 1.2 & --- & 4.8 & 5.2 & 10.2 & 12.3 & --- \\
\hline 14 & 3.6 & --- & 3.0 & --- & 4.9 & 1.1 & --- & --- & 5.2 & 10.8 & 12.5 & $-\cdots$ \\
\hline 15 & 3.5 & -- & 2.6 & --- & 5.2 & 3.4 & --- & 4.9 & 7.4 & 10.7 & --- & $\cdots$ \\
\hline 16 & --- & -- & 4.4 & --- & 5.4 & -+ & -- & 4.1 & 8.5 & 9.7 & 12.4 & -- \\
\hline 17 & 4.1 & --- & 4.8 & --- & 4.9 & -- & --- & 4.1 & 7.7 & 9.3 & 12.4 & --- \\
\hline 18 & 4.4 & --- & 4.5 & --- & 5.5 & --- & --- & 4.2 & 7.4 & --- & 12.1 & $-\cdots$ \\
\hline 19 & 5.9 & --- & 3.6 & --- & 4.2 & -- & --- & 3.8 & 6.7 & --- & 11.0 & $\cdots$ \\
\hline 20 & 7.1 & --- & 2.2 & --- & 3.9 & --- & --- & 3.2 & 7.0 & -- & 9.4 & --- \\
\hline 21 & -- & -- & 1.8 & --- & 4.5 & 5.7 & --- & 4.7 & 6.7 & --- & 9.1 & $-\cdots$ \\
\hline 22 & --- & --- & 2.0 & --- & 3.6 & 4.6 & -- & 5.5 & 6.8 & -- & 8.7 & -- \\
\hline 23 & --- & --- & -- & 2.8 & 3.2 & 5.0 & --- & 5.2 & 7.6 & -- & 8.6 & --- \\
\hline 24 & 7.2 & --- & 1.8 & 3.7 & 2.8 & 6.4 & --- & 4.4 & 8.4 & --- & 8.8 & --- \\
\hline 25 & 7.1 & --- & 1.7 & 7.0 & 2.5 & 7.1 & --- & 4.1 & 8.4 & --- & 8.2 & -- \\
\hline 26 & -- & -- & 2.4 & 6.8 & 2.2 & -- & 1.4 & 5.2 & 9.3 & $\cdots$ & 7.5 & -- \\
\hline 27 & --- & $\cdots$ & 2.4 & 6.3 & 2.0 & --- & 1.2 & 6.3 & 9.5 & --- & 7.1 & --- \\
\hline 28 & --- & --- & 2.1 & 6.0 & 1.8 & $-\cdots$ & 2.1 & 6.7 & 8.9 & $\cdots$ & 7.5 & $\cdots$ \\
\hline 29 & --- & --- & 1.4 & 6.4 & -- & -- & 4.4 & 5.7 & 8.7 & --- & 7.6 & -- \\
\hline 30 & --- & --- & .5 & 6.5 & --- & --- & 4.8 & 5.6 & 9.0 & -- & 9.8 & --- \\
\hline 31 & -- & --- & 1.7 & 6.8 & --- & -- & --- & 4.8 & --- & --- & 9.8 & --- \\
\hline
\end{tabular}

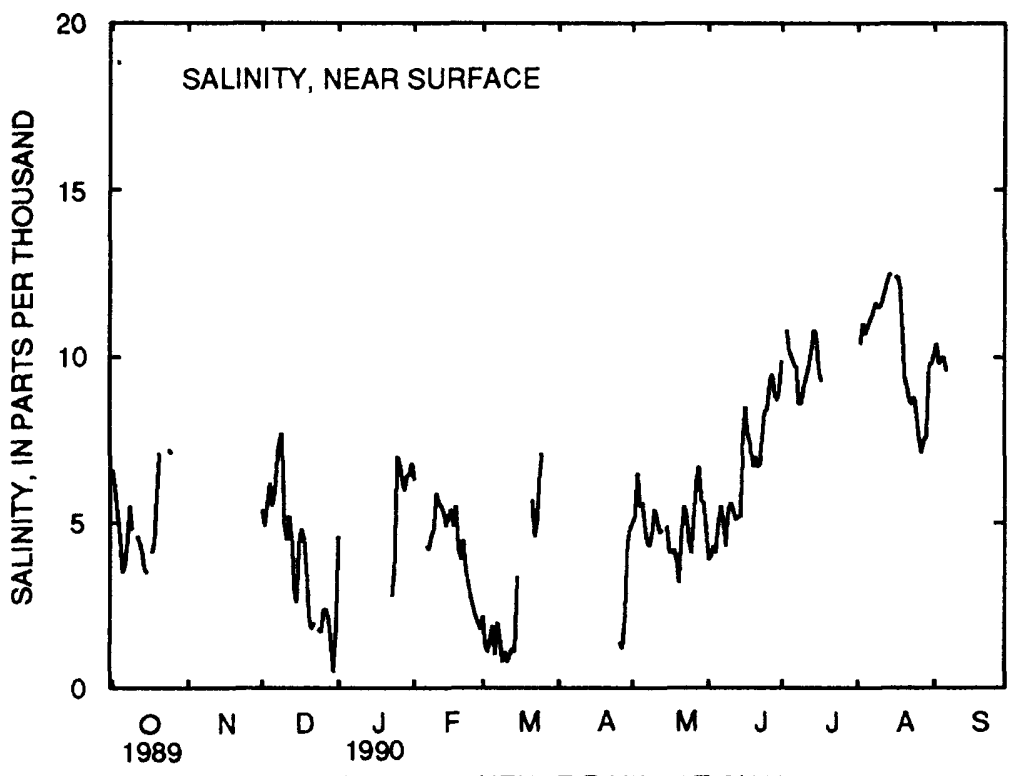

DAILY MEAN SALINITY, NEUSE RIVER AT CHANNEL LIGHT 9 
Table 11.--Daily mean values of salinity, water temperature, and dissolved oxygen in water at site 9, Neuse River at Channel Light 9, May 1989 to September 1990--Continued

[Site 9 is at latitude $34^{\circ} 56^{\prime} 54^{\prime \prime}$, longitude $76^{\circ} 48^{\prime} 36^{\prime \prime}$, Craven County; U.S. Geological Survey downstream order number 0209265810 ; ppt, parts per thousand; ---, no data; ${ }^{\circ} \mathrm{C}$, degrees

Celstus; mg/L, milligrams per liter; a, dally minimum value 1s less than or equal to $2.0 \mathrm{mg} / \mathrm{L}$; b, daily minimum value is less than or equal to $5.0 \mathrm{mg} / \mathrm{L}$ but greater than $2.0 \mathrm{mg} / \mathrm{L}$; <, less than]

SALINITY, NEAR BOTTOM

SENSOR POSITION. -2 feet above streambed.

EXTREMES FOR MAY TO SEPTEMBER 1989.--Max Imum value recorded, 15.7 ppt June 29, 1989; minimum value recorded, 2.3 ppt July 28, 1989.

DAILY MEAN VALUES OF SALINITY, NEAR BOTTOM (IN PARTS PER THOUSAND), MAY TO SEPTEMBER 1989

\begin{tabular}{|c|c|c|c|c|c|}
\hline DAY & MAY & JUNE & JULY & AUG & SEPT \\
\hline 1 & --- & 6.4 & 8.0 & --- & 7.3 \\
\hline 2 & -- & 6.4 & 8.1 & --- & 7.4 \\
\hline 3 & -- & 7.0 & 7.9 & --- & 6.4 \\
\hline 4 & -- & 7.0 & 8.2 & --- & 9.0 \\
\hline 5 & --- & 7.3 & 7.6 & --- & 8.2 \\
\hline 6 & $-\cdots$ & 7.3 & 6.9 & --- & 8.0 \\
\hline 7 & --- & 8.2 & 5.9 & -- & 7.4 \\
\hline 8 & --- & 8.0 & --- & -- & 7.3 \\
\hline 9 & --- & 8.8 & --- & --- & 7.1 \\
\hline 10 & - - & 8.6 & --- & --- & 7.5 \\
\hline 11 & -- & 8.5 & --- & --- & 7.7 \\
\hline 12 & -- & 8.9 & -- & --- & 9.0 \\
\hline 13 & -- & 8.3 & --- & --- & 8.0 \\
\hline 14 & -- & 8.2 & -- & -- & 7.8 \\
\hline 15 & -- & 8.0 & --- & --- & 8.6 \\
\hline 16 & -- & 8.7 & $\ldots$ & --- & 9.7 \\
\hline 17 & -- & 9.0 & --- & -- & 9.7 \\
\hline 18 & - - & 8.8 & --- & --- & 8.9 \\
\hline 19 & -- & 10.9 & --- & -- & 7.4 \\
\hline 20 & -- & 11.4 & --- & --- & 8.7 \\
\hline 21 & --- & 10.3 & 8.6 & --- & 9.1 \\
\hline 22 & -- & 10.8 & 8.7 & -- & 9.5 \\
\hline 23 & -- & 12.2 & 9.1 & 8.9 & 8.5 \\
\hline 24 & --- & 11.5 & 8.5 & 8.6 & 8.2 \\
\hline 25 & --- & 9.4 & 7.0 & 9.0 & 9.0 \\
\hline 26 & -- & 9.3 & 5.2 & 9.6 & 8.0 \\
\hline 27 & -- & 10.0 & 6.8 & 9.6 & 8.1 \\
\hline 28 & -- & 12.5 & 5.9 & 8.7 & 7.7 \\
\hline 29 & --- & 12.8 & 5.6 & 8.4 & 7.0 \\
\hline 30 & --- & 9.3 & --- & 7.4 & 6.4 \\
\hline 31 & 6.9 & -- & --- & 6.7 & $-\cdots$ \\
\hline
\end{tabular}

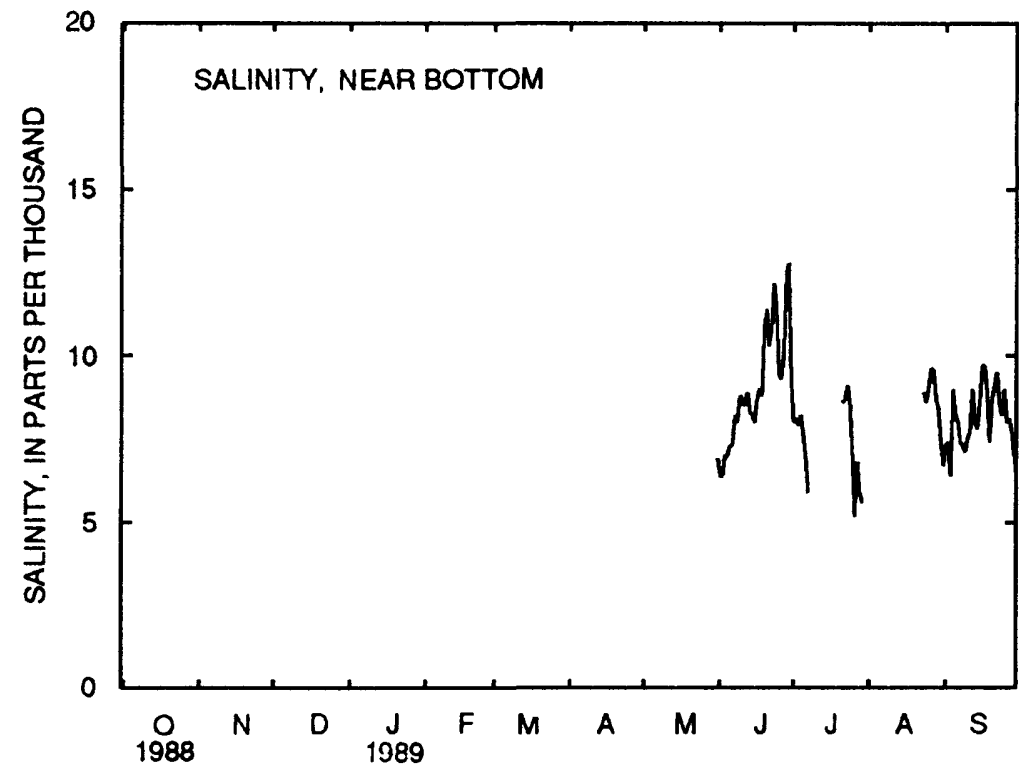

DAILY MEAN SALINITY, NEUSE RIVER AT CHANNEL LIGHT 9 
Table 11.--Daily mean values of salinity, water temperature, and dissolved oxygen in water at site 9, Neuse River at Channel Light 9, May 1989 to september 1990--Continued

[Site 9 is at latitude $34^{\circ} 56^{\prime} 54^{\prime \prime}$, longitude $76^{\circ} 48^{\prime} 36^{\prime \prime}$, Craven County; U.S. Geological Survey downst ream order number 0209265810; ppt, parts per thousand; ---, no data; ${ }^{\circ} \mathrm{C}$, degrees Celsius; mg/L, milligrams per liter; a, dally minimum value is less than or equal to $2.0 \mathrm{mg} / \mathrm{L}$; b, daily minimum value $1 \mathrm{~s}$ less than or equal to $5.0 \mathrm{mg} / \mathrm{L}$ but greater than $2.0 \mathrm{mg} / \mathrm{L}$; <, less than]

SALINITY, NEAR BOTTOM

SENSOR POSITION. -2 feet above streambed.

EXTREMES FOR OCTOBER 1989 TO SEPTEMBER 1990.--Maximum value recorded, 15.1 ppt August 17, 1990; minimum value recorded, 0.5 ppt December 24, 1989.

DAILY MEAN VALUES OF SALINITY, NEAR BOTTOM (IN PARTS PER THOUSAND), OCTOBER 1989 TO SEPTEMBER 1990

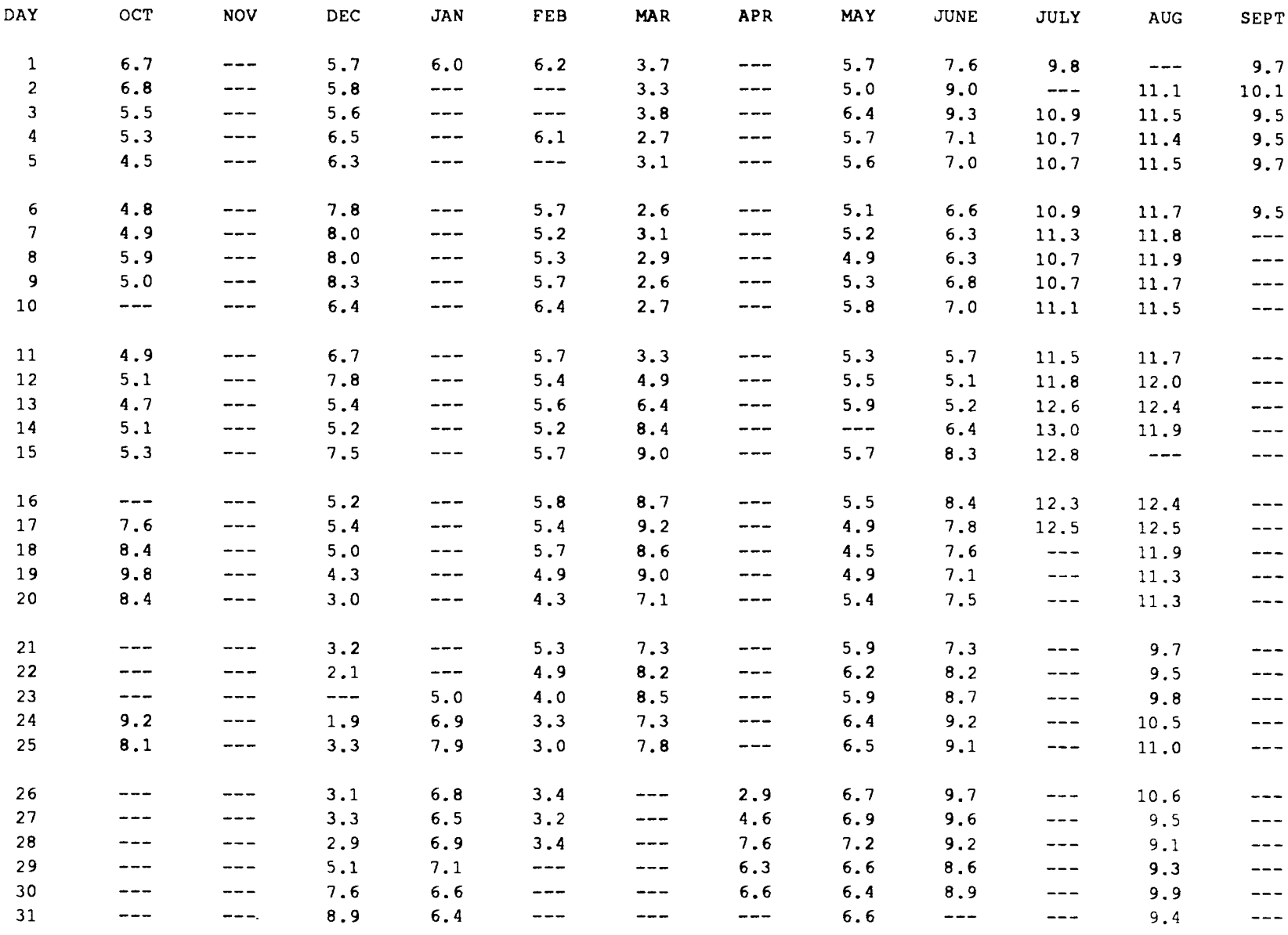

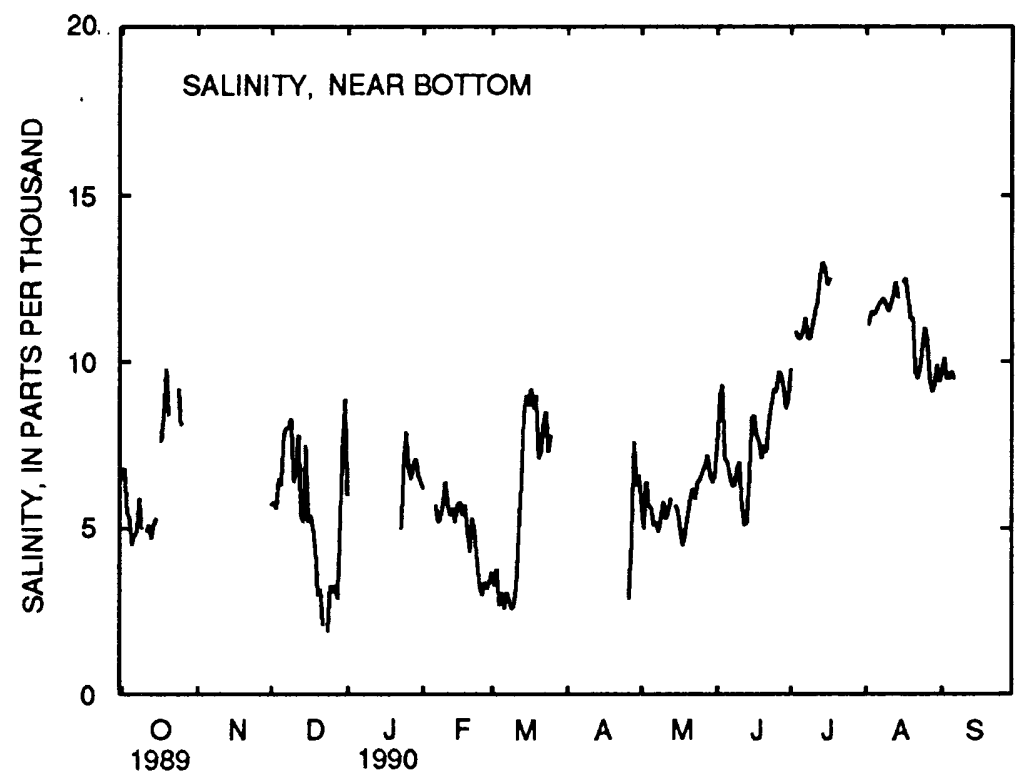

DAILY MEAN SALINITY, NEUSE RIVER AT CHANNEL LIGHT 9 
Table 11.--Dałly mean values of salinity, water temperature, and dissolved oxygen in water at site 9, Neuse River at Channel Light 9, May 1989 to September 1990--Cont1nued

[S1te 9 is at latitude $34^{\circ} 56^{\prime} 54^{\prime \prime}$, longltude $76^{\circ} 48^{\prime} 36^{\prime \prime}$, Craven County; U.S. Geologlcal Survey downstream order number 0209265810 ; ppt, parts per thousand; ---, no data; ${ }^{\circ} \mathrm{C}$, degrees

Celsius; mg/L, milligrams per liter; a, dally minimum value is less than or equal to $2.0 \mathrm{mg} / \mathrm{L} ; \mathrm{b}$, daily minlmum value is less than or equal to $5.0 \mathrm{mg} / \mathrm{L}$ but greater than $2.0 \mathrm{mg} / \mathrm{L}$; <, less than]

WATER TEMPERATURE, NEAR SURFACE

SENSOR POSITION. --8 feet above streambed.

EXTREMES FOR MAY TO SEPTEMBER 1989.--Max1mum value recorded, $32.3^{\circ} \mathrm{C}$ August 6,$1989 ; \mathrm{m} 1 \mathrm{nimum}$ value recorded, 21.8 ${ }^{\circ} \mathrm{C}$ september $28,1989$.

DAILY MEAN VALUES OF WATER TEMPERATURE, NEAR SURFACE (IN DEGREES CELSIUS), MAY TO SEPTEMBER 1989

\begin{tabular}{|c|c|c|c|c|c|}
\hline DAY & MAY & JUNE & JULY & AUG & SEPT \\
\hline 1 & --- & 24.5 & 26.3 & 28.1 & 28.2 \\
\hline 2 & --- & 25.5 & 26.5 & 28.0 & 28.5 \\
\hline 3 & --- & 24.6 & 26.3 & 28.7 & 28.1 \\
\hline 4 & --- & 24.7 & 26.3 & 28.9 & 26.3 \\
\hline 5 & --- & 24.9 & 26.5 & 29.0 & 26.2 \\
\hline 6 & --- & 24.6 & 26.5 & 29.7 & 26.3 \\
\hline 7 & --- & 24.6 & 26.9 & 30.2 & 26.1 \\
\hline 8 & --- & 24.8 & --- & 29.0 & 26.1 \\
\hline 9 & --- & 24.1 & --- & 26.6 & 26.1 \\
\hline 10 & --- & 25.1 & --- & 25.2 & 26.4 \\
\hline 11 & --- & 25.8 & --- & 24.5 & 26.9 \\
\hline 12 & --- & 25.6 & --- & 24.8 & 27.0 \\
\hline 13 & --- & 25.5 & --- & 25.5 & 27.2 \\
\hline 14 & --- & 26.3 & --- & 26.3 & 27.2 \\
\hline 15 & --- & 26.8 & --- & 26.3 & 27.2 \\
\hline 16 & $\cdots$ & 26.9 & -- & 27.1 & 27.3 \\
\hline 17 & -- & 26.5 & --- & 27.7 & 27.1 \\
\hline 18 & --- & 26.5 & --- & 27.3 & 26.3 \\
\hline 19 & --- & 26.7 & --- & 27.1 & 25.6 \\
\hline 20 & $-\cdots$ & 27.0 & --- & 27.2 & 25.7 \\
\hline 21 & --- & 26.9 & 27.7 & 27.8 & 26.2 \\
\hline 22 & --- & 26.7 & 27.7 & 28.3 & 26.7 \\
\hline 23 & --- & 27.0 & 28.7 & 28.8 & 26.6 \\
\hline 24 & --- & 26.8 & 29.1 & 29.0 & 25.0 \\
\hline 25 & --- & 27.0 & 28.7 & 28.7 & 23.3 \\
\hline 26 & --- & 27.8 & 28.8 & 27.6 & 24.0 \\
\hline 27 & --- & 27.9 & 28.8 & 27.6 & 23.5 \\
\hline 28 & --- & 27.9 & 28.6 & 27.6 & 22.4 \\
\hline 29 & --- & 27.7 & 28.9 & 28.2 & 22.6 \\
\hline 30 & --- & 26.5 & 28.4 & 28.3 & 23.2 \\
\hline 31 & 23.4 & --- & 28.4 & 28.1 & --- \\
\hline
\end{tabular}

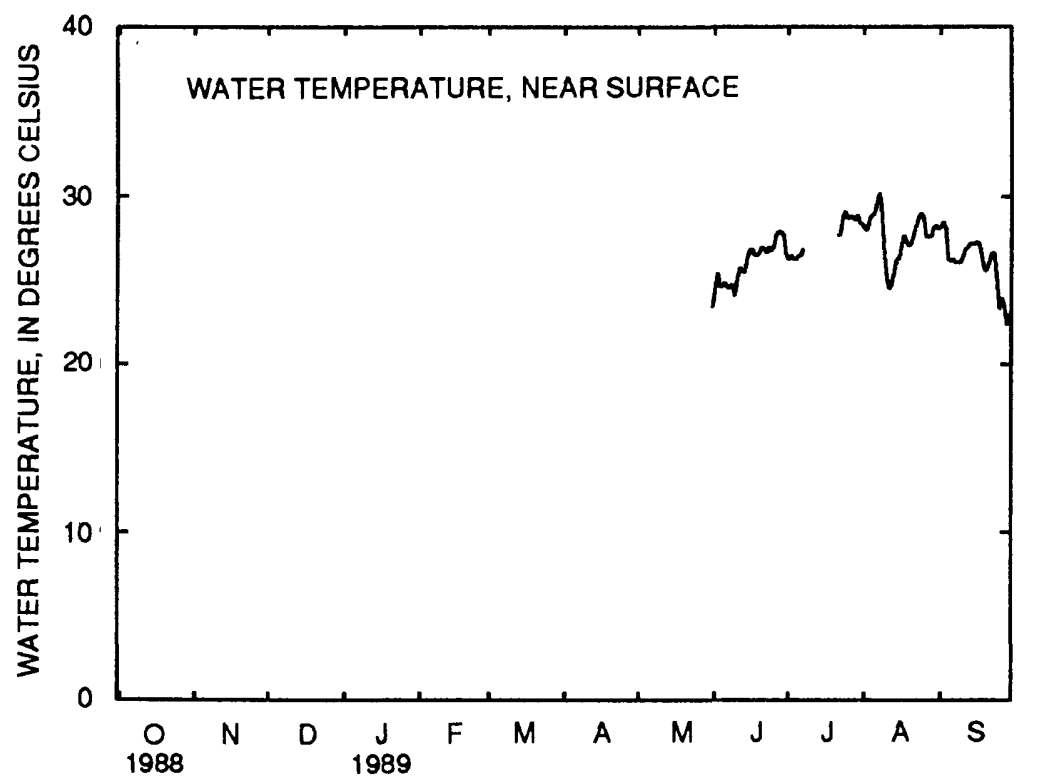

DAILY MEAN WATER TEMPERATURE, NEUSE RIVER AT CHANNEL LIGHT 9 
Table 11.--Dally mean values of salinity, water temperature, and dissolved oxygen in water at site 9 , Neuse River at Channel Light 9, May 1989 to September 1990--Continued

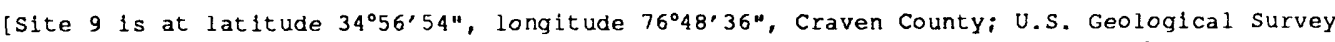
downstream order number 0209265810; ppt, parts per thousand; ---, no data; ${ }^{\circ} \mathrm{C}$, degrees

Celsius; mg/L, m1lligrams per 11ter; a, dally minimum value is less than or equal to $2.0 \mathrm{mg} / \mathrm{L}$; b, daily minimum value is less than or equal to $5.0 \mathrm{mg} / \mathrm{L}$ but greater than $2.0 \mathrm{mg} / \mathrm{L}$; <, less than]

WATER TEMPERATURE, NEAR SURFACE

SENSOR POSITION. --8 feet above streambed.

EXTREMES FOR OCTOBER 1989 TO SEPTEMBER 1990.--Maximum value recorded, $31.8{ }^{\circ} \mathrm{C} \mathrm{August} 27,28$, 1990 ; minimum value recorded, $3.4{ }^{\circ} \mathrm{C}$ December 20, 1989.

DAILY MEAN VALUES OF WATER TEMPERATURE, NEAR SURFACE (IN DEGREES CELSIUS), OCTOBER 1989 TO SEPTEMBER 1990

\begin{tabular}{|c|c|c|c|c|c|c|c|c|c|c|c|c|}
\hline DAY & OCT & NOV & DEC & JAN & FEB & MAR & APR & MAY & JUNE & JULY & AUG & SEPT \\
\hline 1 & 23.0 & $\cdots-$ & 8.9 & --- & 11.7 & 10.9 & --- & 21.6 & 22.3 & 28.9 & --- & 28.2 \\
\hline 2 & 23.4 & --- & 7.8 & --- & --- & 10.9 & --- & 22.0 & 23.8 & --- & 29.1 & 28.4 \\
\hline 3 & 23.9 & -- & 8.7 & -- & -- & 11.5 & --- & 22.5 & 24.3 & 27.8 & 28.5 & 28.4 \\
\hline 4 & 24.6 & --- & 6.7 & --- & 14.2 & 11.8 & -- & 22.3 & 24.7 & 27.6 & 28.6 & 27.7 \\
\hline 5 & 23.1 & --- & 7.6 & --- & -- & 11.6 & -- & 22.5 & 24.2 & 27.5 & 28.3 & 27.2 \\
\hline 6 & 22.4 & --- & 7.4 & --- & 12.8 & 12.5 & --- & 21.8 & 24.2 & 28.4 & 27.9 & 27.2 \\
\hline 7 & 23.1 & -- & 8.7 & -- & 13.4 & 12.4 & $-\cdots$ & 21.6 & 25.5 & 28.5 & 27.8 & $\cdots$ \\
\hline 8 & 21.5 & -- & 8.8 & --- & 13.6 & 11.8 & -- & 21.9 & 26.6 & 28.2 & 27.6 & --- \\
\hline 9 & 19.7 & --- & 8.1 & --- & 14.0 & 12.2 & --- & 21.9 & 26.4 & 28.6 & 27.2 & -- \\
\hline 10 & -- & -- & 7.2 & --- & 14.8 & 13.3 & -- & 22.0 & 26.8 & 28.7 & 27.0 & --- \\
\hline 11 & 18.6 & --- & 6.6 & --- & 14.5 & 14.3 & $-\infty$ & 21.5 & 26.8 & 28.8 & 27.7 & -- \\
\hline 12 & 19.2 & -- & 6.9 & --- & 14.1 & 14.9 & --- & 21.6 & 25.4 & 28.9 & 28.3 & --- \\
\hline 13 & 19.4 & -- & 6.8 & --- & 13.5 & 15.4 & --- & 21.9 & 24.9 & 28.9 & 28.9 & -- \\
\hline 14 & 19.9 & --- & 6.2 & --- & 14.0 & 16.5 & -- & $-m$ & 24.9 & 28.9 & 28.6 & -- \\
\hline 15 & 20.0 & --- & 6.4 & --- & 14.7 & 16.7 & -- & 22.8 & 25.0 & 28.4 & --- & -- \\
\hline 16 & --- & --- & 6.8 & --- & 15.8 & 14.2 & -- & 23.3 & 24.9 & 28.0 & 28.5 & -- \\
\hline 17 & 20.6 & -- & 5.7 & --- & 16.3 & 14.5 & -- & 23.9 & 25.4 & 28.3 & 28.7 & --- \\
\hline 18 & 21.1 & --- & 4.6 & --- & 15.1 & 15.8 & --- & 23.8 & 25.6 & --- & 28.6 & -- \\
\hline 19 & 21.4 & -- & 3.9 & --- & 15.1 & 17.0 & -- & 23.7 & 26.2 & --- & 28.8 & --- \\
\hline 20 & 20.4 & --- & 3.7 & --- & 14.5 & 16.2 & -- & --- & 26.7 & --- & 29.1 & -- \\
\hline 21 & -- & -- & --- & --- & 13.3 & 14.5 & -- & --- & 27.8 & --- & 28.6 & --- \\
\hline 22 & -- & -- & -- & --- & 14.1 & 14.4 & $m$ & --- & 28.1 & --- & 28.4 & - \\
\hline 23 & -- & -- & --- & 10.4 & 14.8 & 15.7 & --- & --- & 27.4 & --- & 28.4 & --- \\
\hline 24 & 15.6 & --- & --- & 10.7 & 14.6 & 16.0 & -- & -- & 27.6 & --- & 28.5 & --- \\
\hline 25 & 16.0 & -- & --- & 10.1 & 12.8 & 15.5 & --- & --- & 27.8 & --- & 28.4 & --- \\
\hline 26 & -- & $-\cdots$ & --- & 10.6 & 10.7 & --- & 22.2 & 21.8 & 27.5 & --- & 28.9 & --- \\
\hline 27 & --- & -- & --- & 9.8 & 10.4 & -- & 22.5 & 22.2 & 27.7 & --- & 30.0 & -- \\
\hline 28 & -- & --- & --- & 9.8 & 10.5 & --- & 22.0 & 21.8 & 28.3 & --- & 30.0 & -- \\
\hline 29 & --- & --- & --- & 10.3 & --- & --- & 21.1 & 22.4 & 28.7 & -- & 29.9 & -- \\
\hline 30 & --- & -- & --- & 11.1 & --- & --- & 20.8 & 21.3 & 28.8 & -- & 29.0 & --- \\
\hline 31 & $-\infty$ & -- & --- & 11.3 & --- & --- & -- & 21.5 & --- & --- & 28.5 & --- \\
\hline
\end{tabular}

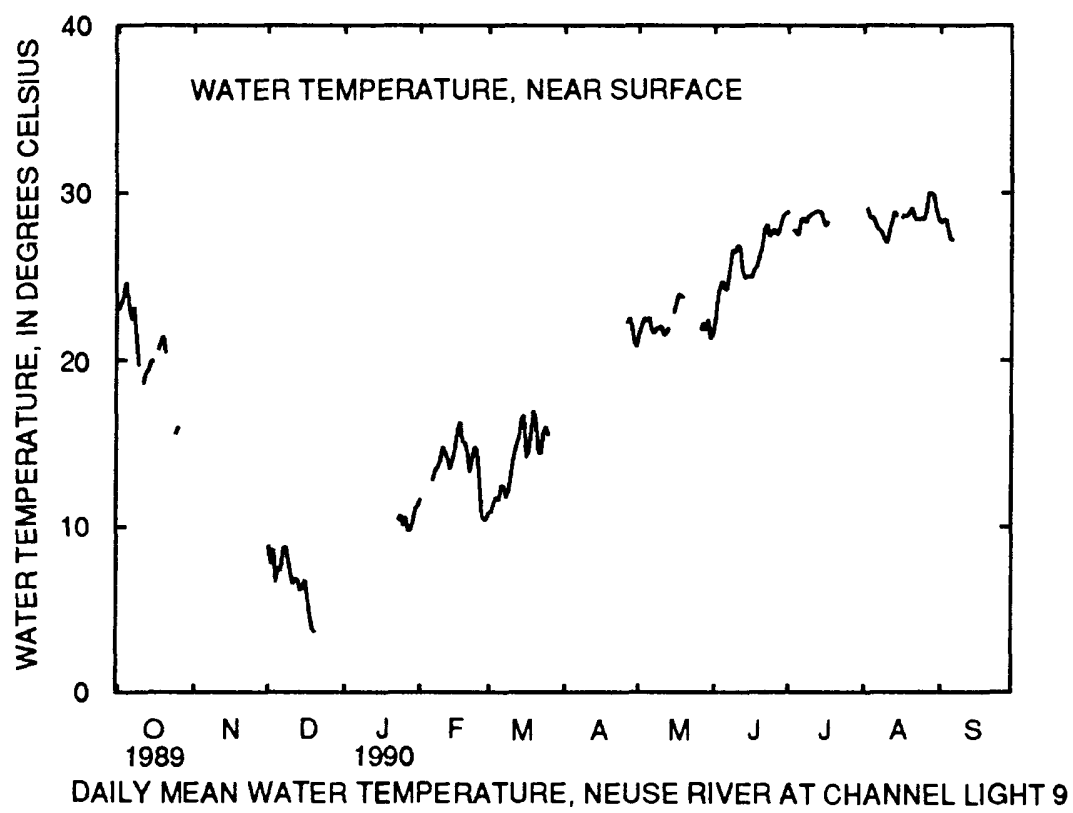


Table 11.--Dafly mean values of salinity, water temperature, and dissolved oxygen in water at site 9 , Neuse River at Channel Light 9, May 1989 to September 1990--Continued

[S1te 9 is at lat1tude $34^{\circ} 56^{\prime} 54^{\prime \prime}$, longltude $76^{\circ} 48^{\prime} 36^{\prime \prime}$, Craven County; U. S. Geological Survey downstream order number 0209265810; ppt, parts per thousand; ---, no data; ${ }^{\circ} \mathrm{C}$, degrees

Celsius; mg/L, milligrams per liter; a, daily minimum value is less than or equal to $2.0 \mathrm{mg} / \mathrm{L}$; b, daily minimum value is less than or equal to $5.0 \mathrm{mg} / \mathrm{L}$ but greater than $2.0 \mathrm{mg} / \mathrm{L}$; <, less thanl

DISSOLVED OXYGEN, NEAR SURFACE

SENSOR POSITION.--8 feet above streambed.

EXTREMES FOR MAY TO SEPTEMBER 1989.--Max1mum value recorded, $13.7 \mathrm{mg} / \mathrm{L}$ September 30 , 1989; minimum value recorded, $1.6 \mathrm{mg} / \mathrm{L}$ June $29,1989$.

DAILY MEAN VALUES OF DISSOLVED OXYGEN, NEAR SURFACE (IN MILLIGRAMS PER LITER), MAY TO SEPTEMBER 1989

\begin{tabular}{|c|c|c|c|c|c|}
\hline DAY & MAY & JUNE & JULY & AUG & SEPT \\
\hline 1 & --- & 8.1 & $6.5 b$ & --- & $6.4 \mathrm{~b}$ \\
\hline 2 & --- & 8.4 & $5.5 b$ & --- & $7.0 \mathrm{~b}$ \\
\hline 3 & -.- & $6.4 b$ & $4.4 \mathrm{a}$ & -- & 7.0 \\
\hline 4 & --- & $7.0 \mathrm{~b}$ & $4.2 b$ & --- & 6.2 \\
\hline 5 & --- & 7.3 & $4.0 \mathrm{~b}$ & --- & $6.1 b$ \\
\hline 6 & -- & 7.6 & $5.6 b$ & --- & 6.8 \\
\hline 7 & -- & 7.5 & $7.4 \mathrm{~b}$ & -- & 7.0 \\
\hline 8 & --- & 8.7 & --- & --- & 8.4 \\
\hline 9 & $-\cdots$ & 7.6 & --- & --- & 8.9 \\
\hline 10 & $-\cdots$ & 9.2 & --- & -- & 9.9 \\
\hline 11 & $\ldots$ & 9.4 & --- & --- & 8.8 \\
\hline 12 & --- & 7.7 & --- & --- & 7.9 \\
\hline 13 & --- & 7.7 & --- & --- & $7.2 b$ \\
\hline 14 & -- & $7.5 b$ & --- & --- & 7.3 \\
\hline 15 & --- & 7.5 & --- & --- & $7.0 \mathrm{~b}$ \\
\hline 16 & -- & 7.3 & -- & --- & $5.9 b$ \\
\hline 17 & --- & 7.0 & --- & --- & $6.8 b$ \\
\hline 18 & -- & 8.1 & --- & --- & $7.0 \mathrm{~b}$ \\
\hline 19 & --- & 7.9 & --- & --- & 7.7 \\
\hline 20 & --- & 7.4 & --- & -- & 7. $2 b$ \\
\hline 21 & --- & 6.3 & --- & --- & 7.3 \\
\hline 22 & --- & $5.9 b$ & --- & --- & 7.8 \\
\hline 23 & --- & $6.5 \mathrm{a}$ & $-\cdots$ & 6.7 & 7.7 \\
\hline 24 & --- & $6.4 \mathrm{~b}$ & --- & $6.7 \mathrm{~b}$ & 7.7 \\
\hline 25 & --- & $6.3 b$ & --- & $6.3 b$ & 7.9 \\
\hline 26 & --- & $7.1 \mathrm{~b}$ & --- & $6.0 \mathrm{~b}$ & 8.5 \\
\hline 27 & --- & $7.1 \mathrm{~b}$ & --- & $6.5 b$ & 9.3 \\
\hline 28 & --- & $6.3 b$ & --- & $6.0 \mathrm{~b}$ & 9.4 \\
\hline 29 & --- & $5.9 a$ & --- & 6.3 & 11.1 \\
\hline 30 & -- & $6.1 \mathrm{~b}$ & --- & $6.2 b$ & 11.7 \\
\hline 31 & 8.3 & -- & --- & 6.2 & -- \\
\hline
\end{tabular}

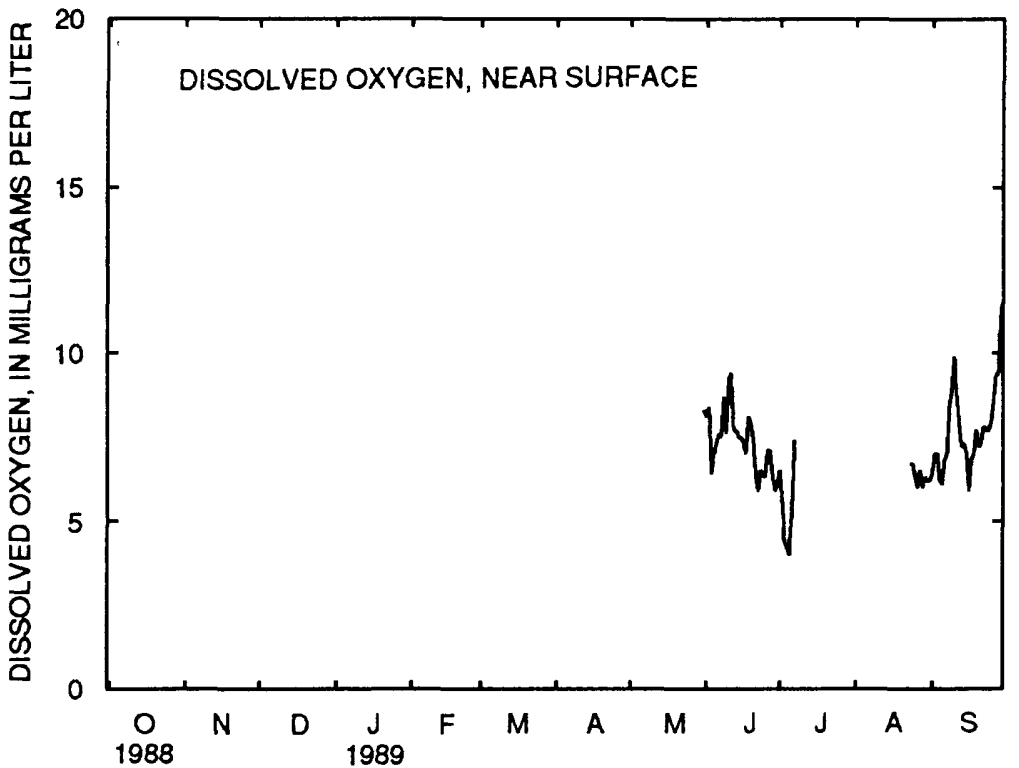

DAILY MEAN DISSOLVED OXYGEN, NEUSE RIVER AT CHANNEL LIGHT 9 
Table 11.--Daily mean values of salinity, water temperature, and dissolved oxygen in water at site 9, Neuse River at Channel Light 9, May 1989 to September 1990--Continued

[Site 9 is at latitude $34^{\circ} 56^{\prime} 54^{\prime \prime}$, long1tude $76^{\circ} 48^{\prime} 36^{\prime \prime}$, Craven County; U.S. Geological Survey downstream order number 0209265810; ppt, parts per thousand; ---, no data; ${ }^{\circ} \mathrm{C}$, degrees

Celsius; mg/L, milligrams per liter; a, dally minlmum value $1 \mathrm{~s}$ less than or equal to $2.0 \mathrm{mg} / \mathrm{L}$; b, daily minimum value is less than or equal to $5.0 \mathrm{mg} / \mathrm{L}$ but greater than $2.0 \mathrm{mg} / \mathrm{L}$; <, less than]

\section{DISSOLVED OXYGEN, NEAR SURFACE}

SENSOR POSITION. --8 feet above streambed.

EXTREMES FOR OCTOBER 1989 TO SEPTEMBER 1990.--Maximum value recorded, 17.6 mg/L February 1 , $1990 ;$ minimum value recorded, <1.0 mg/L June $15,1990$.

DAILY MEAN VALUES OF DISSOLVED OXYGEN, NEAR SURFACE (IN MILLIGRAMS PER LITER), OCTOBER 1989 TO SEPTEMBER 1990

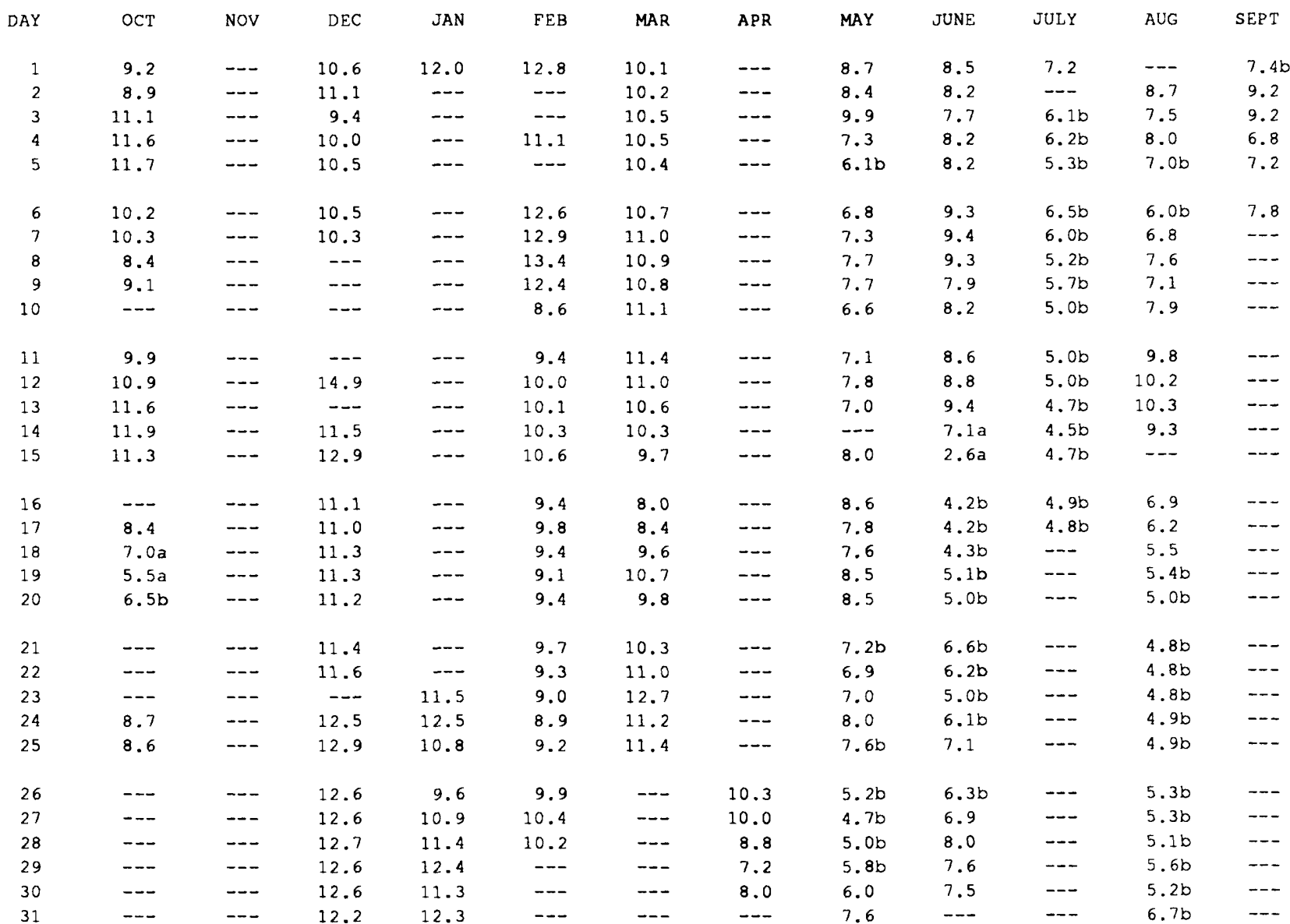

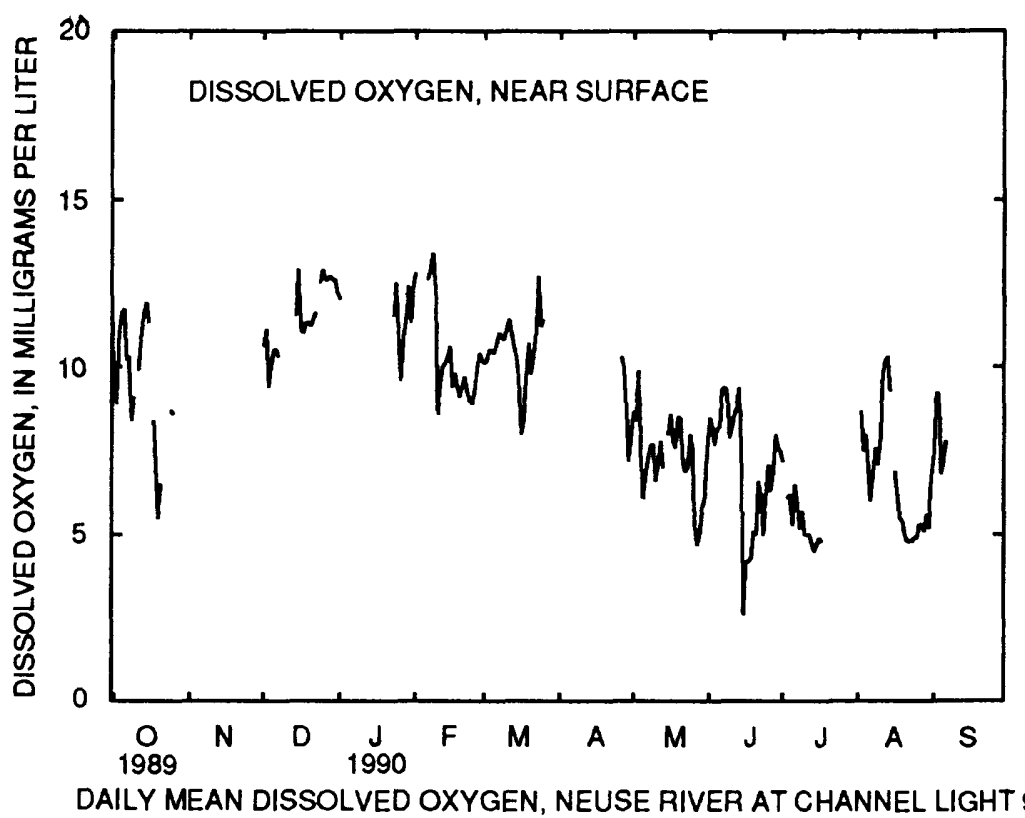


Table 11.--Dally mean values of salinity, water temperature, and dissolved oxygen 1n water at site 9, Neuse River at Channel Light 9, May 1989 to September 1990--Continued

[Site 9 is at latitude $34^{\circ} 56^{\prime} 54^{\prime \prime}$, longitude $76^{\circ} 48^{\prime} 36^{\prime \prime}$, Craven County; U.S. Geological Survey downstream order number 0209265810; ppt, parts per thousand; ---, no data; ${ }^{\circ} \mathrm{C}$, degrees

Celsius; mg/L, milligrams per liter; a, daily minimum value is less than or equal to $2.0 \mathrm{mg} / \mathrm{L}$; b, daily minimum value is less than or equal to $5.0 \mathrm{mg} / \mathrm{L}$ but greater than $2.0 \mathrm{mg} / \mathrm{L}$; <, less than]

DISSOLVED OXYGEN, MID-DEPTH

SENSOR POSITION.--5 feet above streambed.

EXTREMES FOR MAY TO SEPTEMBER 1989.--Maximum value recorded, $13.4 \mathrm{mg} / \mathrm{L}$ september 30 , 1989 ; minimum value recorded, <1.0 mg/L June 12, 13, 15, 16, 20, 21, 1989.

DAILY MEAN VALUES OE DISSOLVED OXYGEN, MID-DEPTH (IN MILLIGRAMS PER LITER), MAY TO SEPTEMBER 1989

\begin{tabular}{|c|c|c|c|c|c|}
\hline DAY & MAY & JUNE & JULY & AUG & SEPT \\
\hline 1 & --- & $6.5 b$ & $5.5 b$ & --- & $5.7 \mathrm{~b}$ \\
\hline 2 & -- & $6.3 b$ & $4.0 b$ & -- & $5.5 \mathrm{~b}$ \\
\hline 3 & -- & $4.6 b$ & $3.7 b$ & -- & 6.9 \\
\hline 4 & --- & $5.8 b$ & $3.5 b$ & -- & 6.5 \\
\hline 5 & -- & $5.9 b$ & $3.2 b$ & -- & 6.5 \\
\hline 6 & -- & $6.2 b$ & $3.2 b$ & -- & 7.2 \\
\hline 7 & -- & $5.7 \mathrm{~b}$ & $5.8 b$ & -- & 7.5 \\
\hline 8 & -- & $5.0 \mathrm{~b}$ & -- & --- & 8.5 \\
\hline 9 & -- & $5.2 b$ & $\ldots$ & -- & 8.3 \\
\hline 10 & --- & $5.6 b$ & $-\infty$ & --- & 8.2 \\
\hline 11 & -- & $4.7 a$ & -- & -- & 8.0 \\
\hline 12 & -- & $2.3 a$ & -- & -- & 7.2 \\
\hline 13 & $-\infty$ & $3.1 \mathrm{a}$ & -- & --- & $7.6 \mathrm{~b}$ \\
\hline 14 & --- & $3.7 \mathrm{a}$ & $\cdots$ & -- & 7.7 \\
\hline 15 & --- & $2.7 a$ & -- & -- & $6.2 b$ \\
\hline 16 & -- & $3.3 \mathrm{a}$ & -- & -- & $5.5 b$ \\
\hline 17 & -- & $3.7 \mathrm{a}$ & -- & -- & $6.1 b$ \\
\hline 18 & -- & $3.6 a$ & -- & -- & $7.3 b$ \\
\hline 19 & --- & $2.9 a$ & -- & --- & 8.1 \\
\hline 20 & $\ldots$ & $2.0 a$ & --- & --- & 7.16 \\
\hline 21 & -- & $2.1 a$ & $\ldots$ & -- & 7.5 \\
\hline 22 & -- & $1.2 a$ & $-\cdots$ & $-\cdots$ & 8.0 \\
\hline 23 & -- & $1.2 \mathrm{a}$ & -- & 5.3 & 7.6 \\
\hline 24 & -- & $2.0 \mathrm{a}$ & -- & 5.5 & 7.8 \\
\hline 25 & -- & $2.9 a$ & -- & 5.7 & 7.9 \\
\hline 26 & -- & $2.9 a$ & -- & 5.6 & 8.5 \\
\hline 27 & -- & $3.8 a$ & $-\cdots$ & 6.1 & 9.5 \\
\hline 28 & $\cdots$ & $3.3 a$ & -- & 5.7 & 9.8 \\
\hline 29 & --- & $4.5 a$ & $-\infty$ & 5.8 & 10.7 \\
\hline 30 & --- & $5.2 b$ & -- & 6.3 & 11.7 \\
\hline 31 & $6.8 b$ & --- & -- & 5.9 & -- \\
\hline
\end{tabular}

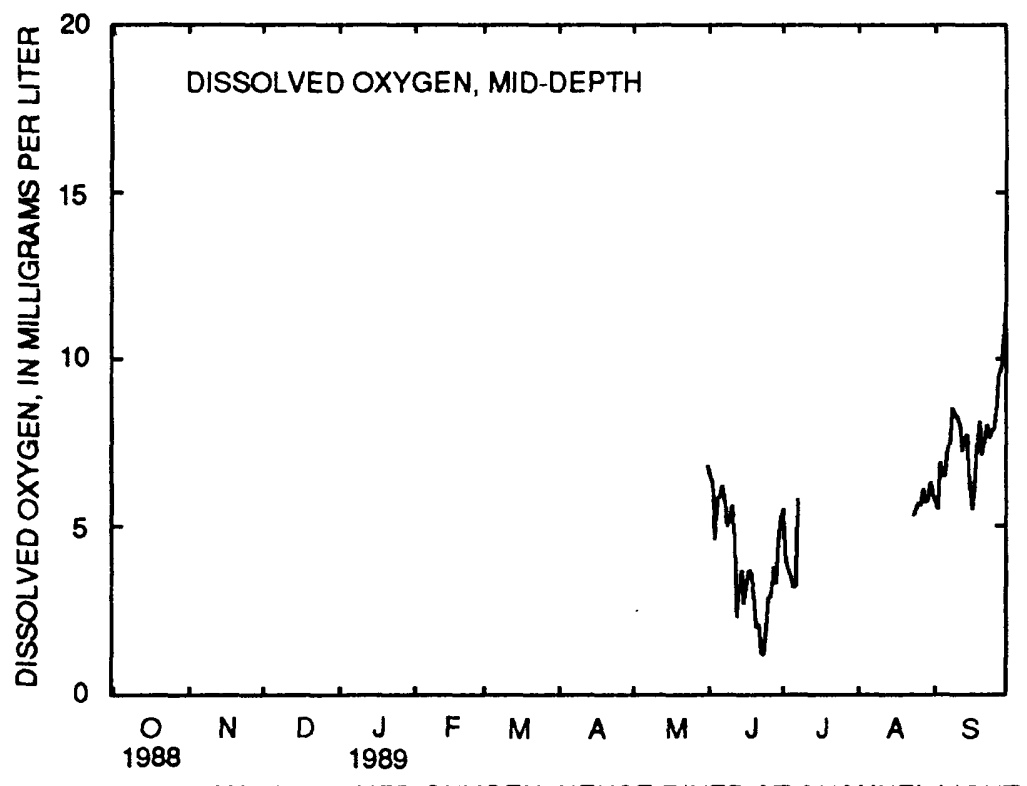

DAILY MEAN DISSOLVED OXYGEN, NEUSE RIVER AT CHANNEL LIGHT 9 
Table 11.--Daily mean values of salinity, water temperature, and dissolved oxygen in water at site 9, Neuse River at Channel Light 9, May 1989 to September 1990--Continued

[Site 9 is at latitude $34^{\circ} 56^{\prime} 54^{\prime \prime}$, longitude $76^{\circ} 48^{\prime} 36^{\prime \prime}$, Craven County; U.S. Geological Survey downstream order number 0209265810; ppt, parts per thousand; ---, no data; ${ }^{\circ} \mathrm{C}$, degrees

Celsius; mg/L, milligrams per liter; a, dally minimum value is less than or equal to $2.0 \mathrm{mg} / \mathrm{L}$; b, daily minimum value is less than or equal to $5.0 \mathrm{mg} / \mathrm{L}$ but greater than $2.0 \mathrm{mg} / \mathrm{L}$; <, less than]

DISSOLVED OXYGEN, MID-DEPTH

SENSOR POSITION. --5 feet above streambed.

EXTREMES FOR OCTOBER 1989 TO SEPTEMBER 1990.--Maximum value recorded, 17.8 mg/L January 31 , 1990 ; minimum value recorded, $1.1 \mathrm{mg} / \mathrm{L}$ June $22,1990$.

DAILY MEAN VALUES OF DISSOLVED OXYGEN, MID-DEPTH (IN MILLIGRAMS PER LITER), OCTOBER 1989 TO SEPTEMBER 1990

\begin{tabular}{|c|c|c|c|c|c|c|c|c|c|c|c|c|}
\hline DAY & OCT & NOV & $\mathrm{DEC}$ & JAN & $F E B$ & MAR & APR & MAY & JUNE & JULY & AUG & SEPT \\
\hline 1 & 9.7 & -- & 10.9 & 12.7 & 13.7 & 10.6 & -- & 8.6 & -- & -- & -- & $\cdots$ \\
\hline 2 & 8.4 & -- & 10.8 & -- & -- & 10.8 & $\rightarrow$ & 8.6 & -- & --- & 7.9 & -- \\
\hline 3 & 11.2 & -- & 10.0 & -- & -- & 10.5 & -- & 9.4 & --- & $5.4 \mathrm{~b}$ & 7.1 & --- \\
\hline 4 & 11.4 & -- & 10.6 & -- & 12.1 & 12.4 & -- & $6.9 \mathrm{~b}$ & --- & $5.5 b$ & 7.1 & -- \\
\hline 5 & 11.5 & $-\infty$ & 11.1 & -- & -- & 11.5 & -- & $5.9 b$ & --- & $4.5 b$ & $6.5 b$ & -- \\
\hline 6 & 10.7 & -- & 10.5 & -- & 12.7 & 10.7 & -- & 6.4 & -- & $5.1 \mathrm{~b}$ & 5.9 & $\ldots$ \\
\hline 7 & 10.8 & -- & 10.5 & -- & 13.4 & 13.0 & -- & 6.8 & -- & $5.1 \mathrm{~b}$ & 6.6 & -- \\
\hline 8 & 9.3 & -- & 10.7 & --- & 13.4 & 12.0 & $\ldots$ & 7.5 & $\ldots$ & $4.0 \mathrm{~b}$ & 7.4 &.- \\
\hline 9 & 10.0 & -- & 10.4 & --- & 11.8 & 11.7 & -- & 7.4 & $\cdots$ & $4.4 \mathrm{~b}$ & 7.1 & -- \\
\hline 10 & -- & -- & 10.6 & -- & 9.3 & 11.2 & -- & 6.5 & -- & $4.1 \mathrm{a}$ & 8.0 & $\ldots$ \\
\hline 11 & 10.6 & -- & 10.7 & -- & 10.2 & 11.2 & -- & 6.9 & -- & $4.1 \mathrm{~b}$ & 9.0 & -- \\
\hline 12 & 11.1 & -- & 10.6 & -- & 10.7 & 10.2 & --- & 7.1 & -- & $4.1 b$ & 9.4 & -- \\
\hline 13 & 11.6 & - & 11.0 & -- & 9.9 & 9.7 & -- & $6.3 \mathrm{~b}$ & -- & $3.8 \mathrm{~b}$ & 9.6 & $\ldots$ \\
\hline 14 & 10.9 & -- & 10.9 & - & 9.9 & 8.6 & -- & -- & --- & $3.8 \mathrm{~b}$ & 8.7 & --- \\
\hline 15 & 10.6 & --- & 11.4 & $\cdots$ & 10.4 & 7.2 & --- & 7.7 & --- & $3.9 b$ & --- & $-\cdots$ \\
\hline 16 & - & -- & 11.1 & --- & 9.7 & 7.4 & -- & 8.7 & $4.9 b$ & $4.1 \mathrm{~b}$ & 8.6 & --- \\
\hline 17 & $5.7 b$ & --- & 11.1 & -- & 10.3 & 8.1 & -- & 8.0 & $4.4 b$ & $3.9 \mathrm{~b}$ & 8.2 & --- \\
\hline 18 & $6.0 \mathrm{~b}$ & -- & 11.6 & --- & 10.6 & 9.5 & -- & 8.0 & $3.9 a$ & -- & 8.0 & --- \\
\hline 19 & $5.4 b$ & --- & 12.0 & --- & 10.1 & 9.8 & -- & 8.6 & $4.7 \mathrm{~b}$ & --- & 8.0 & -- \\
\hline 20 & $7.0 \mathrm{~b}$ & -- & 12.1 & --- & 10.6 & 9.4 & -- & 8.4 & $4.6 a$ & -- & 7.4 & - \\
\hline 21 & -- & -- & 11.9 & --- & 10.7 & 10.2 & -- & $7.5 b$ & $6.3 b$ & -- & 7.0 & --- \\
\hline 22 & $-\infty$ & -- & 12.6 & -- & 9.8 & 10.7 & --- & 7.4 & $4.7 \mathrm{a}$ & -- & 6.6 & --- \\
\hline 23 & $\ldots$ & -- & --- & 11.3 & 9.5 & 11.6 & -- & 7.5 & $5.0 \mathrm{~b}$ & -- & $6.3 b$ & -- \\
\hline 24 & 8.4 & -- & 13.5 & 12.5 & 9.9 & 11.0 & -- & 7.5 & --- & -- & $5.9 b$ & --- \\
\hline 25 & 8.6 & --- & 11.1 & 11.0 & 10.2 & 10.9 & -- & --- & $\cdots$ & $\cdots$ & $3.9 a$ & --- \\
\hline 26 & -- & -- & 12.9 & 10.4 & 10.5 & $\ldots$ & 9.6 & -- & $-\infty$ & -- & -- & --- \\
\hline 27 & -- & -- & 13.2 & 10.2 & 10.2 & --- & 9.0 & -- & -- & --- & --- & $-\cdots$ \\
\hline 28 & --- & -- & 13.2 & 9.8 & 10.7 & -- & $6.1 b$ & -- & -- & -- & -- & -- \\
\hline 29 & --- & --- & 13.0 & 11.2 & -- & --- & $6.6 b$ & --- & -- & $\cdots$ & -- & -- \\
\hline 30 & --- & --- & 13.1 & 10.8 & -- & -- & $7.4 \mathrm{~b}$ & --- & --- & -- & --- & --- \\
\hline 31 & --- & -- & 13.0 & 13.2 & --- & -- & --- & --- & --- & --- & --- & --- \\
\hline
\end{tabular}

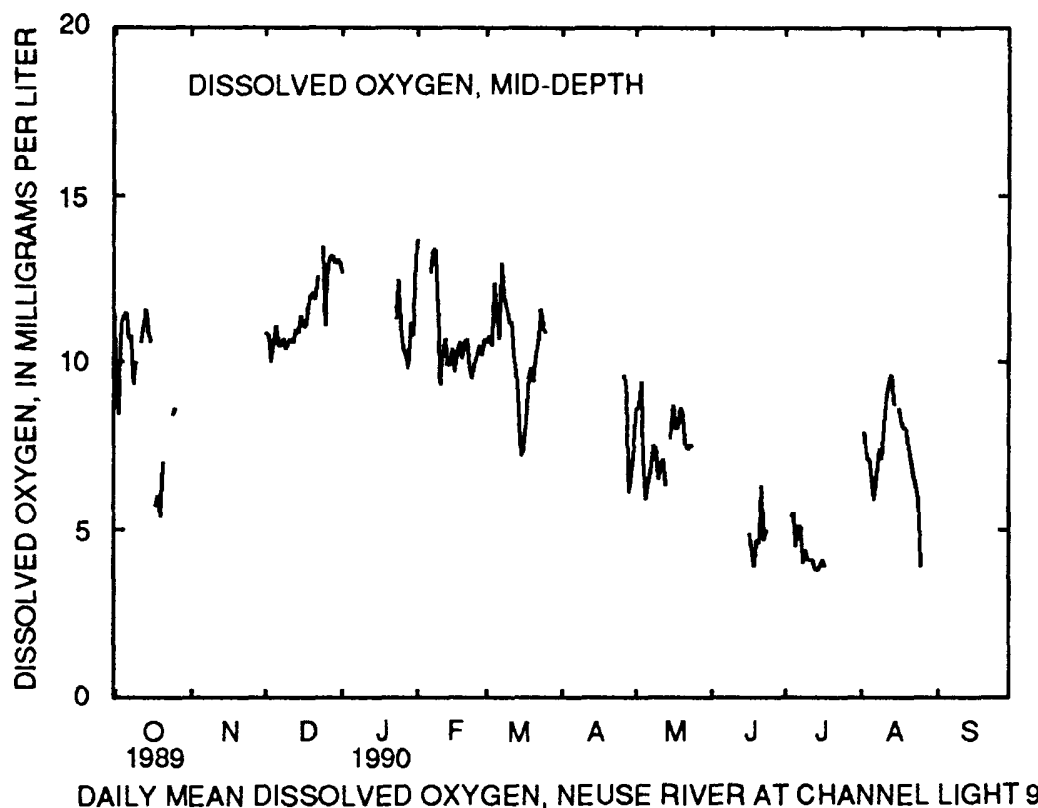


Table 11.--Daily mean values of salinity, water temperature, and dissolved oxygen in water at site 9, Neuse River at Channel Light 9, May 1989 to September 1990--Cont Inued

[S1te 9 is at latitude $34^{\circ} 56^{\prime} 54^{\prime \prime}$, longitude $76^{\circ} 48^{\prime} 36^{\prime \prime}$, Craven County; U.S, Geological Survey downstream order number 0209265810 ; ppt, parts per thousand; ---, no data; ${ }^{\circ} \mathrm{C}$, degrees

Celsius; mg/L, milligrams per liter; a, dally minimum value is less than or equal to $2.0 \mathrm{mg} / \mathrm{L}$; b, daily minimum value is less than or equal to $5.0 \mathrm{mg} / \mathrm{L}$ but greater than $2.0 \mathrm{mg} / \mathrm{L}$; $<$, less than]

DISSOLVED OXYGEN, NEAR BOTTOM

SENSOR POSITION.--2 feet above streambed.

EXTREMES FOR MAY TO SEPTEMBER 1989.--Maximum value recorded, $11.3 \mathrm{mg} / \mathrm{L}$ July 1,$1989 ; \mathrm{m} 1 \mathrm{nimum}$ value recorded, <1.0 $\mathrm{mg} / \mathrm{L}$ May 31, June 1-5, 12, 13, 15-17, August 23, 29, September 2, 1989.

DAILY MEAN VALUES OF DISSOLVED OXYGEN, NEAR BOTTOM (IN MILLIGRAMS PER LITER), MAY TO SEPTEMBER 1989

\begin{tabular}{|c|c|c|c|c|c|}
\hline DAY & MAY & JUNE & JULY & AUG & SEPT \\
\hline 1 & --- & $3.8 a$ & $8.5 b$ & --- & $4.8 \mathrm{a}$ \\
\hline 2 & -- & $2.6 a$ & $7.5 b$ & --- & $3.0 a$ \\
\hline 3 & -- & $3.7 \mathrm{a}$ & $4.9 \mathrm{~b}$ & -- & $6.6 \mathrm{~b}$ \\
\hline 4 & --- & $4.5 a$ & $5.5 b$ & --- & $5.4 b$ \\
\hline 5 & -- & $4.5 a$ & $5.0 \mathrm{~b}$ & --- & $4.6 \mathrm{~b}$ \\
\hline 6 & -- & $5.9 \mathrm{~b}$ & $5.9 b$ & -- & $4.8 b$ \\
\hline 7 & --- & $4.7 \mathrm{a}$ & $6.1 b$ & --- & $5.5 b$ \\
\hline 8 & --- & $4.9 \mathrm{~b}$ & --- & --- & 8.0 \\
\hline 9 & -- & $5.0 \mathrm{~b}$ & --- & --- & 7.5 \\
\hline 10 & --- & $5.4 \mathrm{~b}$ & --- & --- & $5.6 \mathrm{~b}$ \\
\hline 11 & --- & $5.2 a$ & -- & -- & $4.6 \mathrm{~b}$ \\
\hline 12 & --- & $3.1 \mathrm{a}$ & -- & --- & $4.3 b$ \\
\hline 13 & --- & $4.5 a$ & --- & --- & $5.3 a$ \\
\hline 14 & --- & $4.6 a$ & -- & --- & $5.8 b$ \\
\hline 15 & --- & $3.7 \mathrm{a}$ & -- & --- & $2.1 a$ \\
\hline 16 & -- & $1.6 a$ & --- & --- & $1.8 a$ \\
\hline 17 & --- & $3.0 \mathrm{a}$ & --- & --- & $2.5 a$ \\
\hline 18 & --- & $2.0 \mathrm{a}$ & --- & --- & $5.1 a$ \\
\hline 19 & --- & $1.4 \mathrm{a}$ & --- & --- & 6.8 \\
\hline 20 & --- & $1.3 a$ & --- & -- & $5.3 a$ \\
\hline 21 & --- & -- & -- & --- & 6.7 \\
\hline 22 & --- & --- & --- & --- & 7.1 \\
\hline 23 & --- & --- & --- & 5.4 & 6.6 \\
\hline 24 & --- & --- & --- & 6.0 & 6.7 \\
\hline 25 & --- & --- & --- & 7.1 & 6.7 \\
\hline 26 & --- & -- & --- & 7.1 & 6.9 \\
\hline 27 & --- & --- & --- & 7.9 & 7.6 \\
\hline 28 & --- & --- & --- & 6.4 & 7.7 \\
\hline 29 & --- & --- & --- & 5.3 & 7.2 \\
\hline 30 & -- & 6.1 & --- & 7.0 & 7.3 \\
\hline 31 & 4.3 & --- & --- & 6.2 & --- \\
\hline
\end{tabular}

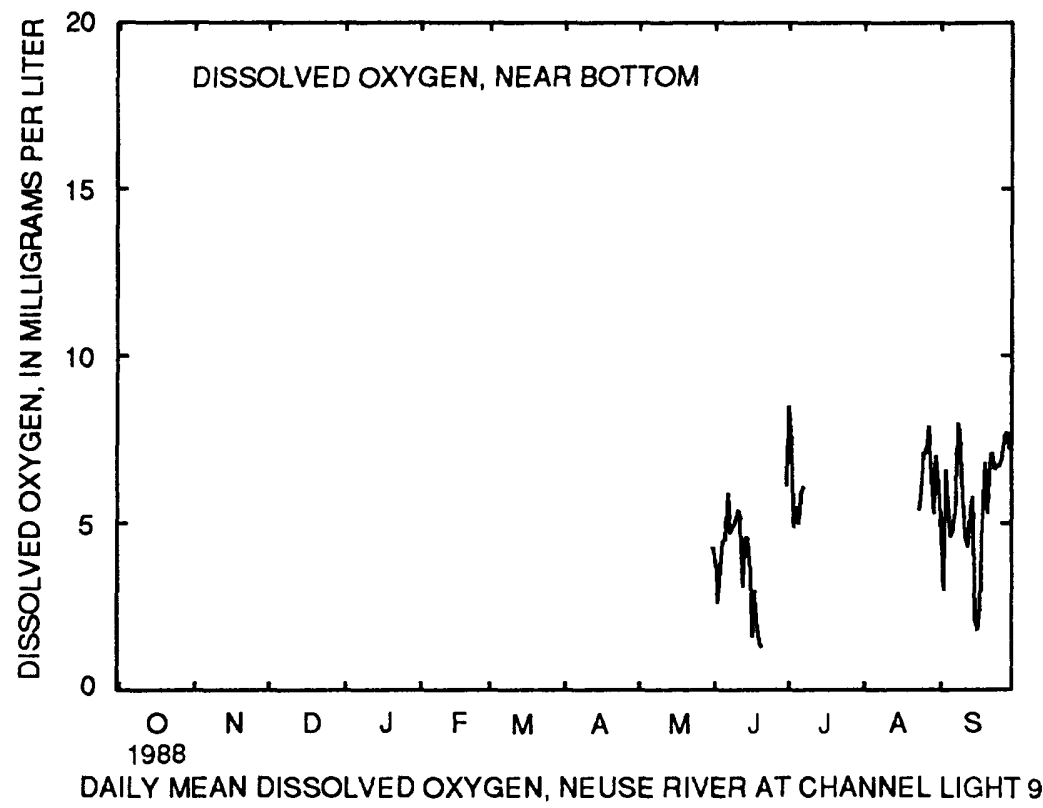


Table 11.--Dally mean values of salinity, water temperature, and dissolved oxygen in water at site 9 , Neuse River at Channel Light 9, May 1989 to September 1990--Continued

[Site 9 is at latitude $34^{\circ} 56^{\prime} 54^{\prime \prime}$, longitude $76^{\circ} 48^{\prime} 36^{\prime \prime}$, Craven County; U.S. Geological Survey downstream order number 0209265810; ppt, parts per thousand; ---, no data; ${ }^{\circ} \mathrm{C}$, degrees Celsius; $\mathrm{mg} / \mathrm{L}$, milligrams per liter; a, daily minimum value is less than or equal to $2.0 \mathrm{mg} / \mathrm{L}$; b, daily minimum value is less than or equal to $5.0 \mathrm{mg} / \mathrm{L}$ but greater than $2.0 \mathrm{mg} / \mathrm{L}$; <, less thanl

\section{DISSOLVED OXYGEN, NEAR BOTTOM}

SENSOR POSITION. --2 feet above streambed.

EXTREMES FOR OCTOBER 1989 TO SEPTEMBER 1990.--Maximum value recorded, 16.3 mg/L January 31, 1990; minimum value recorded, <1.0 mg/L March 23, Apri1 26-30, May 1, 4, 7, 16, 17, 20, 21, June 15, July 9, 1990.

DAILY MEAN VALUES OF DISSOLVED OXYGEN, NEAR BOTTOM (IN MILLIGRAMS PER LITER), OCTOBER 1989 TO SEPTEMBER 1990

\begin{tabular}{|c|c|c|c|c|c|c|c|c|c|c|c|c|}
\hline DAY & OCT & NOV & DEC & JAN & FEB & MAR & APR & MAY & JUNE & JULY & AUG & SEPT \\
\hline 1 & 6.9 & -- & 10.9 & 12.5 & 11.9 & $5.3 b$ & $\ldots$ & $5.0 a$ & $\ldots$ & $6.8 \mathrm{~b}$ & -- & $6.6 \mathrm{~b}$ \\
\hline 2 & $4.7 \mathrm{a}$ & --- & 10.9 & --- & -- & $5.0 \mathrm{~b}$ & -- & 8.2 & --- & -- & $6.4 \mathrm{a}$ & 6.8 \\
\hline 3 & 7.2 & -- & 10.3 & -- & -- & $5.3 b$ & -- & $7.6 \mathrm{~b}$ & -- & $4.9 \mathrm{~b}$ & -- & $7.6 \mathrm{~b}$ \\
\hline 4 & $7.4 \mathrm{~b}$ & -- & 10.8 & --- & 9.5 & -- & -- & $5.3 a$ & -- & $5.3 a$ & -- & $7.6 \mathrm{~b}$ \\
\hline 5 & $6.5 b$ & -- & 10.8 & -- & -- & $\cdots$ & $-\infty$ & $5.7 \mathrm{~b}$ & --- & $4.6 \mathrm{~b}$ & --- & $7.4 \mathrm{~b}$ \\
\hline 6 & $5.8 b$ & -- & 10.2 & --- & 10.4 & -- & -- & $5.9 b$ & -- & $5.0 \mathrm{a}$ & -. & $7.7 b$ \\
\hline 7 & $6.3 b$ & -- & 10.6 & --- & 10.4 & -- & -- & $5.0 a$ & --- & $5.5 b$ & --- & -- \\
\hline 8 & $5.1 \mathrm{~b}$ & -- & 10.8 & -- & 10.0 & -- & $-\cdots$ & $5.3 a$ & -- & $4.1 \mathrm{~b}$ & $\ldots$ & -- \\
\hline 9 & $5.7 \mathrm{~b}$ & --- & 10.7 & -- & --- & -- & -- & $5.2 a$ & --- & $4.2 \mathrm{a}$ & -- & -- \\
\hline 10 & --- & --- & 10.6 & --- & -- & --- & -- & $5.7 a$ & -- & $4.5 a$ & -- & -- \\
\hline 11 & $5.6 b$ & -- & 10.7 & --- & 9.3 & -- & -- & 6.9 & -- & $4.5 b$ & $-\infty$ & -- \\
\hline 12 & $5.4 \mathrm{~b}$ & --- & 10.3 & --- & 10.1 & -- & -- & $6.1 \mathrm{~b}$ & -- & $5.1 \mathrm{~b}$ & -- & -- \\
\hline 13 & --- & --- & 11.1 & -- & 8.6 & 8.3 & $-\infty$ & $5.1 \mathrm{~b}$ & --- & $4.8 b$ & -- & -- \\
\hline 14 & -- & --- & 11.1 & --- & 7.8 & 8.3 & -- & -- & -- & $4.3 a$ & -. & -- \\
\hline 15 & -- & --- & 10.7 & -- & $5.4 a$ & 8.4 & --- & $5.8 b$ & $3.4 \mathrm{a}$ & $4.8 \mathrm{~b}$ & -- & -- \\
\hline 16 & -- & -- & 11.4 & -- & $4.5 b$ & 8.9 & -- & 4.3 & 8.4 & $4.4 b$ & $7.8 \mathrm{~b}$ & -- \\
\hline 17 & $-\infty$ & $-\infty$ & 11.7 & --- & $4.0 \mathrm{~b}$ & 9.2 & -- & $4.9 b$ & 7.3 & $4.1 b$ & $8.1 \mathrm{~b}$ & $-\ldots$ \\
\hline 18 & -- & -- & 12.2 & --- & $4.2 b$ & 10.3 & -- & $7.1 \mathrm{~b}$ & $6.3 b$ & $\cdots$ & 9.6 & -- \\
\hline 19 & -- & -- & 12.5 & --- & $4.2 b$ & $8.3 b$ & -- & $6.1 \mathrm{~b}$ & $7.0 \mathrm{~b}$ & -- & 9.3 & -- \\
\hline 20 & --- & --- & 12.3 & --- & 4. $3 b$ & $9.2 \mathrm{~b}$ & --- & 4.1 & $6.0 \mathrm{~b}$ & --- & $7.8 b$ & -- \\
\hline 21 & --- & -- & 12.0 & --- & $4.5 b$ & $9.8 b$ & --- & $5.9 a$ & $6.6 a$ & -- & $8.2 b$ & --- \\
\hline 22 & --- & --- & 12.8 & --- & $4.8 b$ & $6.8 a$ & --- & $7.6 \mathrm{~b}$ & $4.7 \mathrm{a}$ & $-\cdots$ & $7.0 \mathrm{~b}$ & --- \\
\hline 23 & -- & --- & --- & 9.6 & 5.5 & $6.3 a$ & --- & 8.2 & $6.2 b$ & --- & $6.4 \mathrm{~b}$ & -- \\
\hline 24 & 7.7 & $-\cdots$ & 13.9 & 9.5 & 5.6 & $9.3 a$ & $\cdots$ & -- & 7.7 & --- & $5.6 \mathrm{~b}$ & -- \\
\hline 25 & 8.5 & --- & 13.5 & 8.9 & 5.8 & $8.5 b$ & -- & -- & 8.3 & -- & $3.7 b$ & -- \\
\hline 26 & -- & -- & 13.5 & 9.4 & 5.8 & --- & $5.7 \mathrm{a}$ & -- & $7.3 \mathrm{~b}$ & $-\cdots$ & $3.7 \mathrm{~b}$ & -. \\
\hline 27 & --- & --- & 13.2 & 9.5 & $5.6 b$ & $\ldots$ & $2.6 a$ & $-\cdots$ & 7.7 & --- & $4.9 b$ & -- \\
\hline 28 & --- & --- & 12.9 & 8.3 & $5.0 \mathrm{~b}$ & --- & $1.4 \mathrm{a}$ & -- & $8.0 \mathrm{~b}$ & - & $5.6 \mathrm{~b}$ & -- \\
\hline 29 & --- & -- & 11.6 & 8.4 & -- & -- & $3.3 a$ & -- & $7.3 b$ & --- & $4.3 b$ & -- \\
\hline 30 & --- & -- & 11.0 & 9.1 & -- & --- & $3.2 \mathrm{a}$ & --- & $7.2 \mathrm{~b}$ & --- & $6.1 \mathrm{~b}$ & -- \\
\hline 31 & -- & -- & 12.2 & 11.0 & $\ldots$ & -. & -- & -- & --- & $\ldots$ & $6.5 b$ & -- \\
\hline
\end{tabular}

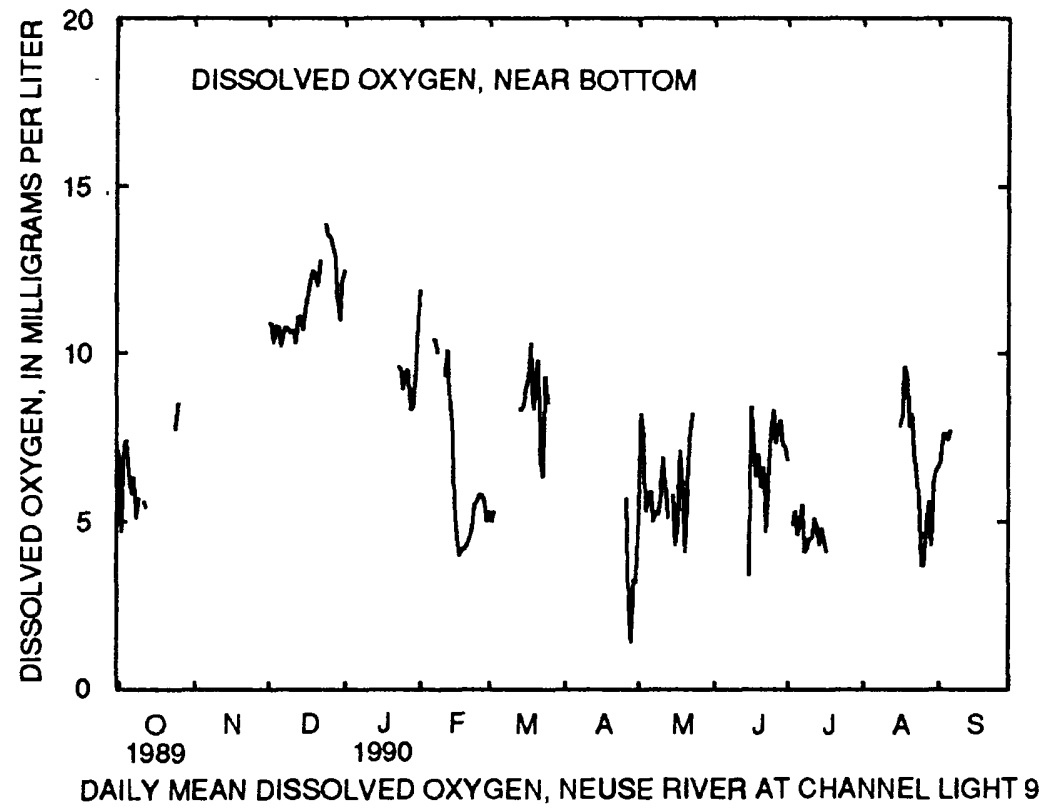


Table 12.--Daily mean values of salinity, water temperature, and dissolved oxygen in water at site 10, Adams Creek at Light 4, June 1989 to September 1990

Site 10 is at latitude $34^{\circ} 57^{\prime} \mathbf{2 4}^{\prime \prime}$, longltude $76^{\circ} 40^{\prime} 54^{\prime \prime}$, Craven County; U.S. Geological Survey downstream order number 0209266925; ppt, parts per thousand; ---, no data; ${ }^{\circ} \mathrm{C}$, degrees

Celsius; mg/L, milligrams per liter; a, dally minimum value is less than or equal to $2.0 \mathrm{mg} / \mathrm{L}$; b, daily minimum value is less than or equal to $5.0 \mathrm{mg} / \mathrm{L}$ but greater than $2.0 \mathrm{mg} / \mathrm{L}$; <, less thanl

SALINITY, NEAR SURFACE

SENSOR POSITION.--6 feet above streambed.

EXTREMES FOR JUNE TO SEPTEMBER 1989.--Maxtmum value recorded, 32.4 ppt June 9, 1989; mintmum value recorded, 4.4 ppt June 27, 1989.

DAILY MEAN VALUES OF SALINITY, NEAR SURFACE (IN PARTS PER THOUSAND), JUNE TO SEPTEMBER 1989

\begin{tabular}{|c|c|c|c|c|}
\hline DAY & JUNE & JULY & AUG & SEPT \\
\hline 1 & 8.9 & 8.6 & --- & 9.3 \\
\hline 2 & 8.3 & 8.8 & -- & 8.2 \\
\hline 3 & 8.3 & 8.8 & --- & 8.2 \\
\hline 4 & 9.5 & 8.8 & -- & 9.3 \\
\hline 5 & 9.4 & 8.8 & -- & 9.8 \\
\hline 6 & 12.0 & 9.3 & -- & 10.0 \\
\hline 7 & 10.2 & 8.7 & --- & 9.8 \\
\hline 8 & 10.1 & --- & --- & 9.9 \\
\hline 9 & 17.2 & --- & -- & 9.9 \\
\hline 10 & 11.1 & -- & --- & 9.0 \\
\hline 11 & 8.8 & --- & -- & 7.5 \\
\hline 12 & 10.1 & --- & -- & 7.4 \\
\hline 13 & 11.7 & --- & -- & 7.9 \\
\hline 14 & 10.9 & -- & --- & 9.3 \\
\hline 15 & 12.3 & --- & -- & 9.0 \\
\hline 16 & 16.2 & --- & --- & 9.2 \\
\hline 17 & 12.5 & --- & -- & 9.6 \\
\hline 18 & 11.1 & -- & --- & 9.6 \\
\hline 19 & 10.2 & -- & --- & 10.3 \\
\hline 20 & 9.9 & $-\cdots$ & --- & 10.4 \\
\hline 21 & 10.6 & -- & --- & 10.8 \\
\hline 22 & 10.2 & -- & --- & 10.8 \\
\hline 23 & 7.9 & -- & 8.3 & 10.6 \\
\hline 24 & 7.3 & -- & 8.3 & 10.5 \\
\hline 25 & 7.8 & $-\cdots$ & 8.7 & 11.5 \\
\hline 26 & 7.4 & --- & 9.6 & 10.0 \\
\hline 27 & 6.6 & --- & 10.0 & 10.0 \\
\hline 28 & 7.2 & -- & 10.2 & 10.7 \\
\hline 29 & 6.2 & $\cdots$ & 10.1 & 10.3 \\
\hline 30 & 8.6 & --- & 9.8 & 9.1 \\
\hline 31 & --- & -- & 9.0 & -- \\
\hline
\end{tabular}

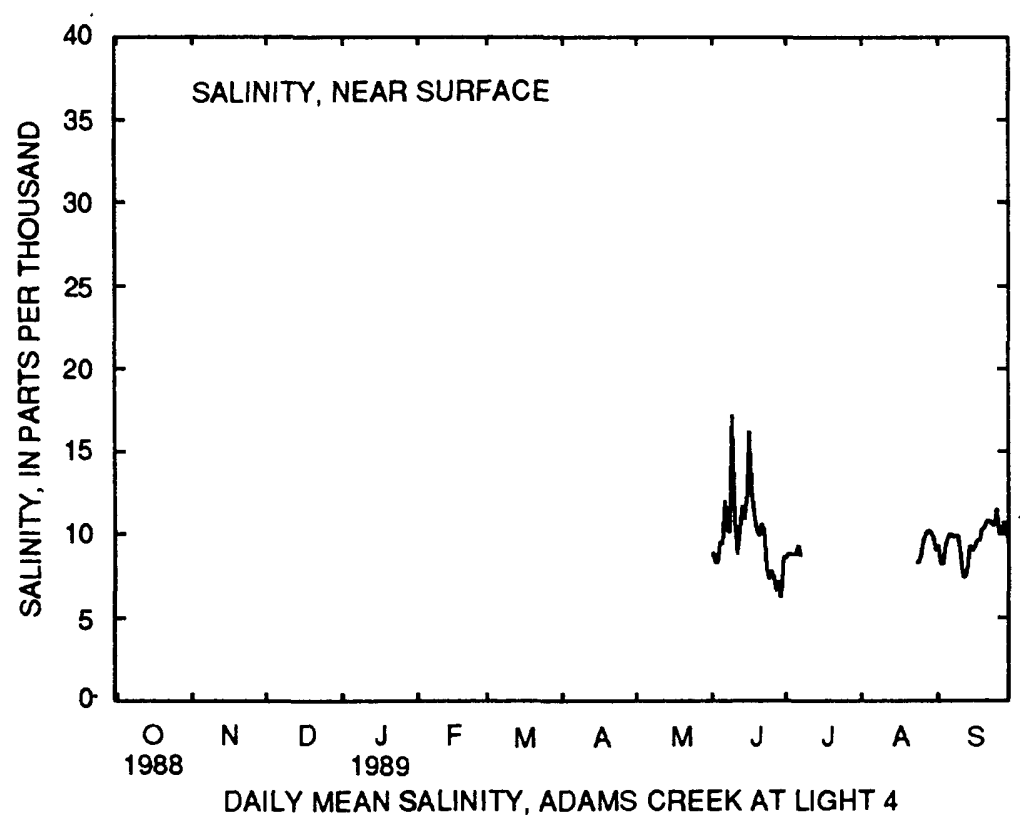


Table 12.--Daily mean values of salinity, water temperature, and dissolved oxygen in water at site 10 , Adams Creek at Light 4, June 1989 to September 1990--Continued

[Site 10 is at latitude $34^{\circ} 57^{\prime} 24^{\prime \prime}$, longitude $76^{\circ} 40^{\prime} 54^{\prime \prime}$, Craven County; U.S. Geological Survey downstream order number 0209266925; ppt, parts per thousand; ---, no data; ${ }^{\circ} \mathrm{C}$, degrees

Celsius; mg/L, milligrams per liter; a, dally minimum value is less than or equal to $2.0 \mathrm{mg} / \mathrm{L}$; b, dally minimum value is less than or equal to $5.0 \mathrm{mg} / \mathrm{L}$ but greater than $2.0 \mathrm{mg} / \mathrm{L}$; <, less thanl

SALINITY, NEAR SURFACE

SENSOR POSITION. --6 feet above st reambed.

EXTREMES FOR OCTOBER 1989 TO SEPTEMBER 1990.--Maximum value recorded, 32.8 ppt March 18, $1990 ;$ minimum value recorded, 0.8 ppt December $31,1989$.

DAILY MEAN VALUES OF SALINITY, NEAR SURFACE (IN PARTS PER THOUSAND), OCTOBER 1989 TO SEPTEMBER 1990

\begin{tabular}{|c|c|c|c|c|c|c|c|c|c|c|c|c|}
\hline DAY & OCT & NOV & $D E C$ & JAN & EEB & MAR & APR & MAY & JUNE & JULY & $A \cup G$ & SEPT \\
\hline 1 & 9.2 & --- & 6.9 & 6.1 & 9.5 & 5.4 & --- & 6.1 & 6.9 & 11.4 & $\cdots$ & 10.9 \\
\hline 2 & 8.9 & --- & 7.1 & --- & 9.0 & 5.8 & 6.3 & 6.4 & 6.4 & 12.0 & 13.3 & 11.0 \\
\hline 3 & 7.9 & -- & 6.2 & --- & 7.5 & 4.9 & --- & 6.8 & 7.1 & 13.2 & 13.8 & 11.1 \\
\hline 4 & 7.6 & --- & 6.4 & -- & 8.6 & 4.6 & 5.0 & 6.9 & 8.9 & 14.1 & 13.7 & 11.3 \\
\hline 5 & 6.7 & --- & 7.6 & -- & 8.0 & 5.8 & 5.5 & 10.2 & 9.0 & 15.0 & 13.8 & 11.5 \\
\hline 6 & 5.9 & --- & 7.7 & -- & 8.3 & 5.5 & --- & 8.2 & 9.2 & 12.9 & 14.0 & 11.3 \\
\hline 7 & 4.9 & --- & --- & --- & 7.2 & 7.0 & 6.2 & 7.5 & 9.0 & 12.8 & 14.4 & --- \\
\hline 8 & 6.2 & --- & --- & --- & 6.5 & 7.2 & 6.5 & 7.5 & 8.1 & 13.7 & 14.1 & -- \\
\hline 9 & 7.0 & --- & -- & --- & 7.1 & 5.7 & -- & 7.7 & 8.9 & 14.4 & 13.9 & -- \\
\hline 10 & 6.8 & -- & 9.4 & --- & 8.2 & 4.2 & 5.9 & 16.0 & 8.9 & 14.7 & 13.6 & -- \\
\hline 11 & 6.6 & --- & 7.1 & --- & 7.6 & 3.8 & --- & 8.7 & 8.3 & 15.3 & 13.3 & --- \\
\hline 12 & 6.6 & -- & 6.6 & -- & 7.7 & 4.2 & 5.0 & 7.0 & 8.7 & -- & 13.0 & --- \\
\hline 13 & 5.9 & --- & 7.0 & -- & 7.3 & 3.7 & 4.9 & 8.9 & 9.1 & --- & 12.8 & --- \\
\hline 14 & 5.4 & -- & 6.8 & --- & 6.7 & 3.7 & 5.1 & --- & 8.9 & 15.1 & 13.0 & --- \\
\hline 15 & 5.4 & --- & 5.9 & --- & 6.7 & 6.0 & 3.7 & 7.5 & 9.1 & 16.5 & --- & --- \\
\hline 16 & 5.1 & --- & 4.8 & -- & 9.1 & 11.2 & 2.7 & --- & 9.6 & 14.6 & 13.6 & -- \\
\hline 17 & --- & --- & 4.8 & --- & 7.2 & 19.7 & 3.1 & --- & 10.3 & --- & 13.9 & --- \\
\hline 18 & 5.4 & --- & 5.8 & --- & 7.6 & 16.2 & 3.4 & --- & 10.3 & -- & 14.0 & --- \\
\hline 19 & 6.3 & -- & 6.9 & --- & 7.5 & 13.2 & --- & -- & 10.1 & --- & 13.8 & -- \\
\hline 20 & 7.7 & --- & 6.4 & --- & 7.1 & 10.6 & 4.2 & --- & 9.8 & --- & 13.2 & --- \\
\hline 21 & 8.2 & --- & 5.6 & --- & 8.1 & 10.1 & -- & -- & 9.6 & --- & 12.2 & -- \\
\hline 22 & 8.6 & --- & 5.4 & --- & 7.7 & 9.0 & -- & -- & 9.6 & -- & 11.8 & --- \\
\hline 23 & 8.0 & --- & 6.6 & 7.2 & 8.3 & 8.4 & -- & --- & 12.0 & -- & 11.8 & --- \\
\hline 24 & 10.4 & --- & 5.7 & 6.8 & 8.4 & 7.4 & 3.4 & --- & 11.5 & -- & 11.2 & --- \\
\hline 25 & 9.7 & --- & 3.2 & 8.1 & 5.5 & 9.4 & 3.2 & --- & 10.9 & --- & 10.4 & --- \\
\hline 26 & -- & -- & 3.7 & 9.8 & 6.9 & 9.7 & 3.3 & 7.7 & 11.4 & --- & 9.8 & --- \\
\hline 27 & --- & --- & 2.8 & 9.5 & 7.1 & 9.6 & 2.8 & 8.6 & 11.5 & --- & 9.3 & --- \\
\hline 28 & --- & -- & 3.1 & 9.0 & 5.5 & -- & 3.2 & 8.5 & 11.4 & -- & 8.9 & --- \\
\hline 29 & --- & --- & --- & 8.4 & --- & 9.6 & 4.4 & 8.5 & 11.7 & --- & 9.1 & $\cdots$ \\
\hline 30 & --- & --- & -- & 9.1 & --- & 8.5 & 5.5 & 7.6 & 12.4 & -- & 9.8 & --- \\
\hline 31 & --- & --- & 3.6 & 9.4 & --- & --- & --- & 7.6 & --- & -- & 11.4 & $\cdots$ \\
\hline
\end{tabular}

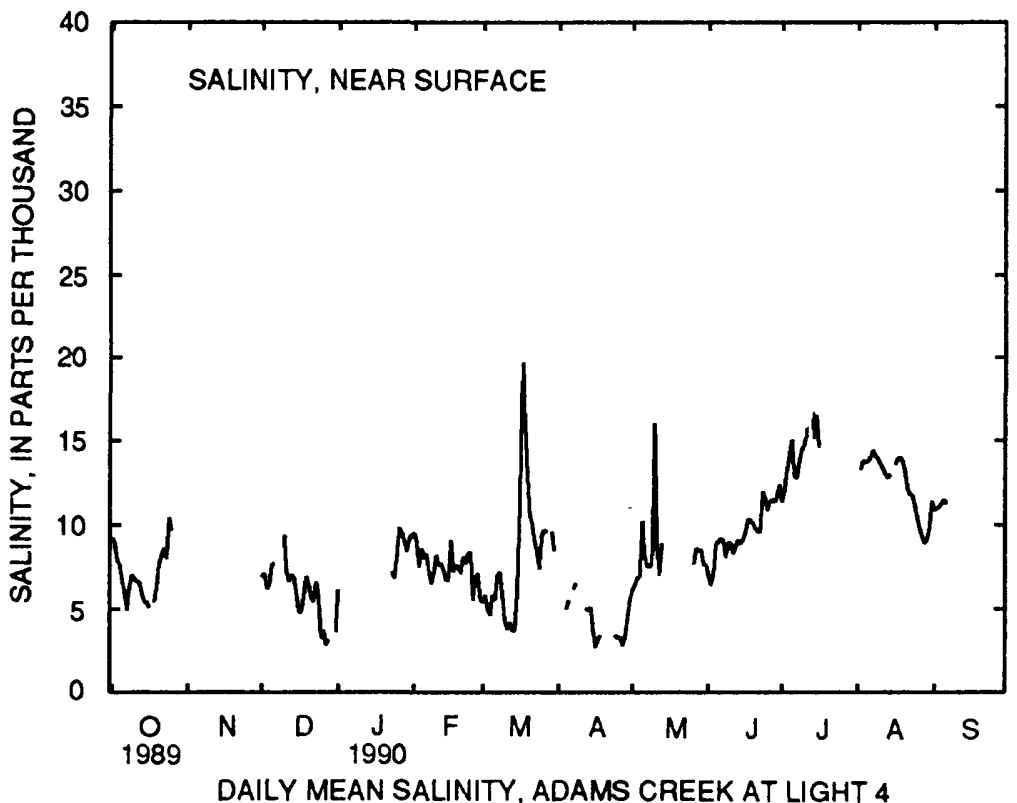


Table 12.--Daily mean values of salinity, water temperature, and dissolved oxygen in water at site 10 , Adams Creek at Light 4, June 1989 to September 1990--Continued

[Site 10 is at latitude $34^{\circ} 57^{\prime} 24^{\prime \prime}$, longltude $76^{\circ} 40^{\prime} 54^{\prime \prime}$, Craven County; U.s. Geological survey downstream order number 0209266925; ppt, parts per thousand; ---, no data; ${ }^{\circ} \mathrm{C}$, degrees

Celsius; mg/L, mlliligrams per liter; a, daily minimum value is less than or equal to $2.0 \mathrm{mg} / \mathrm{L}$; b, daily minimum value is less than or equal to $5.0 \mathrm{mg} / \mathrm{L}$ but greater than $2.0 \mathrm{mg} / \mathrm{L}$; <, less than]

SALINITY, NEAR BOTTOM

SENSOR POSITION. -2 feet above streambed.

EXTREMES FOR JUNE TO SEPTEMBER 1989.--Maximum value recorded, 31.5 ppt June 9, 1989 ; minimum value recorded, 6.4 ppt september 11, 1989.

DAILY MEAN VALUES OF SALINITY, NEAR BOTTOM (IN PARTS PER THOUSAND), JUNE TO SEPTEMBER 1989

\begin{tabular}{|c|c|c|c|c|}
\hline DAY & JUNE & JULY & AUG & SEPT \\
\hline 1 & 8.9 & 12.1 & --- & 10.2 \\
\hline 2 & 8.6 & 12.4 & -- & 8.9 \\
\hline 3 & 8.4 & 12.6 & $-\cdots$ & 8.3 \\
\hline 4 & 10.4 & 12.7 & -- & 9.4 \\
\hline 5 & 11.1 & 13.0 & -- & 9.9 \\
\hline 6 & 15.2 & 13.6 & --- & 9.8 \\
\hline 7 & 13.3 & 12.8 & -- & 9.6 \\
\hline 8 & 13.0 & $m$ & --- & 9.7 \\
\hline 9 & 19.9 & --- & $+\cdots$ & 9.6 \\
\hline 10 & 14.8 & --- & --- & 9.2 \\
\hline 11 & 10.2 & $\cdots$ & $-\cdots$ & 8.5 \\
\hline 12 & 11.2 & -- & --- & 8.6 \\
\hline 13 & 13.0 & -- & $\ldots$ & 9.2 \\
\hline 14 & 12.9 & --- & --- & 9.2 \\
\hline 15 & 13.4 & -- & --- & 8.9 \\
\hline 16 & --- & -- & --- & 9.3 \\
\hline 17 & 14.6 & -- & -- & 9.6 \\
\hline 18 & 12.6 & -- & --- & 9.8 \\
\hline 19 & 11.7 & --- & -- & 10.2 \\
\hline 20 & 11.7 & $\cdots$ & --- & 10.4 \\
\hline 21 & 12.4 & --- & $\ldots$ & 10.9 \\
\hline 22 & 12.1 & --- & -- & 11.0 \\
\hline 23 & 10.1 & --- & 9.5 & 10.8 \\
\hline 24 & 9.4 & $m$ & 9.4 & 10.8 \\
\hline 25 & 10.1 & --- & 9.2 & 11.8 \\
\hline 26 & 9.8 & $\cdots$ & 9.8 & 10.6 \\
\hline 27 & 9.6 & -- & 10.0 & 10.4 \\
\hline 28 & 10.3 & --- & 10.1 & 11.2 \\
\hline 29 & 9.7 & --- & 10.0 & 11.0 \\
\hline 30 & 11.8 & -- & 10.0 & 10.2 \\
\hline 31 & -- & -- & 9.9 & - \\
\hline
\end{tabular}

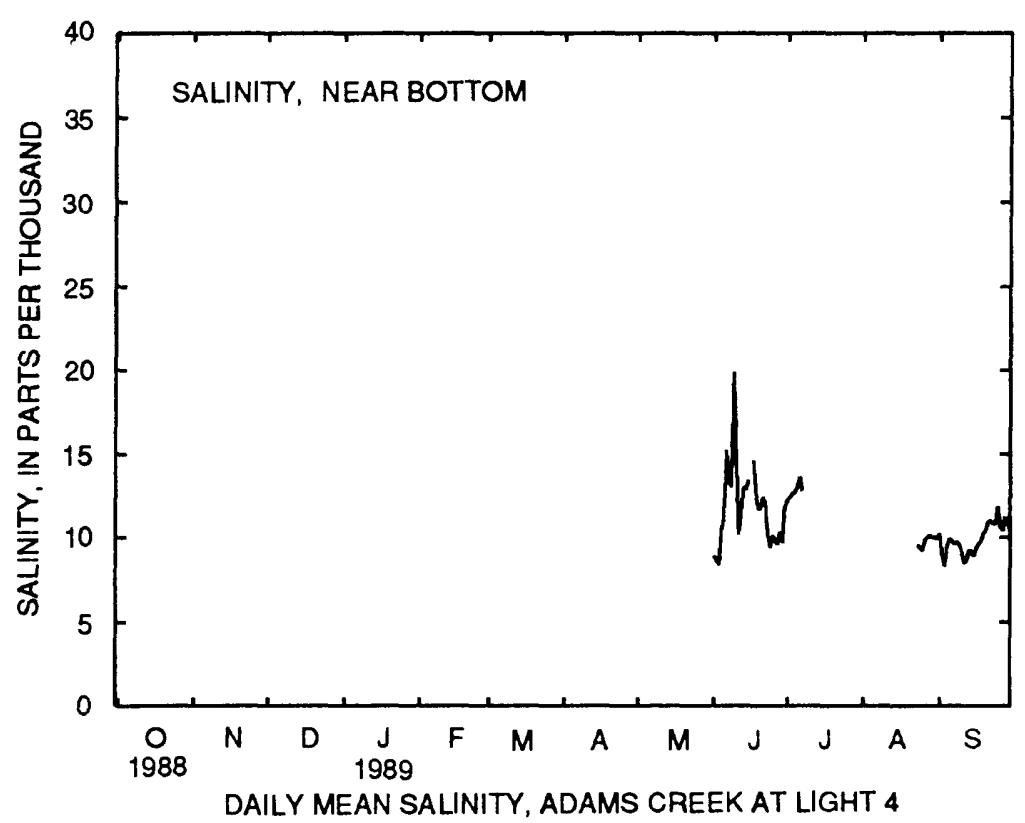


Table 12.--Daily mean values of salinity, water temperature, and dissolved oxygen in water at site 10 , Adams Creek at Light 4, June 1989 to September 1990--Cont i nued

[Site 10 is at latitude $34^{\circ} 57^{\prime} 24^{\prime \prime}$, longitude $76^{\circ} 40^{\circ} 54^{\prime \prime}$, Craven County; U.S. Geologlcal Survey downst ream order number 0209266925 ; ppt, parts per thousand; - --, no data; ${ }^{\circ} \mathrm{C}$, degrees

Celslus; mg/L, milligrams per liter; a, dally minlmum value is less than or equal to $2.0 \mathrm{mg} / \mathrm{L}$; b, daily minimum value is less than or equal to $5.0 \mathrm{mg} / \mathrm{L}$ but greater than $2.0 \mathrm{mg} / \mathrm{L}$; <, less than]

SALINITY, NEAR BOTTOM

SENSOR POSITION.--2 feet above streambed.

EXTREMES FOR OCTOBER 1989 TO SEPTEMBER 1990.--Maximum value recorded, 29.5 ppt June 24 , $1990 ;$ minimum value recorded, 4.5 ppt December 17, 1989, March 10, 11, 1990.

DAILY MEAN VALUES OF SALINITY, NEAR BOTTOM (IN PARTS PER THOUSAND), OCTOBER 1989 TO SEPTEMBER 1990

\begin{tabular}{|c|c|c|c|c|c|c|c|c|c|c|c|c|}
\hline DAY & OCT & NOV & DEC & JAN & FEB & MAR & APR & MAY & JUNE & JULY & AUG & SEPT \\
\hline 1 & 10.1 & -- & 8.1 & 6.9 & 8.6 & 10.0 & -- & -- & 6.7 & 16.1 & -- & 11.9 \\
\hline 2 & 9.9 & --- & 7.5 & --- & 8.8 & 9.7 & $-\infty$ & $-\infty$ & 6.1 & --- & --- & --- \\
\hline 3 & 9.1 & -- & 5.9 & -- & 8.3 & 8.6 & -- & $-\cdots$ & 6.4 & $\cdots$ & -- & --- \\
\hline 4 & 8.7 & --- & 7.5 & --- & 8.3 & 6.8 & -- & --- & 7.5 & --- & --- & --- \\
\hline 5 & 8.1 & -- & 18.9 & --- & 7.9 & 6.7 & -- & --- & 9.1 & --- & -- & 12.1 \\
\hline 6 & 7.8 & --- & 21.7 & -- & 8.1 & 6.4 & -- & --- & 10.1 & -- & -- & -- \\
\hline 7 & 7.2 & -- & 14.6 & -- & 7.9 & 6.1 & -- & -- & 9.5 & $\cdots$ & --- & --- \\
\hline 8 & 8.0 & -- & 9.7 & --- & 7.4 & 6.9 & -- & --- & 8.7 & $\cdots$ & -- & --- \\
\hline 9 & 8.1 & --- & 10.1 & $\cdots$ & 7.6 & 6.3 & $-\infty$ & -- & 9.4 & --- & -- & -- \\
\hline 10 & 8.1 & --- & 10.0 & -- & 8.4 & 5.2 & -- & --- & 15.8 & --- & --- & --- \\
\hline 11 & 8.0 & -- & 8.4 & --- & 9.7 & 5.0 & -- & --- & 13.0 & --- & -- & --- \\
\hline 12 & 8.0 & --- & 7.3 & -- & 8.9 & --- & -- & -- & 9.6 & --- & --- & $\ldots$ \\
\hline 13 & 7.6 & -- & 7.2 & --- & 8.4 & $-\cdots$ & $-\cdots$ & -- & 9.1 & --- & --- & $\cdots$ \\
\hline 14 & 7.1 & --- & 7.3 & --- & 8.5 & -- & -- & -- & 9.0 & --- & --- & -- \\
\hline 15 & 7.2 & --- & 6.5 & -- & 7.9 & $\cdots$ & -- & $\cdots$ & 9.2 & $-\cdots$ & --- & --- \\
\hline 16 & 7.0 & -- & 5.2 & --- & 10.6 & --- & $\ldots$ & -- & 10.0 & $-\cdots$ & --- & -- \\
\hline 17 & --- & -- & 4.7 & --- & 17.3 & --- & -- & --- & 9.7 & -- & --- & --- \\
\hline 18 & 8.5 & -- & 5.4 & --- & 10.5 & -- & -- & -- & 9.4 & $\ldots$ & --- & --- \\
\hline 19 & 10.9 & --- & 6.1 & $-\cdots$ & 8.2 & --- & -- & --- & 11.2 & --- & $-\cdots$ & -- \\
\hline 20 & 12.2 & --- & 6.0 & --- & 7.1 & -- & -- & -- & 13.4 & --- & --- & --- \\
\hline 21 & 13.0 & --- & 5.9 & --- & 8.2 & -- & -- & -- & 11.9 & --- & -- & -- \\
\hline 22 & 14.0 & $\ldots$ & 5.4 & --- & 8.1 & - & -- & --- & 12.2 & --- & --- & --- \\
\hline 23 & 10.1 & --- & 5.3 & 7.1 & 8.4 & -- & -- & --- & 15.6 & --- & --- & --- \\
\hline 24 & 11.4 & --- & 5.6 & 7.2 & 12.7 & --- & -- & -- & 22.0 & --- & --- & --- \\
\hline 25 & 10.3 & --- & 6.7 & 7.5 & 14.0 & --- & -- & --- & 14.6 & -- & --- & $\cdots$ \\
\hline 26 & -- & -- & 6.8 & 11.1 & 12.2 & --- & -- & 6.9 & 13.3 & -- & --- & -- \\
\hline 27 & -- & -- & 7.2 & 11.1 & 11.5 & --- & --- & 11.7 & 13.1 & -- & --- & -- \\
\hline 28 & --- & --- & 6.5 & 8.9 & 10.7 & --- & -- & 10.7 & 12.6 & --- & --- & - - \\
\hline 29 & -- & $\ldots$ & 6.7 & 8.4 & $\ldots$ & -- & -- & 9.4 & 12.8 & -- & --- & --- \\
\hline 30 & -- & --- & 6.8 & 8.5 & --- & -- & --- & 7.9 & 14.4 & --- & -- & --- \\
\hline 31 & $\cdots$ & --- & 7.6 & 8.5 & -- & -- & -- & 7.0 & --- & $\cdots$ & --- & $\cdots$ \\
\hline
\end{tabular}

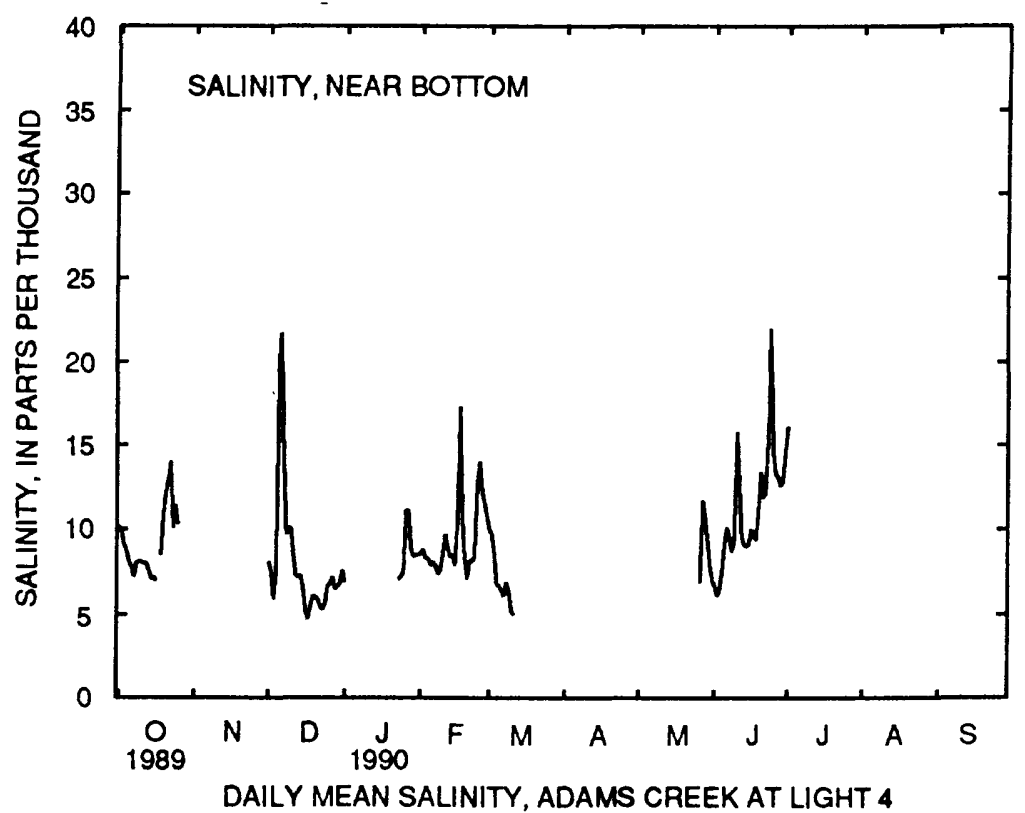


Table 12.--Dally mean values of salinity, water temperature, and dissolved oxygen in water at site 10 , Adams Creek at Light 4, June 1989 to September 1990--Cont Inued

Site 10 is at latitude $34^{\circ} 57^{\prime} 24^{\prime \prime}$, longitude $76^{\circ} 40^{\prime} 54^{\prime \prime}$, Craven County; U.S. Geological Survey downstream order number 0209266925; ppt, parts per thousand; ---, no data; ${ }^{\circ} \mathrm{C}$, degrees Celsius; mg/L, milligrams per liter; a, daily minimum value is less than or equal to $2.0 \mathrm{mg} / \mathrm{L}$; b, daily minimum value is less than or equal to $5.0 \mathrm{mg} / \mathrm{L}$ but greater than $2.0 \mathrm{mg} / \mathrm{L}$; <, less than]

WATER TEMPERATURE, NEAR SURFACE

SENSOR POSITION. -6 feet above streambed.

EXTREMES FOR JUNE TO SEPTEMBER 1989.--Maximum value recorded, $30.7{ }^{\circ} \mathrm{C}$ August 24 , September 1 , 1989 ; minimum value recorded, $19.8{ }^{\circ} \mathrm{C}$ september $28,1989$.

DAILY MEAN VALUES OF WATER TEMPERATURE, NEAR SURFACE (IN DEGREES CELSIUS), JUNE TO SEPTEMBER 1989

\begin{tabular}{|c|c|c|c|c|}
\hline DAY & JUNE & JULY & AUG & SEPT \\
\hline 1 & --- & 26.7 & -- & 28.5 \\
\hline 2 & --- & 27.0 & -- & 28.8 \\
\hline 3 & $-\cdots$ & 27.1 & -- & 27.9 \\
\hline 4 & -- & 27.1 & -. & 26.0 \\
\hline 5 & $-\cdots$ & 27.5 & -- & 25.8 \\
\hline 6 & $-\ldots$ & 27.2 & -- & 26.0 \\
\hline 7 & --- & 27.4 & -- & 25.7 \\
\hline 8 & -- & -- & -- & 25.8 \\
\hline 9 & -- & -- & $\ldots$ & 26.1 \\
\hline 10 & --- & --- & -- & 26.3 \\
\hline 11 & -- & -- & $-\cdots$ & 27.2 \\
\hline 12 & --- & -- & -- & 27.7 \\
\hline 13 & --- & -- & -- & 27.5 \\
\hline 14 & --- & --- & -- & 27.2 \\
\hline 15 & 28.1 & --- & -- & 27.1 \\
\hline 16 & 28.3 & -- & -- & 27.4 \\
\hline 17 & 26.9 & -- & $-\cdots$ & 27.1 \\
\hline 18 & 26.8 & -- & -- & 26.2 \\
\hline 19 & 27.7 & --- & -- & 25.3 \\
\hline 20 & 28.0 & -- & $-\cdots$ & 25.5 \\
\hline 21 & 28.3 & -- & -- & 26.2 \\
\hline 22 & 27.7 & --- & $-\infty$ & 26.5 \\
\hline 23 & 27.7 & -- & 29.3 & 26.4 \\
\hline 24 & 27.8 & -- & 29.4 & 23.8 \\
\hline 25 & 27.6 & -- & 28.7 & 21.9 \\
\hline 26 & 28.3 & -- & 27.8 & 23.0 \\
\hline 27 & 28.8 & --- & 27.7 & 22.5 \\
\hline 28 & 29.2 & -- & 27.9 & 20.9 \\
\hline 29 & 28.7 & $\cdots$ & 28.4 & 21.6 \\
\hline 30 & 27.0 & --- & 28.6 & 22.2 \\
\hline 31 & --- & -- & 28.2 & --- \\
\hline
\end{tabular}

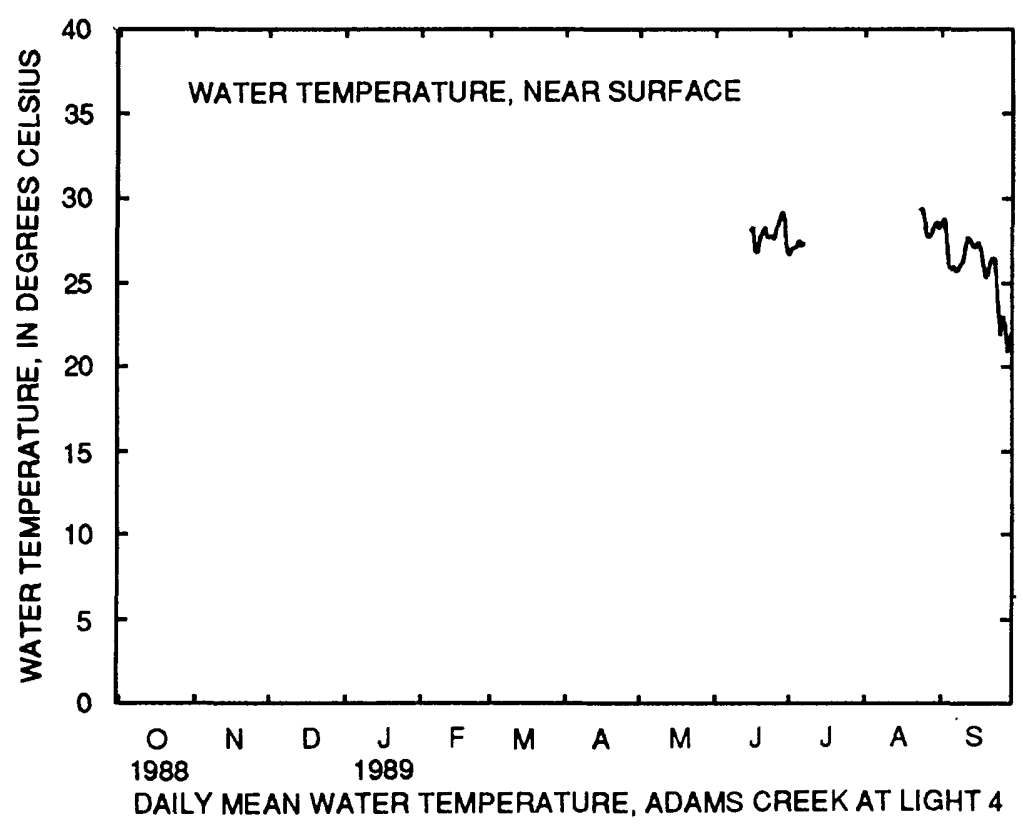


Table 12.--Dally mean values of salinity, water temperature, and dissolved oxygen in water at site 10 , Adams Creek at Light 4, June 1989 to September 1990--Continued

[Site 10 is at latitude $34^{\circ} 57^{\prime} 24^{\prime \prime}$, longltude $76^{\circ} 40^{\prime} 54^{\prime \prime}$, Craven County; U.S. Geological Survey downstream order number 0209266925 ; ppt, parts per thousand; ---, no data; ${ }^{\circ} \mathrm{C}$, degrees

Celsius; mg/L, milligrams per liter; a, dally minimum value is less than or equal to $2.0 \mathrm{mg} / \mathrm{L}$; b, daily minimum value is less than or equal to $5.0 \mathrm{mg} / \mathrm{L}$ but greater than $2.0 \mathrm{mg} / \mathrm{L}$; <, less than]

WATER TEMPERATURE, NEAR SURFACE

SENSOR POSITION. --6 feet above streambed.

EXTREMES FOR OCTOBER 1989 TO SEPTEMBER 1990.--Maximum value recorded, $32.8{ }^{\circ} \mathrm{C}$ August 19,29 , 1990 ; minimum value recorded, $0.1{ }^{\circ} \mathrm{C}$ December $24,1989$.

DAILY MEAN VALUES OF WATER TEMPERATURE, NEAR SURFACE (IN DEGREES CELSIUS), OCTOBER 1989 TO SEPTEMBER 1990

\begin{tabular}{|c|c|c|c|c|c|c|c|c|c|c|c|c|}
\hline DAY & OCT & NOV & DEC & JAN & FEB & MAR & APR & MAY & JUNE & JULY & AUG & SEPT \\
\hline 1 & 21.9 & --- & 10.5 & 4.8 & 11.7 & 10.2 & --- & 22.5 & 25.0 & 29.3 & --- & 29.5 \\
\hline 2 & 22.3 & --- & 10.5 & --- & 13.1 & 10.3 & 15.8 & 23.0 & 25.0 & 28.9 & 30.2 & 29.5 \\
\hline 3 & 23.1 & --- & 9.1 & -- & 14.2 & 11.4 & --- & 22.3 & 25.8 & 28.7 & 29.4 & 29.8 \\
\hline 4 & 23.4 & --- & 6.6 & --- & 15.6 & 11.6 & 14.7 & 23.1 & 26.3 & 28.7 & 29.1 & 28.5 \\
\hline 5 & 22.3 & --- & 7.1 & --- & 14.1 & 11.2 & 15.3 & 24.2 & 25.6 & 29.0 & 29.0 & 27.8 \\
\hline 6 & 22.4 & --- & 8.1 & --- & 13.2 & 11.7 & --- & 22.3 & 25.4 & 29.1 & 28.8 & 28.3 \\
\hline 7 & 23.1 & --- & 8.9 & --- & 13.5 & 11.5 & 16.3 & 21.9 & 26.4 & 29.2 & 28.6 & --- \\
\hline 8 & 21.7 & --- & 8.5 & --- & 13.5 & 10.6 & 15.8 & 22.1 & 28.0 & 29.3 & 28.0 & --- \\
\hline 9 & 19.7 & --- & 7.9 & --- & 13.6 & 11.2 & --- & 22.6 & 28.7 & 29.8 & 27.7 & -- \\
\hline 10 & 19.2 & --- & 7.3 & --- & 15.2 & 12.6 & 16.3 & 22.6 & 28.6 & 30.2 & 27.6 & --- \\
\hline 11 & 19.4 & --- & 7.0 & --- & 14.1 & 13.7 & --- & 21.4 & 27.7 & 30.3 & 28.1 & -- \\
\hline 12 & 19.9 & --- & 6.8 & --- & 13.0 & 15.2 & 16.6 & 21.5 & 25.8 & 30.1 & 28.7 & --- \\
\hline 13 & 20.5 & -- & 6.5 & $-\infty$ & 12.8 & 16.4 & 16.8 & 22.2 & 25.0 & 29.6 & 29.1 & --- \\
\hline 14 & 20.9 & --- & 6.3 & --- & 13.2 & 17.6 & 16.8 & --- & 25.3 & 29.9 & 29.2 & -- \\
\hline 15 & 21.4 & --- & 6.2 & --- & 14.1 & 17.7 & 17.5 & 22.7 & 25.6 & 29.5 & $\cdots$ & --- \\
\hline 16 & 21.8 & --- & 5.9 & --- & 15.9 & 16.6 & 18.6 & --- & 25.6 & 28.8 & 31.0 & -- \\
\hline 17 & --- & --- & 4.8 & --- & 16.1 & 19.0 & 19.5 & --- & 26.2 & --- & 30.8 & --- \\
\hline 18 & 22.9 & --- & 4.1 & --- & 14.0 & 17.3 & 18.0 & -- & 26.7 & --- & 30.9 & --- \\
\hline 19 & 23.2 & --- & 3.8 & --- & 13.8 & 16.7 & --- & --- & 27.4 & --- & 31.2 & --- \\
\hline 20 & 21.8 & $\cdots$ & 4.1 & --- & 13.3 & 15.6 & 17.4 & -- & 27.6 & --- & 31.4 & --- \\
\hline 21 & 17.9 & --- & 3.9 & --- & 11.7 & 12.8 & --- & --- & 28.5 & -- & 30.8 & -- \\
\hline 22 & 17.0 & --- & 3.2 & --- & 12.9 & 13.7 & --- & --- & 29.4 & --- & 30.6 & $-\cdots$ \\
\hline 23 & 16.9 & --- & 1.4 & 10.9 & 14.2 & 14.8 & --- & --- & 29.5 & --- & 30.9 & -- \\
\hline 24 & 17.2 & --- & .3 & 11.2 & 14.1 & 15.4 & 20.7 & --- & 28.8 & --- & 30.4 & --- \\
\hline 25 & 17.3 & -- & .4 & 12.4 & 11.4 & 14.7 & 21.5 & --- & 28.6 & --- & 30.1 & --- \\
\hline 26 & -- & --- & .7 & 12.6 & 9.7 & 14.0 & 22.2 & 24.7 & 28.2 & --- & 30.3 & -- \\
\hline 27 & --- & -- & .9 & 10.2 & 9.7 & 13.7 & 23.3 & 24.9 & 28.0 & --- & 30.9 & --- \\
\hline 28 & -- & --- & 1.1 & 9.9 & 10.0 & --- & 23.8 & 23.9 & 28.1 & --- & 31.5 & -- \\
\hline 29 & --- & --- & 1.5 & 10.5 & --- & 13.7 & 23.4 & 24.5 & 28.8 & --- & 32.0 & --- \\
\hline 30 & $-\cdots$ & -- & 2.0 & 11.3 & --- & 14.2 & 22.3 & 24.4 & 29.2 & --- & 31.3 & -- \\
\hline 31 & -- & -- & 2.8 & 11.2 & --- & -- & $-\infty$ & 24.5 & --- & --- & 29.6 & --- \\
\hline
\end{tabular}

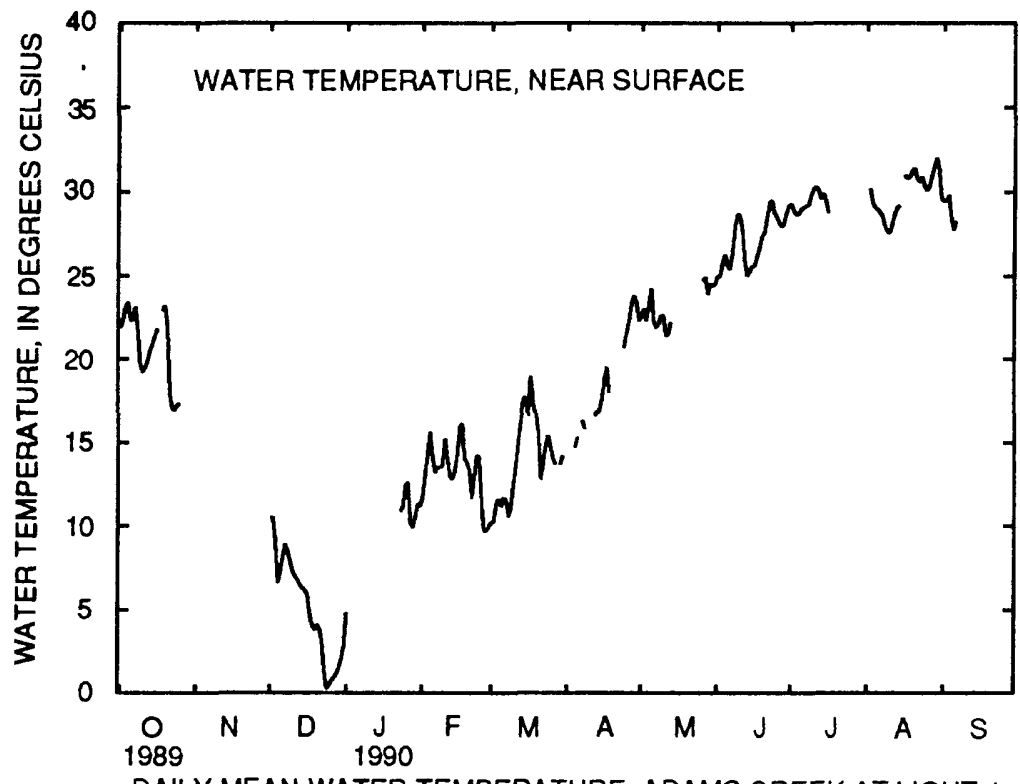


Table 12.--Daily mean values of salinity, water temperature, and dissolved oxygen in water at site 10, Adams Creek at Light 4, June 1989 to September $1990-$-Cont inued

[S1te 10 is at latitude $34^{\circ} 57^{\prime} 24^{\prime \prime}$, longltude $76^{\circ} 40^{\prime} 54^{\prime \prime}$, Craven County; U.S. Geologica] Survey downst ream order number 0209266925; ppt, parts per thousand; ---, no data; ${ }^{\circ} \mathrm{C}$, degrees

Celsius; mg/L, milligrams per liter; a, daily minimum value is less than or equal to $2.0 \mathrm{mg} / \mathrm{L}$; b, daily minimum value is less than or equal to $5.0 \mathrm{mg} / \mathrm{L}$ but greater than $2.0 \mathrm{mg} / \mathrm{L} ;<$, less than]

\section{DISSOLVED OXYGEN, NEAR SURFACE}

SENSOR POSITION. --6 feet above streambed.

EXTREMES FOR JUNE TO SEPTEMBER 1989.--Maximum value recorded, 12.6 mg/L September 29, 1989; m1nimum value recorded, $2.6 \mathrm{mg} / \mathrm{L}$ June $26,1989$.

DAILY MEAN VALUES OF DISSOLVED OXYGEN, NEAR SURFACE (IN MILLIGRAMS PER LITER), JUNE TO SEPTEMBER 1989

\begin{tabular}{|c|c|c|c|c|}
\hline DAY & JUNE & JULY & AUG & SEPT \\
\hline 1 & 7.2 & 7.4 & -- & 9.0 \\
\hline 2 & 7.8 & $6.8 \mathrm{~b}$ & --- & 8.9 \\
\hline 3 & 7.7 & $6.0 \mathrm{~b}$ & -- & 7.9 \\
\hline 4 & 7.3 & $5.9 b$ & -- & 8.2 \\
\hline 5 & 7.7 & $6.0 \mathrm{~b}$ & -- & 8.3 \\
\hline 6 & 8.2 & $6.6 b$ & -- & 8.0 \\
\hline 7 & 8.0 & 7.9 & -- & 8.3 \\
\hline 8 & 8.2 & --- & -- & 8.1 \\
\hline 9 & 8.2 & -- & -- & 8.3 \\
\hline 10 & 8.9 & --- & --- & 8.7 \\
\hline 11 & 8.8 & --- & -- & 8.6 \\
\hline 12 & 7.7 & -- & -- & 8.2 \\
\hline 13 & 7.6 & --- & -- & 7.4 \\
\hline 14 & 7.9 & -- & $-\cdots$ & $6.6 \mathrm{~b}$ \\
\hline 15 & 7.4 & -- & --- & 7.2 \\
\hline 16 & 6.7 & --- & -- & 7.3 \\
\hline 17 & $6.4 \mathrm{~b}$ & --- & -- & $5.7 \mathrm{~b}$ \\
\hline 18 & $7.0 \mathrm{~b}$ & --- & --- & $6.1 \mathrm{~b}$ \\
\hline 19 & 7.3 & --- & --- & 6.1 \\
\hline 20 & $6.8 b$ & --- & $-\cdots$ & 6.9 \\
\hline 21 & $6.7 b$ & --- & $-\cdots$ & 7.9 \\
\hline 22 & $5.4 \mathrm{~b}$ & --- & --- & 8.0 \\
\hline 23 & $6.2 b$ & $-\cdots$ & 6.9 & 7.4 \\
\hline 24 & $6.4 \mathrm{~b}$ & -- & 6.8 & 8.5 \\
\hline 25 & $6.1 \mathrm{~b}$ & --- & 6.9 & 8.7 \\
\hline 26 & $5.7 b$ & $\cdots$ & 8.0 & 8.7 \\
\hline 27 & $5.8 \mathrm{~b}$ & --- & 8.1 & 9.0 \\
\hline 28 & $5.6 b$ & --- & 7.8 & 9.6 \\
\hline 29 & $6.7 b$ & -- & 7.8 & 10.0 \\
\hline 30 & 7.3 & --- & 7.8 & 9.8 \\
\hline 31 & --- & -- & 8.5 & -- \\
\hline
\end{tabular}

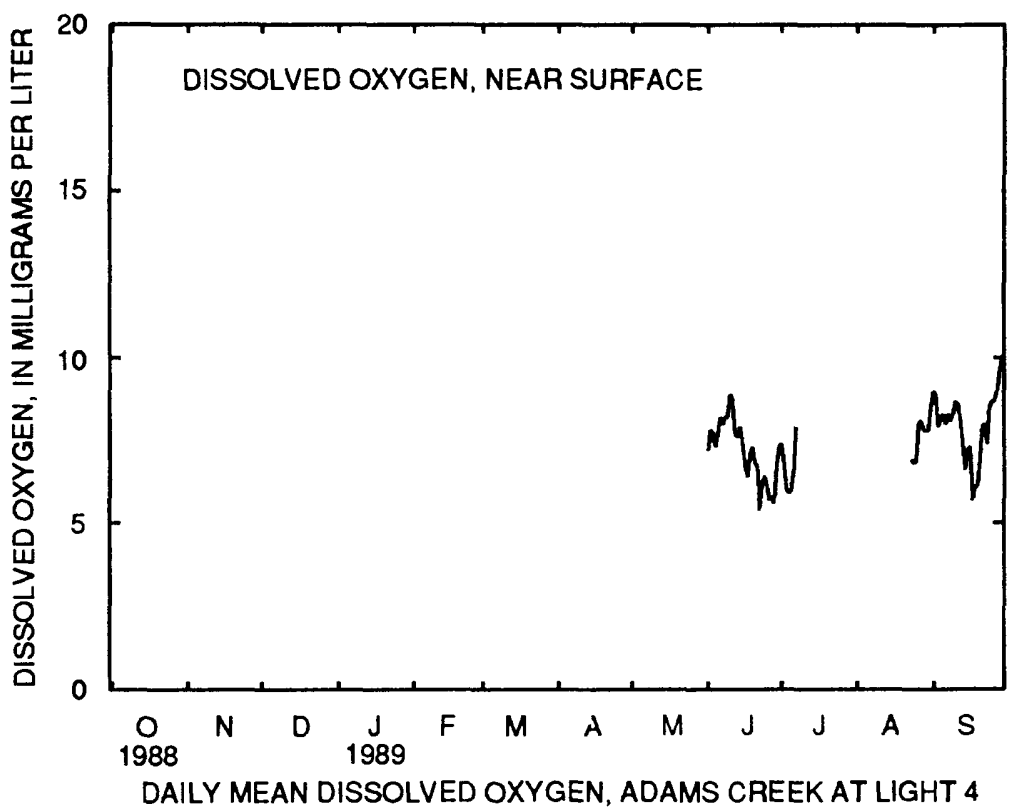


Table 12.--Daily mean values of salinity, water temperature, and dissolved oxygen in water at site 10 , Adams Creek at Light 4, June 1989 to September 1990--Continued

[Site 10 is at latitude $34^{\circ} 57^{\prime} 24^{\prime \prime}$, longitude 76 $40^{\prime} 54^{\prime \prime}$, Craven Countyi U.S. Geological Survey downst ream order number 0209266925 ; ppt, parts per thousand; ---, no data; ${ }^{\circ} \mathrm{C}$, degrees

Celsius; mg/L, milligrams per liter; a, dally minimum value is less than or equal to $2.0 \mathrm{mg} / \mathrm{L}$; b, dally minimum value is less than or equal to $5.0 \mathrm{mg} / \mathrm{L}$ but greater than $2.0 \mathrm{mg} / \mathrm{L}$; <, less than]

DISSOLVED OXYGEN, NEAR SURFACE

SENSOR POSITION. --6 feet above st reambed.

EXTREMES FOR OCTOBER 1989 TO SEPTEMBER 1990.--Maximum value recorded, 19.9 mg/L January 28 , 1990 ; minimum value recorded, $3.1 \mathrm{mg} / \mathrm{L}$ July $3,15,1990$.

DAILY MEAN VALUES OF DISSOLVED OXYGEN, NEAR SURFACE (IN MILLIGRAMS PER LITER), OCTOBER 1989 TO SEPTEMBER 1990

\begin{tabular}{|c|c|c|c|c|c|c|c|c|c|c|c|c|}
\hline DAY & OCT & NOV & DEC & JAN & FEB & MAR & APR & MAY & JUNE & JULY & AUG & SEPT \\
\hline 1 & 8.3 & -- & $-\infty$ & -- & 12.0 & 11.5 & -- & 7.6 & --- & 8.6 & --- & -- \\
\hline 2 & 7.6 & $-\infty$ & --- & --- & 12.0 & 11.7 & 8.6 & 7.4 & $-\infty$ & 7.3 & 7.3 & --- \\
\hline 3 & 9.0 & --- & --- & -- & 12.1 & 12.2 & -- & 6.9 & -- & $5.3 b$ & 6.9 & -- \\
\hline 4 & 9.5 & --- & --- & $-\cdots$ & 10.5 & 11.6 & 8.5 & 6.5 & -- & $5.1 b$ & 8.1 & --- \\
\hline 5 & 9.8 & -- & $-\infty$ & -- & 10.2 & 11.3 & 8.4 & 7.0 & -- & $5.7 b$ & 7.4 & -- \\
\hline 6 & 9.6 & --- & --- & -- & 10.7 & 12.0 & -- & 7.7 & --- & $6.2 b$ & 7.0 & -- \\
\hline 7 & 9.5 & --- & --- & $-\cdots$ & 11.8 & 10.8 & 8.3 & 8.5 & -- & $6.2 b$ & 7.2 & -- \\
\hline 8 & 7.8 & -- & --- & --- & 12.0 & 11.2 & 8.8 & 8.6 & -- & $5.7 b$ & 8.2 & $-\infty$ \\
\hline 9 & 8.3 & -- & --- & -- & 11.0 & 11.7 & -- & 8.3 & -- & $6.0 \mathrm{~b}$ & 8.4 & --- \\
\hline 10 & 8.7 & --- & --- & --- & 9.3 & 12.0 & --- & 7.5 & --- & $6.3 \mathrm{~b}$ & 8.8 & -- \\
\hline 11 & 8.7 & --- & -- & --- & 10.2 & 12.4 & -- & 8.6 & --- & $6.0 \mathrm{~b}$ & 9.3 & --- \\
\hline 12 & 8.6 & $-\infty$ & --- & --- & 11.1 & 11.2 & 8.0 & 8.8 & -- & $5.2 b$ & 9.2 & -- \\
\hline 13 & 9.3 & --- & --- & -- & 11.6 & 10.8 & 7.8 & 7.7 & --- & $4.6 \mathrm{~b}$ & 8.4 & -- \\
\hline 14 & 9.3 & --- & --- & $-\cdots$ & 12.2 & 10.8 & 6.7 & --- & -- & $4.5 b$ & 7.0 & --- \\
\hline 15 & 9.0 & --- & 9.6 & --- & 11.7 & 9.5 & $7.0 \mathrm{~b}$ & 7.1 & 10.4 & $4.1 \mathrm{~b}$ & -- & -- \\
\hline 16 & 9.0 & --- & 9.1 & -- & 9.7 & 7.3 & $7.5 b$ & -- & 8.6 & $6.2 \mathrm{~b}$ & 7.7 & -- \\
\hline 17 & --- & --- & 8.9 & --- & 10.8 & 6.6 & $6.4 \mathrm{~b}$ & -- & 8.3 & -- & 6.8 & --- \\
\hline 18 & 9.1 & --- & -- & -- & 10.3 & 8.4 & 7.4 & --- & 7.4 & -- & $7.6 b$ & --- \\
\hline 19 & 8.4 & --- & --- & -- & 10.6 & 8.9 & -- & --- & 8.0 & --- & $7.2 \mathrm{~b}$ & -- \\
\hline 20 & 7.8 & --- & -- & -- & 10.4 & 8.9 & --- & --- & 8.1 & -- & $6.9 b$ & -- \\
\hline 21 & 8.9 & -- & 12.4 & --- & 10.5 & 9.3 & $-\cdots$ & --- & 7.9 & -- & --- & -- \\
\hline 22 & 9.4 & --- & 12.4 & -- & 10.1 & 9.5 & -- & -- & 7.6 & -- & -- & --- \\
\hline 23 & 9.5 & -- & --- & 11.6 & 9.1 & 9.6 & -- & -- & 7.0 & -- & --- & -- \\
\hline 24 & 8.8 & --- & --- & 11.9 & 9.0 & 10.1 & --- & --- & 7.1 & -- & --- & --- \\
\hline 25 & 9.0 & -- & --- & 10.7 & 10.5 & 8.5 & -- & --- & $6.3 b$ & $-\cdots$ & --- & --- \\
\hline 26 & -- & --- & --- & 9.1 & 11.1 & 8.0 & 9.5 & --- & $5.6 \mathrm{~b}$ & -- & $\cdots$ & -- \\
\hline 27 & --- & --- & -- & 11.3 & 11.3 & 7.9 & 9.2 & --- & $5.4 \mathrm{~b}$ & -- & --- & -- \\
\hline 28 & --- & --- & --- & 14.6 & 11.6 & --- & 8.6 & --- & $5.9 b$ & -- & -- & --- \\
\hline 29 & -- & -- & --- & 14.0 & --- & 8.0 & 7.5 & -- & $5.5 b$ & -- & --- & -- \\
\hline 30 & --- & $-\infty$ & --- & 11.6 & --- & 8.3 & 7.4 & -- & $6.7 \mathrm{~b}$ & -- & --- & -- \\
\hline 31 & -- & --- & 10.5 & 11.5 & --- & --- & --- & --- & --- & -- & --- & $-\infty$ \\
\hline
\end{tabular}

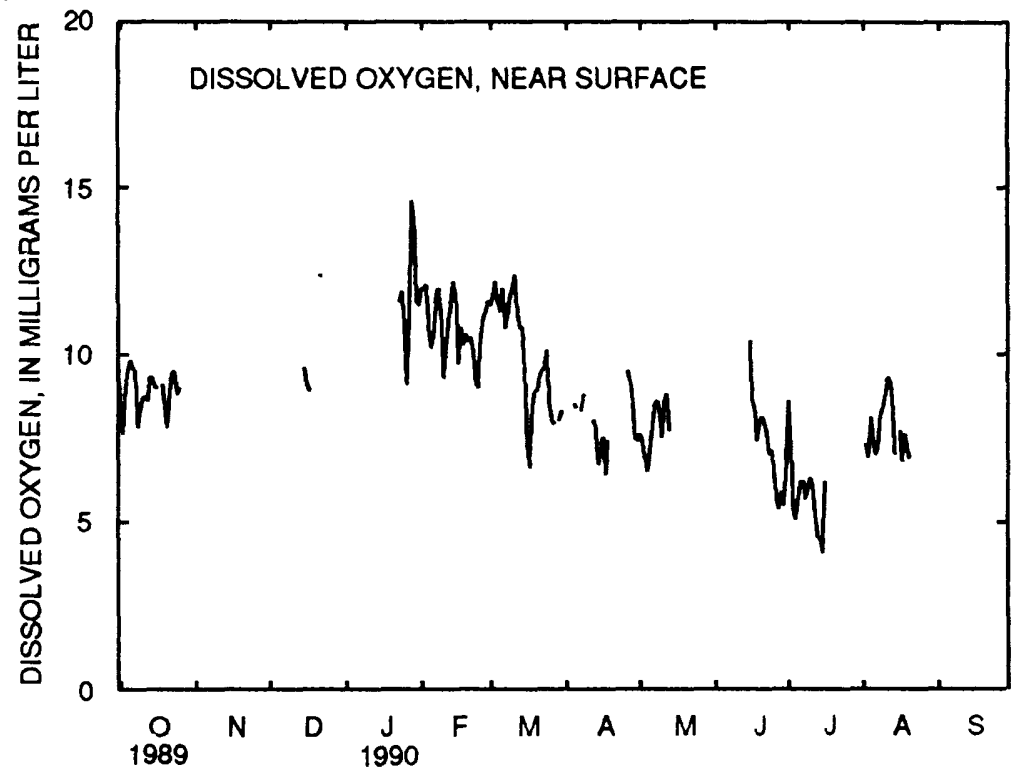

DAILY MEAN DISSOLVED OXYGEN, ADAMS CREEK AT LIGHT 4 
Table 12.--Daily mean values of salinity, water temperature, and dissolved oxygen in water at site 10 , Adams Creek at Light 4, June 1989 to September 1990--Continued

[Site 10 is at latitude $34^{\circ} 57^{\prime} 24^{\prime \prime}$, longitude $76^{\circ} 40^{\circ} 54^{\prime \prime}$, Craven County; U.S. Geological Survey downstream order number 0209266925; ppt, parts per thousand; ---, no data; ${ }^{\circ} \mathrm{C}$, degrees

Celsius; mg/L, milligrams per liter; a, dally minimum value is less than or equal to $2.0 \mathrm{mg} / \mathrm{L}$; b, daily minimum value is less than or equal to $5.0 \mathrm{mg} / \mathrm{L}$ but greater than $2.0 \mathrm{mg} / \mathrm{L}$; <, less thanl

DISSOLVED OXYGEN, MID-DEPTH

SENSOR POSITION. --4 feet above streambed.

EXTREMES FOR JUNE TO SEPTEMBER 1989.--Maximum value recorded, $11.5 \mathrm{mg} / \mathrm{L}$ September 29, 1989 ; minimum value recorded, <1.0 mg/L June 25, July 2-5, 1989.

DAILY MEAN VALUES OF DISSOLVED OXYGEN, MID-DEPTH (IN MILLIGRAMS PER LITER), JUNE TO SEPTEMBER 1989

\begin{tabular}{|c|c|c|c|c|}
\hline DAY & JUNE & JULY & AUG & SEPT \\
\hline 1 & 7.4 & $4.8 \mathrm{~b}$ & -- & $6.7 \mathrm{~b}$ \\
\hline 2 & 7.9 & $3.7 a$ & -- & $7.1 \mathrm{~b}$ \\
\hline 3 & 7.5 & $2.8 a$ & -- & 6.7 \\
\hline 4 & 7.0 & $3.2 \mathrm{a}$ & $\cdots$ & 6.9 \\
\hline 5 & 7.2 & $2.7 \mathrm{a}$ & --- & 6.9 \\
\hline 6 & 7.5 & $3.4 \mathrm{a}$ & -- & 7.0 \\
\hline 7 & 6.8 & $5.3 b$ & -- & 7.5 \\
\hline 8 & 6.6 & -- & --- & 7.7 \\
\hline 9 & 5.8 & -- & -- & 7.9 \\
\hline 10 & 6.2 & $-\infty-$ & $-\cdots$ & 7.8 \\
\hline 11 & $5.5 b$ & -- & -- & 7.8 \\
\hline 12 & $4.2 b$ & -- & -- & 7.5 \\
\hline 13 & $4.2 b$ & --- & --- & $6.1 \mathrm{~b}$ \\
\hline 14 & $6.2 \mathrm{~b}$ & --- & -- & $6.0 \mathrm{~b}$ \\
\hline 15 & 6.8 & --- & -- & 6.3 \\
\hline 16 & $6.2 \mathrm{~b}$ & -- & -- & $6.1 \mathrm{~b}$ \\
\hline 17 & $6.0 \mathrm{~b}$ & $-\cdots$ & $-\cdots$ & $5.8 b$ \\
\hline 18 & $6.2 b$ & $\cdots$ & $-\cdots$ & $6.5 b$ \\
\hline 19 & $6.5 b$ & --- & -- & 6.4 \\
\hline 20 & $5.7 \mathrm{~b}$ & -- & --- & 6.8 \\
\hline 21 & $5.5 \mathrm{a}$ & -- & $-\infty$ & 7.6 \\
\hline 22 & $4.3 a$ & $-\cdots$ & $-\infty$ & 7.6 \\
\hline 23 & -- & --- & $5.7 b$ & 7.3 \\
\hline 24 & $5.3 a$ & -- & $5.9 \mathrm{~b}$ & 8.2 \\
\hline 25 & $4.7 \mathrm{a}$ & -- & $6.3 b$ & 8.4 \\
\hline 26 & $-\cdots$ & $-\cdots$ & $6.2 b$ & 8.4 \\
\hline 27 & $4.1 \mathrm{~b}$ & -- & $5.8 b$ & 8.8 \\
\hline 28 & $3.7 \mathrm{a}$ & -- & $5.8 \mathrm{~b}$ & 9.3 \\
\hline 29 & $5.2 \mathrm{a}$ & -- & $5.7 \mathrm{~b}$ & 9.4 \\
\hline 30 & $5.2 b$ & -- & $5.7 \mathrm{~b}$ & 9.3 \\
\hline 31 & --- & $-\infty$ & 6.7 & --- \\
\hline
\end{tabular}

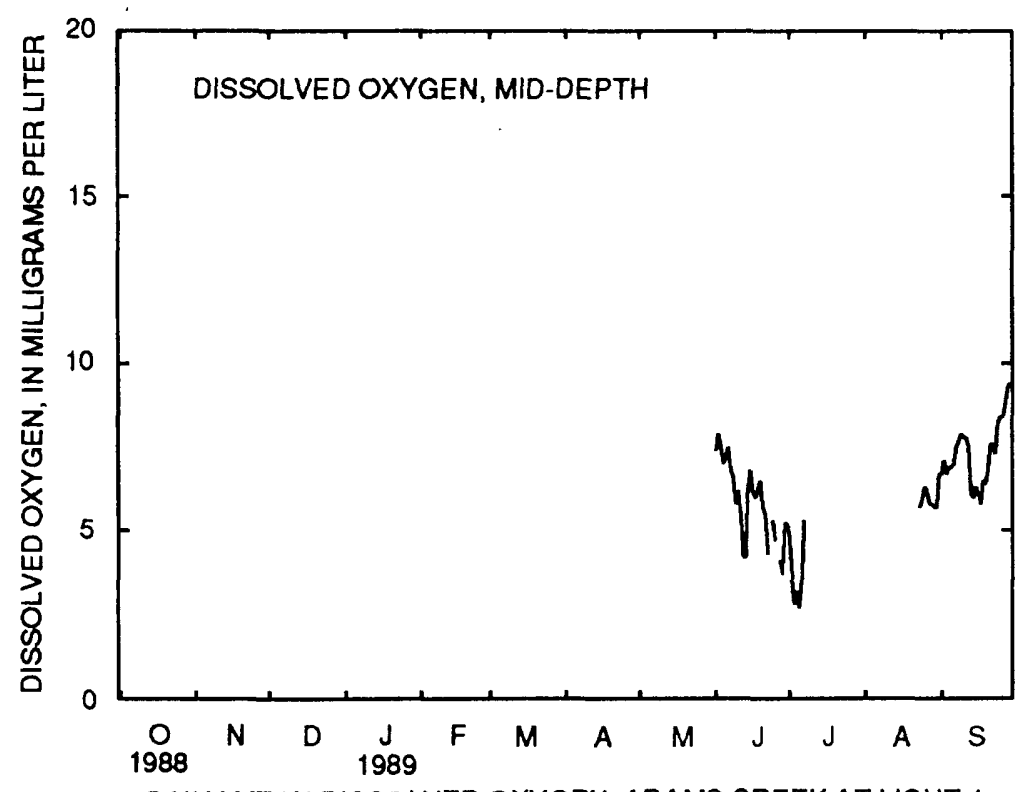

DAILY MEAN DISSOLVED OXYGEN, ADAMS CREEK AT LIGHT 4 
Table 12.--Daily mean values of salinity, water temperature, and dissolved oxygen in water at site 10 , Adams Creek at Light 4, June 1989 to September 1990--Continued

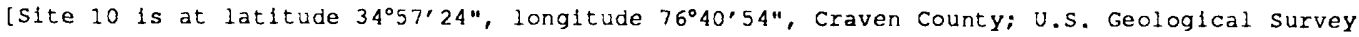
downstream order number 0209266925; ppt, parts per thousand; - - no data; ${ }^{\circ} \mathrm{C}$, degrees

Celsius; mg/L, milligrams per liter; a, daily minimum value is less than or equal to $2.0 \mathrm{mg} / \mathrm{L}$; b, daily minimum value is less than or equal to $5.0 \mathrm{mg} / \mathrm{L}$ but greater than $2.0 \mathrm{mg} / \mathrm{L}$; <, less than]

DISSOLVED OXYGEN, MID-DEPTH

SENSOR POSITION. -4 feet above streambed.

EXTREMES FOR OCTOBER 1989 TO SEPTEMBER 1990.--Maximum value recorded, 19.4 mg/L January 28 , $1990 ; \mathrm{minimum}$ recorded, <1.0 mg/L May 2, 1990.

DAILY MEAN VALUES OF DISSOLVED OXYGEN, MID-DEPTH (IN MILLIGRAMS PER LITER), OCTOBER 1989 TO SEPTEMBER 1990

\begin{tabular}{|c|c|c|c|c|c|c|c|c|c|c|c|c|}
\hline DAY & OCT & NOV & $D E C$ & JAN & FEB & MAR & APR & MAY & JUNE & JULY & AUG & SEPT \\
\hline 1 & 8.3 & --- & 9.9 & 14.3 & 12.7 & --- & $\ldots$ & $5.7 \mathrm{~b}$ & $7.3 \mathrm{~b}$ & $4.8 b$ & --- & -- \\
\hline 2 & 7.7 & -- & 10.1 & -- & 12.6 & -- & -- & $5.8 a$ & $6.9 b$ & $6.3 \mathrm{~b}$ & 6.7 & $\cdots$ \\
\hline 3 & 8.6 & -- & 9.9 & --- & 12.1 & -- & -- & $5.9 b$ & $6.8 \mathrm{~b}$ & $7.3 \mathrm{~b}$ & 6.8 & -- \\
\hline 4 & 9.5 & -- & 10.3 & -- & 10.6 & -- & -- & $5.8 \mathrm{~b}$ & $6.6 \mathrm{~b}$ & 7.0 & 7.8 & -- \\
\hline 5 & 9.3 & --- & $10.4 \mathrm{a}$ & --- & 10.1 & -- & $-\cdots$ & 7.2 & 7.9 & 7.0 & 7.1 & -- \\
\hline 6 & 9.0 & -- & 10.8 & --- & 10.6 & -- & --- & 8.7 & $7.1 \mathrm{~b}$ & $7.0 \mathrm{~b}$ & 6.6 & --1 \\
\hline 7 & 8.7 & -- & --- & $\ldots$ & 11.7 & -- & - & 10.9 & $6.8 \mathrm{~b}$ & $7.3 \mathrm{~b}$ & 6.6 & --- \\
\hline 8 & 8.1 & -- & --- & $\cdots$ & 12.0 & --- & --- & 12.1 & 7.8 & $6.4 b$ & $7.4 \mathrm{~b}$ & -- \\
\hline 9 & 9.0 & --- & $\cdots$ & --- & 11.2 & --- & --- & 12.4 & $6.7 \mathrm{~b}$ & $6.5 b$ & 7.5 & $-\cdots$ \\
\hline 10 & 9.3 & --- & 11.1 & --- & 9.7 & --- & --- & 11.2 & $6.5 b$ & $6.4 \mathrm{~b}$ & 7.6 & $-\cdots$ \\
\hline 11 & 9.4 & --- & 11.7 & --- & 10.5 & -- & --- & 10.7 & 7.3 & $5.7 \mathrm{~b}$ & 8.0 & -- \\
\hline 12 & 9.3 & --- & 10.6 & --- & 11.2 & --- & -- & 10.6 & 7.8 & $4.9 b$ & 8.1 & --- \\
\hline 13 & 9.5 & --- & 11.3 & --- & --- & 8.1 & --- & $7.5 b$ & 7.5 & $5.1 \mathrm{~b}$ & 7.6 & -- \\
\hline 14 & 9.4 & -- & 12.1 & --- & --- & 6.5 & -- & --- & 7.6 & $4.8 b$ & 6.8 & --- \\
\hline 15 & 9.3 & --- & 11.9 & --- & --- & $6.0 \mathrm{~b}$ & --- & $7.2 \mathrm{~b}$ & $6.8 \mathrm{~b}$ & $4.5 b$ & --- & --- \\
\hline 16 & 8.6 & --- & 11.8 & --- & --- & $5.5 b$ & --- & -- & $7.2 \mathrm{~b}$ & $5.8 \mathrm{~b}$ & --- & - \\
\hline 17 & -- & --- & 12.1 & --- & --- & $5.7 b$ & -- & --- & 7.5 & --- & --- & -- \\
\hline 18 & 8.4 & --- & 12.4 & --- & --- & 5.9 & --- & --- & 6.8 & -- & --- & -- \\
\hline 19 & $7.0 \mathrm{~b}$ & --- & 12.8 & --- & --- & 6.0 & --- & --- & 7.4 & --- & --- & -- \\
\hline 20 & 7.1 & --- & 12.9 & --- & --- & 6.1 & -- & --- & 7.4 & --- & --- & --- \\
\hline 21 & 8.2 & --- & 13.0 & --- & --- & 6.2 & --- & -- & 7.0 & -- & -- & -- \\
\hline 22 & 8.9 & --- & 13.5 & -- & --- & 6.6 & -- & --- & 7.1 & -- & --- & --- \\
\hline 23 & 9.2 & $-\cdots$ & 14.1 & 12.0 & --- & 7.3 & --- & -- & $6.5 b$ & --- & --- & --- \\
\hline 24 & 8.8 & --- & 14.6 & 12.5 & --- & 8.0 & -- & --- & $7.2 \mathrm{~b}$ & --- & --- & --- \\
\hline 25 & 8.9 & --- & 14.6 & 11.2 & --- & 8.3 & $\cdots$ & --- & $6.8 b$ & --- & -- & --- \\
\hline 26 & --- & --- & 14.8 & 9.1 & -- & --- & 8.2 & 7.5 & $6.9 \mathrm{~b}$ & -- & --- & --- \\
\hline 27 & --- & --- & 14.7 & 11.6 & --- & $\cdots$ & $7.6 b$ & $7.3 \mathrm{~b}$ & $7.0 \mathrm{~b}$ & --- & --- & -- \\
\hline 28 & -- & --- & 14.7 & 14.6 & --- & --- & $6.7 \mathrm{~b}$ & 7.1 & $7.5 b$ & --- & --- & -- \\
\hline 29 & --- & --- & --- & 14.7 & --- & -- & $5.7 \mathrm{~b}$ & $7.0 \mathrm{~b}$ & $5.6 \mathrm{~b}$ & -- & -- & --- \\
\hline 30 & --- & --- & --- & 11.8 & --- & -- & $5.5 b$ & 7.4 & $4.1 \mathrm{~b}$ & -- & -- & --- \\
\hline 31 & -- & --- & 14.5 & 12.0 & -- & --- & --- & 7.9 & --- & --- & --- & $\cdots$ \\
\hline
\end{tabular}

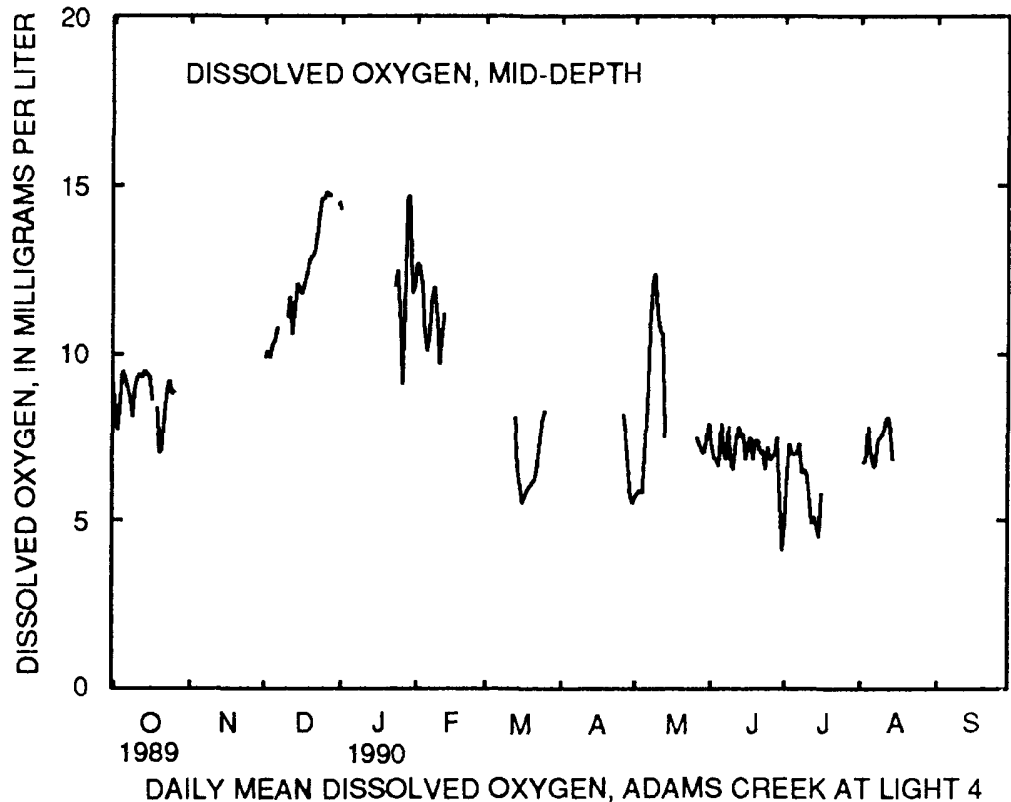




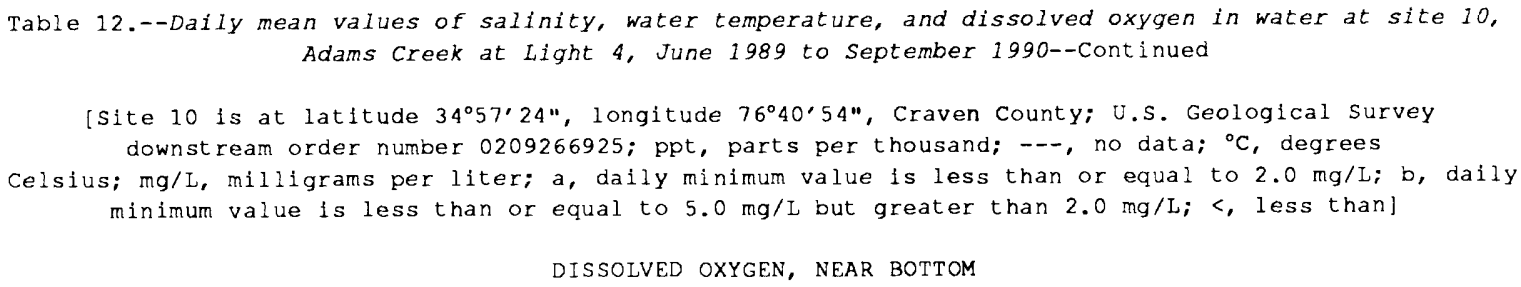

SENSOR POSITION. -2 feet above streambed.

EXTREMES FOR JUNE TO SEPTEMBER 1989. --Maximum value recorded, $14.2 \mathrm{mg} / \mathrm{L}$ June 10, 1989; minimum value recorded, $<1.0 \mathrm{mg} / \mathrm{L}$ June 26 , July $3-5,1989$.

DAILY MEAN VALUES OF DISSOLVED OXYGEN, NEAR BOTTOM (IN MILLIGRAMS PER LITER), JUNE TO SEPTEMBER 1989

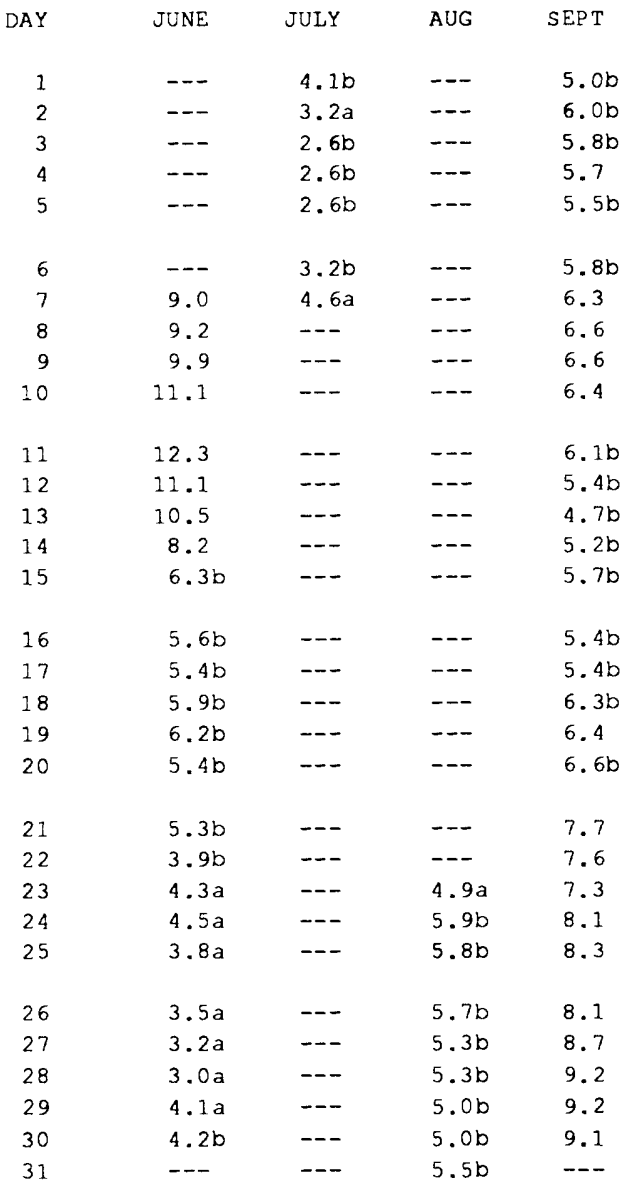

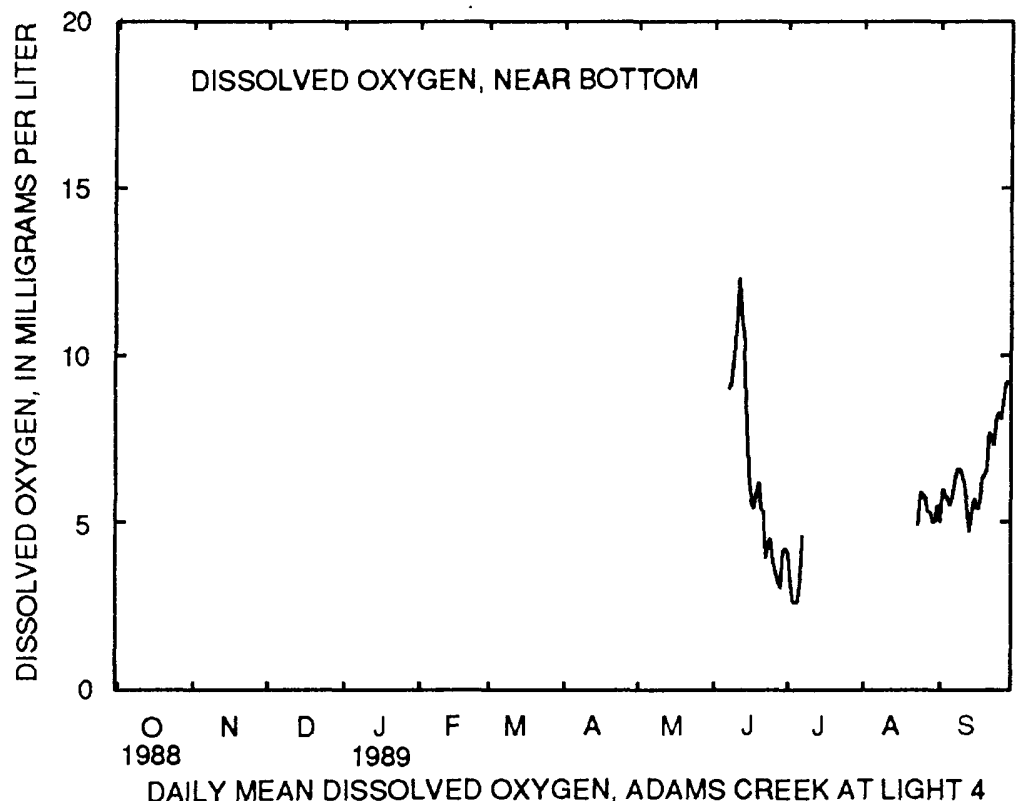

DAILY MEAN DISSOLVED OXYGEN, ADAMS CREEK AT LIGHT 4

141 
Table 12.--Daily mean values of salinity, water temperature, and dissolved oxygen in water at site 10 , Adams Creek at Light 4, June 1989 to September 1990--Continued

[Site 10 is at latitude $34^{\circ} 57^{\prime} 24^{\prime \prime}$, longitude $76^{\circ} 40^{\prime} 54^{\prime \prime}$, Craven County; U.S. Geological Survey downst ream order number 0209266925; ppt, parts per thousand; ---, no data; ${ }^{\circ} \mathrm{C}$, degrees Celsius; mg/L, milligrams per liter; a, dally minimum value is less than or equal to $2.0 \mathrm{mg} / \mathrm{L}$; b, daily minimum value is less than or equal to $5.0 \mathrm{mg} / \mathrm{L}$ but greater than $2.0 \mathrm{mg} / \mathrm{L}$; <, less than]

DISSOLVED OXYGEN, NEAR BOTTOM

SENSOR POSITION. --2 feet above streambed.

EXTREMES FOR OCTOBER 1989 TO SEPTEMBER 1990.--Maximum value recorded, 18.6 mg/L January 28, 1990; minimum value recorded, $1.1 \mathrm{mg} / \mathrm{L}$ May 2, 1990.

DAILY MEAN VALUES OF DISSOLVED OXYGEN, NEAR BOTTOM (IN MILLIGRAMS PER LITER), OCTOBER 1989 TO SEPTEMBER 1990

\begin{tabular}{|c|c|c|c|c|c|c|c|c|c|c|c|c|}
\hline DAY & $\mathrm{OCT}$ & NOV & DEC & JAN & $F E B$ & MAR & APR & MAY & JUNE & JULY & AUG & SEPT \\
\hline 1 & 8.3 & --- & -- & 14.4 & 12.9 & 10.6 & --- & $5.3 b$ & $6.2 b$ & $5.1 b$ & $\ldots$ & $\ldots$ \\
\hline 2 & 7.7 & -- & -- & -- & 12.9 & 10.5 & -- & $5.0 \mathrm{a}$ & $5.7 b$ & $7.0 \mathrm{~b}$ & $5.5 b$ & -- \\
\hline 3 & 8.4 & -- & --- & -- & 12.3 & 11.2 & -- & $5.5 b$ & $5.4 b$ & $7.1 b$ & $5.9 b$ & -- \\
\hline 4 & 9.3 & -- & -- & --- & 11.1 & 10.4 & -- & $5.3 b$ & $5.8 b$ & 7.4 & 6.5 & -- \\
\hline 5 & 8.9 & -- & --- & --- & 10.6 & 10.3 & -- & $5.8 \mathrm{~b}$ & $7.0 \mathrm{~b}$ & $6.4 \mathrm{~b}$ & $5.5 b$ & --- \\
\hline 6 & 8.7 & -- & -- & -- & 10.9 & 10.9 & -- & $5.8 \mathrm{~b}$ & $5.6 \mathrm{~b}$ & $6.2 \mathrm{~b}$ & --- & -- \\
\hline 7 & 8.1 & -- & --- & -- & 12.1 & 10.8 & -- & $6.4 \mathrm{~b}$ & $5.8 b$ & $7.8 \mathrm{~b}$ & $-\cdots$ & -- \\
\hline 8 & 7.9 & $-\cdots$ & --- & -- & 12.1 & 10.7 & --- & $6.6 \mathrm{~b}$ & $6.4 \mathrm{~b}$ & $7.1 \mathrm{~b}$ & -- & -- \\
\hline 9 & 8.8 & --- & --- & --- & 11.2 & 10.9 & --- & $6.4 \mathrm{~b}$ & $5.9 b$ & 7.6 & --- & -- \\
\hline 10 & 9.2 & $\cdots$ & --- & --- & 9.6 & 11.1 & --- & $6.3 \mathrm{~b}$ & $4.7 \mathrm{~b}$ & $6.2 \mathrm{~b}$ & --- & --- \\
\hline 11 & 9.2 & --- & --- & --- & 10.2 & 11.0 & -- & $6.1 \mathrm{~b}$ & $6.5 b$ & $5.3 b$ & --- & \\
\hline 12 & 9.2 & -- & --- & --- & 11.0 & 10.2 & --- & $6.6 \mathrm{~b}$ & 7.5 & $4.0 \mathrm{~b}$ & --- & -- \\
\hline 13 & 8.9 & --- & --- & --- & 11.1 & 9.6 & -- & $5.8 \mathrm{~b}$ & 7.5 & $3.3 \mathrm{~b}$ & --- & \\
\hline 15 & 8.8 & --- & 11.9 & --- & 10.9 & $7.3 b$ & -- & 8.4 & $6.2 b$ & $3.3 b$ & --- & --- \\
\hline 16 & 8.4 & --- & 12.0 & --- & 9.2 & $6.4 \mathrm{~b}$ & -- & -- & $6.4 \mathrm{~b}$ & $3.7 \mathrm{~b}$ & $5.7 b$ & \\
\hline 17 & --- & --- & 12.3 & -- & $8.6 \mathrm{~b}$ & $6.6 \mathrm{~b}$ & -- & -- & $7.3 b$ & --- & $5.9 b$ & --- \\
\hline 18 & 7.4 & --- & 12.7 & --- & 9.7 & $6.9 \mathrm{~b}$ & --- & --- & 6.8 & --- & $6.0 \mathrm{~b}$ & --- \\
\hline 19 & $6.0 \mathrm{~b}$ & --- & 13.0 & --- & 9.3 & $7.8 \mathrm{~b}$ & -- & -- & $6.9 b$ & --- & $6.0 \mathrm{~b}$ & -- \\
\hline 20 & $6.5 b$ & --- & 13.1 & -- & 10.1 & $8.5 b$ & -- & --- & $6.5 b$ & --- & $5.5 b$ & --- \\
\hline 21 & 8.0 & --- & 13.2 & --- & 10.1 & 9.0 & --- & -- & $6.3 b$ & --- & $4.9 b$ & --- \\
\hline 22 & 8.5 & -- & 13.8 & - & 9.8 & 8.6 & --- & -- & $6.0 \mathrm{~b}$ & --- & --- & -- \\
\hline 23 & 8.9 & --- & 14.4 & 12.1 & 8.8 & 8.6 & --- & -- & $5.1 \mathrm{~b}$ & --- & --- & -- \\
\hline 24 & 8.7 & --- & 14.9 & 12.6 & 7.8 & $10.0 \mathrm{~b}$ & $-\cdots$ & --- & $4.1 \mathrm{~b}$ & --- & --- & --- \\
\hline 25 & 8.8 & --- & 14.8 & 11.3 & 9.0 & 8.7 & --- & --- & $3.9 b$ & --- & --- & --- \\
\hline 26 & --- & --- & 15.0 & 9.2 & 10.4 & -- & $7.1 \mathrm{~b}$ & $6.4 \mathrm{~b}$ & $2.9 b$ & -- & -- & -- \\
\hline 27 & --- & $\cdots$ & 14.7 & 11.7 & 10.4 & --- & $6.6 \mathrm{~b}$ & $5.6 \mathrm{~b}$ & $2.8 \mathrm{~b}$ & $\ldots$ & -- & --- \\
\hline 28 & --- & --- & 14.7 & 14.3 & 10.1 & -- & $6.0 \mathrm{~b}$ & $5.7 b$ & $4.2 \mathrm{~b}$ & --- & -- & -- \\
\hline 29 & --- & --- & --- & 14.8 & -- & --- & $5.3 b$ & $5.6 \mathrm{~b}$ & $3.8 \mathrm{~b}$ & --- & -- & -- \\
\hline 30 & --- & --- & --- & 12.0 & --- & -- & $5.2 b$ & $6.0 \mathrm{~b}$ & $3.3 b$ & -- & --- & --- \\
\hline 31 & --- & $\cdots$ & 14.4 & 12.2 & --- & --- & --- & $6.2 b$ & --- & --- & --- & \\
\hline
\end{tabular}

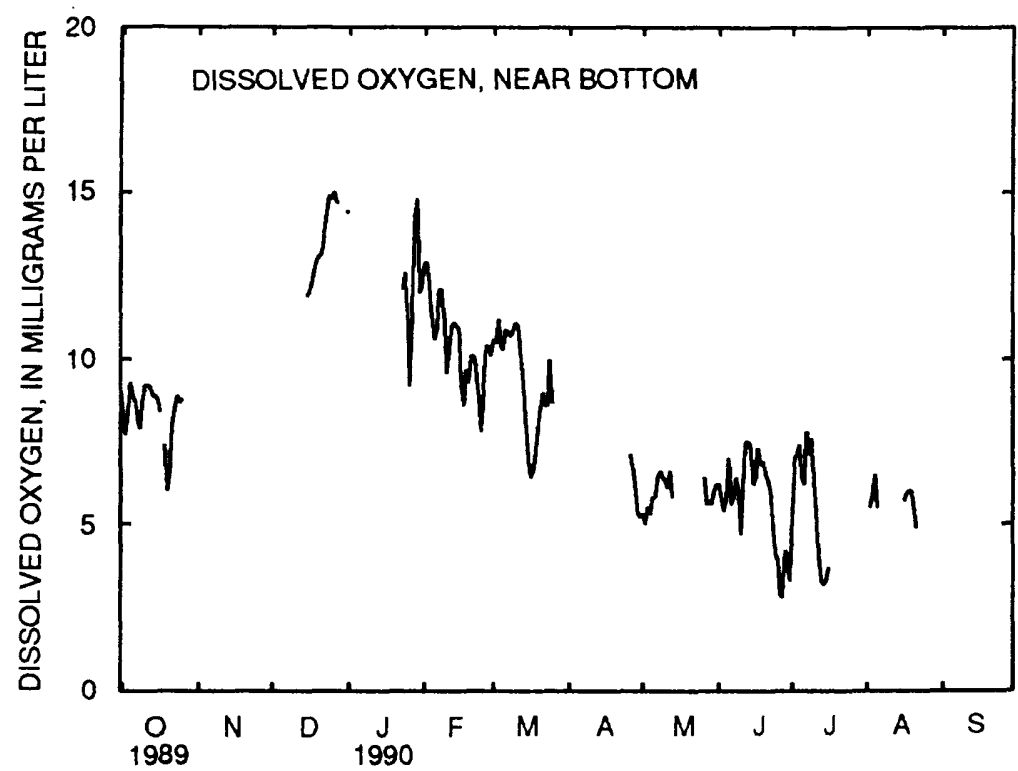


Table 13.--Daily mean values of salinity, water temperature, and dissolved oxygen in water at site 11 , Neuse River at Junction Light, May to September 1990

[Site 11 is at latitude $35^{\circ} 08^{\prime} 42^{\prime \prime}$, longltude 76 30.06", Pamlico County; U.S. Geological Survey downstream order number 0209269000 ; ppt, parts per thousand; ---, no data; ${ }^{\circ} \mathrm{C}$, degrees

Celsius; mg/L, milligrams per liter; a, dally minimum value is less than or equal to 2.0 mg/L; b, daily minimum value is less than or equal to $5.0 \mathrm{mg} / \mathrm{L}$ but greater than $2.0 \mathrm{mg} / \mathrm{L}$; <, less than]

SALINITY, NEAR SURFACE

SENSOR POSITION. -12 feet above streambed.

EXTREMES FOR MAY TO SEPTEMBER 1990.--Maximum value recorded, 17.0 ppt September 21, 1990; minimum value recorded, 10.6 ppt July 28, 1990.

DAILY MEAN VALUES OF SALINITY, NEAR SURFACE (IN PARTS PER THOUSAND), MAY TO SEPTEMBER 1990

\begin{tabular}{|c|c|c|c|c|c|}
\hline DAY & MAY & JUNE & JULY & AUG & SEPT \\
\hline 1 & -- & --- & 12.2 & 15.8 & -- \\
\hline 2 & -- & --- & 13.6 & 15.5 & -- \\
\hline 3 & --- & --- & 14.6 & 14.7 & --- \\
\hline 4 & --- & --- & 14.5 & 14.3 & $-\infty$ \\
\hline 5 & -- & --- & 14.0 & 14.7 & --- \\
\hline 6 & -- & -- & 14.1 & 15.1 & -- \\
\hline 7 & -- & -- & 13.8 & 14.1 & --- \\
\hline 8 & --- & --- & 12.9 & 13.5 & -- \\
\hline 9 & --- & --- & 13.1 & 13.7 & $\cdots$ \\
\hline 10 & -- & -- & 12.7 & 13.2 & --- \\
\hline 11 & $\ldots$ & --- & 12.7 & 12.7 & -- \\
\hline 12 & --- & --- & 12.5 & 13.5 & 16.4 \\
\hline 13 & -- & --- & 12.1 & 14.2 & 16.1 \\
\hline 14 & --- & -- & 12.2 & 14.5 & 15.7 \\
\hline 15 & -- & --- & --- & --- & 16.0 \\
\hline 16 & -- & 12.8 & 11.4 & --- & 15.6 \\
\hline 17 & -- & 12.7 & 11.7 & -- & 15.7 \\
\hline 18 & --- & 13.2 & 11.9 & --- & 14.5 \\
\hline 19 & --- & 13.4 & 12.1 & --- & 14.7 \\
\hline 20 & --- & 14.4 & 12.3 & --- & 15.7 \\
\hline 21 & -- & 14.9 & 13.4 & $-\cdots$ & 15.8 \\
\hline 22 & --- & 15.4 & 13.6 & --- & 14.2 \\
\hline 23 & --- & 15.4 & 13.9 & --- & 12.8 \\
\hline 24 & -- & 14.8 & 14.5 & --- & 11.7 \\
\hline 25 & --- & 14.0 & --- & -- & 12.2 \\
\hline 26 & --- & 12.5 & 13.3 & --- & 12.0 \\
\hline 27 & -- & 11.4 & 12.1 & --- & 12.1 \\
\hline 28 & --- & 11.5 & 11.8 & --- & 12.2 \\
\hline 29 & 12.1 & 11.8 & 12.7 & --- & 11.9 \\
\hline 30 & -- & 12.1 & 14.4 & $-\cdots$ & 11.3 \\
\hline 31 & 12.9 & --- & 14.9 & --- & -- \\
\hline
\end{tabular}

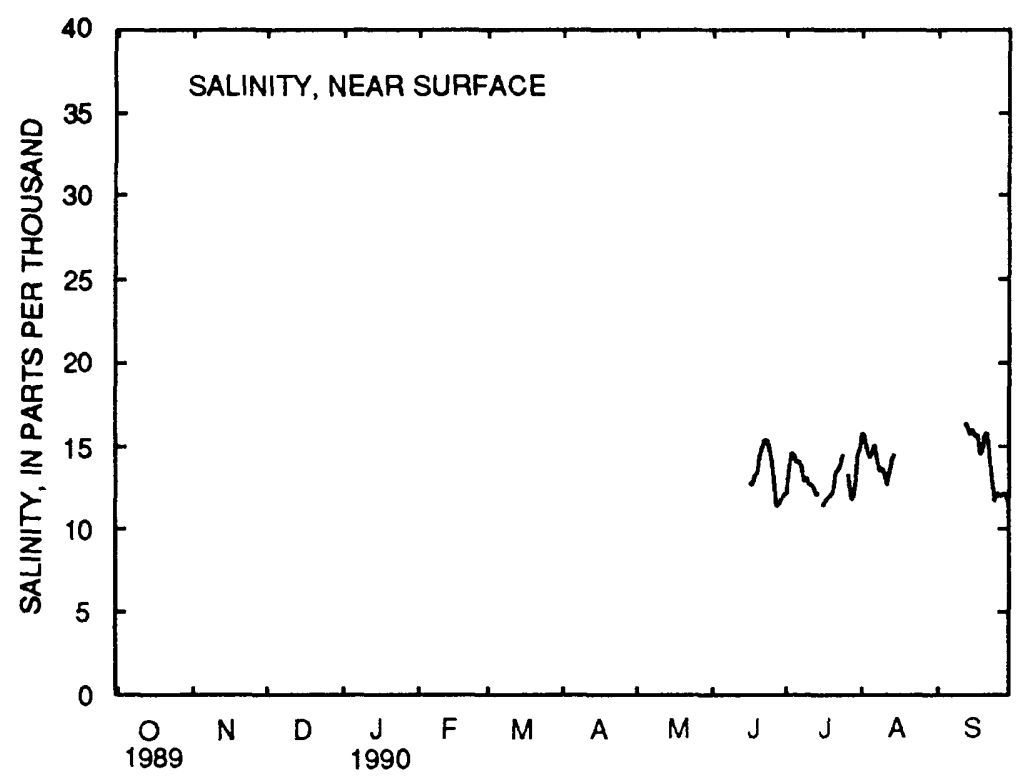

DAILY MEAN SALINITY, NEUSE RIVER AT JUNCTION LIGHT 
Table 13.--Dally mean values of salinity, water temperature, and dissolved oxygen in water at site 11, Neuse River at Junction Light, May to September 1990--Continued

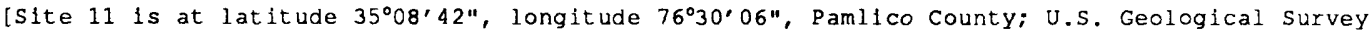
downst ream order number 0209269000 ; ppt, parts per thousand; ---, no data; ${ }^{\circ} \mathrm{C}$, degrees

Celsius; mg/L, milligrams per liter; a, dally minimum value is less than or equal to 2.0 mg/L; b, daily minlmum value is less than or equal to $5.0 \mathrm{mg} / \mathrm{L}$ but greater than $2.0 \mathrm{mg} / \mathrm{L} ;<$, less than

\section{SALINITY, NEAR BOTTOM}

SENSOR POSITION. --2 feet above streambed.

EXTREMES FOR MAY TO SEPTEMBER 1990.--Maxlmum value recorded, 20.7 ppt June 29, 1990; minimum value recorded, 10.7 ppt May 17, 1990.

DAILY MEAN VALUES OF SALINITY, NEAR BOTTOM (IN PARTS PER THOUSAND), MAY TO SEPTEMBER 1990

\begin{tabular}{|c|c|c|c|c|c|}
\hline DAY & MAY & JUNE & JULY & AUG & SEPT \\
\hline 1 & -- & -- & 14.1 & --- & -- \\
\hline 2 & $-\cdots$ & -- & 14.7 & --- & --- \\
\hline 3 & --- & --- & 14.5 & --- & --- \\
\hline 4 & --- & --- & 14.4 & -- & -- \\
\hline 5 & -- & --- & 14.3 & $-\cdots$ & --- \\
\hline 6 & --- & --- & 14.7 & --- & --- \\
\hline 7 & --- & --- & 14.4 & -- & --- \\
\hline 8 & --- & --- & 13.9 & --- & --- \\
\hline 9 & --- & --- & 13.9 & -- & -- \\
\hline 10 & --- & --- & 13.5 & --- & -- \\
\hline 11 & --- & --- & 13.7 & -- & --- \\
\hline 12 & --- & --- & 12.9 & --- & 17.6 \\
\hline 13 & --- & --- & 13.1 & -- & 17.4 \\
\hline 14 & --- & --- & 12.9 & --- & 17.1 \\
\hline 15 & --- & --- & --- & --- & 17.0 \\
\hline 16 & 11.4 & 12.8 & 12.4 & --- & 17.0 \\
\hline 17 & 11.4 & 13.1 & 13.3 & --- & 17.3 \\
\hline 18 & 12.5 & 13.2 & 12.8 & -- & 16.8 \\
\hline 19 & 13.8 & 13.7 & 13.2 & --- & 16.8 \\
\hline 20 & 13.6 & 14.7 & 14.1 & -- & 16.8 \\
\hline 21 & 13.1 & 15.8 & 15.8 & --- & 17.0 \\
\hline 22 & 13.2 & 16.1 & 14.8 & $\cdots$ & 16.8 \\
\hline 23 & 13.0 & 15.2 & 14.7 & -- & 16.9 \\
\hline 24 & 12.7 & 15.1 & 14.8 & --- & 17.3 \\
\hline 25 & --- & 15.9 & --- & --- & 17.8 \\
\hline 26 & -- & 16.6 & 14.6 & -- & 17.6 \\
\hline 27 & 12.6 & 16.9 & 14.3 & -- & 17.3 \\
\hline 28 & 13.2 & 16.2 & 14.2 & --- & 17.4 \\
\hline 29 & 13.1 & 15.5 & 14.4 & -- & 17.0 \\
\hline 30 & --- & 14.0 & 15.0 & --- & 16.8 \\
\hline 31 & 13.9 & --- & $-\cdots$ & -- & -- \\
\hline
\end{tabular}

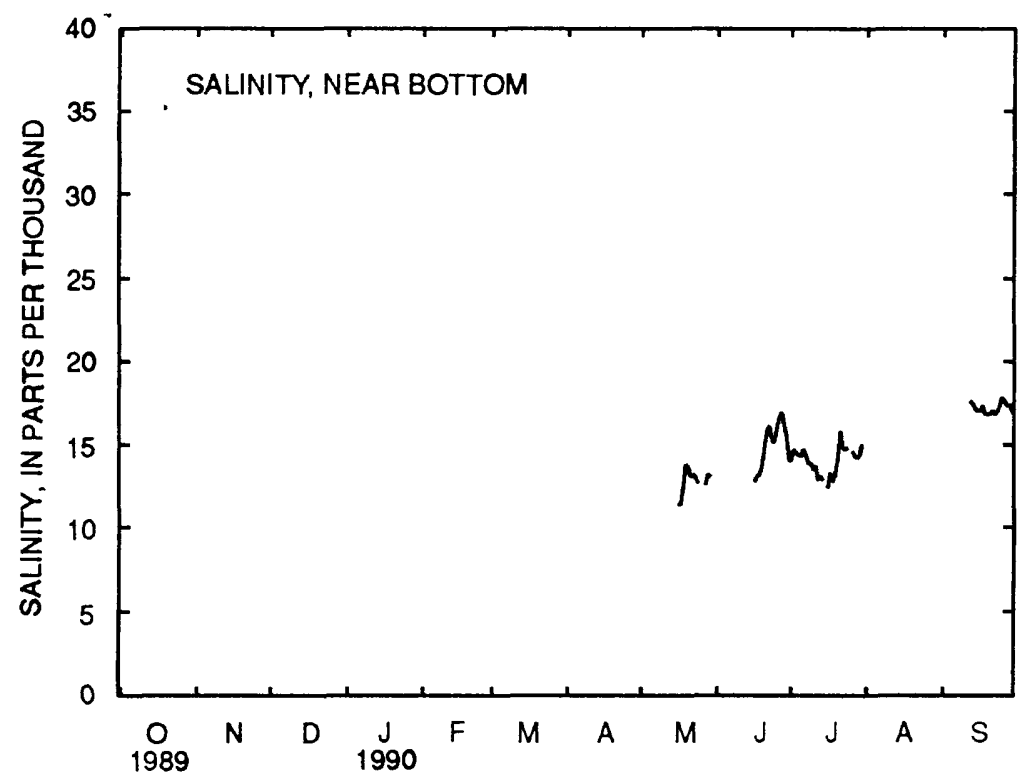

DAILY MEAN SALINITY, NEUSE RIVER AT JUNCTION LIGHT 
Table 13.--Daily mean values of salinity, water temperature, and dissolved oxygen in water at site 11 , Neuse River at Junction Light, May to september 1990--Continued

[Site 11 is at latitude $35^{\circ} 08^{\prime} 42^{\prime \prime}$, longitude $76^{\circ} 30^{\prime} 06^{\prime \prime}$, Pamlico County; U.S. Geological Survey downst ream order number 0209269000 ; ppt, parts per thousand; ---, no data; ${ }^{\circ} \mathrm{C}$, degrees

Celsius; mg/L, milligrams per liter; a, dally minimum value is less than or equal to $2.0 \mathrm{mg} / \mathrm{L}$; b, dally minimum value is less than or equal to $5.0 \mathrm{mg} / \mathrm{L}$ but greater than $2.0 \mathrm{mg} / \mathrm{L}$; <, less than]

WATER TEMPERATURE, NEAR SURFACE

SENSOR POSITION.--12 feet above st reambed.

EXTREMES FOR MAY TO SEPTEMBER 1990.--Maximum value recorded, $29.5{ }^{\circ} \mathrm{C}$ June 28,$1990 ; \mathrm{minimum}$ value recorded, 19.8 ${ }^{\circ} \mathrm{C}$ May 24, 1990.

DAILY MEAN VALUES OF WATER TEMPERATURE, NEAR SURFACE (IN DEGREES CELSIUS), MAY TO SEPTEMBER 1990

\begin{tabular}{|c|c|c|c|c|c|}
\hline DAY & MAY & JUNE & JULY & AUG & SEPT \\
\hline 1 & -- & 22.1 & -- & 28.1 & -- \\
\hline 2 & -- & 22.8 & -- & 28.1 & --- \\
\hline 3 & -- & 23.0 & --- & 27.9 & --- \\
\hline 4 & -- & 23.6 & -- & 27.9 & -- \\
\hline 5 & -- & 23.1 & --- & 27.9 & -- \\
\hline 6 & -- & 23.2 & -- & --- & -- \\
\hline 7 & --- & 23.9 & -- & -- & -- \\
\hline 8 & -- & -- & --- & --- & --- \\
\hline 9 & -- & -- & --- & --- & --- \\
\hline 10 & --- & 25.2 & --- & -- & --- \\
\hline 11 & --- & --- & --- & -- & -- \\
\hline 12 & --- & 23.9 & --- & --- & 27.0 \\
\hline 13 & -- & 23.3 & --- & -- & 27.0 \\
\hline 14 & --- & 23.4 & --- & --- & 26.8 \\
\hline 15 & --- & --- & --- & --- & 26.7 \\
\hline 16 & 22.8 & 24.4 & $--\cdots$ & --- & 26.6 \\
\hline 17 & 23.0 & 24.8 & --- & --- & 25.8 \\
\hline 18 & 22.9 & 25.6 & -- & -- & 24. \\
\hline 19 & 22.6 & 26.1 & --- & --- & 23.8 \\
\hline 20 & 22.7 & 26.5 & --- & --- & 23.8 \\
\hline 21 & 23.1 & 27.0 & -- & --- & 23.6 \\
\hline 22 & 22.6 & 27.5 & --- & --- & 23.5 \\
\hline 23 & 21.0 & 27.1 & --- & -- & 23.6 \\
\hline 24 & 20.8 & 27.3 & -- & --- & 22.8 \\
\hline 25 & --- & 27.7 & --- & --- & 22.4 \\
\hline 26 & --- & 27.4 & --- & --- & 22.6 \\
\hline 27 & 21.8 & 27.6 & --- & --- & 22.6 \\
\hline 28 & 21.5 & 28.2 & --- & --- & 22.7 \\
\hline 29 & 21.7 & --- & --- & --- & 22.8 \\
\hline 30 & -- & --- & --- & --- & 22.9 \\
\hline 31 & 21.7 & --- & --- & --- & --- \\
\hline
\end{tabular}

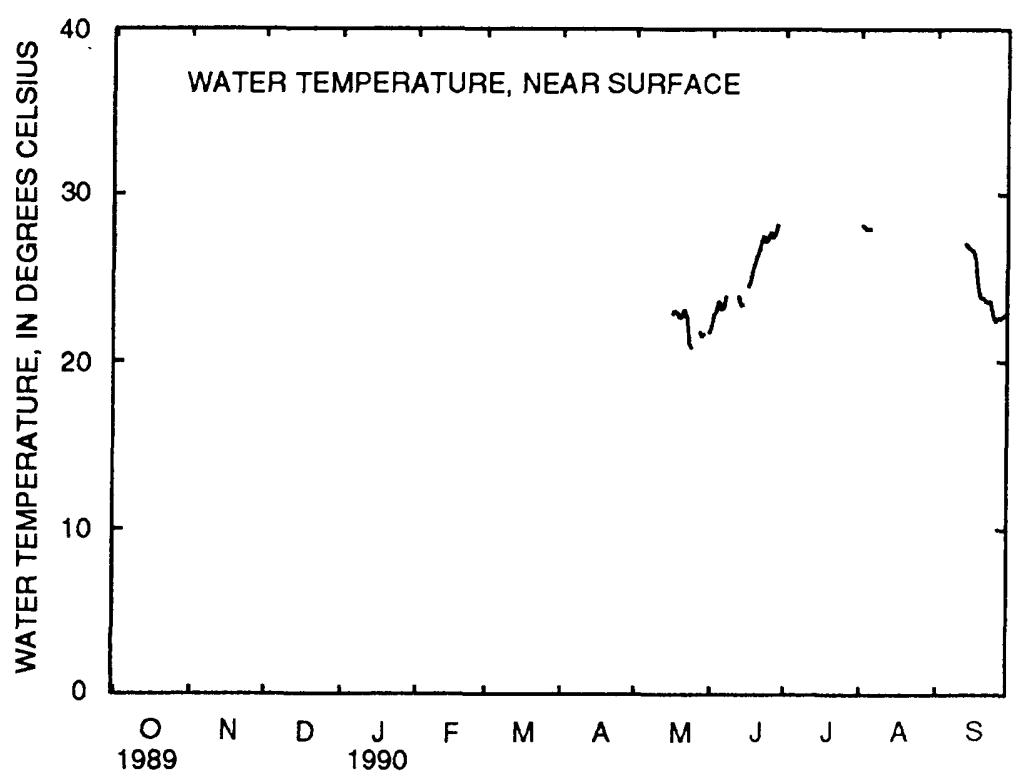


Table 13.--Dally mean values of salinity, water temperature, and dissolved oxygen in water at site 11 , Neuse River at Junction Light, May to September 1990--Continued

[Site 11 is at latitude $35^{\circ} 08^{\prime} 42^{\prime \prime}$, longitude $76^{\circ} 30^{\circ} 06^{\prime \prime}$, Pamlico County; U.S. Geological Survey downst ream order number 0209269000; ppt, parts per thousand; ---, no data; ${ }^{\circ} \mathrm{C}$, degrees

Celsius; mg/L, mllifgrams per liter; a, daily minimum value is less than or equal to $2.0 \mathrm{mg} / \mathrm{L} ; \mathrm{b}$, daily minimum value is less than or equal to $5.0 \mathrm{mg} / \mathrm{L}$ but greater than $2.0 \mathrm{mg} / \mathrm{L}$; <, less thanl

DISSOLVED OXYGEN, NEAR SURFACE

SENSOR POSITION.--12 feet above streambed.

EXTREMES FOR MAY TO SEPTEMBER 1990.--Maximum value recorded, $11.2 \mathrm{mg} / \mathrm{L}$ July 19, $1990 ; \mathrm{minimum}$ value recorded, 3.0 $\mathrm{mg} / \mathrm{L}$ JulY $12,13,1990$.

DAILY MEAN VALUES OF DISSOLVED OXYGEN, NEAR SURFACE (IN MILLIGRAMS PER LITER), MAY TO SEPTEMBER 1990

\begin{tabular}{|c|c|c|c|c|c|}
\hline DAY & MAY & JUNE & JULY & AUG & SEPT \\
\hline 1 & --- & 6.8 & --- & 6.7 & --- \\
\hline 2 & --- & 6.7 & --- & 6.4 & --- \\
\hline 3 & --- & 6.4 & --- & $6.0 \mathrm{~b}$ & --- \\
\hline 4 & --- & 6.2 & --- & $5.6 b$ & --- \\
\hline 5 & -- & 6.6 & -- & $5.6 b$ & --- \\
\hline 6 & --- & --- & $5.0 \mathrm{~b}$ & 5.4 & --- \\
\hline 7 & --- & --- & $4.3 b$ & 4.7 & --- \\
\hline 8 & --- & --- & $3.9 b$ & 4.4 & --- \\
\hline 9 & --- & --- & $4.0 \mathrm{~b}$ & 4.5 & --- \\
\hline 10 & $\cdots$ & --- & $4.1 b$ & 4.7 & $-\cdots$ \\
\hline 11 & --- & --- & $4.0 \mathrm{~b}$ & --- & --- \\
\hline 12 & --- & --- & $3.6 \mathrm{~b}$ & --- & -- \\
\hline 13 & --- & --- & $3.5 b$ & --- & --- \\
\hline 14 & -- & --- & --- & -- & --- \\
\hline 15 & --- & --- & --- & --- & --- \\
\hline 16 & --- & 7.9 & --- & $-\cdots$ & \\
\hline 17 & --- & 7.9 & -- & --- & --- \\
\hline 18 & $-\cdots$ & 8.0 & --- & --- & -- \\
\hline 19 & -- & 7.4 & 9.4 & --- & -- \\
\hline 20 & --- & 7.7 & 9.4 & -- & -- \\
\hline 21 & --- & 7.5 & 8.5 & --- & \\
\hline 22 & --- & 6.9 & 8.7 & -- & \\
\hline 23 & --- & 5.8 & 8.3 & --- & \\
\hline 24 & -- & $5.2 b$ & 7.4 & --- & \\
\hline 25 & --- & --- & --- & --- & \\
\hline 26 & --- & --- & 6.3 & --- & \\
\hline 27 & 6.4 & --- & 6.0 & --- & --- \\
\hline 28 & 6.0 & --- & 6.2 & -- & \\
\hline 29 & 6.3 & --- & 7.2 & --- & \\
\hline 30 & --- & --- & 8.0 & --- & 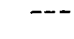 \\
\hline 31 & 6.7 & --- & 7.7 & --- & \\
\hline
\end{tabular}

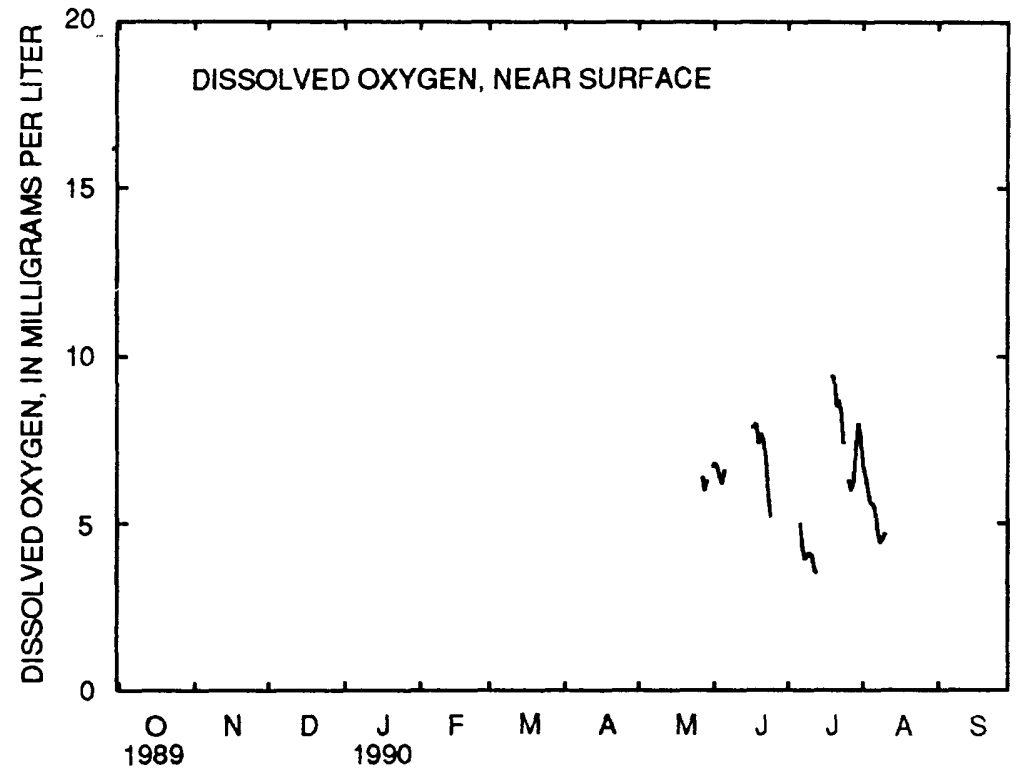


Table 13.--Daily mean values of salinity, water temperature, and dissolved oxygen in water at site 11, Neuse River at Junction Light, May to september 1990--Continued

[S1te 1 is at latitude $35^{\circ} 08^{\prime} 42^{\prime \prime}$, longltude $76^{\circ} 30^{\circ} 06^{\prime \prime}$, Pamilco County; U.S. Geological Survey downstream order number 0209269000; ppt, parts per thousand; ---, no data; ${ }^{\circ} \mathrm{C}$, degrees

Celsius; mg/L, mflligrams per liter; a, dally minimum value ls less than or equal to $2.0 \mathrm{mg} / \mathrm{L}$; b, daily minimum value is less than or equal to $5.0 \mathrm{mg} / \mathrm{L}$ but greater than $2.0 \mathrm{mg} / \mathrm{L}$; <, less than)

DISSOLVED OXYGEN, MID-DEPTH

SENSOR POSITION.--8 feet above streambed.

EXTREMES FOR MAY TO SEPTEMBER 1990.--Maximum value recorded, $11.3 \mathrm{mg} / \mathrm{L}$ July 23, $1990 ; \mathrm{mln} 1 \mathrm{mum}$ value recorded, 4.2 mg/L June 5, 1990.

DAILY MEAN VALUES OF DISSOLVED OXYGEN, MID-DEPTH (IN MILLIGRAMS PER LITER), MAY TO SEPTEMBER 1990

\begin{tabular}{|c|c|c|c|c|c|}
\hline DAY & MAY & JUNE & JULY & AUG & SEPT \\
\hline 1 & --- & 7.1 & --- & 6.4 & --- \\
\hline 2 & --- & 7.0 & --- & 6.4 & --- \\
\hline 3 & --- & 6.7 & -- & $6.0 b$ & --- \\
\hline 4 & -- & 6.1 & --- & $6.1 b$ & --- \\
\hline 5 & --- & $5.2 b$ & --- & $6.4 b$ & $-\infty$ \\
\hline 6 & --- & --- & 7.5 & 6.3 & -- \\
\hline 7 & -- & -- & 7.5 & -- & --- \\
\hline 8 & -- & --- & 7.5 & --- & --- \\
\hline 9 & --- & -- & 8.1 & --- & --- \\
\hline 10 & --- & --- & 8.1 & --- & --- \\
\hline 11 & --- & -- & 8.1 & -- & -- \\
\hline 12 & --- & --- & 7.5 & --- & 6.3 \\
\hline 13 & --- & --- & 7.4 & --- & 6.4 \\
\hline 14 & --- & -- & 7.5 & --- & $5.6 b$ \\
\hline 15 & -- & --- & --- & --- & 5.8 \\
\hline 16 & 8.0 & 7.6 & 8.0 & --- & 6.0 \\
\hline 17 & 7.4 & 7.5 & 8.5 & --- & 6.1 \\
\hline 18 & 7.5 & 7.8 & 9.3 & --- & 6.0 \\
\hline 19 & 7.7 & 7.1 & 9.6 & --- & 6.1 \\
\hline 20 & 7.5 & 7.7 & 9.7 & --- & 5.5 \\
\hline 21 & 7.1 & 7.5 & 9.0 & -- & 5.6 \\
\hline 22 & 6.8 & 7.0 & 9.1 & -- & 5.5 \\
\hline 23 & 7.3 & 6.0 & 9.5 & --- & $5.3 b$ \\
\hline 24 & 7.9 & 6.1 & 9.3 & -- & 5.8 \\
\hline 25 & -- & 7.0 & --- & --- & 6.2 \\
\hline 26 & -- & 6.1 & 8.5 & -- & 6.1 \\
\hline 27 & 6.9 & $5.5 b$ & 8.3 & --- & 5.9 \\
\hline 28 & 6.5 & 6.2 & 8.4 & --- & 5.9 \\
\hline 29 & 6.6 & --- & 8.6 & --- & 5.8 \\
\hline 30 & --- & --- & 9.0 & --- & $5.3 b$ \\
\hline 31 & $6.7 \mathrm{~b}$ & --- & 7.5 & --- & --- \\
\hline
\end{tabular}

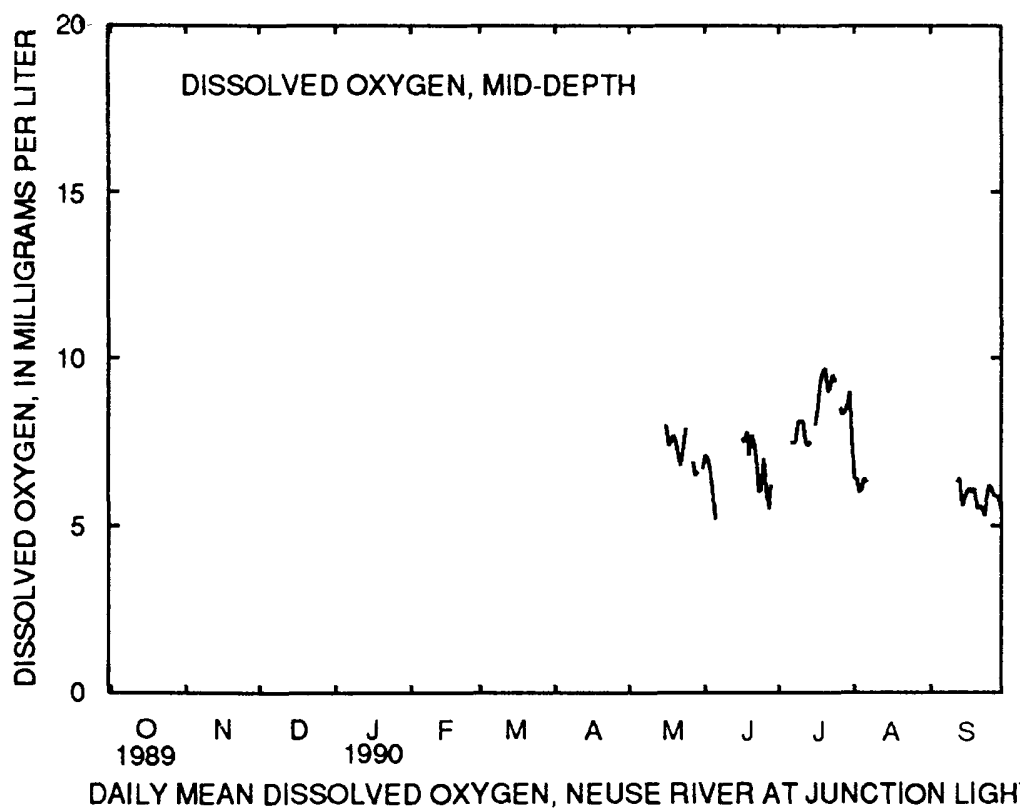


Table 13.--Daily mean values of salinity, water temperature, and dissolved oxygen in water at site 11 , Neuse River at Junction Light, May to September 1990--Continued

[Site 11 is at latitude $35^{\circ} 08^{\prime} 42^{\prime \prime}$, longitude $76^{\circ} 30^{\prime} 06^{\prime \prime}$, Pamlico County; U.S. Geological Survey downst ream order number 0209269000; ppt, parts per thousand; - - , no data; ${ }^{\circ} \mathrm{C}$, degrees

Celsius; mg/L, milligrams per liter; a, daily minimum value is less than or equal to $2.0 \mathrm{mg} / \mathrm{L}$; b, daily minimum value is less than or equal to $5.0 \mathrm{mg} / \mathrm{L}$ but greater than $2.0 \mathrm{mg} / \mathrm{L}$; <, less than]

DISSOLVED OXYGEN, NEAR BOTTOM

SENSOR POSITION. --2 feet above streambed.

EXTREMES FOR MAY TO SEPTEMBER 1990.--Maximum value recorded, 14.8 mg/L June 16, 1990; m1n1mum value recorded, $<1.0 \mathrm{mg} / \mathrm{L}$ May 20, July 21, 27, 1990.

DAILY MEAN VALUES OF DISSOLVED OXYGEN, NEAR BOTTOM (IN MILLIGRAMS PER LITER), MAY TO SEPTEMBER 1990

\begin{tabular}{|c|c|c|c|c|c|}
\hline DAY & MAY & JUNE & JULY & AUG & SEPT \\
\hline 1 & -- & -- & -- & $6.5 b$ & --- \\
\hline 2 & $m$ & -- & -- & $8.0 b$ & -- \\
\hline 3 & -- & --- & --- & $6.0 \mathrm{~b}$ & --- \\
\hline 4 & -- & --- & --- & $5.3 b$ & --- \\
\hline 5 & -- & $-\cdots$ & -- & $5.4 a$ & --- \\
\hline 6 & --- & -- & $6.3 b$ & 5.7 & --- \\
\hline 7 & -- & -- & $6.7 \mathrm{~b}$ & $5.2 b$ & -- \\
\hline 8 & --- & --- & 6.2 & $6.9 b$ & -- \\
\hline 9 & -- & --- & 6.6 & $7.5 b$ & --- \\
\hline 10 & -- & --- & 7.2 & $5.9 b$ & --- \\
\hline 11 & -- & --- & $6.8 b$ & -- & --- \\
\hline 12 & --- & --- & 7.7 & --- & 6.0 \\
\hline 13 & -- & -- & 7.5 & --- & 5.7 \\
\hline 14 & --- & $-\infty$ & 7.5 & -- & $4.6 a$ \\
\hline 15 & --- & --- & --- & --- & $4.8 \mathrm{~b}$ \\
\hline 16 & $5.4 b$ & 8.2 & 8.1 & $\ldots$ & $4.5 b$ \\
\hline 17 & $5.7 b$ & 7.6 & 8.2 & --- & $5.1 b$ \\
\hline 18 & $5.0 \mathrm{~b}$ & $5.9 \mathrm{~b}$ & $7.8 b$ & -- & $5.2 b$ \\
\hline 19 & $5.7 a$ & $6.0 \mathrm{~b}$ & $8.7 b$ & -- & $5.0 \mathrm{~b}$ \\
\hline 20 & $5.1 \mathrm{~b}$ & $6.4 \mathrm{~b}$ & $8.7 b$ & -- & $4.8 \mathrm{~b}$ \\
\hline 21 & $5.3 b$ & $5.0 \mathrm{~b}$ & $6.0 \mathrm{~b}$ & --- & $4.8 b$ \\
\hline 22 & $5.2 b$ & $4.1 \mathrm{~b}$ & $8.4 a$ & -- & $4.3 b$ \\
\hline 23 & 5.3 & $4.9 b$ & $9.2 b$ & -- & $4.1 \mathrm{~b}$ \\
\hline 24 & $4.9 b$ & $5.0 \mathrm{~b}$ & 10.8 & $\cdots$ & $4.5 b$ \\
\hline 25 & -- & $5.4 b$ & --- & --- & $4.5 b$ \\
\hline 26 & --- & --- & 7.9 & -- & $4.4 b$ \\
\hline 27 & $5.3 b$ & --- & $5.0 \mathrm{~b}$ & $\cdots$ & $3.7 a$ \\
\hline 28 & $4.8 b$ & --- & $6.3 b$ & +- & $4.4 b$ \\
\hline 29 & $4.0 \mathrm{~b}$ & -- & $6.1 \mathrm{~b}$ & -- & $4.6 b$ \\
\hline 30 & --- & --- & $5.6 a$ & --- & $3.9 b$ \\
\hline 31 & $3.1 \mathrm{a}$ & --- & $6.1 \mathrm{~b}$ & -- & --- \\
\hline
\end{tabular}

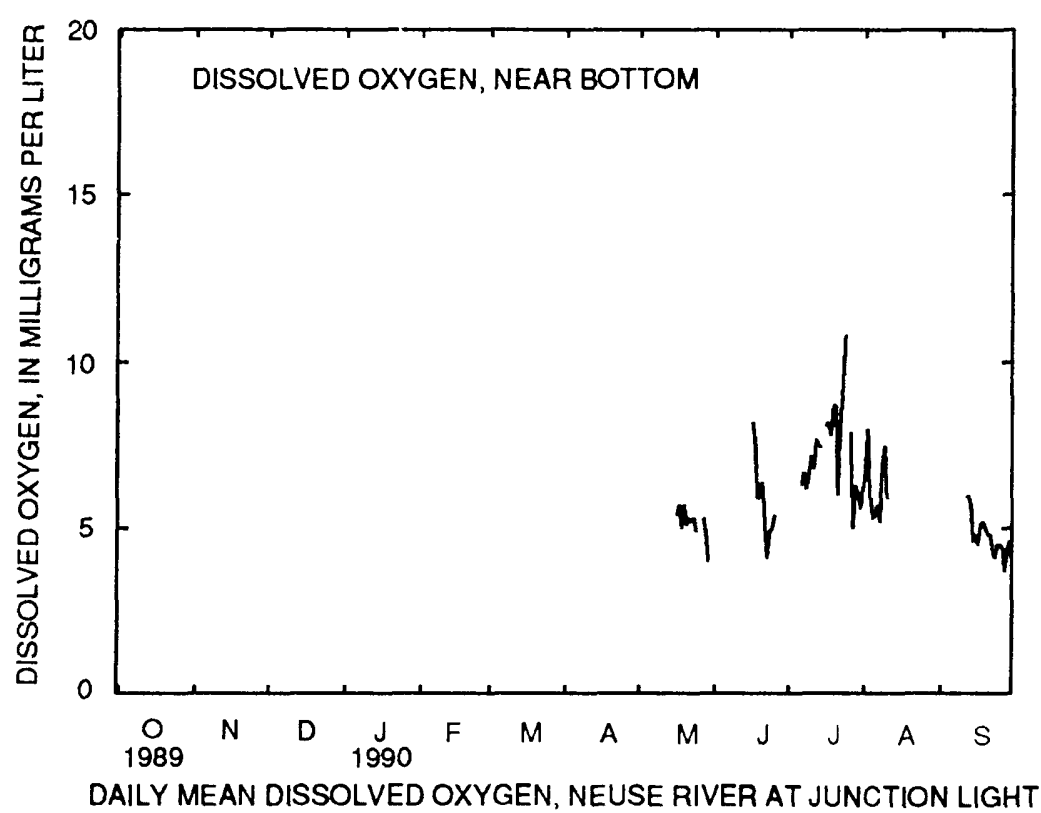




\section{REFERENCES}

Bales, J.D., 1990, Data-collection program for Pamlico River estuary model calibration and validation in Spaulding, M.L., ed., Estuarine and Coastal Modeling: New York, American Society of Civil Engineers, p. 492- 501 .

Bellis, V., O'Connor, M.P., and Riggs, S.R., 1975, Estuarine shoreline erosion in the Albemarle-Pamlico region of North Carolina: Raleigh, University of North Carolina Sea Grant College Program, North Carolina State University, Report No. UNC-SG-75-29, 67 p.

Campbe11 Scientific, Inc., 1988, CR10 measurement and control module operator's manual: Logan, Utah.

1987, SM192/SM176 storage module preliminary instruction manual: Logan, Utah.

Copeland, B.J., Hodson, R.G., Riggs, S.R., and Pendleton, E.C., 1984, The ecology of the Pamlico River, North Carolina--an estuarine profile: Washington, D.C., U.S. Fish and Wildlife Service, Report No. FWS/OBS$82 / 06,83 \mathrm{p}$.

Ficken, J.H., and Scott, C.T., 1989, Operating manual for the U.S. Geological Survey minimonitor, 1988 edition, analog-voltage model: U.S. Geological Survey Open-File Report 89-403, 92 p.

Giese, G.L., Wilder, H.B., and Parker, G.G., 1985, Hydrology of major estuaries and sounds of North Carolina: U.S. Geological Survey WaterSupply Paper 2221, 108 p.

Gordon, A.B., and Katzenbach, M., 1983, Guidelines for use of water quality monitors: U.S. Geological Survey Open-File Report 83-681, 94 p.

Hardy, A.V., and Hardy, J.D., 1971, Weather and climate in North Carolina: Raleigh, Agricultural Experiment Station, North Carolina State University, Bulletin 396. 
Harned, D.A., and Davenport, M.S., 1990, Water-quality trends and basin activities and characteristics for the Albemarle-Pamiico estuarine system, North Carolina and Virginia: U.S. Geological Survey Open-File Report 90-398, $164 \mathrm{p}$.

Holland, A.F., 1990, Near coastal program plan for 1990--estuaries: Narragansett, Rhode Island, Environmental Research Laboratory, U.S. Environmenta1 Protection Agency, Report No. EPA/600/4-90/033.

Holman, R.E., 1988 (revised 1989), Albemarle-Pamlico estuarine study baseline water quality monitoring plan: Raleigh, North Carolina Department of Environment, Health, and Natural Resources, Project No. $88-01 / 02,32$ p.

Martin Marietta Environmental Systems, 1987a, Chesapeake Bay mainstem monitoring program statistical and analytical support contract--final report, volume I: Annapolis, Maryland, Chesapeake Bay Program, Report No. CBP/TRS $12 / 87,49 \mathrm{p}$.

1987b, Chesapeake Bay mainstem monitoring program statistical and analytical support contract--final report, volume II: Annapolis, Maryland, Report No. CBP/TRS 13/87, 535 p.

Miller, R.L., Bradford, W.L., and Peters, N.E., 1988, Specific conductance-theoretical considerations and application to analytical quality control: U.S. Geological Survey Water-Supply Paper 2311, 16 p.

National Academy of Sciences, 1977, Environmental monitoring--a report to the U.S. Environmental Protection Agency: Washington, D.C., 181 p.

National Research Council, 1990, Managing troubled waters--the role of marine environmental monitoring: Washington, D.C., National Academy Press, Committee on a Systems Assessment of Marine Environmental Monitoring, Marine Board, Commission on Engineering and Technical Systems, $125 \mathrm{p}$. 
Noga, E.J., and Stanley, D.W., 1989, Determining the relationship between water quality and ulcerative mycosis in Atlantic menhaden in Holman, R.E., ed., Project abstracts for the period 1989-1990: Raleigh, Albemarle-Pamlico Estuarine Study, North Carolina Department of Environment, Health, and Natural Resources, Project No. 89/08, p. 30.

Pearce, J.B., and Despres-Patanjo, L., 1988, A review of monitoring strategies and assessments of estuarine pollution: Aquatic Toxicology, v. 11, p. $323-343$.

We11s, J.T., 1989, A scoping study of the distribution, composition, and dynamics of water-column and bottom sediments--Albemarle-Pamlico estuarine system: Raleigh, Albemarle-Pamlico Estuarine Study, North Carolina Department of Natural Resources and Community Development, Project No. 89-05, 39 p.

Wilder, H.B., Robison, T.M., and Lindskov, K.L., 1978, Water resources of northeast North Carolina: U.S. Geological Survey Water-Resources Investigations $77-81,113 \mathrm{p}$. 